\title{
Evaluating the Intra-variability and Inter-variability of Fibers in Cotton T-shirts using Microspectrophotometry
}

Brianne Miller

West Virginia University

Follow this and additional works at: https://researchrepository.wvu.edu/etd

\section{Recommended Citation}

Miller, Brianne, "Evaluating the Intra-variability and Inter-variability of Fibers in Cotton T-shirts using Microspectrophotometry" (2013). Graduate Theses, Dissertations, and Problem Reports. 557.

https://researchrepository.wvu.edu/etd/557

This Thesis is protected by copyright and/or related rights. It has been brought to you by the The Research Repository @ WVU with permission from the rights-holder(s). You are free to use this Thesis in any way that is permitted by the copyright and related rights legislation that applies to your use. For other uses you must obtain permission from the rights-holder(s) directly, unless additional rights are indicated by a Creative Commons license in the record and/ or on the work itself. This Thesis has been accepted for inclusion in WVU Graduate Theses, Dissertations, and Problem Reports collection by an authorized administrator of The Research Repository @ WVU. For more information, please contact researchrepository@mail.wvu.edu. 
Evaluating the Intra-variability and Inter-variability of Fibers in Cotton T-shirts using Microspectrophotometry

\author{
Brianne Miller
}

\begin{abstract}
Thesis submitted to the Eberly College of Arts and Sciences

at West Virginia University in partial fulfillment of the requirements for the degree of
\end{abstract}

\author{
Master of Science \\ in \\ Forensic and Investigative Science \\ Keith Morris, Ph.D., Chair \\ Patrick Buzzini, Ph.D. \\ Suzanne Bell, Ph.D.
}

Department of Forensic and Investigative Science

Morgantown, West Virginia

2013

Keywords: Fibers, cotton, Colorimetry, Microspectrophotmetry 


\title{
ABSTRACT \\ Evaluating the Intra-variability and Inter-variability of Fibers in Cotton T-shirts using Microspectrophotometry
}

\author{
Brianne Miller
}

The objective of this study was to evaluate and compare the differentiation of cotton fibers by the means of intra-garment and inter-garment comparison while utilizing Microspectrophotometry. Five different colors of cotton fibers were used: gold, kelly green, orange, red and royal. Ten tshirts of each color were used in this study. Phase I of this study involved using the CRAIC ® QDI QDS IITM Stereomicrospectrophotometer to produce a spectrum of the front, back, sleeve and armpit of the t-shirt. These sections were then compared to the color chips in the Munsell Book of Color by observing them with the naked eye. Phase II of this study involved varying the boxcar width, the average scans and the integration time to find the optimal settings that should be used when collecting spectra on the CRAIC® QDI 1000 Microspectrophotometer. A time trial was completed during Part 1 and Part 2 of Phase III to determine the proper time the instrument's lamp should be allowed to warm-up prior to collecting spectra of samples. Phase IV of this study involved preparing 200 slides of fibers from the front, back, armpit and sleeve of each color repeated for all ten t-shirts to allow for the intra-garment comparison. Phase V used the front section of each t-shirt and a fiber from that section was cut into three different sections providing 150 slides to demonstration the inter-garment comparison. To conclude the study, the five colors used in this study were compared to each other to determine if they could be differentiated. 


\section{Acknowledgements}

I would like to thank Dr. Keith Morris, Dr. Patrick Buzzini, and Dr. Suzanne Bell for all of their help and time that they have dedicated to this thesis research. I also would like to thank them for helping me achieve my goal of receiving my Master's degree here at West Virginia University. I would especially like to thank my parents, Scott and Vickie Miller. Without their encouragement and inspiration I would have never been able to accomplish my goals and dreams. 


\section{Table of Contents}

Title Page

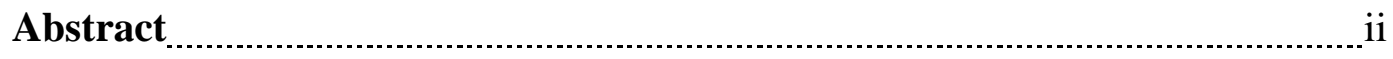

Acknowledgements

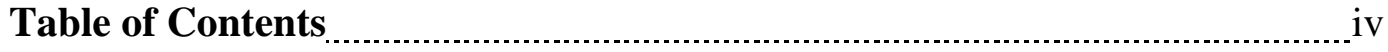

List of Figures

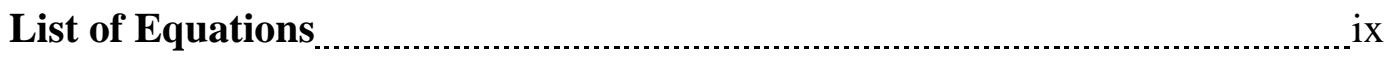

List of Tables $\ldots \ldots \ldots$

List of Photos

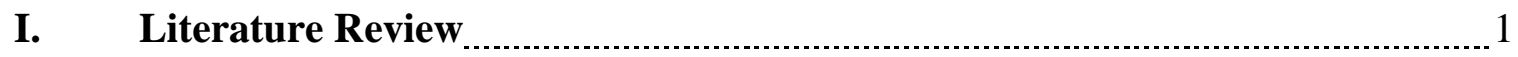

II. Materials/Methods

Materials

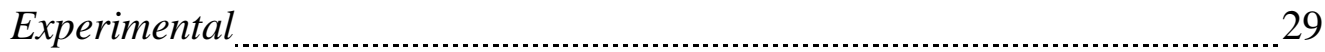

III. Results 33

IV. Discussion 122

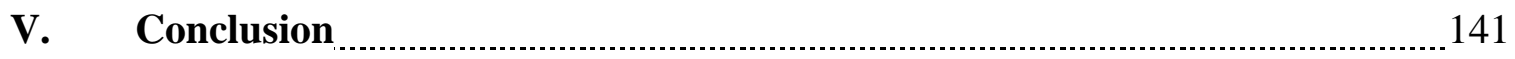

VI. Future Research

VII. References

VIII. Appendix A - Figures $\ldots$

IX. Appendix B - Data Tables $\quad 179$ 


\section{List of Figures}

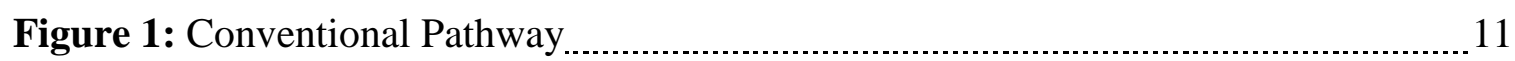

Figure 2: Alternative Pathway

Figure 3: Dark scan using the CRAIC® QDI QDS Stereomicrospectrophotometer $\ldots . . . . .18$

Figure 4: Reference scans using the CRAIC® QDI QDS

Stereomicrospectrophotometer 18

Figure 5: Dark scan using the CRAIC® QDI 1000 Microspectrophotometer _................ 20

Figure 6: Reference scan using the CRAIC@ QDI 1000 Microspectrophotometer _........ 20

Figure 7: Didymium filter on the CRAIC® QDI QDS

Stereomicrospectrophotomer $\ldots$

Figure 8: Holmium filter on the CRAIC® QDI QDS

Stereomicrospectrophotometer 22

Figure 9: ND 0.1filter on the CRAIC@ QDI QDS Stereomicrospectrophotometer _...... 22

Figure 10: ND 0.5 filter on the CRAIC@ QDI QDS

Stereomicrospectrophotomeer

Figure 11: ND 1.0 filter on the CRAIC® QDI QDS

Stereomicrospectrophotometer

Figure 12: Holmium oxide filter on the CRAIC® QDI 1000

Microspectrophotometer 26

Figure 13: Didymium filter on the CRAIC® QDI 1000 Microspectrophotometer ........ 26

Figure 14: ND 0.1 filter on the CRAIC® QDI 1000 Microspectrophotometer _............ 27

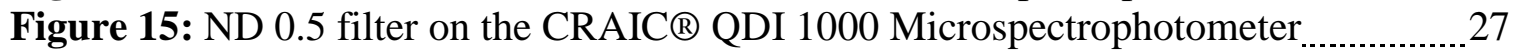

Figure 16: ND 1.0 filter on the CRAIC® QDI 1000 Microspectrophotometer ...............28

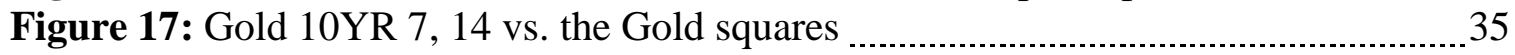

Figure 18: Kelly Green 7.5G 4, 8 vs. the Kelly Green squares .................................... 37

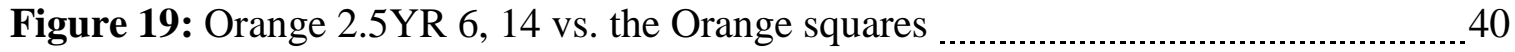

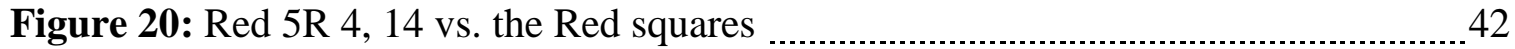

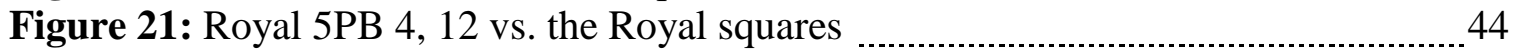

Figure 22: Average scan variation of Kelly Green A_F $\ldots$

Figure 23: Average scan variation of Kelly Green A_F using scans 10, 15, $20 \ldots \ldots \ldots \ldots$

Figure 24: Least squared absorbance values for each average scan variation …............. 47

Figure 25: The sum of the squares for each average scan variation $\ldots$

Figure 26: Boxcar width variation of Kelly Green A_F

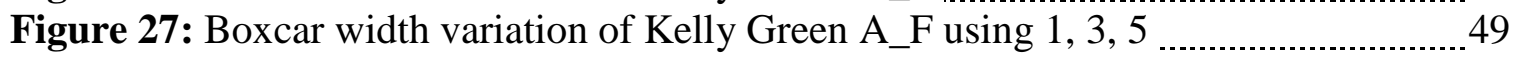

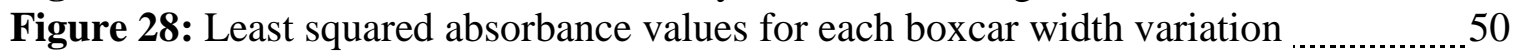

Figure 29: The sum of the squares for each boxcar width variation $\quad 50$

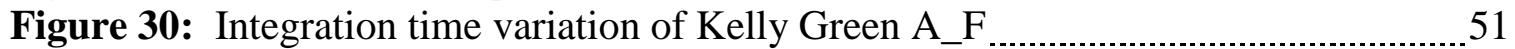

Figure 31: Integration time variation of Kelly Green A_F using 20, 30, 50, $100 \ldots \ldots \ldots . . . .52$

Figure 32: Least squared absorbance values for each integration time variation .................. 53

Figure 33: The sum of the squares for each integration time variation …........................ 53

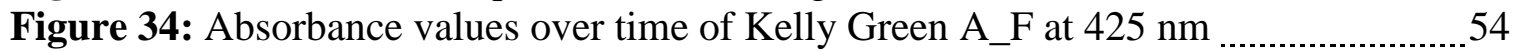

Figure 35: Absorbance values over time of Kelly Green A_F at $520 \mathrm{~nm} \ldots \ldots \ldots \ldots \ldots \ldots . . . . . . . . . . . . .55$

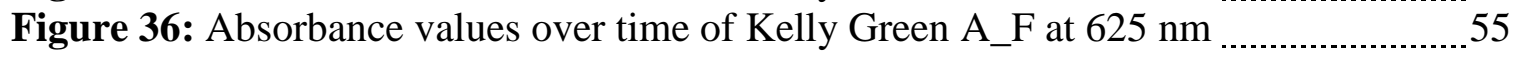

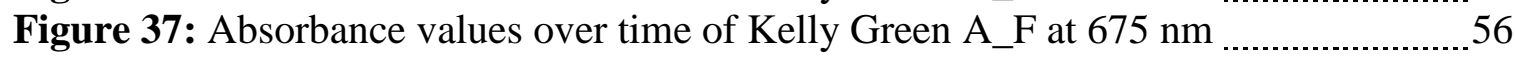

Figure 38: Absorbance values over time of Kelly Green A_F at $700 \mathrm{~nm}$....................... 56 
Figure 39: Absorbance values over time of Kelly Green A_ every 10 minutes _............. 57

Figure 40: Absorbance values over time of Kelly Green A_F at $425 \mathrm{~nm} \ldots \ldots \ldots \ldots . . . . . . . . . . . . . . . . . . . .58$

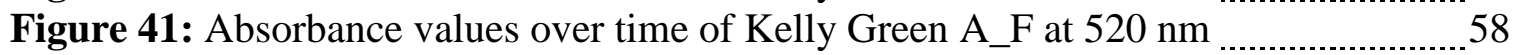

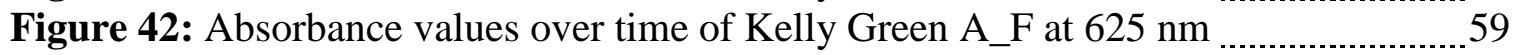

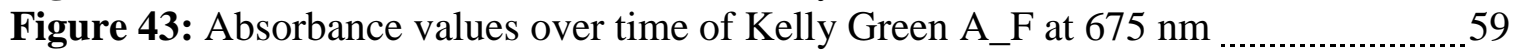

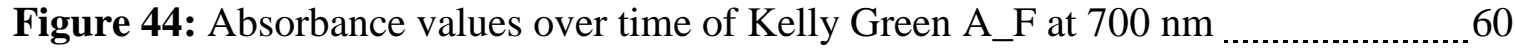

Figure 45: Absorbance values over time of Kelly Green A_F every 10 minutes _........... 60

Figure 46: Spectra of Gold A-J shirts for armpit, back, front and sleeve for all ten

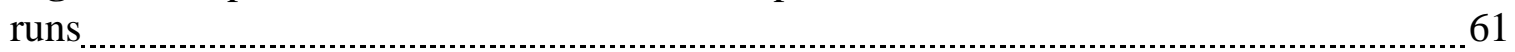

Figure 47: Spectra of the armpit, back, front and sleeve for all Gold A-J shirts $\ldots . . . \ldots . . . . . .62$

Figure 48: Spectra of average absorbance, UCL and LCL for all 4 regions of

Gold A Shirts

Figure 49: Spectra of average absorbance, UCL and LCL for all 4 regions of

Gold B Shirts

Figure 50: Spectra of average absorbance, UCL and LCL for every Gold Shirt $\ldots . . . . . . . . . . .63$

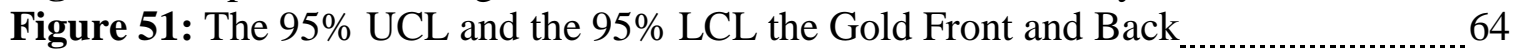

Figure 52: The 95\% UCL and the 95\% LCL the Gold Front and Sleeve ......................... 64

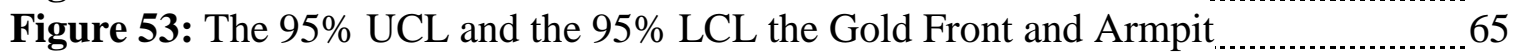

Figure 54: The 95\% UCL and the 95\% LCL the Gold Back and Sleeve _.......................... 65

Figure 55: The 95\% UCL and the 95\% LCL the Gold Back and Armpit _....................... 66

Figure 56: The 95\% UCL and the 95\% LCL the Gold Sleeve and Armpit _.................. 66

Figure 57: Spectra of Kelly Green A-J shirts for armpit, back, front and sleeve for all ten runs

Figure 58: Spectra of the armpit, back, front and sleeve for all Kelly Green A-

J7shirts

Figure 59: Spectra of average absorbance, UCL and LCL for all 4 regions of

Kelly Green C Shirts

Figure 60: Spectra of average absorbance, UCL and LCL for all 4 regions of

Kelly Green D Shirts

Figure 61: Spectra of average absorbance, UCL and LCL for every Kelly Green

Shirt 69

Figure 62: The 95\% UCL and the 95\% LCL the Kelly Green Front and Back 70

Figure 63: The 95\% UCL and the 95\% LCL the Kelly Green Front and Sleeve 70

Figure 64: The 95\% UCL and the 95\% LCL the Kelly Green Front and Armpit

Figure 65: The 95\% UCL and the 95\% LCL the Kelly Green Back and Sleeve

Figure 66: The 95\% UCL and the 95\% LCL the Kelly Green Back and Armpit 71

Figure 67: The 95\% UCL and the 95\% LCL the Kelly Green Sleeve and Armpit ............ 72

Figure 68: Spectra of Orange A-J shirts for armpit, back, front and sleeve for all

ten runs 73

Figure 69: Spectra of the armpit, back, front and sleeve for all Orange A-J shirts _......... 74

Figure 70: Spectra of average absorbance, UCL and LCL for all 4 regions of Orange E Shirts

Figure 71: Spectra of average absorbance, UCL and LCL for all 4 regions of Orange F Shirts 75

Figure 72: Spectra of average absorbance, UCL and LCL for every Orange Shirt _........ 75

Figure 73: The 95\% UCL and the 95\% LCL the Orange Front and Back 
Figure 74: The 95\% UCL and the 95\% LCL the Orange Front and Sleeve 76

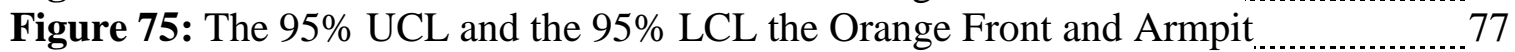

Figure 76: The 95\% UCL and the 95\% LCL the Orange Back and Sleeve _.................. 77

Figure 77: The 95\% UCL and the 95\% LCL the Orange Back and Armpit _........................ 78

Figure 78: The 95\% UCL and the 95\% LCL the Orange Sleeve and Armpit ................ 78

Figure 79: Spectra of Red A-J shirts for armpit, back, front and sleeve for all ten runs

Figure 80: Spectra of the armpit, back, front and sleeve for all Red A-J shirts $\quad 80$

Figure 81: Spectra of average absorbance, UCL and LCL for all 4 regions of Red

G Shirts

Figure 82: Spectra of average absorbance, UCL and LCL for all 4 regions of Red H Shirts

Figure 83: Spectra of average absorbance, UCL and LCL for every Red Shirt $\ldots . . . . . . . . . . . .81$

Figure 84: The 95\% UCL and the 95\% LCL the Red Front and Back _.................................. 82

Figure 85: The 95\% UCL and the 95\% LCL the Red Front and Sleeve _.......................... 82

Figure 86: The 95\% UCL and the 95\% LCL the Red Front and Armpit _....................... 83

Figure 87: The 95\% UCL and the 95\% LCL the Red Back and Sleeve _....................... 83

Figure 88: The 95\% UCL and the 95\% LCL the Red Back and Armpit ........................... 84

Figure 89: The 95\% UCL and the 95\% LCL the Red Sleeve and Armpit _..................... 84

Figure 90: Spectra of Royal A-J shirts for armpit, back, front and sleeve for all

ten runs

Figure 91: Spectra of the armpit, back, front and sleeve for all Royal A-J shirts 86

Figure 92: Spectra of average absorbance, UCL and LCL for all 4 regions of

Royal I Shirts

Figure 93: Spectra of average absorbance, UCL and LCL for all 4 regions of

Royal J Shirts

Figure 94: Spectra of average absorbance, UCL and LCL for every Royal Shirt _.............87

Figure 95: The 95\% UCL and the 95\% LCL the Royal Front and Back _.....................................

Figure 96: The 95\% UCL and the 95\% LCL the Royal Front and Sleeve _..................... 88

Figure 97: The 95\% UCL and the 95\% LCL the Royal Front and Armpit _.................. 89

Figure 98: The 95\% UCL and the 95\% LCL the Royal Back and Sleeve _.................... 89

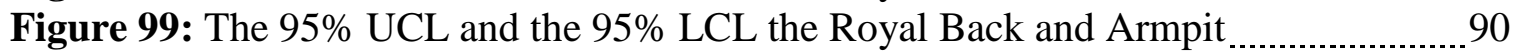

Figure 100: The 95\% UCL and the 95\% LCL the Royal Sleeve and Armpit _................90

Figure 101: Spectra of Gold A-J shirts for section1, 2 and 3 for all ten runs _................. 92

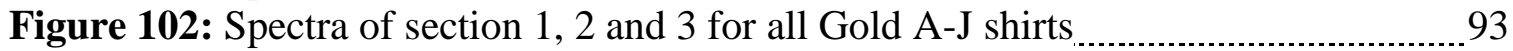

Figure 103: Spectra of average absorbance, UCL and LCL for all 3 regions of

Gold I Shirts

Figure 104: Spectra of average absorbance, UCL and LCL for all 3 regions of

Gold J Shirts

Figure 105: Spectra of average absorbance, UCL and LCL for every Gold Shirt

Figure 106: The 95\% UCL and the 95\% LCL of Gold Section 1 and Section $2 \ldots \ldots \ldots . . . .95$

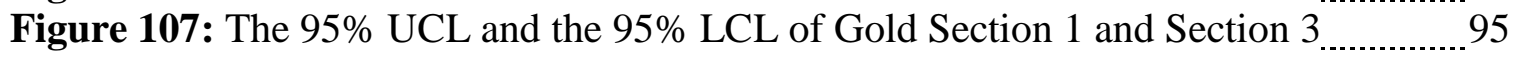

Figure 108: The 95\% UCL and the 95\% LCL of Gold Section 2 and Section $3 \ldots \ldots . . . . . .96$

Figure 109: Spectra of Kelly Green A-J shirts for section1, 2 and 3 for all ten runs

Figure 110: Spectra of section 1, 2 and 3 for all Kelly Green A-J shirts _....................... 98 
Figure 111: Spectra of average absorbance, UCL and LCL for all 3 regions of Kelly Green G Shirts

Figure 112: Spectra of average absorbance, UCL and LCL for all 3 regions of Kelly Green H Shirts

Figure 113: Spectra of average absorbance, UCL and LCL for every Kelly Green

Shirt

Figure 114: The 95\% UCL and the 95\% LCL of Kelly Green Section 1 and Section 2 100

Figure 115: The 95\% UCL and the 95\% LCL of Kelly Green Section 1 and Section 3

Figure 116: The 95\% UCL and the 95\% LCL of Kelly Green Section 2 and Section 3 101

Figure 117: Spectra of Orange A-J shirts for section 1, 2 and 3 for all ten runs 102

Figure 118: Spectra of section 1, 2 and 3 for all Orange A-J shirts 103

Figure 119: Spectra of average absorbance, UCL and LCL for all 3 regions of Orange E Shirts

Figure 120: Spectra of average absorbance, UCL and LCL for all 3 regions of Orange F Shirts 104

Figure 121: Spectra of average absorbance, UCL and LCL for every Orange Shirt ..... 104 Figure 122: The 95\% UCL and the 95\% LCL of Orange Section 1 and Section 2 _...... 105 Figure 123: The 95\% UCL and the 95\% LCL of Orange Section 1 and Section $3 \ldots \ldots . . . .105$ Figure 124: The 95\% UCL and the 95\% LCL of Orange Section 2 and Section $3 \ldots 106$ Figure 125: Spectra of Red A-J shirts for section1, 2 and 3 for all ten runs ................... 107 Figure 126: Spectra of section 1, 2 and 3 for all Red A-J shirts $\ldots \ldots$ Figure 127: Spectra of average absorbance, UCL and LCL for all 3 regions of Red C Shirts 108

Figure 128: Spectra of average absorbance, UCL and LCL for all 3 regions of Red D Shirts 109

Figure 129: Spectra of average absorbance, UCL and LCL for every Red Shirt Figure 130: The 95\% UCL and the 95\% LCL of Red Section 1 and Section 2 Figure 131: The 95\% UCL and the 95\% LCL of Red Section 1 and Section 3 Figure 132: The 95\% UCL and the 95\% LCL of Red Section 2 and Section 3 Figure 133: Spectra of Royal A-J shirts for section1, 2 and 3 for all ten runs Figure 134: Spectra of section 1, 2 and 3 for all Royal A-J shirts

Figure 135: Spectra of average absorbance, UCL and LCL for all 3 regions of Royal A Shirts

Figure 136: Spectra of average absorbance, UCL and LCL for all 3 regions of Royal B Shirts

Figure 137: Spectra of average absorbance, UCL and LCL for every Royal Shirt

Figure 138: The 95\% UCL and the 95\% LCL of Royal Section 1 and Section 2

Figure 139: The 95\% UCL and the 95\% LCL of Royal Section 1 and Section 3

Figure 140: The 95\% UCL and the 95\% LCL of Royal Section 2 and Section 3

Figure 141: The 95\% UCL and the 95\% LCL of all Orange and Kelly Green shirts

Figure 142: The 95\% UCL and the 95\% LCL of all Orange and Red shirts 
Figure 144: The 95\% UCL and the 95\% LCL of all Orange and Royal shirts 119

Figure 145: The 95\% UCL and the 95\% LCL of all Kelly Green and Red shirts 119

Figure 146: The 95\% UCL and the 95\% LCL of all Kelly Green and Gold shirts ....... 120

Figure 147: The 95\% UCL and the 95\% LCL of all Kelly Green and Royal shirts ....... 120

Figure 148: The 95\% UCL and the 95\% LCL of all Red and Gold shirts .................... 121

Figure 149: The 95\% UCL and the 95\% LCL all Red and Royal shirts ....................... 121

Figure 150: The 95\% UCL and the 95\% LCL of all Royal and Gold shirts ................ 122

Figure 151: Absorbance Spectrum of Kelly Green A_F using average scan of 5

Figure 152: Absorbance Spectrum of Kelly Green A_F using average scan of $20 \ldots \ldots \ldots 127$

Figure 153: Absorbance Spectrum of Kelly Green A_F using average scan of 50 _....... 127

Figure 154: Absorbance Spectrum of Kelly Green A_F using boxcar width of $5 \ldots \ldots \ldots$

Figure 155: Absorbance Spectrum of Kelly Green A_F using boxcar width of $25 \ldots 129$

Figure 156: Absorbance Spectrum of Kelly Green A_F using boxcar width of 50 ....... 130

Figure 157: Absorbance Spectrum of Kelly Green A_F using integration time of

$20 \mathrm{~ms}$

Figure 158: Absorbance Spectrum of Kelly Green A_F using integration time of $100 \mathrm{~ms}$

Figure 159: Absorbance Spectrum of Kelly Green A_F after 5 minutes $\ldots 134$

Figure 160: Absorbance Spectrum of Kelly Green A_F after 30 minutes ...................... 134

Figure 161: Absorbance Spectrum of Kelly Green A_F after 40 minutes _...................... 135

Figure 162: Absorbance Spectrum of Kelly Green A_F after 10 minutes _..................... 136

Figure 163: Absorbance Spectrum of Kelly Green A_F after 30 minutes $\ldots \ldots \ldots \ldots \ldots . . . . . . . . . .137$

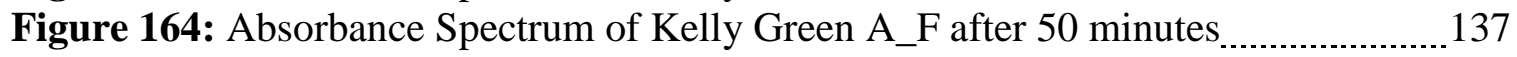

\section{List of Equations}

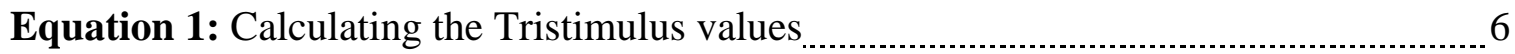

Equation 2: Calculating the Chromaticity values $\ldots$

Equation 3: Calculating absorbance values $\ldots \ldots \ldots$

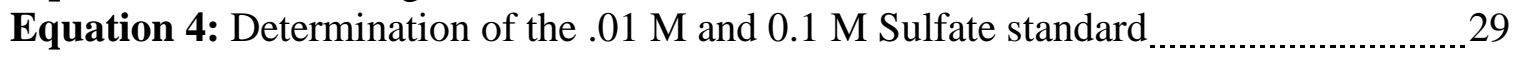

Equation 5: Calculation of the Least Squares Method $\ldots 31$

Equation 6: Calculating the Upper and Lower Confidence Bounds at 95\% confidence 32 


\section{List of Tables}

Table 1: The color of the five t-shirt using the Munsell Book of Color

Table 2: NIST ${ }^{\circledR}$ certified standards for the Holmium oxide filter ……....................... 24

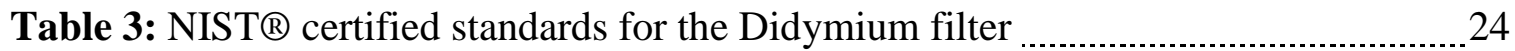

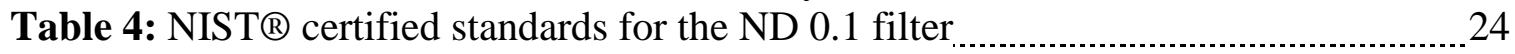

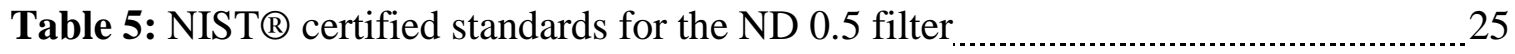

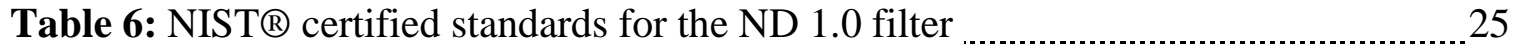

Table 7: Minimum and Maximum wavelength and absorbance values for Gold _............ 34

Table 8: Minimum and Maximum wavelength and absorbance values for Kelly

Green

Table 9: Minimum and Maximum wavelength and absorbance values for Orange _...........39

Table 10: Minimum and Maximum wavelength and absorbance values for Red _........... 41

Table 11: Minimum and Maximum wavelength and absorbance values for Royal _......... 43

Table 12: The OR functions results of the ten comparisons of all five colors _............... 143

\section{List of Photos}

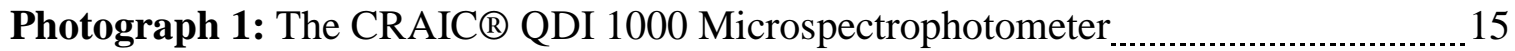

Photograph 2: Kelly Green A_F

Photograph 3: Kelly Green A_F Munsell Chip 7.5G 4, 8

Photograph 4: Kelly Green A_F on the QDI QDS Stereomicrospectrophotometer......... 17

Photograph 5: Gold 10YR 7, 14 Munsell Chip _............................................ 34

Photograph 6: Gold A_A square $\ldots$

Photograph 7: Kelly Green 7.5G 4, 8 Mnsell Chip

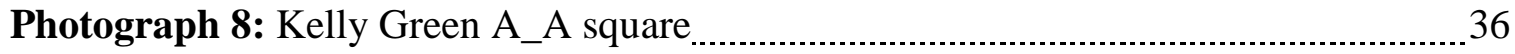

Photograph 9: Orange 2.5YR 6, 14 Musell Chip _.................................................. 38

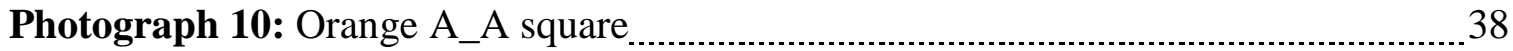

Photograph 11: Red 5R 4, 14 Munsell Chip ___ _ _ _ $\quad 41$

Photograph 12: Red A_A square _............... $\quad 41$

Photograph 13: Royal 5PB 4, 12 Munsell Chip _......................................... 43

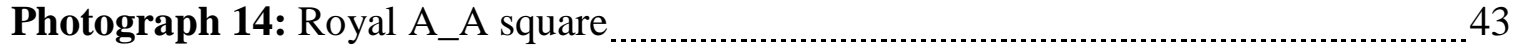




\section{Literature Review}

The importance of textile fibers can be dated back 3000 years. More recently, textile fibers have been found to have a wider application including architectural and industrial textiles [1]. There are two categories of textile fibers, including man-made and natural fibers. Man-made fibers are synthesized through a chemical process and can be subdivided into synthetic fibers and regenerated fibers. Synthetic fibers are made from fibers that have been spun from synthesized fiber-forming polymers [1]. Regenerated fibers are produced from naturally occurring polymers by a technique that includes the regeneration of the original polymer structure [1]. Natural fibers are ones that occur in nature and have three classifications, including animal (protein), vegetable (cellulose) and mineral (asbestos).

Synthetic fibers include polyamides, aramids, polyesters, polyurethane, polyolefin and polyvinyl derivatives. Nylon 6.6 and Nylon 6 are the two most widely manufactured polyamides [1]. Nylon fibers and fabrics are the most abrasion-resistant of all the common textile fibers. Their smooth and regular appearance makes them shiny and translucent. Nylon can be dyed with a variety of dye stuffs making it ideal for the carpeting industry. Nomex and Kevlar are the most commonly encountered aramids [1]. Aramid fibers are resistant to stretching and have a high chemical and heat resistance. Nomex is primarily used for fire/heat retardant purposes such as heat protective clothing. The application of Kevlar includes conveyor belts and reinforcement fibers in sporting goods because it is five times stronger than steel and ten times stronger than aluminum [1]. Man-made fibers cannot be identified based on their morphology alone. Instead, identification is achieved through the determination of the fiber's optical properties, such as refractive index, estimation of birefringence and determination of the sign of elongation [2]. 
Animal (protein) fibers are the most important substance in living matter [1]. Proteins are chainlike molecules formed by $\alpha$-amino acids and peptide linkage. The difference in the proteins is determined by the differences between the side groups $(\mathrm{R})$ that are attached to the main chain. There are 20 different amino acids with different side groups that are known [1]. Examples of animal fibers are silk and wool. Silk is very strong, resistant to creasing and has a luxurious appearance. Wool is made from the protein keratin, which is produced in the fiber follicle in the skin of sheep. They are a rough, scaly fiber making them readily transferrable during contact. The use of mineral fibers (asbestos) has been greatly reduced in industry due to its health hazards. Asbestos is replaced whenever possible by glass or Nomex [1]. The most important form of asbestos is called chrysotile making up $90 \%$ of the production. Vegetable (cellulose) fibers are divided into three groups which are based on the section of the plant from which they were harvested; including seed, bast (stem) and leaf [1]. Cotton is an example of a seed fiber. It is attached to the seeds on the genus Gossypium which is found in mostly all subtropical climates where there are seas, lakes, and large rivers [1]. The cotton fiber is greatly elongated and consists of protoplasmic material bounded by a primary wall with the cuticle on its exterior surface. During the maturing period, the fiber is thickened which occurs by the deposition of cellulose on the interior surface of the primary wall forming the secondary wall [1]. As drying continues the walls of the fiber shrink, the lumen becomes smaller and flatter and the fiber develops convolutions or twists [1]. The convolutions are important morphological features that are observed in the microscopic examination of cotton. Flax (linen) and hemp are two examples of bast fibers and seal and abaca (Manila hemp) are examples of leaf fibers. There are three important aspects of natural fiber identification. They include the authentic reference specimens, sample preparation and careful examination of the most characteristic features by appropriate 
means [2]. Cotton can be recognized by its flattened and twisted appearance and its failure to be extinct when between crossed polars. Cotton fibers should be mounted in water or glyercine. This media helps to swell the fibers to display their morphological characteristics [2].

The examination of trace evidence which include fibers, paints and hair is one of the most important types of physical evidence in the forensic field. Fibers are interesting in forensic science due in part because they have a high degree of variability within their different colors and shades. They also can be transferred during contact [3]. This is based on the ideas of Edmond Locard. His theory simply states that every contact leaves a trace [4]. Fibers that are left at the crime scene can either link a known suspect to the crime or provide investigative leads if the suspect is unknown. Fiber evidence can also provide reconstruction information that is designed to answer a specific question regarding the case and can offer clues about the case or sequence of events. To demonstration the importance of reconstruction, there was a particular case involving a theft and murder. Suzuki [3] explains that the suspect admitted to entering into the house and finding the body while committing the theft. The actual crime scene was reconstructed in accordance to the man's confession. The characteristic dark yellowish-green woolen fibers that were worn by the suspect while entering the home were chosen as the target fibers. All the fibers that presented the same morphological similarities were further examined using microspectrophotometry (MSP) in the ultraviolet-visible range. The number of fibers that were found at each area of the body, mattress, and blankets were compared to the reconstructed scene and the actual investigation. A significant difference was found. This result concluded that there was a strong contact between the victim and the suspect. There were significant discrepancies between the described behavior of the suspect and the analyzed distribution of the dark yellowish-green woolen fibers. 
For the collection of fiber evidence, or any other type of trace evidence from the crime scene, multiple techniques are utilized. Hand retrieval can be used when an alternative light source makes the fiber visible [4]. Adhesive tape lifting is the most common and effective method of collecting trace evidence from the crime scene because it is simple, inexpensive and non-destructive. Combing or brushing is generally used when removing trace evidence from hair [4]. Vacuuming is used to collect the evidence in a special trap that holds a paper. Vacuuming had proven to be ideal for surfaces that are damp or dirty, although vacuuming tends to pick up a large amount of material making the search for specific fibers tedious. Whatever technique is employed, it must be easy for the evidence collector to work with so that tangible results are obtainable [4]. Once the trace evidence has been properly collected from the crime scene using the mentioned techniques, appropriate comparison examinations are carried out in the laboratory.

Determination and comparison of the fiber color is the first applied technique (after general microscopy) that should be carried out in the forensic laboratory. Colorimetry is the science of measurement of the observed color and its translation into a mathematical form to determine and specify color [5]. The recognition of color by humans is not objective, thus problems with human color vision can lead to certain restrictions for the forensic fiber examiner. Fabrics and fibers can share the same hue. Hue is the property of light by which the color of an object is classified as red, blue, green, or yellow in reference to the spectrum, even though they were dyed with varying dyes or mixtures of dyes [5]. This is known as metamerism. Metamerism occurs when two or more colored items are produced with different dyes, pigments or coloring agents that appear to be the same color under certain light conditions [6]. The general concern in the field of forensic science whether or not the colors can be differentiated through the UV-VIS spectra. In 1980, Chamberlin and Chamberlin established that two different approaches that 
would assist in defining and measuring color. The Munsell Color System is used for visual comparison. It uses standards which are accepted as references and the CIE System is an instrumental measurement of parts of the color in terms of the relative amounts of each wavelength present. The Munsell Color System divided three-dimensional color space into hue $(\mathrm{H})$, value (V) and chroma (C) [7]. The hues are arranged in a circle and are divided into five primary hues plus five intermediate hues. In each hue region, $\mathrm{H}$ is normally denoted in four areas; $2.5,5,7.5$ and 10 [8]. The vertical axis symbolizes V ranging from black to white. The $\mathrm{C}$ varies from 0 to 12 , with 12 being the most saturated color of the given $\mathrm{H}$ and $\mathrm{V}$ [8]. The Munsell Book of Color now has over 1500 color samples. The Munsell specifications contain over 2000 color samples, which are colored chips dyed with pigments [5]. According to the CIE System, the human eye can only distinguish three dimensions of color- hue, saturation and intensity. This model is developed based upon the tristimulus theory of color perception. The color of an object is determined by type of light source, the viewing conditions present and the color response of the observer. [5] The cell receptors in the eye are responsible for distinguishing levels of brightness and the color receptors in the eye are responsible for color vision. At the Common Internaltionale de L'Eclairage Conference in 1931, the mathematical treatment of color data was designed. In forensic science Illuminant A (incandescent light), Illuminant B (noon sunshine) and Illuminant D65 (daylight) are of the most interest. [5] However, once this is accomplished, it is found that any color can be expressed in terms of the two color coordinates $\mathrm{x}$ and $y$. The colors which can be matched by combining a given set of three primary colors are represented on the chromaticity diagram by a triangle joining the coordinates for the three colors. Along with the determination of color, it is also essential to use different instruments and techniques during the examination of fiber evidence. 


$$
\begin{aligned}
& X={ }_{\lambda} L_{\lambda} * x_{\lambda} * \Delta \lambda \\
& Y={ }_{\lambda} L_{\lambda} * y_{\lambda} * \Delta \lambda \\
& Z={ }_{\lambda} L_{\lambda} * Z * \Delta \lambda
\end{aligned}
$$

Equation 1: Calculating the Trimstimulus values

$$
\begin{aligned}
& x=X /(X+Y+Z) \\
& y=Y /(X+Y+Z) \\
& z=Z /(X+Y+Z)
\end{aligned}
$$

Equation 2: Calculating the chromaticity coordinates

Microscopy is an essential examination technique in all aspects of forensic examination and comparison of fibers [2]. Microscopes are necessary to observe fiber evidence no matter what tests or observations are being completed. When preparing a fiber for a specific examination in the laboratory, it is important to understand both the microscope and the type of fiber that one may be dealing with [2]. The stereomicroscope is utilized for the searching of small items of evidence and to manipulate individual fibers. To directly compare fiber properties, such as diameter, color and morphology, the comparison microscope is used [2]. Fluorescence microscopy can be used to detect illumination of the fibers originating from the dyes in the fiber. 
UV-VIS MSP is used to distinguish the colors of fibers that have different spectral colors, but appear similar to the naked eye.

MSP is a method that has been is use for over 40 years in the forensic field, but has been used in biological disciplines for more than 70 years. Casperson developed MSP for biological science in order to locate and identify chemical constituents within the cell [9]. The application of MSP for the examination of fibers was first described by Amsler in 1959 [5]. MSP was established as a quick and non-destructive method for examination of trace evidence. This instrument is used as a comparative technique when examining fibers rather than for identification purposes. The microspectrophotometer allows measurement of the absorption of electromagnetic radiation by microscopic amounts of many kinds of materials in the visible region $(400-800 \mathrm{~nm})$ and the ultraviolet region $(200-400 \mathrm{~nm})$ of the electromagnetic radiation spectrum [5]. Absorption can be described by an electron becoming excited if the frequency of the incident electromagnetic radiation matches or closely corresponds to the difference in energy between two electronic states causing electronic transition from the highest occupied molecular orbital to the lowest unoccupied molecular orbital [5]. The choromophore, which is the colored part of the molecule, is responsible for the absorption of the radiation in the UV-VIS region. When using the MSP for the examination of fibers, two parameters are used; the transmission (T) and the absorbance (A). The physical result of absorption measurement is a spectrum. An absorbance spectrum that has multiple points of identification can be compared successfully, but if the spectrum is broad and has little detail it is considered less discriminating. Wiggins [10] uses the first derivative of an absorbance spectra to determine if it can provide additional discrimination for fiber evidence. In natural fibers, there is a higher level of intra-sample variation. In the black cotton fibers, the original spectra showed little variation meaning the first 
derivative also showed little variation. In this study, it was determined that calculating the first derivative can provide extra points of comparison aiding the interpretation of the evidence when the absorbance spectra is tightly grouped with regard to the absorbance level. The spectrum that is produced by the MSP provides important information about the conjugated electron systems, but does not provide any information about the spatial arrangement [5].

MSP is an important technique not only used for the comparison of fiber evidence, but for paints and hairs as well. Kopchick [11] analyzed 120 black and gray vehicle paints. During this study it was found that MSP provided discrimination information in the achromatic paint samples due to the secondary chromatic pigments added in the achromatic paint formula. It can be concluded that MSP should be incorporated in the analytical scheme when black and gray topcoats are included. In the study conducted by Barrett [12] 55 different red hair dyes were analyzed using UV-VIS MSP. It was found that the presence of hair dyes reduces the intrasample variability of the hair color. Even though all these different studies have been conducted, trace evidence continues to be corroborative evidence Therefore, trace evidence can only confirm or infirm preexisting hypotheses.

To demonstrate the significance of trace evidence examination ensuring its acceptance by the forensic science community two methods have been developed; population studies and target studies. Population studies are used to describe the sample demographics of a certain type of trace evidence. They also provide feedback either confirming or altering the analyst's prospects on the number of materials that may be transferred or persisted [13]. Target fiber studies use a specific trace evidence sample type and then the items are searched for examples that are indistinguishable. Target studies are used to answer specific case-related questions of transfer, persistence and significance. In the study conducted by Houck [13] twenty unrelated 
trace evidence cases were selected at random from the author's casework. These cases were then split into three categories (bank robbery, kidnapping and homicides) and were cross-compared. The fibers were further divided into natural and manufactured groups as well as by their color. They were then analyzed using light microscopy, fluorescent microscopy and MSP. 2083 fibers were cross-compared making 2,163,603 comparisons. No two fibers were found to exhibit the same characteristics when examined using the techniques mentioned above. Therefore, it can be stated that it is rare to find two unrelated items having foreign fibers that are analytically indistinguishable.

The objective of this study is to evaluate the differentiation, if any, in the color of the cotton t-shirt samples (royal, kelly green, orange, red and gold), which included an intra-garment fiber comparison and an inter-garment comparison. The intra-garment comparison will determine if there is variability in the spectra within the same garment in different areas of the same garment, for example the front, back, armpit and sleeve. The inter-garment comparison will determine if there is variability in the spectra between the same fiber. In the study performed by Wiggins and Drummond [14] the spectra were divided into matching sub-populations by overlaying the spectra and observing the differences between the spectra. These include the trough or decrease in the absorbance and the characteristics of the peaks and the noise level. The Munsell Color System will provide a prerequisite for determining and specifying the color of the fibers that are being analyzed. Conducting a fiber study, will hopefully provide more conclusive and significant results that are needed in the trace evidence realm.

Much useful information has resulted from target and population studies and examination of the blocks of color in both natural and man-made fibers. The study of the color blocks can provide useful information about natural fibers. They often rely on color as the main comparative 
examination technique, because they lack a wide range of morphological characteristics [15]. All of the research carried out has shown that man-made fibers, such as polyester, exhibit a very high degree of polymorphisms, meaning they have multiple forms, providing specific examples with a high degree of individuality [15]. In the study conducted by Grieve [15] 263 blue polyester fibers were obtained. Blue was chosen because it is the most common fiber color in forensic casework and polyester is found in a wide variety of textiles. The fibers were first examined using bright field microscopy and classified according to their cross-section and texture. Spectra from each group were recorded using a Zeiss 800 spectrophotometer. Based on the results that were obtained from this study, it could be determined that the chance of two blue polyester fibers matching is very low, giving them a high discriminating power.

A study conducted by Biermann [16] showed the discriminating power and evidential value of blue and red cotton fiber. This value can be very high when using light microscopy, fluorescence microscopy and UV-VIS MSP. Three hundred and fifty-one blue and 287 red cotton fibers were collected and the Munsell Color System was applied to make sure that the fibers selected were in the correct block of color. Ninety-four percent of the blue cotton fibers examined could be differentiated. The discriminating power of the method using both fluorescent microscopy and MSP was determined to by 0.9996 . Out of 275 red cotton fibers examined $93 \%$ of them could be differentiated. Using the same techniques, the discriminating power was found to be 0.9995 . Although blue and red cotton fibers make up a high percentage of textile fibers, these results prove that when using the aforementioned examination techniques fibers can still provide excellent evidence when involved in forensic fiber cases. Palmer [17] aims to complement current studies to determine to what degree the UV-VIS MSP can discriminate the blue non-denim blue cotton fibers. Out of 100 garments that were gathered, 9 different items of 
clothing were sampled: trousers, skirts, short and long-sleeved shirts and t-shirts, jumpers, hooded jumpers and polo shirts. From 78 different brands represented, 23 of them were classified as mid-blue and 61 of them were classified as dark blue. When comparing the spectra of these samples, it was determined that these outcomes are in accordance with the results obtained by Biermann. Also evidenced was that UV-VIS MSP alone can provide a similar discriminating power when combined with microscopy. With the evidential value demonstrated, the problem that remains is the proper pathways that should be taken from the initial search to the comparison. It is expected that with the data now available, laboratories will re-evaluate their analytical pathways.

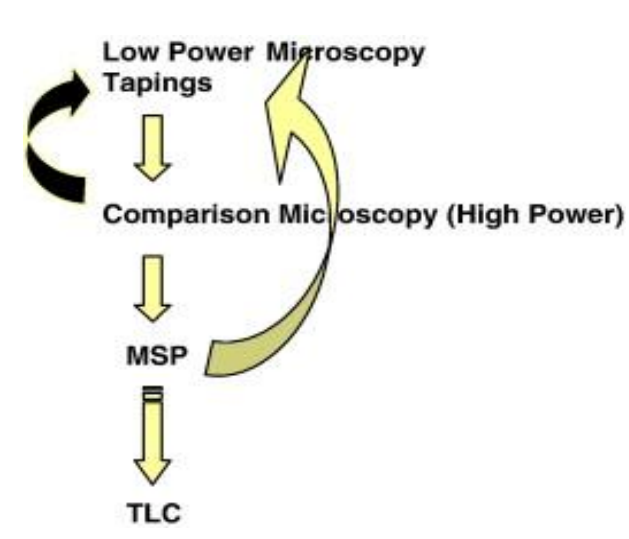

Figure 1: Conventional Pathway [17]

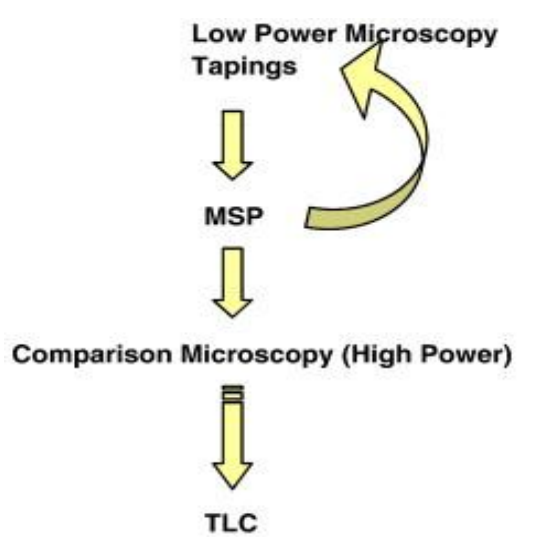

Figure 2: Alternative Pathway [17]

Through the use of this alternative technique the results of this study provide more discrimination in cotton fibers. This proposed method provides shorter analysis time and more effective examination in the forensic laboratory. 


\section{Materials/Methods}

\section{Materials}

\section{T-shirts used}

Five varying non-worn (adult small) cotton shirts were obtained from Underground Printing ${ }^{\circledR}$ in Morgantown, West Virginia. The colors of the t-shirts were chosen based on their current popularity in forensic casework today which includes; Gold, Kelly Green, Orange, Red and Royal. Ten shirts of each color (50 total) were obtained. Two inch by 2 inch squares were cut out from the front, back, sleeve and armpit of each t-shirt. The hue (describing the color), value (overall intensity to how light or dark a color is) and chroma (strength or dominance of the hue) of the t-shirts were determined by using the Munsell Book of Color.

\begin{tabular}{cccc}
\hline & Hue & Value & Chroma \\
\hline Red & $5 R$ & 4 & 14 \\
Orange & 2.5 YR & 6 & 14 \\
Gold & 10 YR & 7 & 14 \\
Kelly Green & 7.5 G & 4 & 8 \\
Royal & 5 PB & 4 & 12 \\
\hline
\end{tabular}

Table 1: The colors of the five t-shirts based on the Munsell Color Book

Sample Prep

Each 2 inch by 2 inch square of the t-shirt was labeled according to which segment of the t-shirt it came from, for example, Kelly Green A_F. This label indicates it was the first of the ten shirts and originated the front square. For the first part, each of the 200 Fisherbrand ${ }^{\circledR}$ microscopic slides were prepared by extracting between 5 and 10 fibers from the square using 
tweezers and labeled the same way as the t-shirt squares. My initials (BM) were also included on each slide. The microscopic slides were placed under the stereomicroscope for clear visualization of the fibers and to place them on the slide. One to two drops of the mounting medium Entellan was then placed on each slide. Lastly, Fisherbrand ${ }^{\circledR}$ cover slips were used to secure the fibers onto the slides and allowed to dry. For the second part, 150 microscopic slides were prepared by only using the front square of every color t-shirt. One fiber was pulled from the square and then cut into three sections. The microscopic slides were prepared the same way as the first part, but labeled according to the section. For example, Kelly Green A_1, which signifies the first kelly green t-shirt and the first section. The tweezers and stage of the stereomicroscope were cleaned with isopropyl alcohol after each sample was prepared.

\section{Instruments Used}

After the t-shirts were cut and observed using the Munsell Color system, a CRAIC® QDI QDS II TM Stereomicrospectrophotometer was used to collect spectra of each square. This instrument consists of a microscope with a stabilized light source, a spectral dispersive element (monochromator), a detector, and a data processing device. The microscope which is coupled with the spectrophotometer is what allows the visualization of the fibers on the slides. The microscope is set to 40x objective for proper visualization of the fibers. The light source emits all of the wavelengths of the ultraviolet and visible light via a Xenon lamp source that is set to 75 $\mathrm{W}$. The power lamp source is turned on prior to running any samples and allowed to warm up for 30 minutes before use. The monochromator separates this light into its different components, namely the different wavelengths and different colors, before it reaches the sample. The monochromator produces monochromatic light of a certain spectral bandwidth. The sample is scanned step by step, and a spectrum is created point- by- point. The detector component is what converts the light into an electric signal. The data processing device represents the CRAIC® software, which allows for the display of the spectrum and the management of the parameters. When this light, which is given a wavelength $(\lambda)$ and intensity $(I)$, hits the sample, it can then be absorbed. For example, in this study, when spectrum was collected in absorbance mode, the minimum point on the spectrum (from $350 \mathrm{~nm}$ to $750 \mathrm{~nm}$ ) is where the instrument is transmitting the color of the fiber and the maximum point on the spectrum is where the instrument is absorbing the color (complimentary color of the sample). 


$$
A_{\lambda}=-\log T=\log \frac{I_{0}}{I}
$$

\section{A- Absorbance T- Transmittance I- Intensity}

Equation 3: Calculating the absorbance values

The CRAIC® QDI 1000 Microspectrophotometer was used to collect the spectra and the chromaticity values of the slides that were previously prepared. This instrument is configured and works in a similar way as the a CRAIC® QDI QDS II TM Stereomicrospectrophotometer. The microscope also coupled with the spectrophotometer, is what allows the visualization of the fibers on the slides. The microscope is set to $360 \mathrm{X}$ objective for proper visualization of the fibers. The light source in this instrument emits all of the wavelengths of the ultraviolet and visible light via a Xenon lamp source that is set to $75 \mathrm{~W}$. 


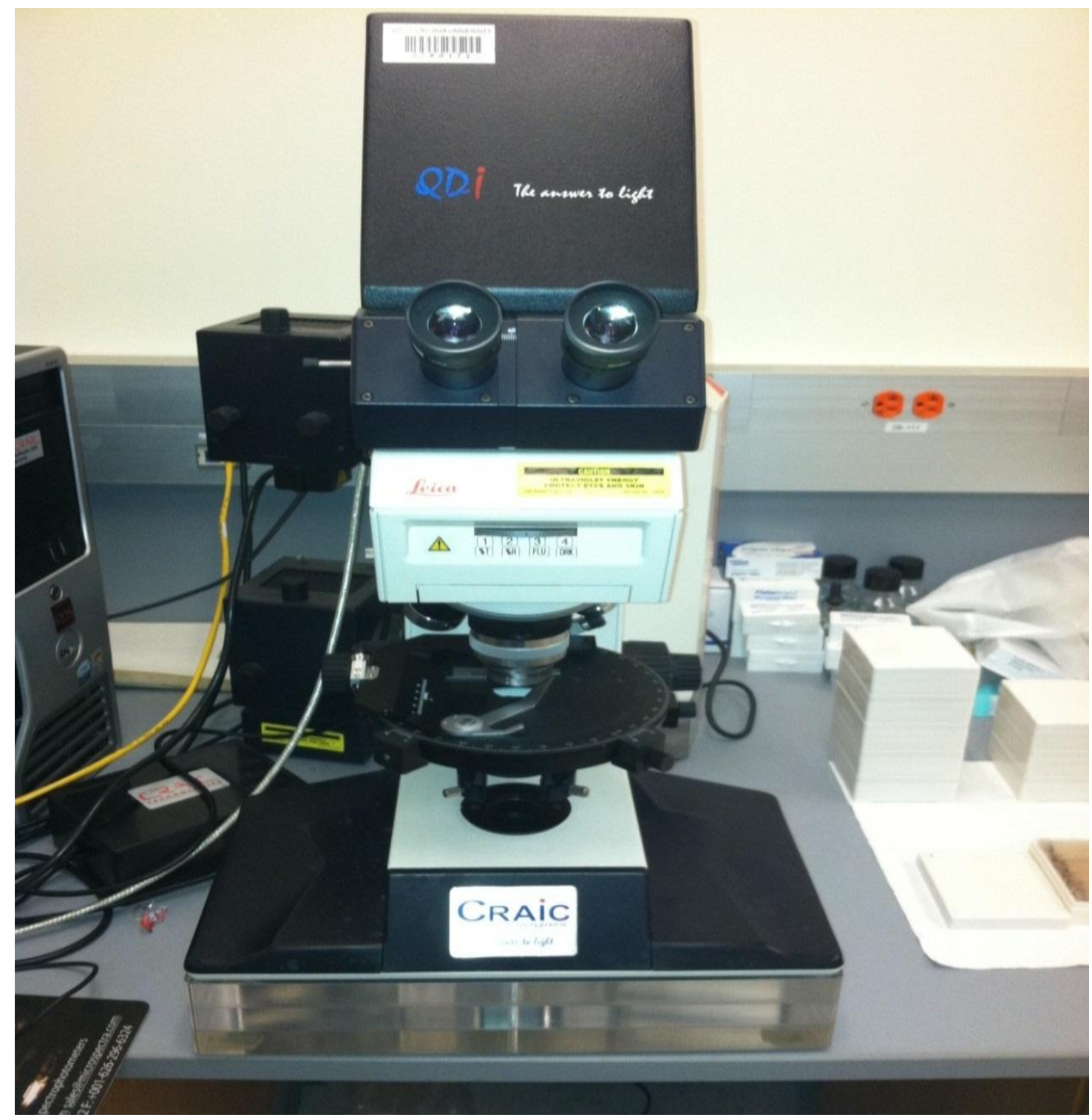

Photograph 1: The CRAIC® QDI 1000 Microspectrophotometer

This instrument contains three different light paths; the transmitted light, the incident light and the image light. The transmitted light path contains the lamp housing for the $100 \mathrm{~W}$ mercury or the $75 \mathrm{~W}$ xenon lamp, the aperture diaphragm, the imaging system of the aperture diaphragm, the field diaphragm, the polarizer and the condenser. The selected lamp is turned on before running any samples and allowed to warm up, depending on the mode chosen to collect the spectra; absorbance, transmittance, reflectance or fluorescence. The aperture diaphragm determines the resolution, the depth of field and the contrast of the microscope image. The best resolution is obtained when the apertures of the objective and condenser are approximately the same. The incident light path contains the light source, the aperture diaphragm, the field diaphragm, and the reflector or filter cube. The image light path consists of the objective, the tube optics, the tube 
and the eyepiece. The instrument also contains a stage where the slides are placed, as well as, a course and fine adjustment knob for focusing of the sample.

\section{Software}

The CRAIC® CCD Image Software was used for both the QDS QDI II ${ }^{\mathrm{TM}}$ Stereomicrospectrophotometer and the QDI 1000 Microspectrophotometer to view and take photos of the fibers, t-shirt squares and the Munsell Color chips placed on the stage of the instruments. Once the sample is placed on the stage, the focus and zoom control knobs are operated to locate it. A black square is placed in the center of the screen. This square represents the location from where the instrument collects the spectrum. The software provides a window that allows the user to choose what resolution they want to use, being full resolution of $1280 \mathrm{X}$ 1024 to the smallest resolution of $320 \times 240$. The user is also able to change the exposure of the photo by pushing the Auto button or positioning the slider bar to a suitable level. The white balance and saturation may also be adjusted by the user. The "live" mode at the top of the screen allows the user to visualize the sample and the "capture" mode allows for the image to be taken and stored.

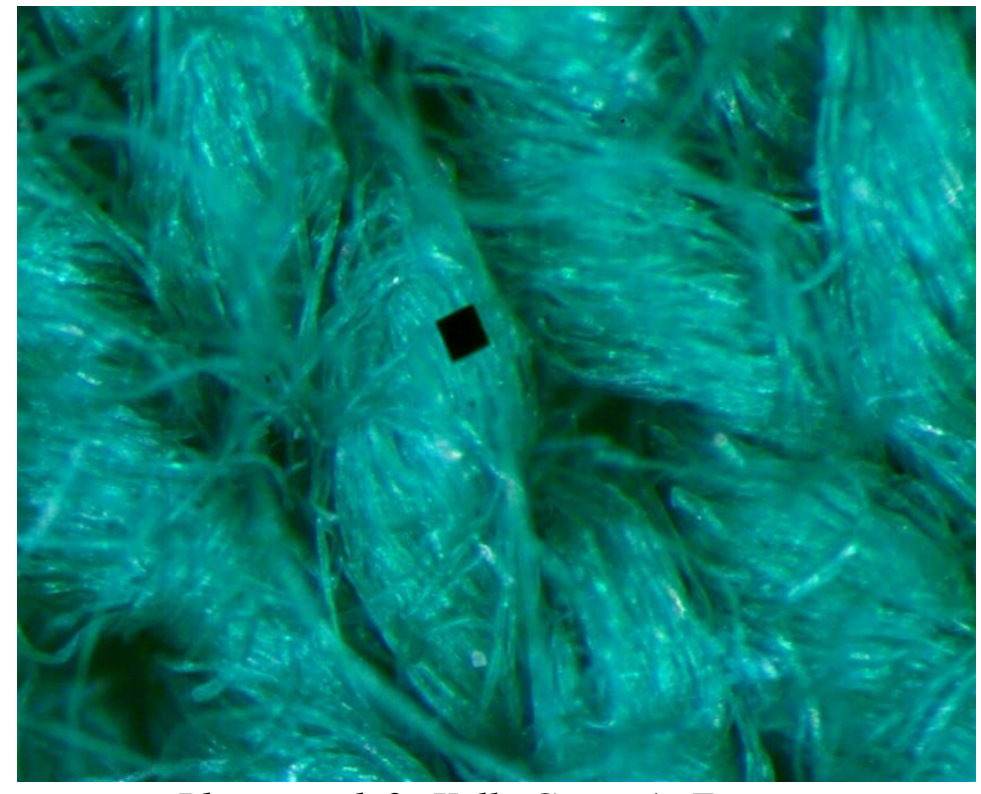

Photograph 2: Kelly Green A_F

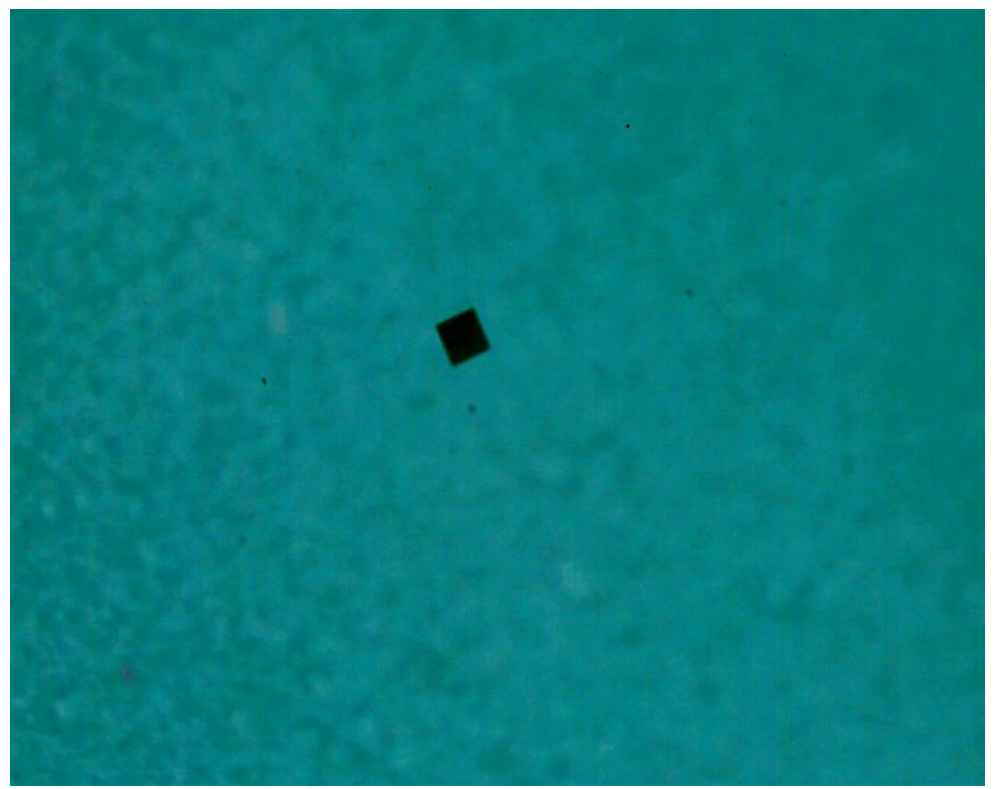

Photograph 3:Munsell Chip 7.5G 4,8 


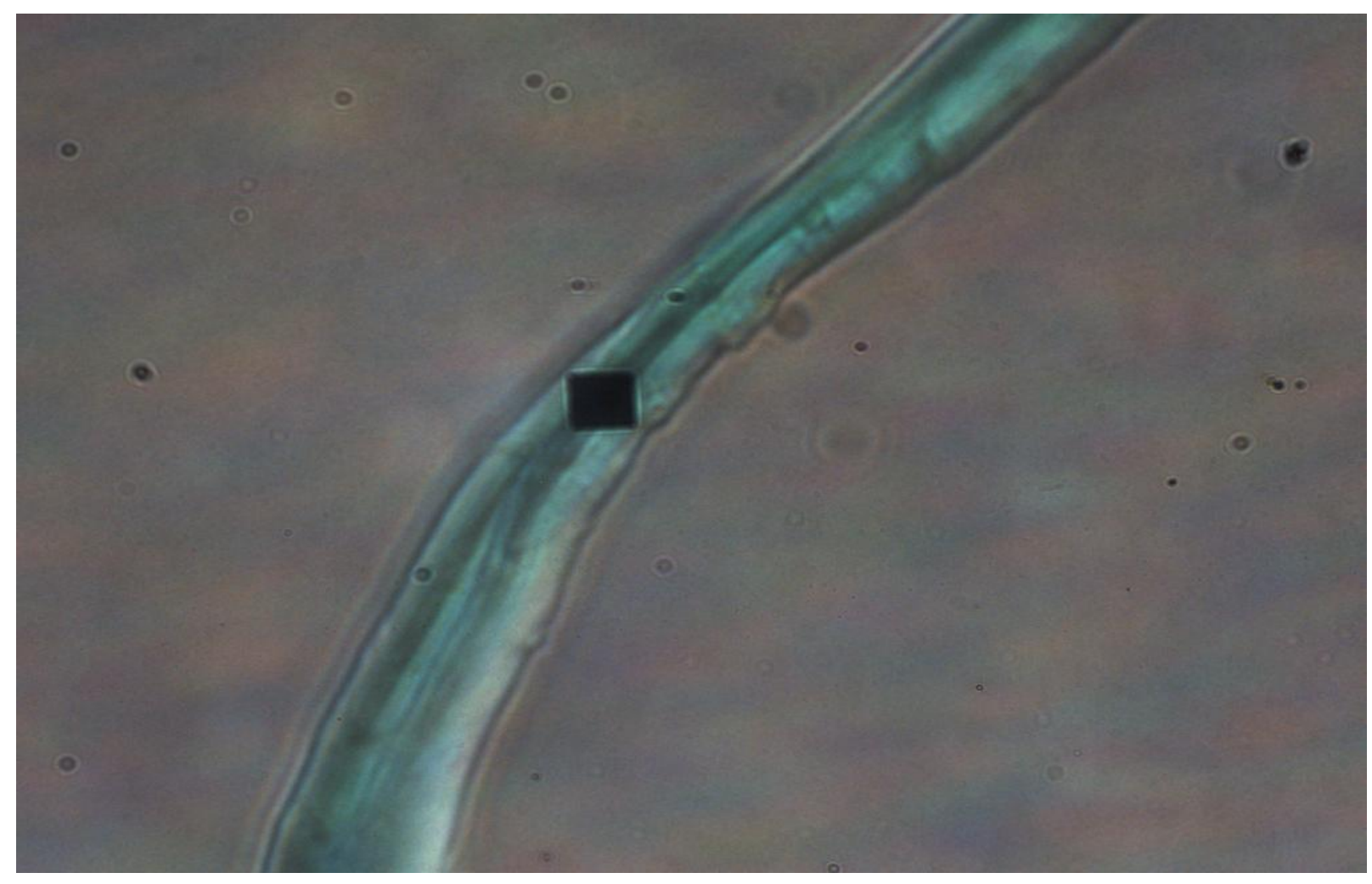

Photograph 4: Kelly Green A_F on the CRAIC® QDS QDI II TM Stereomicrospectrophotometer

Operating the QDS QDI II ${ }^{\mathrm{TM}}$ Stereomicrospectrophotometer, the CRAIC® Data Acquisition Software is used. This program provides different rows that contain all the instrument controls and parameters. The first row exhibits the program name and the second row enumerates the menu which contains the file, view and edit drop down items. The third row shows the toolbar which allows for the opening and closing of the spectrum or spectra, saving the spectrum, auto scaling the spectrum in the $\mathrm{x}$ and $\mathrm{y}$ direction, and illustrate and edit the peak markings. The spectrum is then displayed. The units are revealed as well as the file name, the scan time, and the objective. The fourth row contains the instrument controls. The "My Folder" button is where the user can set the experiment ID chosen for the particular task. The "AutoSet" Optimize" button optimizes the instrument. "Collect Darkscan" will collect the instruments background spectrum, as when the light is totally blocked from the spec head and "Collect Reference" will collect a reference spectrum, allowing light to pass through the spec head. The "Collect SampleScan" is used to collect the spectrum of the sample. The "AutoCal 
Wavelength Check" and the "AutoCal ${ }^{\mathrm{TM}}$ Photometric Check" is used to check the instruments calibration with the NIST® Traceable Standards.

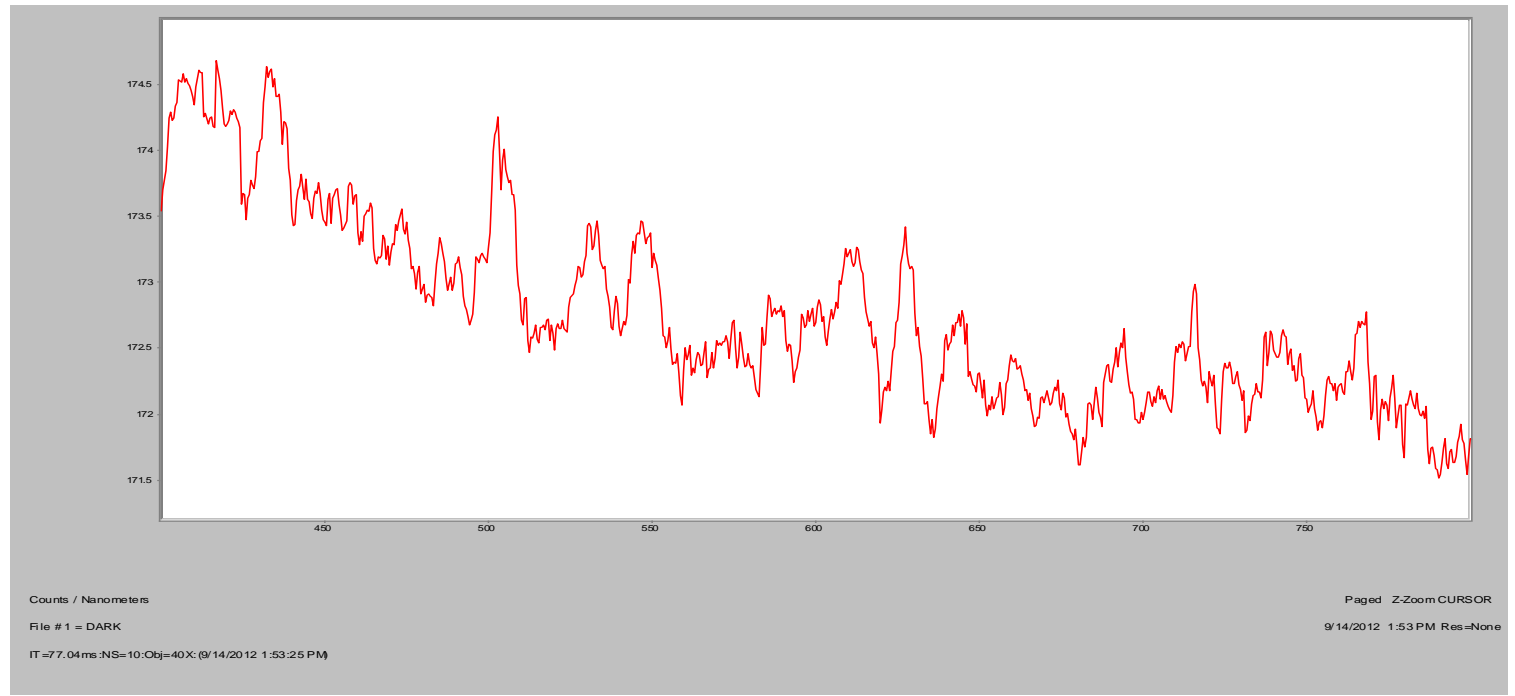

Figure 3: Dark Scan on the CRAIC® QDS QDI II TM Stereomicrospectrophotometer

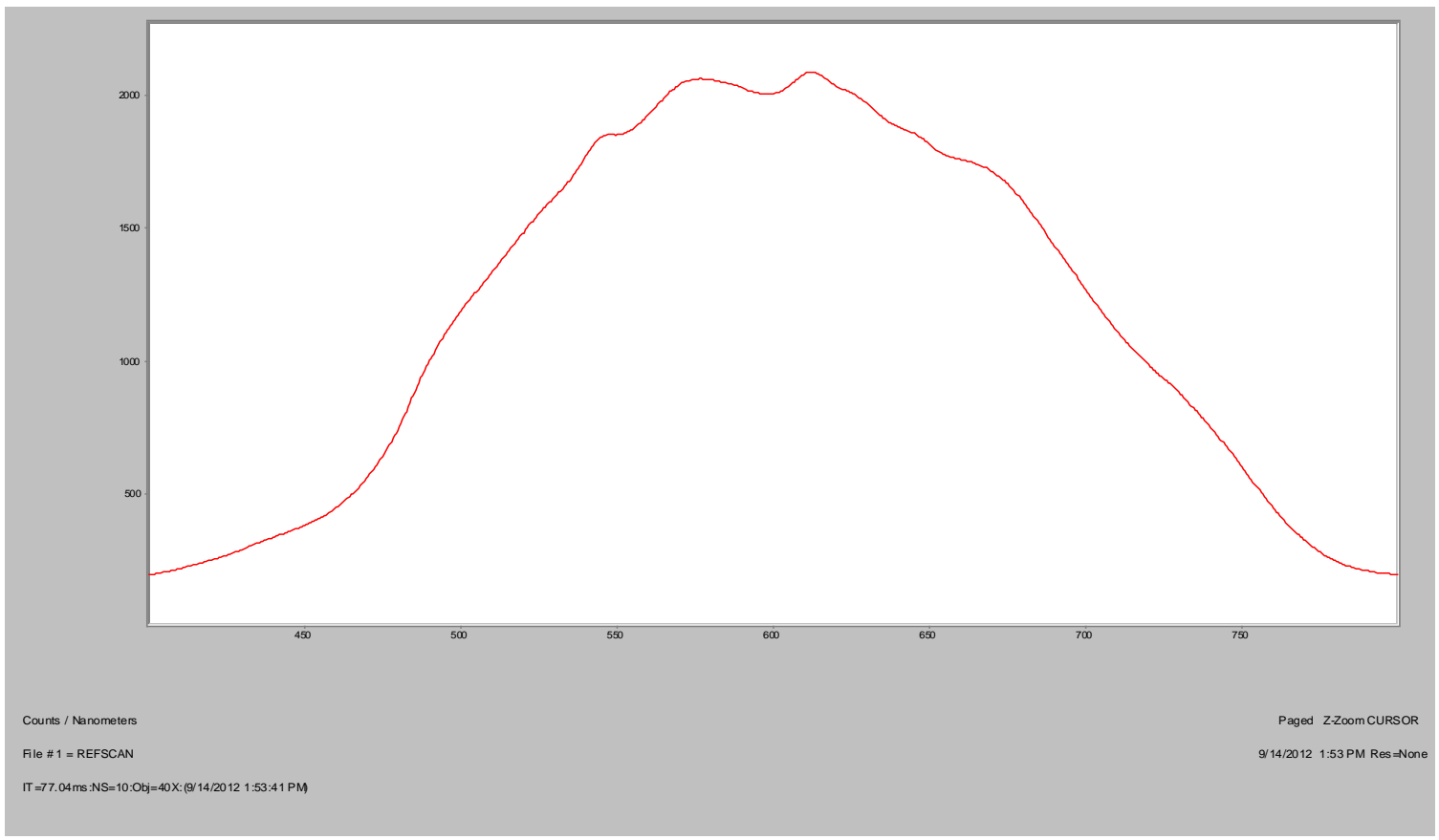

Figure 4: Reference Scan on the CRAIC® QDS QDI II TM Stereomicrospectrophotometer 
For the CRIAC® QDI 1000 Microspectrophotometer, Ocean Optics ${ }^{\circledR}$ SpectraSuite ${ }^{\circledR}$ Spectrophotometer Operating Software was used for the collecting of the spectrum for each sample to determine the intra and inter-variability of the fiber samples. This software contains three rows of controls and parameters. In the "File" drop down box, the type of measurement that the user wants to perform, which can be either absorbance, transmission, reflection, relative irradiance or fluorescence measurement is found here. In the "View" drop down box, the user is able to view the different graph layering options. Annotations, legends, a trend line, or peaks can be added to the spectrum using this tool. The "Processing" drop down box allows the user to edit the color of the spectrum. The acquisition tool bar consists of the integration time, the scans to average and the boxcar width. The integration time is the measurement of the detector monitoring the photons, thus the longer the integration time the longer the detector is monitoring. The scan to average specifies the number of spectral acquisitions that the device driver accumulates before the software receives a scan. The higher the scans to average value, signifies the better signal to noise ratio. The boxcar width sets the boxcar smoothing width, which averages across the spectral data. The greater the boxcar width value, the smoother the data and the higher the signal to noise ratio. A loss in spectral resolution will result if the boxcar width value is set too high. The color space button that appears on the acquisition tool bar is used to measure the color of the sample being analyzed. This setting of the software allows the user to choose the reflectance settings, the standard observer, and the standard Illuminant. Using this setting also provides the tristimulus values (X, Y, Z), 1931 color space chromaticity coordinates $(\mathrm{x}, \mathrm{y}, \mathrm{z})$ and the chromaticity diagram for the sample. Once the spectrum that appears in the center of the screen, there are buttons that allow the user to save the spectrum, copy the absorbance and wavelength values into a Microsoft ${ }^{\circledR}$ Excel or copy the spectrum into a Microsoft@ Word document. The user can also change the range of the $\mathrm{x}$ and $\mathrm{y}$ values in the spectrum by selecting the magnifying glass button. The black light bulb button is used to collect a dark scan and the yellow light bulb button is used to collect a reference scan. 


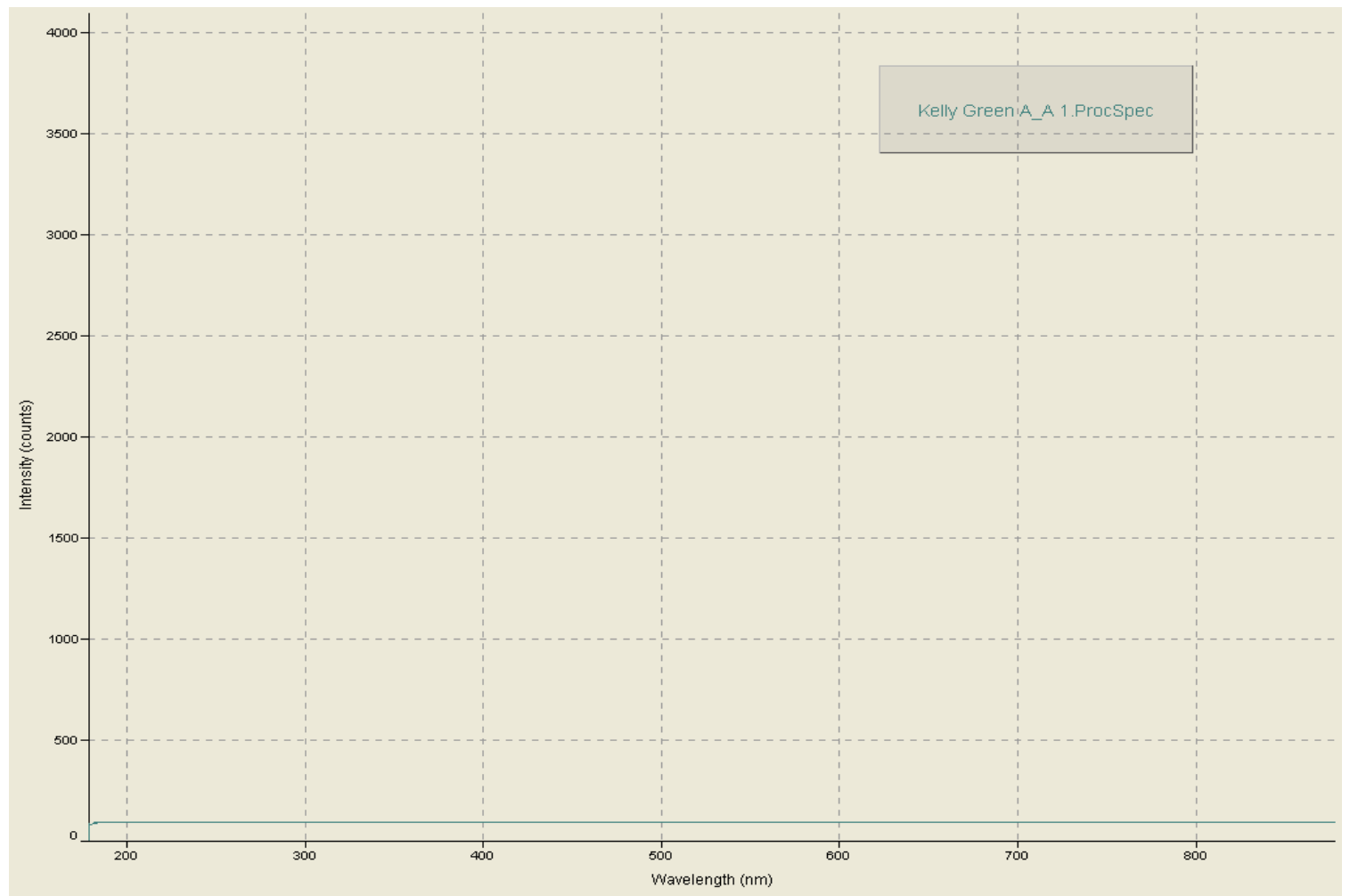

Figure 5: Dark scan on the CRIAC® QDI 1000 Microspectrophotometer

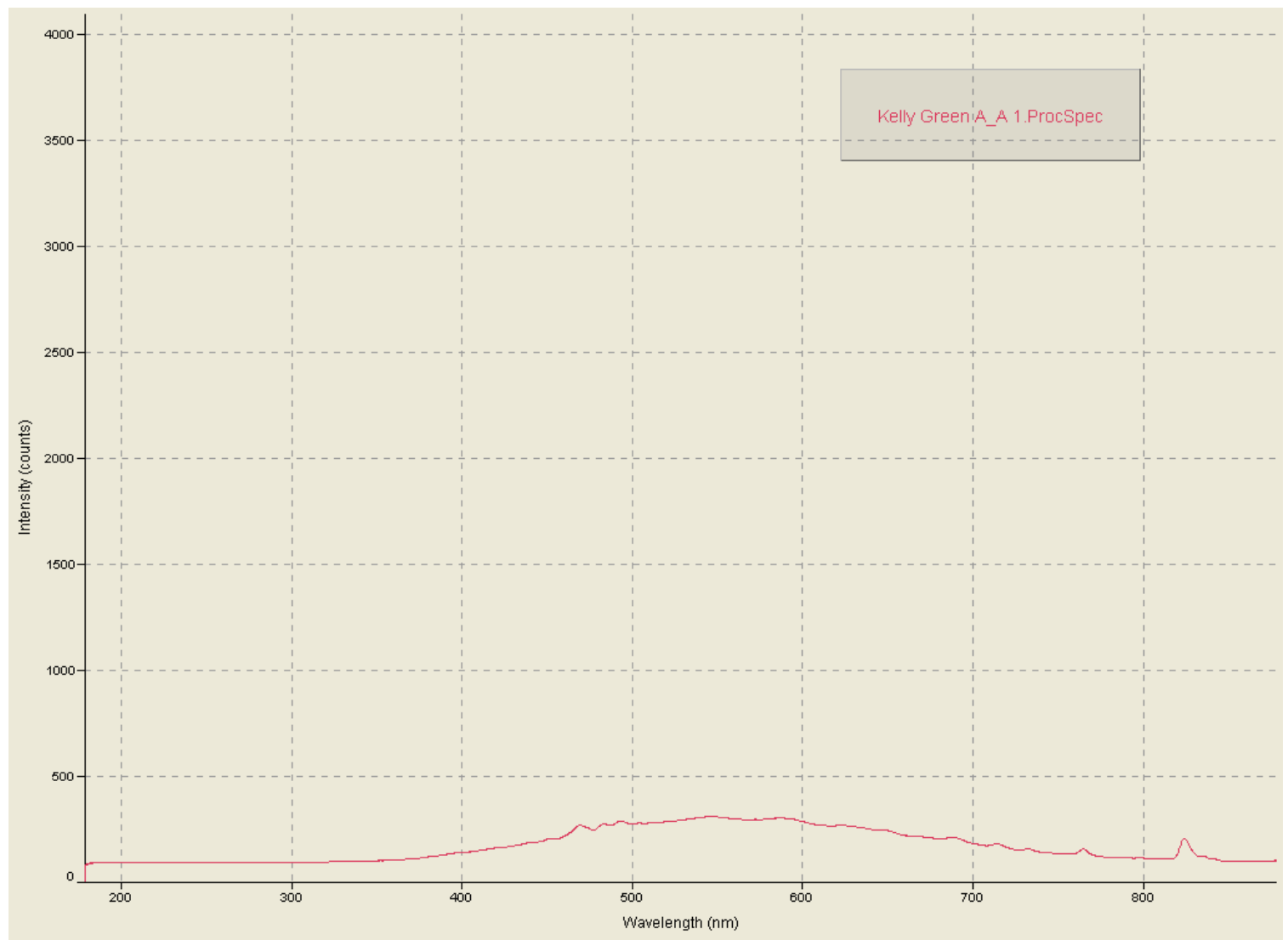

Figure 6: Reference scan on the CRIAC® QDI 1000 Microspectrophotometer 


\section{Calibration of Instrument}

To calibrate and validate the CRAIC ® QDI QDS II ${ }^{\mathrm{TM}}$ Stereomicrospectrophotometer and the CRIAC® QDI 1000 Microspectrophotometer, NIST® Traceable Standards were used to check wavelength calibration and photometric calibration of the instrument. A Holmium Oxide filter and a Didymium filter was utilized to test the wavelength calibration and ND 0.1, 0.5 and 1.0 filter were used to check the photometric calibration. NIST® provides a list of the certified wavelengths in nanometers $(\mathrm{nm})$ and the combined uncertainties that must be met in order to properly validate and calibrate the instrument before use. This procedure was done by placing each filter below the stage of the instrument. The "Auto Cal ${ }^{\text {TM }}$ Wavelength" button was selected on the CRAIC@ QDI data acquisition software. An absorbance spectrum was collected for the photometric filter and transmission mode used for the wavelength filters. If a "smiley face" appeared, the instrument was within the established calibration limits. If a "frowning face" appeared, the steps had to be repeated. When using the Ocean Optics® software, the absorbance and wavelength values obtained were compared to the ones that were provided by NIST®.

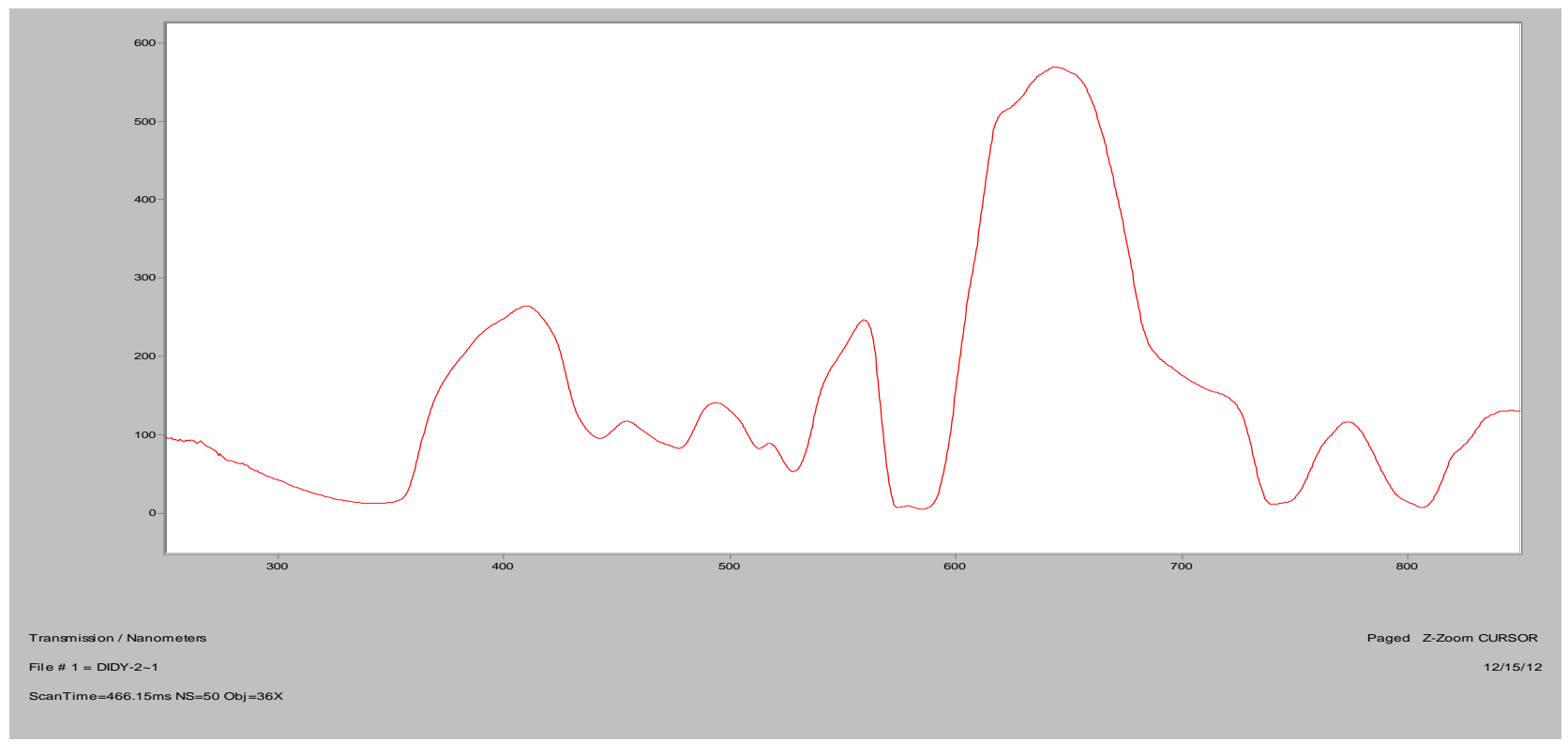

Figure 7: Didymium Filter on the CRAIC ${ }^{\circledR} Q D I Q D S I I^{\mathrm{TM}}$ Stereomicrospectrophotometer 


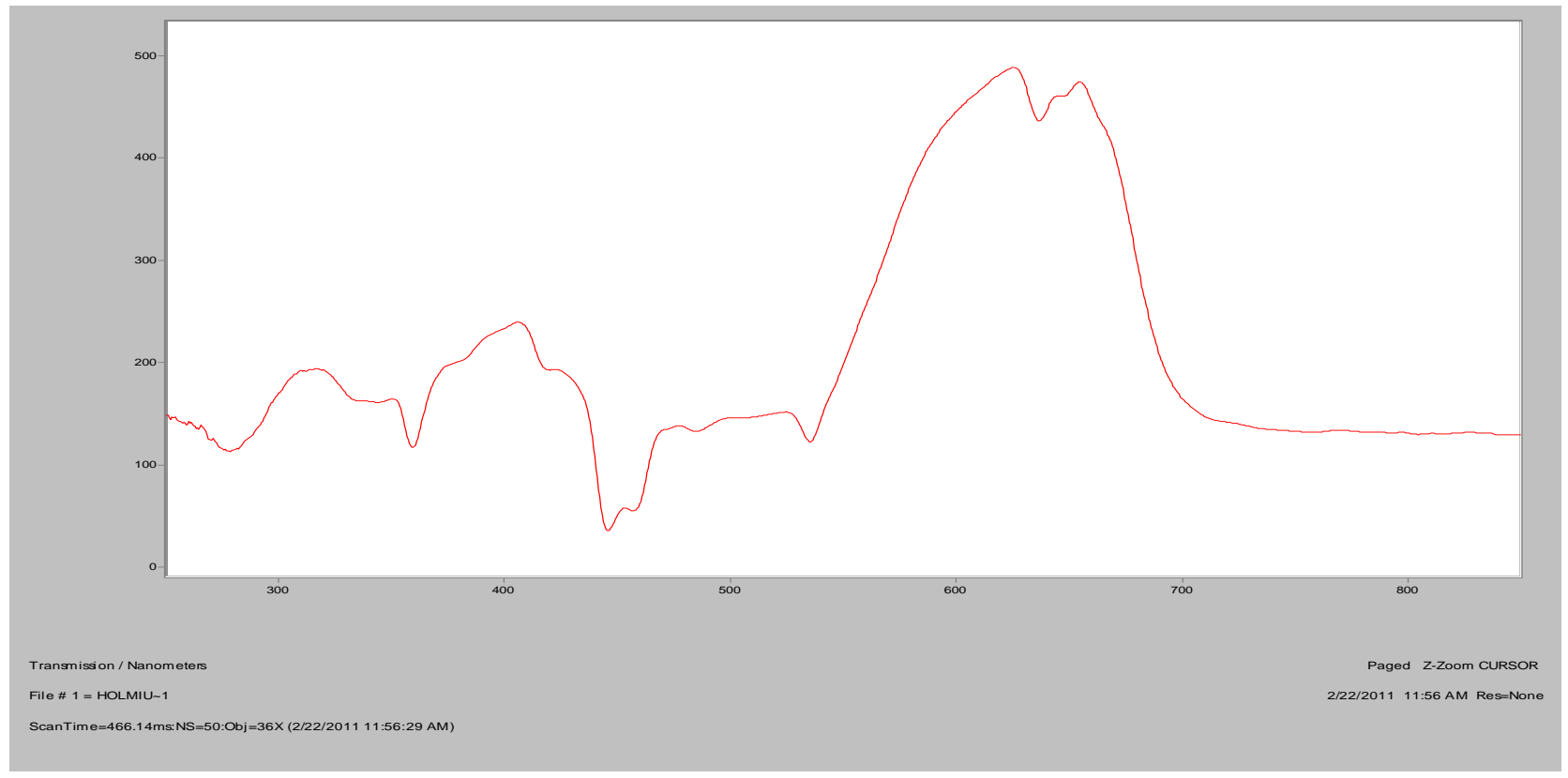

Figure 8: Holmium Oxide filter on the CRAIC ${ }^{\circledR} Q D I Q D S I I^{\mathrm{TM}}$ Stereomicrospectrophotometer

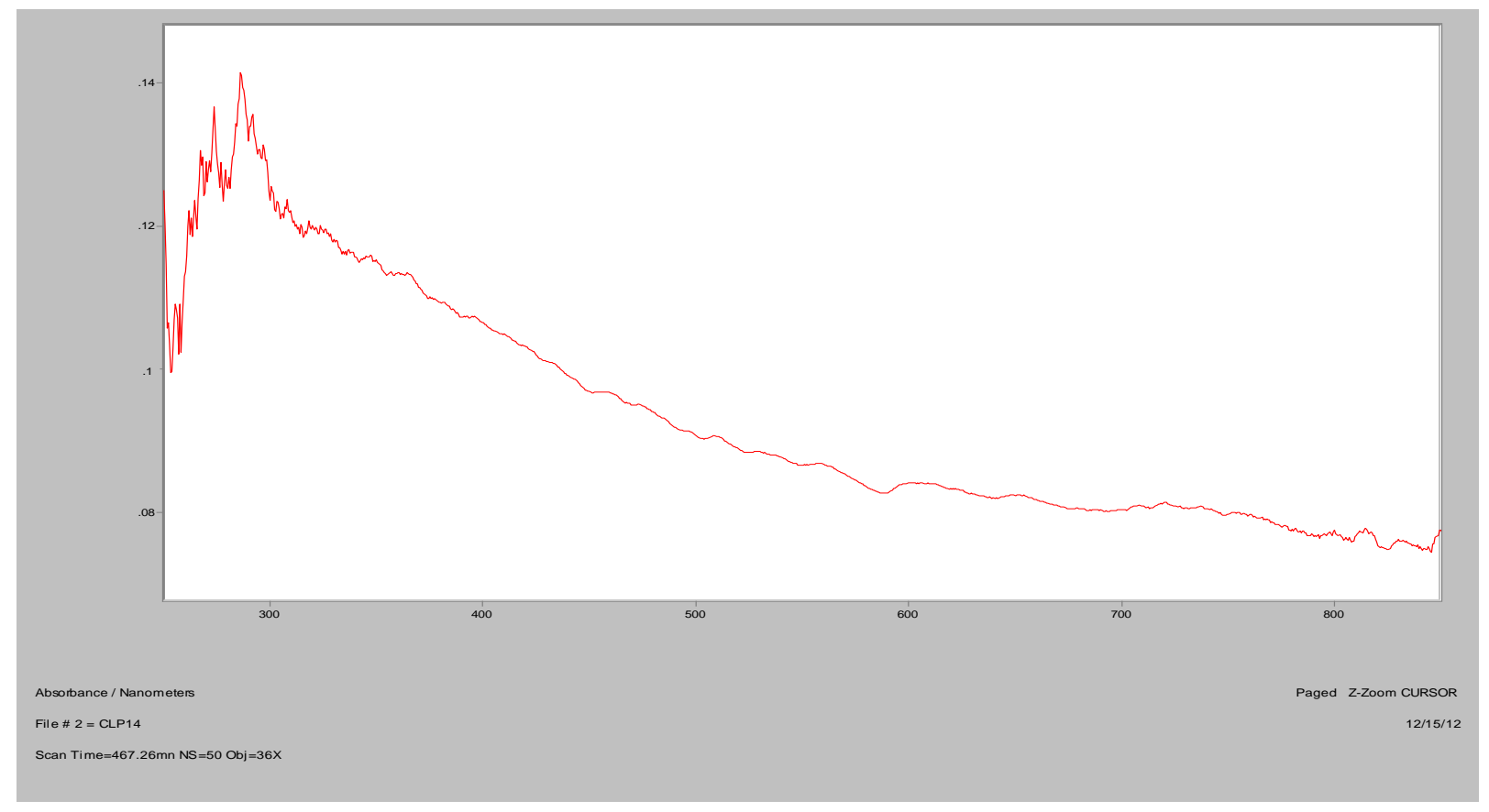

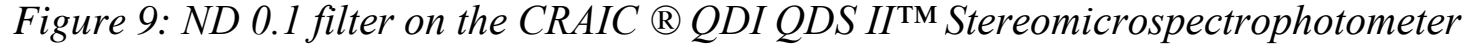




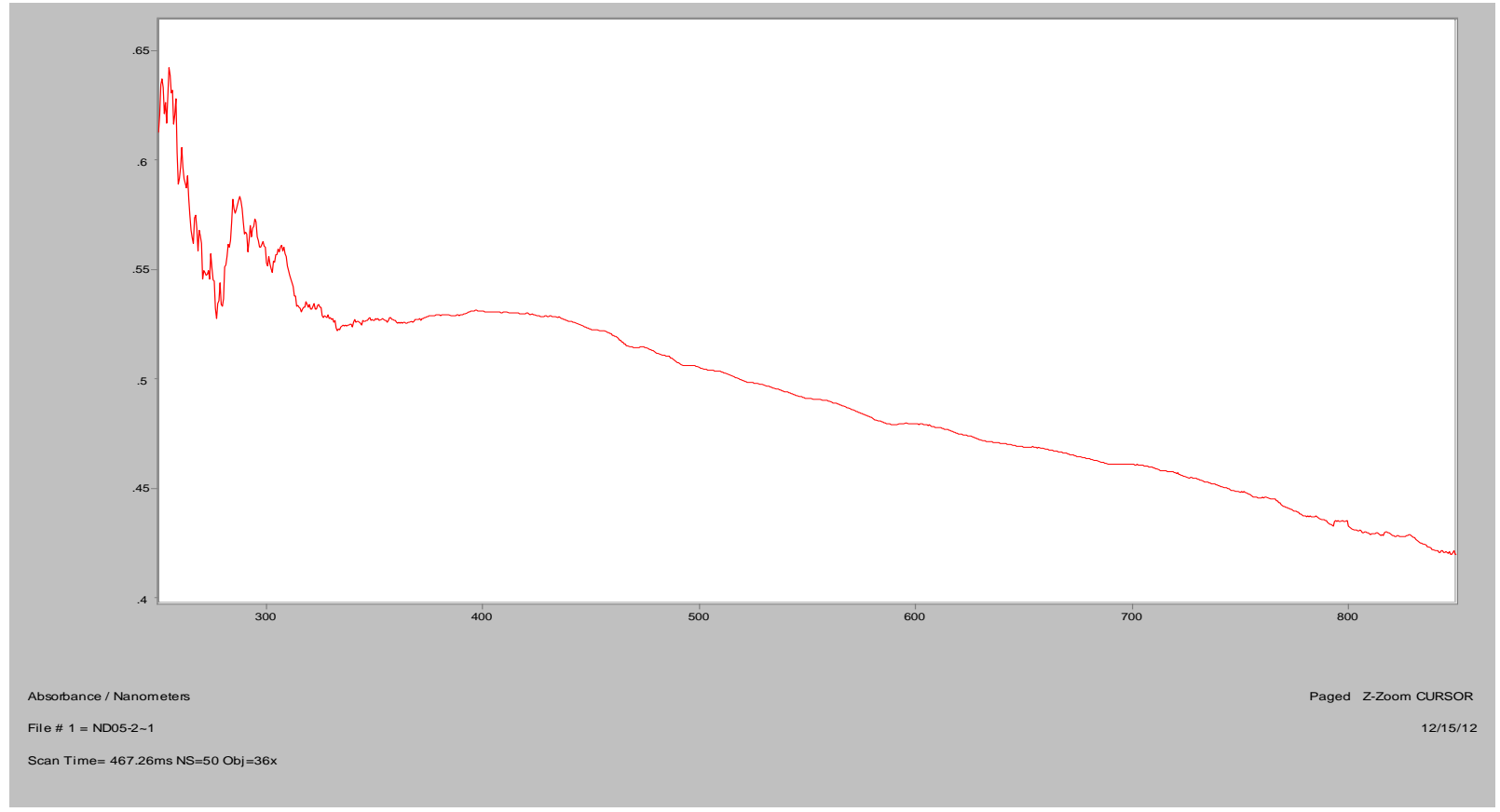

Figure 10: ND 0.5 filter on the CRAIC ${ }^{\circledR} Q D I Q D S I I^{\mathrm{TM}}$ Stereomicrospectrophotometer

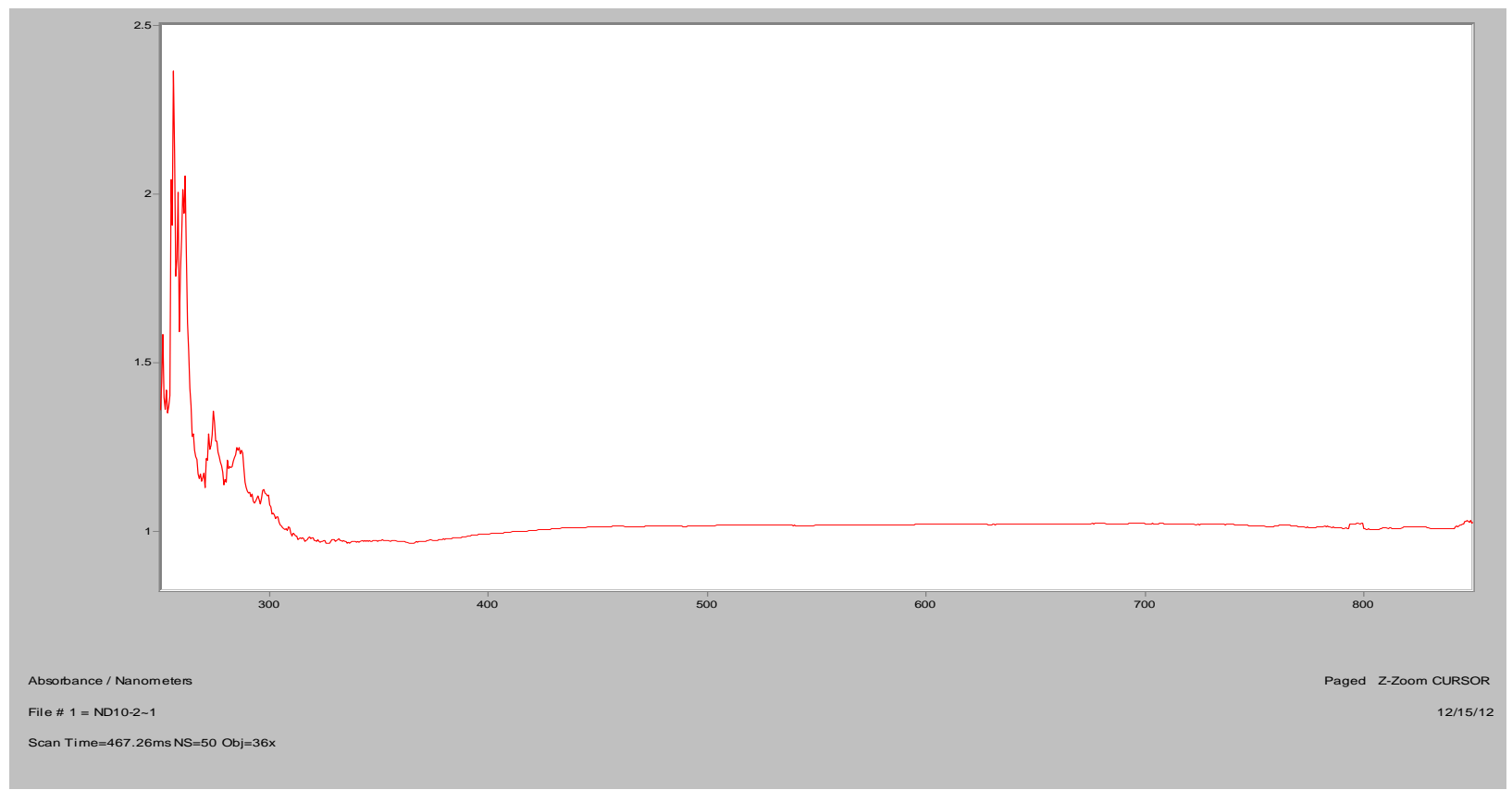

Figure 11: ND 1.0 filter on the CRAIC ${ }^{\circledR} Q D I Q D S$ II ${ }^{\mathrm{TM}}$ Stereomicrospectrophotometer 


\begin{tabular}{|l|l|l|l|}
\hline NIST Peak & MSP Value & Difference & Passed? \\
\hline 360.2 & 359.4 & 0.8 & Yes \\
\hline 418.5 & 417.2 & 1.3 & Yes \\
\hline 445.8 & 445.5 & 0.3 & Yes \\
\hline 536.0 & 535.8 & 0.2 & Yes \\
\hline 637.2 & 636.5 & 0.7 & Yes \\
\hline
\end{tabular}

Table 2: The NIST® certified wavelengths for the Holmium Oxide Filter

\begin{tabular}{|l|l|l|l|}
\hline NIST Peak & MSP Value & Difference & Passed? \\
\hline 441.2 & 440.9 & 0.3 & Yes \\
\hline 513.5 & 512.9 & 0.6 & Yes \\
\hline 684.4 & 683.5 & 0.9 & Yes \\
\hline 806.8 & 806.2 & 0.6 & Yes \\
\hline
\end{tabular}

Table 3: The NIST® certified wavelengths for the Didymium Filter

\begin{tabular}{|l|l|l|l|l|}
\hline Wavelength & NIST & MSP & Difference & Passed? \\
\hline 400 & 0.106 & 0.109 & -0.003 & Yes \\
\hline 500 & 0.095 & 0.093 & 0.002 & Yes \\
\hline 635 & & & & Yes \\
\hline
\end{tabular}

Table 4: The NIST® certified wavelengths for the ND 0.1Filter 


\begin{tabular}{|l|l|l|l|l|}
\hline Wavelength & NIST & MSP & Difference & Passed? \\
\hline 400 & 0.524 & 0.534 & -0.010 & Yes \\
\hline 500 & 0.510 & 0.509 & 0.001 & Yes \\
\hline 635 & 0.474 & 0.476 & -0.002 & Yes \\
\hline
\end{tabular}

Table 5: The NIST® certified wavelengths for the ND 0.5 Filter

\begin{tabular}{|l|l|l|l|l|}
\hline Wavelength & NIST & MSP & Difference & Passed? \\
\hline 400 & 0.977 & 0.996 & -0.019 & Yes \\
\hline 500 & 1.008 & 1.021 & -0.013 & Yes \\
\hline 635 & & & & Yes \\
\hline
\end{tabular}

Table 6: The NIST® certified wavelengths for the ND 1.0 Filter 


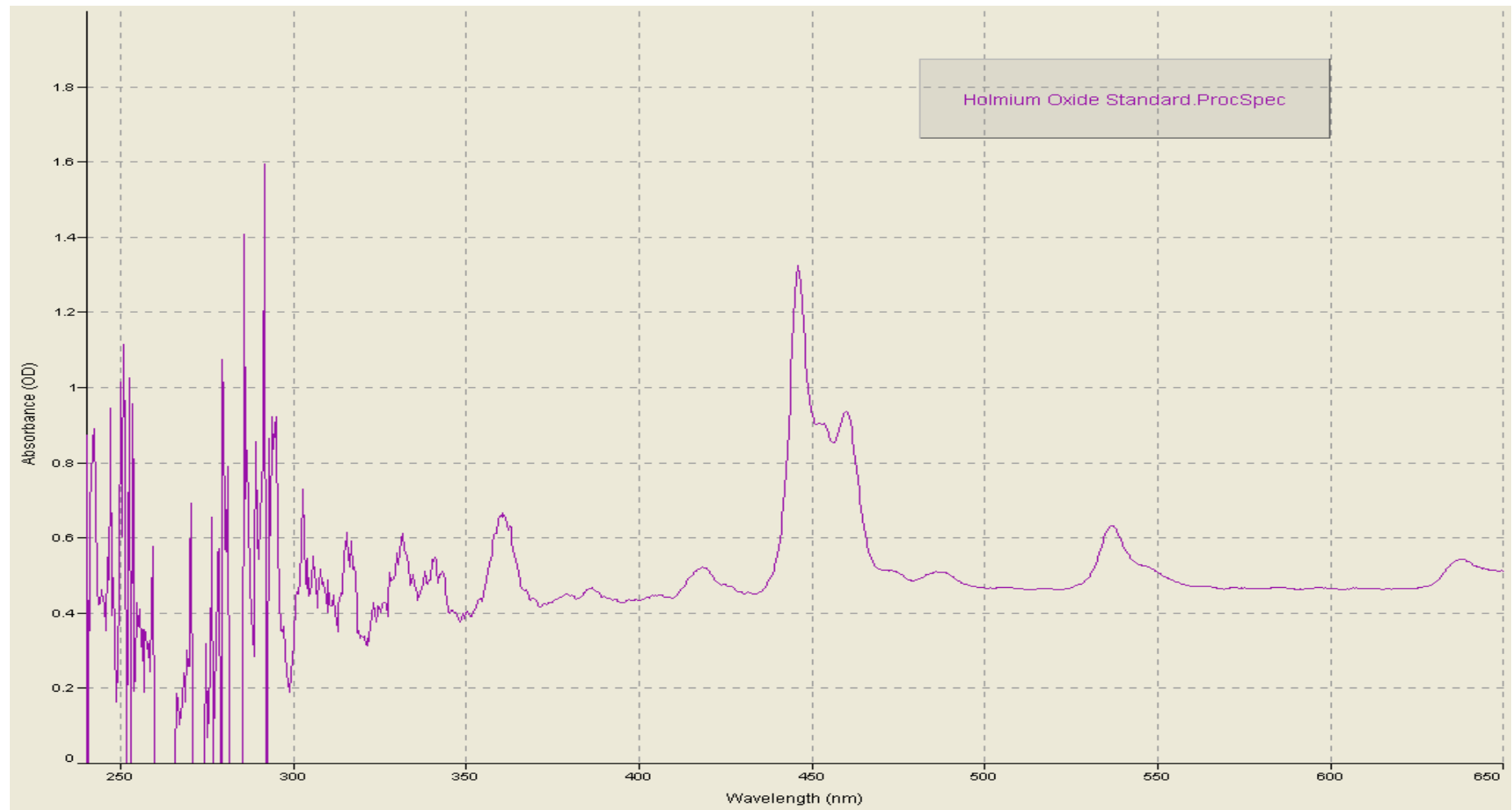

Figure 12: Holmium Oxide filter on the CRAIC® QDI 1000 Microspectrophotometer

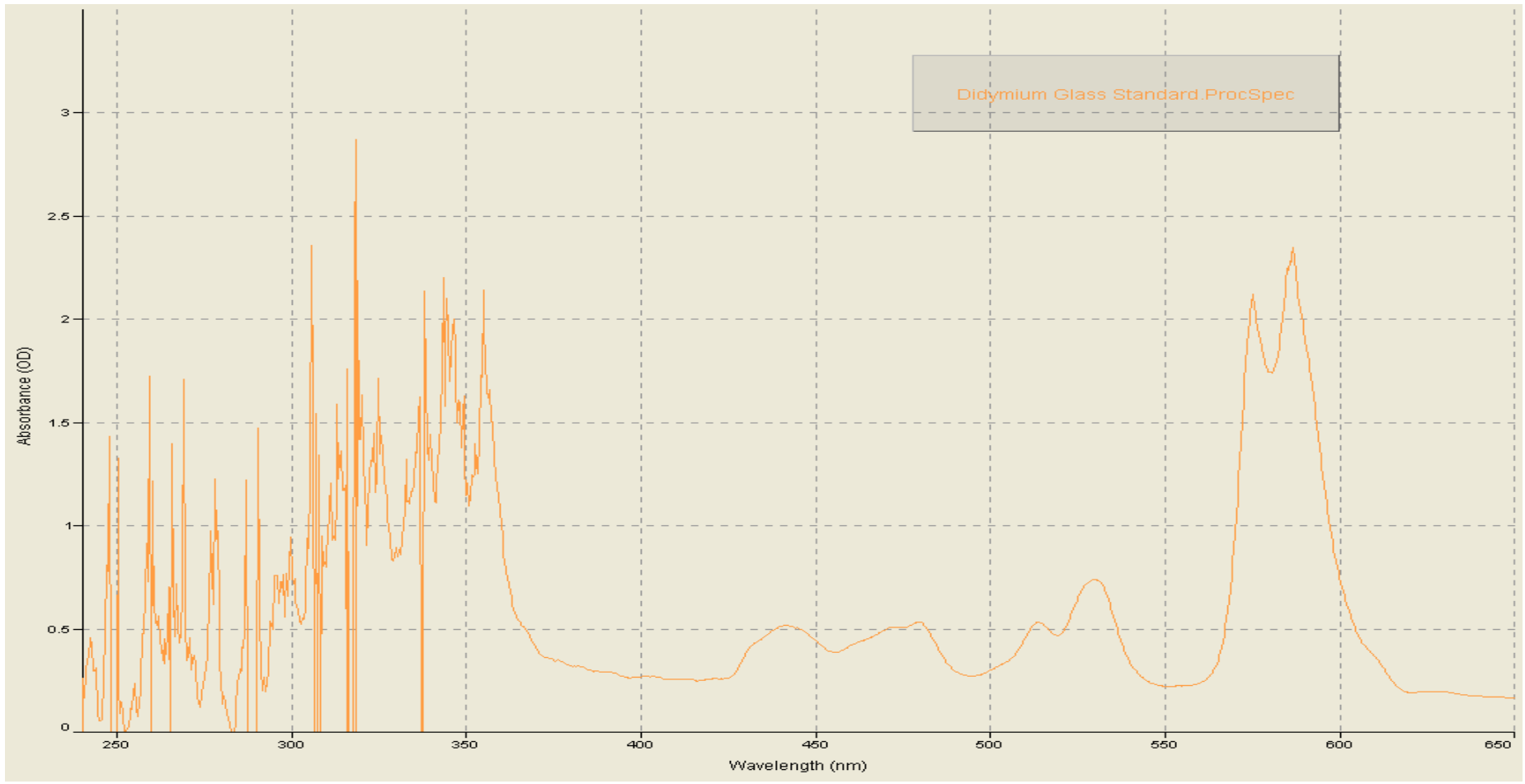

Figure 13: Didymium filter on the CRAIC® QDI 1000 Microspectrophotometer 


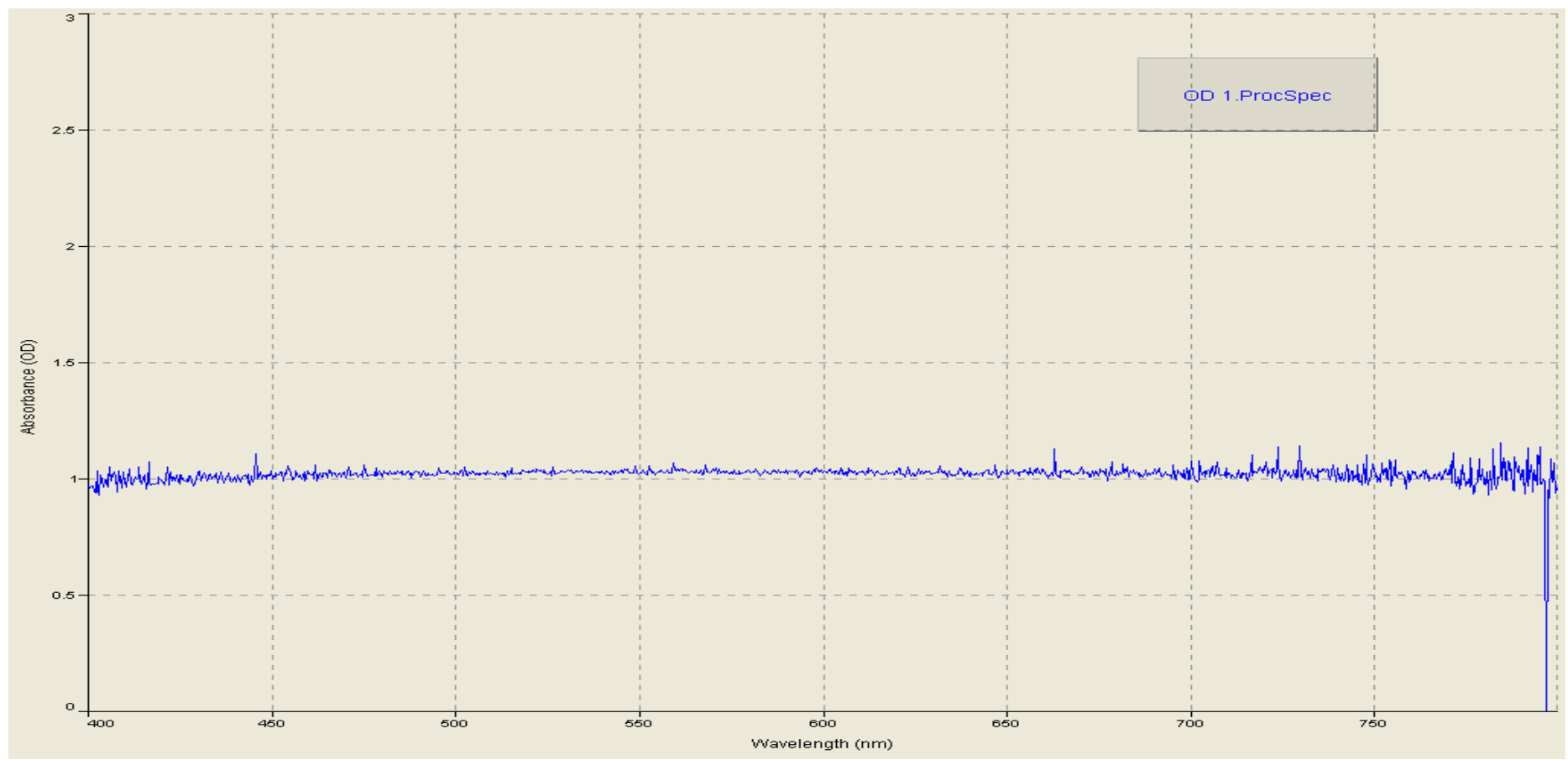

Figure 14: ND 0.1 filter on the CRAIC® QDI 1000 Microspectrophotometer

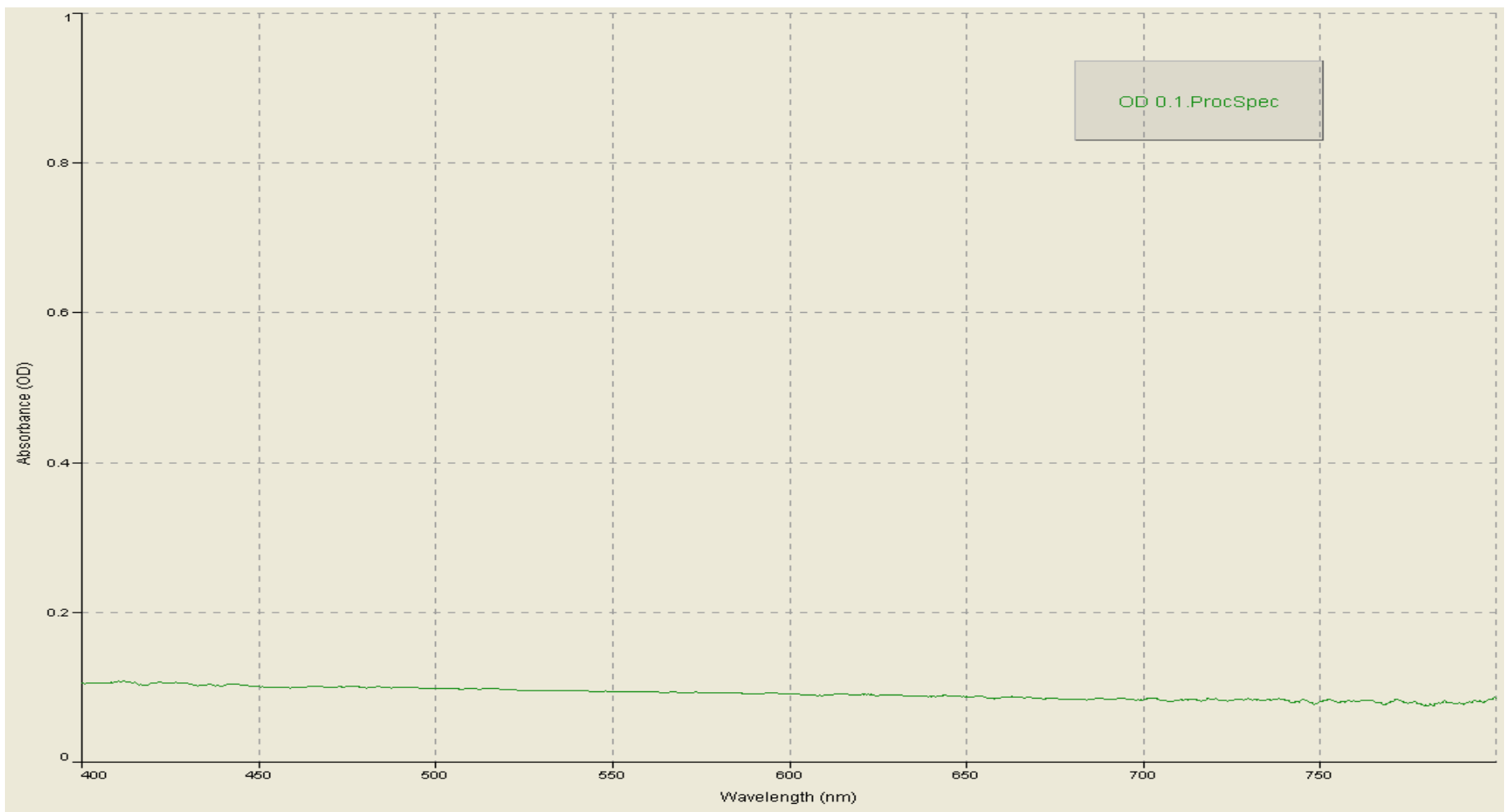

Figure 15: ND 0.5 filter on the CRAIC® QDI 1000 Microspectrophotometer 


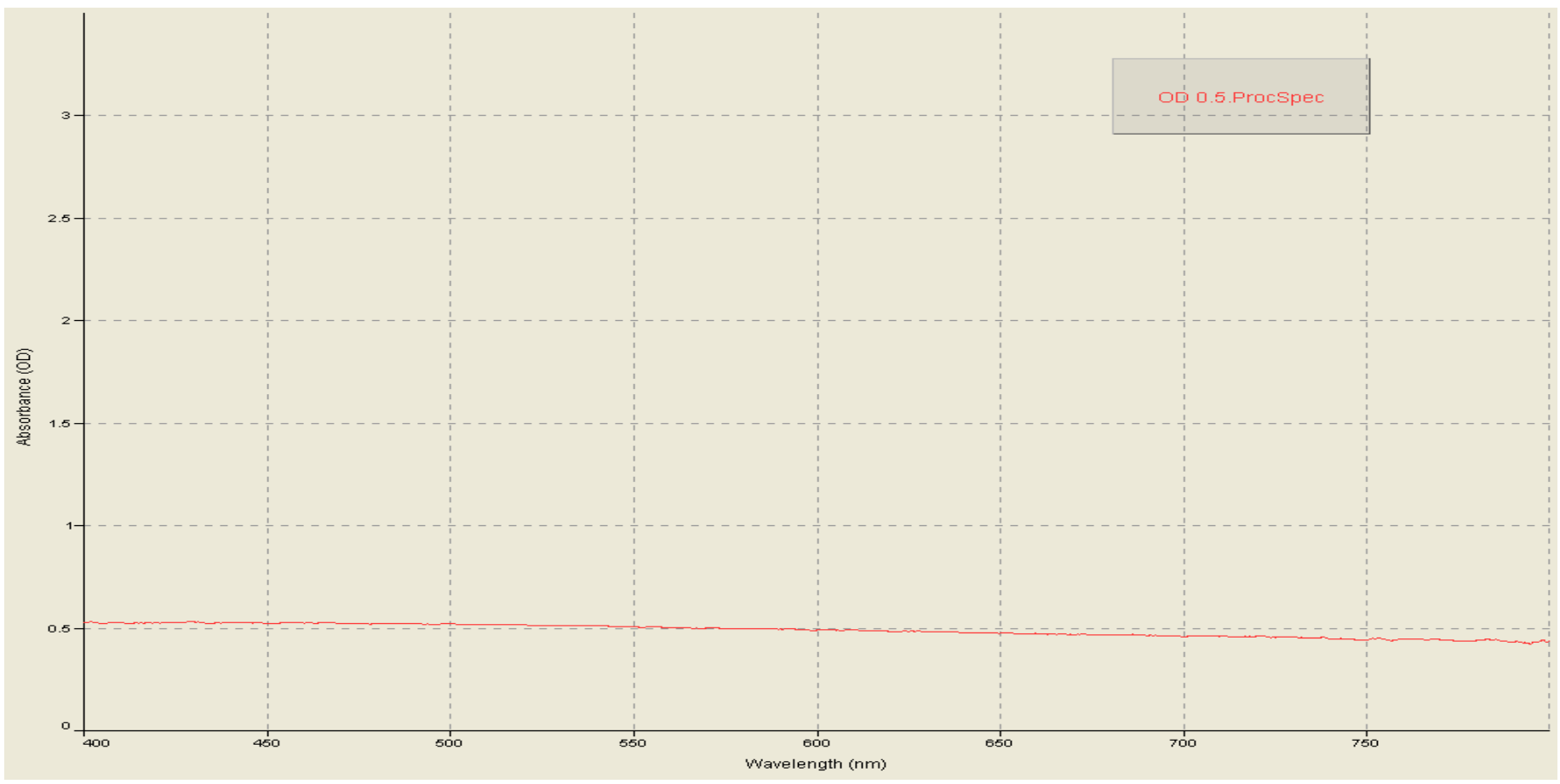

Figure 16: ND 1.0 filter on the CRAIC@ QDI 1000 Microspectrophotometer

When looking at the spectra that were collected for the wavelength and photometric filters on the CRAIC® QDI 1000 Microspectrophotometer, there is noise being produced from $250 \mathrm{~nm}$ to $350 \mathrm{~nm}$. This noise is produced here because the glass microscopic slide does not absorb UV light, which is from $20 \mathrm{~nm}$ to $400 \mathrm{~nm}$ in the visible light spectrum. For the QDI QDS II ${ }^{\mathrm{TM}}$ Stereomicrospectrophotometer, a sulfate standard was required in order to collect the reference and dark scans. To prepare this standard, Fisherbrand ${ }^{\circledR}$ Aluminum sulfate, $\mathrm{Al}_{2}\left(\mathrm{SO}_{4}\right)_{3}$, and Fisherbrand $®$ Barium nitrate, $\mathrm{Ba}\left(\mathrm{NO}_{3}\right)_{2}$ was obtained from Clark Hall of West Virginia University®. The standard was prepared by making .01 M solution using .059 g of $\mathrm{A}_{2}\left(\mathrm{SO}_{4}\right)_{3}$ and $.026 \mathrm{~g}$ of $\mathrm{Ba}\left(\mathrm{NO}_{3}\right)_{2}$. The resulting powders were placed into two different $100 \mathrm{~mL}$ beakers. Ten $\mathrm{mL}$ of distilled water was added to each beaker and mixed until dissolved. A filter was inserted in a plastic funnel and placed into a new $100 \mathrm{~mL}$ beaker. The two solutions were mixed and poured into the filter paper until all the liquid had passed through. The filter paper was then placed into an oven at $120^{\circ} \mathrm{C}$ for two hours for drying. The powder left on the filter paper was scraped onto the paper, placed upon a concave dish and then was pressed and flattened in the 
center of the dish. These steps were repeated to allow for a sufficient amount of prepared standard.

$$
\begin{aligned}
& \frac{594 \mathrm{~g}}{\mathrm{~mol}} \times \frac{.01 \mathrm{~mol}}{\mathrm{~L}} \times \frac{1 \mathrm{~L}}{1000 \mathrm{~mL}} \times 10 \mathrm{~mL}=.0594 \mathrm{~g} \text { of Aluminum sulfate } \\
& \frac{261.34 \mathrm{~g}}{\mathrm{~mol}} \times \frac{.01 \mathrm{~mol}}{\mathrm{~L}} \times \frac{1 \mathrm{~L}}{1000 \mathrm{~mL}} \times 10 \mathrm{~mL}=.0261 \mathrm{~g} \text { of Barium nitrate } \\
& \frac{594 \mathrm{~g}}{\mathrm{~mol}} \times \frac{.1 \mathrm{~mol}}{\mathrm{~L}} \times \frac{1 \mathrm{~L}}{1000 \mathrm{~mL}} \times 10 \mathrm{~mL}=.594 \mathrm{~g} \text { of Aluminum sulfate } \\
& \frac{261.34 \mathrm{~g}}{\mathrm{~mol}} \times \frac{1 \mathrm{~mol}}{\mathrm{~L}} \times \frac{1 \mathrm{~L}}{1000 \mathrm{~mL}} \times 10 \mathrm{~mL}=.261 \mathrm{~g} \text { of Barium nitrate }
\end{aligned}
$$

Equation 4: Determination of .01 $\mathrm{M}$ and .10 M sulfate standard

\section{Experimental}

Phases and Methods

\section{Phase I}

For preliminary examination of the 2 by 2 inch squares of every t-shirt, the QDI QDS II ${ }^{\mathrm{TM}}$ Stereomicrospectrophotometer was used. This was accomplished by placing each square on the stage of the instrument. A spectrum was collected of each of the Munsell chips that best matched each color of the squares, represented in Table 1. The Munsell Chip was placed on the stage of the instrument. A dark scan and a reference scan were collected before the sample spectrum. The absorbance and wavelength values were recorded and plotted into Microsoft® Excel for every spectra $400 \mathrm{~nm}$ to $800 \mathrm{~nm}$, revealing an integration time of $77.04 \mathrm{~ms}$ and an average scan of 20. To properly compare the Munsell Chips to their respective color t-shirt squares, the absorbance and wavelength values that were obtained from the QDI QDS IITM Stereomicrospectrophotometer were plotted against one another to determine if their minimum 
and maximum wavelength values fell into their respected color region in the visible light spectrum.

\section{Phase II}

The Ocean Optics® Spectral Suites software had never previously been used before coupled with the QDI 1000 Microspectrophotometer. A key factor before using the software was the required validation in order to obtain the best possible spectrum. This was accomplished by fluctuating three different settings in the software previously mentioned, which are the average scans, the boxcar width and the integration time. The sample Kelly Green A_F was used for this part of the experiment. The instrument was permitted to warm up for 30 minutes before any spectra were collected. The Kelly Green A_F slide was placed on the stage of the instrument. A dark and reference scan was collected and saved before colleting every spectra. For the first experiment, the boxcar width was changed while the average scans were kept constant at 20 and the integration time was kept constant at 100ms. The boxcar width was set to $1,3,5,7,9,11,15$, 25, 50, 100 and 150. For the second experiment, the average scans were changed while the integration time was kept constant at $100 \mathrm{~ms}$ and the boxcar width was kept constant at 5 . The average scans were set to 2, 5, 10, 15, 20, 30, 50 and 100. For the third part of this experiment, the integration time was changed while the boxcar width was kept constant at 5 and the average scans were kept constant at 20. The integration time was set to $10 \mathrm{~ms}, 20 \mathrm{~ms}, 30 \mathrm{~ms}, 50 \mathrm{~ms}, 100$ ms, $200 \mathrm{~ms}, 300 \mathrm{~ms}, 500 \mathrm{~ms}$ and $1000 \mathrm{~ms}$. A spectrum was collected using wavelengths from $380 \mathrm{~nm}$ to $780 \mathrm{~nm}$ and absorbance from 0 to 1 . These values were plotted into Microsoft® Excel for each different variation in absorbance mode. The least squares method was used for all three sets of data to determine which average scan, boxcar width and integration time had the minimal deviations squared from the data sets that were collected. An example of the average scan calculation is shown below. 


$$
\text { Average scans: }=\left(A_{\max \operatorname{scan}}-A_{\operatorname{scan} x}+k\right)^{2}
$$

\section{Equation 5: Calculation of the Least Squares Method}

The absorbance values that were obtained from the highest number of average scans were used as the reference spectrum. The rest of the average scans $(5,10,15,20,30,50$ and 100) were subtracted from the absorbance values of the reference spectrum. The sum of the squared absorbance values was then calculated. The Solver ${ }^{\circledR}$ add in from Microsoft ${ }^{\circledR}$ Excel was used to adjust the baseline to minimize the sum of the squares. Solver® uses an objective of the sum of the squares and minimizes by varying the value, $\mathrm{k}$. These steps were repeated for the boxcar width variation and the integration time variation.

\section{Phase III}

For this phase of the experiment, a time trial was completed to determine exactly how long the QDI 1000 Microspectrophotometer's 75W Xenon lamp source needed to warm up before use. This was done by using the Kelly Green A_F slide. First, a spectrum was collected every minute for an hour. An integration time of $100 \mathrm{~ms}$, an average scan of 20 and a boxcar width of 5 were used to collect every spectrum based on the results of that were obtained from the previous phase. A dark scan and a reference scan were collected before the first spectrum was gathered of the fibers. A spectrum was collected using wavelengths from $380 \mathrm{~nm}$ to $780 \mathrm{~nm}$ and absorbance from 0 to 1 . These values were plotted into Microsoft ${ }^{\circledR}$ Excel for each different time setting in absorbance mode. Second, a dark scan, a reference scan and a sample spectrum of Kelly Green A_F was collected every minute for an hour. The same settings were used for this part of the experiment. For both parts of this phase, a certain wavelength was chosen and the number of minutes at which the absorbance values were collected was plotted.

\section{Phase IV}

During this phase of the study, 200 slides that represented the of fibers from the front, back, armpit and sleeve of each color repeated for all ten t-shirts was prepared to allow for the 
intra-garment comparison. Each slide was placed on the stage of the CRAIC® QDI 1000 Microspectrophotometer. An integration time of $100 \mathrm{~ms}$, an average scan of 20 and a boxcar width of 5 were used to collect every spectrum. A dark scan and a reference scan were collected

before the spectrum was collected of the fibers. A spectrum for each sample was collected using wavelengths from $380 \mathrm{~nm}$ to $780 \mathrm{~nm}$ and absorbance from 0 to 1 . Ten spectra were collected from a different part of the fiber for every slide that was prepared. The average absorbance, the standard deviation and the upper and lower confidence limits (UCL and LCL) were calculated at the $95 \%$ confidence level for each of the four sections of all 10 shirts of every color. The upper and lower confidences limits were calculated based on the equation below. 1.960 was chosen from the critical values for $\mathrm{t}$ - distribution, because the degree of freedom (n-1) was greater than 500. The program RandFriends ${ }^{\circledR}$ was used to generate a XY conditioning graph to display the absorbance values from the different sections of each shirt. An Add-Ins tab on the toolbar of Microsoft $\circledast$ Excel was used to start the R® program and the R® Commander. The UCL and the LCL for front, back, sleeve and armpit of all five different colors were also calculated and the resulting data was graphed using Microsoft $\circledast$ Excel.

Upper Confidence Limit:

$$
=A_{\text {Average }}+1.960 \times \text { StandardDeviation }_{\text {Average }}
$$

Lower Confidence Limit:

$$
=A_{\text {Average }}-1.960 \times \text { StandardDeviation }_{\text {Average }}
$$

Equation 6: Calculating the Upper and Lower Confidence Bounds at 95\% confidence

\section{Phase V}

In Phase V of this study, a fiber from the front section of every color t-shirt was cut into three different sections providing 150 slides to demonstration the inter-garment comparison. Each slide was placed on the stage of QDI 1000 Microspectrophotometer. An integration time of $100 \mathrm{~ms}$, an average scan of 20 and a boxcar width of 5 were used to collect every spectrum. A 
dark scan and a reference scan were collected before the spectrum was collected of the fibers. A spectrum for each sample was collected using wavelengths from $380 \mathrm{~nm}$ to $780 \mathrm{~nm}$ and absorbance from 0 to 1 . Ten spectra were collected from a different part of the fiber for every slide that was prepared. The average absorbance, the standard deviation and the upper and lower confidence limits were calculated at the $95 \%$ confidence level for section 1, section 2 and section 3 for all ten shirts of every color. 1.960 was still used for the critical values for t- distribution and the program RandFriends ${ }^{\circledR}$ was used to generate a XY conditioning graph to display the absorbance values from the different sections of each shirt. An Add-Ins tab on the toolbar of Microsoft $₫$ Excel was used to start the R® program and the R® Commander. The UCL and the LCL for section 1, 2 and 3 of all five different colors were also calculated and the resulting data was graphed using Microsoft® Excel.

\section{$\underline{\text { Results }}$}

\section{Phase 1}

For the preliminary determination if there was any differentiation between the Munsell chips and their respective color t-shirt squares, the maximum and minimum absorbance were recorded, as well as the wavelength which these minimums and maximums occurred for each of the spectra. Also, the absorbance values for the Munsell chips for each color were also plotted against the average absorbance values of all ten of the t-shirt squares using a wavelength from $400 \mathrm{~nm}$ to $700 \mathrm{~nm}$. Furthermore, the absorbance spectra that were collected for each of the Munsell chips and the t-shirt squares and the wavelength and absorbance values are displayed in Appendix A. 


\section{Gold}

Photograph 5: Gold 10YR 7, 14 Munsell Chip

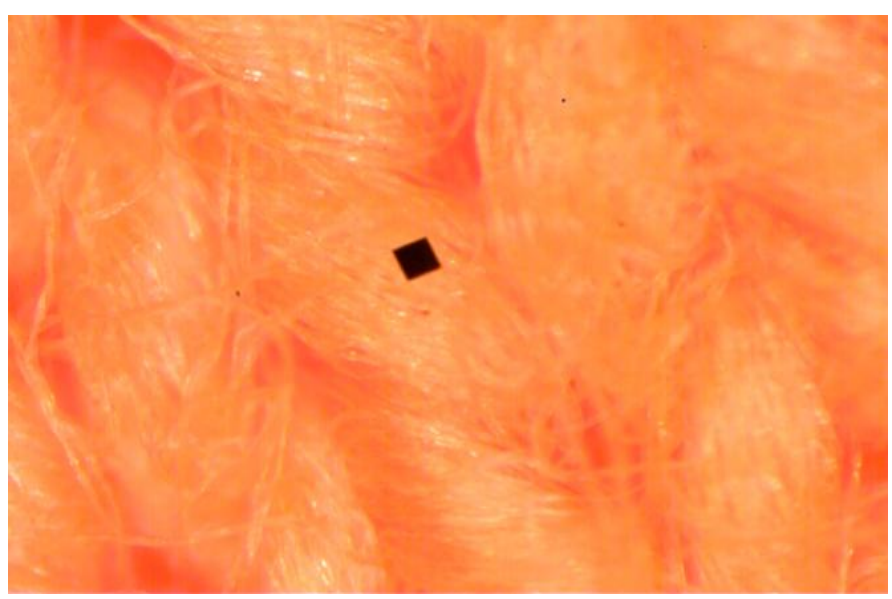

Photograph 6: Gold A_A square

\begin{tabular}{|c|c|c|c|c|}
\hline & Wavelength (nm) & Minimum Abs & Wavelength (nm) & Maximum Abs \\
\hline Gold 10 YR & 620 & 0.00 & 400.21 & 0.889 \\
\hline 7,14 & & & & 0.882 \\
\hline Gold A & 625 & -0.118 & 440.08 & 0.997 \\
\hline Gold C & 660 & -0.001 & 441.91 & 1.125 \\
\hline Gold D & 646 & -0.017 & 443.16 & 1.046 \\
\hline Gold E & 646 & -0.003 & 430.5 & 1.127 \\
\hline Gold F & 658 & -0.007 & 400.21 & 1.161 \\
\hline Gold G & 661 & -0.001 & 400.21 & 1.161 \\
\hline Gold H & 662 & -0.014 & 400.21 & 1.154 \\
\hline Gold I & 661 & -0.014 & 400.21 & 1.273 \\
\hline Gold J & 642 & -0.031 & 400.21 & \\
\hline
\end{tabular}

Table 7: Minimum and Maximum wavelength and absorbance values for Gold 
Photograph 5 and Photograph 6 show a side by side comparison of the Gold 10YR 7, 14 Munsell chip that was chosen out of the Munsell Book of Color and the first gold armpit t-shirt square. This view shows how closely the fibers from the t-shirt square were able to be matched to the chips. Table 7 depicts the minimum and maximum absorbance values that were recorded at their respective wavelength values for the Munsell chip and the gold t-shirt squares. The average absorbance was calculated for the front, back, sleeve and armpit. Those values were used to represent Gold A through Gold $\mathrm{J}$ in the table.

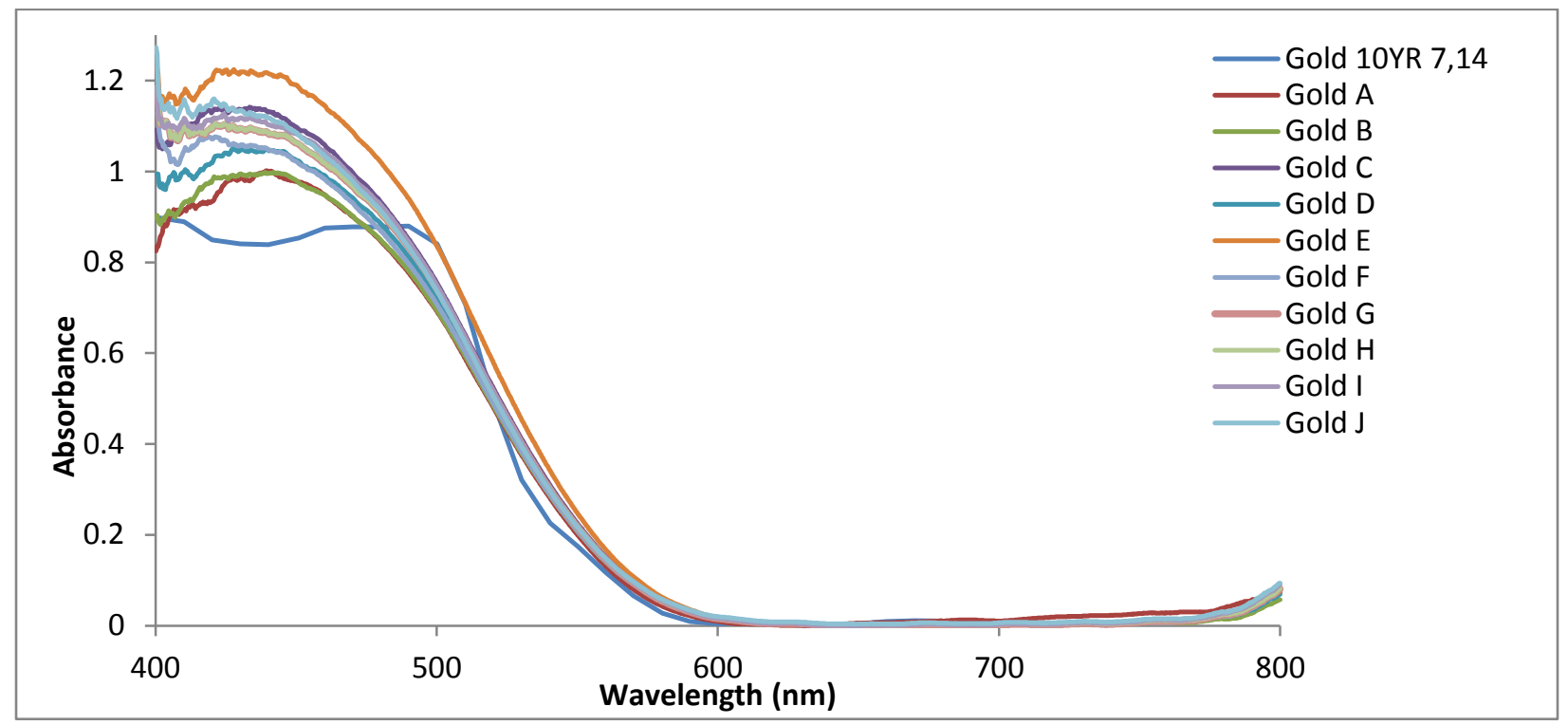

Figure 17: Gold 10YR 7, 14 vs. the Gold squares

In Figure 17, the average absorbance values for Gold A through Gold J were plotted against the absorbance values that were recorded for the Munsell chip. The wavelength and absorbance values that are collected by the instrument and are shown in the graph were collected from $400 \mathrm{~nm}$ to $700 \mathrm{~nm}$ incrementally every $.37 \mathrm{~nm}$. A linear backline correction was used to eliminate the negative absorbance values that were recorded by taking the lowest value and adding it to all the other absorbance values. 

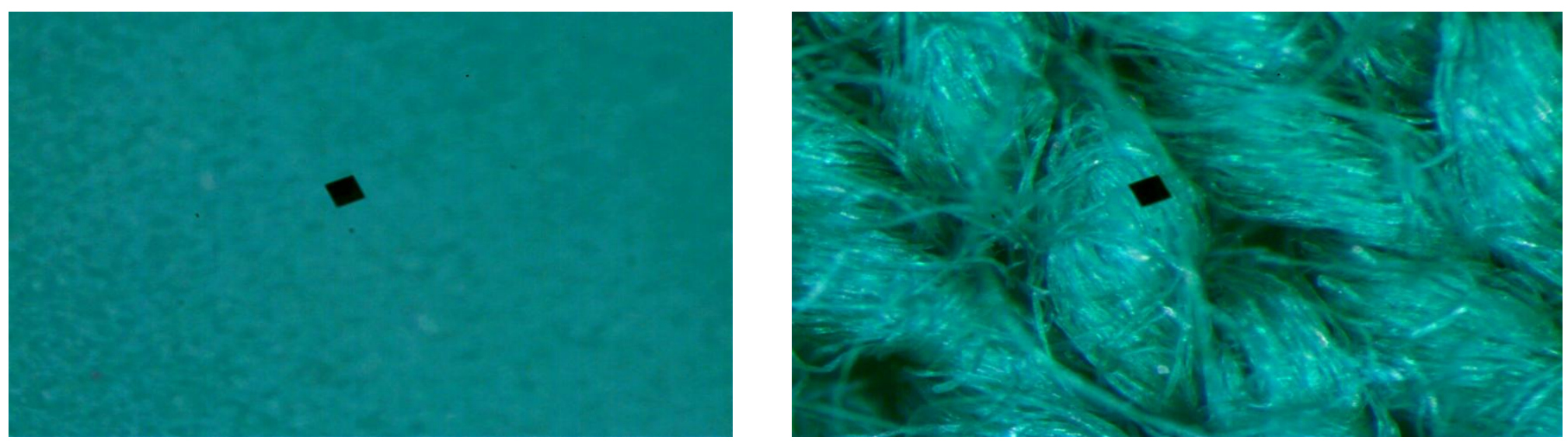

Photograph 7: Kelly Green 7.5G 4, 8 Munsell Chip $\quad$ Photograph 8: Kelly Green A_A square

\begin{tabular}{|c|c|c|c|c|}
\hline & Wavelength (nm) & Minimum Abs & Wavelength (nm) & Maximum Abs \\
\hline Kelly Green 7.5G & 506 & 0.166 & 718.19 & 1.280 \\
\hline 4,8 & & & 677.42 & 1.400 \\
\hline Kelly Green A & 507 & 0.005 & 682.65 & 1.301 \\
\hline Kelly Green C & 511 & 0.099 & 679.46 & 1.302 \\
\hline Kelly Green D & 508 & 0.073 & 686.24 & 1.221 \\
\hline Kelly Green E & 510 & 0.012 & 680.82 & 1.401 \\
\hline Kelly Green F & 508 & 0.050 & 687.93 & 1.302 \\
\hline Kelly Green G & 517 & 0.159 & 683.19 & 1.260 \\
\hline Kelly Green H & 513 & 0.079 & 678.10 & 1.304 \\
\hline Kelly Green I & 509 & 0.040 & 688.61 & 1.318 \\
\hline Kelly Green J & 510 & 0.089 & 683.19 & \\
\hline
\end{tabular}

Table 8: Minimum and Maximum absorbance and wavelength values for Kelly Green 
Photograph 7 and Photograph 8 show a side by side comparison of the Kelly Green 7.5 , 8 Munsell chip that was chosen out of the Munsell Book of Color and the first kelly green armpit t-shirt square. This view shows how closely the fibers from the t-shirt square were able to be matched to the chips. Table 8 depicts the minimum and maximum absorbance values that were recorded at their respective wavelength values for the Munsell chip and the kelly green t-shirt squares. The average absorbance was calculated for the front, back, sleeve and armpit. Those values were used to represent Kelly Green A through Kelly Green J in the table.

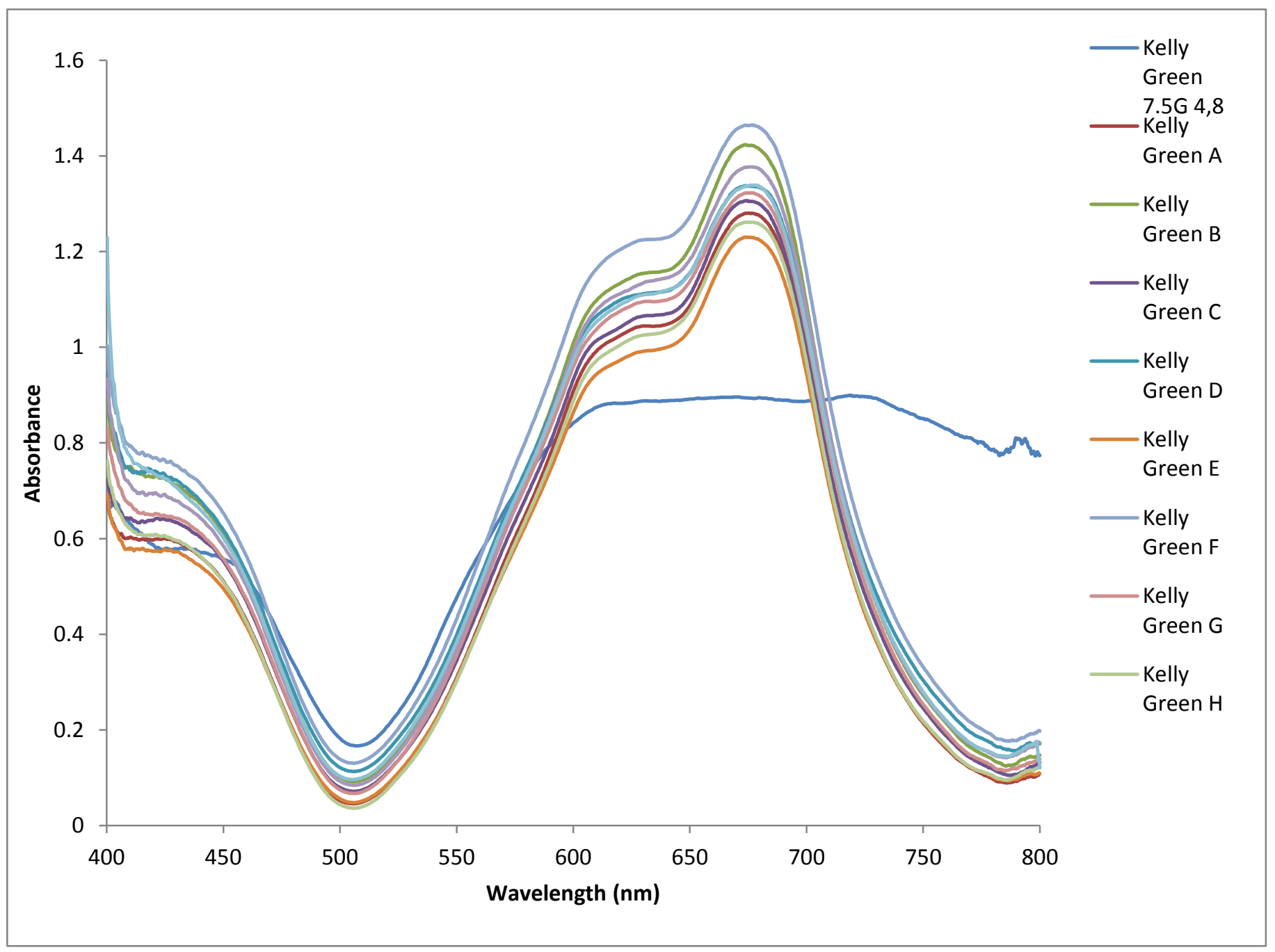

Figure 18: Kelly Green 7.5G 4, 8 vs. the Kelly Green squares 
In Figure 18, the average absorbance values for Kelly Green A through Kelly Green J were plotted against the absorbance values that were recorded for the Munsell chip. The wavelength and absorbance values that are collected by the instrument and are shown in the graph were collected from $400 \mathrm{~nm}$ to $700 \mathrm{~nm}$ incrementally every $.37 \mathrm{~nm}$.

Orange

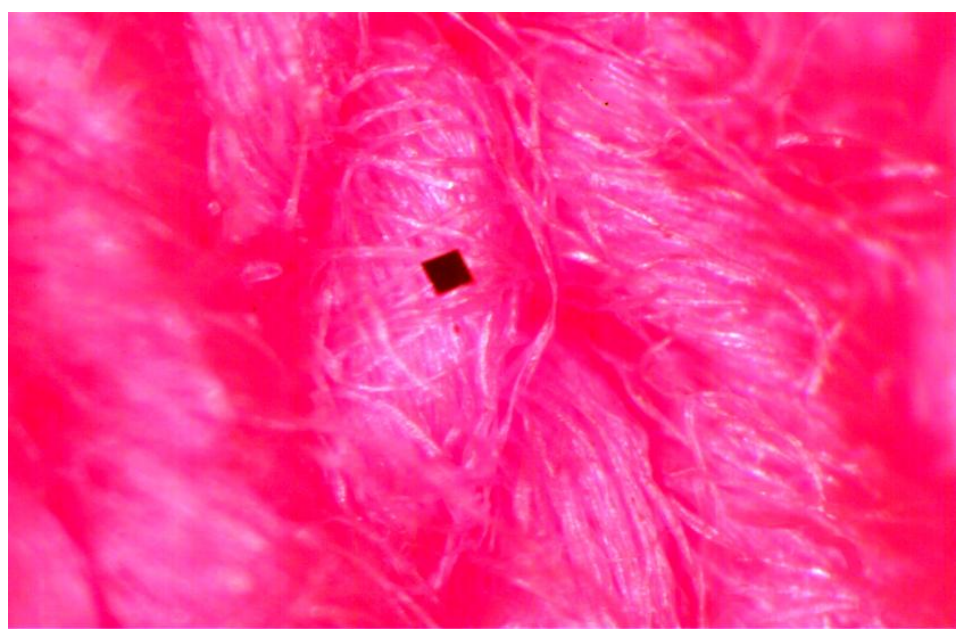

Photograph 9: Orange 2.5 YR 6, 14 Munsell Chip

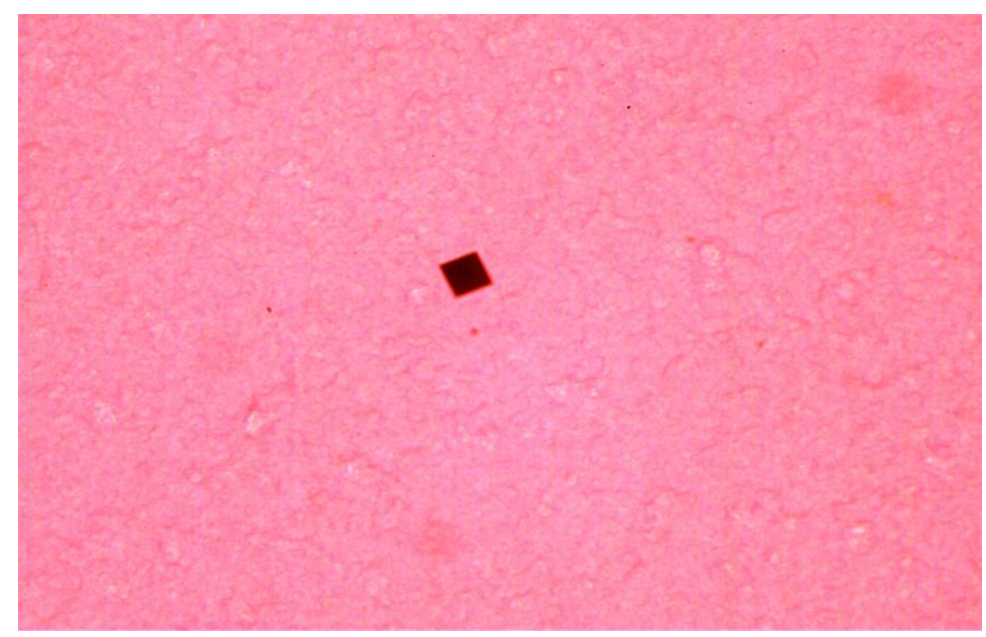

Photograph 10: Orange A_A square 


\begin{tabular}{|l|c|c|c|c|}
\hline & Wavelength (nm) & Minimum Abs & Wavelength (nm) & Maximum Abs \\
\hline Orange 2.5YR & 580 & 0.00 & 487 & 0.7401 \\
\hline Orange A & 598 & -0.163 & 512 & 0.922 \\
\hline Orange B & 598 & -0.169 & 508 & 1.000 \\
\hline Orange C & 598 & -0.168 & 506 & 1.085 \\
\hline Orange D & 597 & -0.170 & 504 & 1.001 \\
\hline Orange E & 597 & -0.168 & 514 & 1.006 \\
\hline Orange F & 599 & -0.166 & 532 & 1.050 \\
\hline Orange G & 596 & -0.169 & 511 & 0.960 \\
\hline Orange H & 589 & -0.141 & 509 & 1.170 \\
\hline Orange I & 584 & -0.295 & 503 & 0.800 \\
\hline Orange J & & & & \\
\hline
\end{tabular}

Table 9: Minimum and Maximum absorbance and wavelength values for Orange

Photograph 9 and Photograph 10 show a side by side comparison of the Orange 2.5YR 6, 14 Munsell chip that was chosen out of the Munsell Book of Color and the first orange armpit tshirt square. This view shows how closely the fibers from the t-shirt square were able to be matched to the chips. Table 9 depicts the minimum and maximum absorbance values that were recorded at their respective wavelength values for the Munsell chip and the orange t-shirt squares. The average absorbance was calculated for the front, back, sleeve and armpit. Those values were used to represent Orange A through Orange $\mathrm{J}$ in the table. 


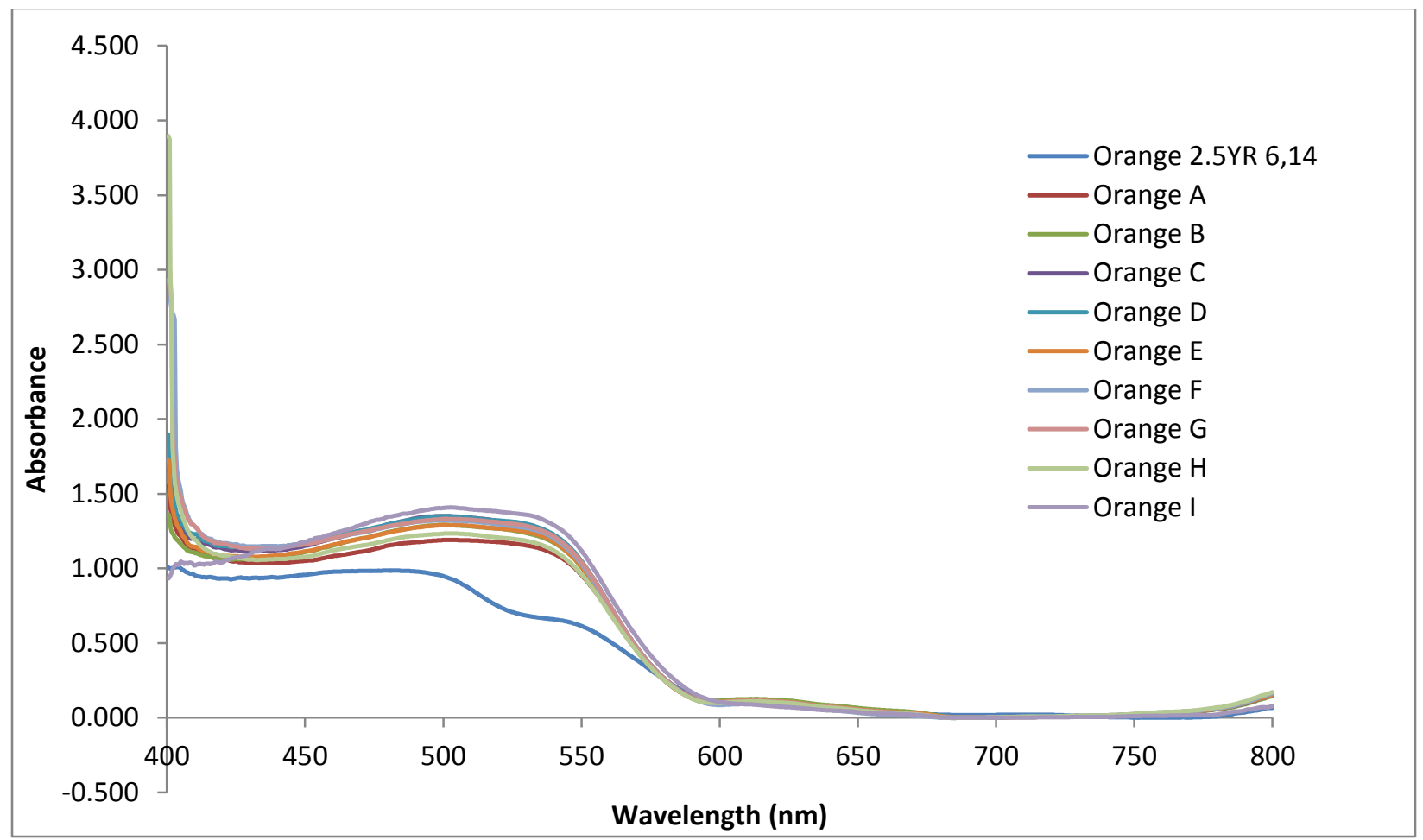

Figure 19: Orange 2.5YR 6, 14 Munsell chip vs. the Orange squares

In Figure 19, the average absorbance values for Orange A through Orange $\mathbf{J}$ were plotted against the absorbance values that were recorded for the Munsell chip. The wavelength and absorbance values that are collected by the instrument and are shown in the graph were collected from $400 \mathrm{~nm}$ to $700 \mathrm{~nm}$ incrementally every $.37 \mathrm{~nm}$. A linear backline correction was used to eliminate the negative absorbance values that were recorded by taking the lowest value and adding it to all the other absorbance values. 


\begin{tabular}{|l|c|c|c|c|}
\hline & Wavelength (nm) & Minimum Abs & Wavelength (nm) & Maximum Abs \\
\hline Red 5R 4, 14 & 622 & -0.118 & 545 & 0.875 \\
\hline Red A & 626 & -0.324 & 514 & 0.980 \\
\hline Red B & 626 & -0.331 & 520 & 0.937 \\
\hline Red C & 624 & -0.112 & 512 & 1.282 \\
\hline Red D & 627 & -0.320 & 512 & 0.986 \\
\hline Red E & 626 & -0.295 & 513 & 0.975 \\
\hline Red F & 625 & -0.287 & 514 & 1.011 \\
\hline Red G & 626 & -0.276 & 516 & 1.171 \\
\hline Red H & 627 & -0.304 & 518 & 1.020 \\
\hline Red I & 624 & -0.269 & 520 & 519 \\
\hline Red J & 622 & -0.282 & & 0.991 \\
\hline
\end{tabular}

Table 10: Minimum and Maximum absorbance and wavelength values for Red 
Photograph 11 and Photograph 12 show a side by side comparison of the Red 5R 4, 14 Munsell chip that was chosen out of the Munsell Book of Color and the first red armpit t-shirt square. This view shows how closely the fibers from the t-shirt square were able to be matched to the chips. Table 10 depicts the minimum and maximum absorbance values that were recorded at their respective wavelength values for the Munsell chip and the red t-shirt squares. The average absorbance was calculated for the front, back, sleeve and armpit. Those values were used to represent Red A through Red J in the table.

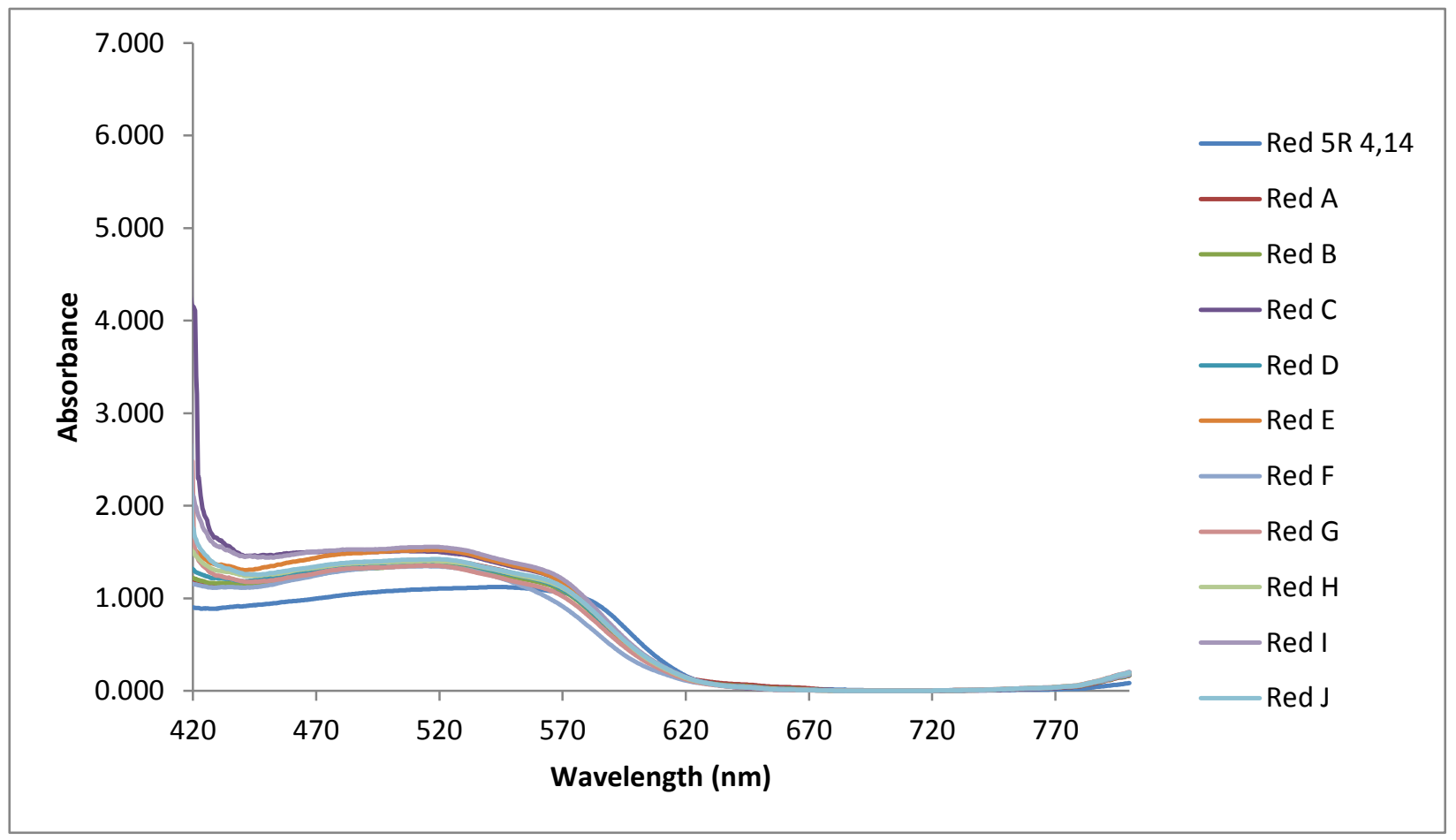

Figure 20: Red 5R 6, 14 Munsell chip vs. the Red squares

In Figure 20, the average absorbance values for Red A through Red J were plotted against the absorbance values that were recorded for the Munsell chip. The wavelength and absorbance values that are collected by the instrument and are shown in the graph were collected from 400 $\mathrm{nm}$ to $700 \mathrm{~nm}$ incrementally every $.37 \mathrm{~nm}$. A linear backline correction was used to eliminate the negative absorbance values that were recorded by taking the lowest value and adding it to all the other absorbance values. 


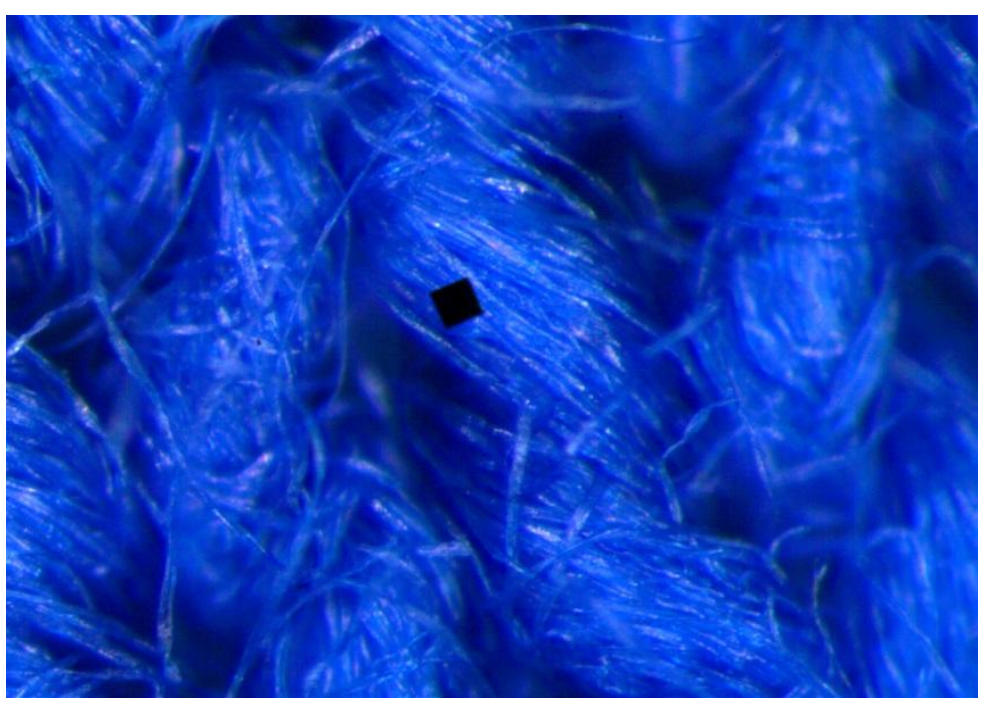

Photograph 14: Royal A_A square

\begin{tabular}{|l|c|c|c|c|}
\hline & Wavelength (nm) & Minimum Abs & Wavelength (nm) & Maximum Abs \\
\hline Royal 5PB 4, 12 & 459 & -0.141 & 612 & 0.828 \\
\hline Royal A & 450 & -0.331 & 612 & 0.704 \\
\hline Royal B & 446 & -0.320 & 610 & 0.699 \\
\hline Royal C & 446 & -0.268 & 609 & 0.747 \\
\hline Royal D & 447 & -0.314 & 609 & 0.745 \\
\hline Royal E & 445 & -0.289 & 612 & 0.642 \\
\hline Royal F & 477 & -0.301 & 612 & 0.731 \\
\hline Royal G & 446 & -0.330 & 627 & 0.951 \\
\hline Royal H & 448 & -0.293 & 612 & 0.735 \\
\hline Royal I & 445 & -0.219 & 613 & 611 \\
\hline Royal J & 446 & -0.295 & & 0.75 \\
\hline
\end{tabular}

Table 11: Minimum and Maximum wavelength and absorbance values for Royal 
Photograph 13 and Photograph 14 show a side by side comparison of the Royal 5 PB 4, 12 Munsell chip that was chosen out of the Munsell Book of Color and the first royal armpit tshirt square. This view shows how closely the fibers from the t-shirt square were able to be matched to the chips. Table 11 depicts the minimum and maximum absorbance values that were recorded at their respective wavelength values for the Munsell chip and the royal t-shirt squares. The average absorbance was calculated for the front, back, sleeve and armpit. Those values were used to represent Royal A through Royal J in the table.

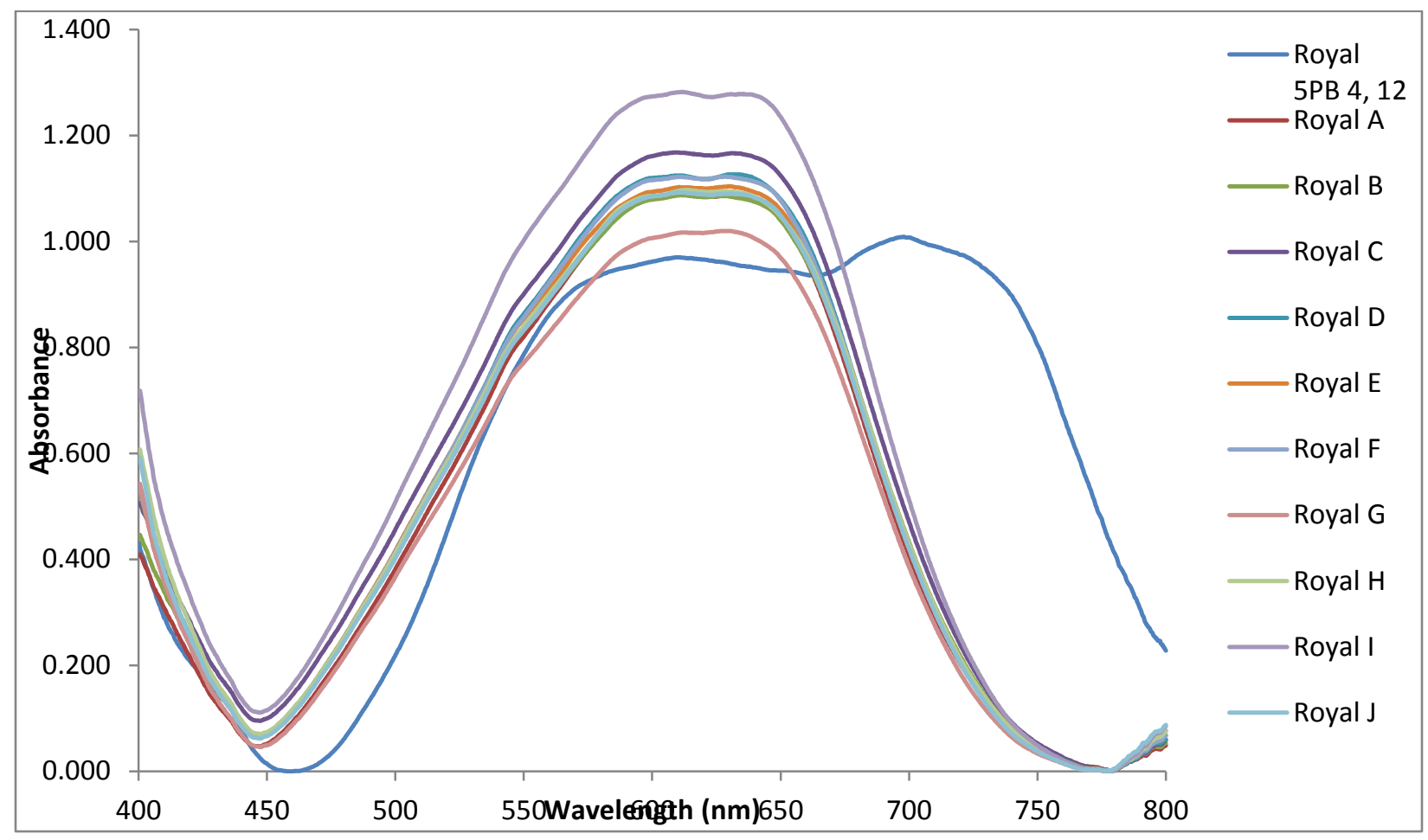

Figure 21: Royal 5PB 4, 12 Munsell chip vs. the Royal squares

In Figure 21, the average absorbance values for Royal A through Royal J were plotted against the absorbance values that were recorded for the Munsell chip. The wavelength and absorbance values that are collected by the instrument and are shown in the graph were collected from $400 \mathrm{~nm}$ to $700 \mathrm{~nm}$ incrementally every $.37 \mathrm{~nm}$. 
Phase II

Average Scans

To better determine the scans to average when collecting spectra using the Ocean Optics ${ }^{\circledR}$ software when coupled with the QDI 1000 Microspectrophotometer, spectra were collected from $400 \mathrm{~nm}$ to $700 \mathrm{~nm}$ incrementally every $.37 \mathrm{~nm}$ using average scans of 2, 5, 10, 15, 20, 30, 50 and 100, which can be seen in Figure 22 and Figure 23. The absorbance values that were recorded into Microsoft $®$ Excel along with the spectra that were collected can be viewed in Appendix A.

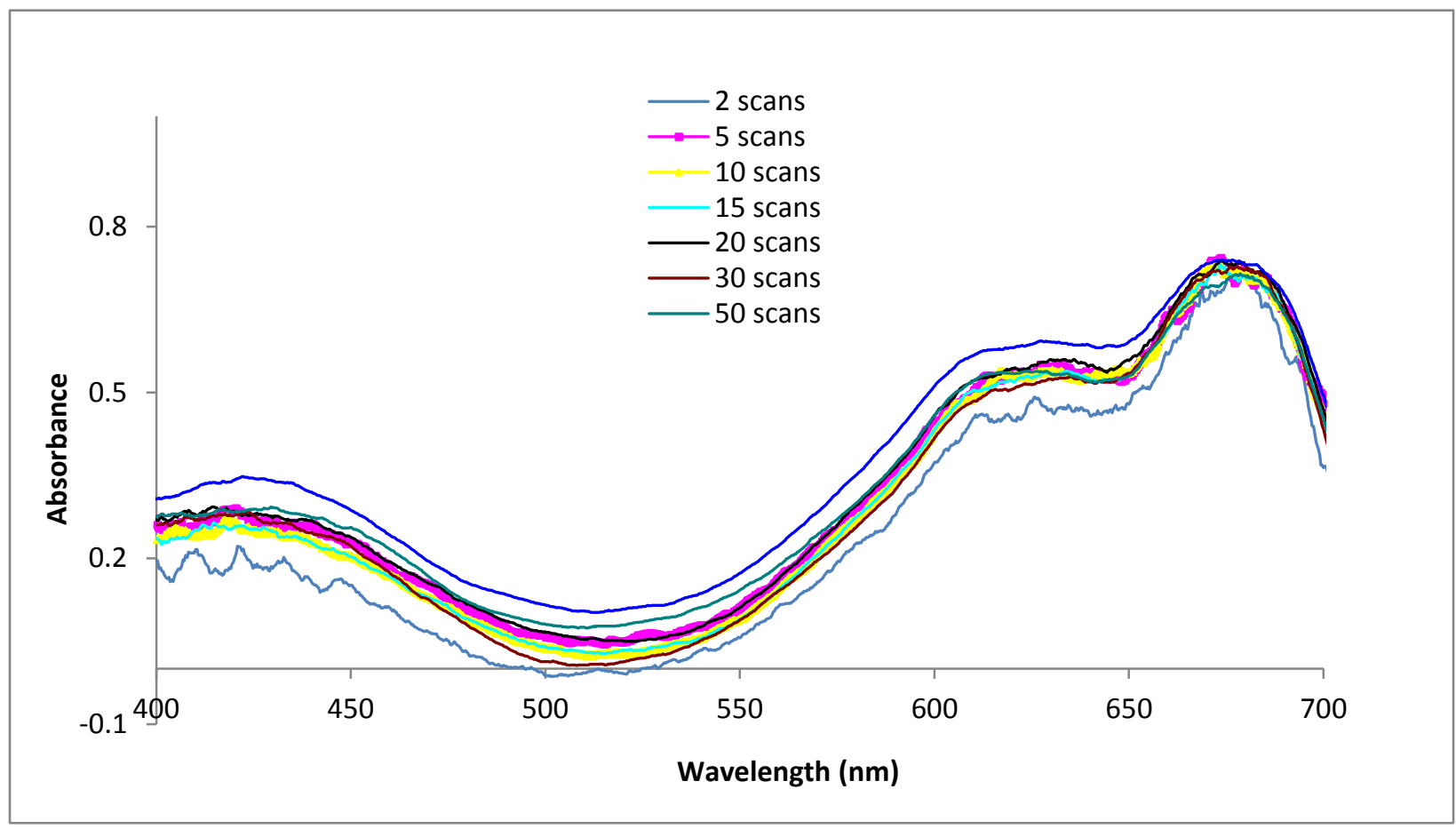

Figure 22: Average scan variation of Kelly Green $A_{-} F$ 


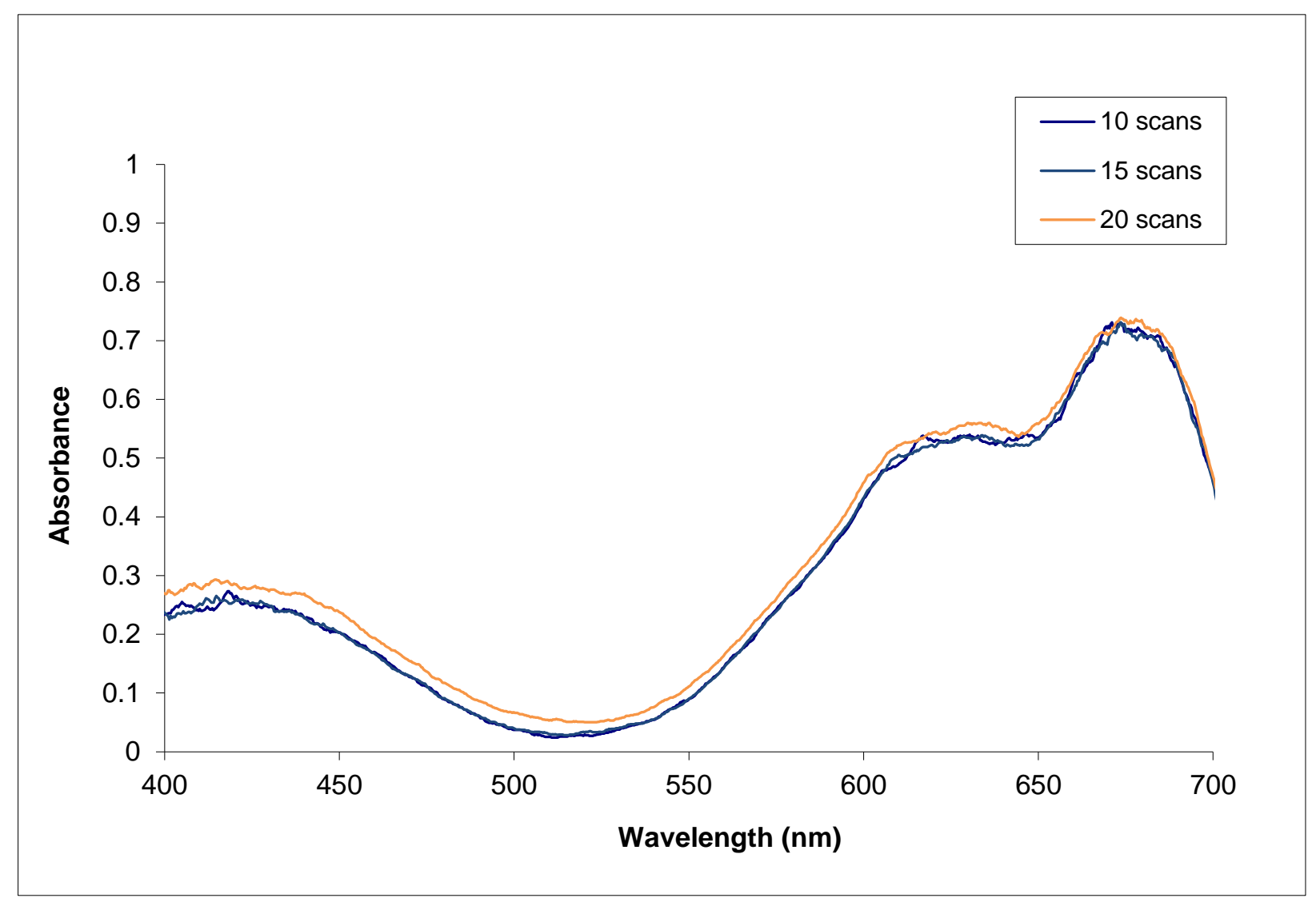

Figure 23: Average scan variation of Kelly Green A_F using scans 10, 15 and 20

Figure 24 show the results the new absorbance values that were calculated for the average scan variation data when the least squares method was used. These values are plotted at their respective wavelength values from $400 \mathrm{~nm}$ to $700 \mathrm{~nm}$. Figure 25 displays the sum of the squared values that were calculated for the different average scan values that were chosen. 


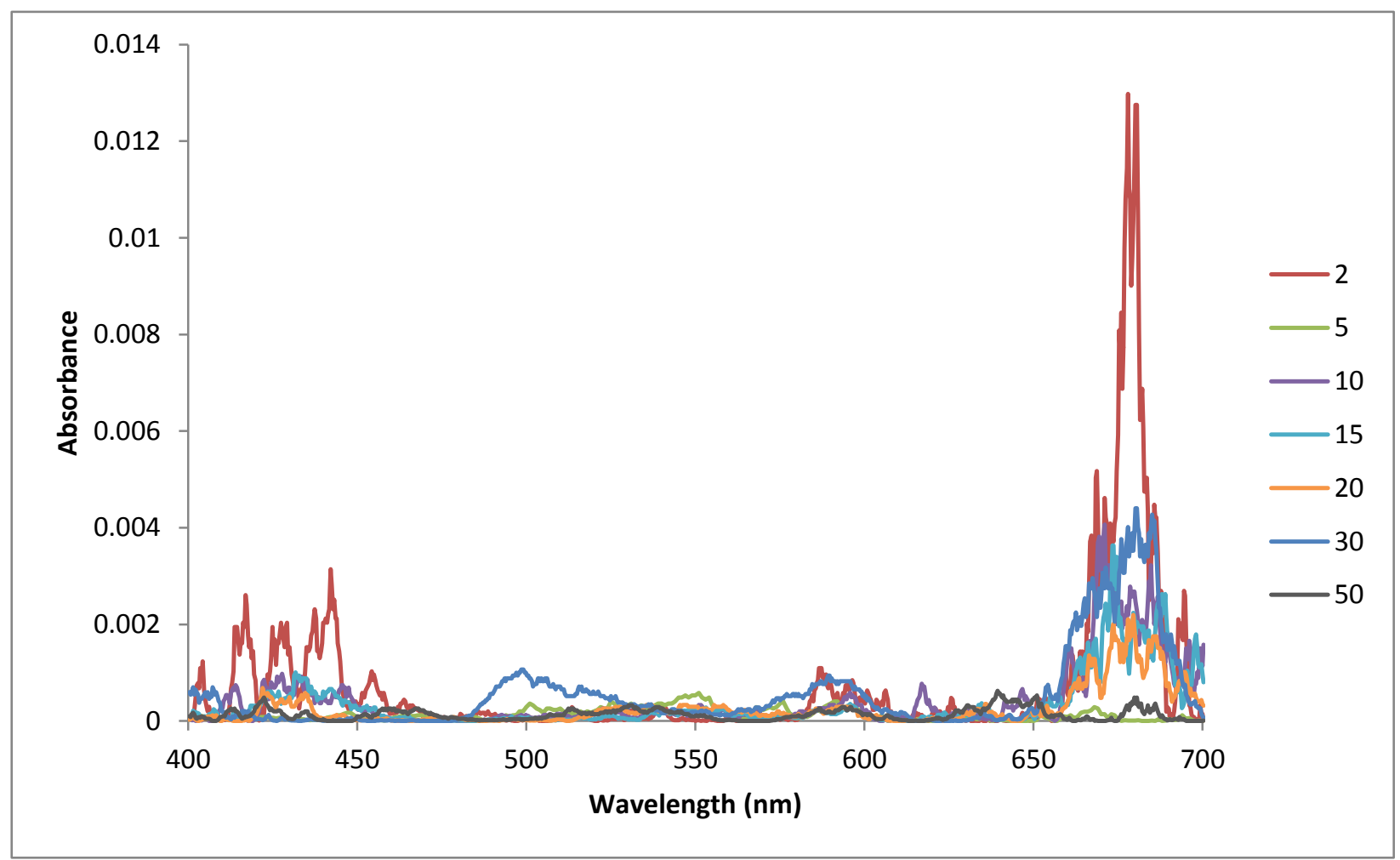

Figure 24: Least squared absorbance values for each average scan variation

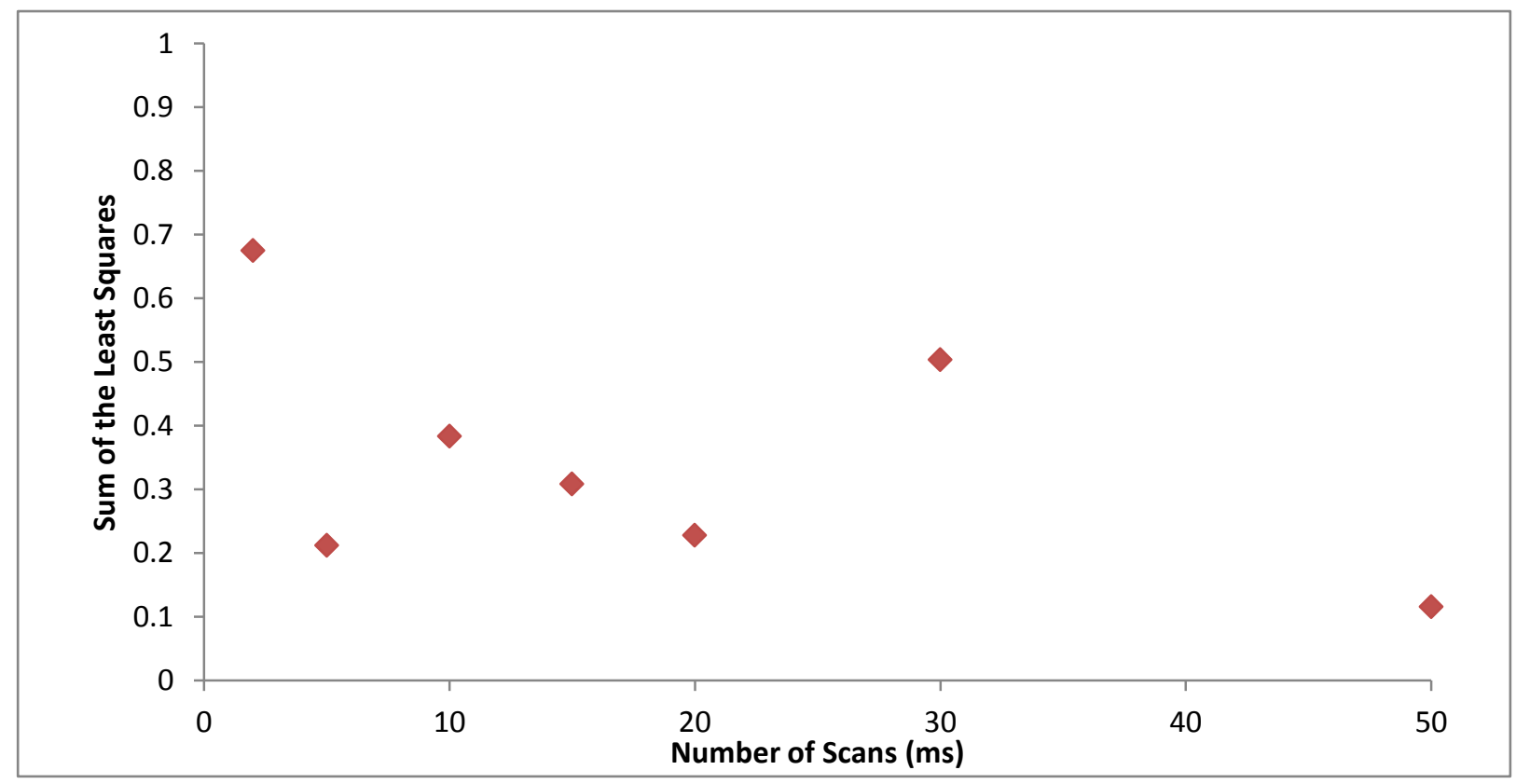

Figure 25: The sum of the squares values for each scan variation 


\section{Boxcar Width}

To better determine the boxcar width when collecting spectra using the Ocean Optics® software when coupled with the QDI 1000 Microspectrophotometer, spectra were collected from $400 \mathrm{~nm}$ to $700 \mathrm{~nm}$ incrementally every $.37 \mathrm{~nm}$ using a boxcar width of 1, 3, 5, 7, 9, 11, 15, 25, 50, 100 and 150, which can be seen in Figure 26 and Figure 27. The absorbance values that were recorded into Microsoft ${ }^{\circledR}$ Excel along with the spectra that were collected can be viewed in Appendix A.

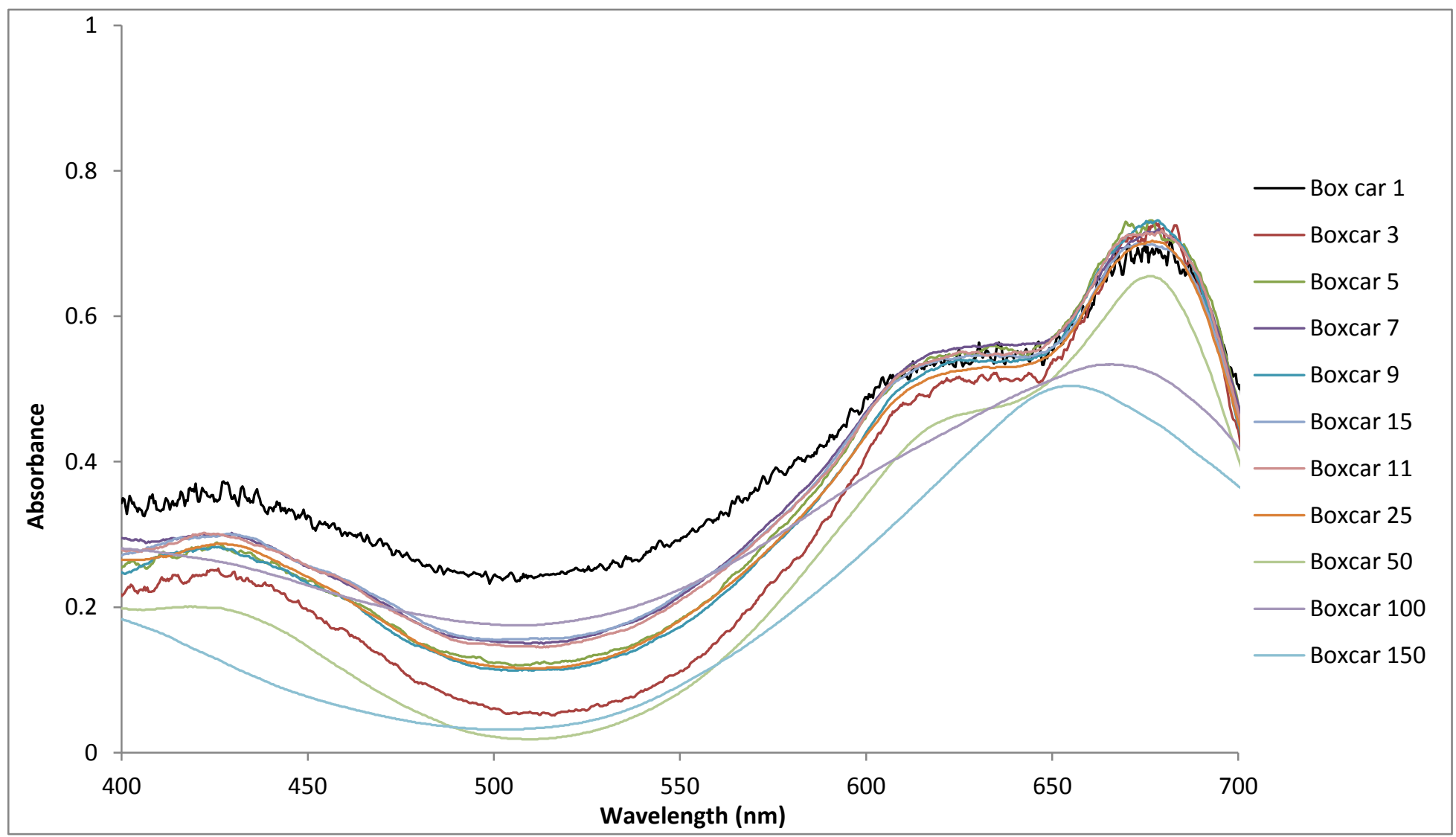

Figure 26: Boxcar width variation of Kelly Green A_F 


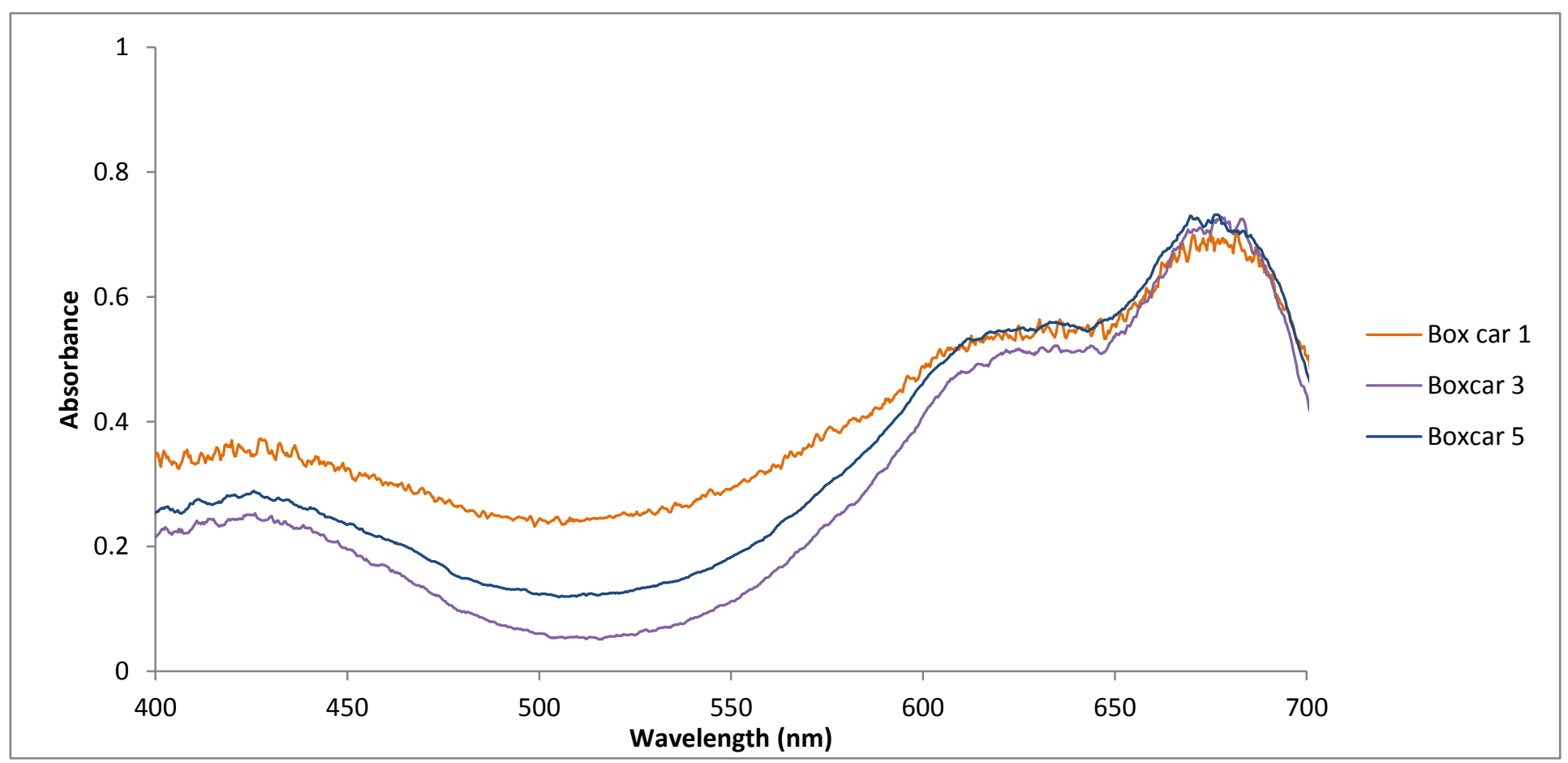

Figure 27: Boxcar width variation of Kelly Green $A_{-} F$ using boxcar width of 1, 3 and 5

Figure 28 show the results the new absorbance values that were calculated for the boxcar width variation data when the least squares method was used. These values are plotted at their respective wavelength values from $400 \mathrm{~nm}$ to $700 \mathrm{~nm}$. Figure 29 displays the sum of the squares values that were calculated for the different boxcar width values that were chosen. 


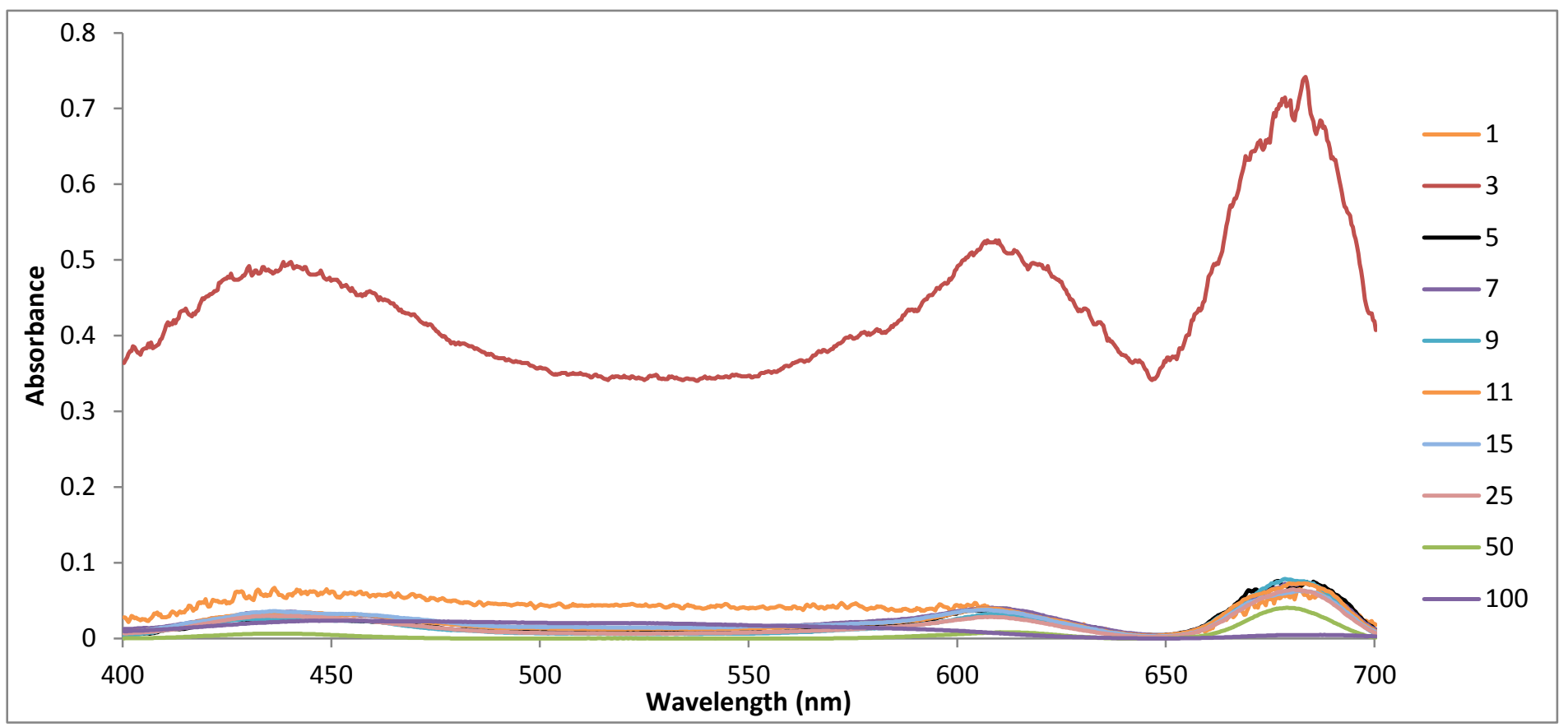

Figure 28: Least squared absorbance values for each boxcar width variation

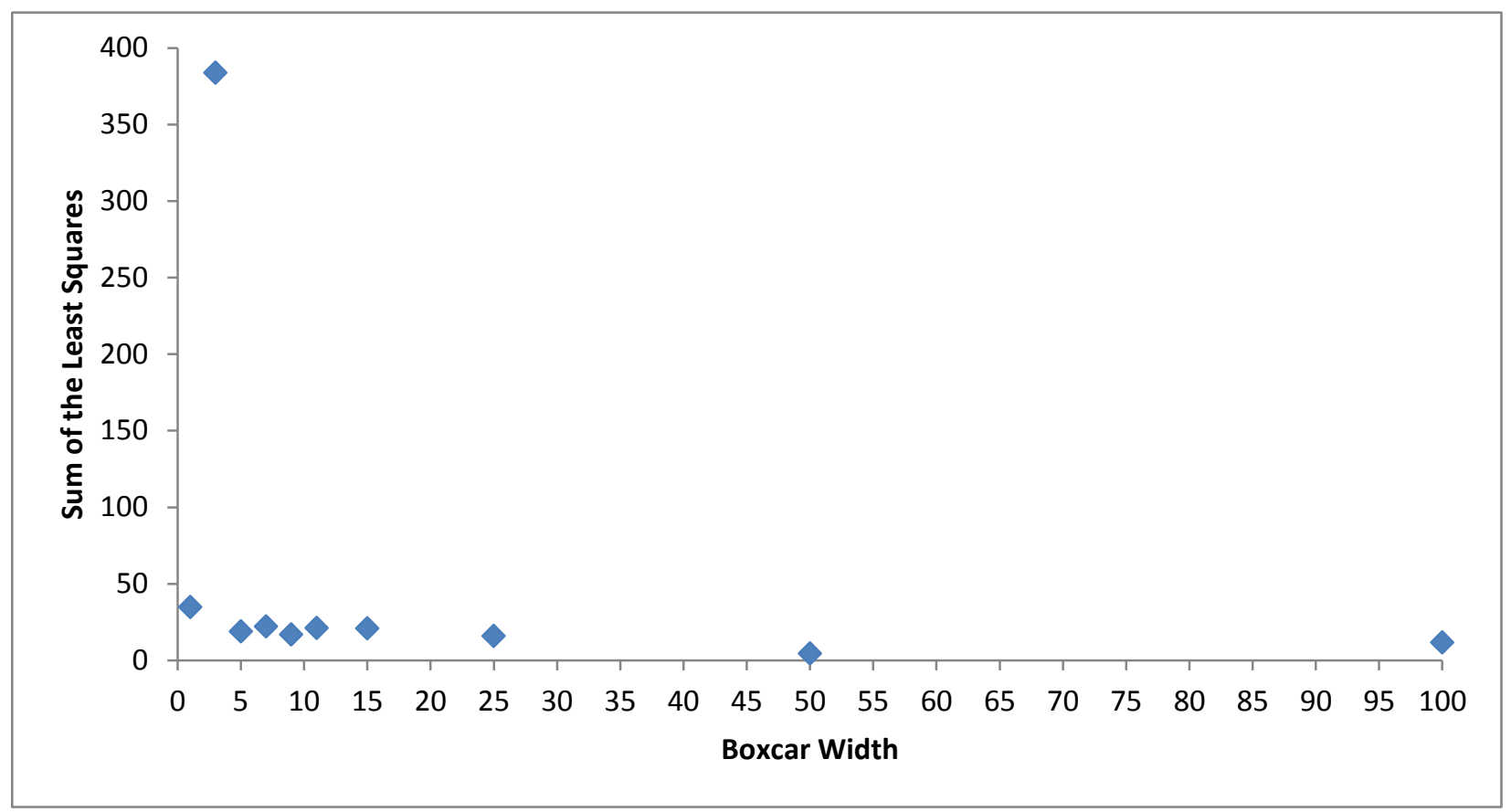

Figure 29: The sum of the squares for each boxcar width variation 


\section{Integration Time}

To better determine the integration time when collecting spectra using the Ocean Optics® software when coupled with the QDI 1000 Microspectrophotometer, spectra were collected from $400 \mathrm{~nm}$ to $700 \mathrm{~nm}$ incrementally every $.37 \mathrm{~nm}$ using integration times of $10 \mathrm{~ms}, 20 \mathrm{~ms}, 30 \mathrm{~ms}, 50 \mathrm{~ms}, 100 \mathrm{~ms}, 200 \mathrm{~ms}, 300 \mathrm{~ms}, 500 \mathrm{~ms}$ and $1000 \mathrm{~ms}$, which can be seen in Figure 30 and Figure 31. The absorbance values that were recorded into Microsoft ${ }^{\circledR}$ Excel along with the spectra that were collected can be viewed in Appendix A.

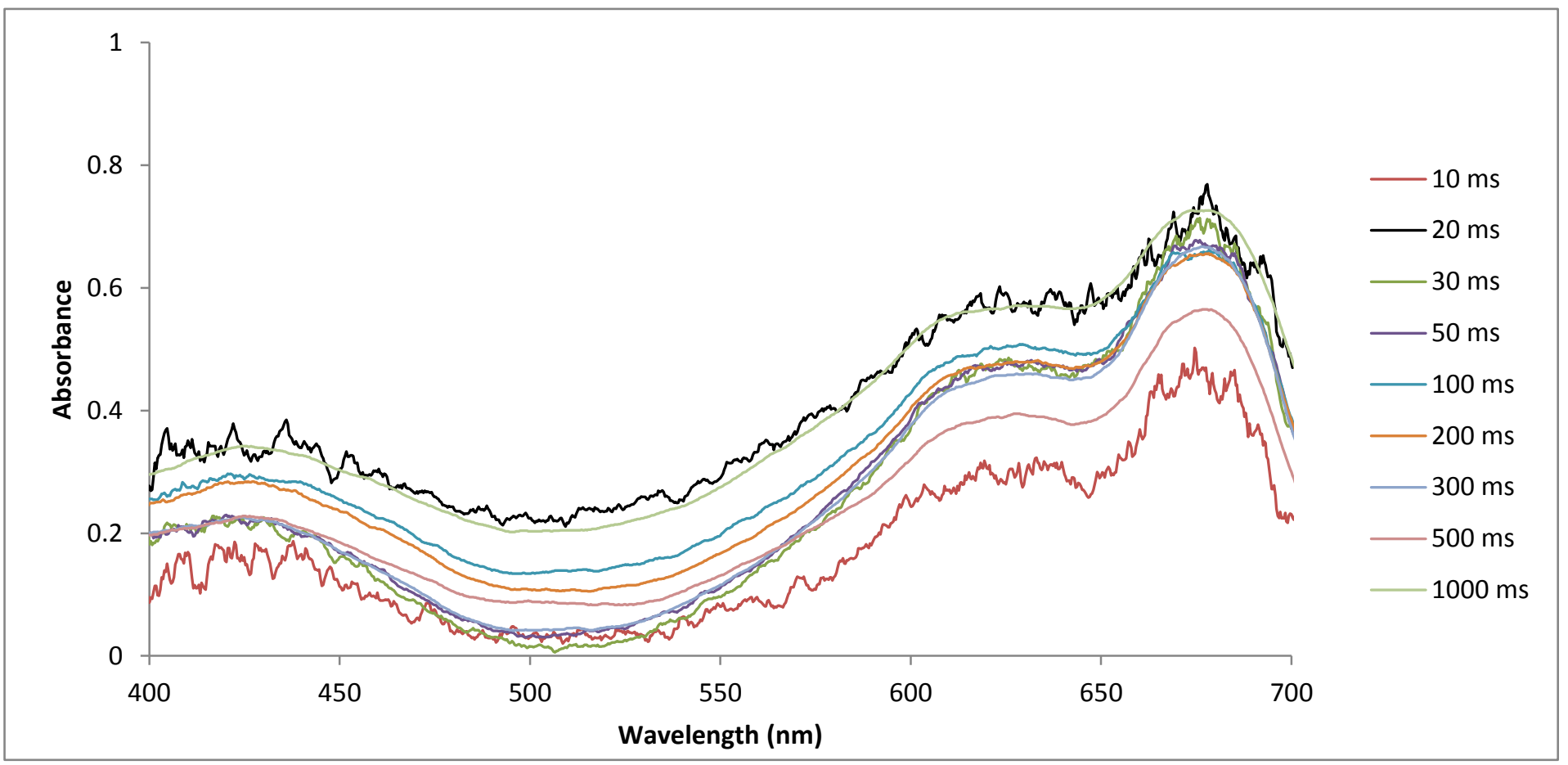

Figure 30: Integration time variation of Kelly Green A_F 


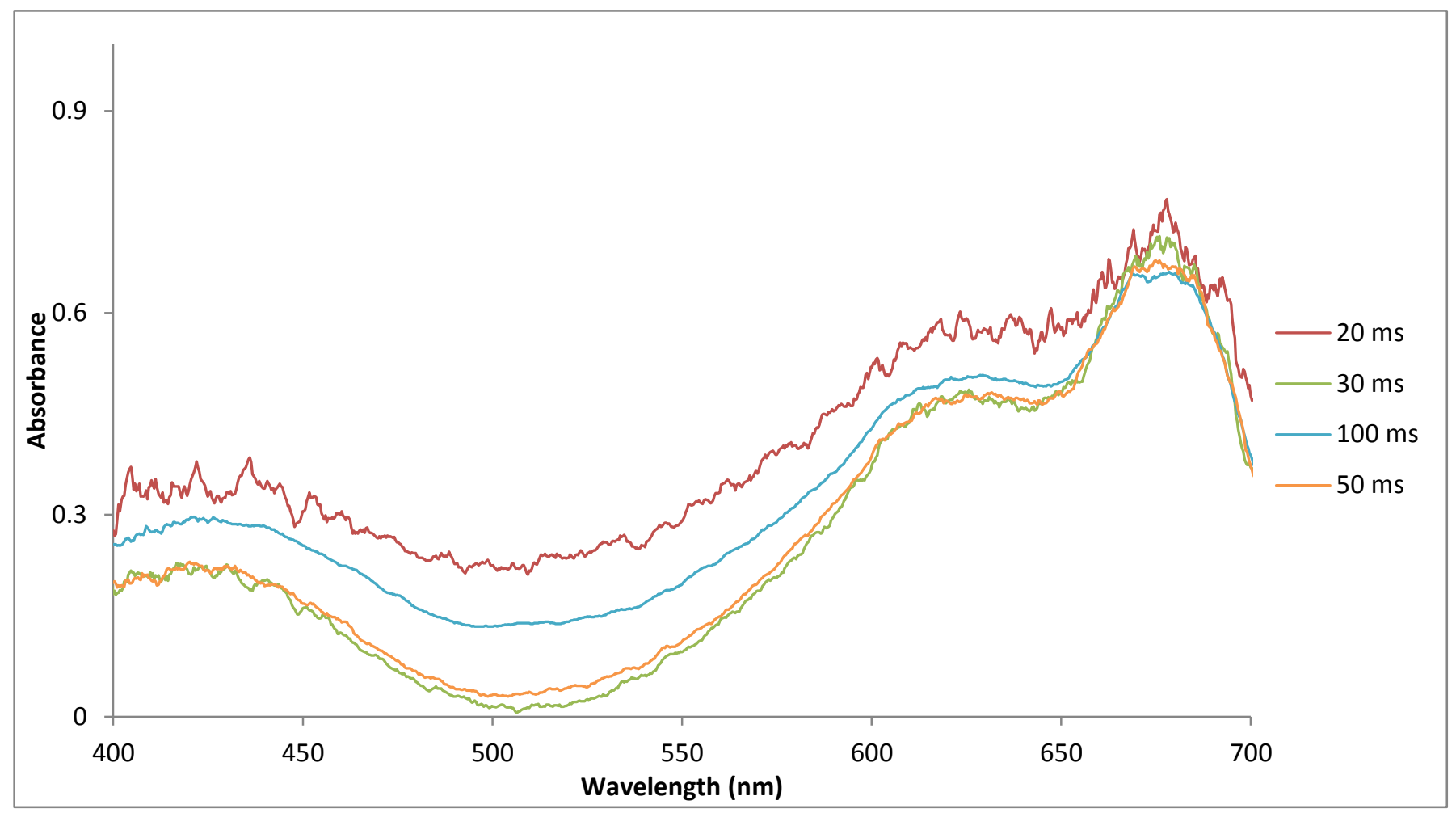

Figure 31: Integration time variation of Kelly Green $A_{-} F$ using times of 20,30, 50 and 100

Figure 32 show the results the new absorbance values that were calculated for the integration time variation data when the least squares method was used. These values are plotted at their respective wavelength values from $400 \mathrm{~nm}$ to $700 \mathrm{~nm}$. Figure 33 displays the sum of the squared values that were calculated for the different integration time values that were chosen. 


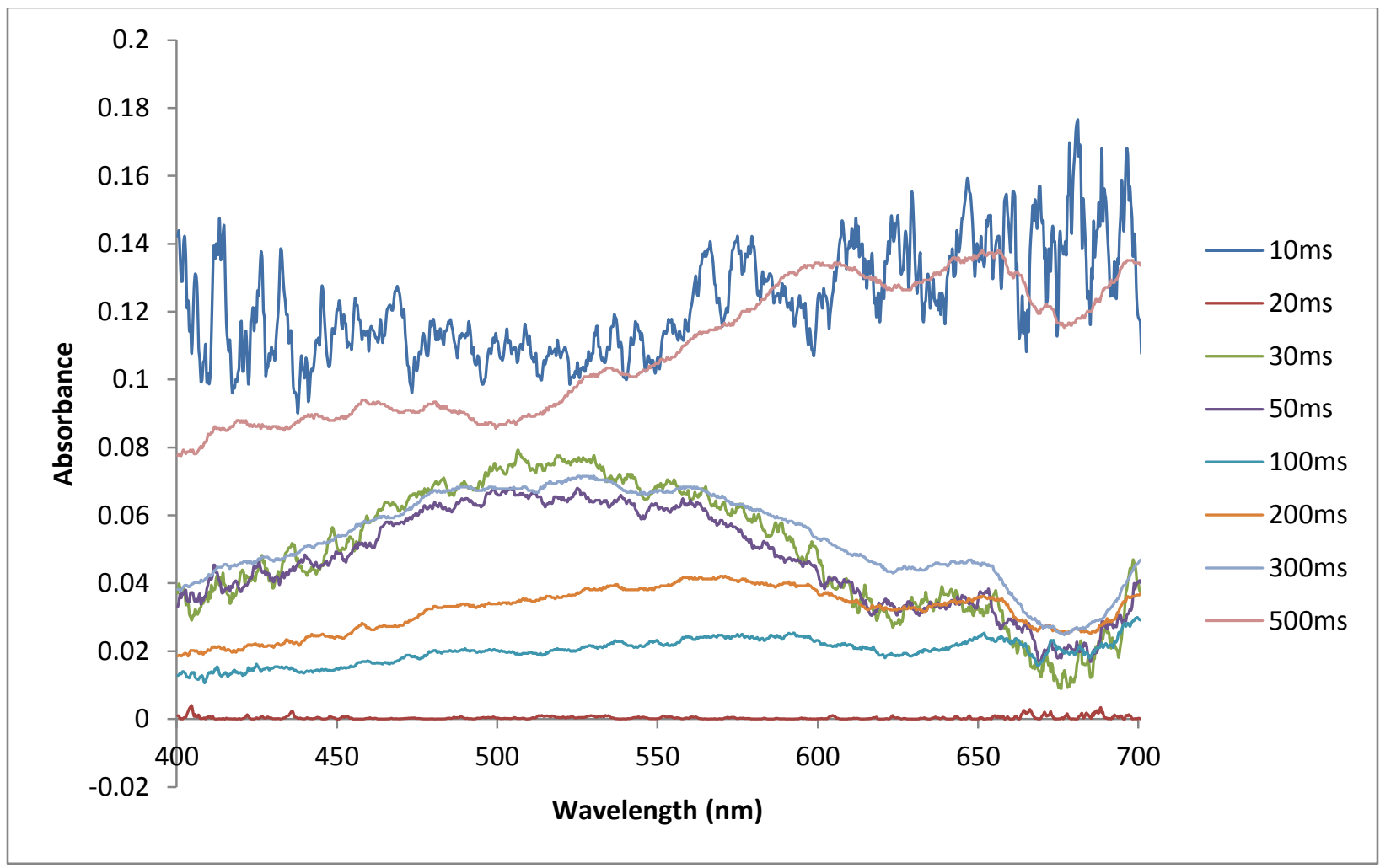

Figure 32: Least squared absorbance values for each integration time variation

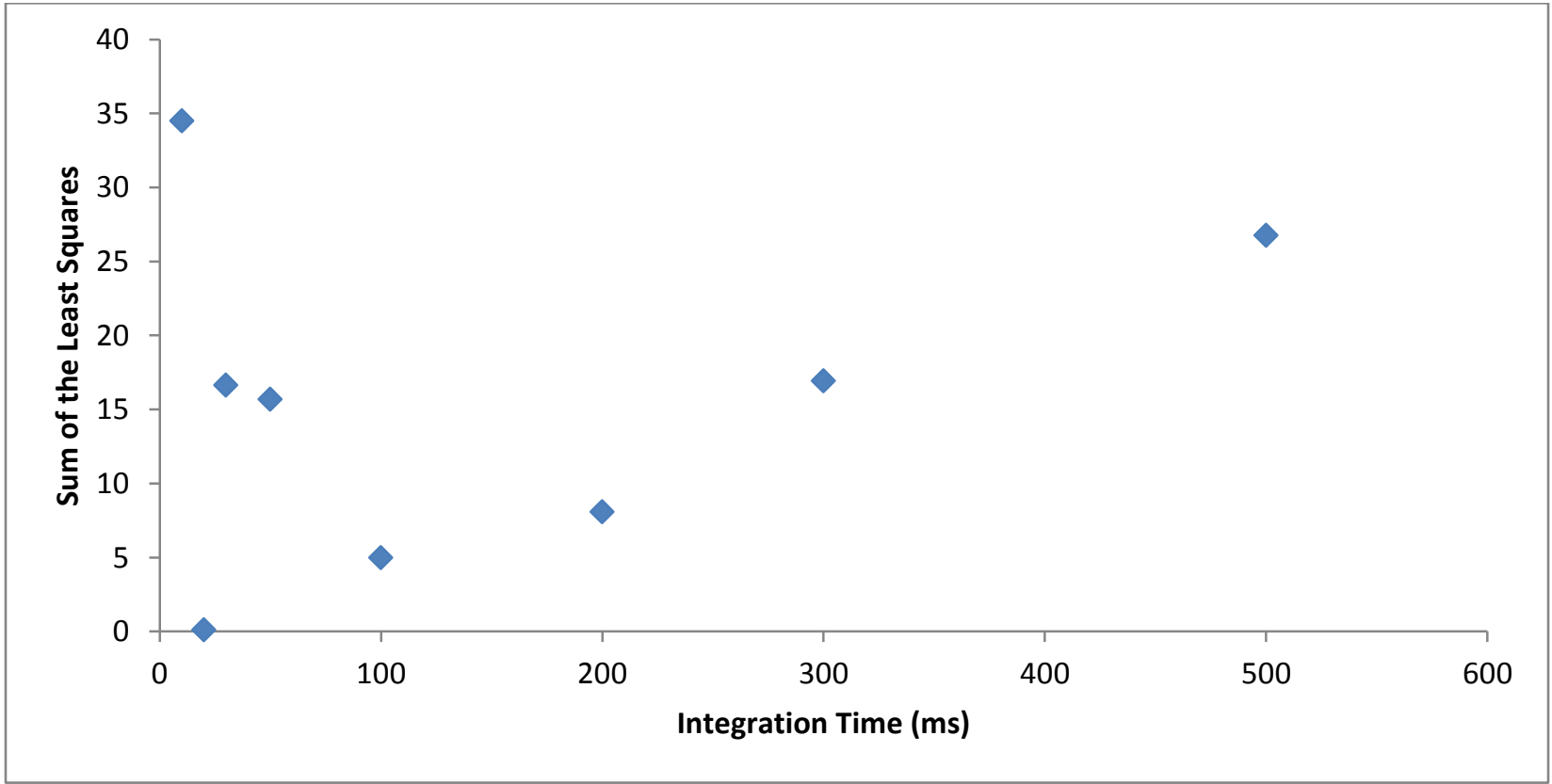

Figure 33: The sum of the squares for each integration time variation 


\section{Phase III}

\section{Time Trial Part 1}

To determine the proper warm-up time for the QDI 1000

Microspectrophotometer's 75 W Xenon lamp, a time trial was performed during this phase. A sample spectrum was collected of Kelly Green A_F every minute for an hour using a wavelength from $380 \mathrm{~nm}$ to $700 \mathrm{~nm}$. The absorbance values were recorded incrementally every $.37 \mathrm{~nm}$. The absorbance values that were recorded at $425 \mathrm{~nm}, 520$ $\mathrm{nm}, 625 \mathrm{~nm}, 675 \mathrm{~nm}$ and $700 \mathrm{~nm}$ were plotted against the time in which they were collected, which are displayed in Figures 34-39.

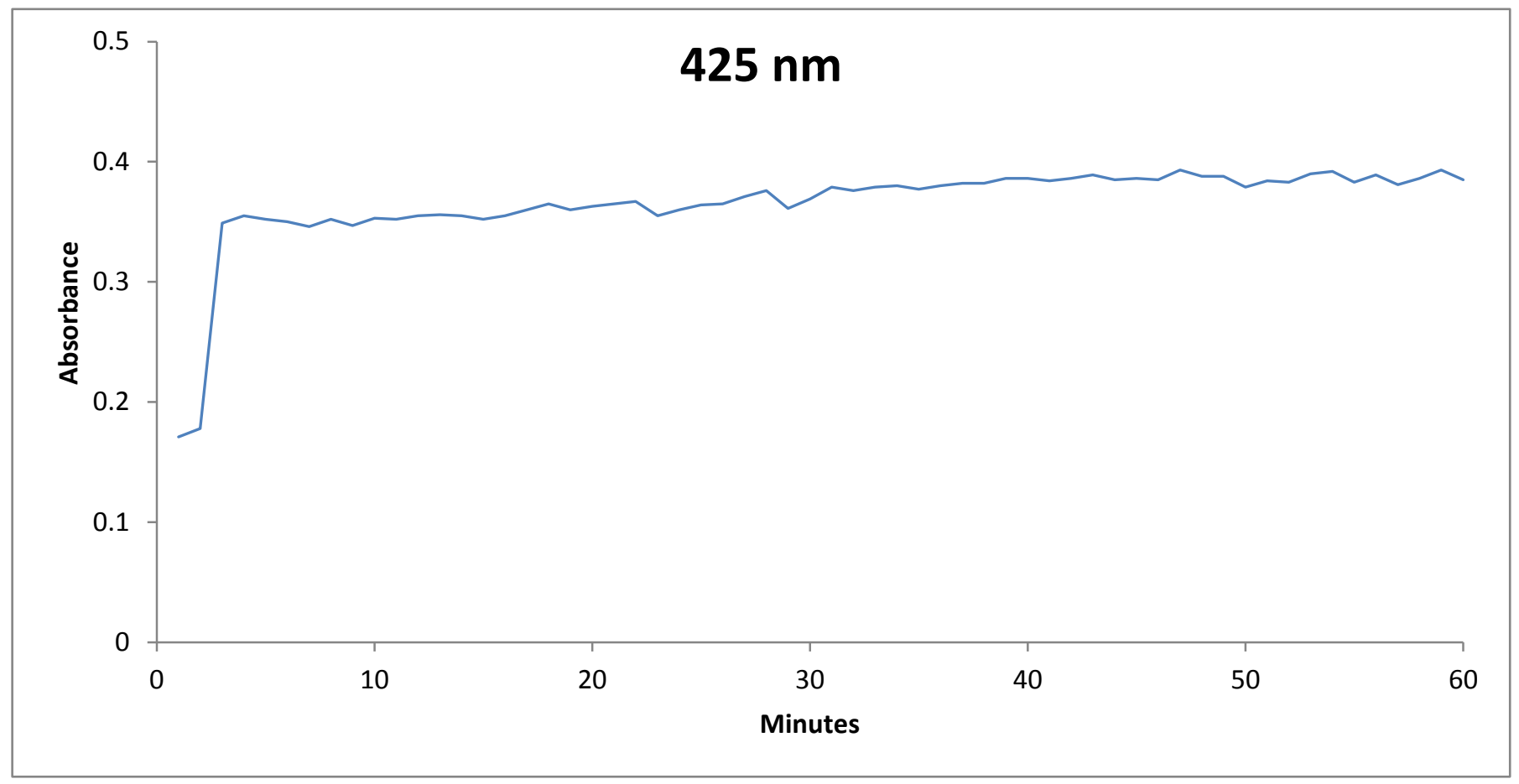

Figure 34: Absorbance values over time of Kelly Green A_F at 425 nm 


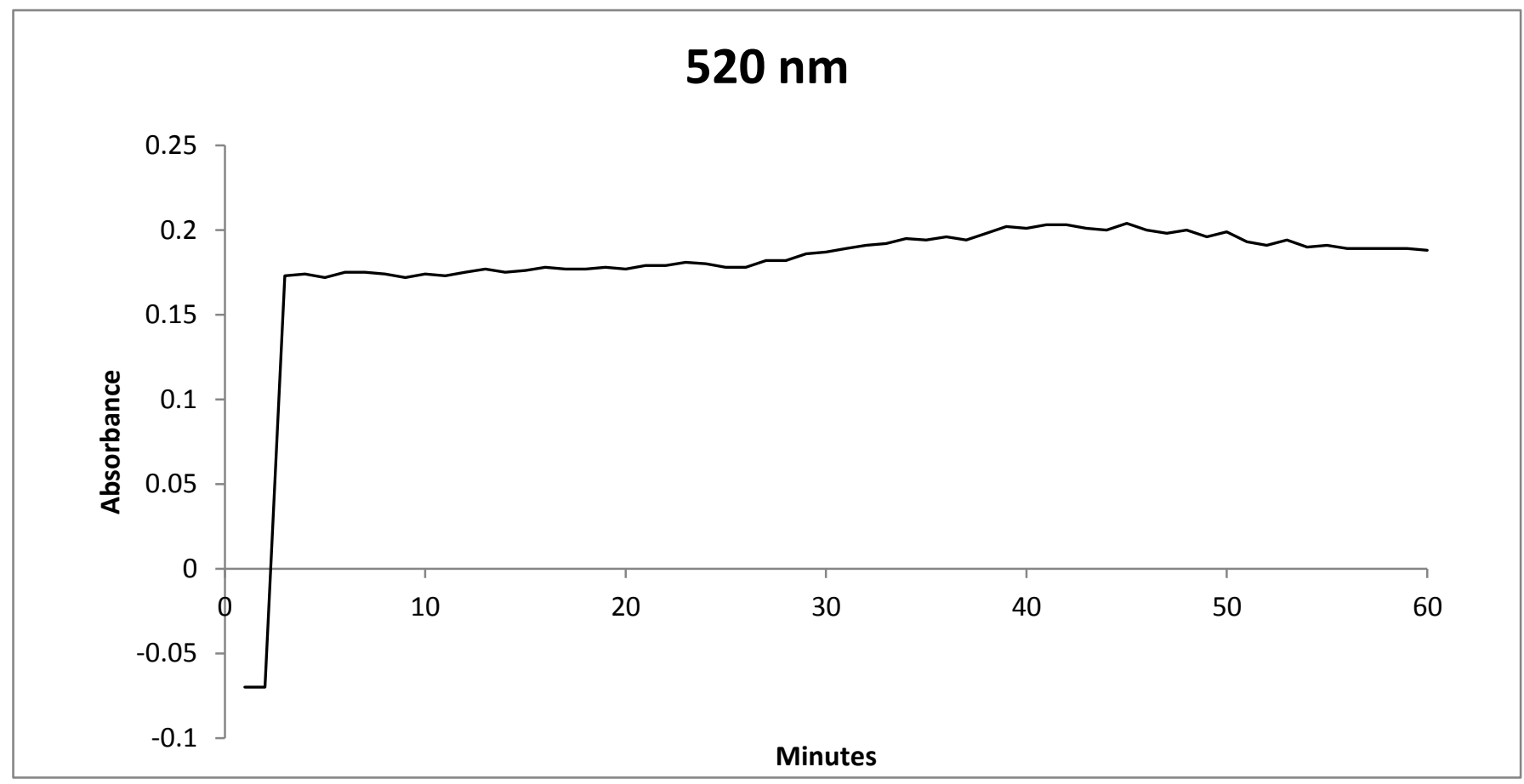

Figure 35: Absorbance values over time of Kelly Green A_F at 520 nm

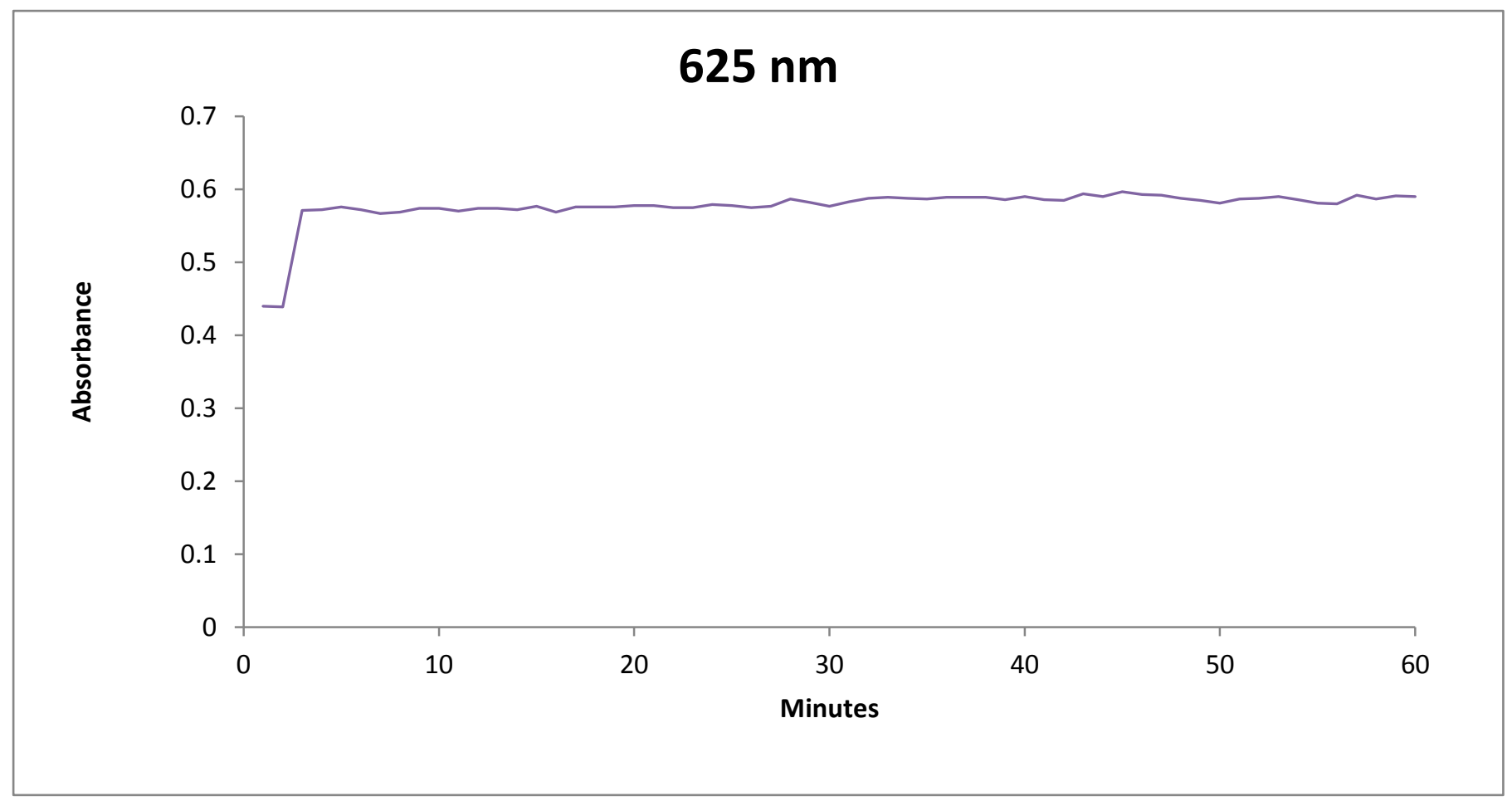

Figure 36: Absorbance values over time of Kelly Green A_F at 625 nm 


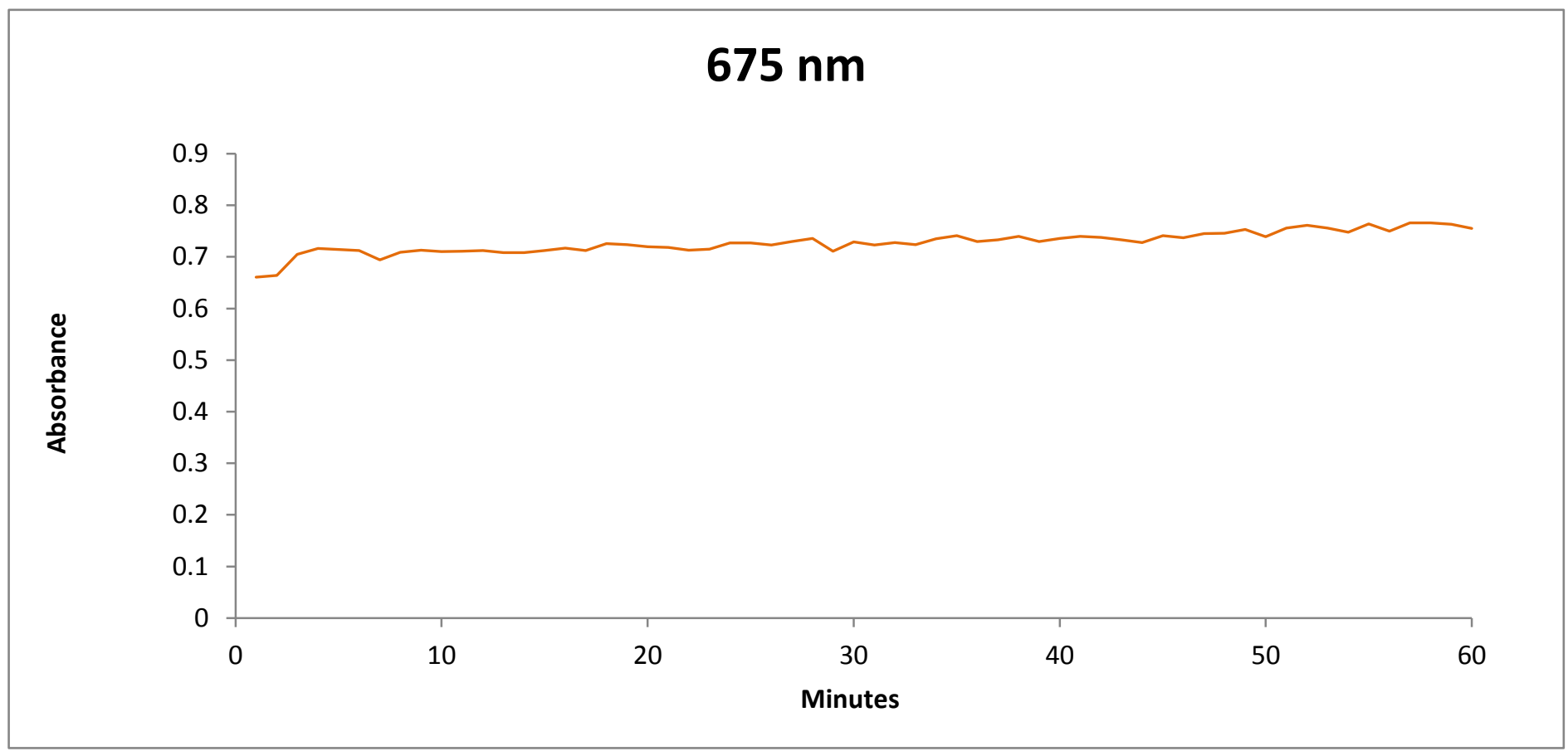

Figure 37: Absorbance values over time of Kelly Green A_F at 675 nm

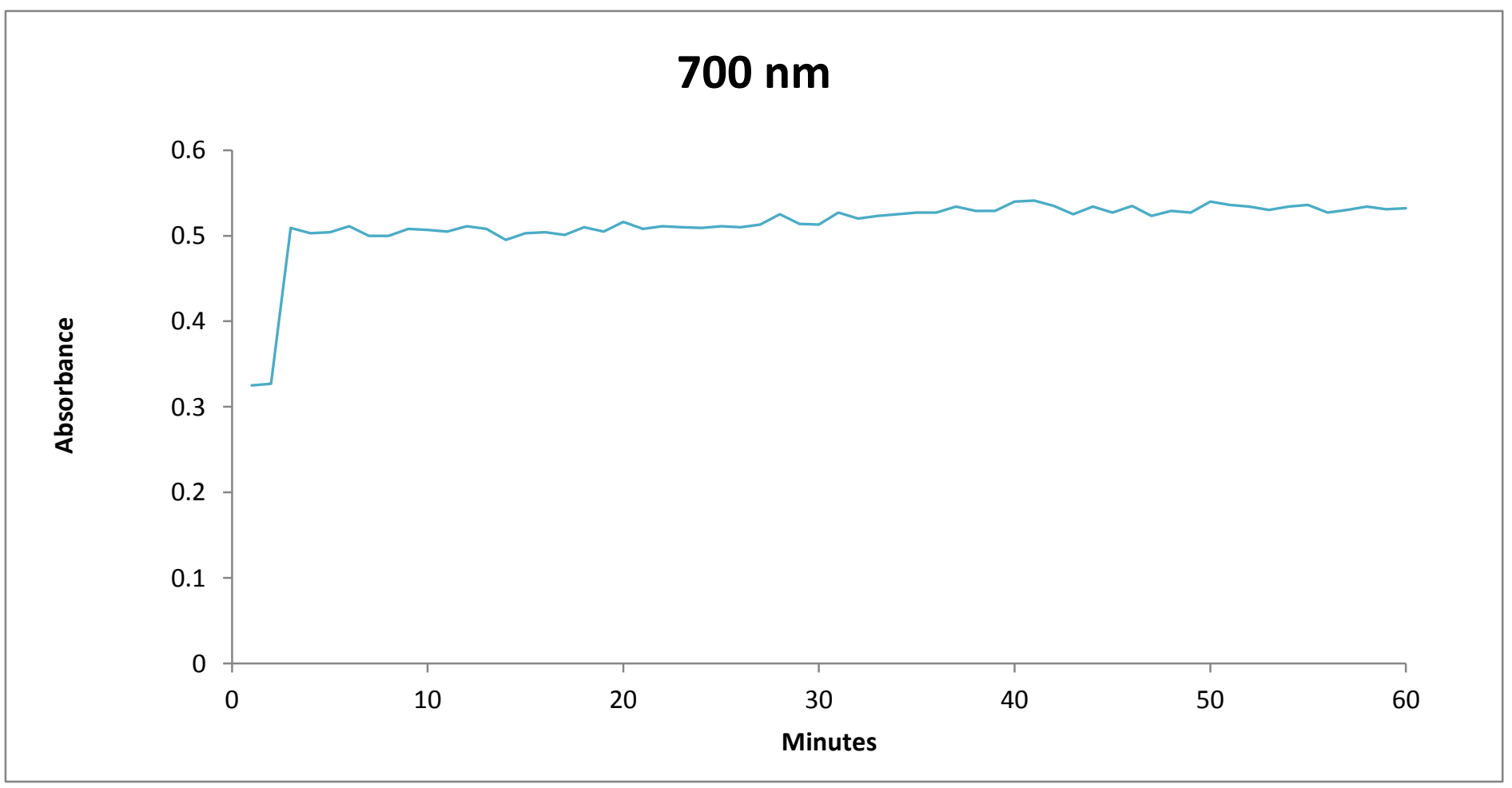

Figure 38: Absorbance values over time of Kelly Green A_F at 700 nm 


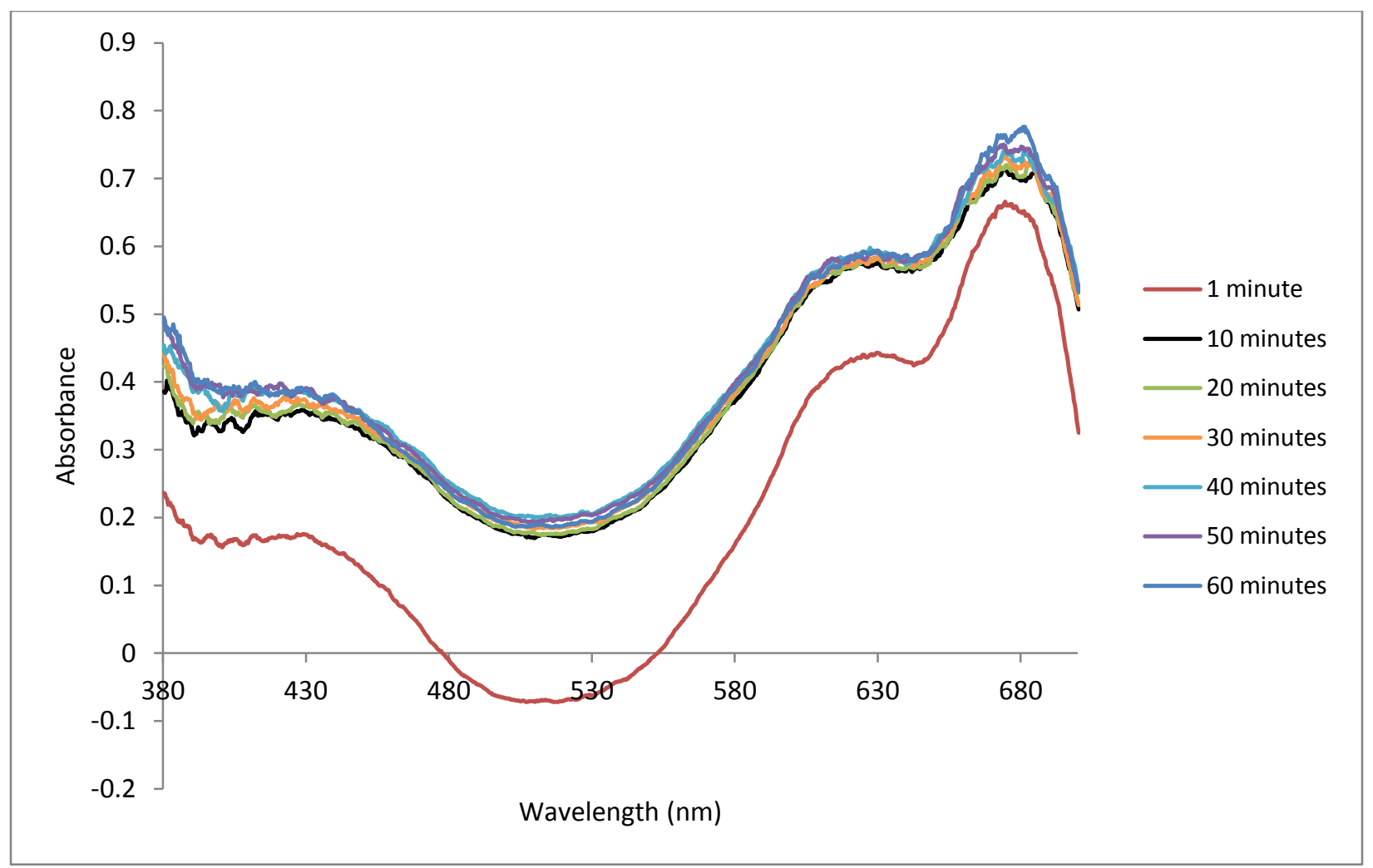

Figure 39: Absorbance values of Kelly Green A_F every ten minutes

\section{Time Trial Part II}

In this part of the time trial, a reference scan and a dark scan were collected before the sample spectrum was collected of Kelly Green A_F every minute for an hour using a wavelength from $380 \mathrm{~nm}$ to $700 \mathrm{~nm}$. The absorbance values were recorded incrementally every $.37 \mathrm{~nm}$. The absorbance values that were recorded at $425 \mathrm{~nm}, 520$ $\mathrm{nm}, 625 \mathrm{~nm}, 675 \mathrm{~nm}$ and $700 \mathrm{~nm}$ were plotted against the time in which they were collected, which are displayed in Figures 40-45. 


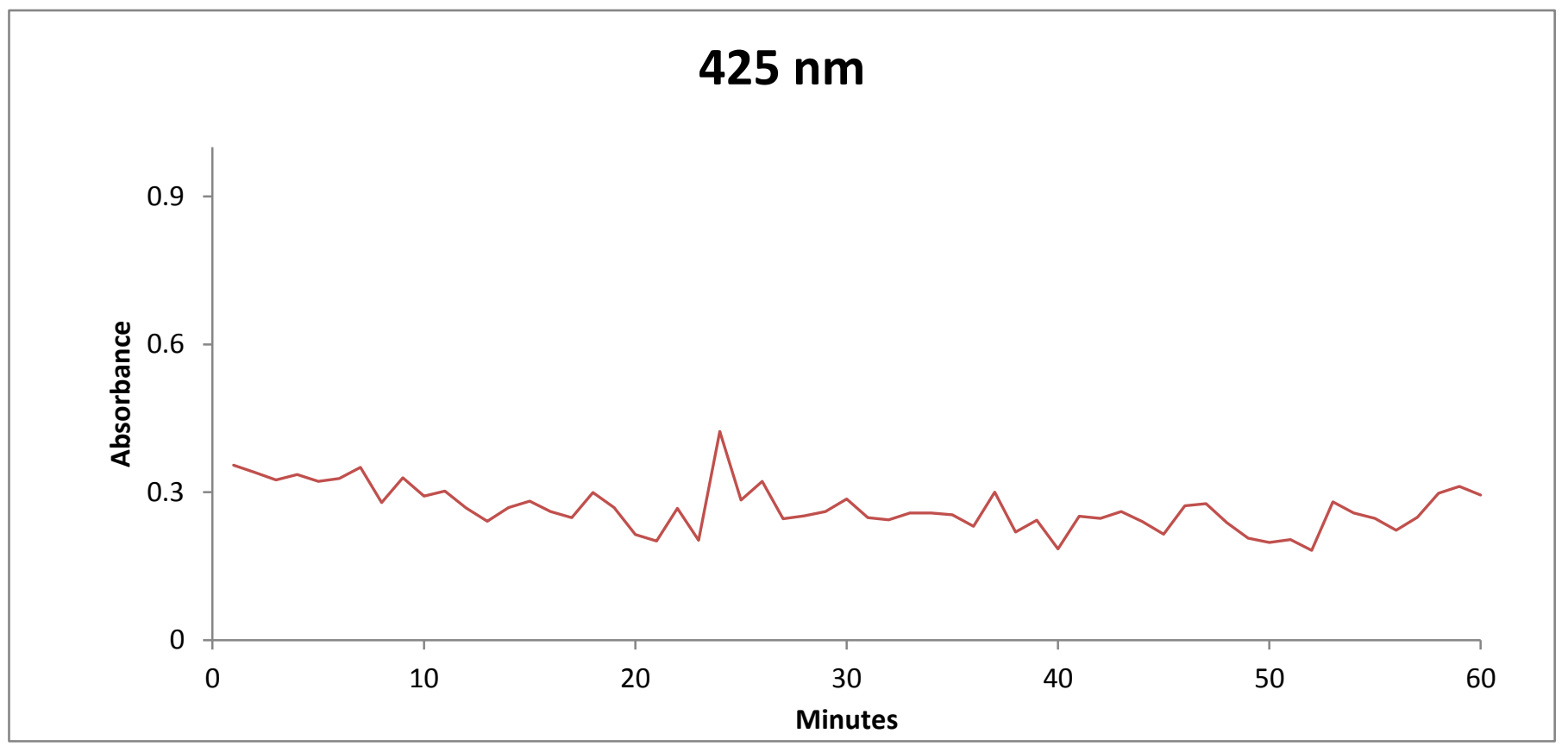

Figure 40: Absorbance values over time of Kelly Green A_F at $425 \mathrm{~nm}$

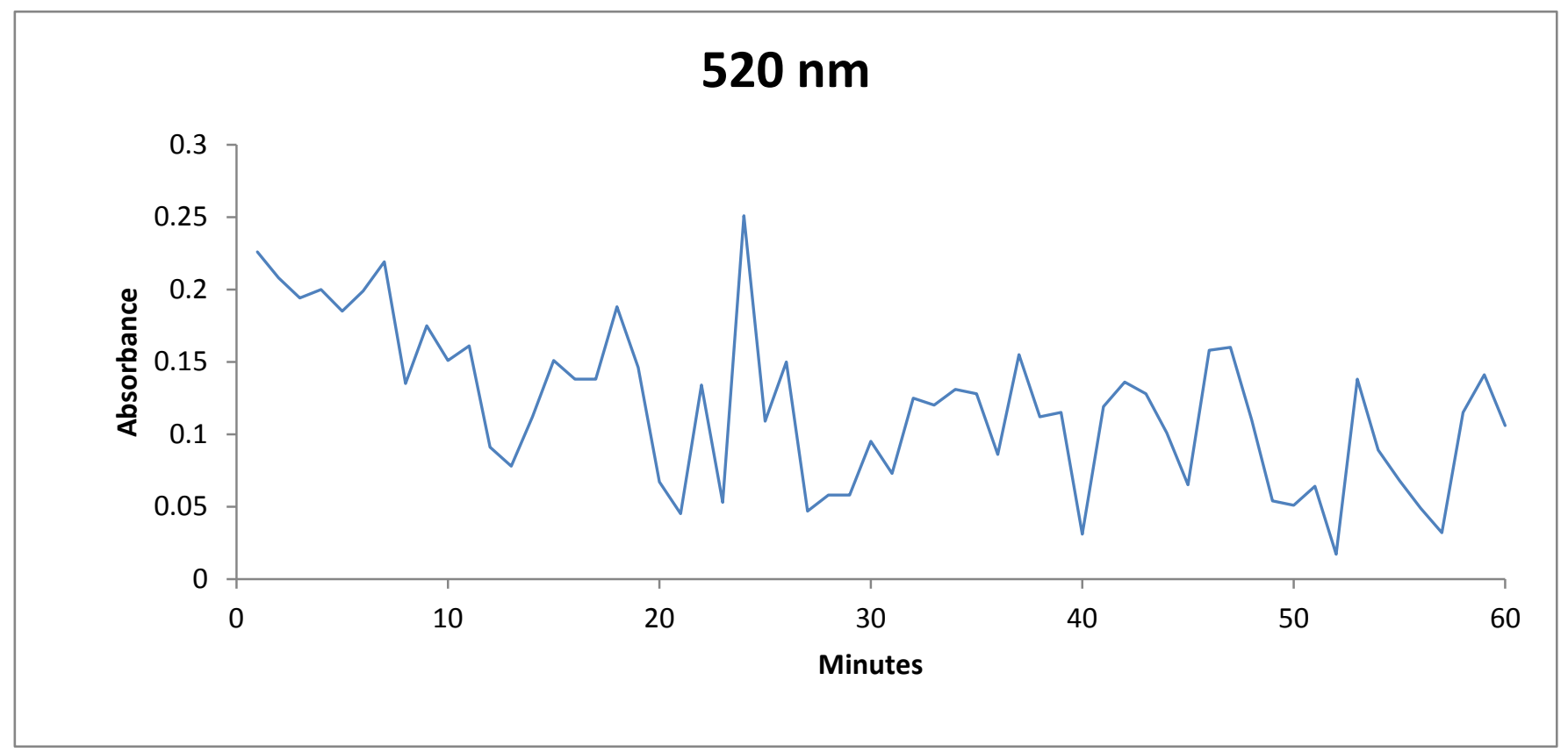

Figure 41: Absorbance values over time of Kelly Green A_F at 520 nm 


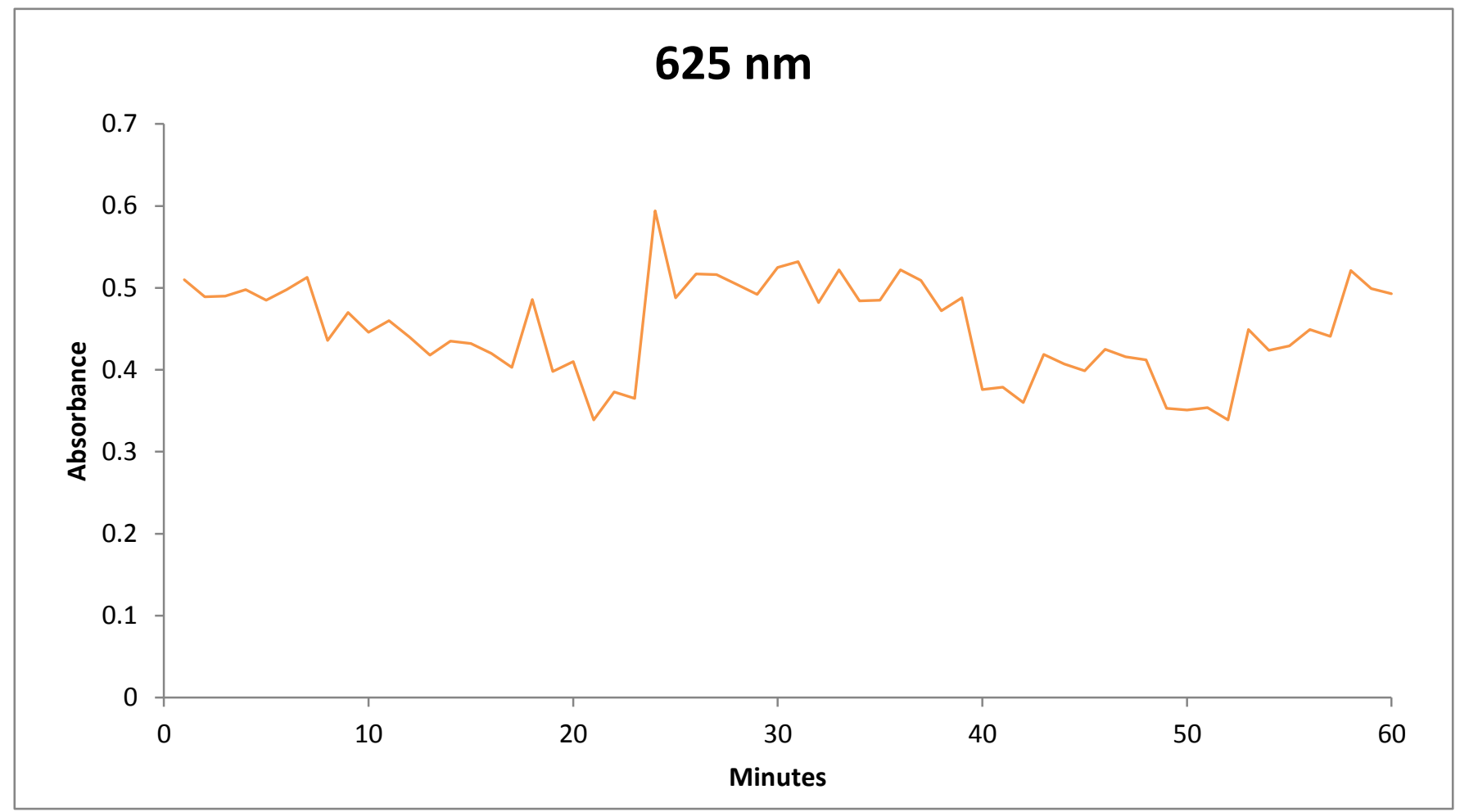

Figure 42: Absorbance values over time of Kelly Green A_F at 625 nm

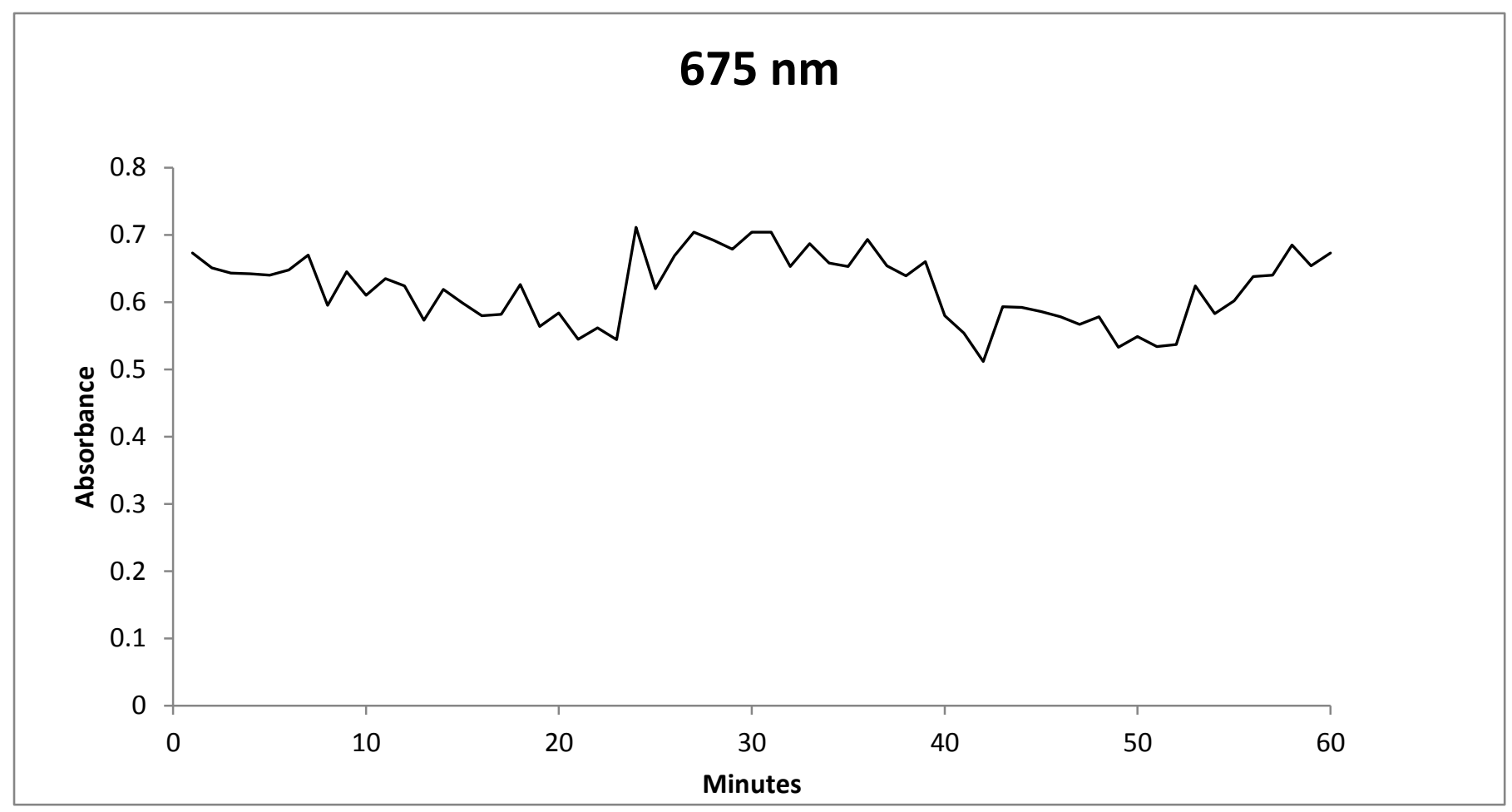

Figure 43: Absorbance values over time of Kelly Green A_F at 675 nm 


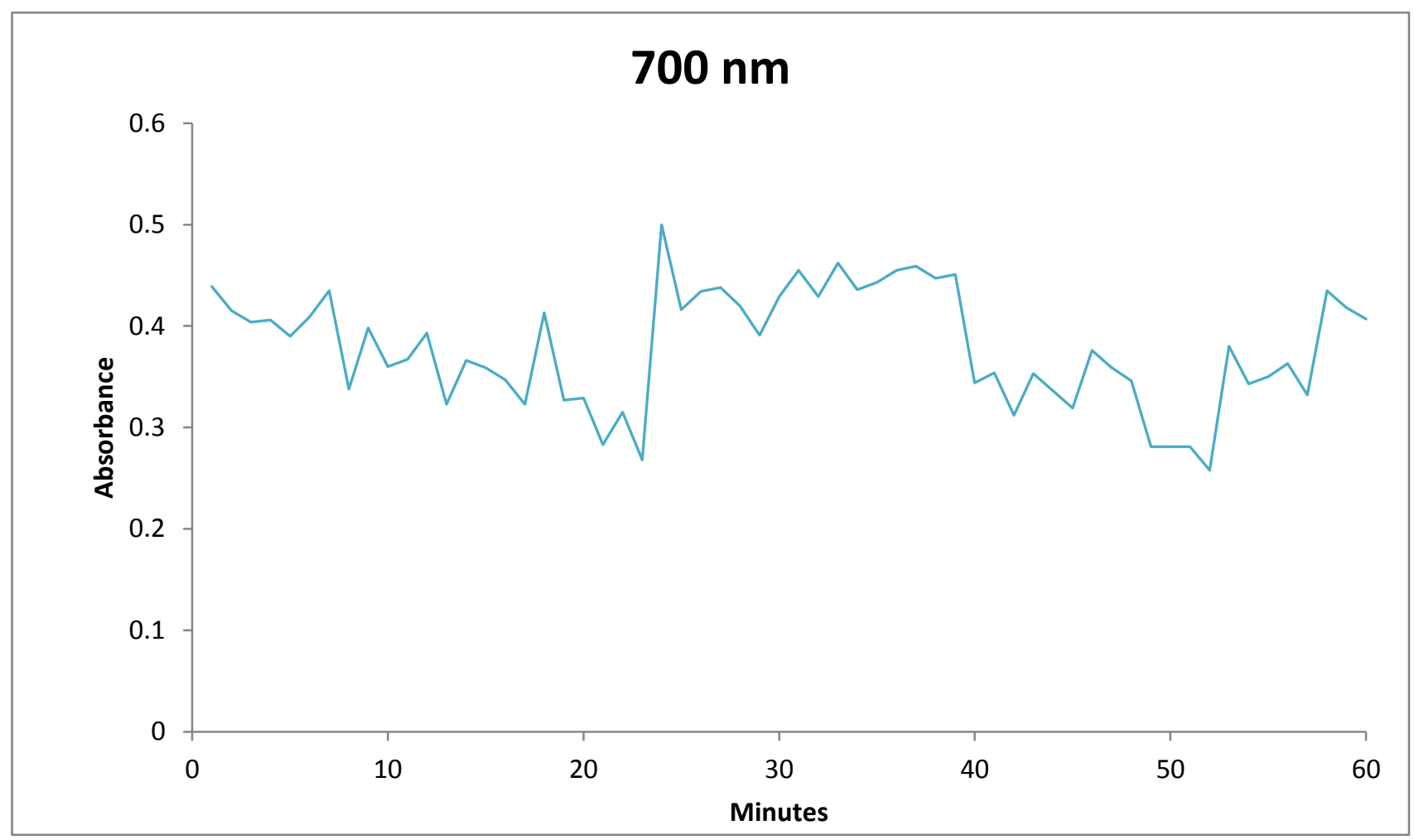

Figure 44: Absorbance values over time of Kelly Green A_F at 700 nm

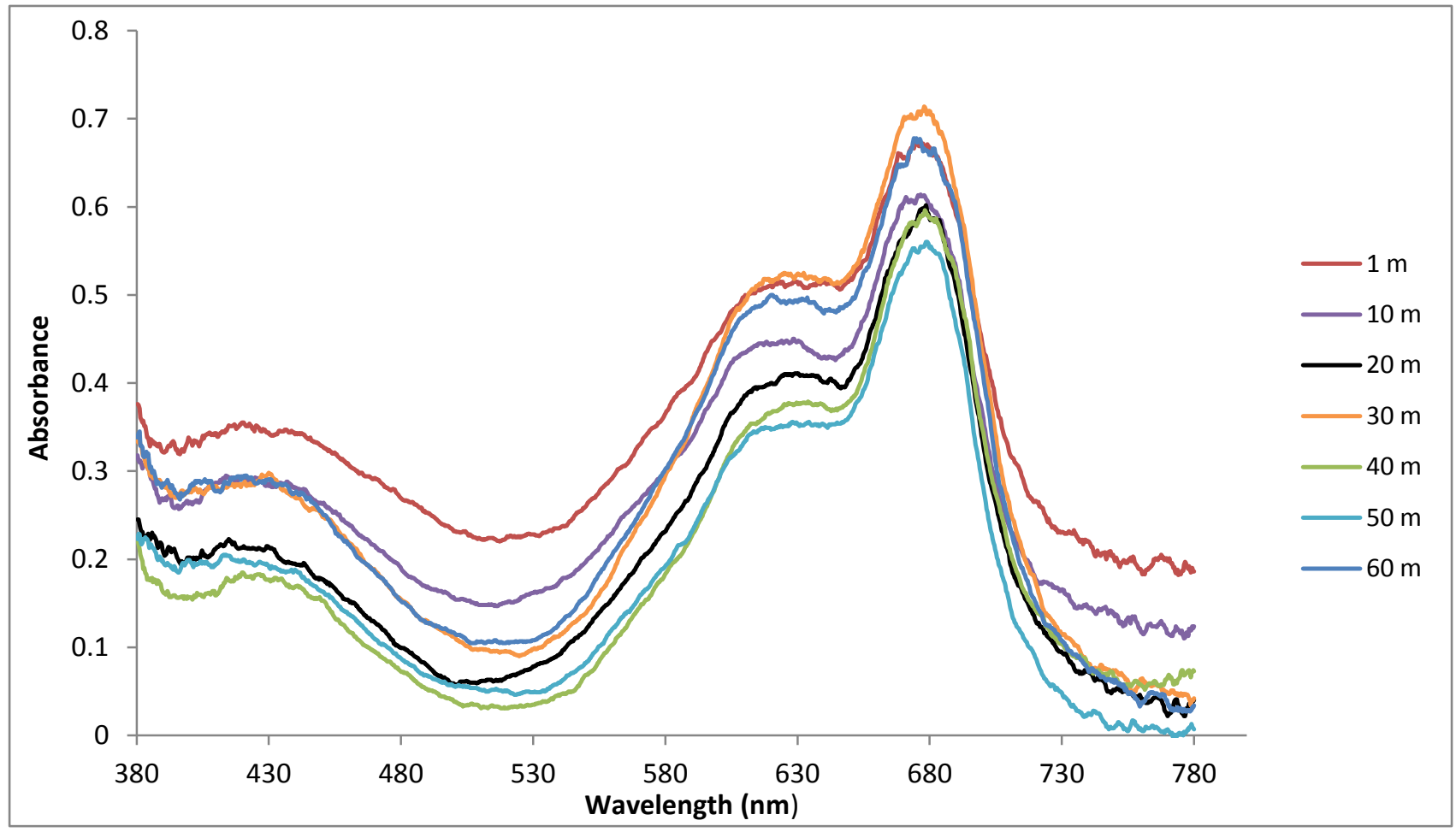

Figure 45: Absorbance values of Kelly Green A_F every ten minutes 
Phase IV

Gold

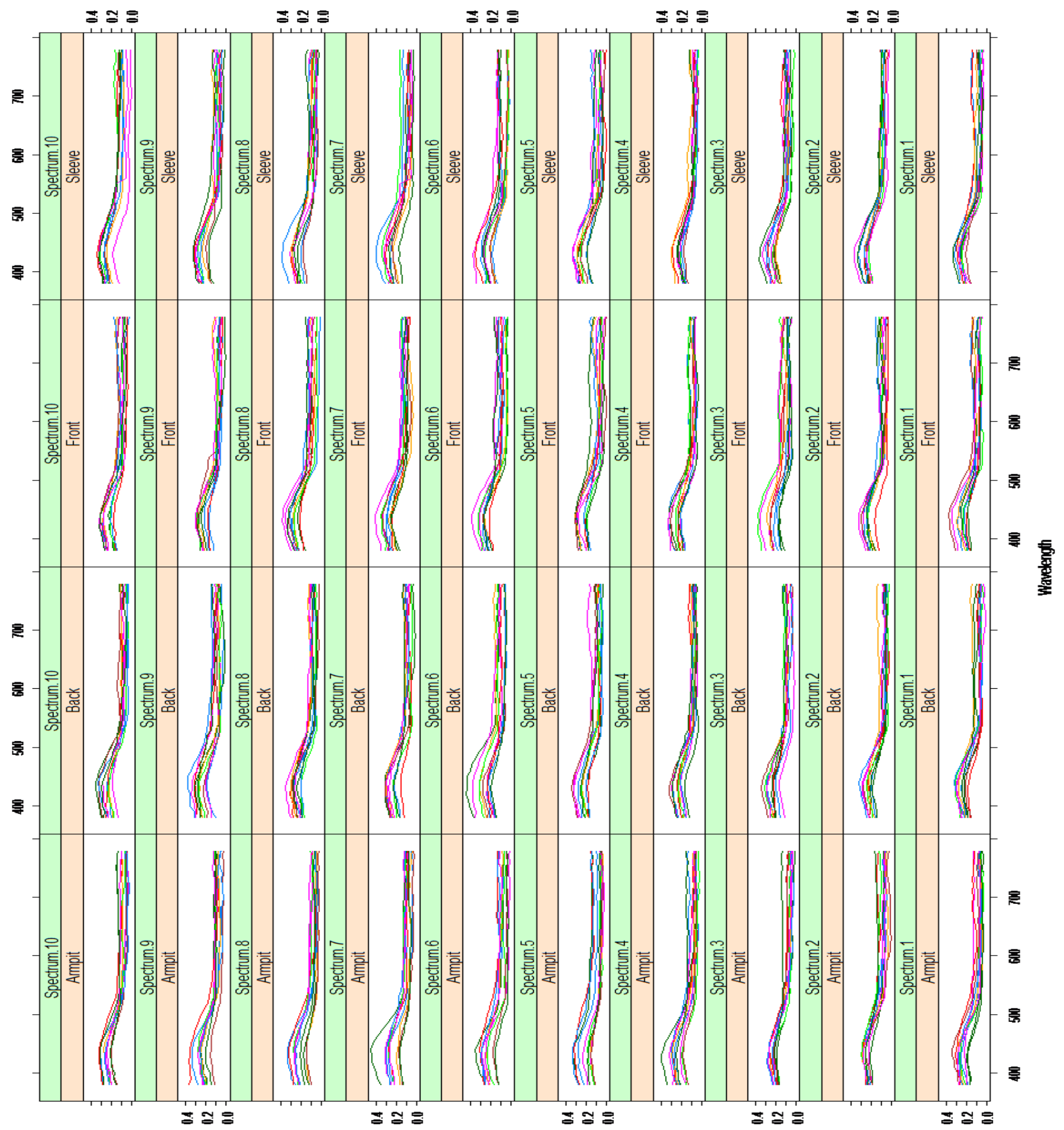

Figure 46: Spectra the Gold A-J shirts for armpit, back, front and sleeve of all ten runs 


\begin{tabular}{|l|}
\hline Armpit : \\
Back : \\
Front : \\
Sleeve
\end{tabular}

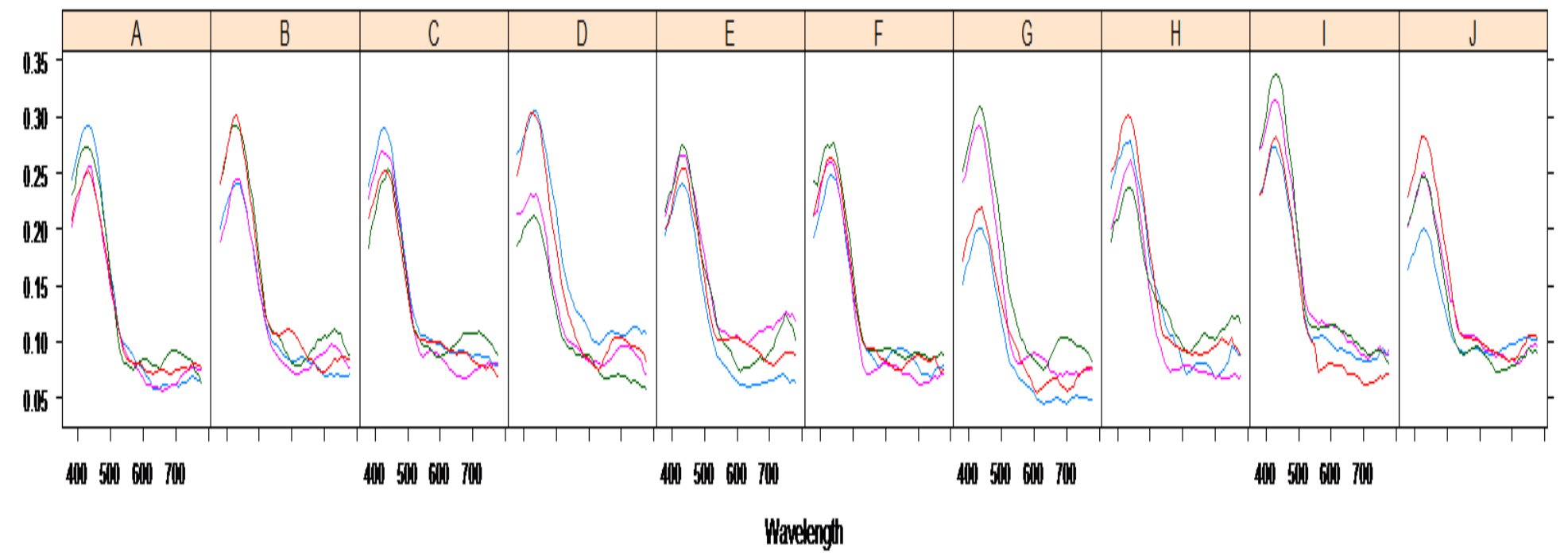

Figure 47: Spectra of the armpit, back, front and sleeve for Gold A-J shirts

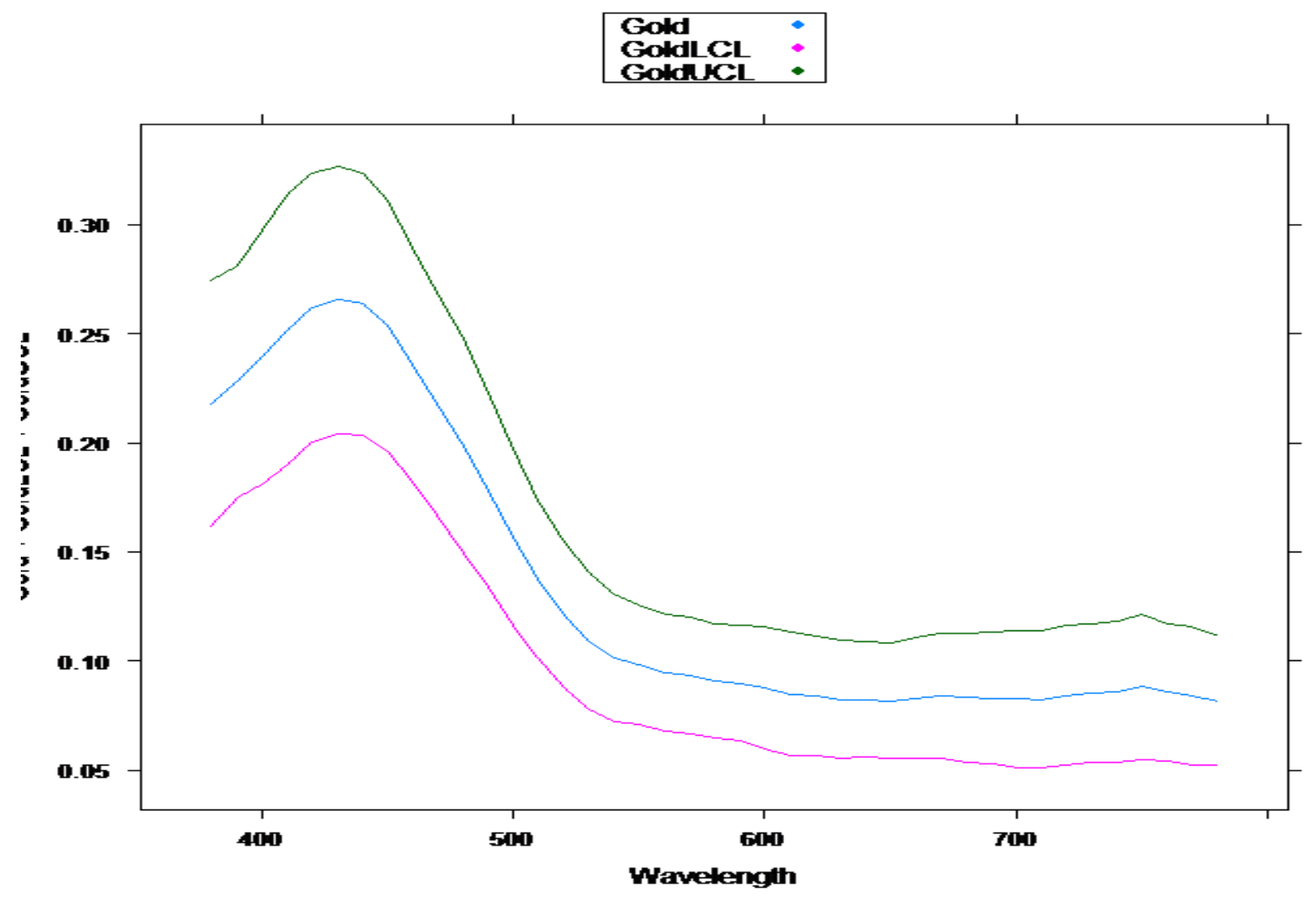

Figure 48: Spectra of the Average Absorbance, UCL and the LCL for 4 all regions of the Gold A shirts 


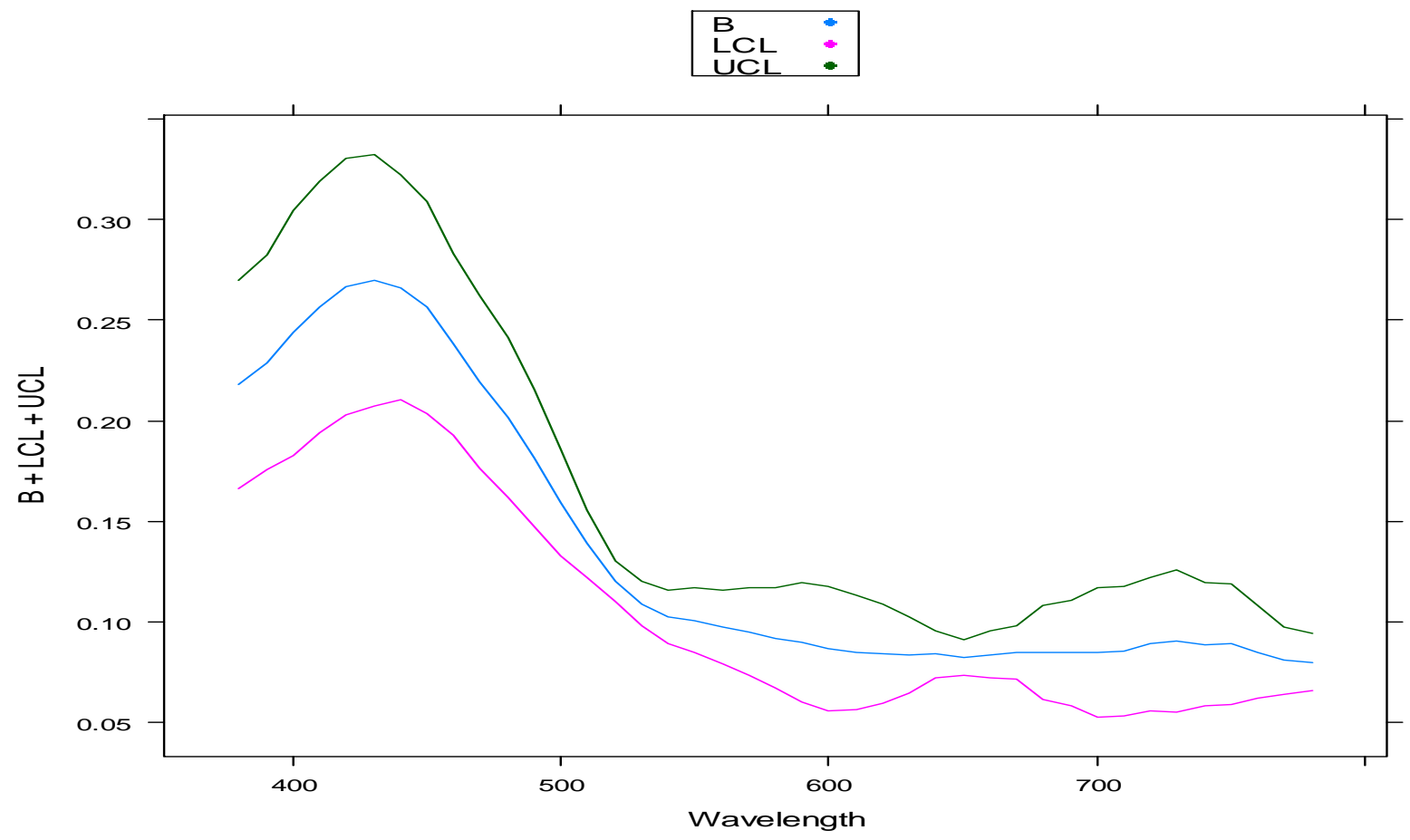

Figure 49: Spectra of the Average Absorbance, UCL and the LCL for all 4 regions of the Gold B shirts

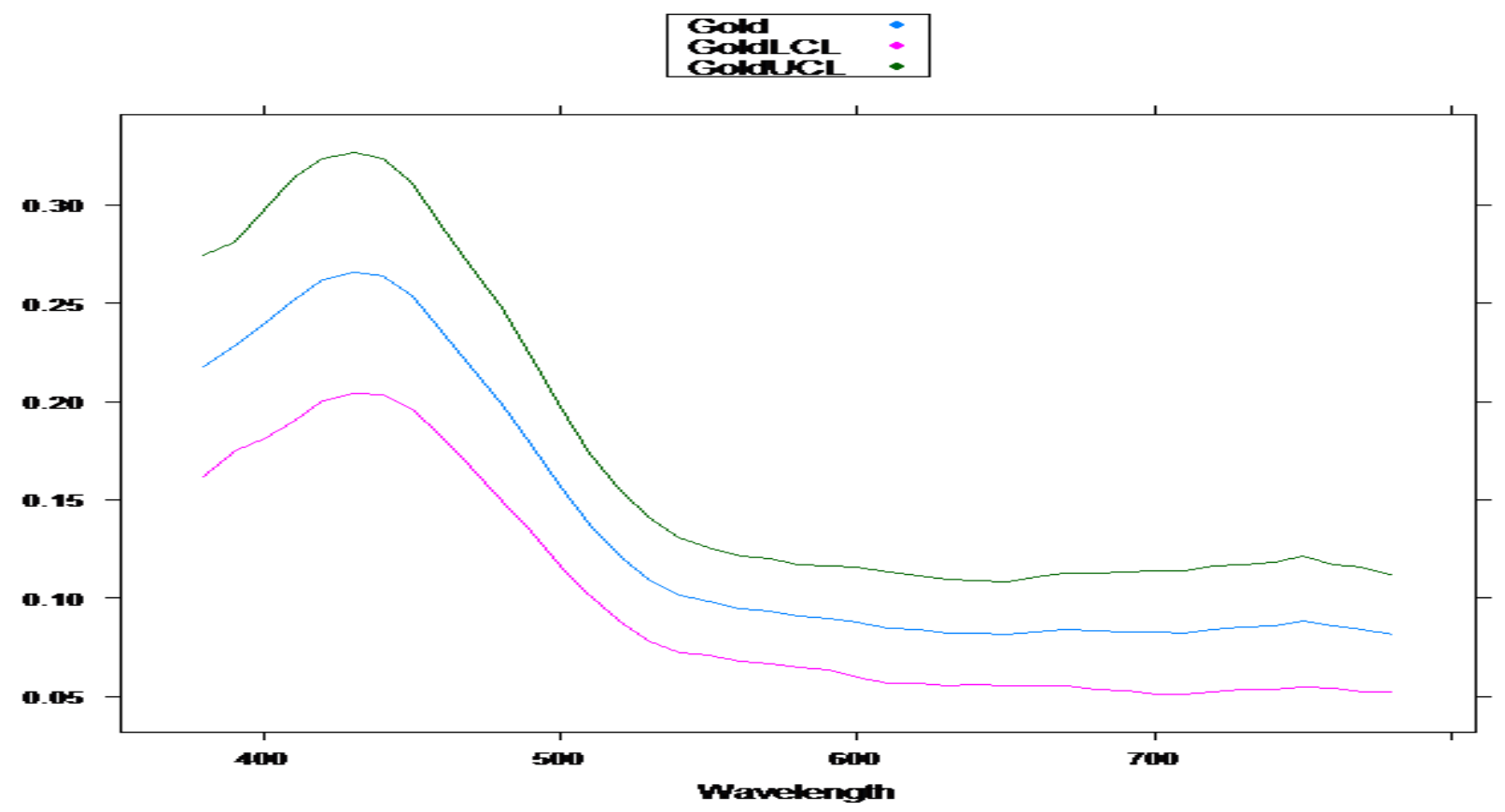

Figure 50: Spectra of the Average Absorbance, UCL and the LCL for every Gold Shirt 


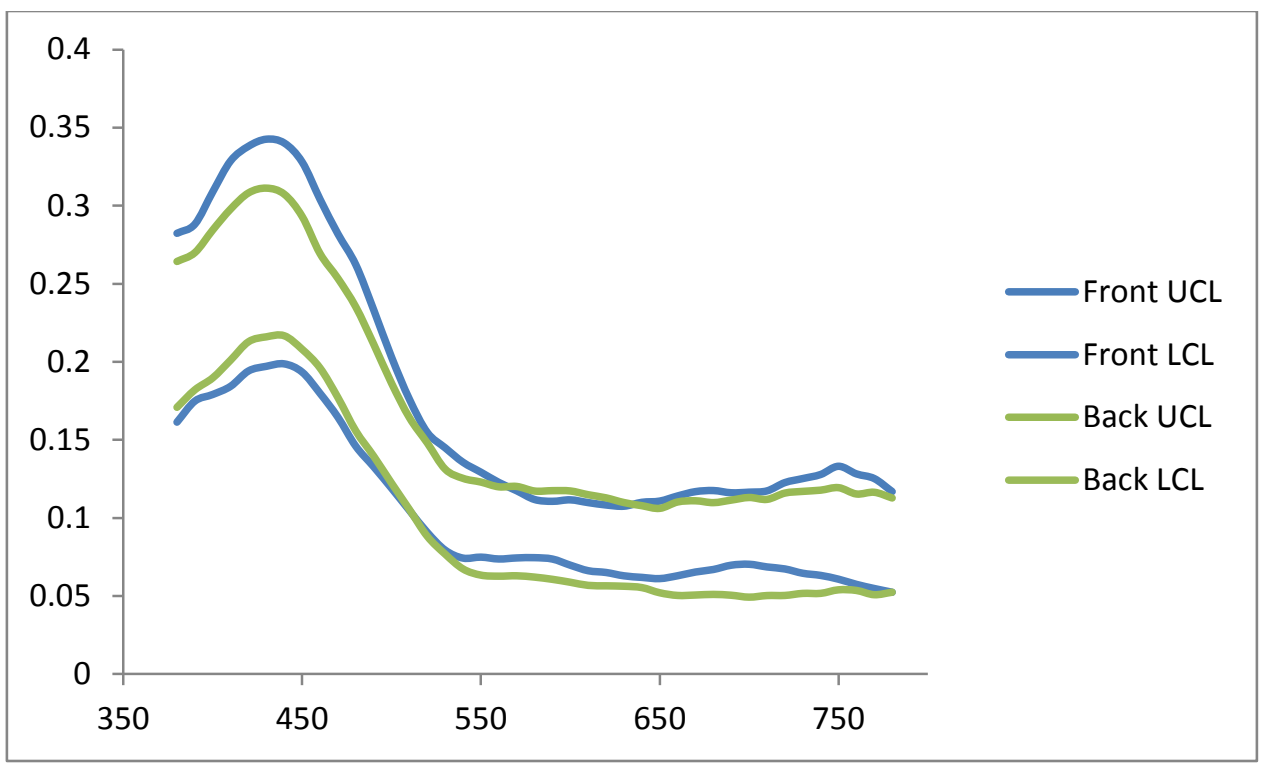

Figure 51: The 95\% UCL and the 95\% LCL for the Gold Front and Back

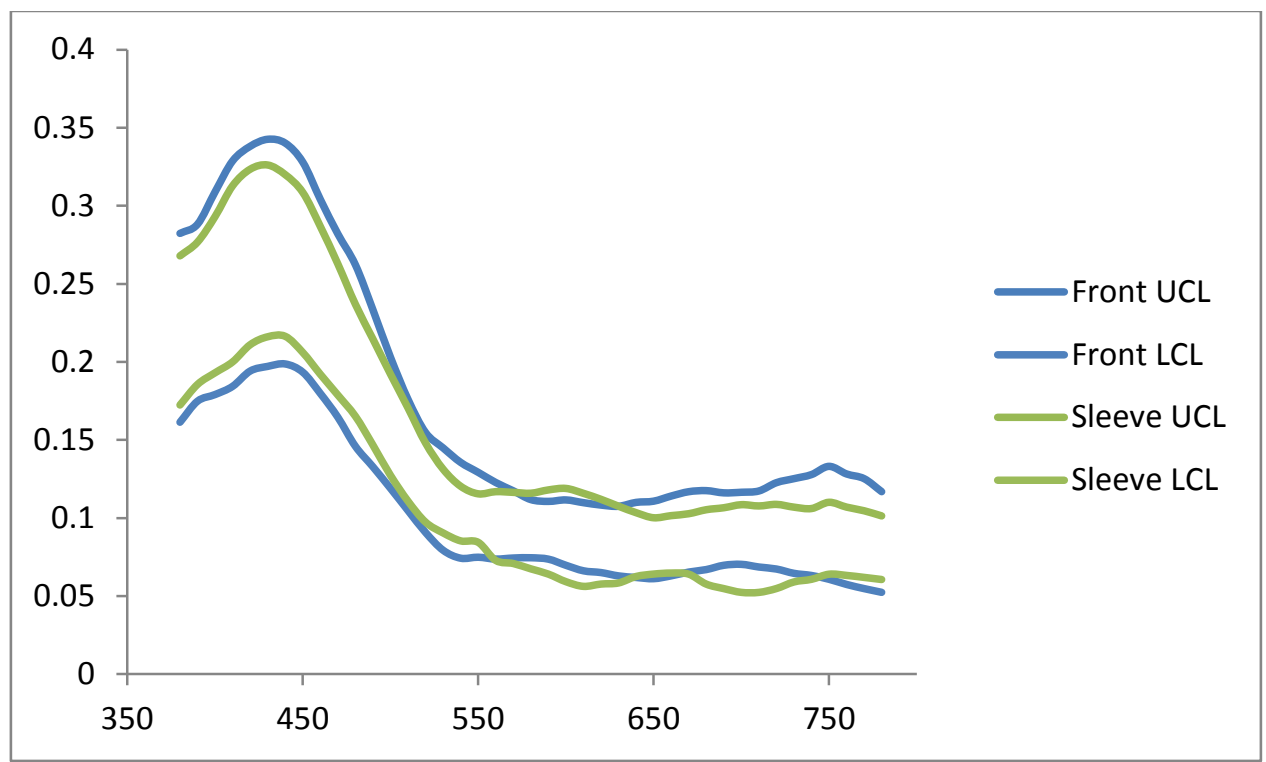

Figure 52: The 95\% UCL and the 95\% LCL for the Gold Front and Sleeve 


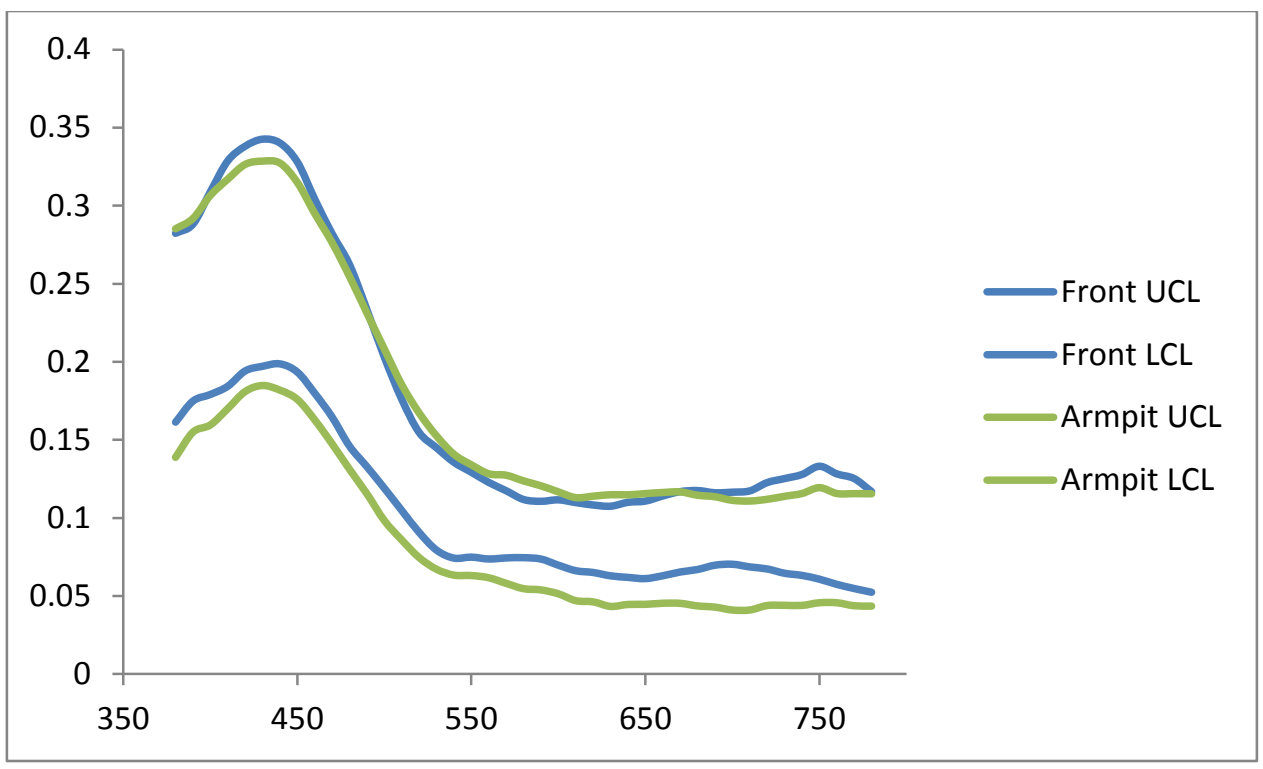

Figure 53: The 95\% UCL and the 95\% LCL for the Gold Front and Armpit

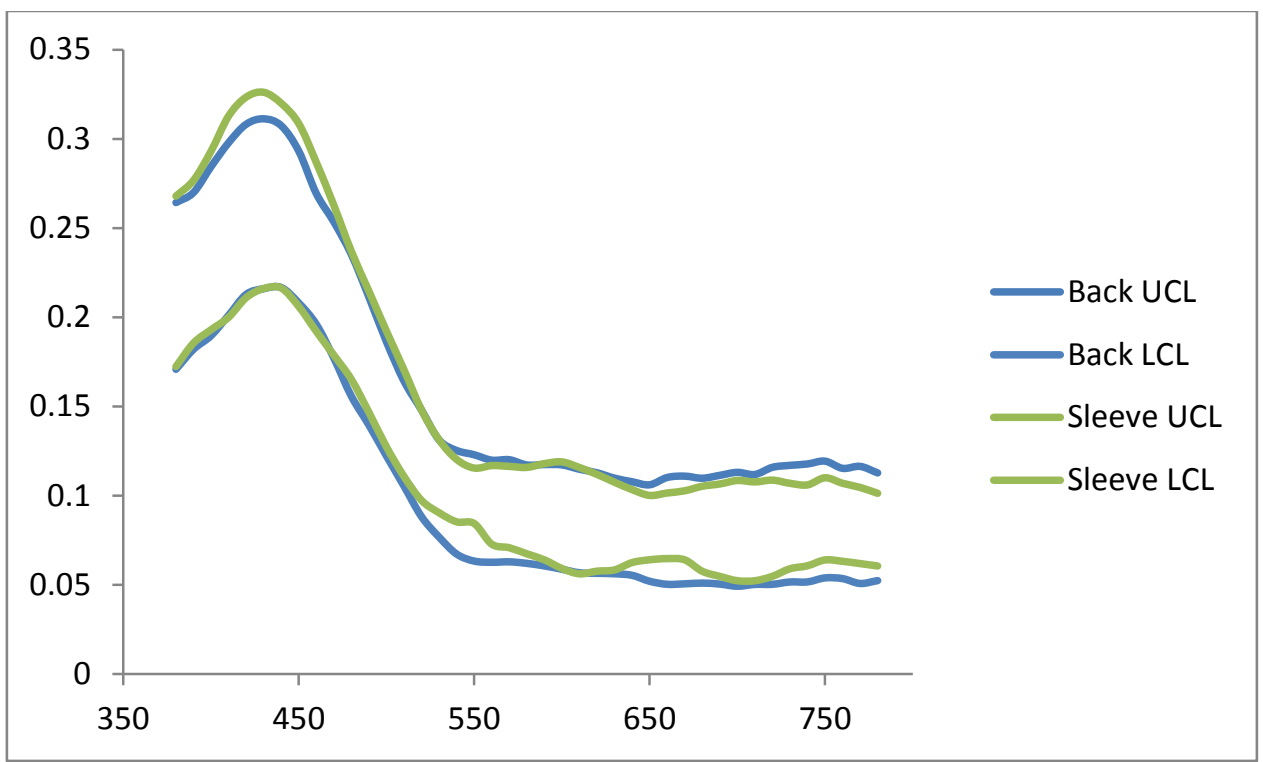

Figure 54: The 95\% UCL and the 95\% LCL for the Gold Back and Sleeve 


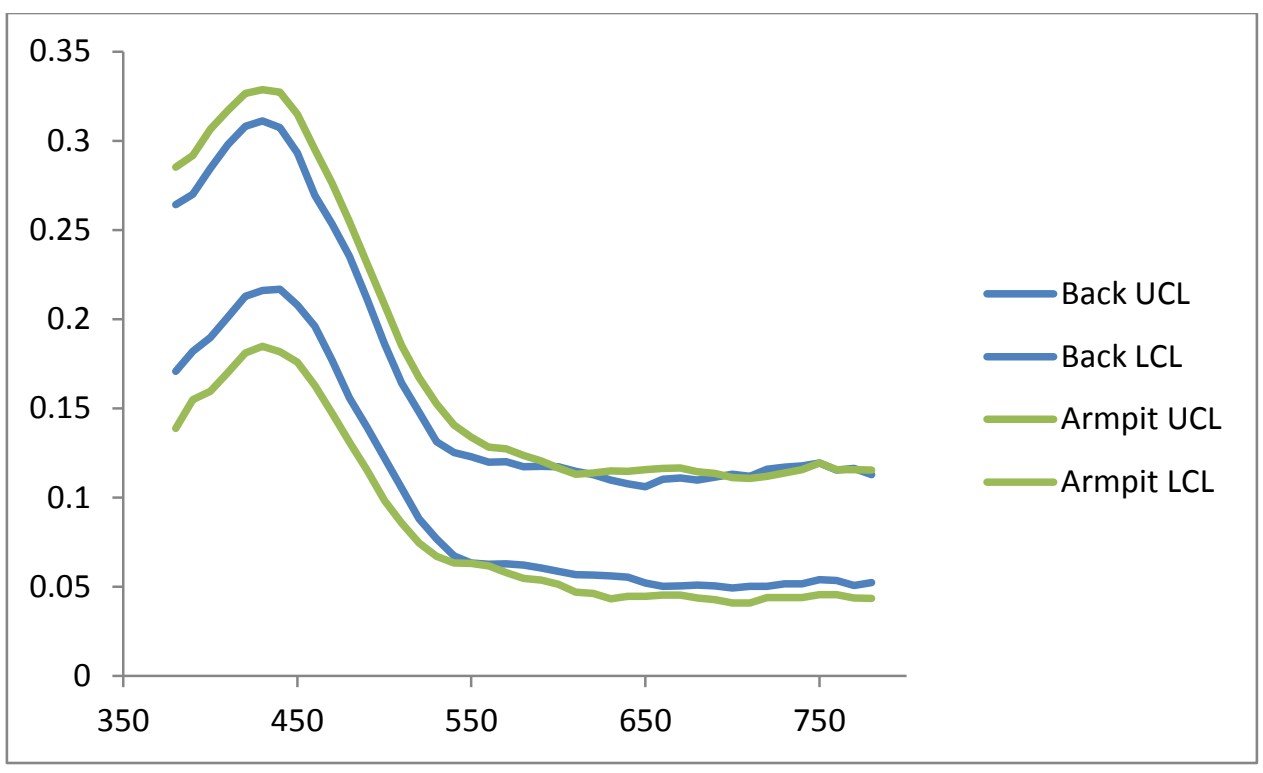

Figure 55: The 95\% UCL and the 95\% LCL for the Gold Back and Armpit

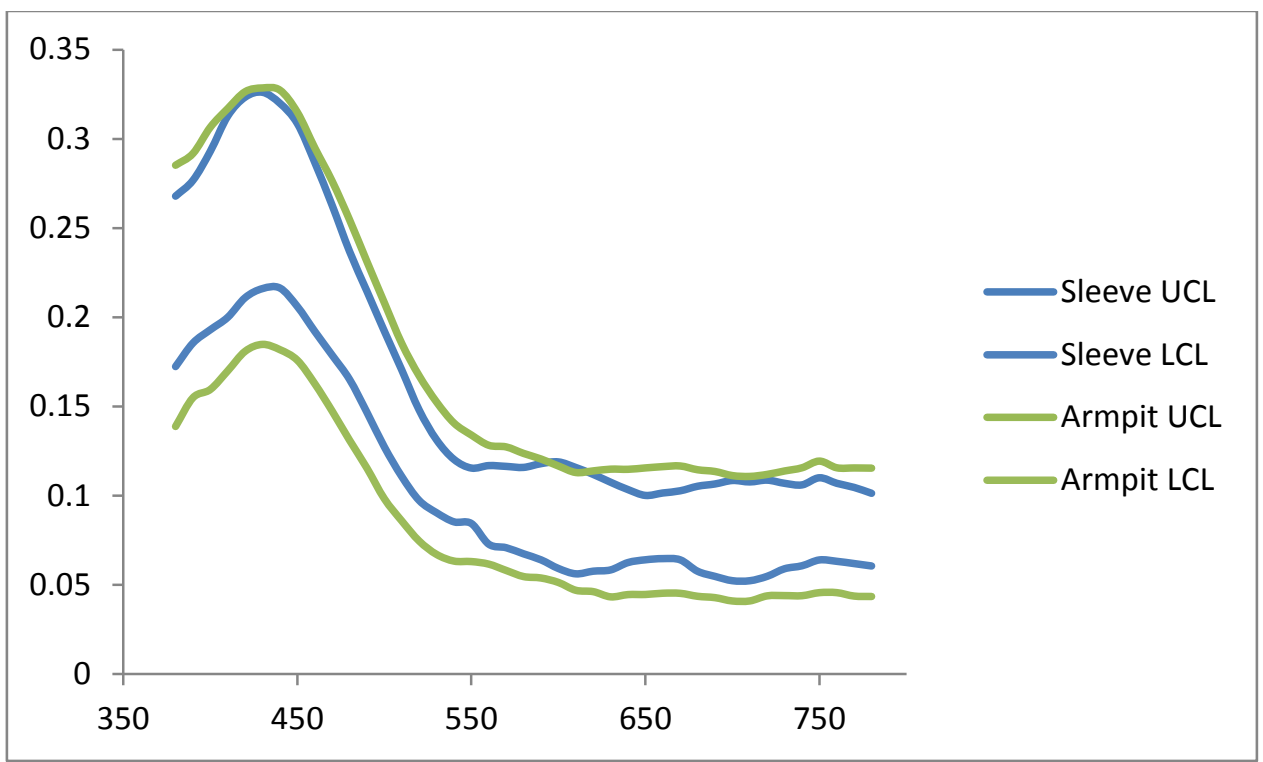

Figure 56: The 95\% UCL and the 95\% LCL for the Gold Armpit and Sleeve 


\section{Kelly Green}

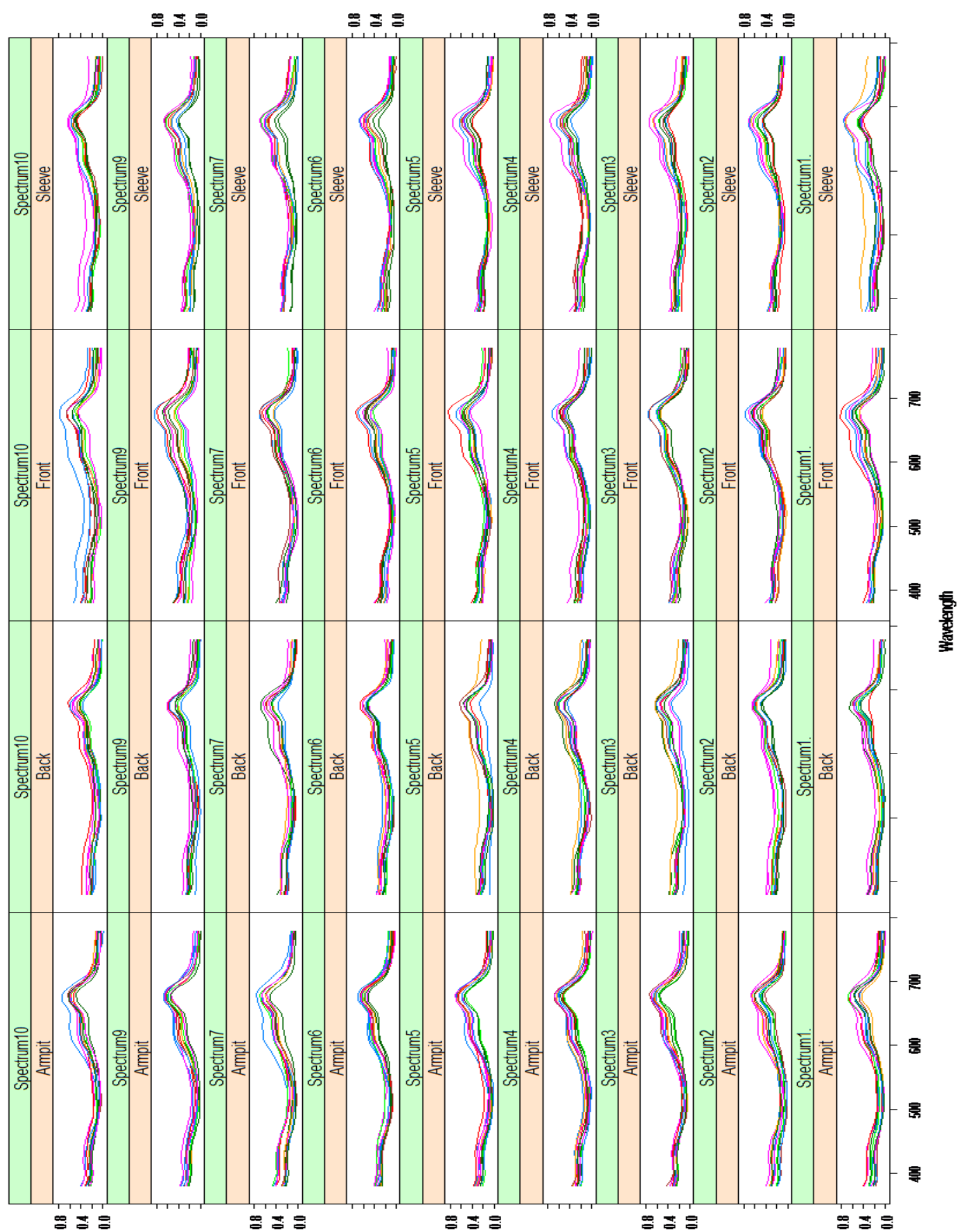

Figure 57: Spectra the Kelly Green A-J shirts for armpit, back, front and sleeve of all ten runs 


\begin{tabular}{|l|}
\hline Armpit : \\
Back : \\
Front : \\
Sleeve \\
\hline
\end{tabular}

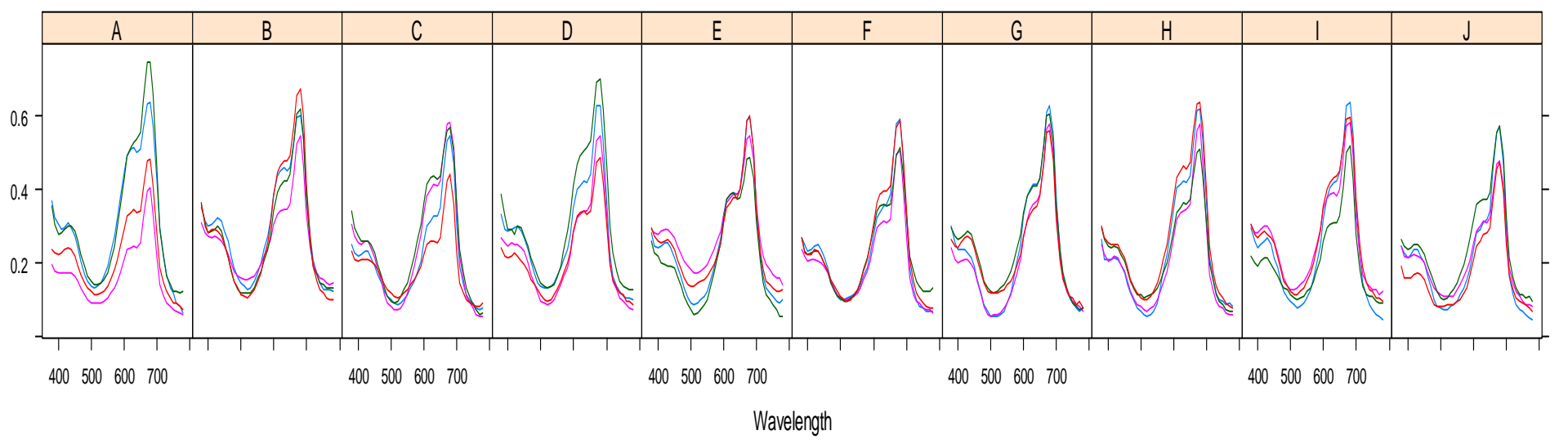

Figure 58: Spectra of the armpit, back, front and sleeve for Kelly Green A-J shirts

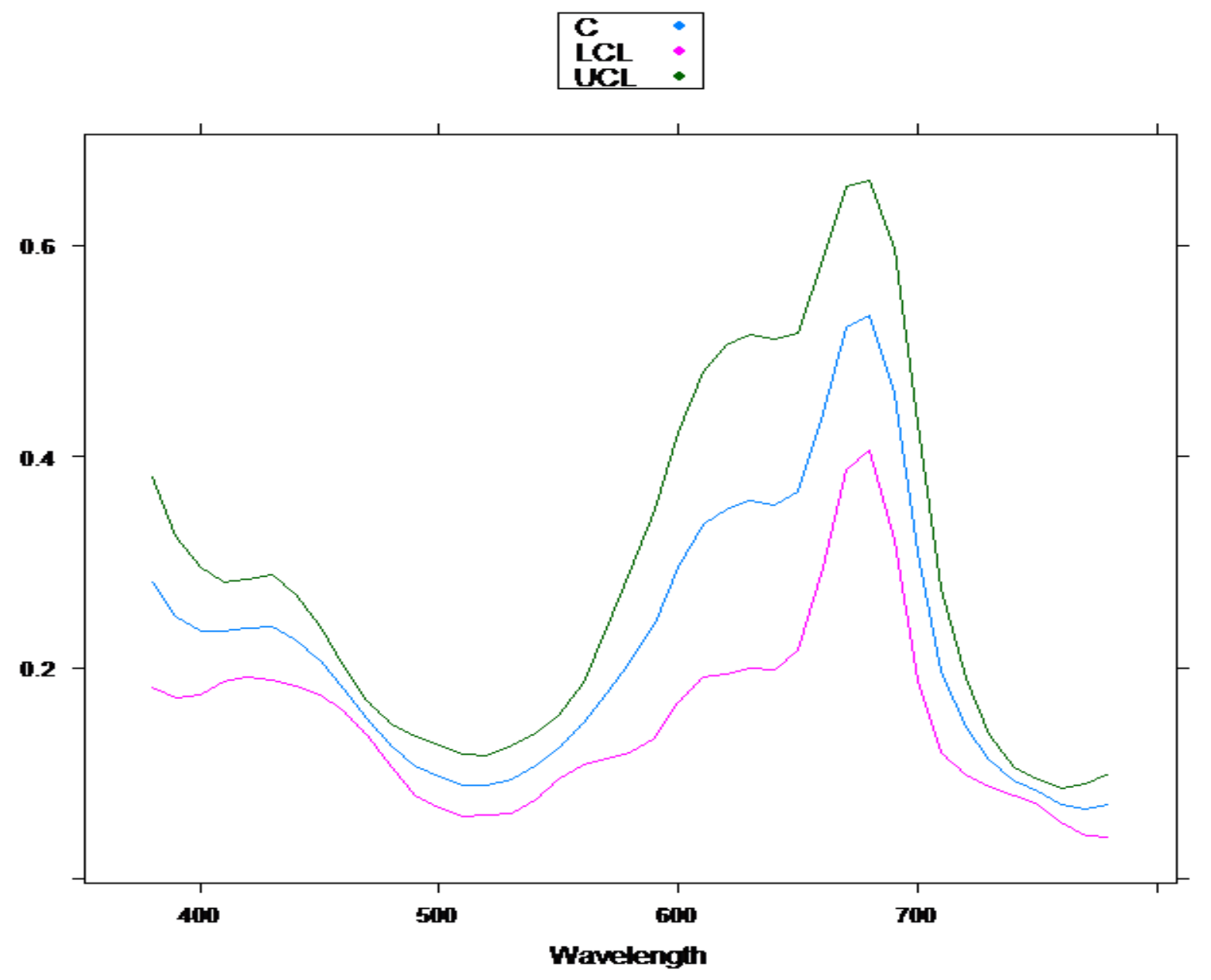

Figure 59: Spectra of the Average Absorbance, UCL and the LCL for 4 all regions of the Kelly Green C shirts 


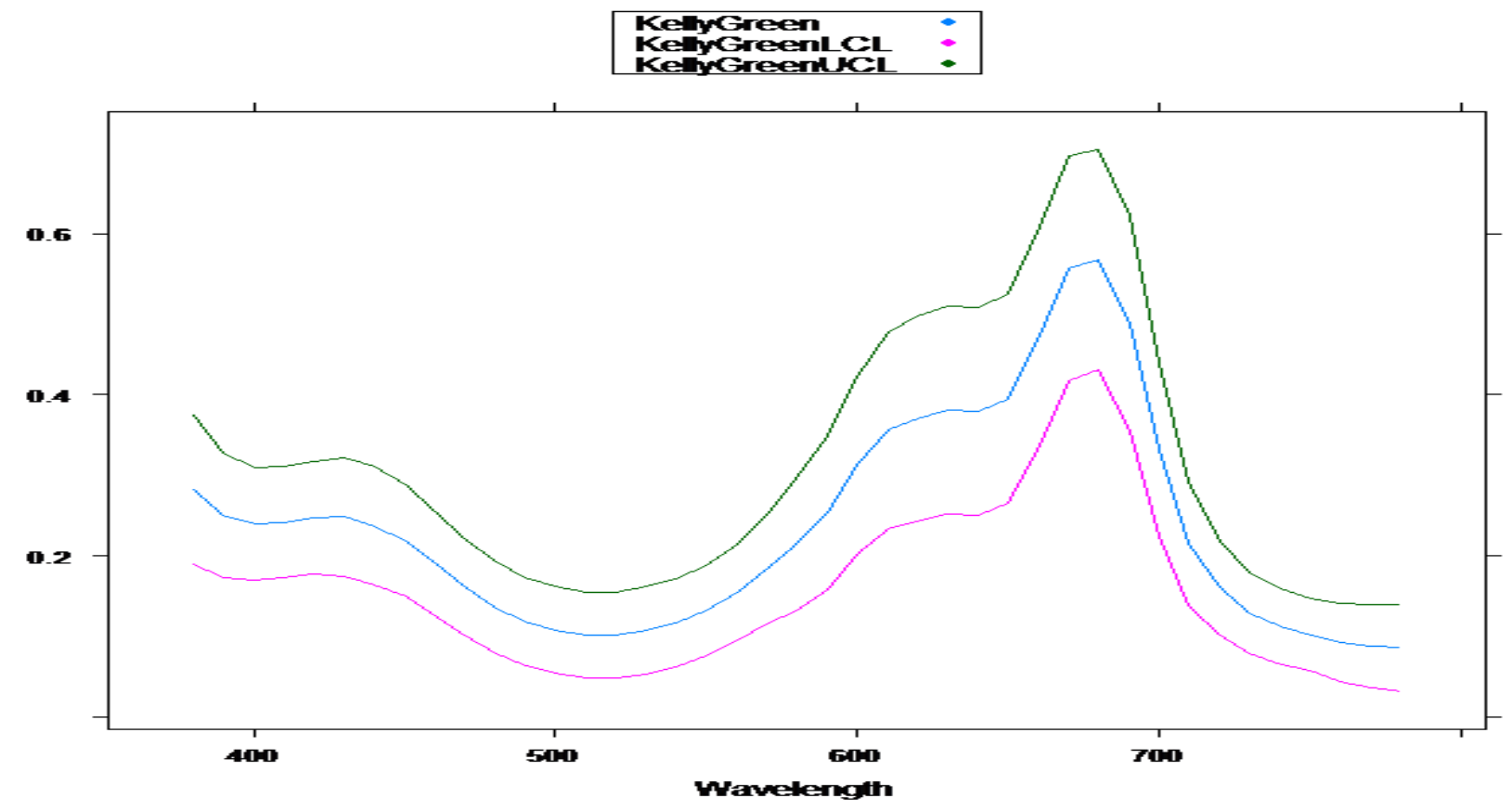

Figure 60: Spectra of the Average Absorbance, UCL and the LCL for 4 all regions of the Kelly Green D shirts

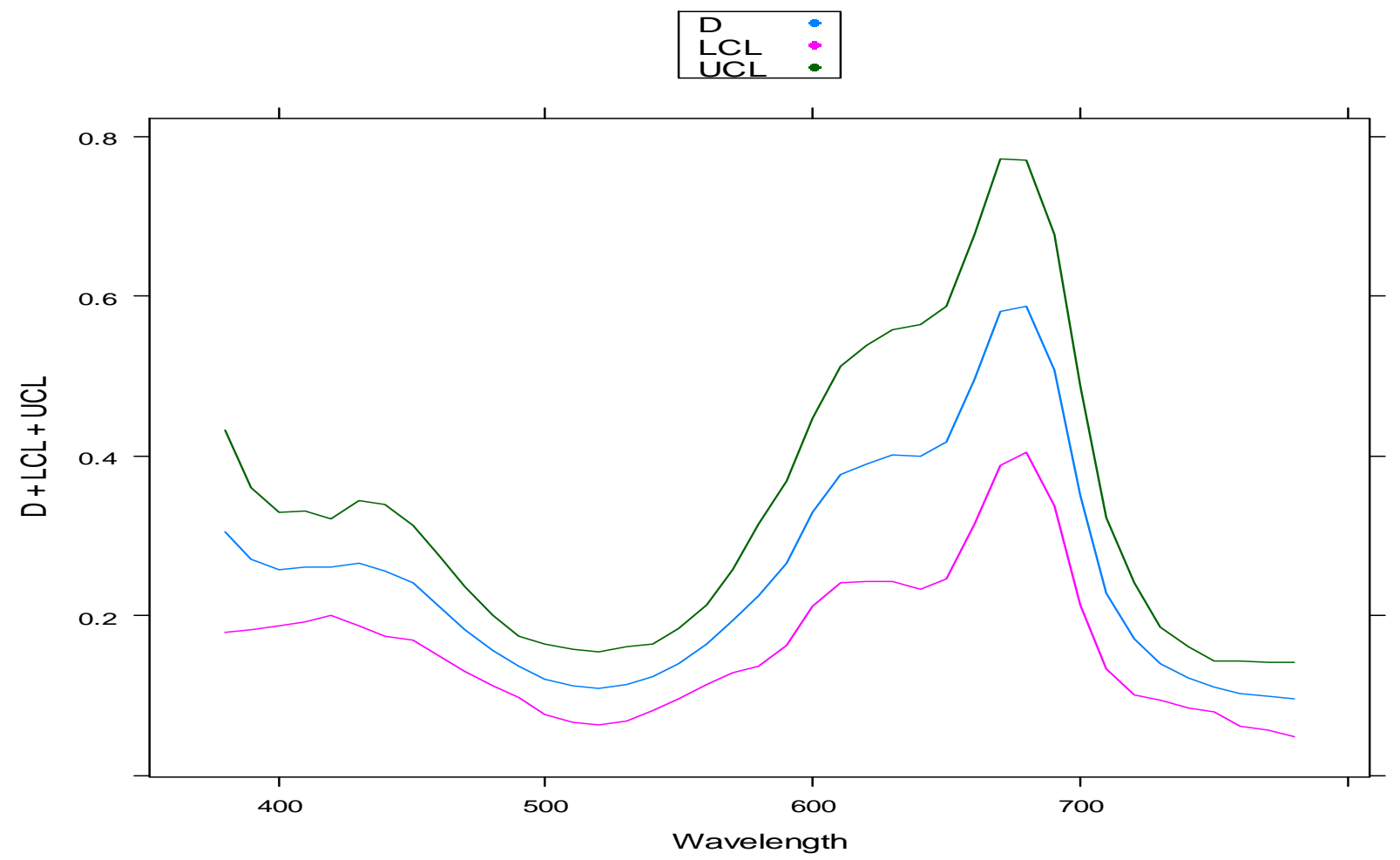

Figure 61: Spectra of the Average Absorbance, UCL and the LCL for every Kelly Green Shirt 


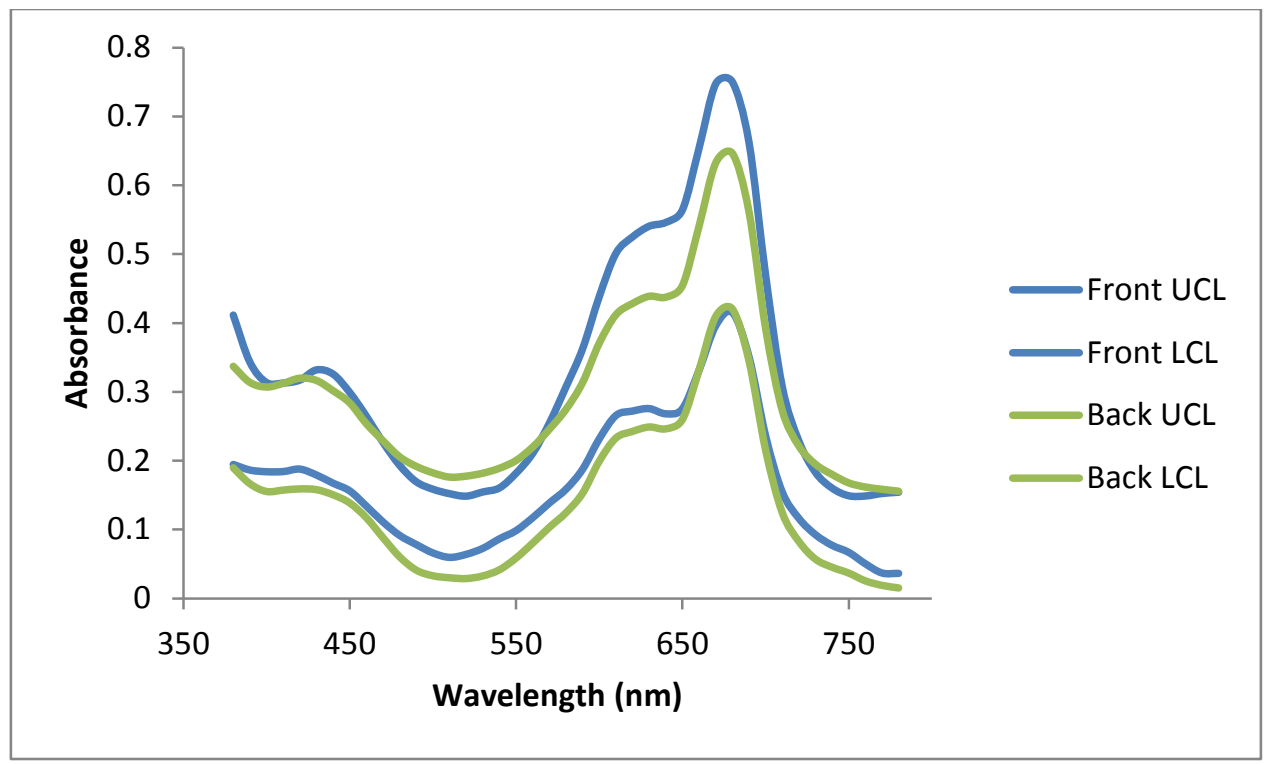

Figure 62: The 95\% UCL and the 95\% LCL for the Kelly Green Front and Back

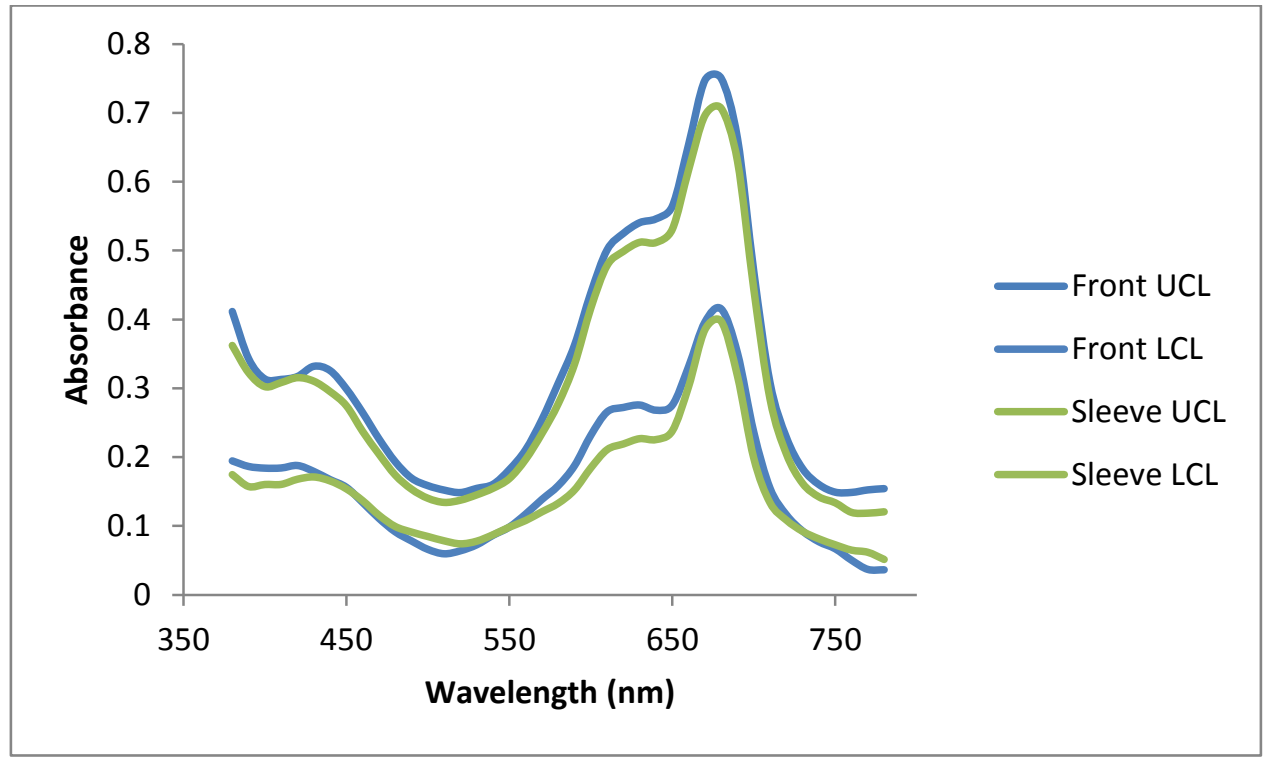

Figure 63: The 95\% UCL and the 95\% LCL for the Kelly Green Front and Sleeve 


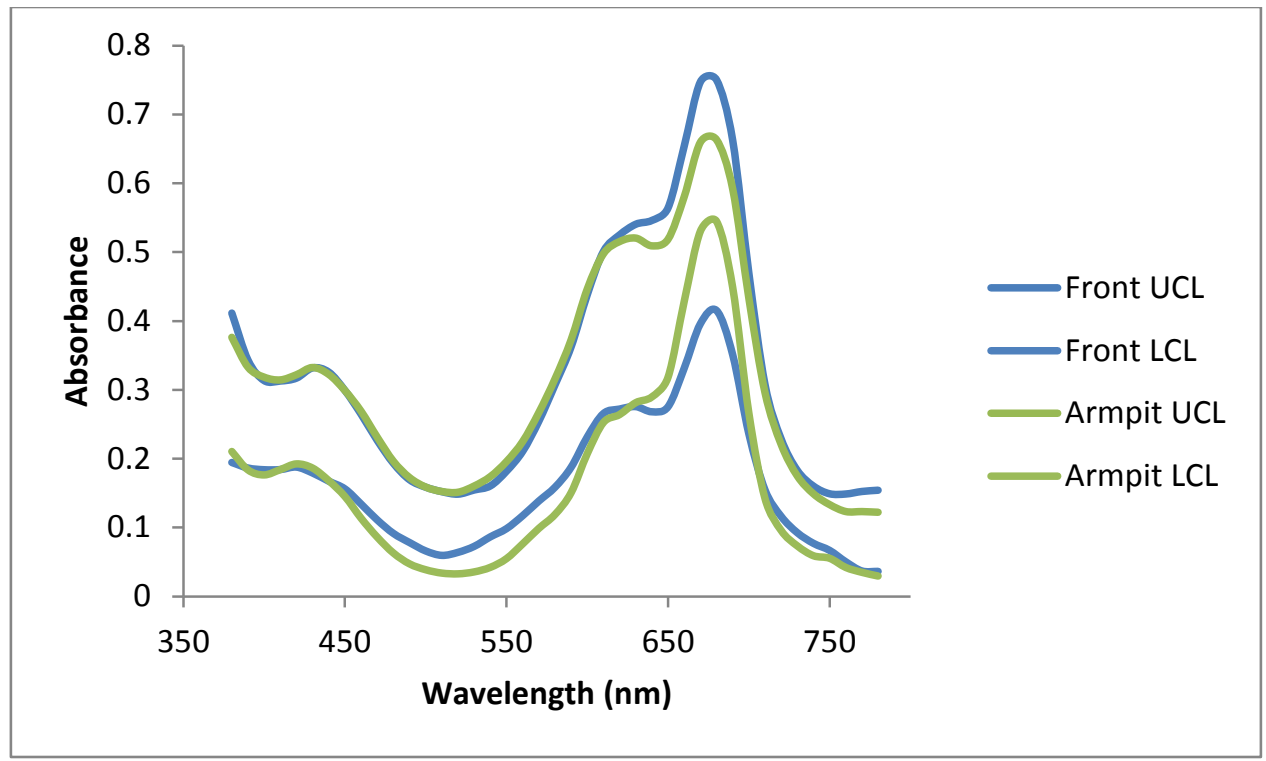

Figure 64: The 95\% UCL and the 95\% LCL for the Kelly Green Front and Armpit

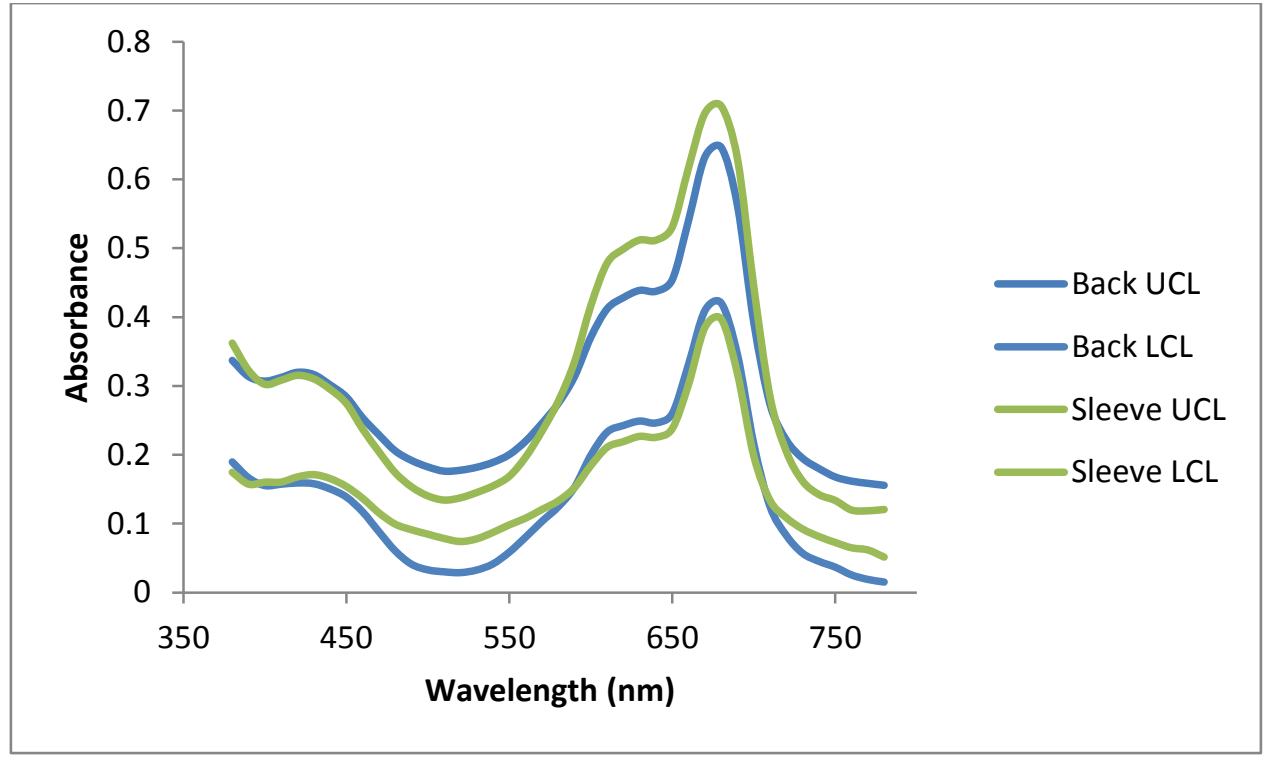

Figure 65: The 95\% UCL and the 95\% LCL for the Kelly Green Back and Sleeve 


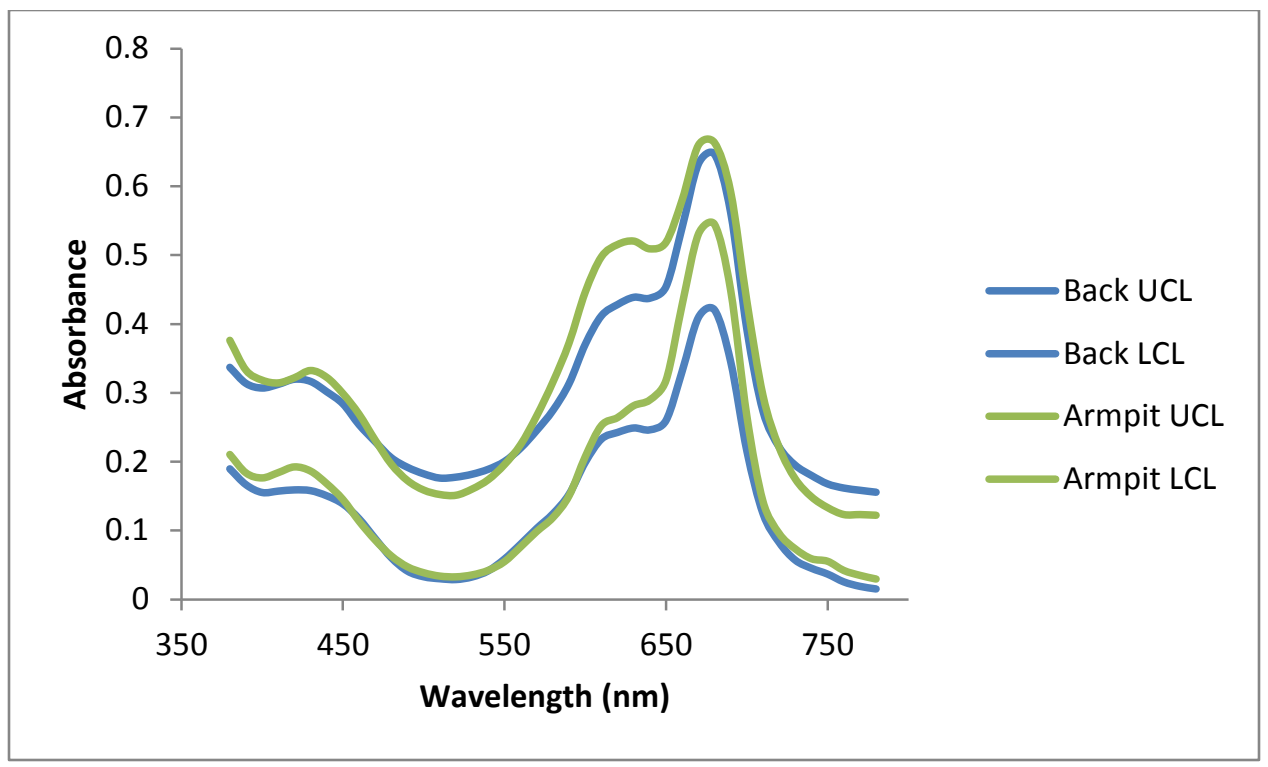

Figure 66: The 95\% UCL and the 95\% LCL for the Kelly Green Back and Armpit

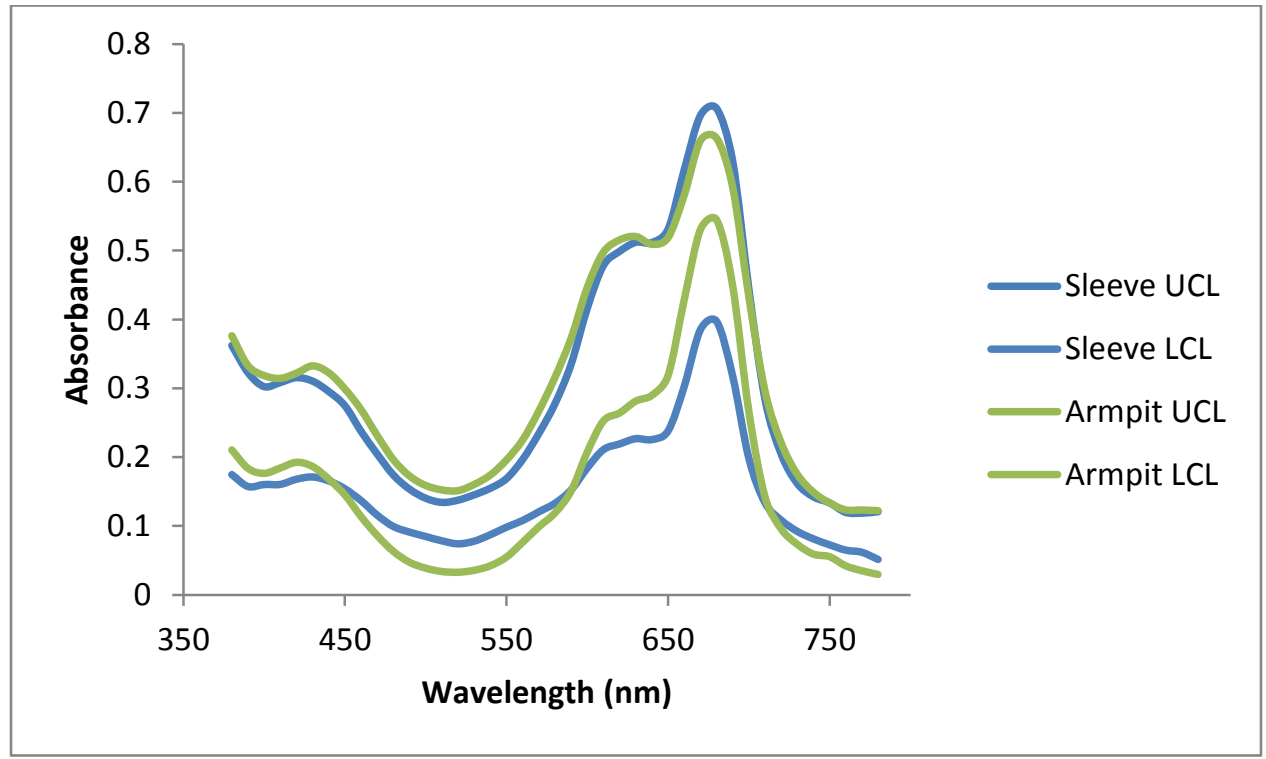

Figure 67: The 95\% UCL and the 95\% LCL for the Kelly Green Sleeve and Armpit 


\section{Orange}

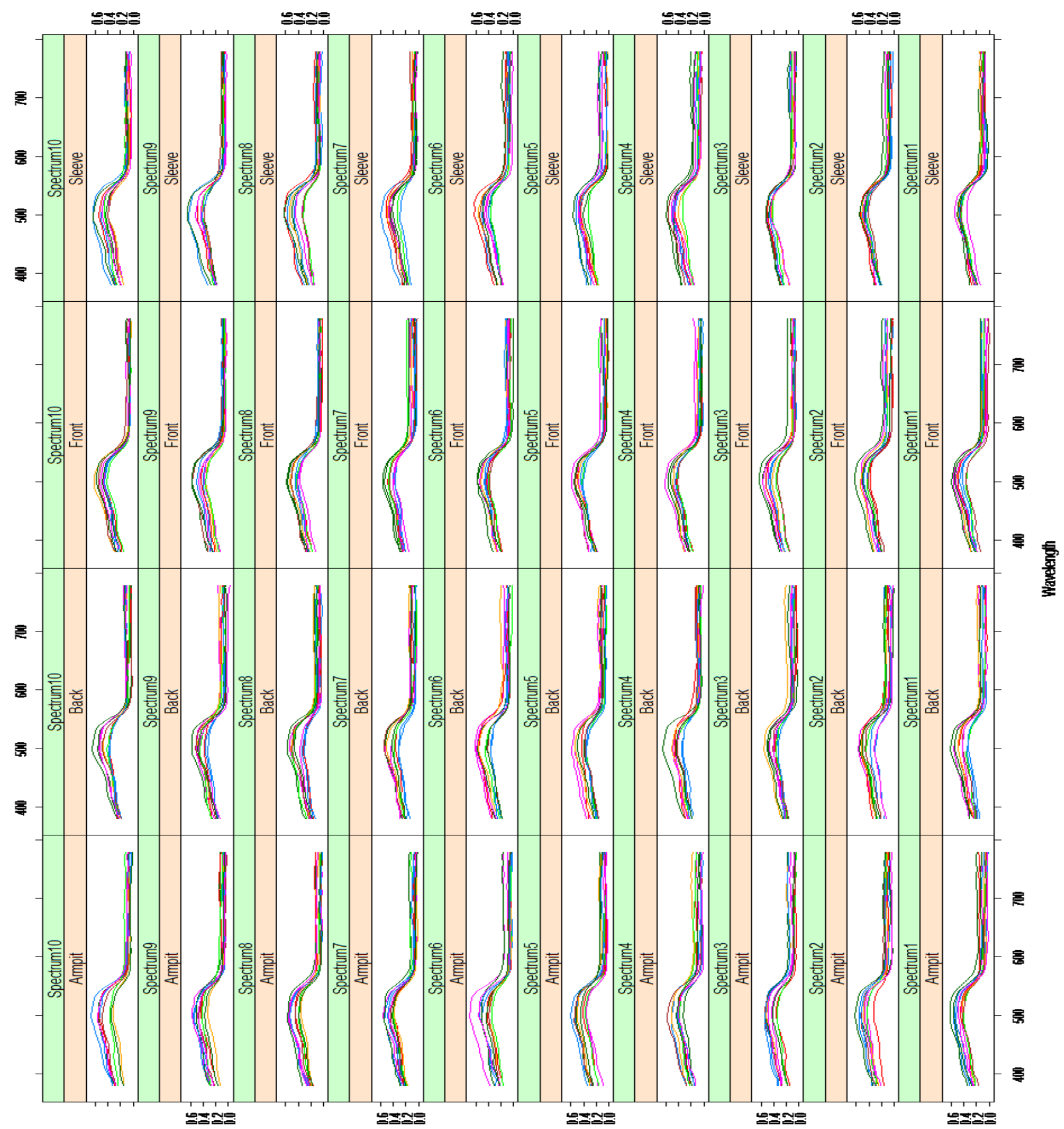

Figure 68: Spectra the Orange A-J shirts for armpit, back, front and sleeve of all ten runs 


\begin{tabular}{|l|}
\hline Armpit : \\
Back : \\
Front : \\
Sleeve \\
\hline
\end{tabular}

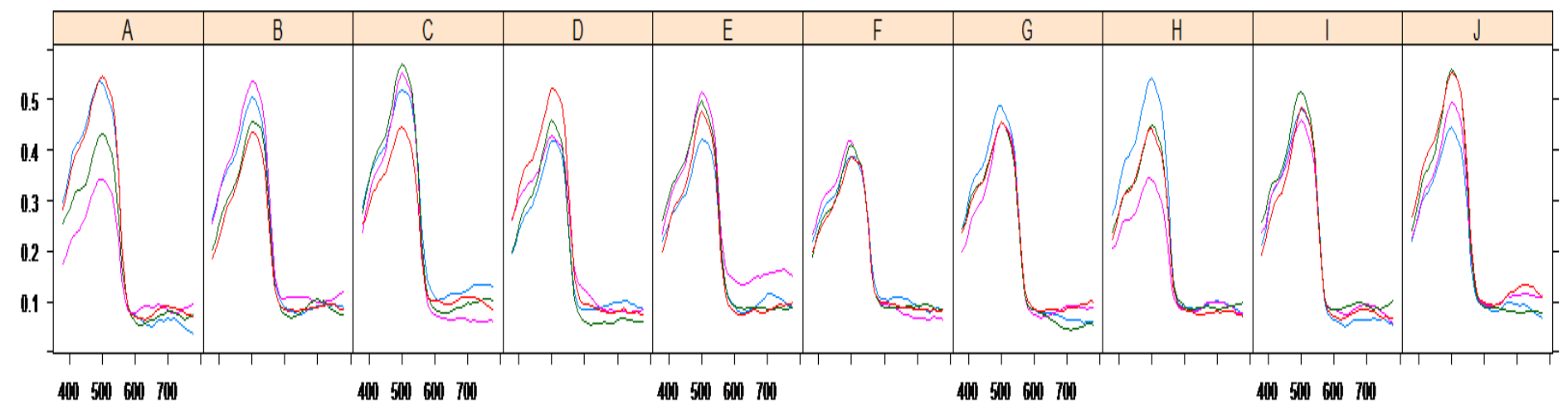

Figure 69: Spectra of the armpit, back, front and sleeve for Orange A-J shirts

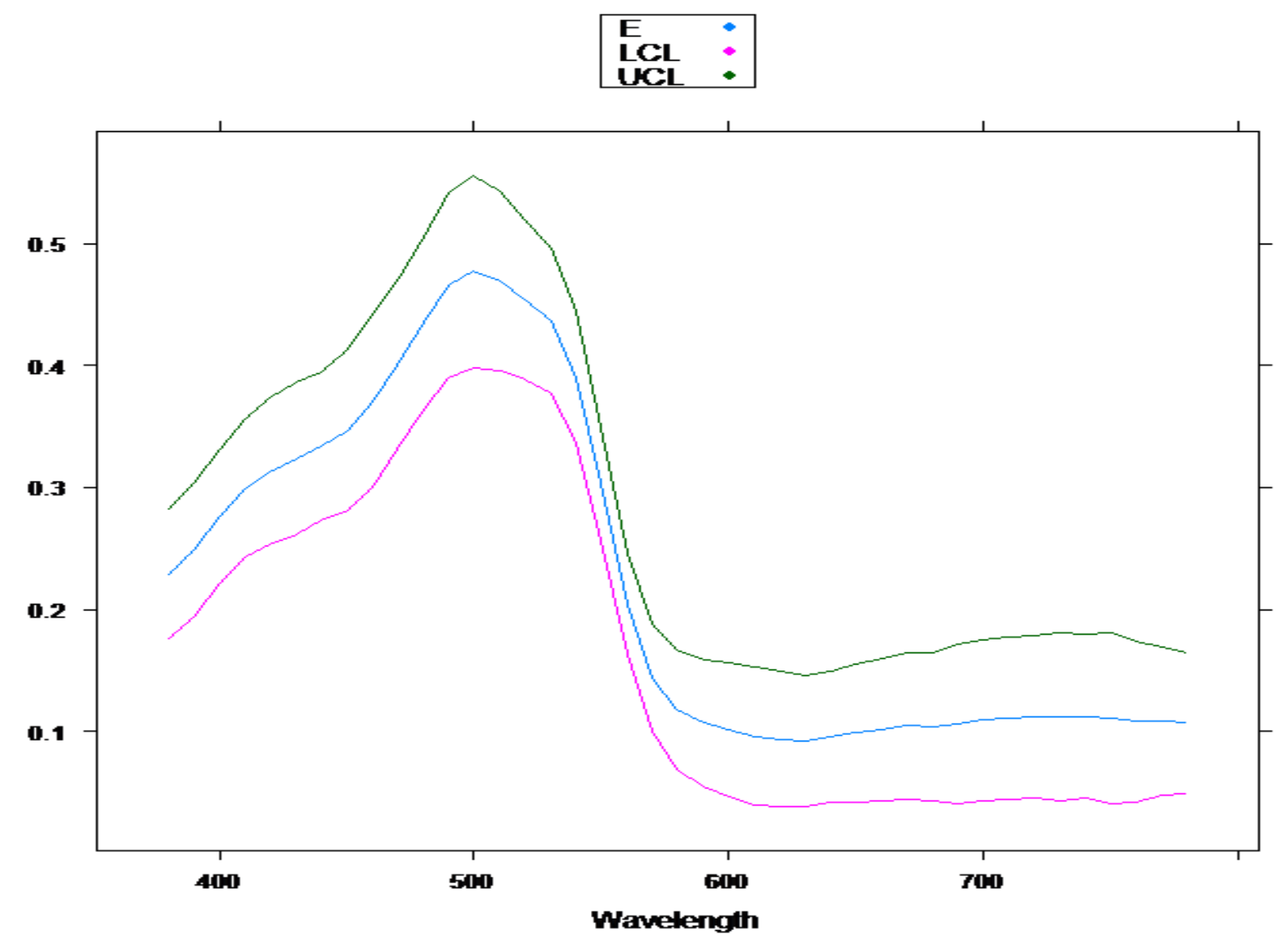

Figure 70: Spectra of the Average Absorbance, UCL and the LCL for 4 all regions of the Orange E Shirts 


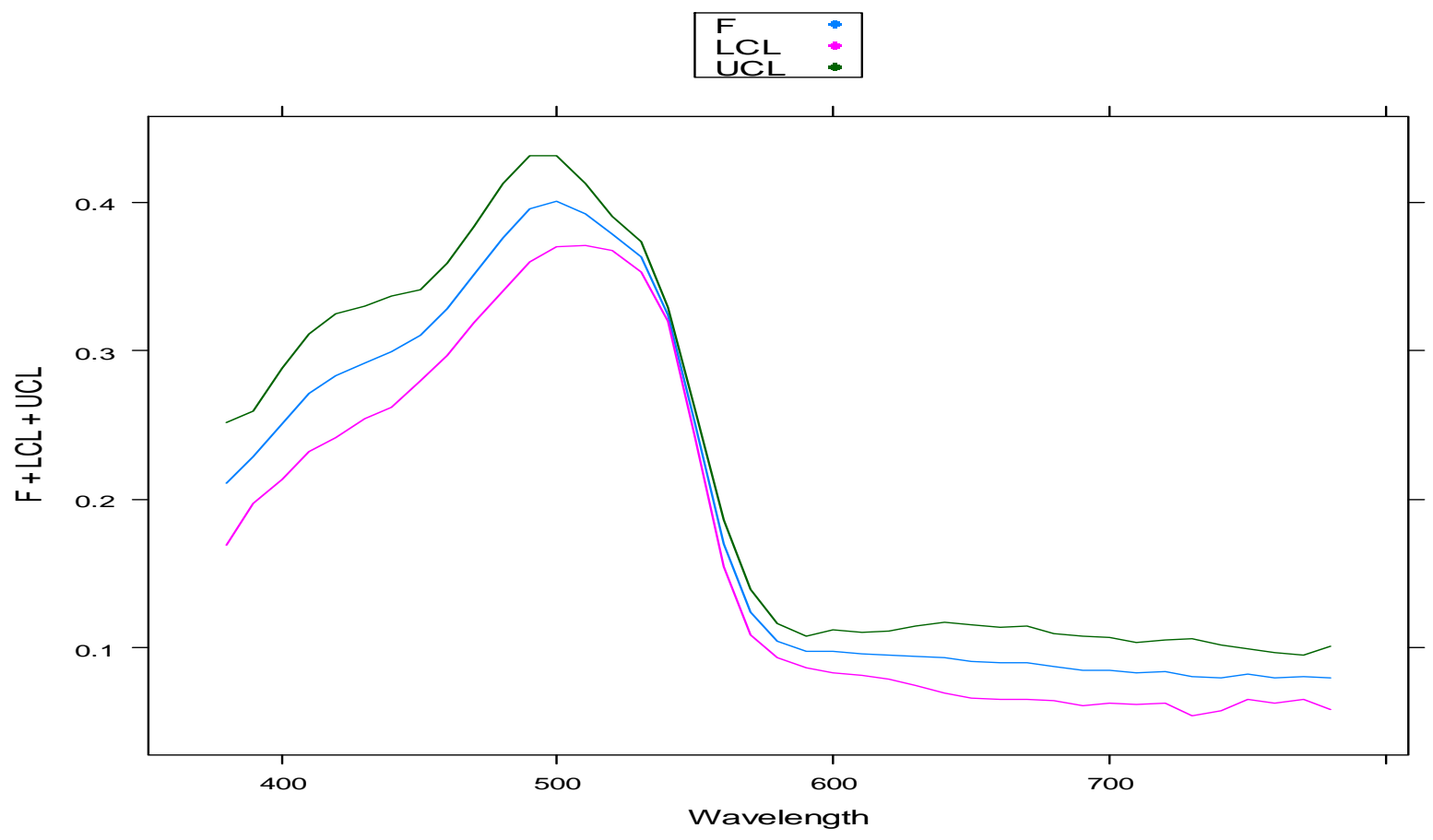

Figure 71: Spectra of the Average Absorbance, UCL and the LCL for 4 all regions of the Orange F Shirts

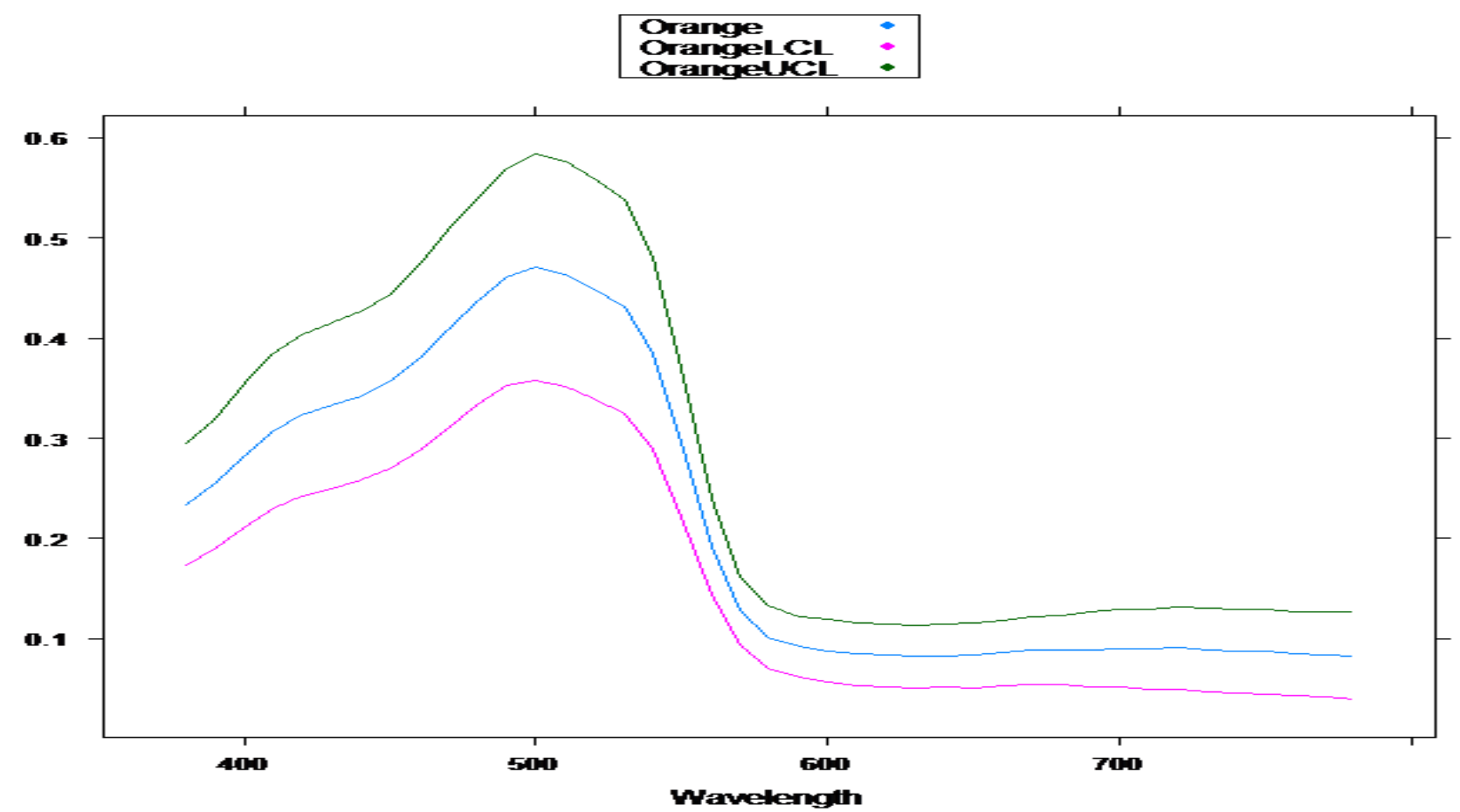

Figure 72: Spectra of the Average Absorbance, UCL and the LCL for every Orange Shirt 


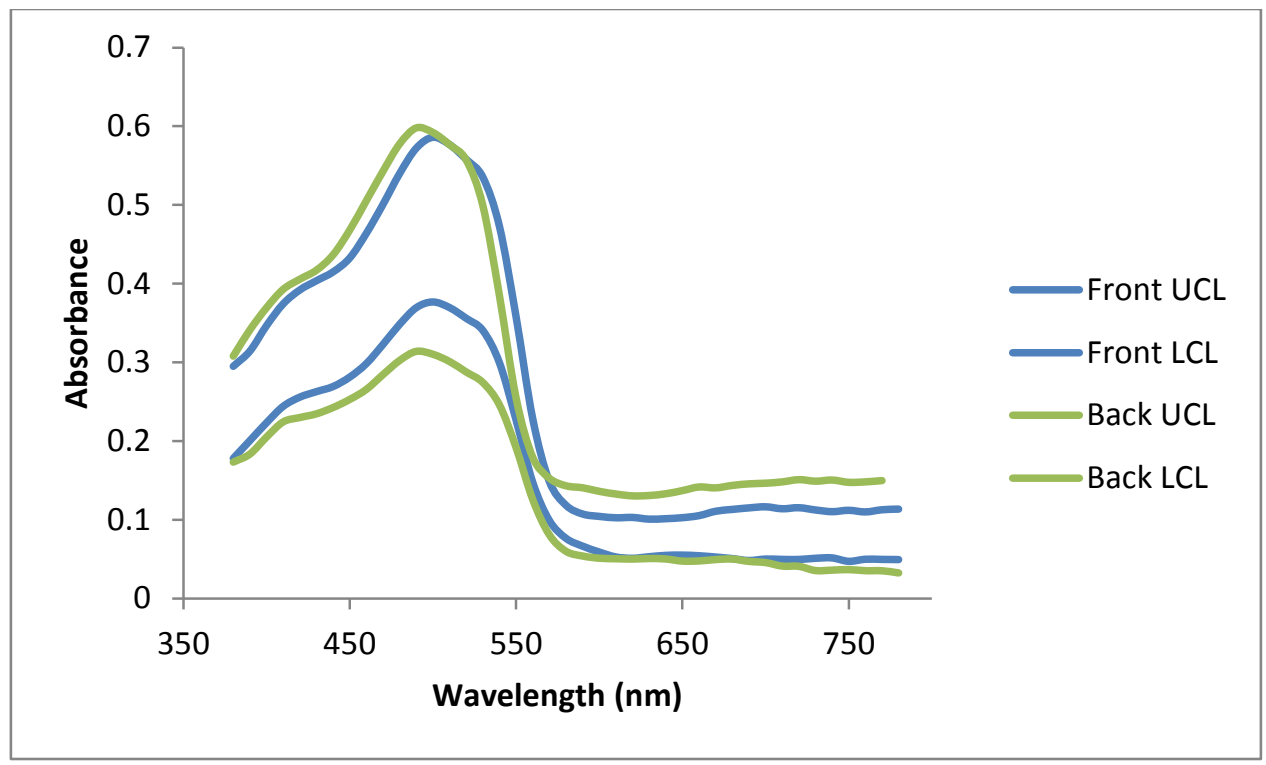

Figure 73: The 95\% UCL and the 95\% LCL for the Orange Front and Back

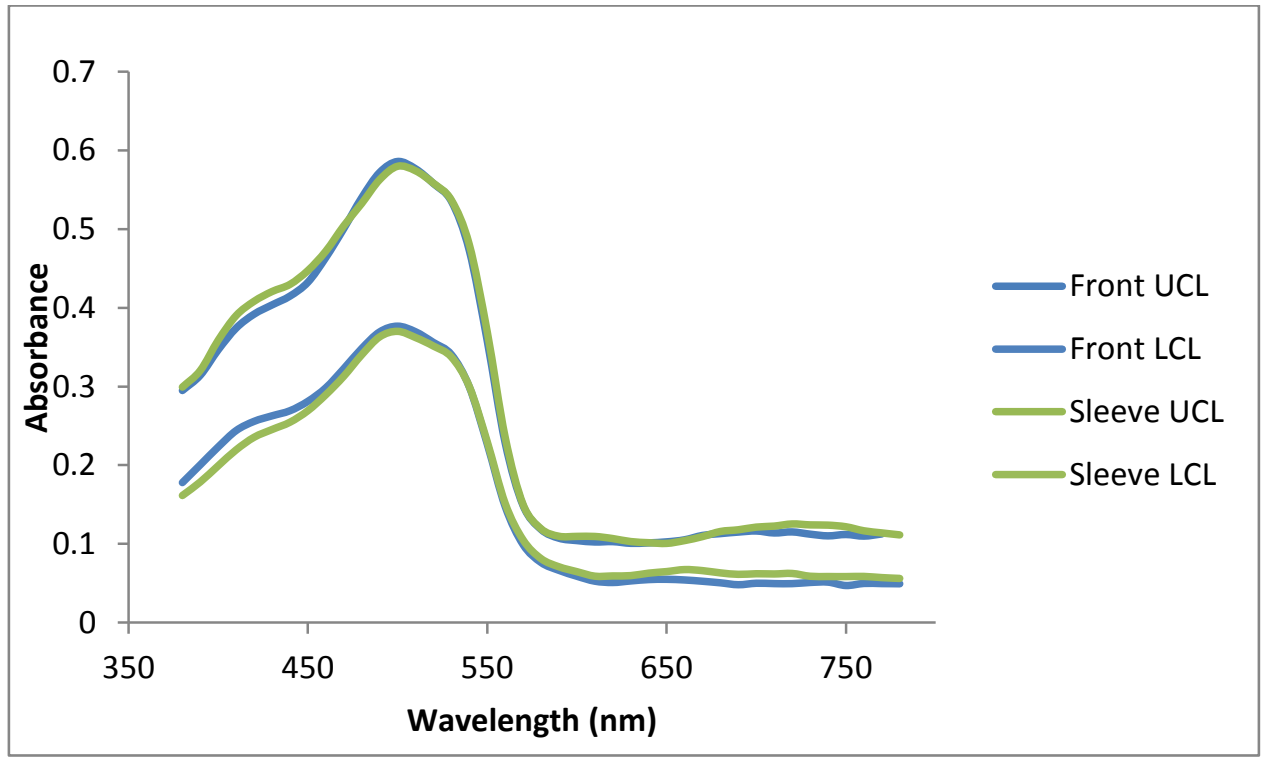

Figure 74: The 95\% UCL and the 95\% LCL for the Orange Front and Sleeve 


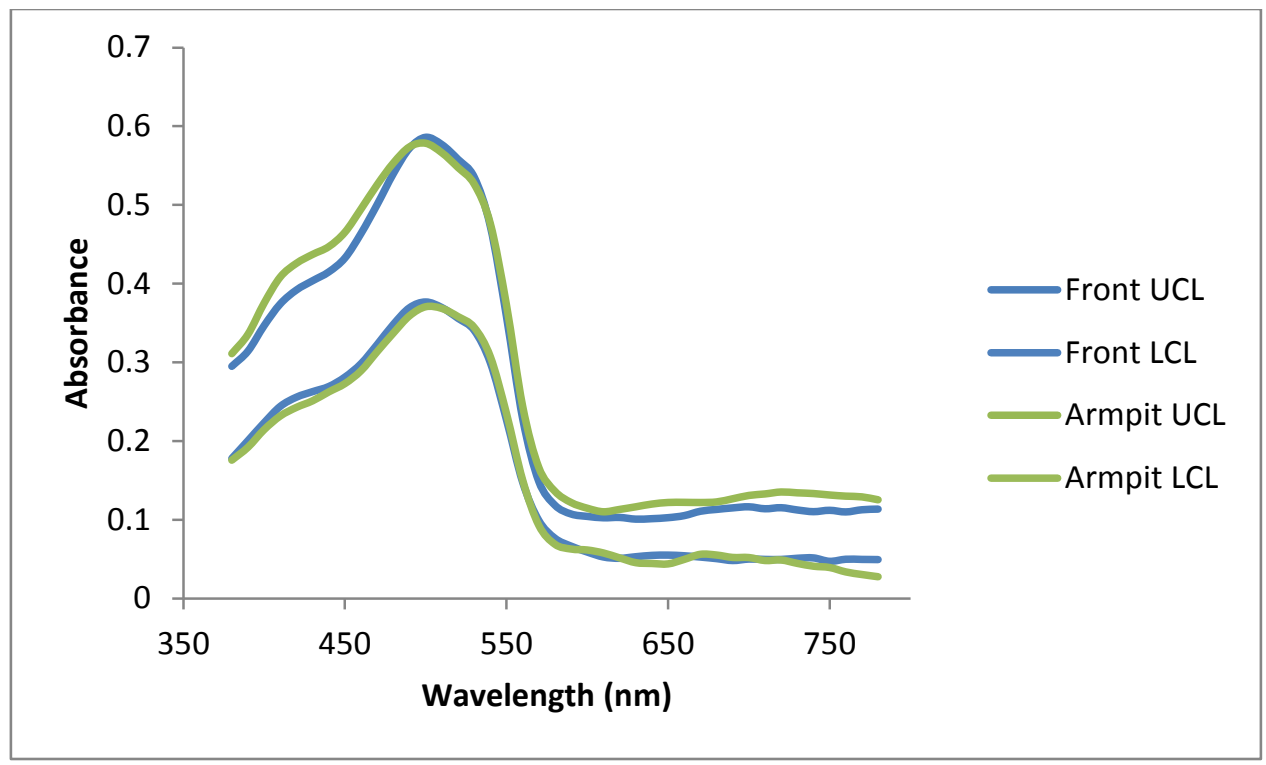

Figure 75: The 95\% UCL and the 95\% LCL for the Orange Front and Armpit

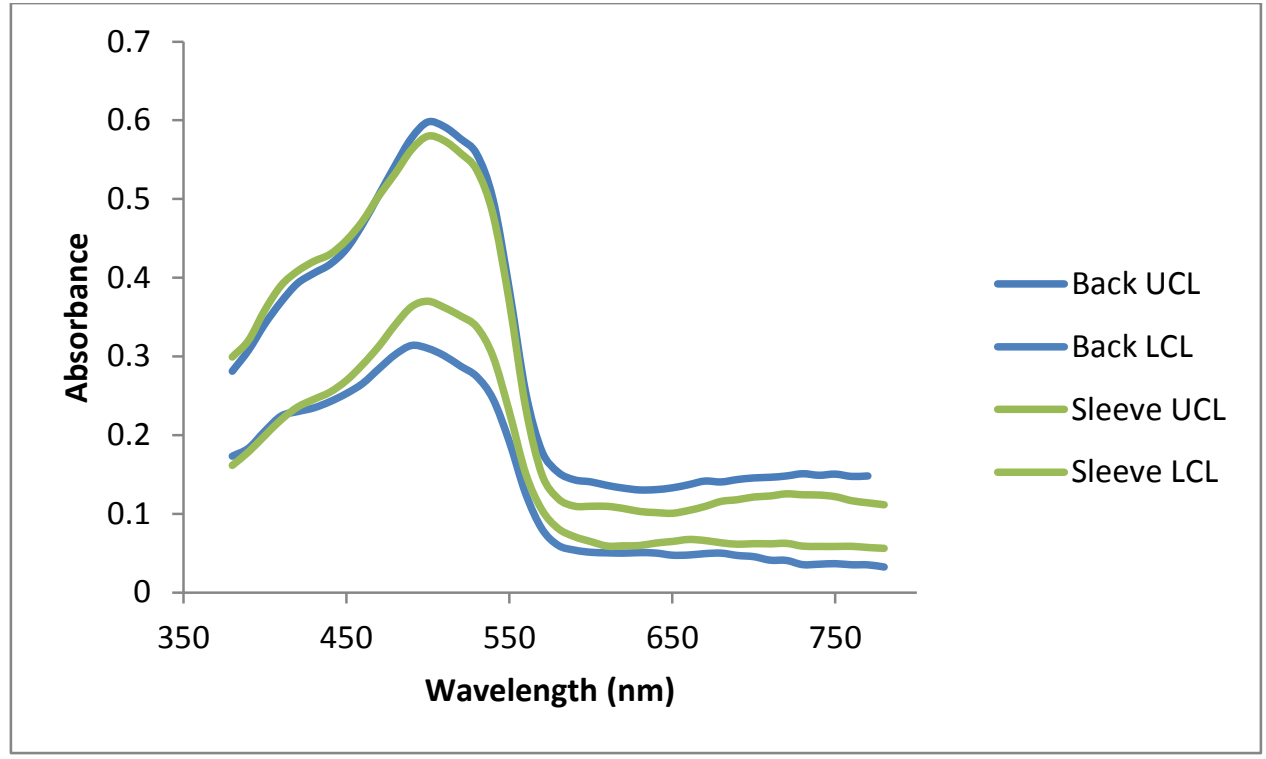

Figure 76: The 95\% UCL and the 95\% LCL for the Orange Back and Sleeve 


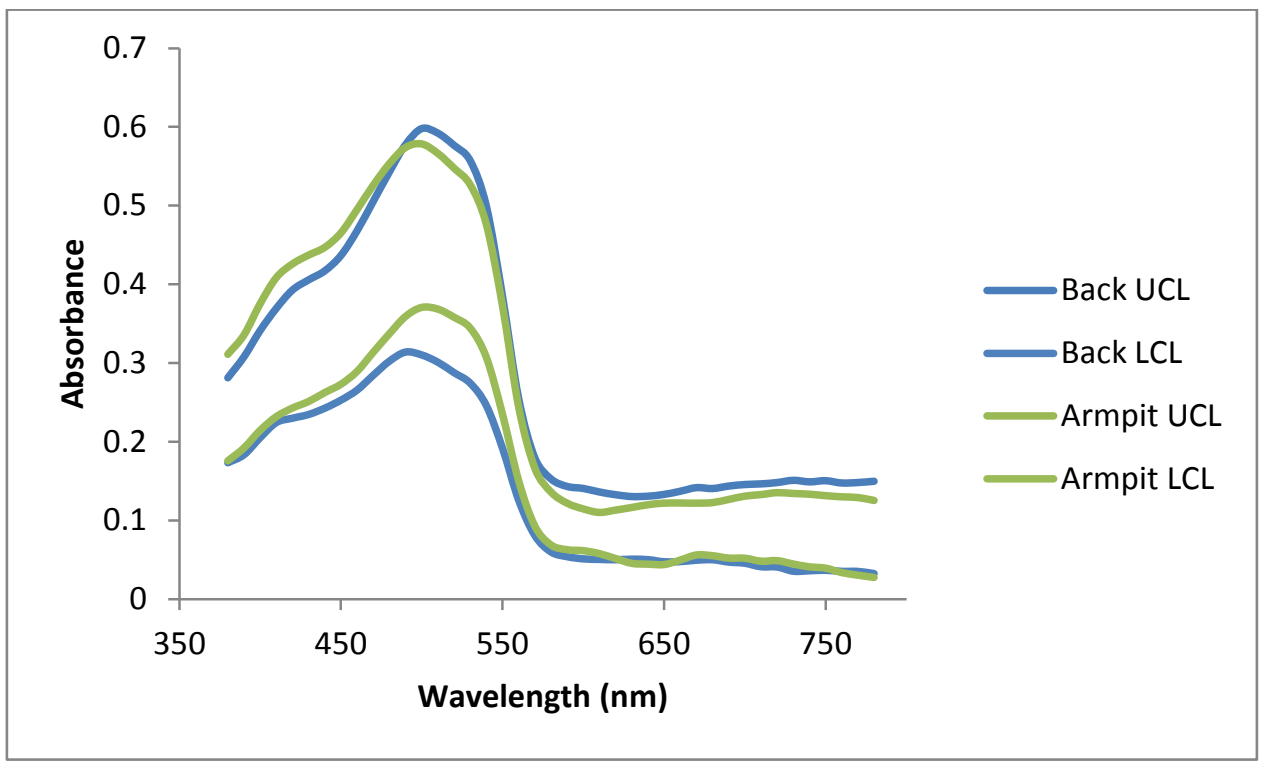

Figure 77: The 95\% UCL and the 95\% LCL for the Orange Back and Armpit

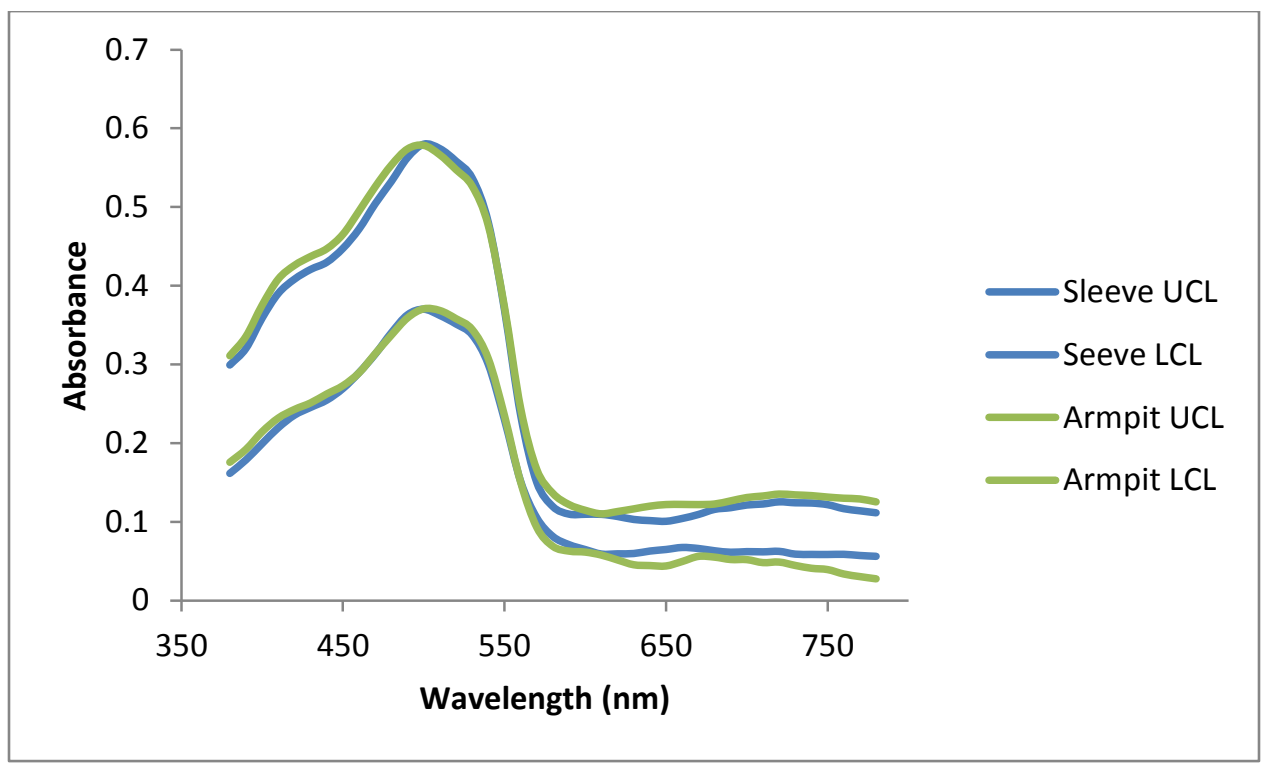

Figure 78: The 95\% UCL and the 95\% LCL for the Orange Sleeve and Armpit 
Red

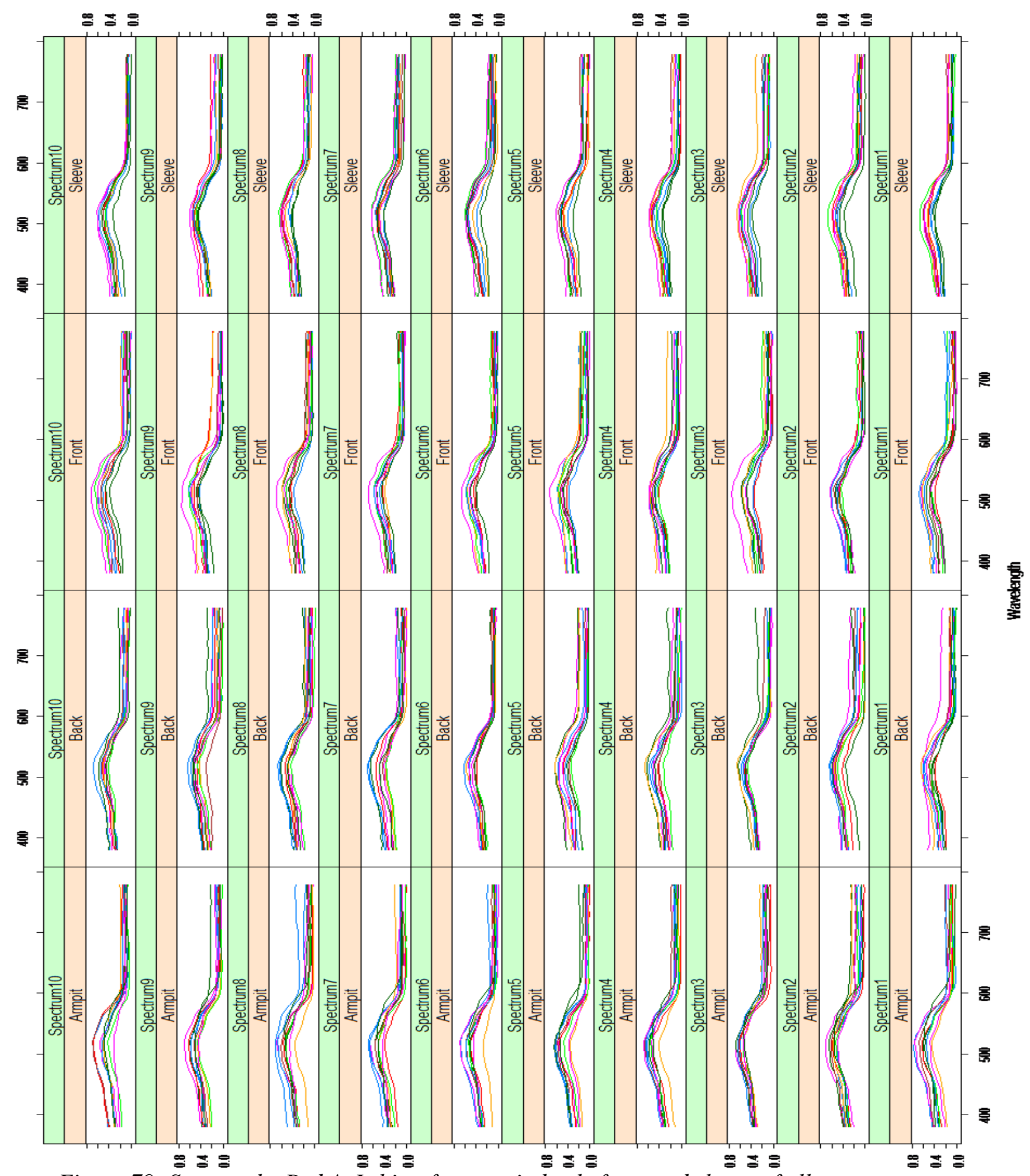

Figure 79: Spectra the Red A-J shirts for armpit, back, front and sleeve of all ten runs 


\begin{tabular}{|l|}
\hline Armpit : \\
Back : \\
Front : \\
Sleeve \\
\hline
\end{tabular}

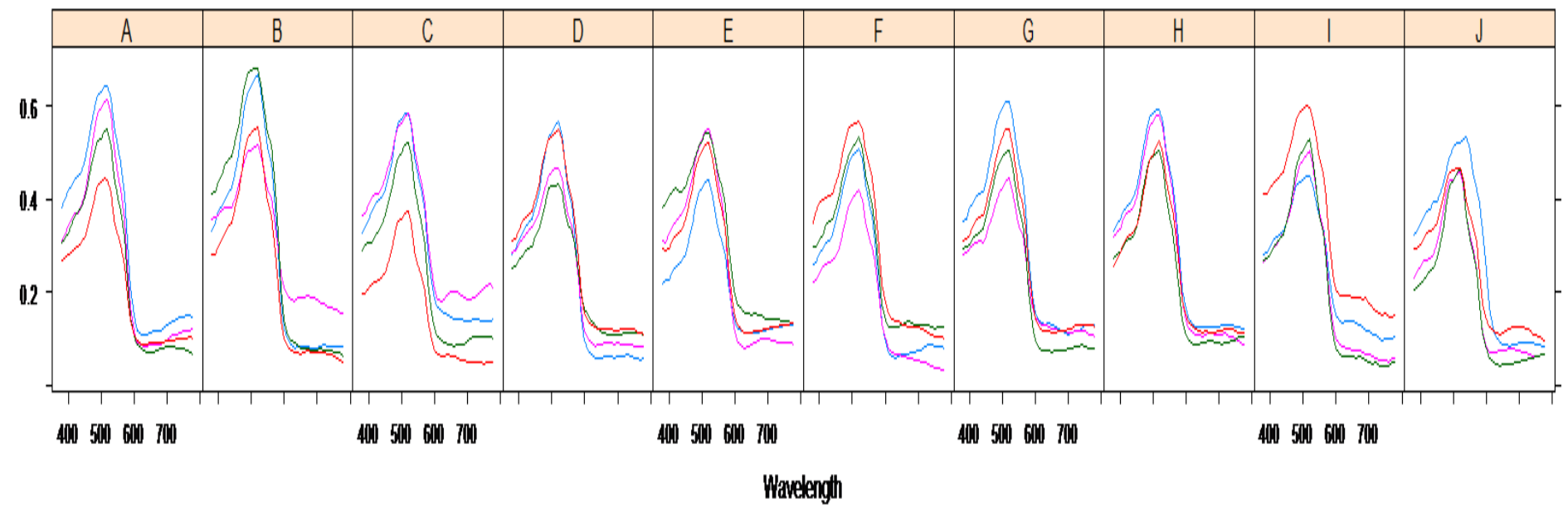

Figure 80: Spectra of the armpit, back, front and sleeve for Red A-J shirts

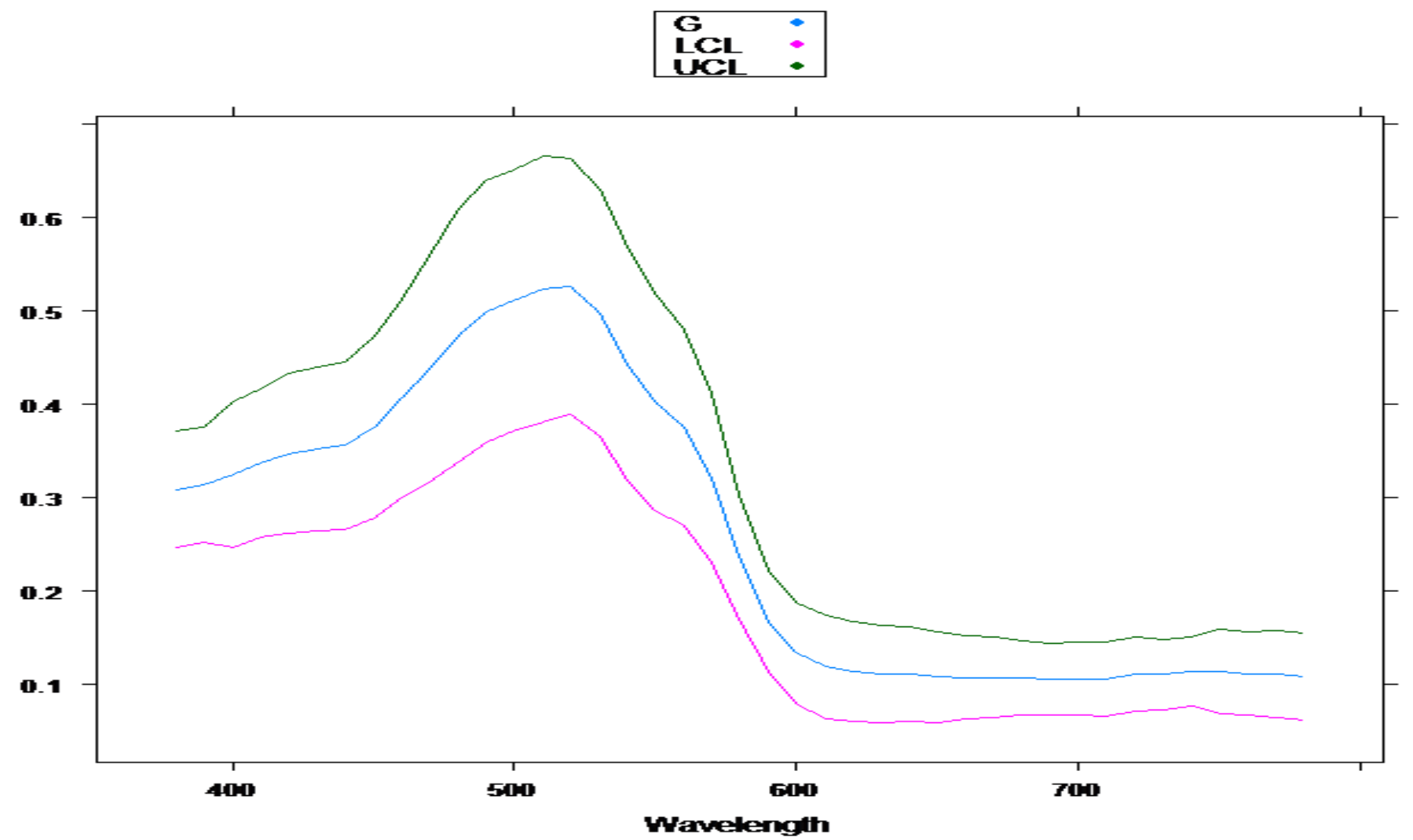

Figure 81: Spectra of the Average Absorbance, UCL and the LCL for 4 all regions of the Red G shirts 


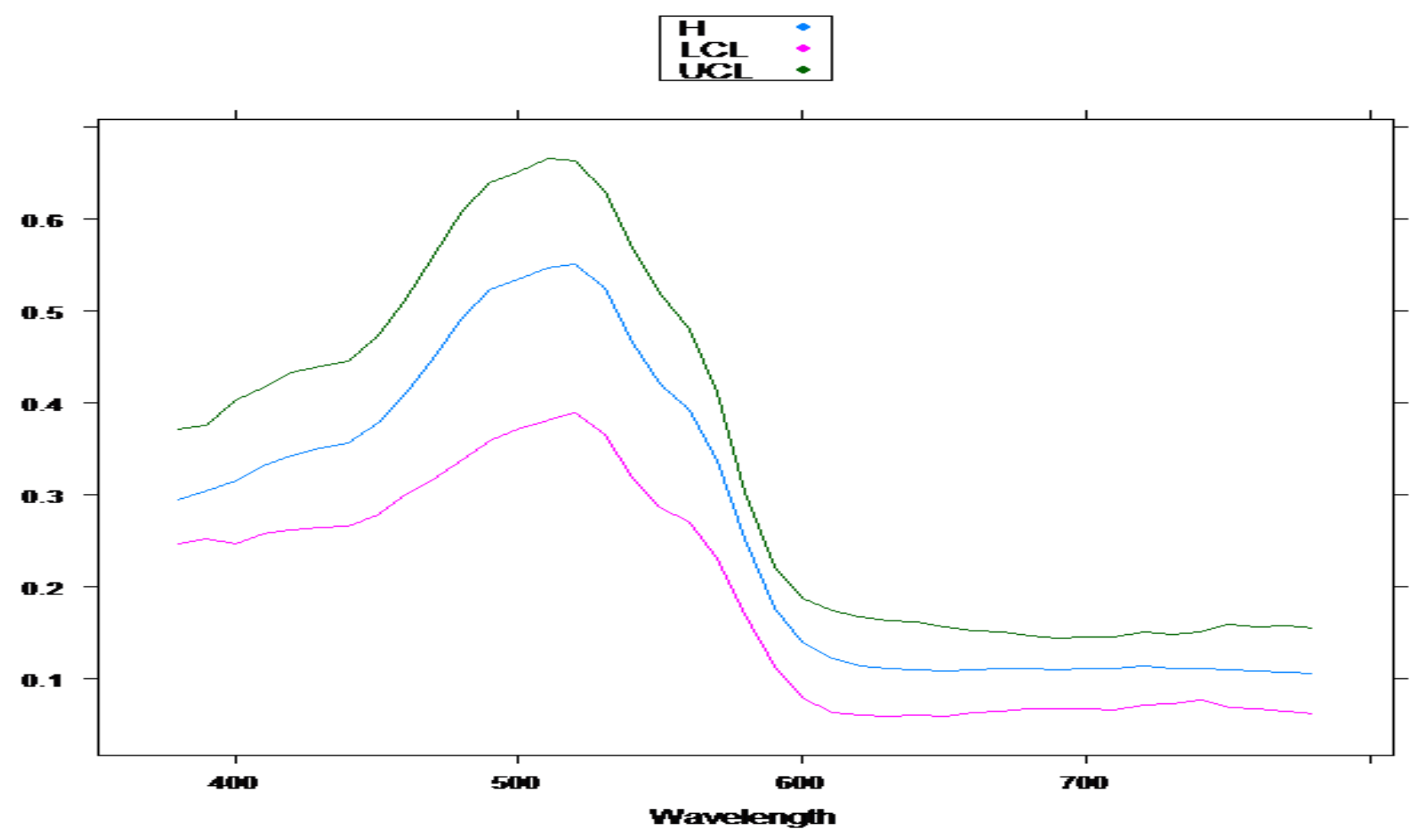

Figure 82: Spectra of the Average Absorbance, UCL and the LCL for 4 all regions of the Red H shirts

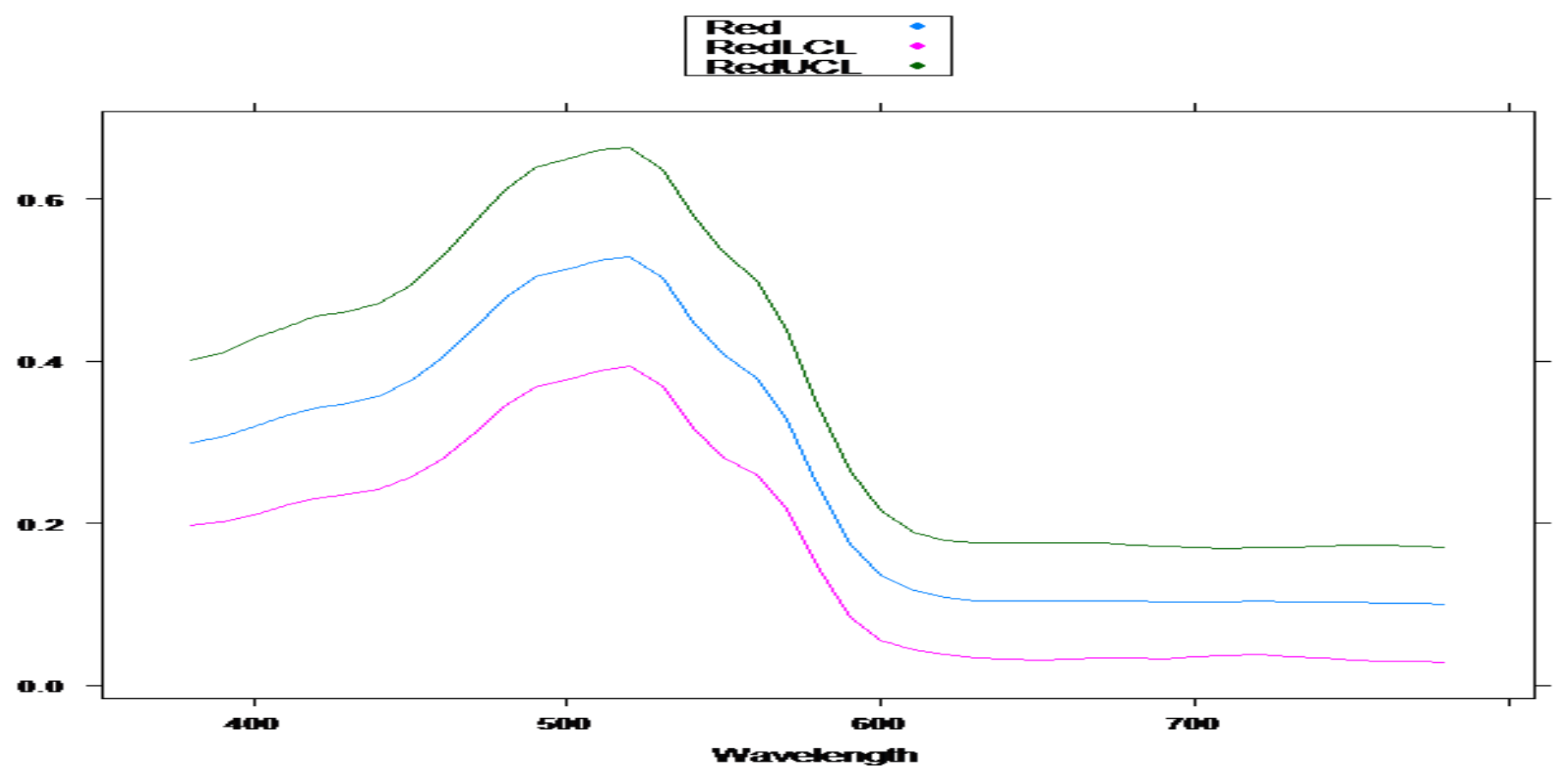

Figure 83: Spectra of the Average Absorbance, UCL and the LCL for every Red Shirt 


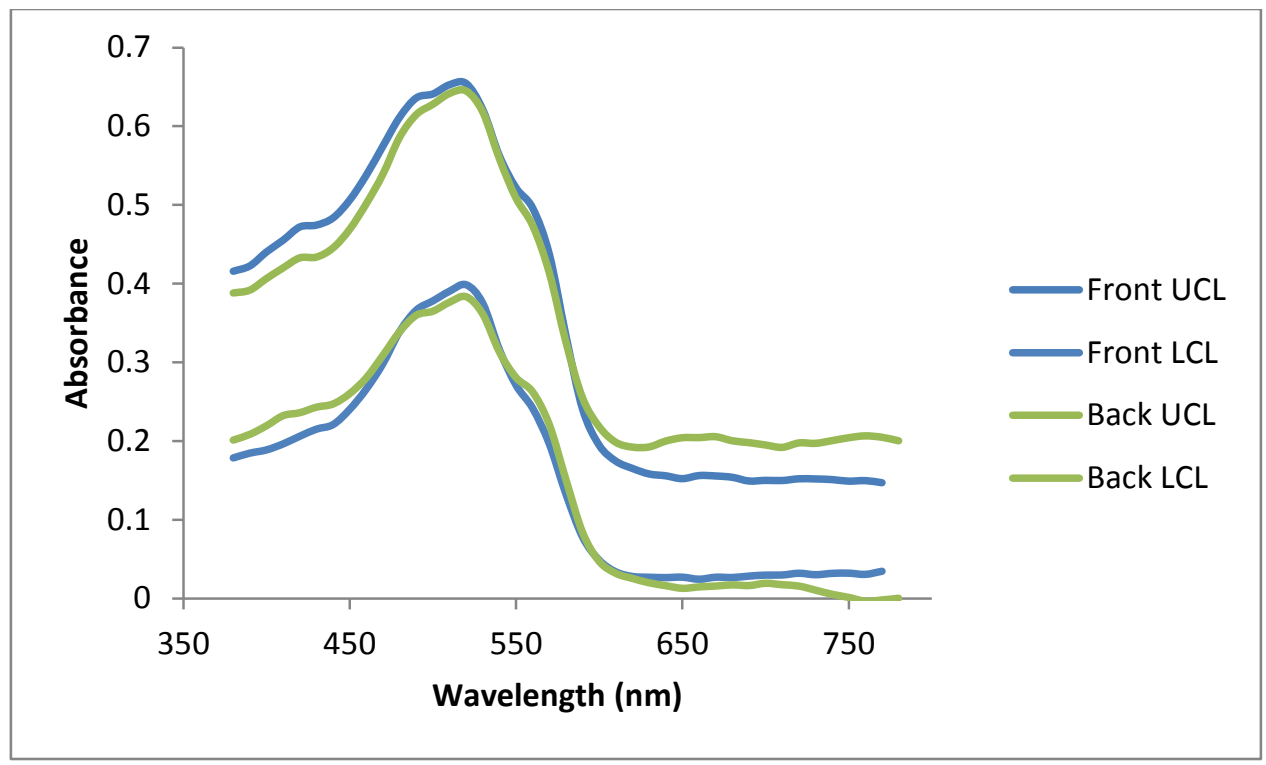

Figure 84: The 95\% UCL and the 95\% LCL for the Red Front and Back

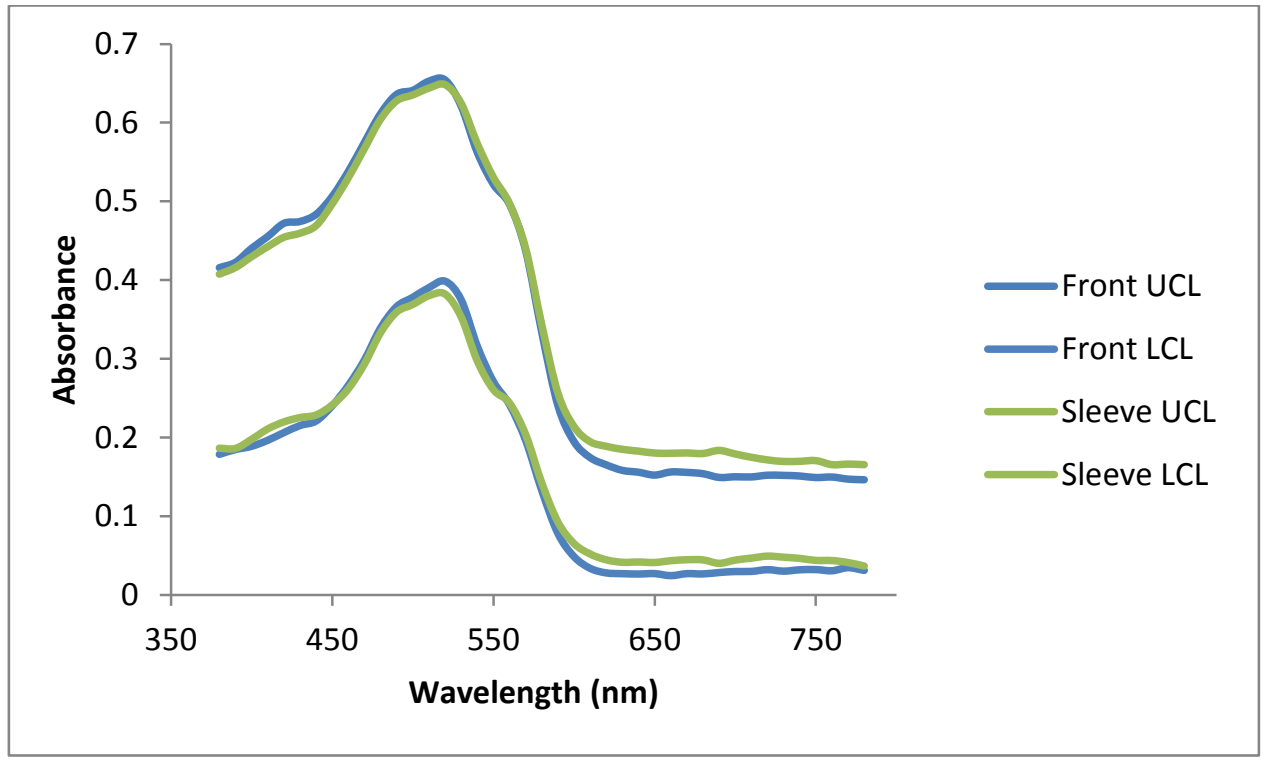

Figure 85: The 95\% UCL and the 95\% LCL for the Red Front and Sleeve 


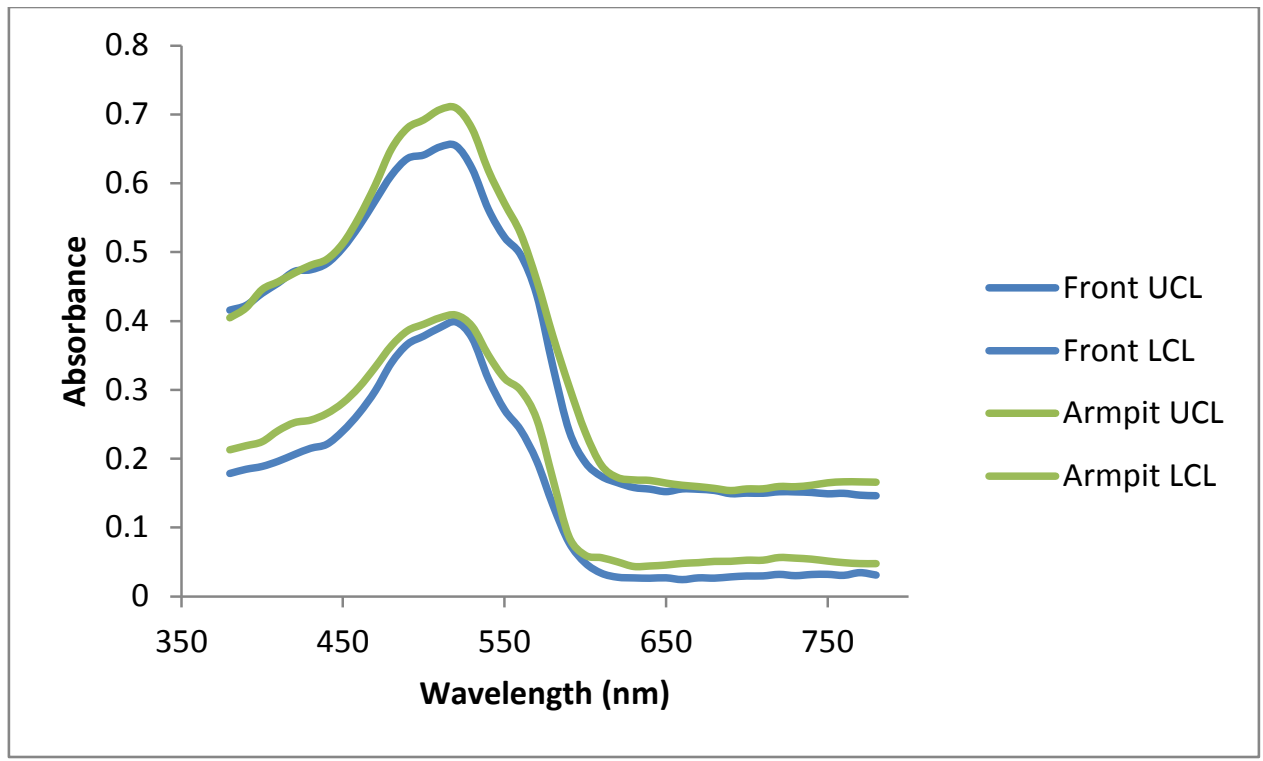

Figure 86: The 95\% UCL and the 95\% LCL for the Red Front and Armpit

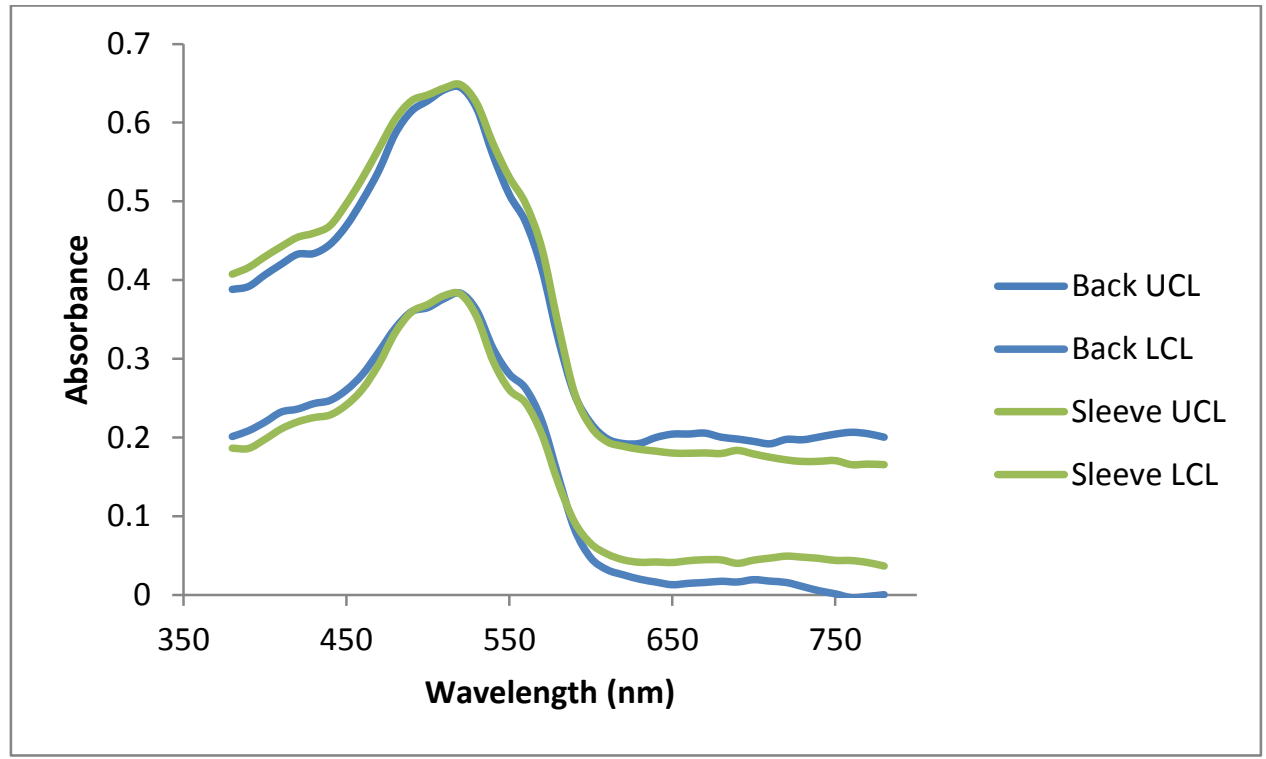

Figure 87: The 95\% UCL and the 95\% LCL for the Red Back and Sleeve 


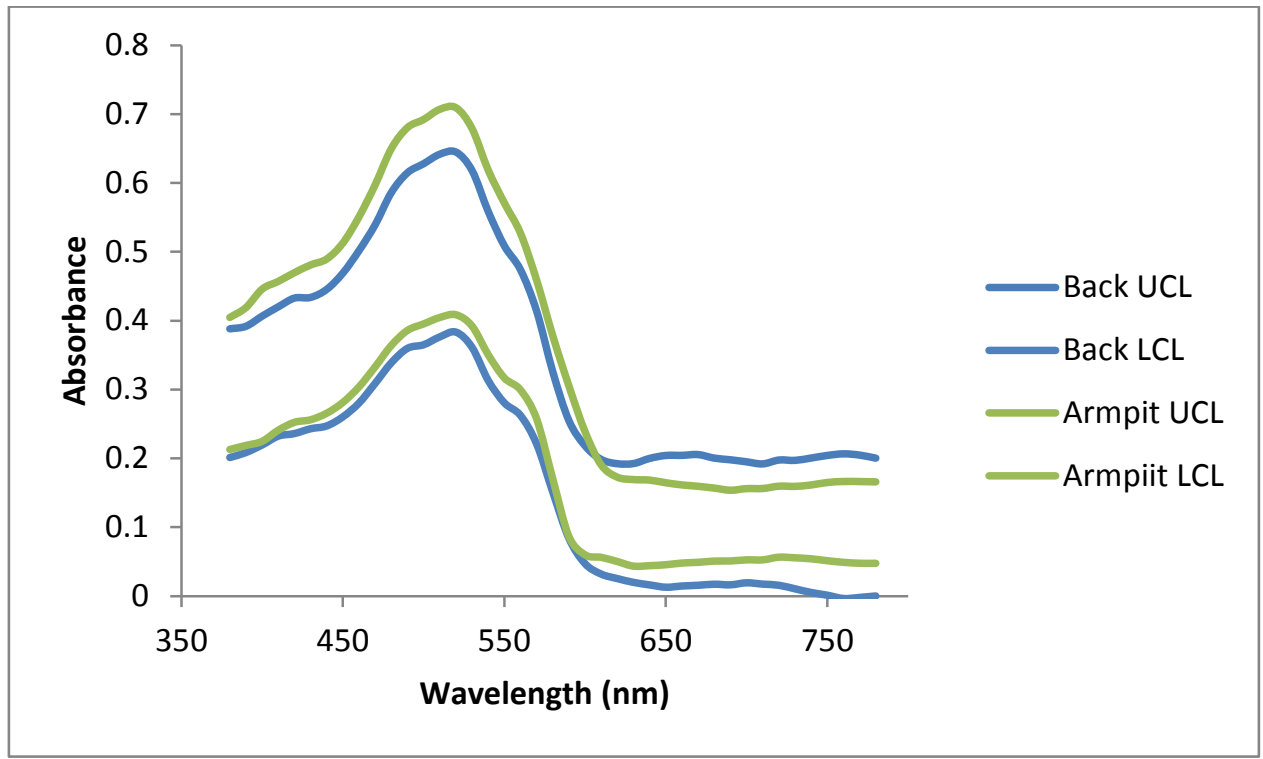

Figure 88: The 95\% UCL and the 95\% LCL for the Red Back and Armpit

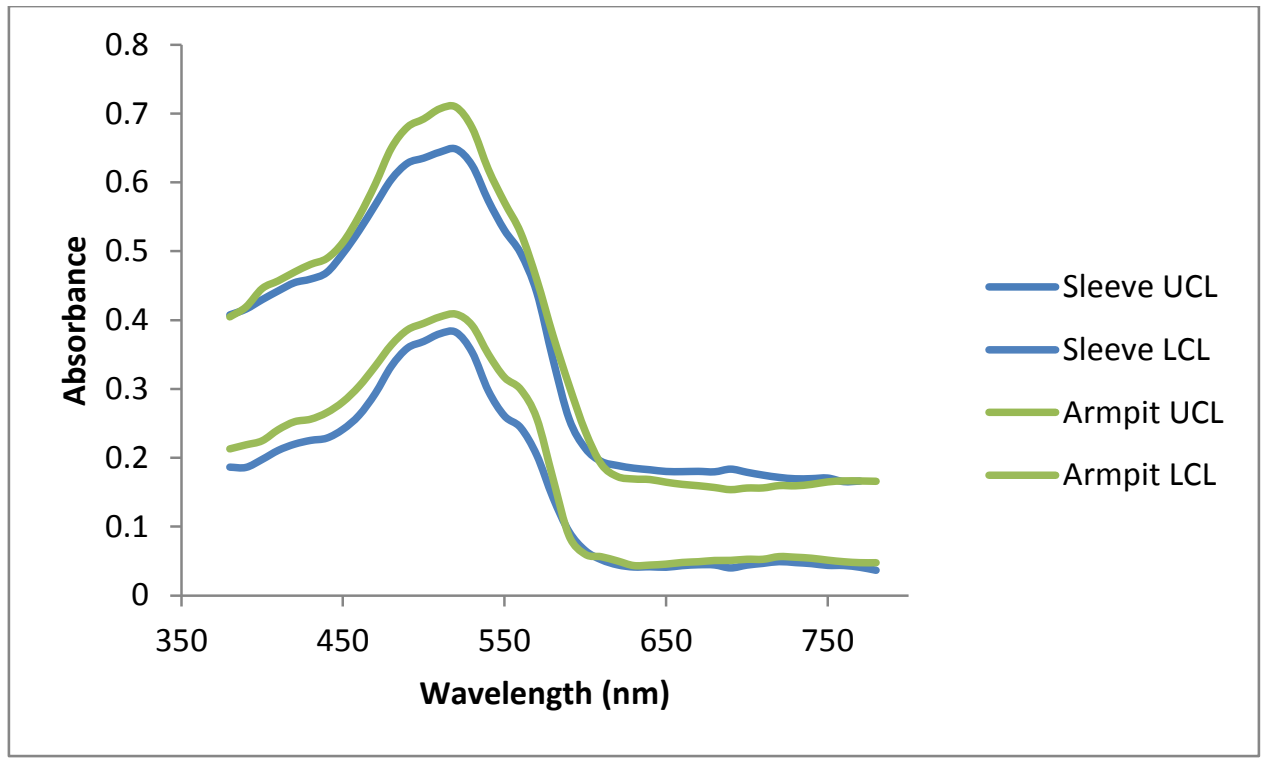

Figure 89: The 95\% UCL and the 95\% LCL for the Red Sleeve and Armpit 


\section{Royal}

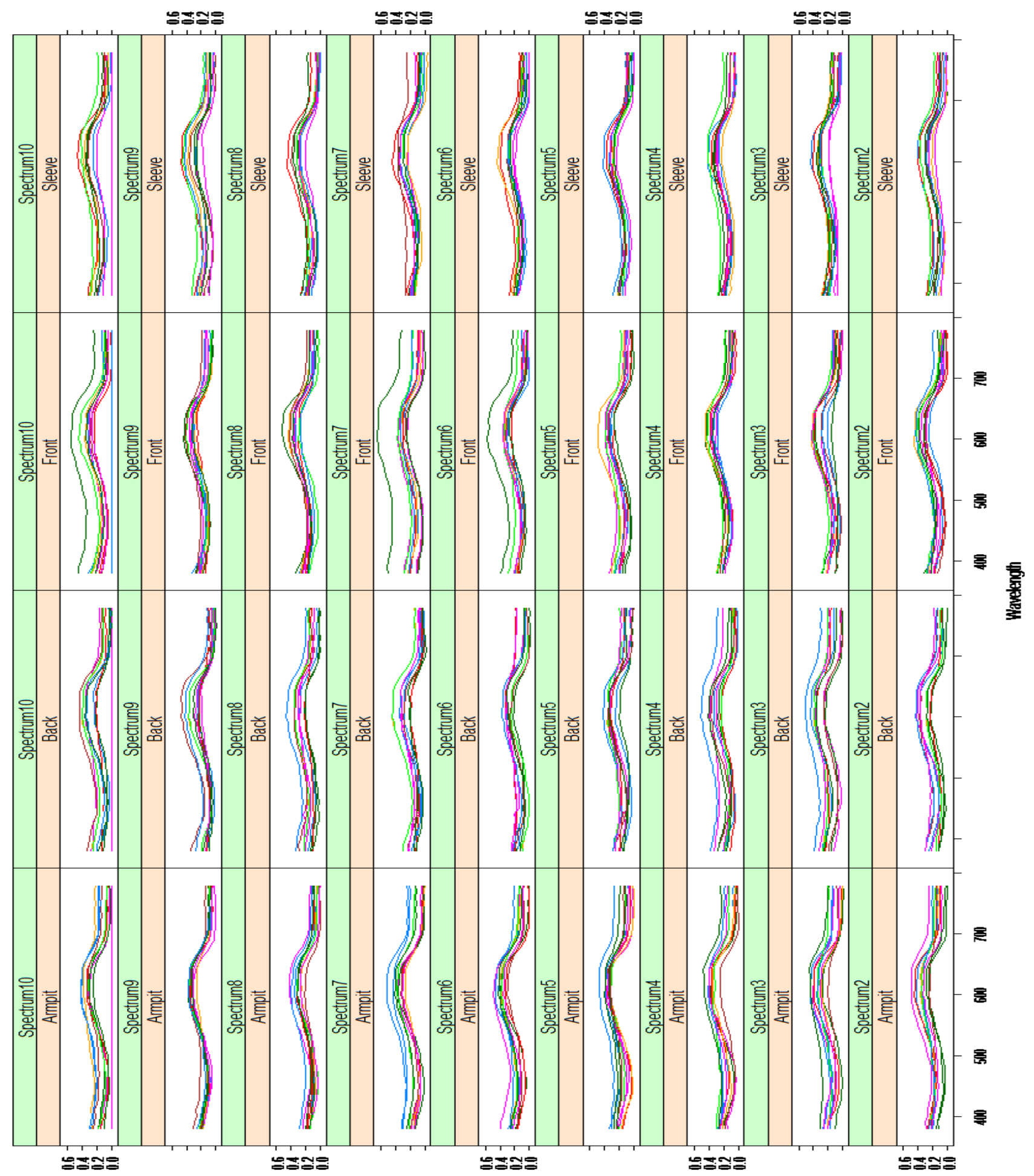

Figure 90: Spectra the Royal A-J shirts for armpit, back, front and sleeve of all ten runs 


\begin{tabular}{|l|}
\hline Armpit : \\
Back \\
Front : \\
Sleeve \\
\hline
\end{tabular}

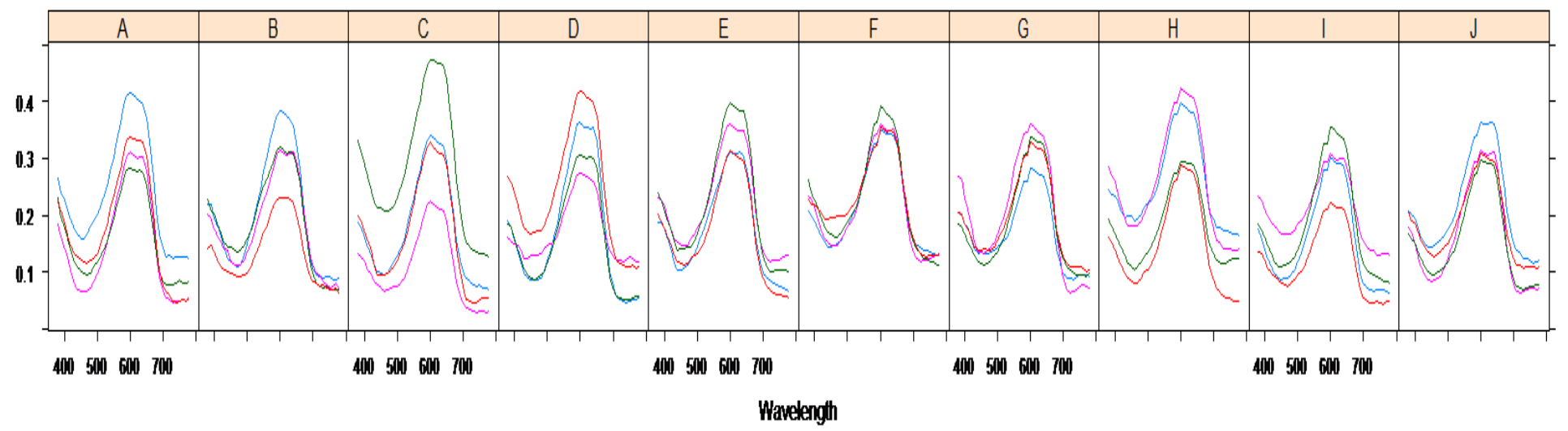

Figure 91: Spectra of the armpit, back, front and sleeve for Royal A-J shirts

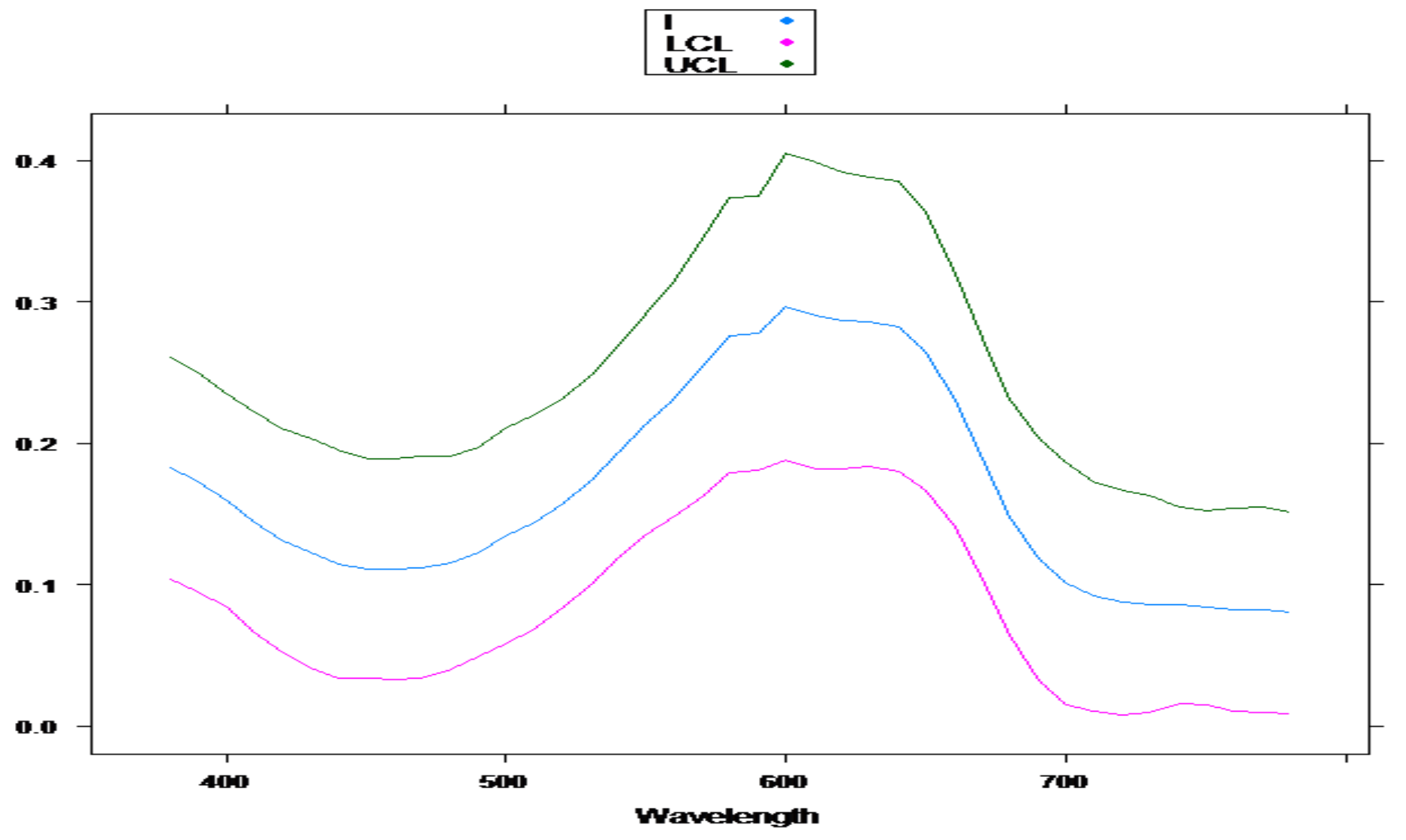

Figure 92: Spectra of the Average Absorbance, UCL and the LCL for 4 all regions of the Royal I shirts 


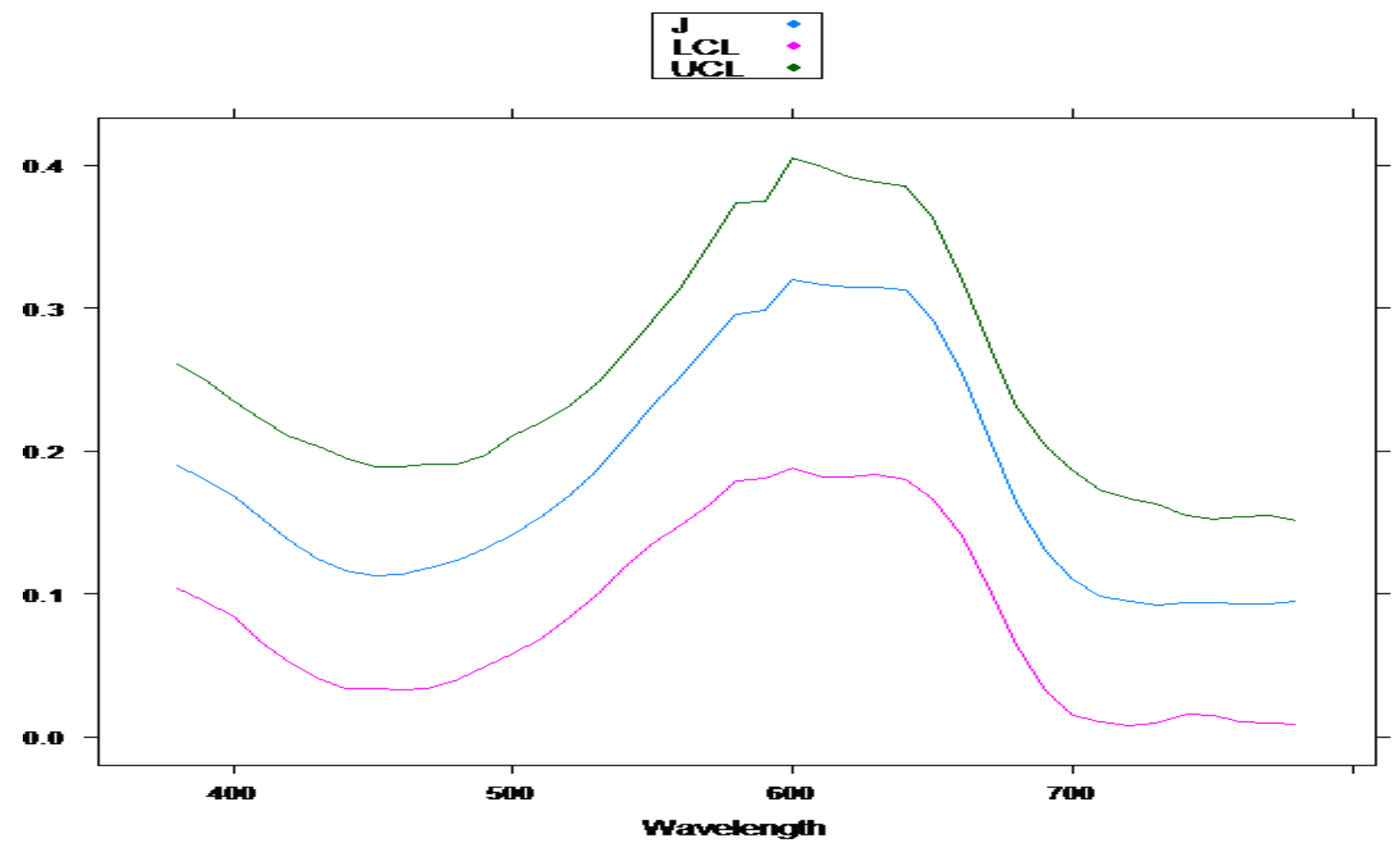

Figure 93: Spectra of the Average Absorbance, UCL and the LCL for 4 all regions of the Royal

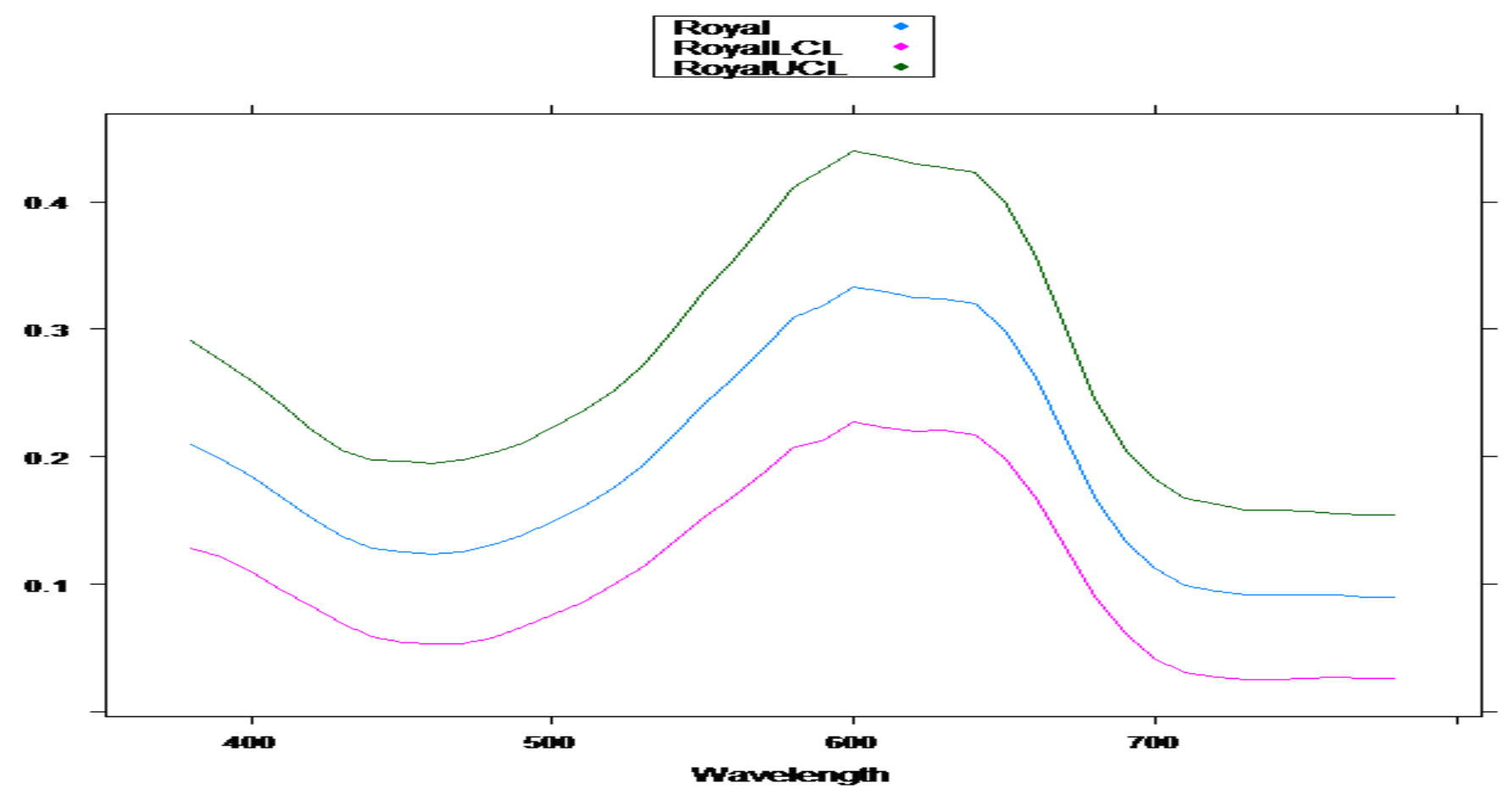

Figure 94: Spectra of the Average Absorbance, UCL and the LCL for every Royal Shirt 


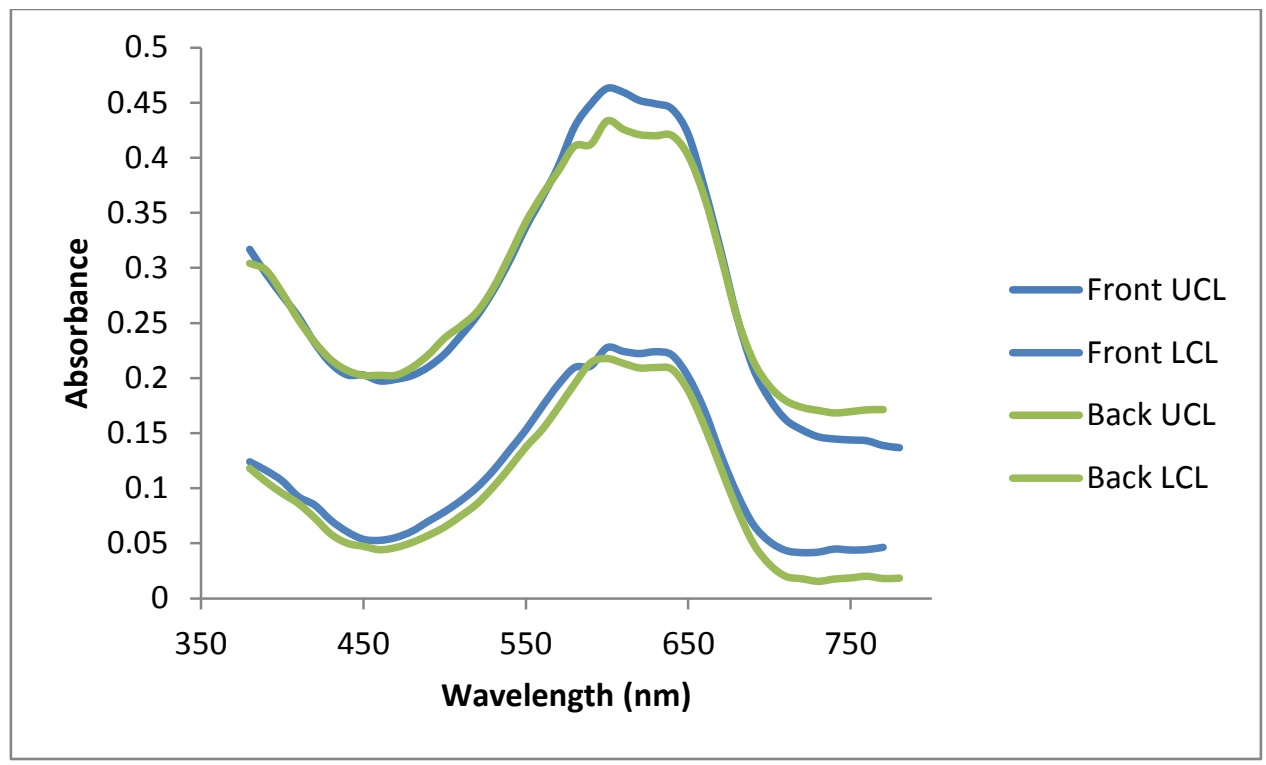

Figure 95: The 95\% UCL and the 95\% LCL for the Royal Front and Back

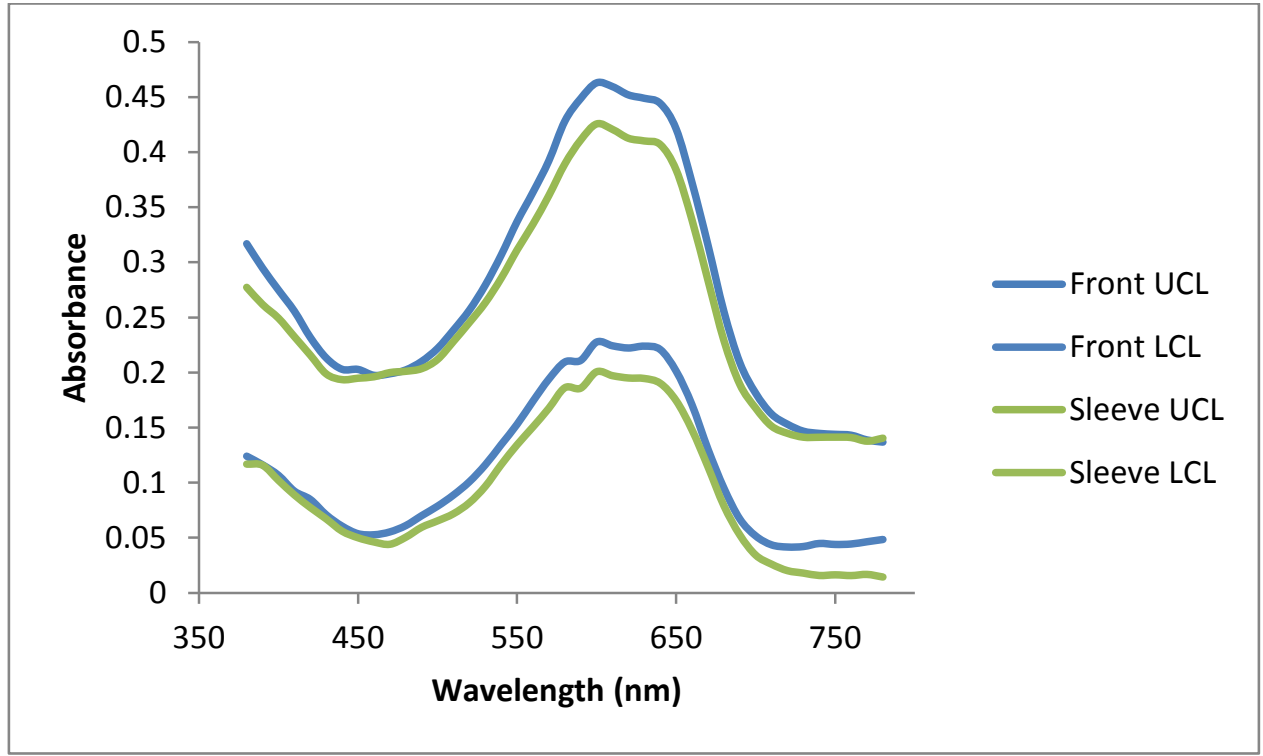

Figure 96: The 95\% UCL and the 95\% LCL for the Royal Front and Sleeve 


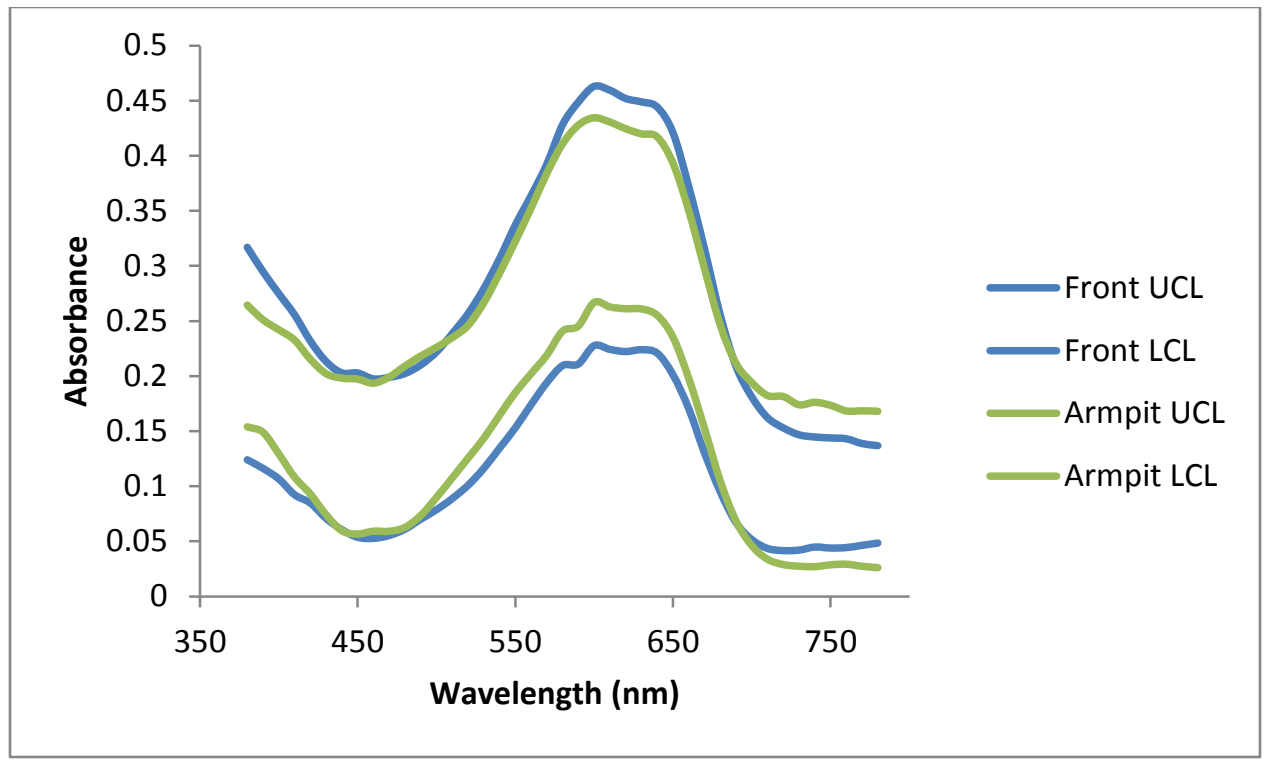

Figure 97: The 95\% UCL and the 95\% LCL for the Royal Front and Armpit

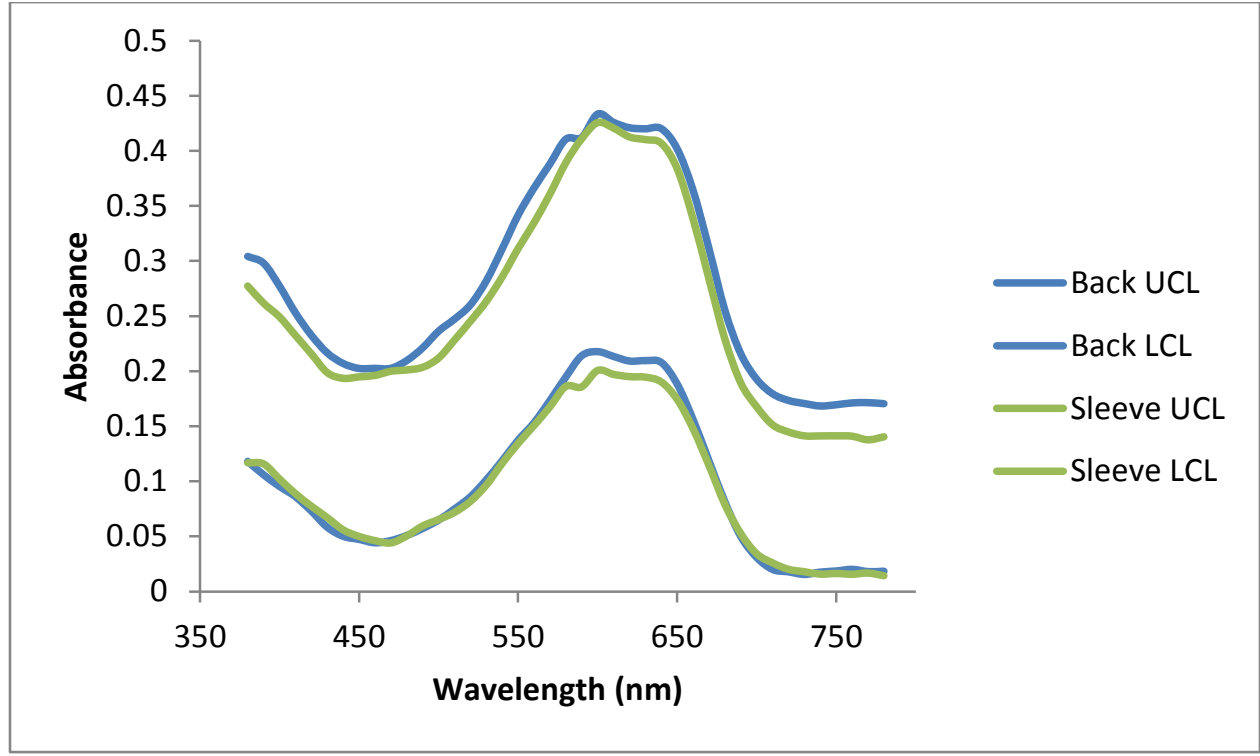

Figure 98: The 95\% UCL and the 95\% LCL for the Royal Back and Sleeve 


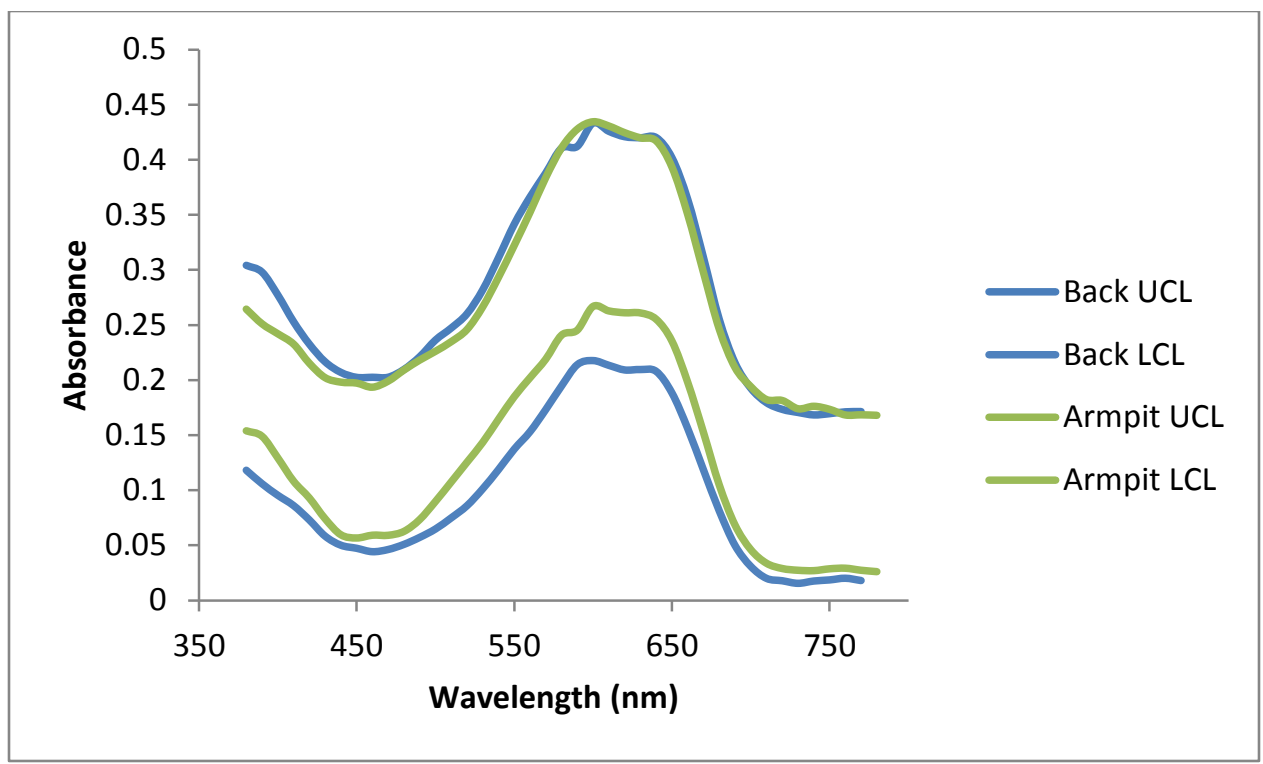

Figure 99: The 95\% UCL and the 95\% LCL for the Royal Back and Armpit

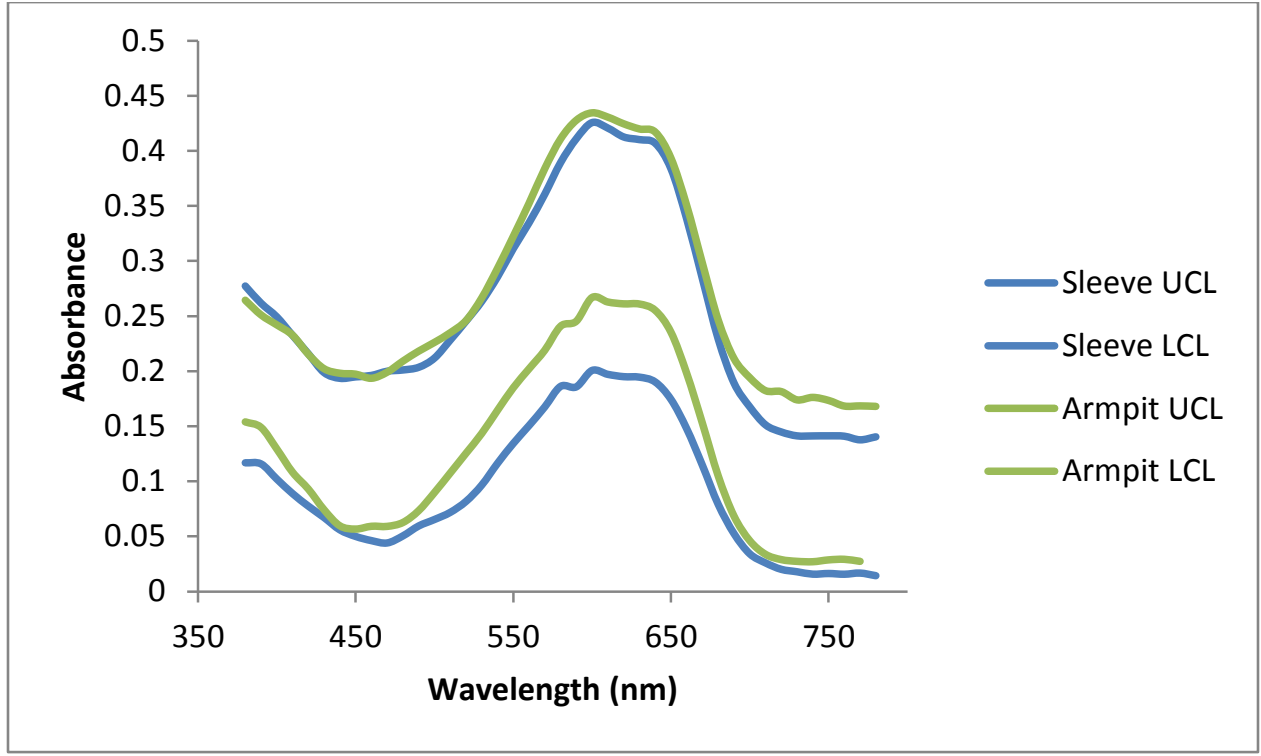

Figure 100: The 95\% UCL and the 95\% LCL for the Royal Sleeve and Armpit 
All the figures in the results section of Phase IV were generated by a XY conditioning plot using RandFriends ${ }^{\circledR} 2.15 .2$ when configured with RExcel ${ }^{\circledR}$ 3.2.9. Figures 46, 57, 68, 79 and 90 were created for the front, back, sleeve and armpit of all five t-shirt colors by using wavelength for the $\mathrm{x}$ axis and the absorbance values as the $\mathrm{y}$ axis that were collected for all ten runs. The number of columns was set to four and the number of rows was set to ten. Figures 47, $58,69,80$ and 91 were created for the front, back, sleeve and armpit of all five t-shirt colors by using the $\mathrm{x}$ axis as wavelength and the $\mathrm{y}$ axis as the average absorbance values that were calculated. The number of rows was set to ten. Figure 48 and Figure 49 were generated to represent the average absorbance, the upper confidence limit and the lower confidence limit for all the absorbance values for the Gold A t-shirt and the Gold B t-shirt. Figure 59 and Figure 60 were generated to represent the average absorbance, the upper confidence limit and the lower confidence limit for all the absorbance values for Kelly Green C t-shirt and Kelly Green D tshirt. Figure 70 and Figure 71 were generated to represent the average absorbance, the upper confidence limit and the lower confidence limit for all the absorbance values for Orange $\mathrm{E} t$-shirt and Orange F t-shirt. Figure 81 and Figure 82 were generated to represent the average absorbance, the upper confidence limit and the lower confidence limit for all the absorbance values for Red G t-shirt and Red H t-shirt. Figure 93 and Figure 94 were generated to represent the average absorbance, the upper confidence limit and the lower confidence limit for all the absorbance values for Royal I t-shirt and Royal J t-shirt. Figures 50, 61, 72, 83 and 94 were created to represent the average absorbance, the upper confidence limit and the lower confidence limit for all the absorbance values for all ten shirts for all five colors. Figure 51-56, Figure 62-67, Figure 73-78, Figure 84-89 and Figure 95-100 were created in Microsoft ${ }^{\circledR}$ Excel to displays all possible ways to compare the front, back, sleeve and armpit of the five different colors by plotting the UCL and the LCL at 95\% confidence. 


\section{Phase V}

\section{Gold}

\begin{tabular}{|c|c|c|}
\hline & 400 & \\
\hline$\stackrel{1}{\text { Spectrum10 }}$ & $\frac{1}{\text { Spectrum10 }}$ & Spectrum10 \\
\hline Section1 & Section 2 & Section 3 \\
\hline$\Rightarrow=0 \cos x=0$ & $\Rightarrow$ & $\mathrm{z}=$ \\
\hline Spectrum9 & Spectrum9 & Spectrum9 \\
\hline Section1 & Section 2 & Section 3 \\
\hline$\equiv=0$ & $\mathrm{~B}=$ & $\mathrm{g}=0$ \\
\hline Spectrum7 & Spectrum7 & Spectrum7 \\
\hline Section1 & Section 2 & Section 3 \\
\hline $\mathrm{F}=\mathrm{z}=\mathrm{m}$ & $=\bar{z}=-=$ & $\mathrm{OP}=$ \\
\hline Spectrum6 & Spectrum6 & Spectrum6 \\
\hline Section1 & Section 2 & Section 3 \\
\hline 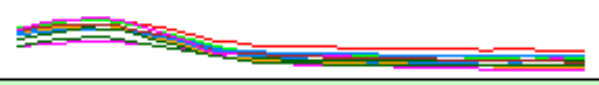 & 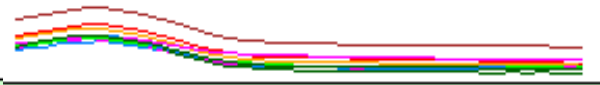 & $\Rightarrow$ \\
\hline Spectrum5 & Spectrum5 & Spectrum5 \\
\hline Section1 & Section 2 & Section 3 \\
\hline 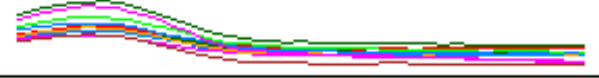 & $\equiv \equiv \equiv$ & $\Rightarrow=0$ \\
\hline Spectrum4 & Spectrum4 & Spectrum4 \\
\hline Section1 & Section 2 & Section 3 \\
\hline 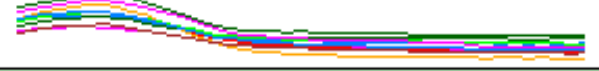 & $\Rightarrow$ & $\Rightarrow=\Rightarrow$ \\
\hline Spectrum3 & Spectrum3 & Spectrum3 \\
\hline Section1 & Section 2 & Section 3 \\
\hline$\Rightarrow$ & $E=\Rightarrow$ & $\Rightarrow=0$ \\
\hline Spectrum2 & Spectrum2 & Spectrum2 \\
\hline Section1 & Section 2 & Section 3 \\
\hline$\Rightarrow=0$ & $\Rightarrow==$ & $=0$ \\
\hline Spectrum1 & Spectrum1 & Spectrum1 \\
\hline Section1 & Section 2 & Section 3 \\
\hline E= & $\equiv=\overline{\equiv=}=0$ & $\Rightarrow=0=\Rightarrow$ \\
\hline \multirow[t]{2}{*}{400} & & $400 \quad 500 \quad 600$ \\
\hline & 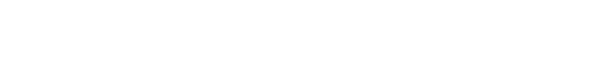 & Wavelength \\
\hline
\end{tabular}

Figure 101: Spectra the Gold A-J shirts for section 1, 2 and 3 of all ten runs 


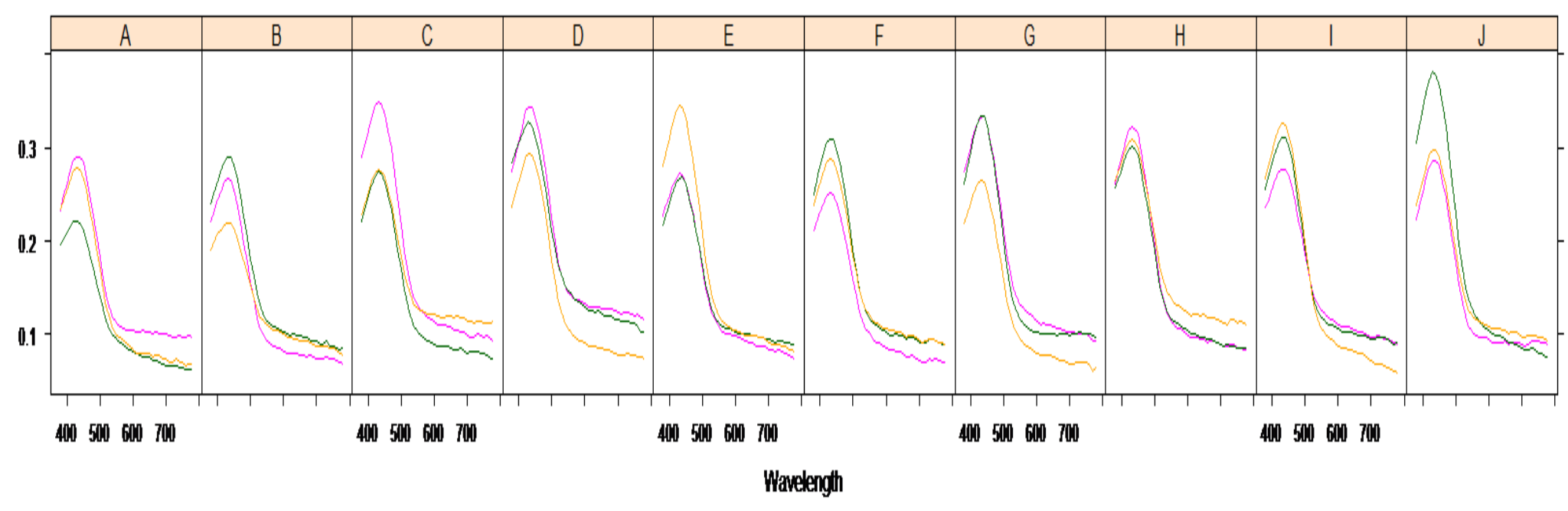

Figure 102: Spectra of section 1, 2 and 3 for Gold A-J shirts

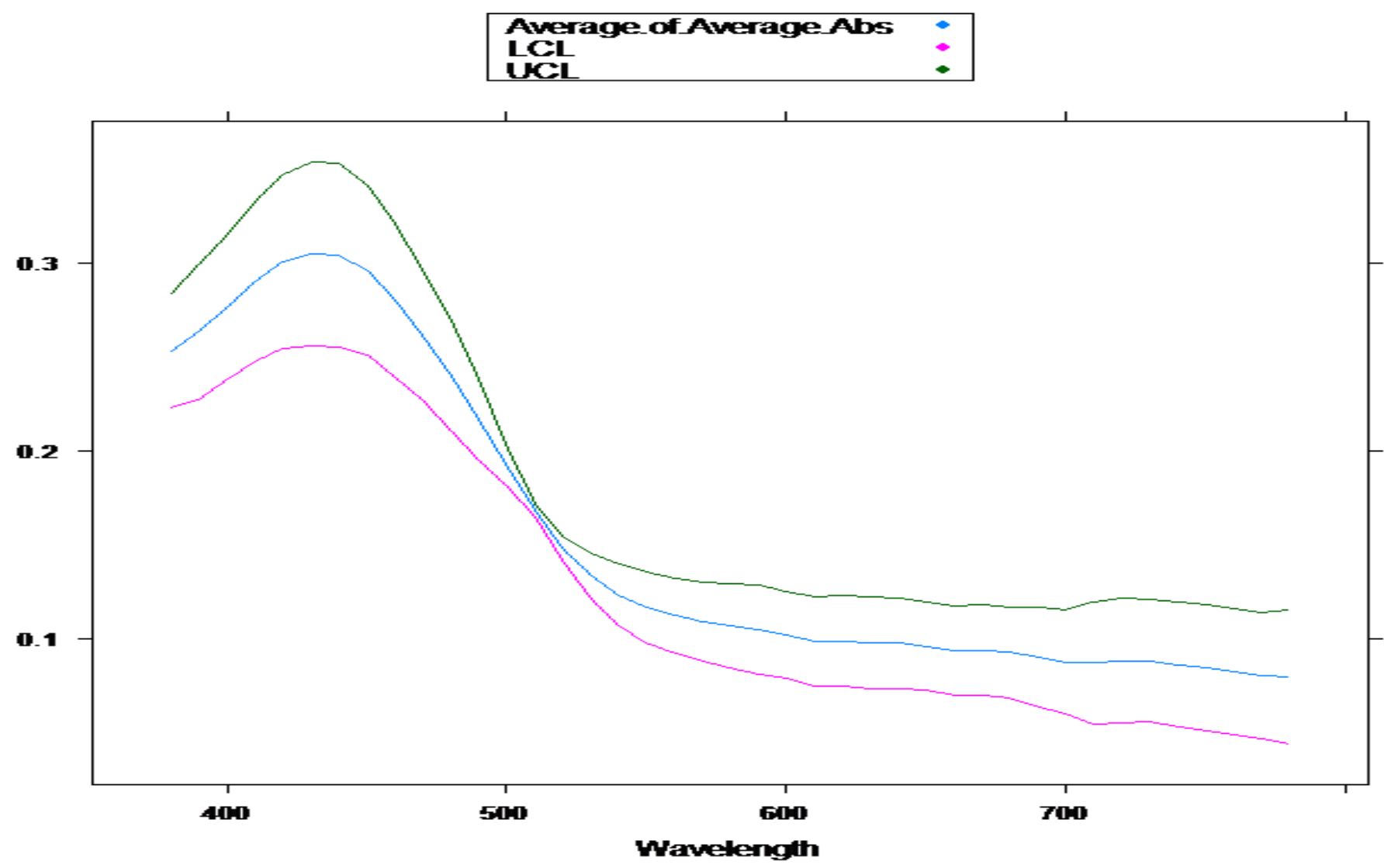

Figure 103: Spectra of the Average Absorbance, UCL and the LCL for 3 all sections of the Gold I shirts 


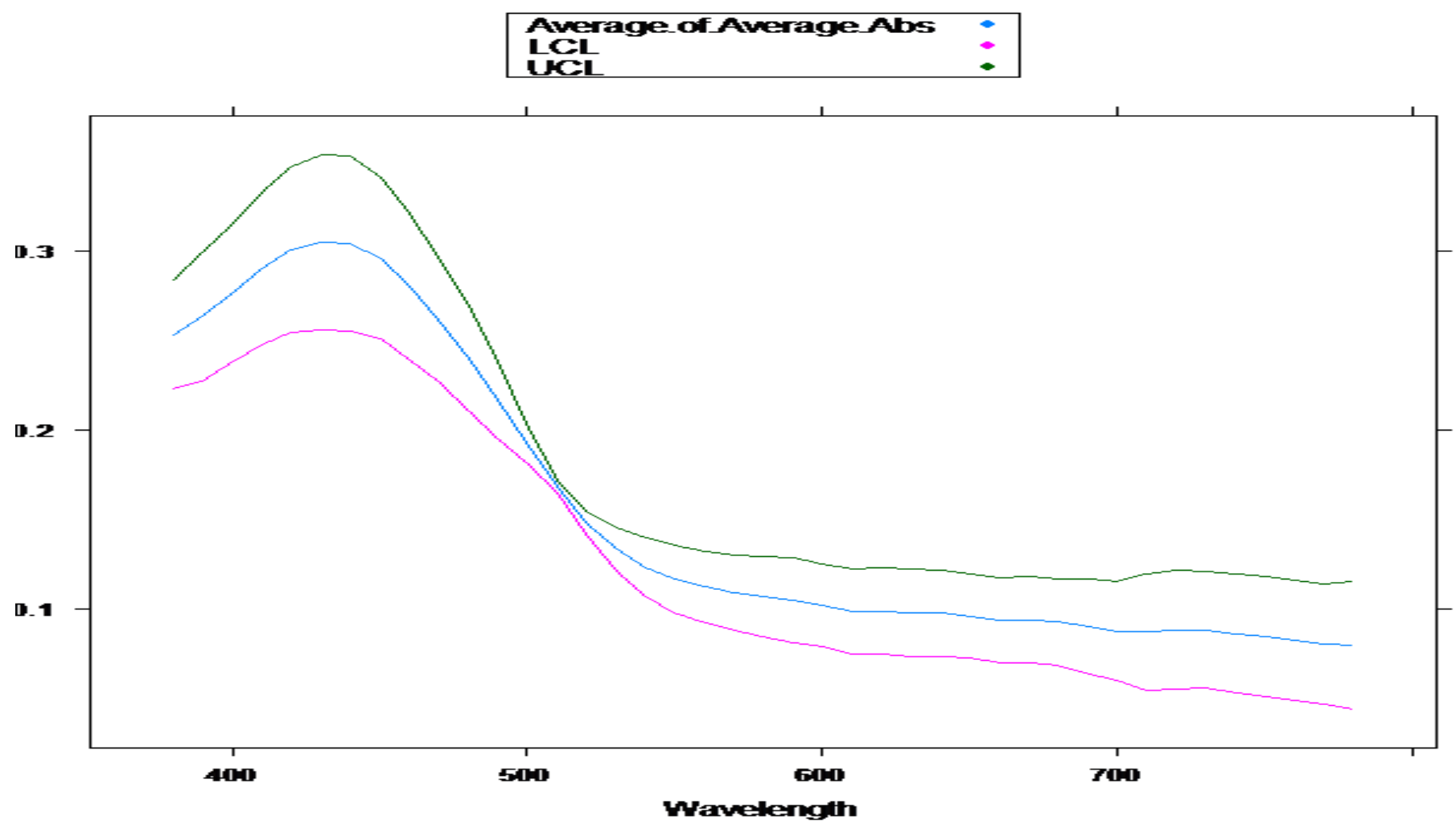

Figure 104: Spectra of the Average Absorbance, UCL and the LCLfor 3 all sections of the Gold $J$ shirts

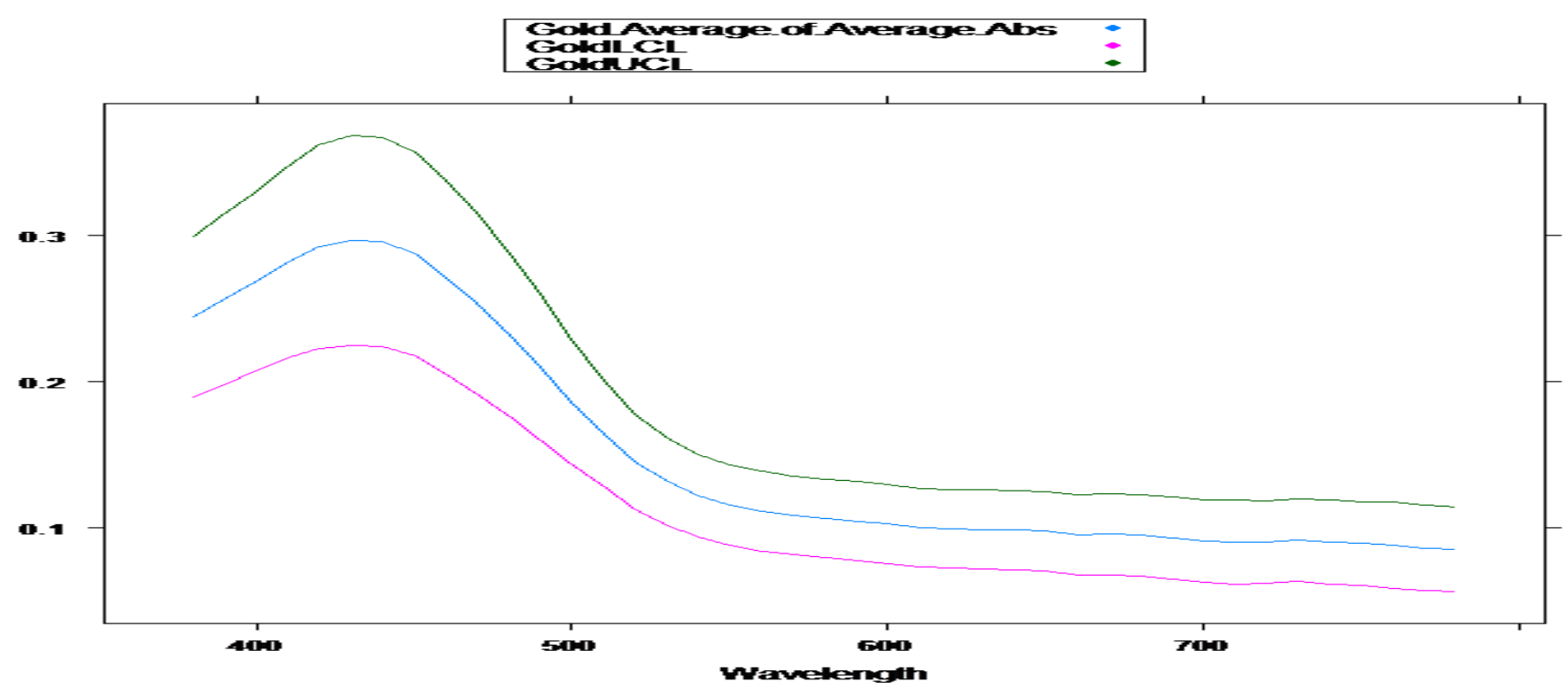

Figure 105: Spectra of the Average Absorbance, UCL and the LCL for every front Gold Shirt 


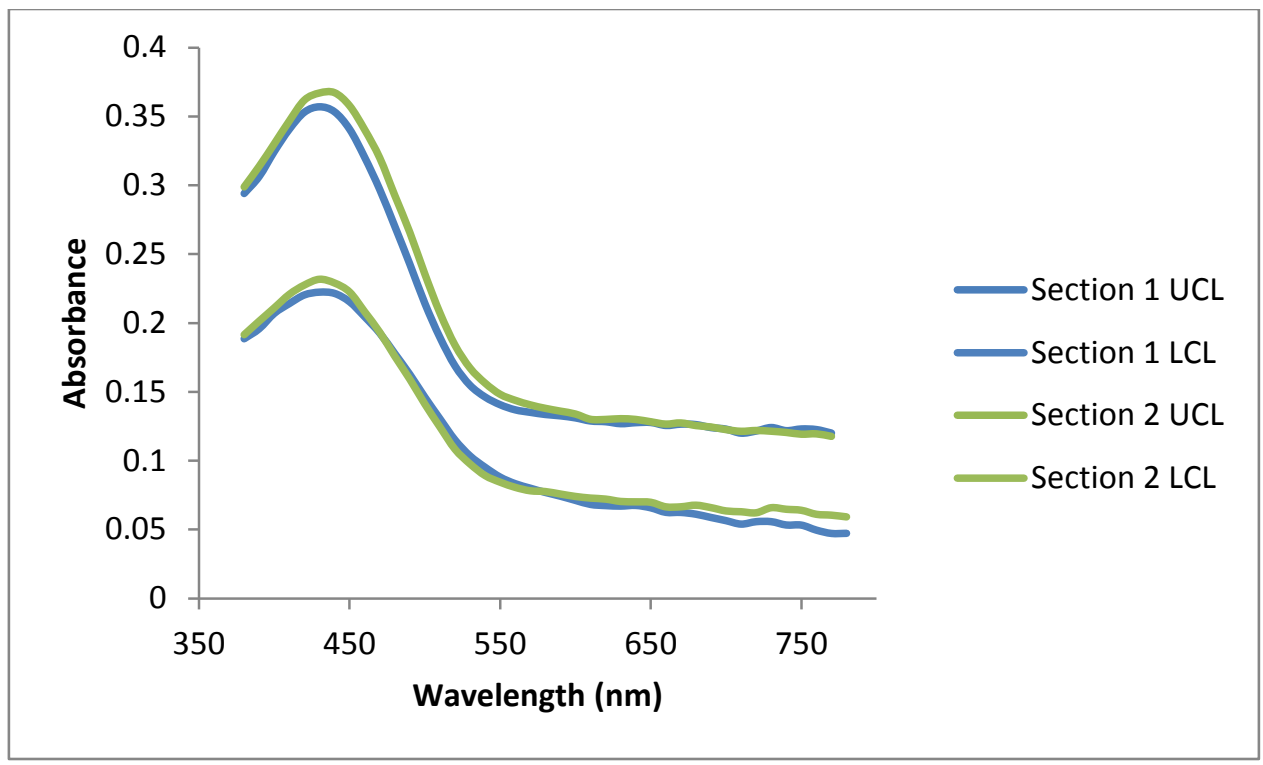

Figure 106: The 95\% UCL and the 95\% LCL of Gold Section 1 and Section 2

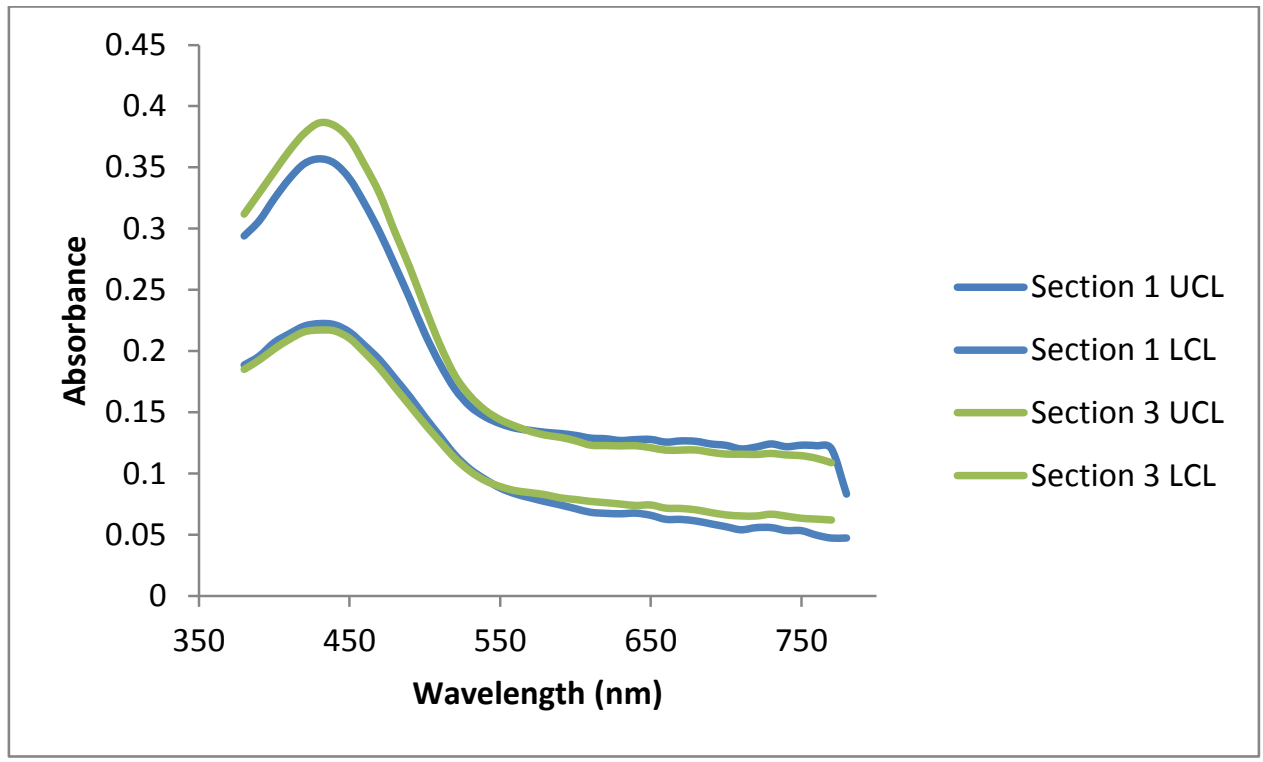

Figure 107: The 95\% UCL and the 95\% LCL of Gold Section 1 and Section 3 


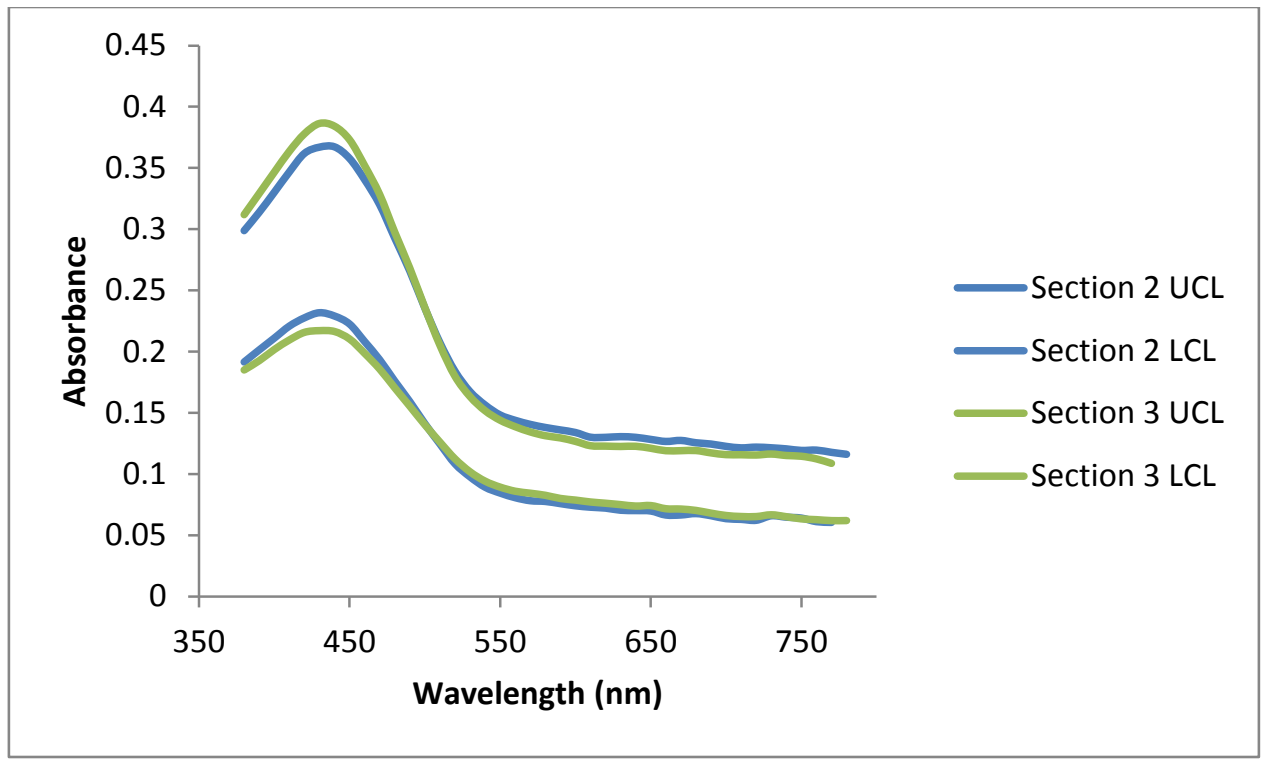

Figure 108: The 95\% UCL and the 95\% LCL of Gold Section 2 and Section 3 


\section{Kelly Green}

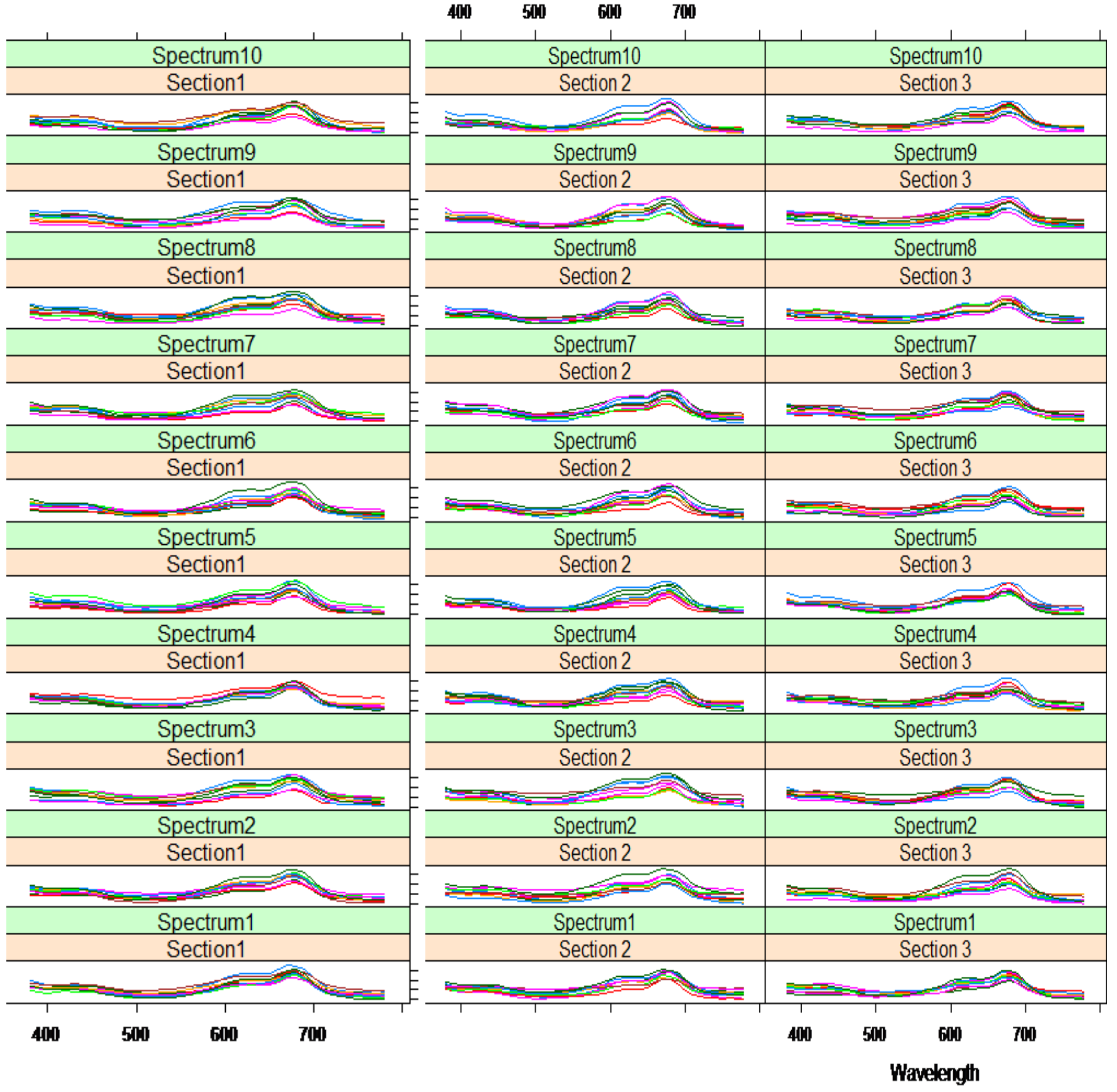

Figure 109: Spectra the Kelly Green A-J shirts for section 1, 2 and 3 of all ten runs 


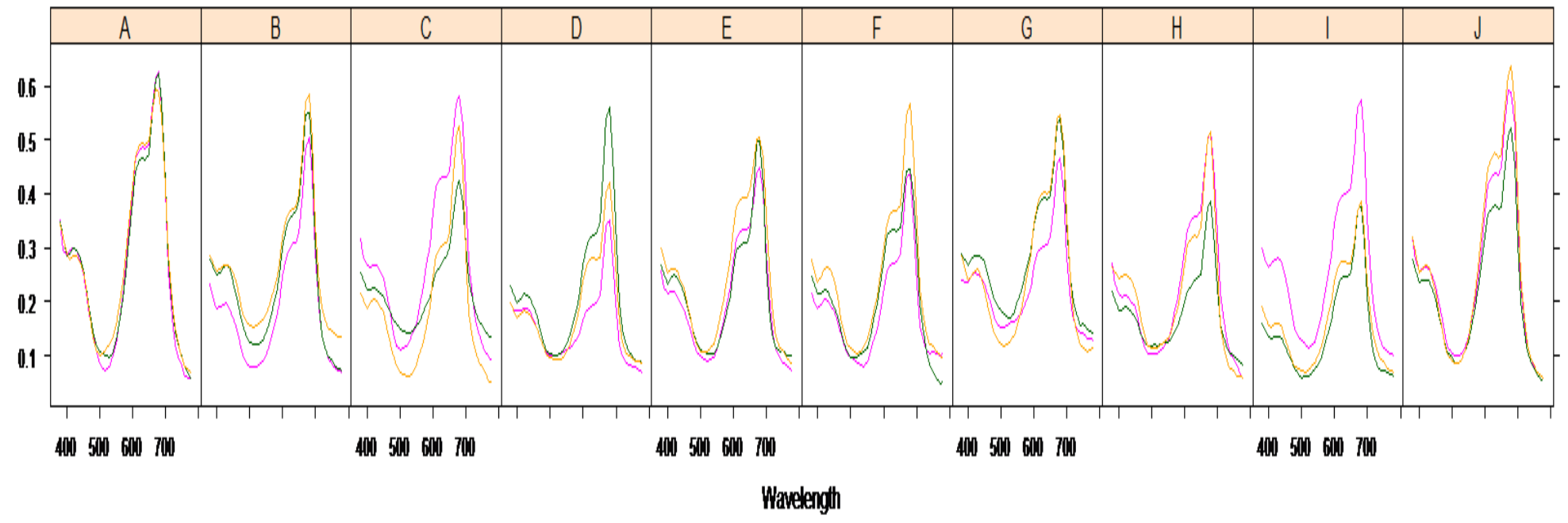

Figure 110: Spectra of section 1, 2 and 3 for Kelly Green A-J shirts

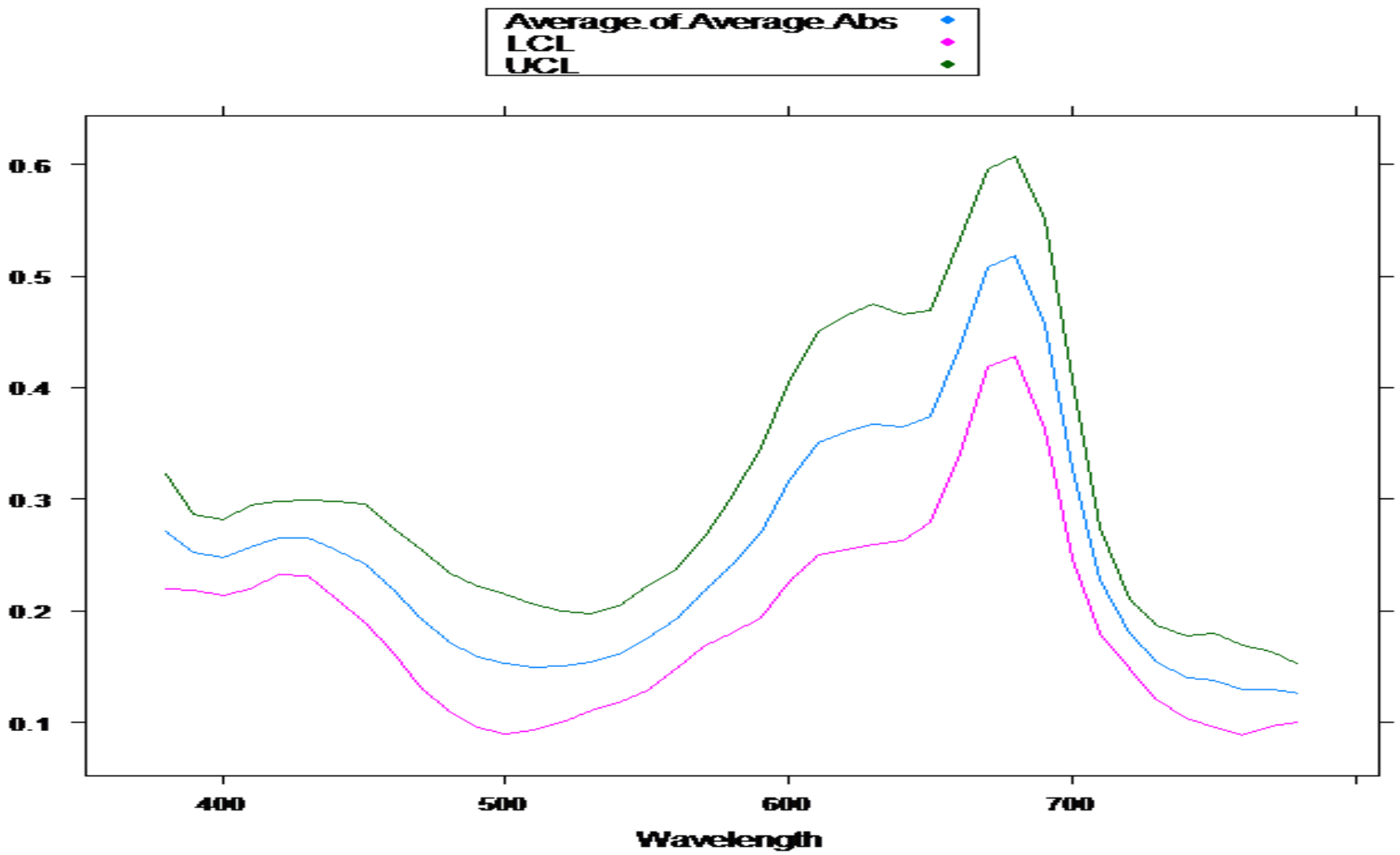

Figure 111: Spectra of the Average Absorbance, UCL and the LCLfor 3 all sections of the Kelly Green G shirts 


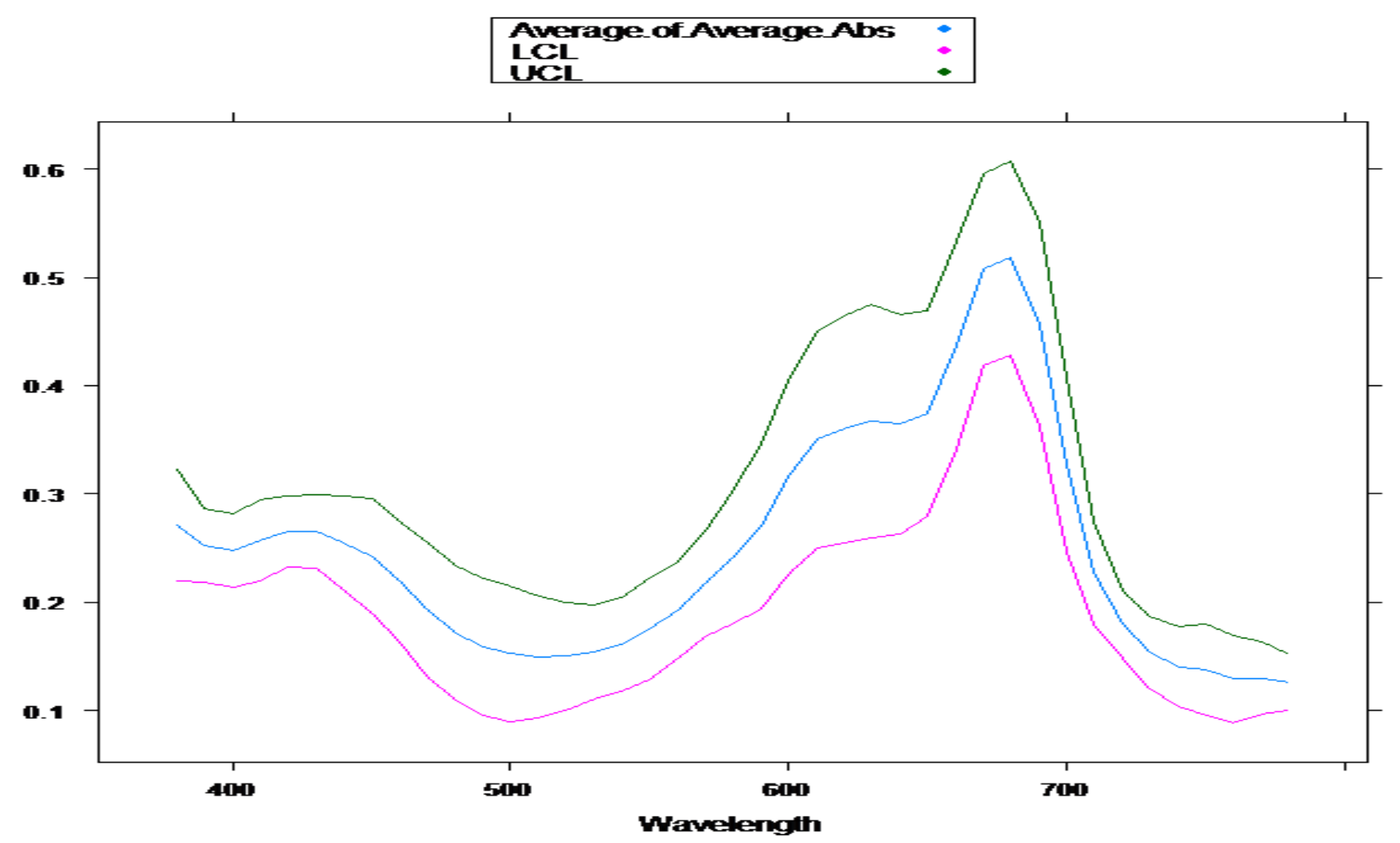

Figure 112: Spectra of the Average Absorbance, UCL and the LCLfor 3 all sections of the Kelly Green $H$ shirts

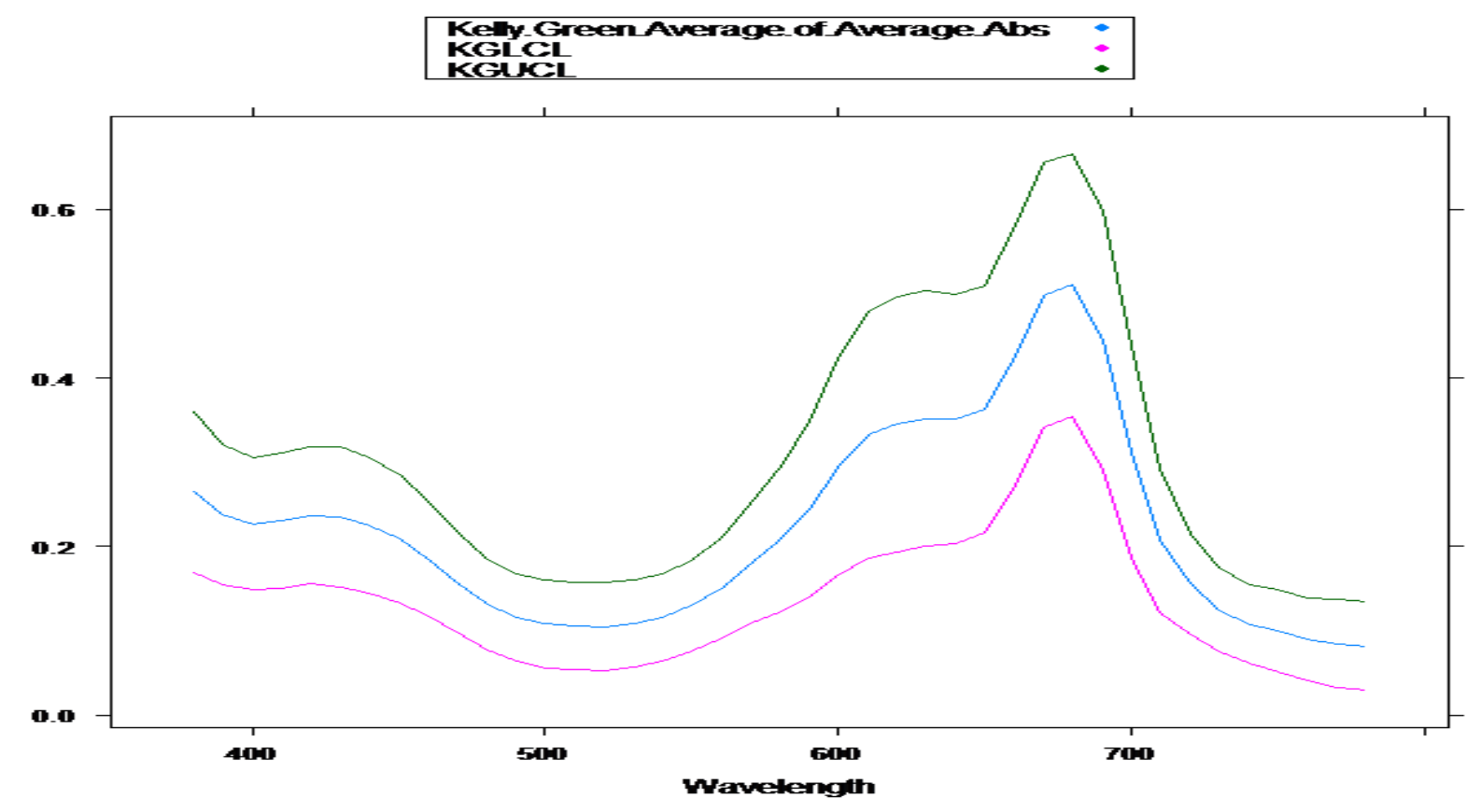

Figure 113: Spectra of the Average Absorbance, UCL and the LCL for every front Kelly Green Shirt 


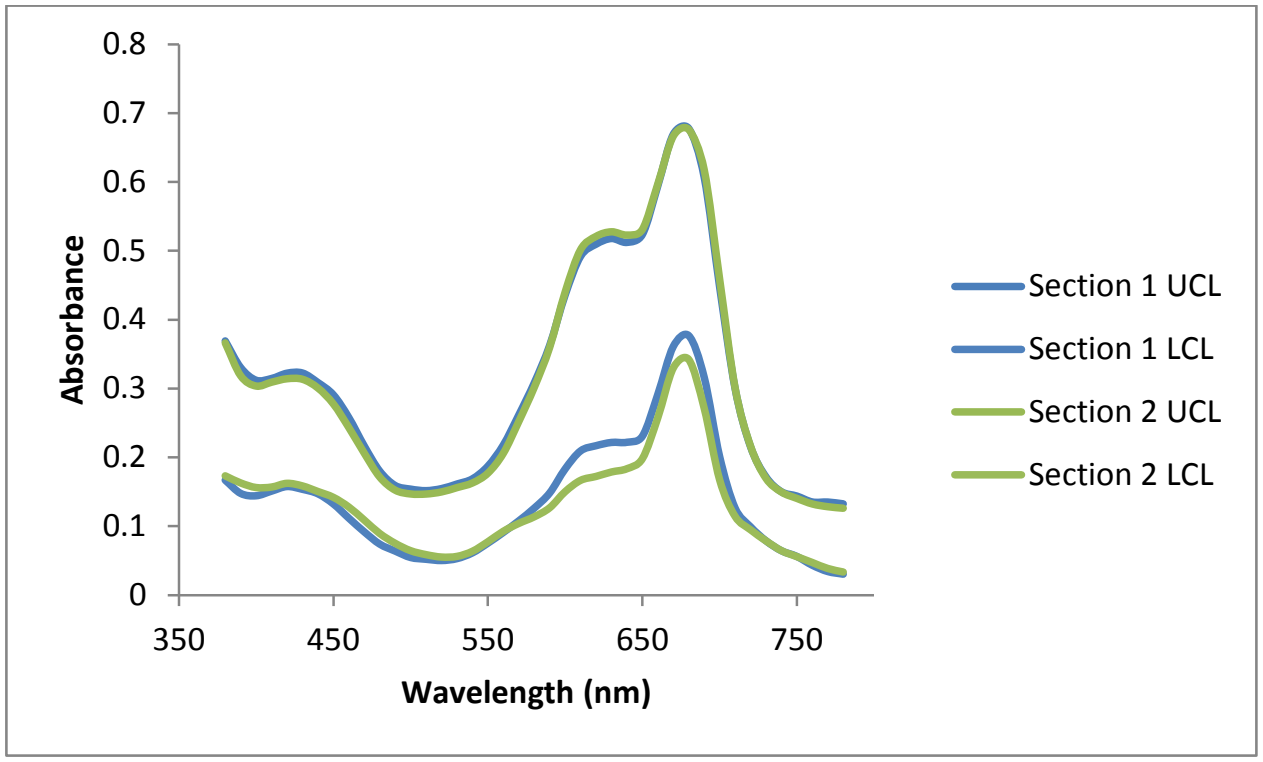

Figure 114: The 95\% UCL and the 95\% LCL of Kelly Green Section 1 and Section 2

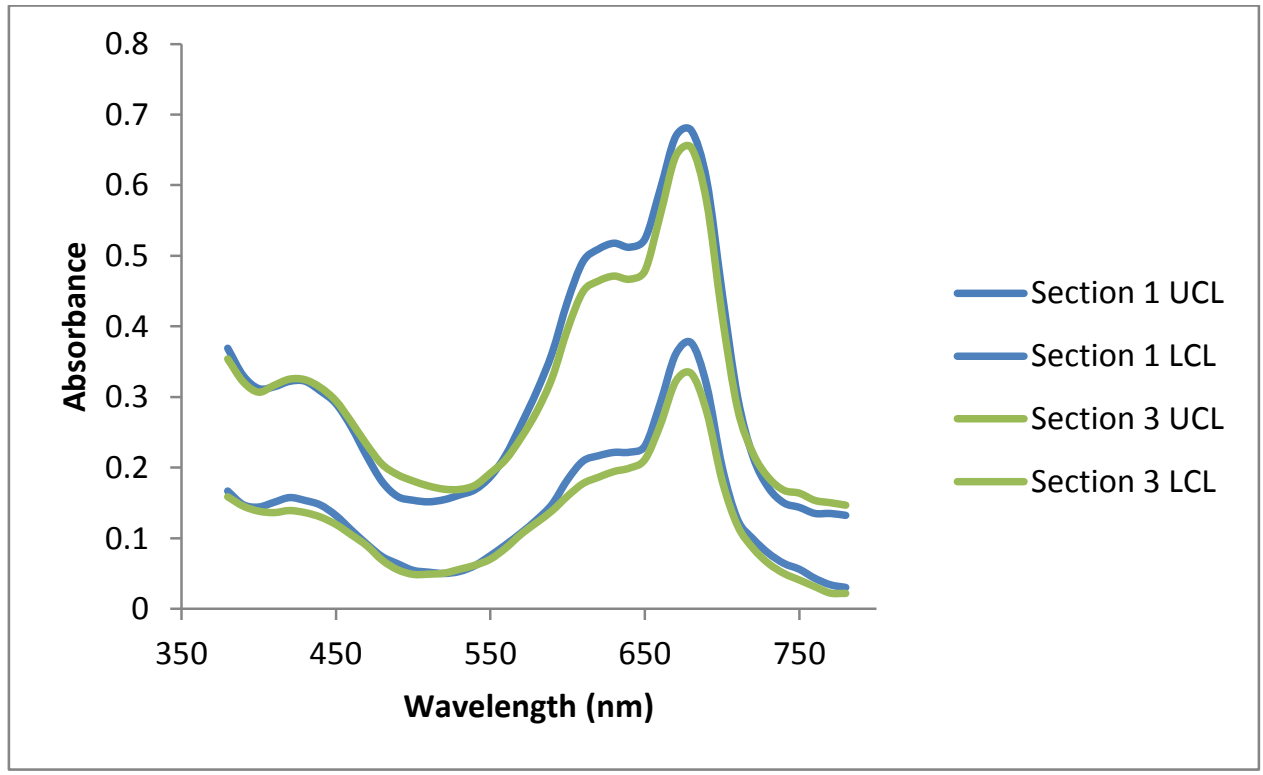

Figure 115: The 95\% UCL and the 95\% LCL of Kelly Green Section 1 and Section 3 


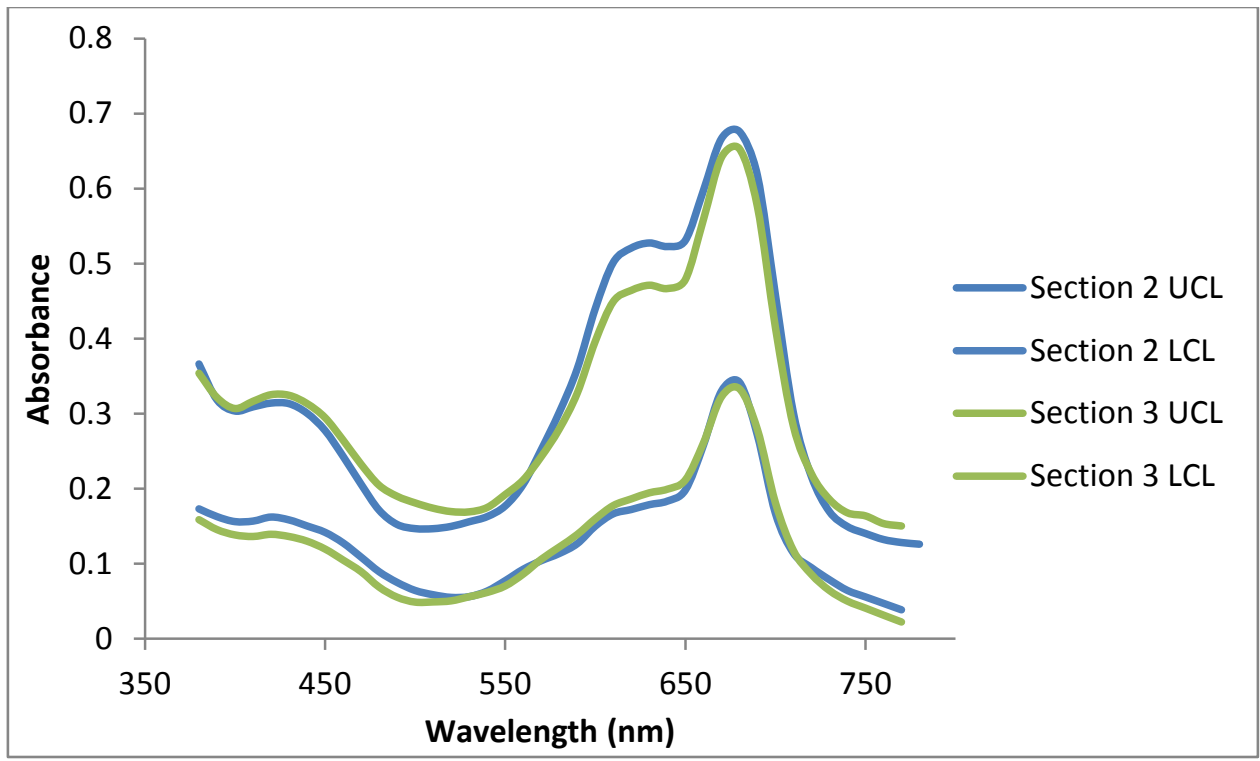

Figure 116: The 95\% UCL and the 95\% LCL of Kelly Green Section 2 and Section 3 


\section{Orange}

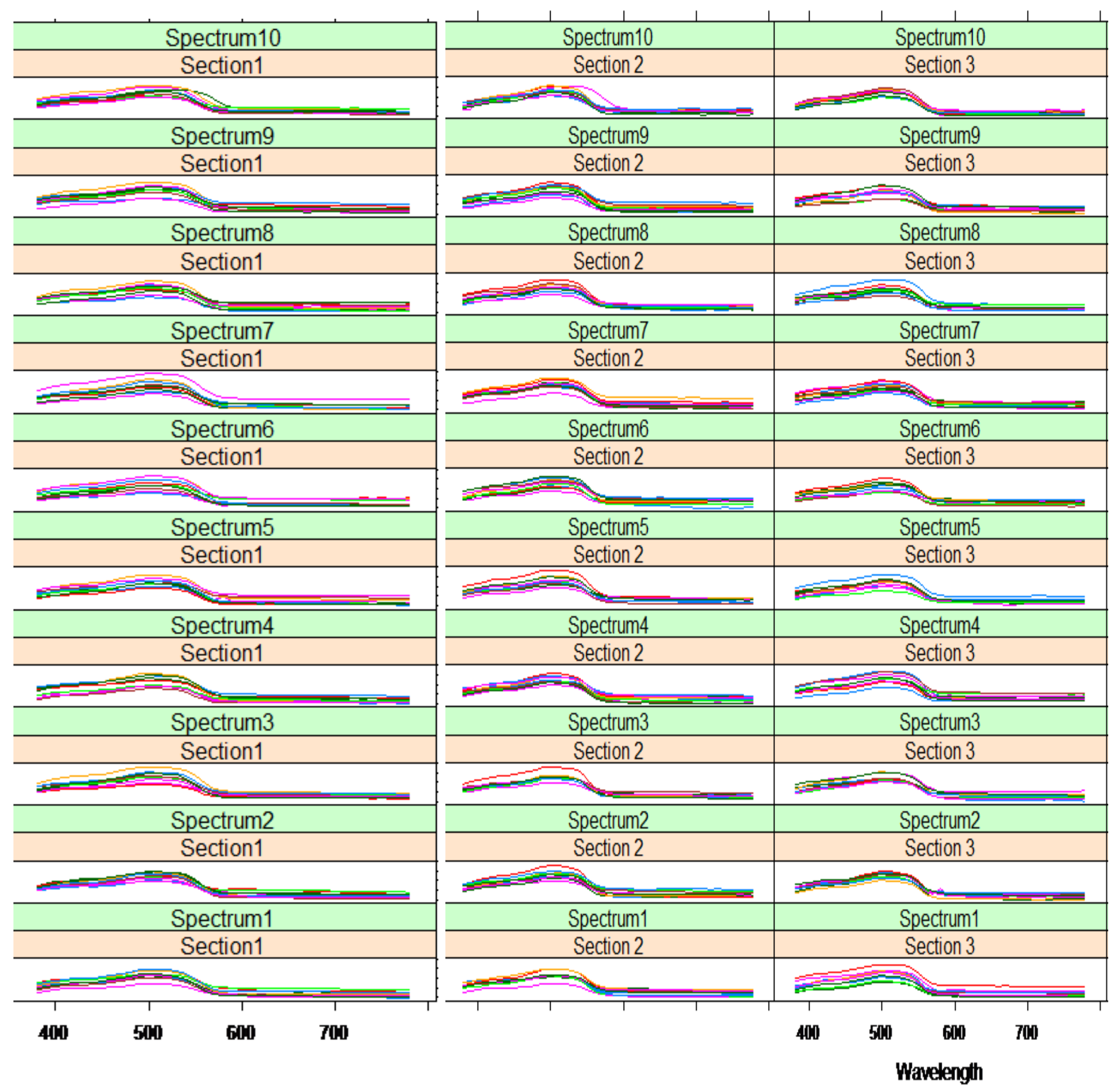

Figure 117: Spectra the Orange A-J shirts for section 1, 2 and 3 of all ten runs 


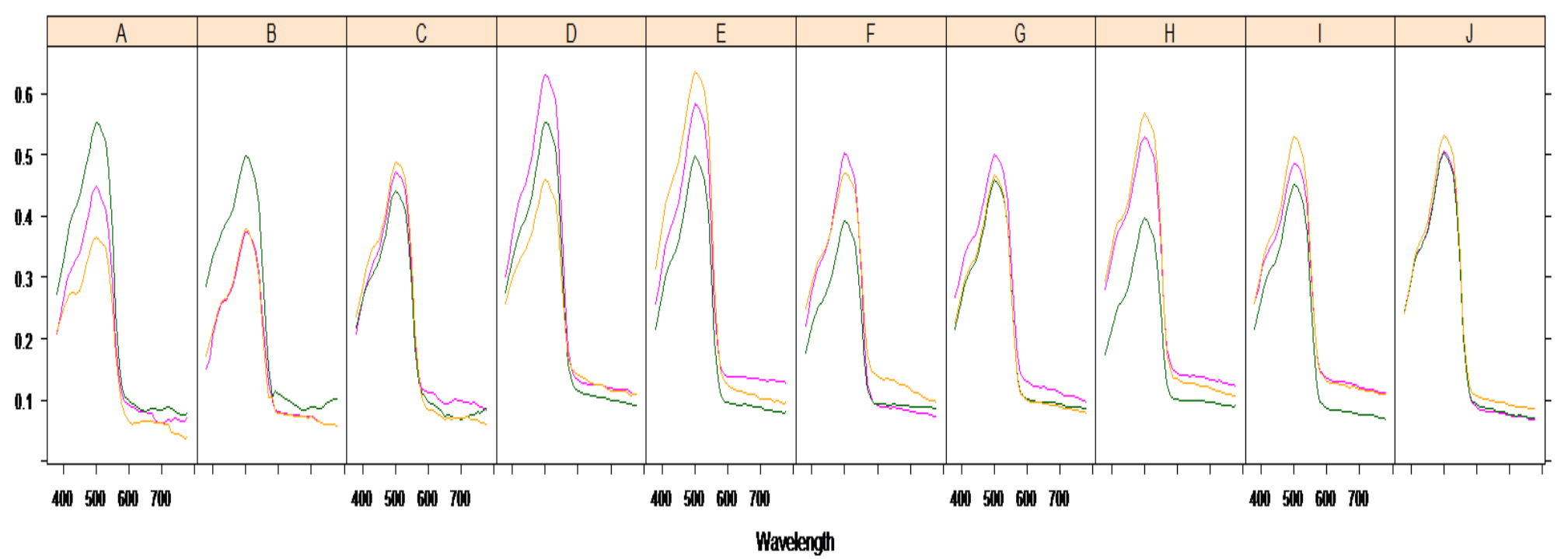

Figure 118: Spectra of section 1, 2 and 3 for Orange A-J shirts

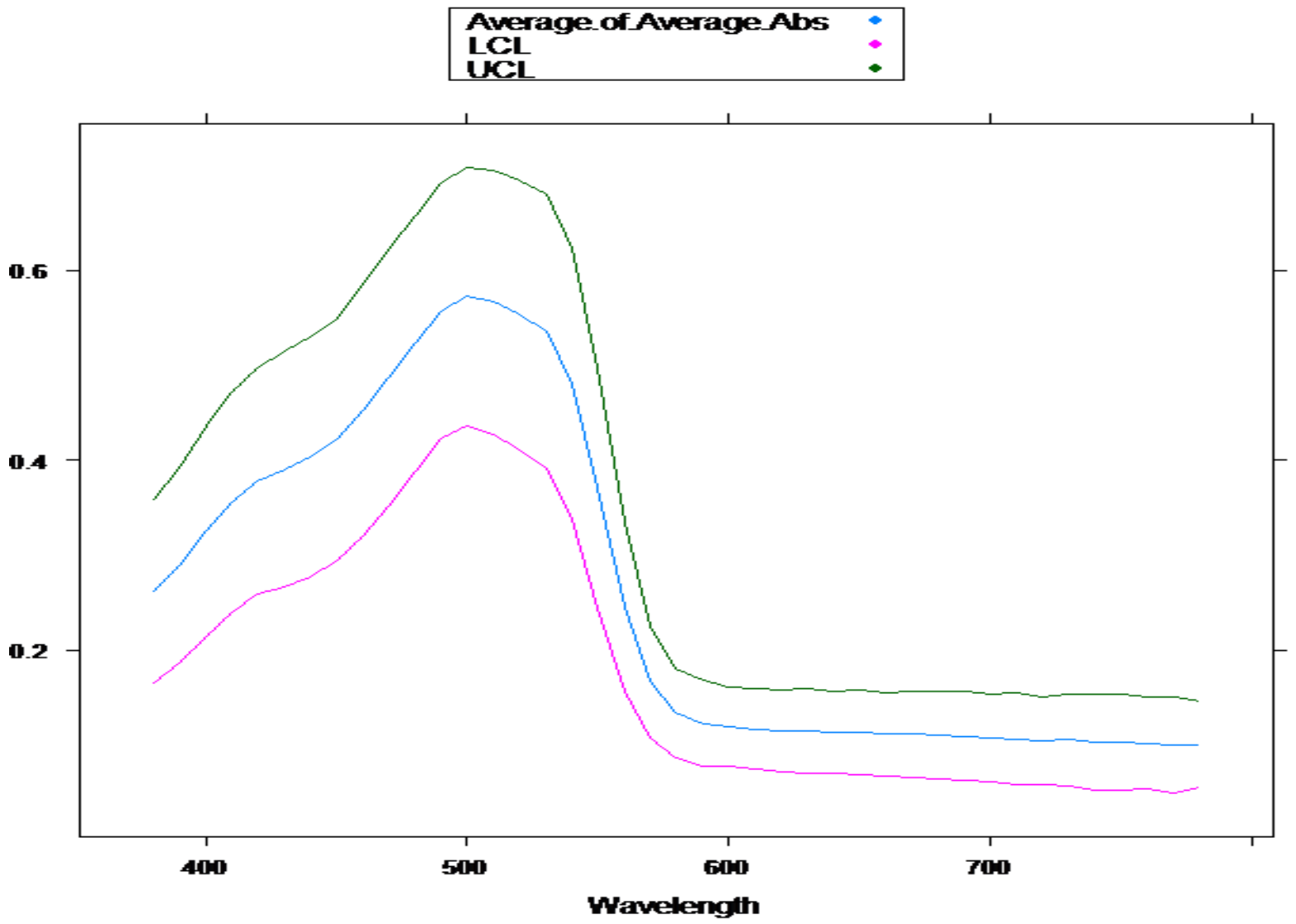

Figure 119: Spectra of the Average Absorbance, UCL and the LCL for 3 all sections of the Orange E shirts 


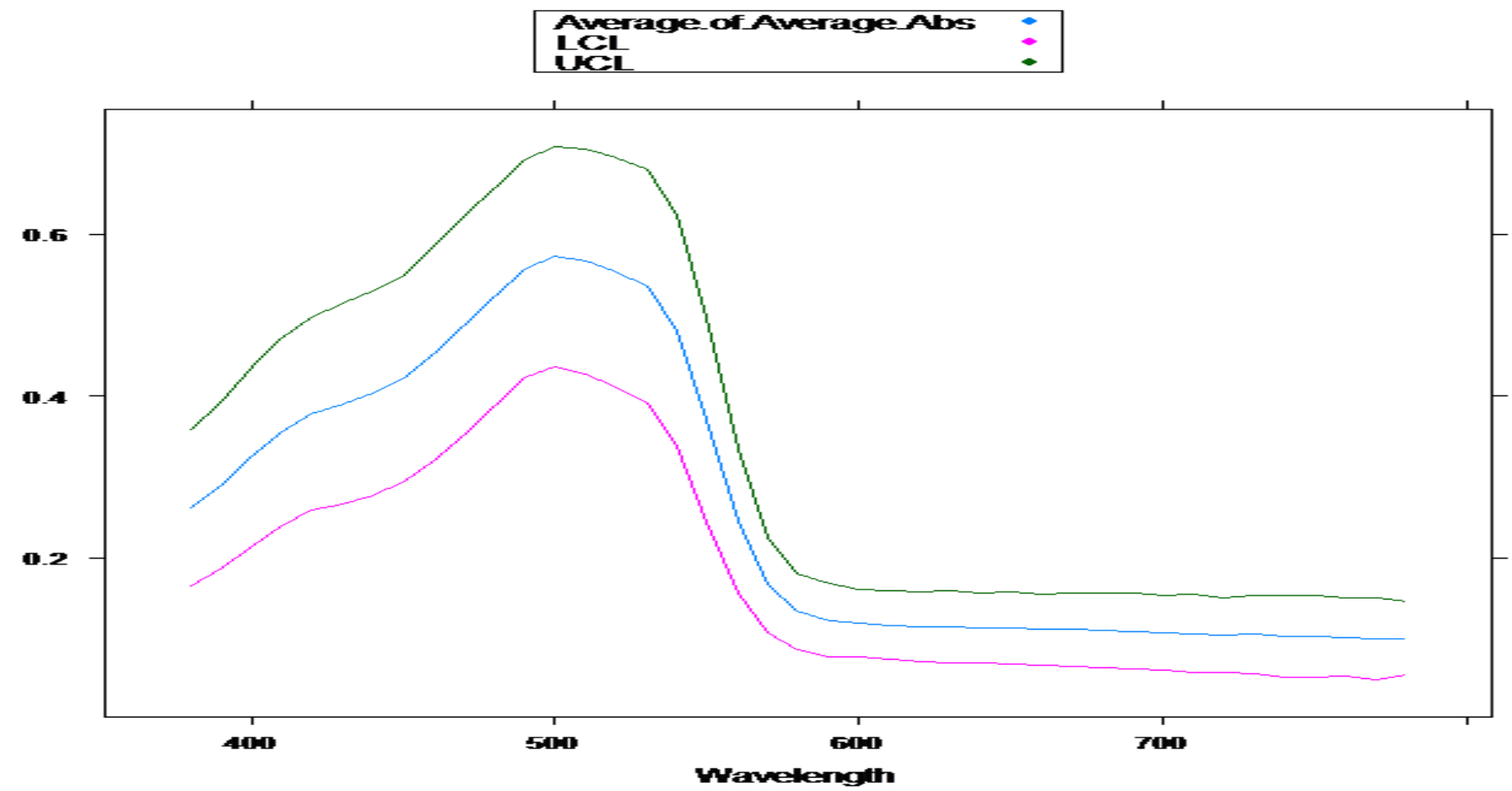

Figure 120: Spectra of the Average Absorbance, UCL and the LCLfor 3 all sections of the Orange F shirts

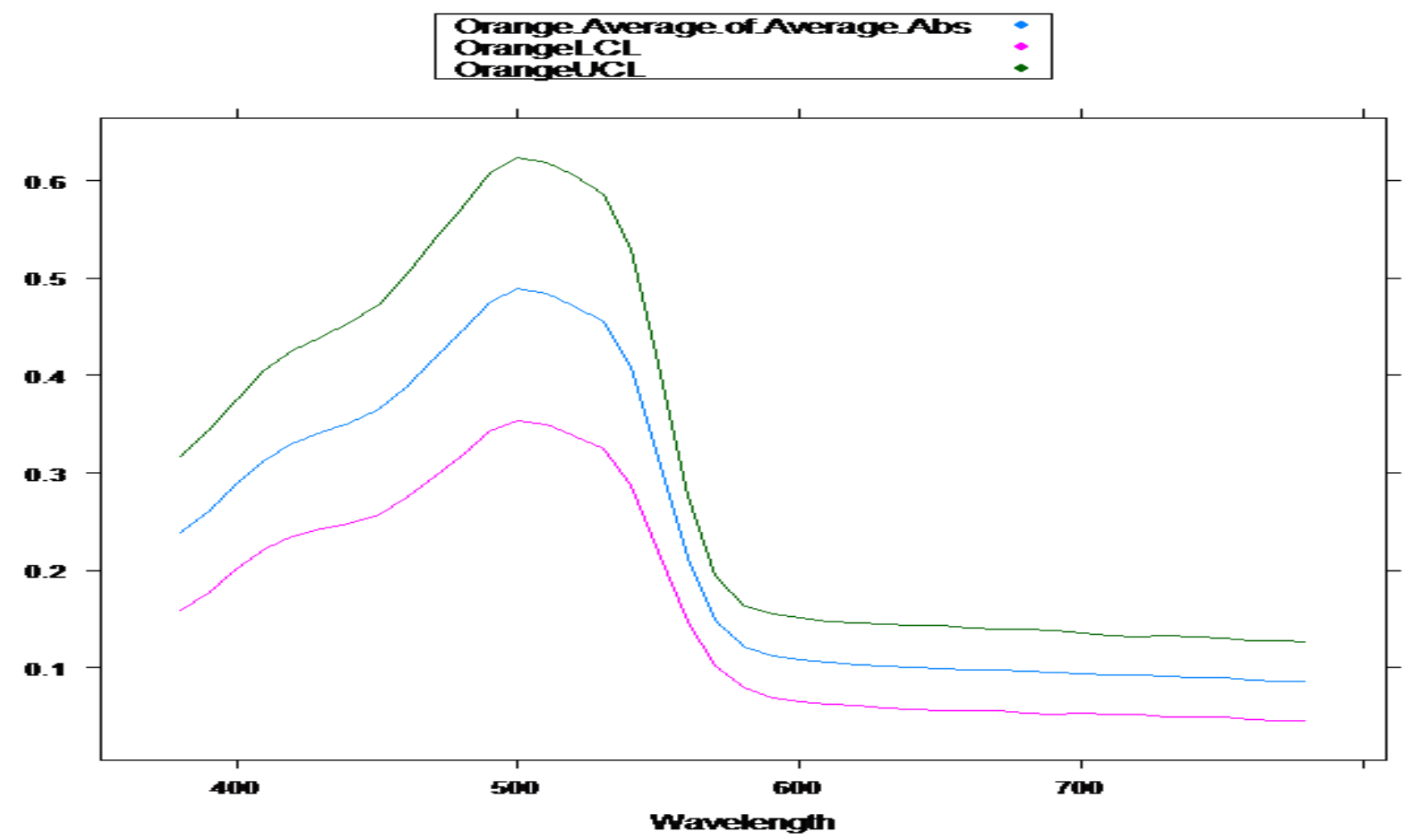

Figure 121: Spectra of the Average Absorbance, UCL and the LCL for every front Orange Shirt 


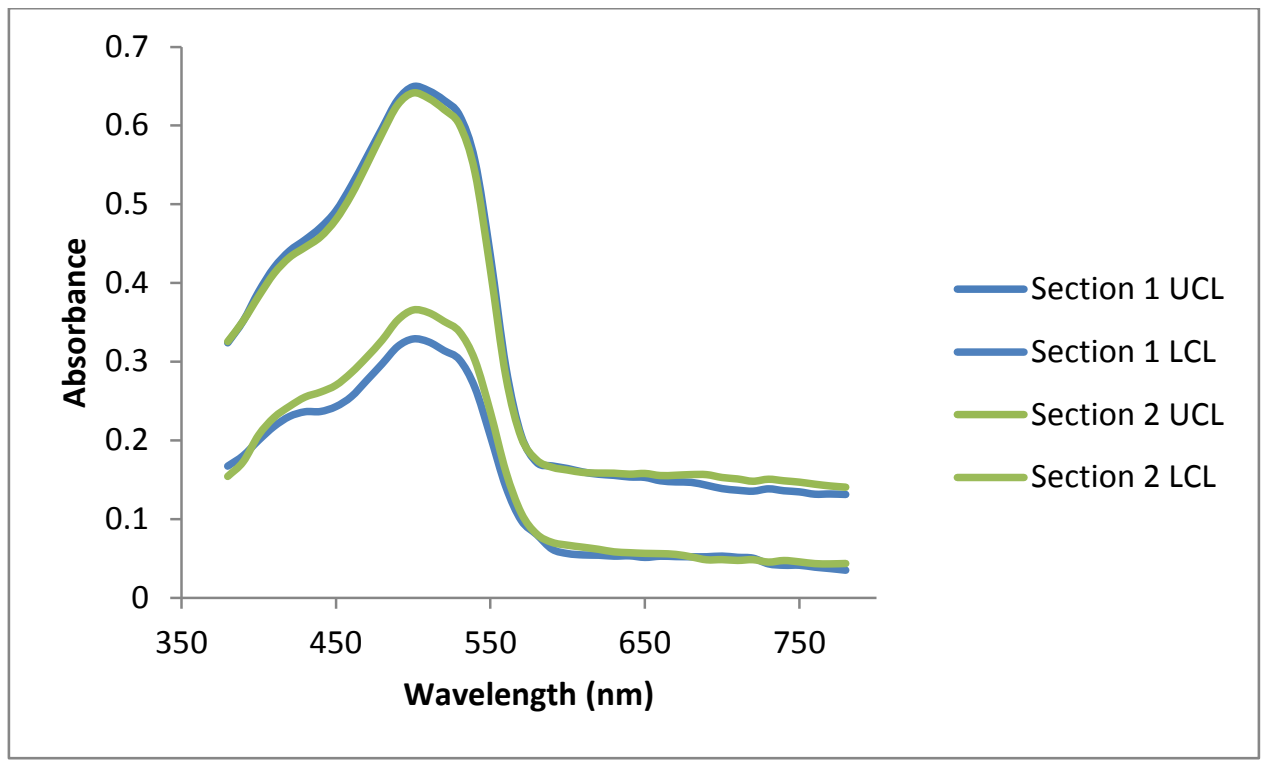

Figure 122: The 95\% UCL and the 95\% LCL of Orange Section 1 and Section 2

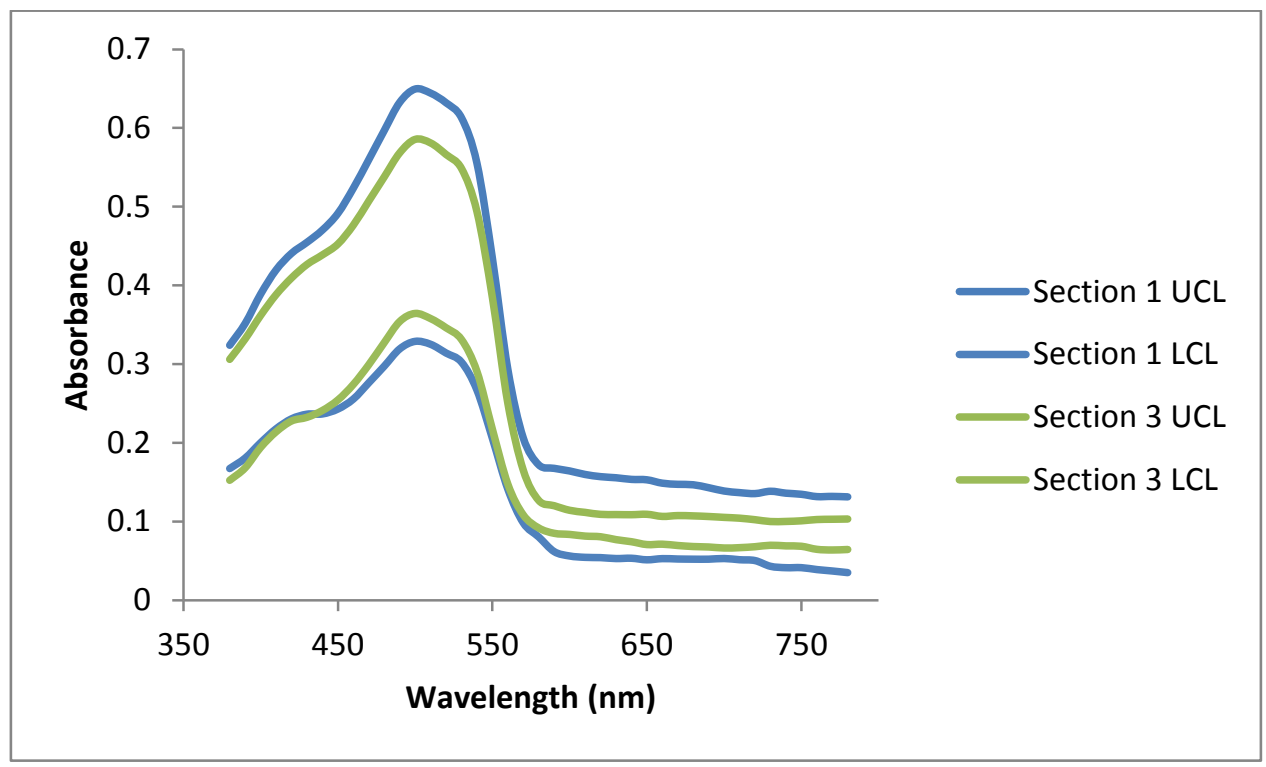

Figure 123: The 95\% UCL and the 95\% LCL of Orange Section 1 and Section 3 


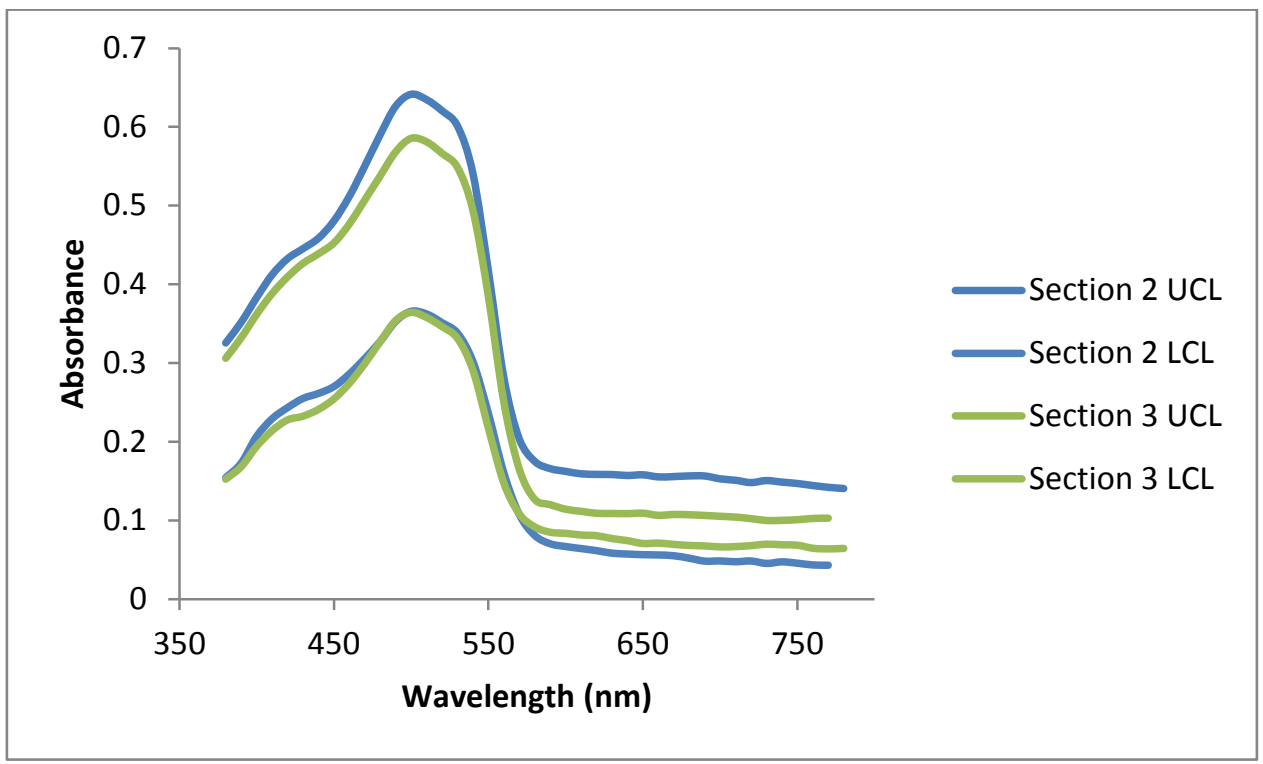

Figure 124: The 95\% UCL and the 95\% LCL of Orange Section 2 and Section 3 


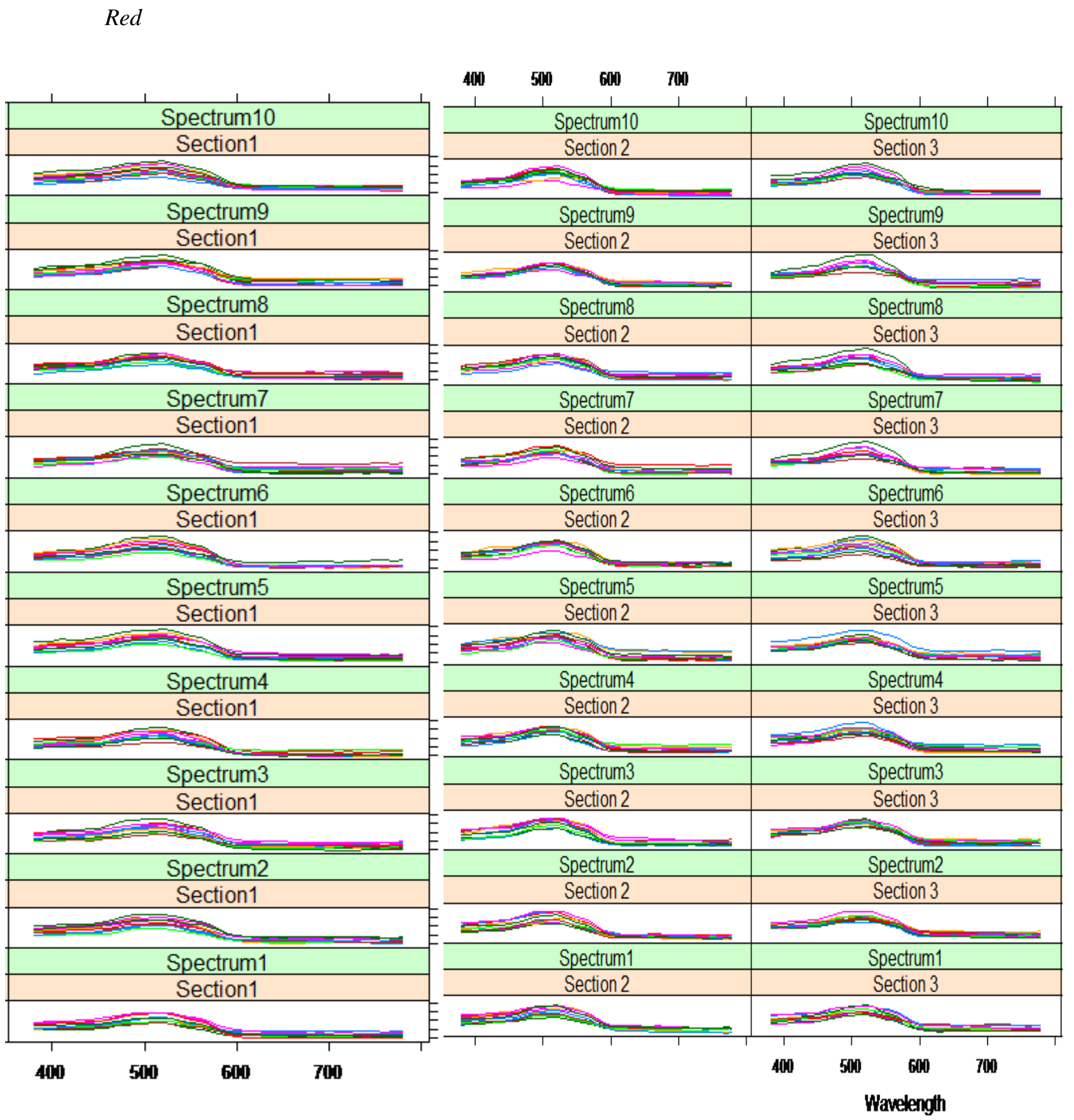

Figure 125: Spectra of the Red A-J shirts for section 1, 2 and 3 of all ten runs 


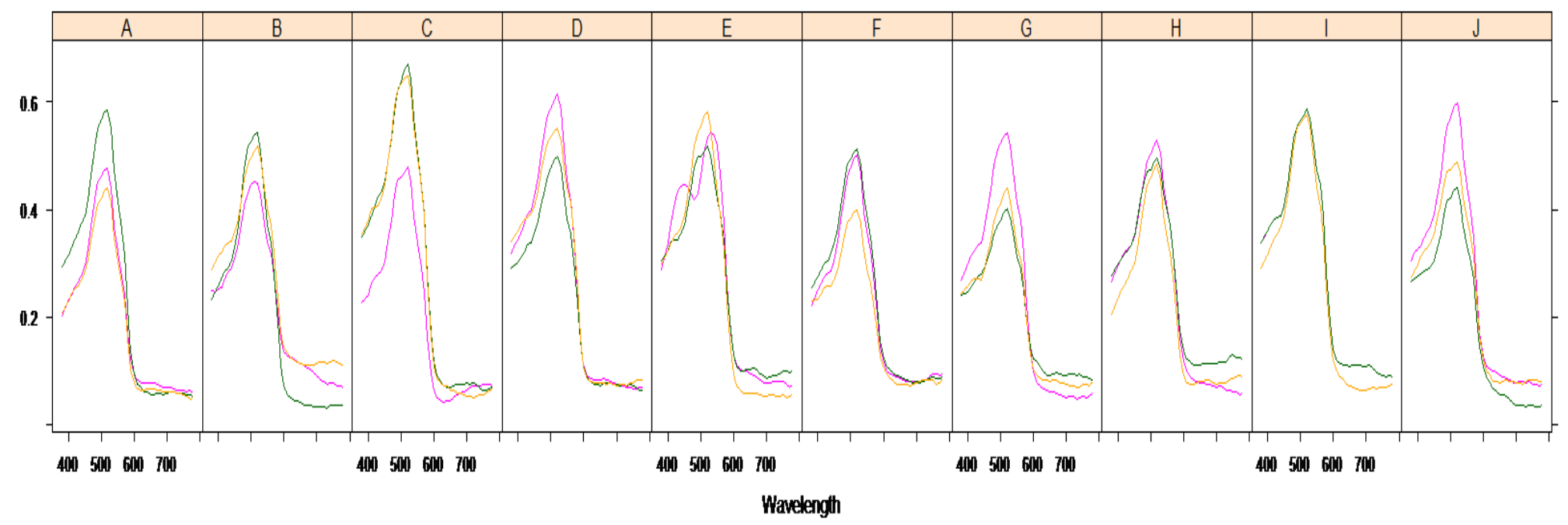

Figure 126: Spectra of section 1, 2 and 3 for Red A-J shirts

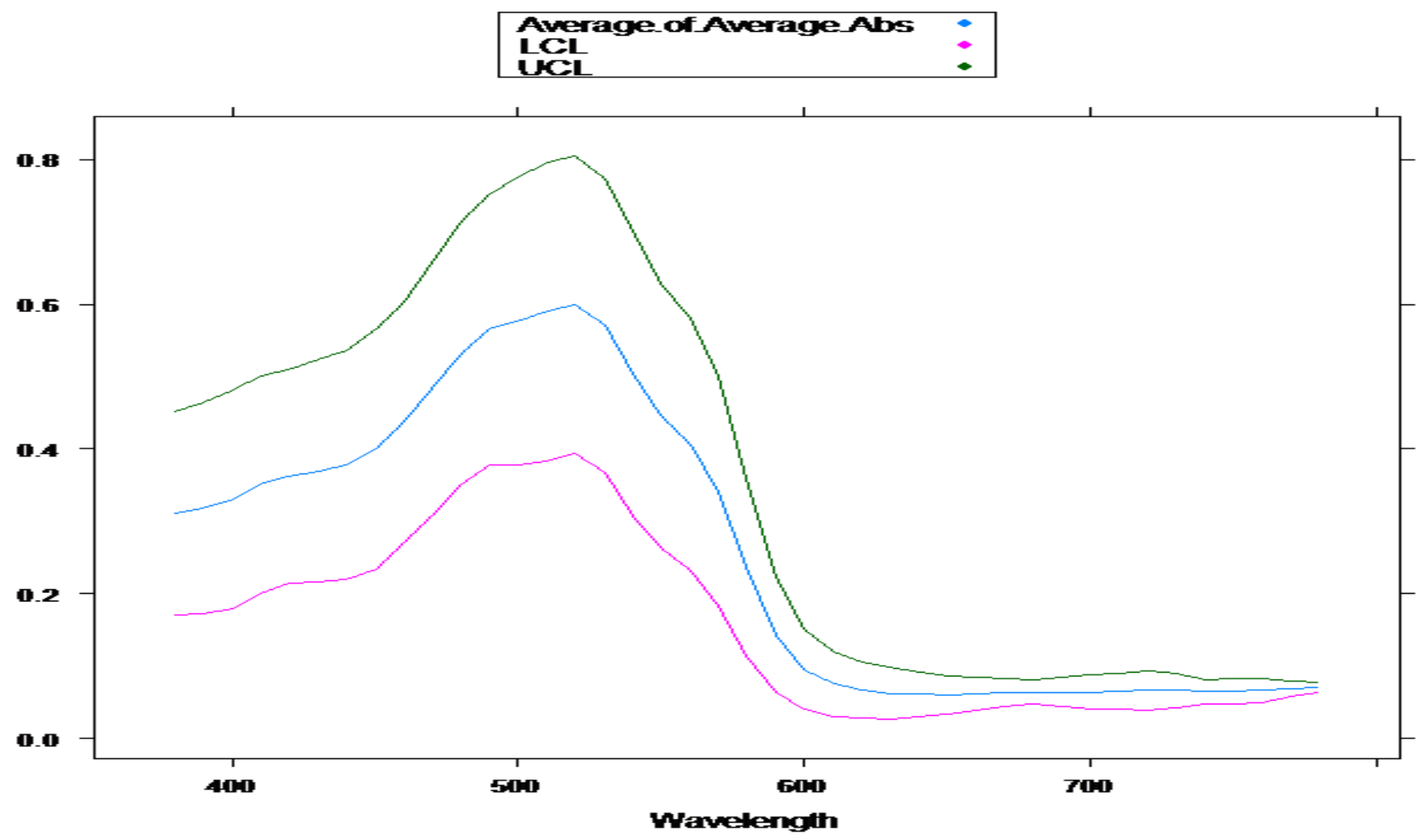

Figure 127: Spectra of the Average Absorbance, UCL and the LCL for 3 all sections of the Red C shirts 


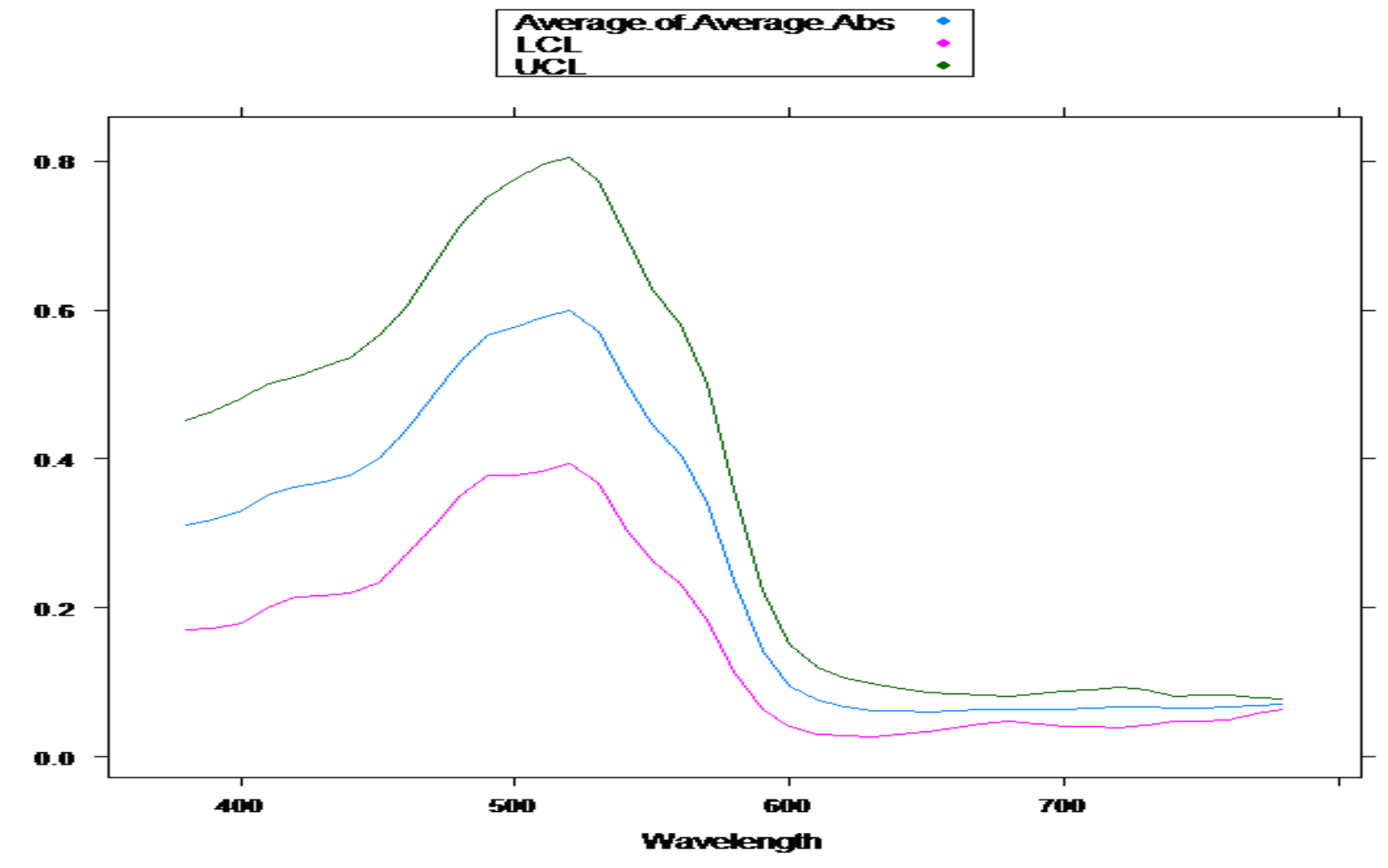

Figure 128: Spectra of the Average Absorbance, UCL and the LCLfor 3 all sections of the Red $D$ shirts

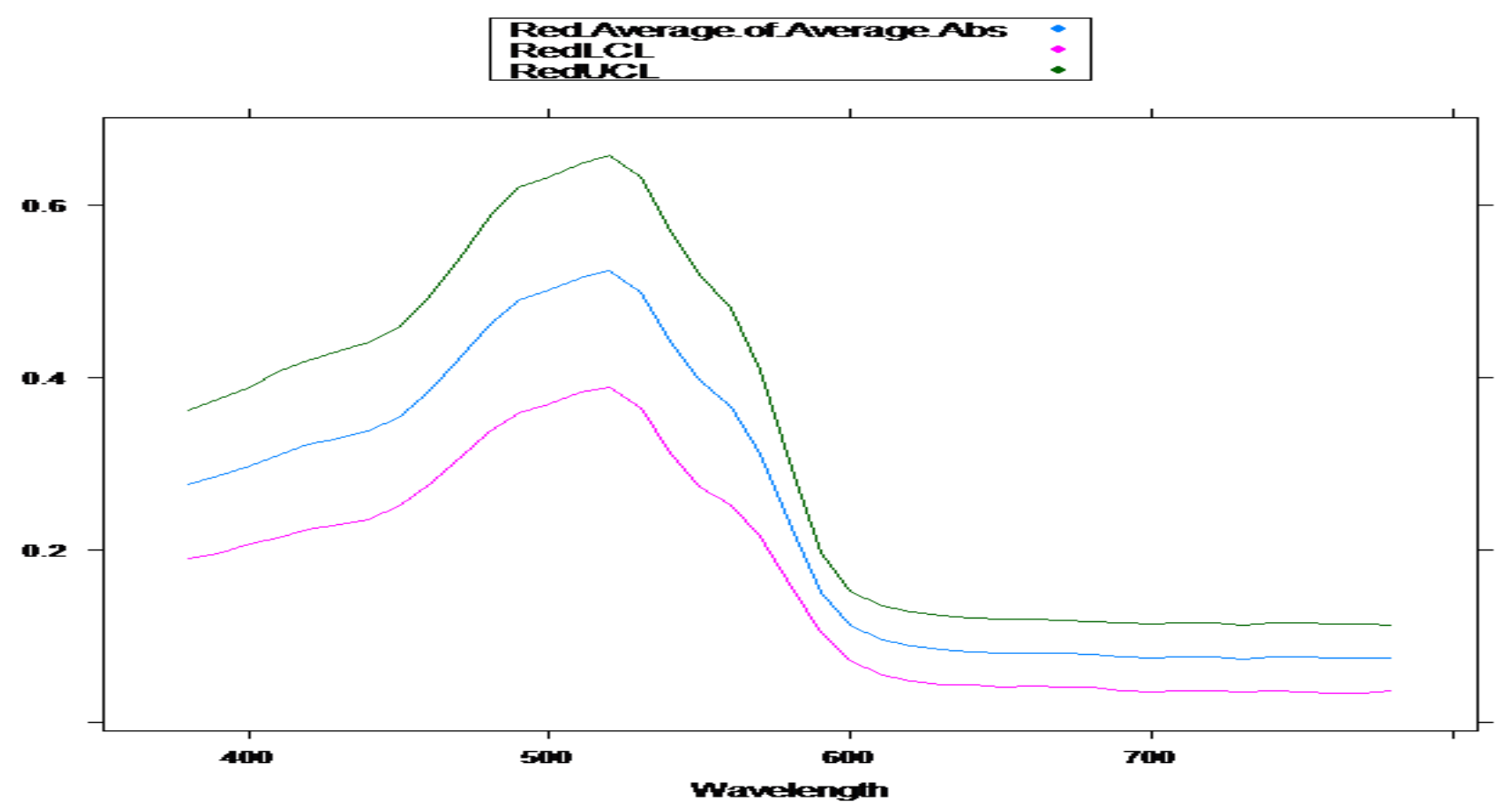

Figure 129: Spectra of the Average Absorbance, UCL and the LCL for every front Red Shirt 


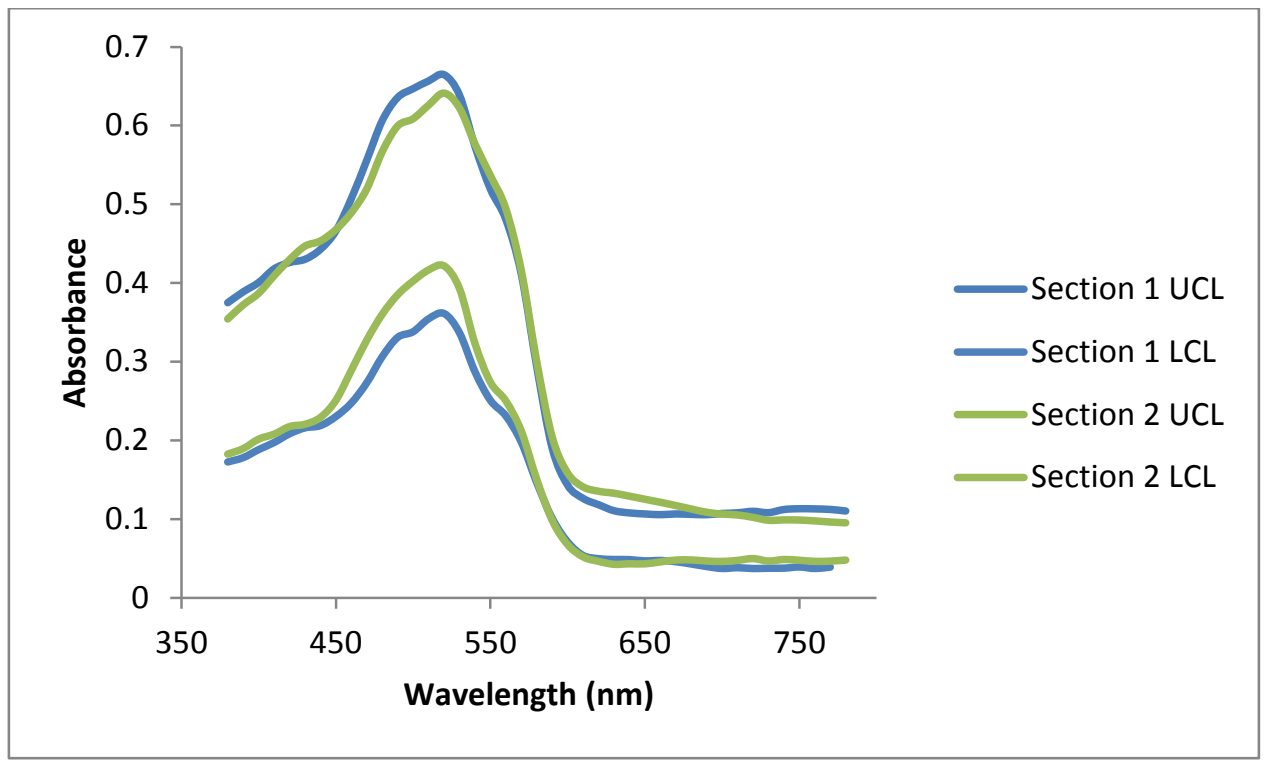

Figure 130: The 95\% UCL and the 95\% LCL of Red Section 1 and Section 2

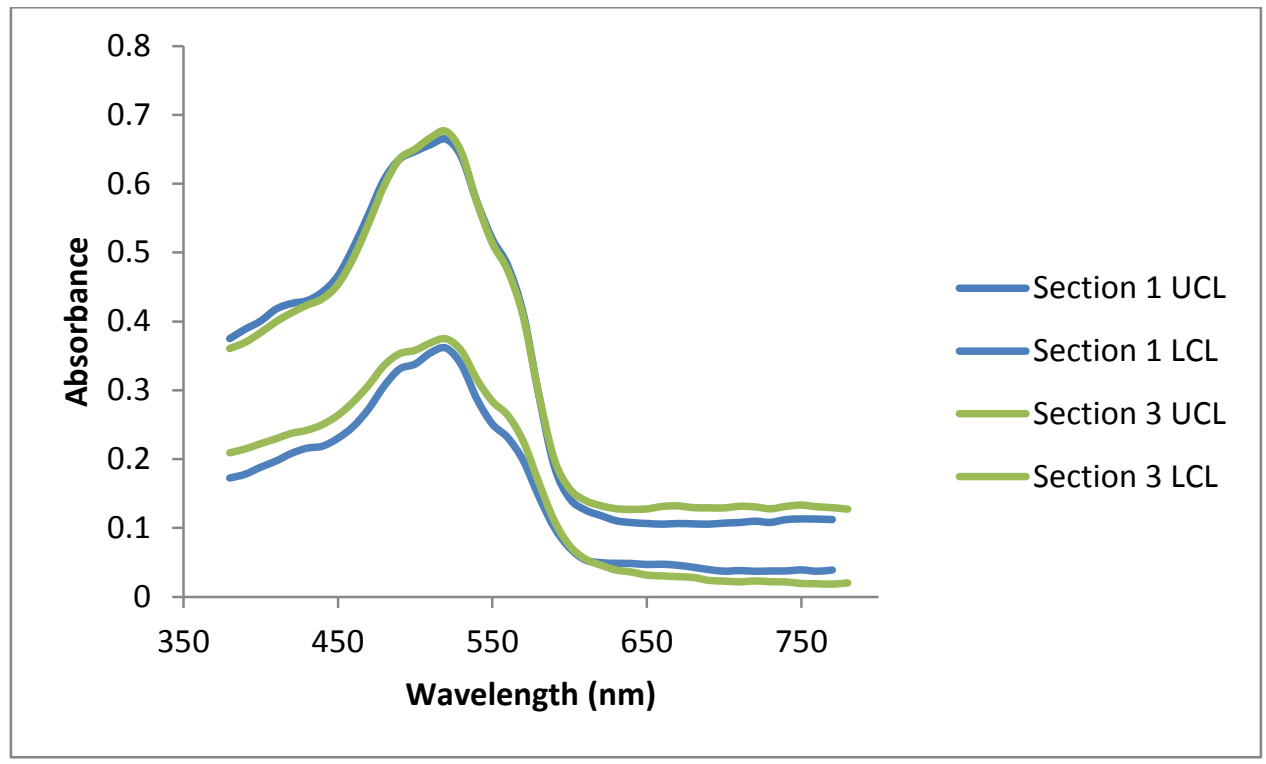

Figure 131: The 95\% UCL and the 95\% LCL of Red Section 1 and Section 3 


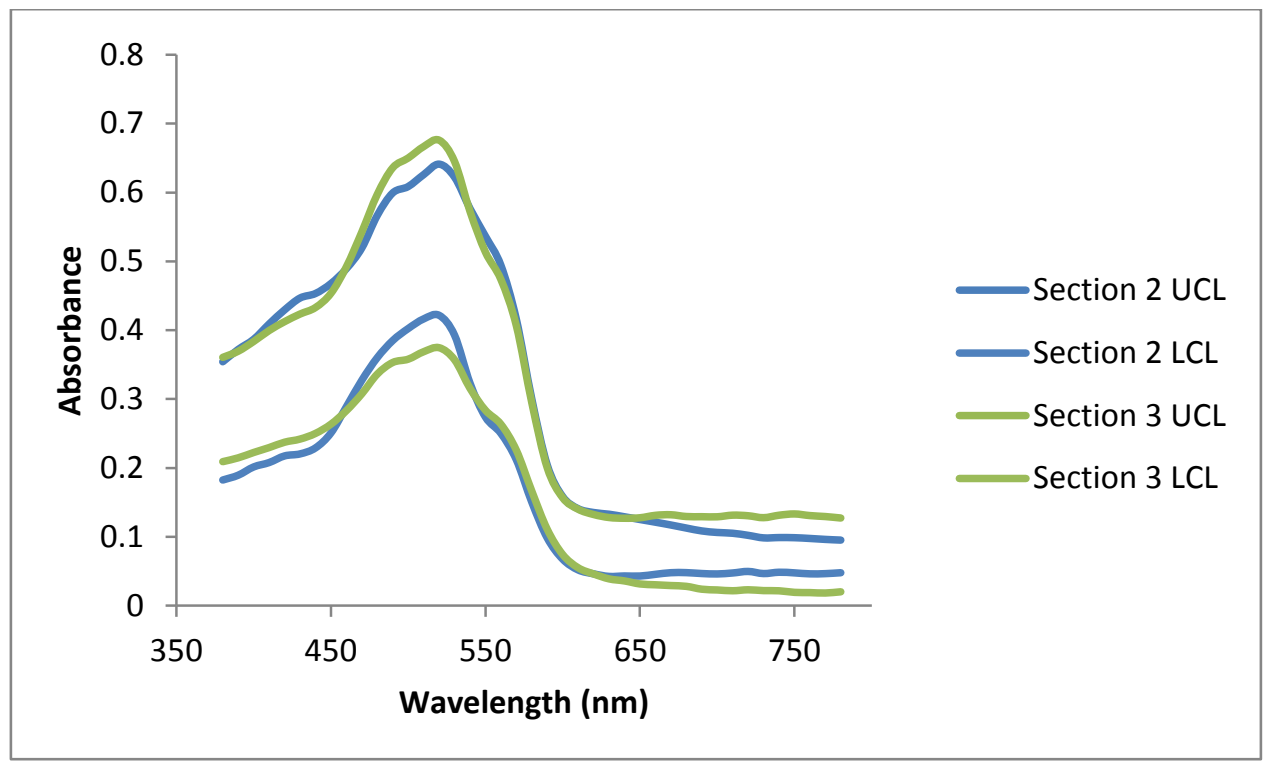

Figure 132: The 95\% UCL and the 95\% LCL of Red Section 2 and Section 3 
Royal

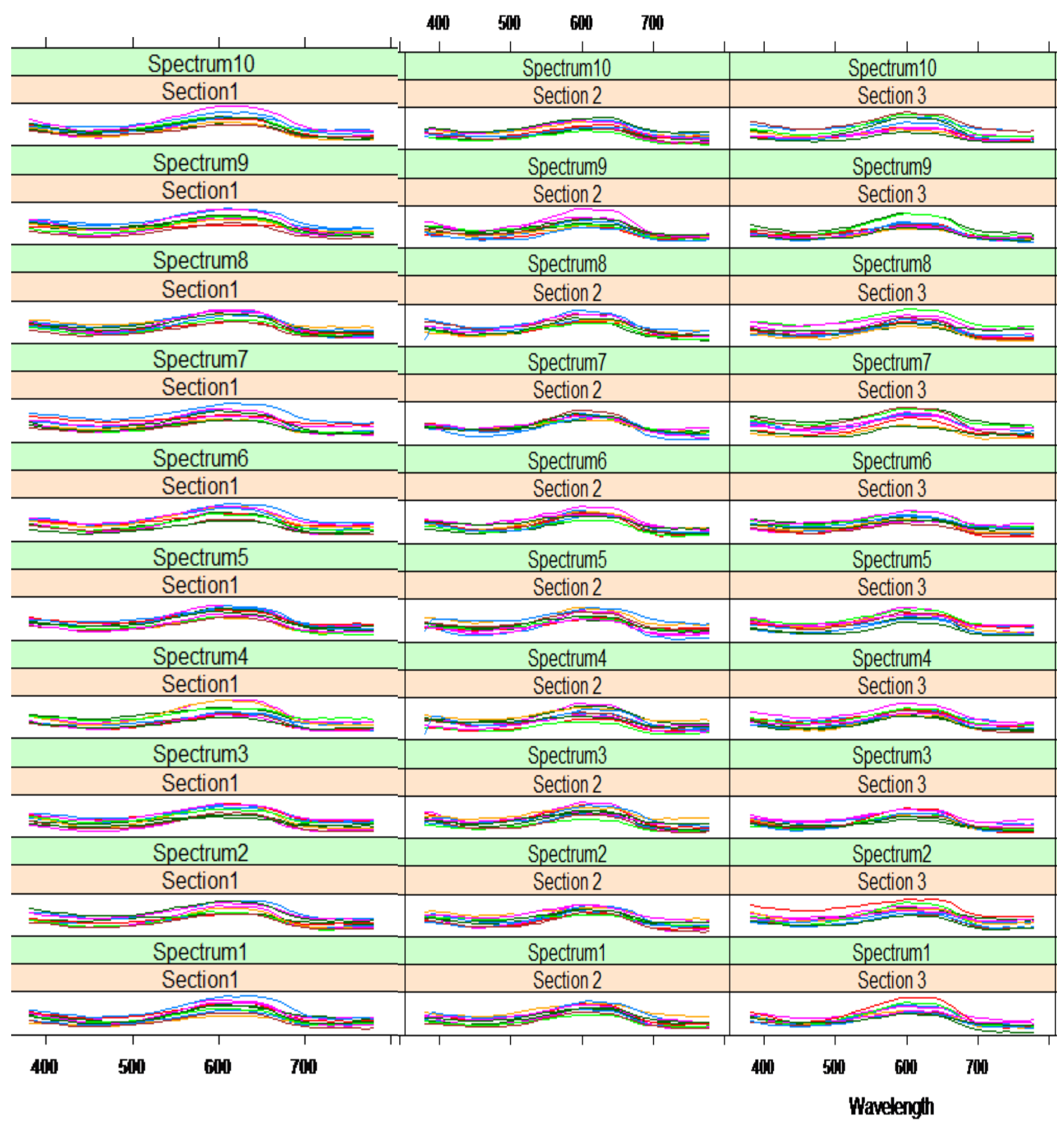

Figure 133: Spectra of the Royal A-J shirts for section 1, 2 and 3 of all ten runs 


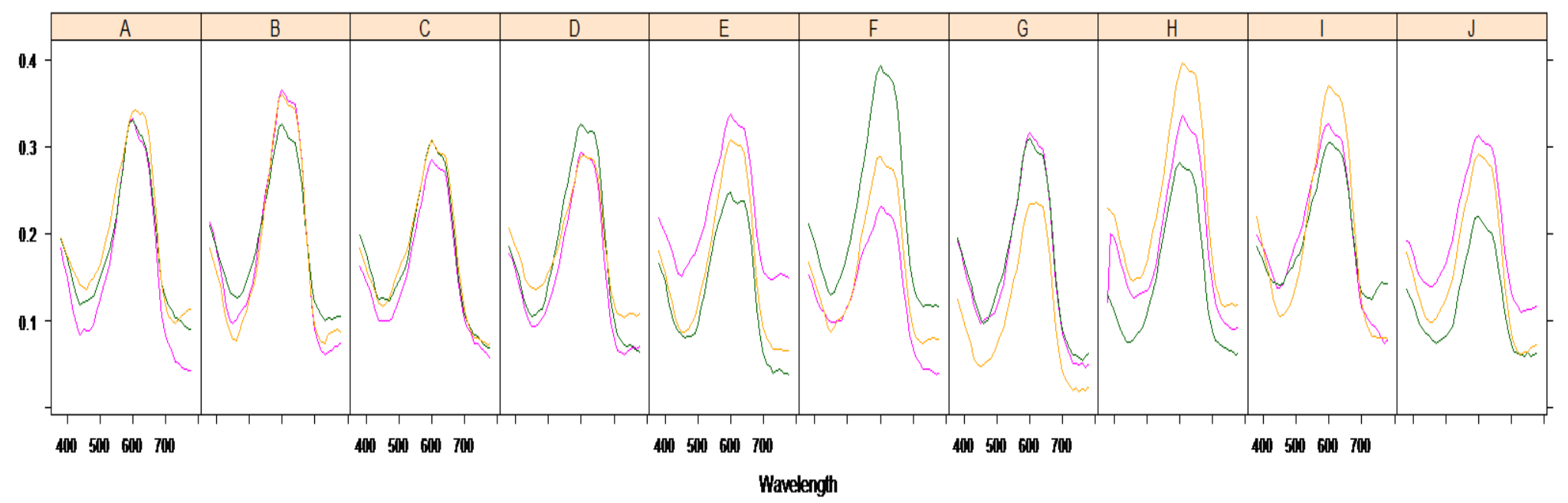

Figure 134: Spectra of section 1, 2 and 3 for Royal A-J shirts

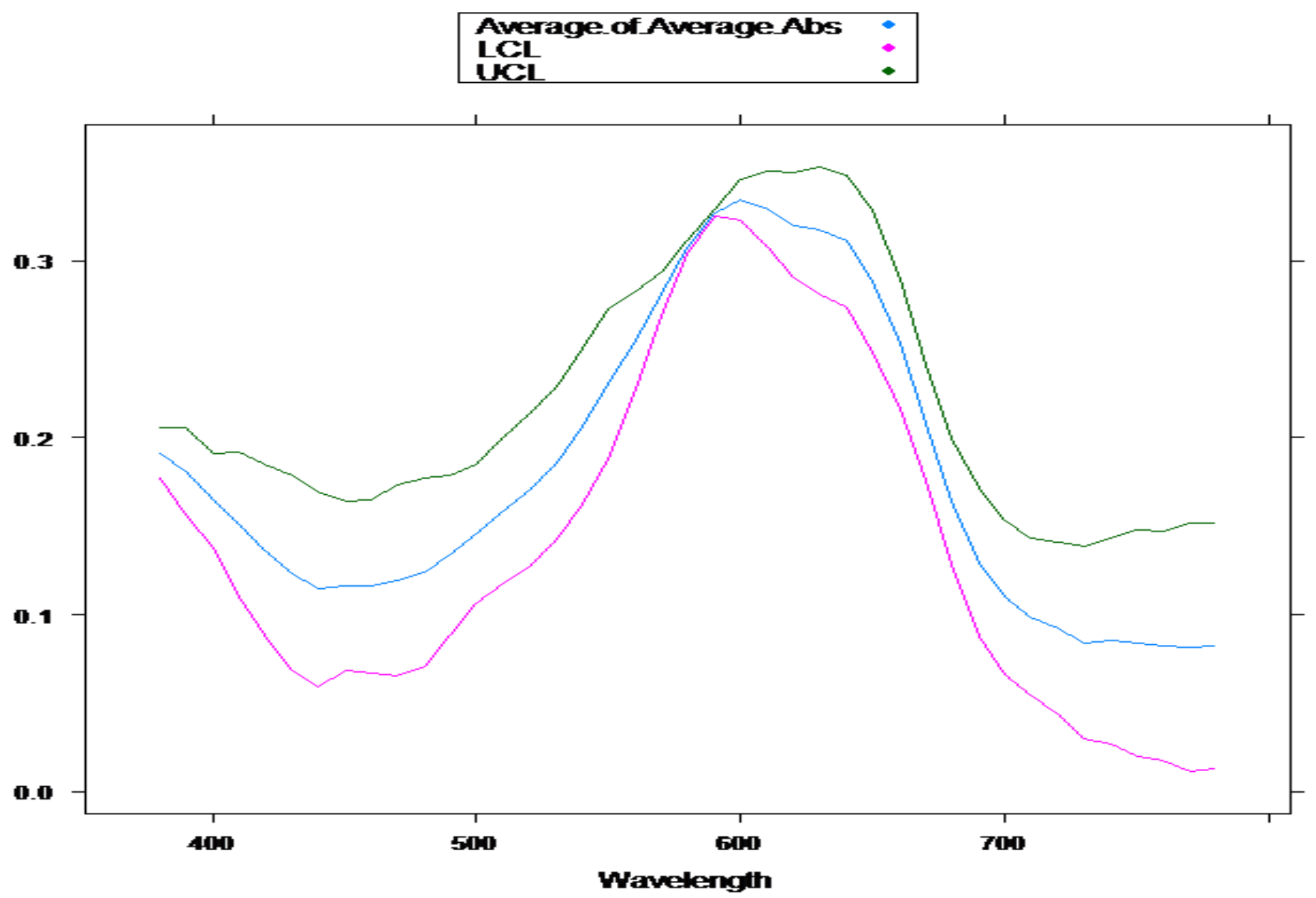

Figure 135: Spectra of the Average Absorbance, UCL and the LCL for 3 all sections of the Royal A shirts 


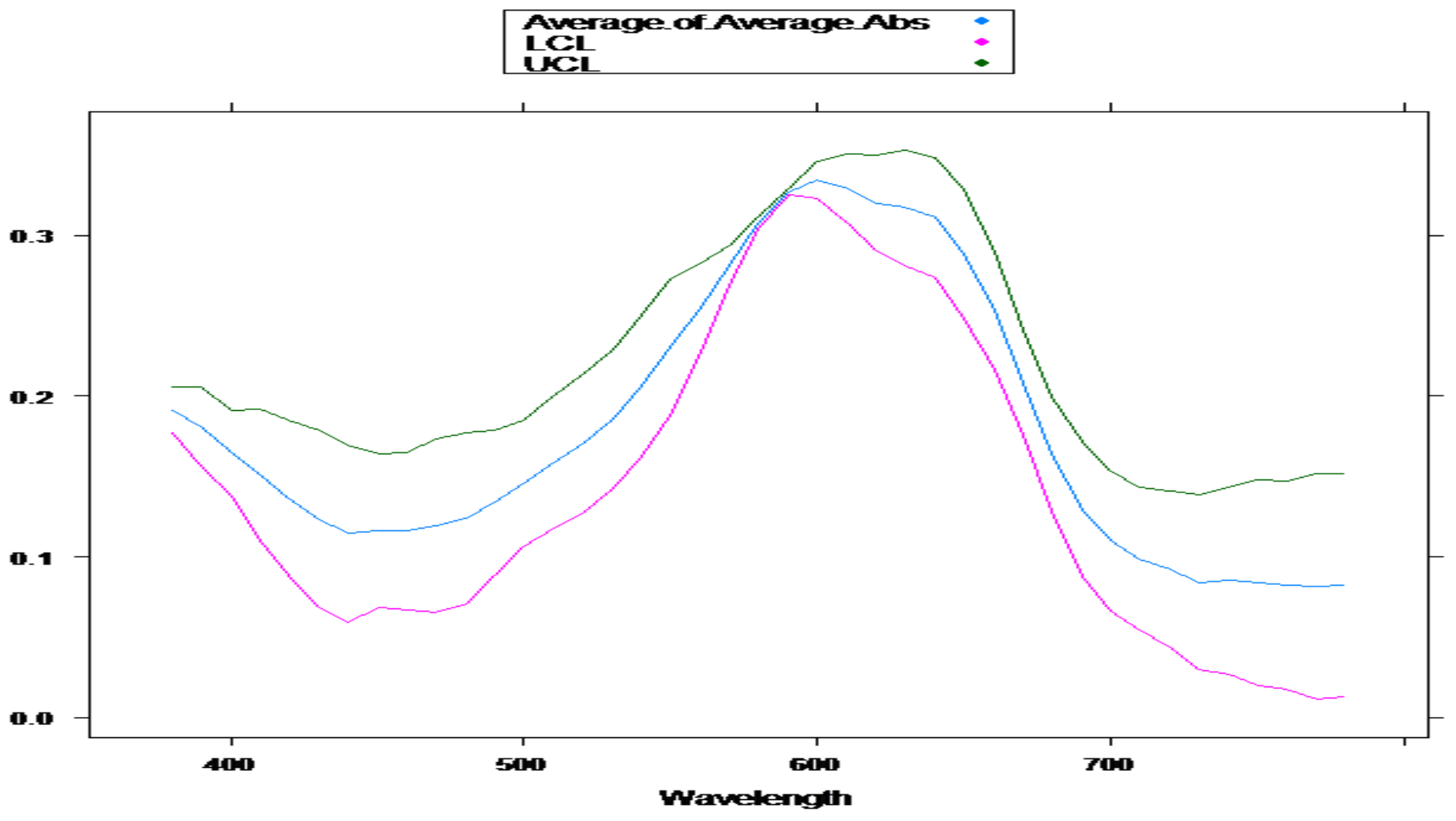

Figure 136: Spectra of the Average Absorbance, UCL and the LCL for 3 all sections of the Royal $B$ shirts

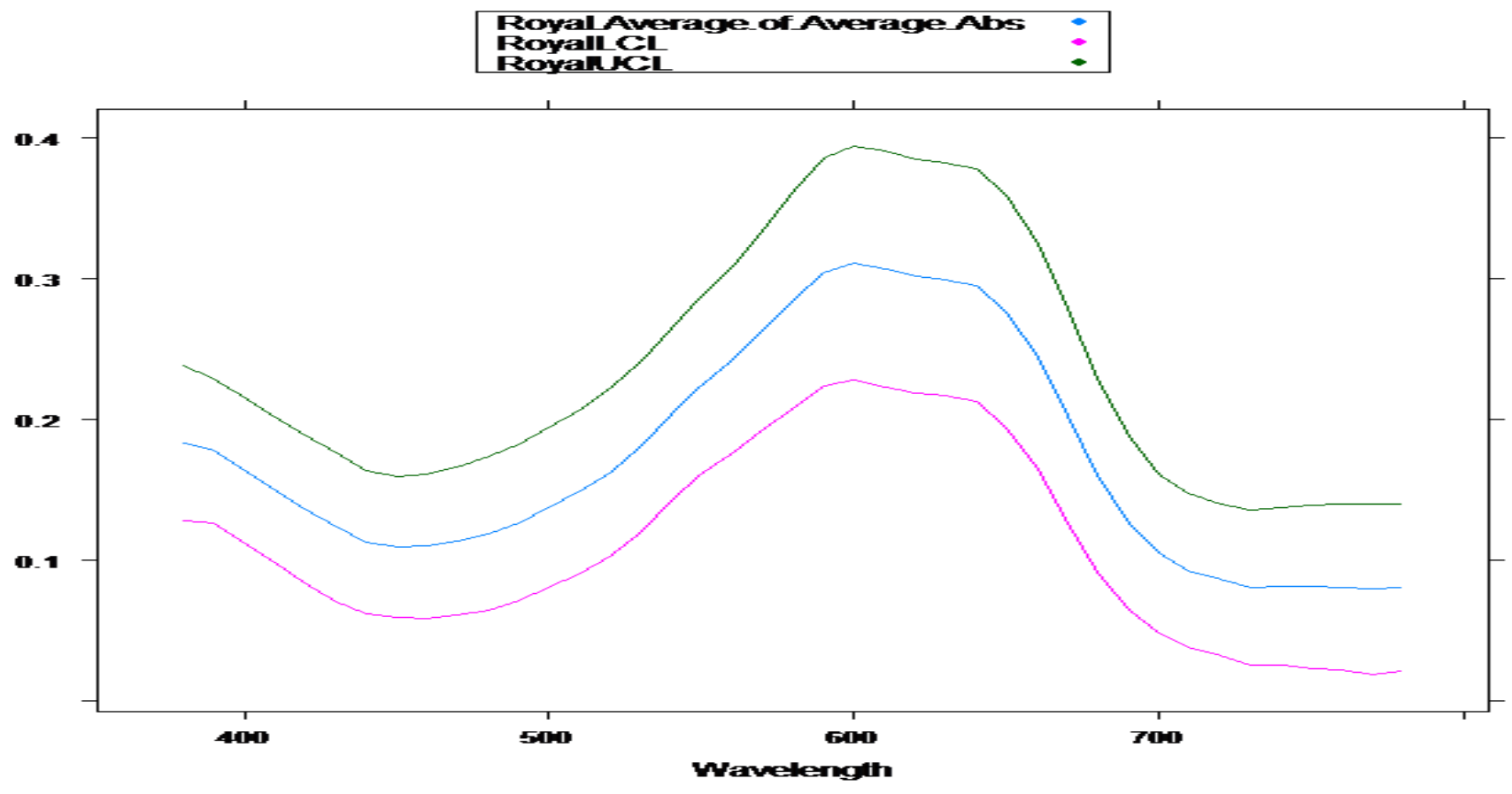

Figure 137: Spectra of the Average Absorbance, UCL and the LCL for every front Royal Shirt 


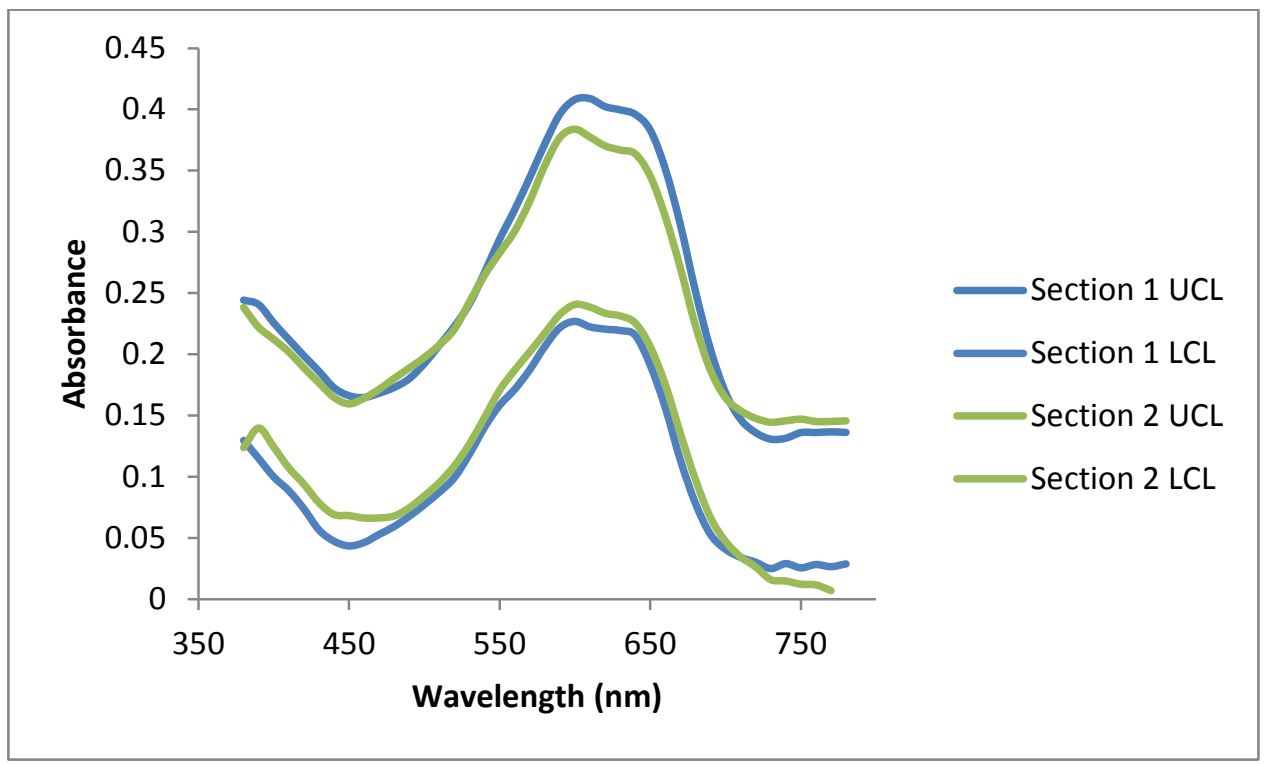

Figure 138: The 95\% UCL and the 95\% LCL of Royal Section 1 and Section 2

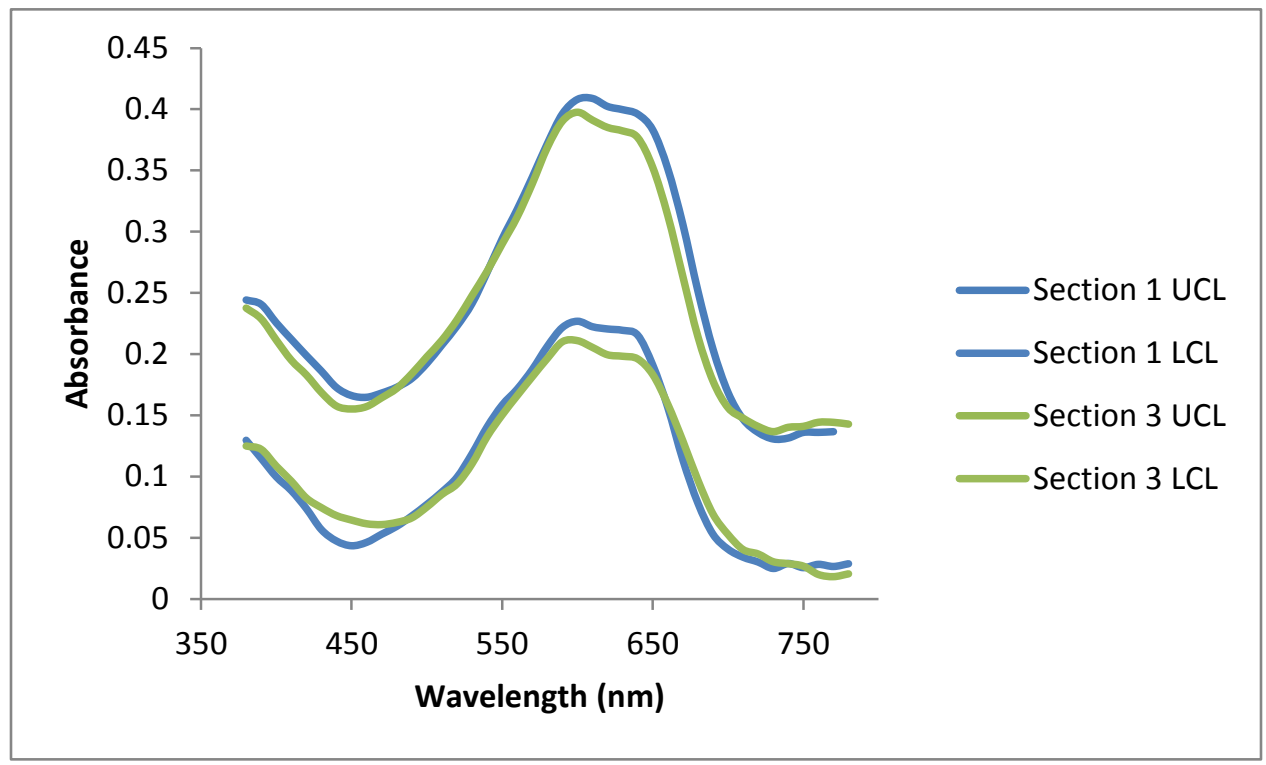

Figure 139: The 95\% UCL and the 95\% LCL of Royal Section 1 and Section 3 


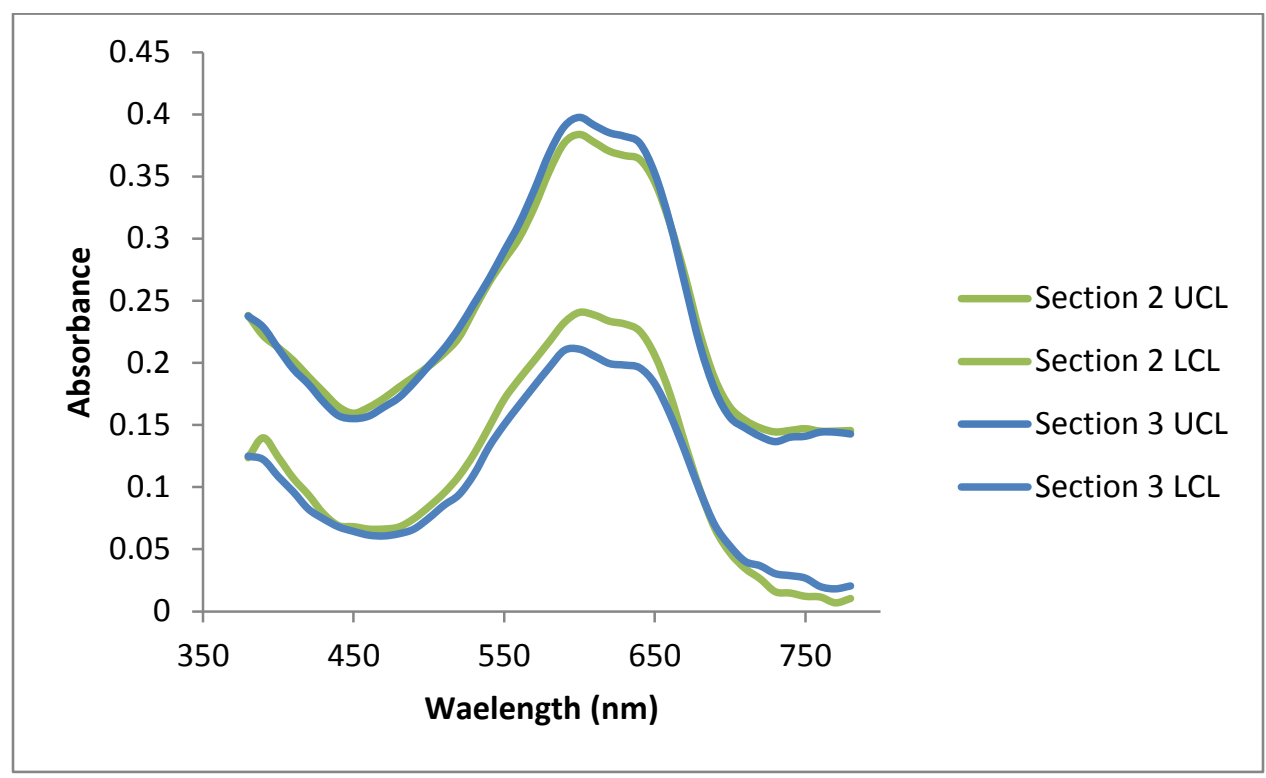

Figure 140: The 95\% UCL and the 95\% LCL of Royal Section 2 and Section 3

All the figures in the results section of Phase IV were generated by a XY conditioning plot using RandFriends® 2.15.2 when configured with RExcel® 3.2.9. Figures 101, 109, 117, 125 and 133 were created for Section 1, 2 and 3 of all five t-shirt colors by using the $\mathrm{x}$ axis as wavelength and the $y$ axis as the absorbance values that were collected for all ten runs. The number of columns was set to 4 and the number of rows was set to 10. Figures 102, 110, 118, 126 and 134 were created for a Section 1, 2 and 3 of all five t-shirt colors by using the $\mathrm{x}$ axis as wavelength and the $y$ axis as the average absorbance values that were calculated. The number of rows set to 10. Figure 103 and Figure 104 were generated to represent the average absorbance, the upper confidence limit and the lower confidence limit for all the absorbance values for front of the Gold I t-shirt and Gold J t-shirt. Figure 111 and Figure 112 were generated to represent the average absorbance, the upper confidence limit and the lower confidence limit for all the absorbance values for front of the Kelly Green $\mathrm{G}$ t-shirt and Kelly Green H t-shirt. Figure 119 and Figure 120 were generated to represent the average absorbance, the upper confidence limit and the lower confidence limit for all the absorbance values for front of the Orange E t-shirt and Orange F t-shirt. Figure 127 and Figure 128 were generated to represent the average absorbance, 
the upper confidence limit and the lower confidence limit for all the absorbance values for front of the Red C t-shirt and Red D t-shirt. Figure 135 and Figure 136 were generated to represent the average absorbance, the upper confidence limit and the lower confidence limit for all the absorbance values for front of the Royal A t-shirt and Royal B t-shirt. Figures 105, 113, 121, 139 and 137 were created to represent the average absorbance, the upper confidence limit and the lower confidence limit for all the absorbance values for the front of all ten shirts for all five colors.

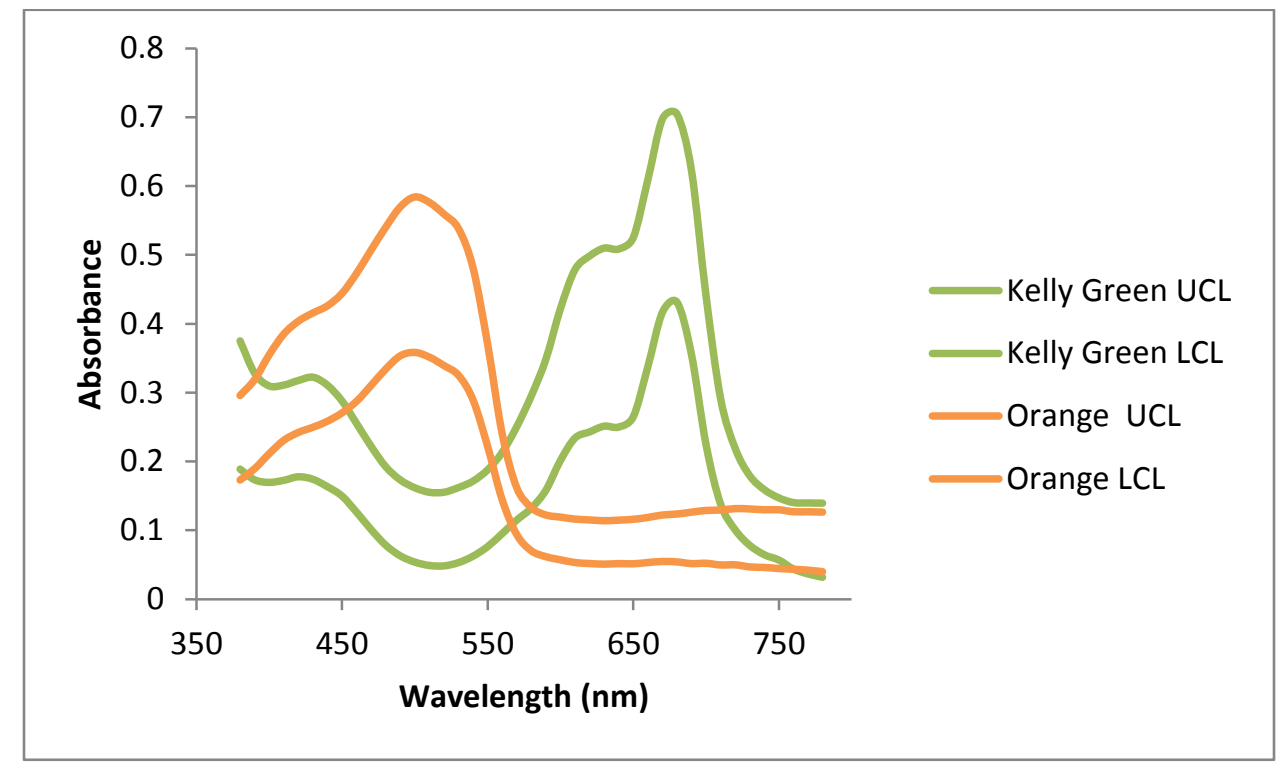

Figure 141: The 95\% UCL and the 95\% LCL at of all Orange and Kelly Green Shirts 


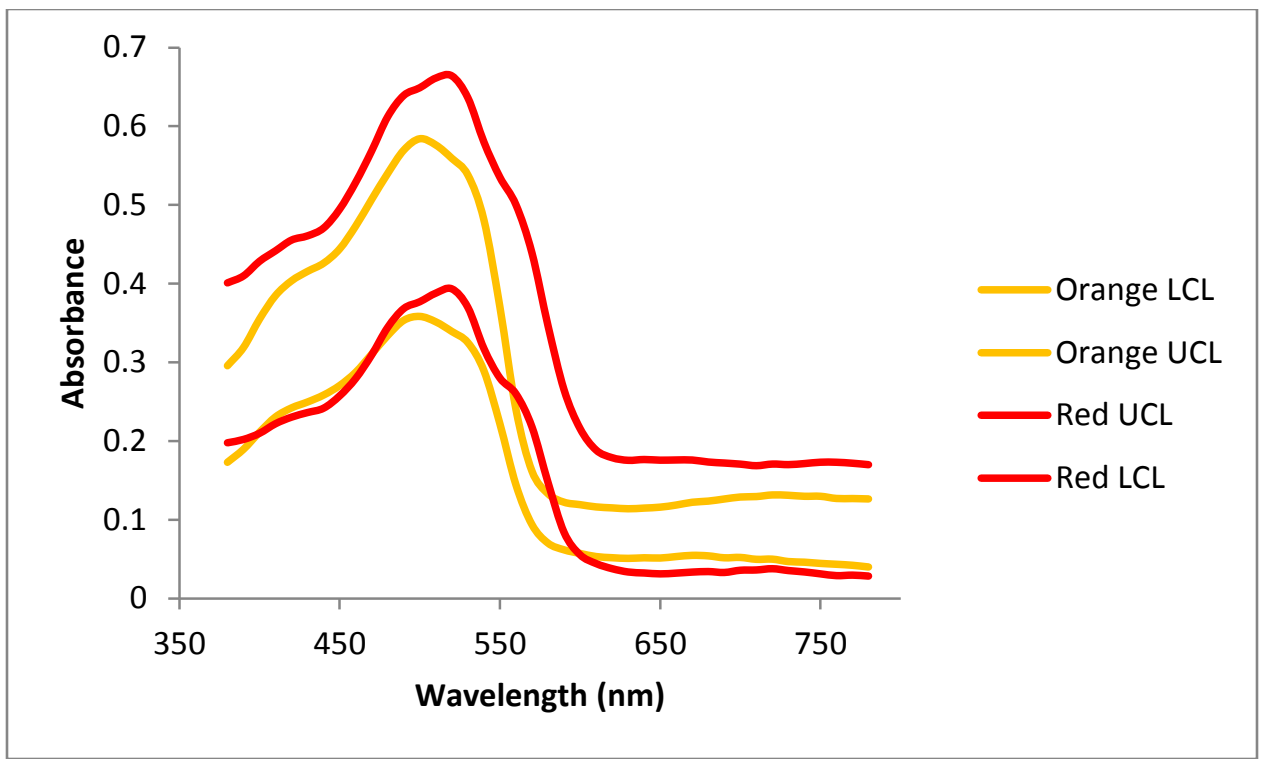

Figure 142: The 95\% UCL and the 95\% LCL of all Orange and Red Shirts

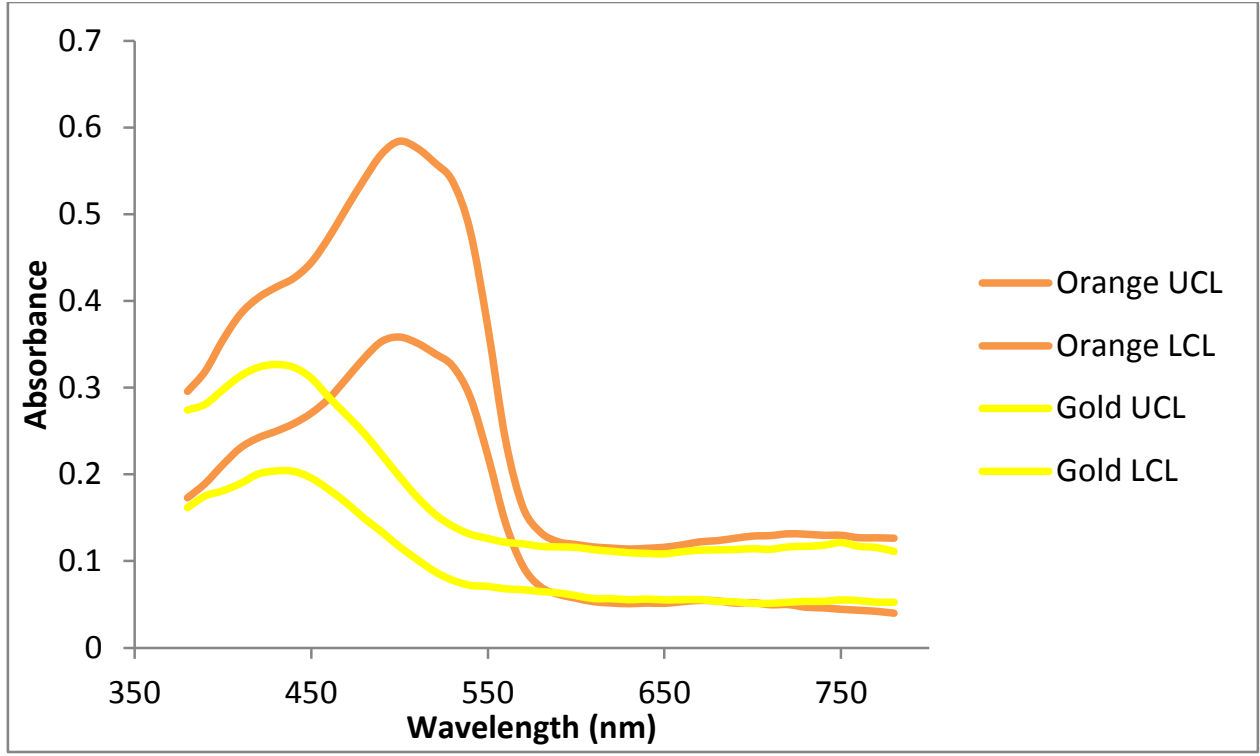

Figure 143: The 95\% UCL and the 95\% LCL of all Orange and Gold Shirts 


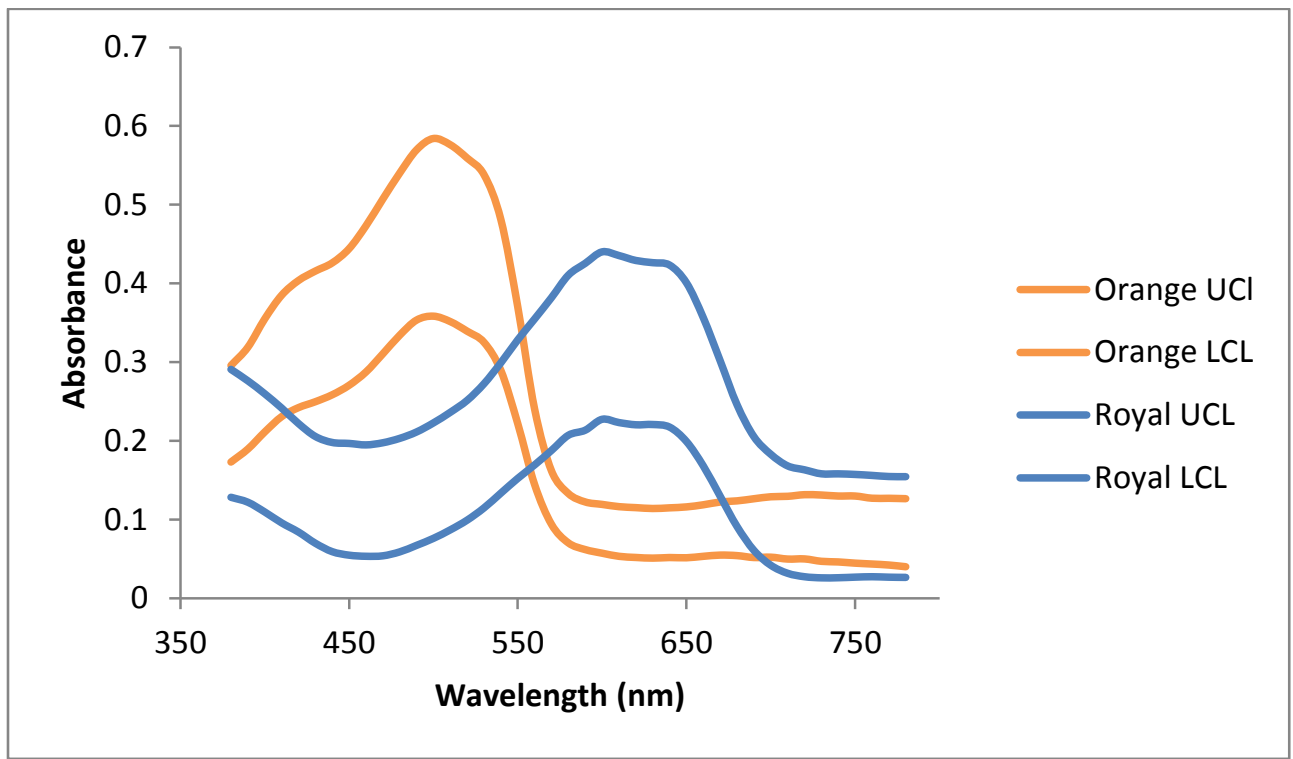

Figure 144: The 95\% UCL and the 95\% LCL of all Orange and Royal Shirts

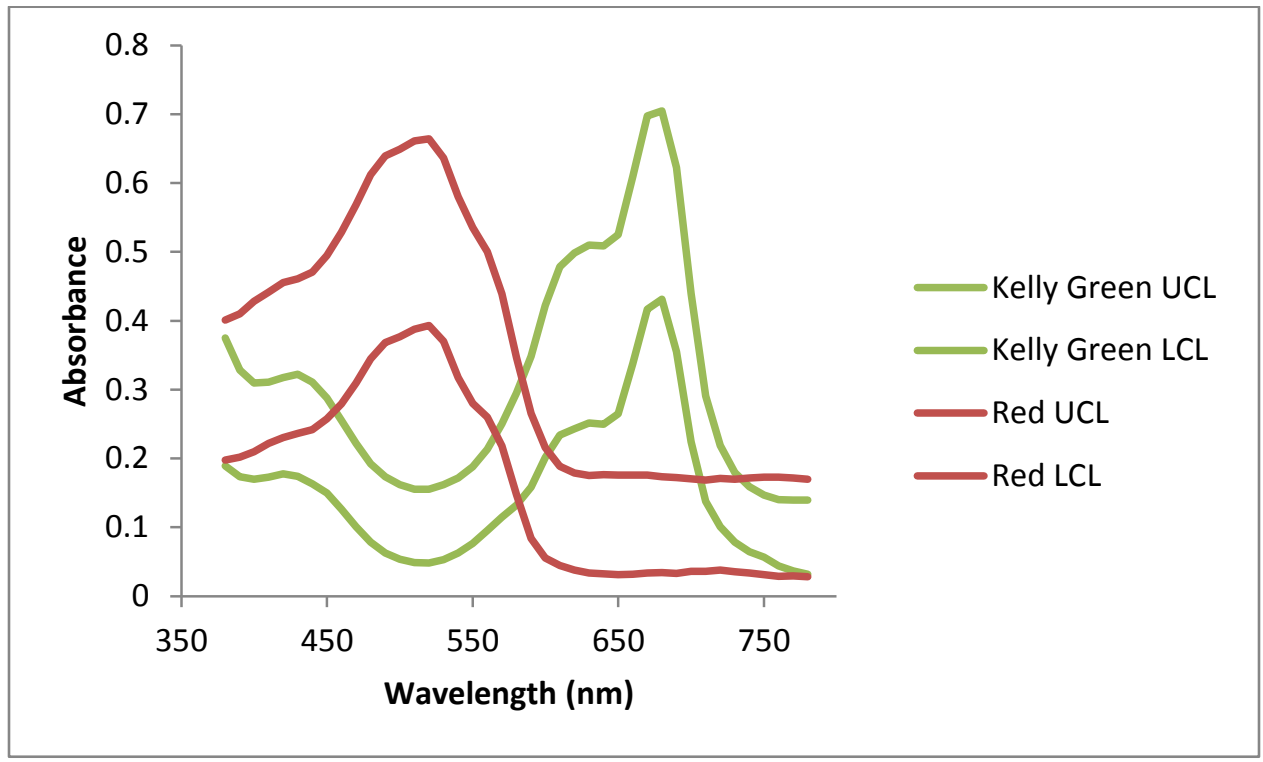

Figure 145: The 95\% UCL and the 95\% LCL of all Kelly Green and Red Shirts 


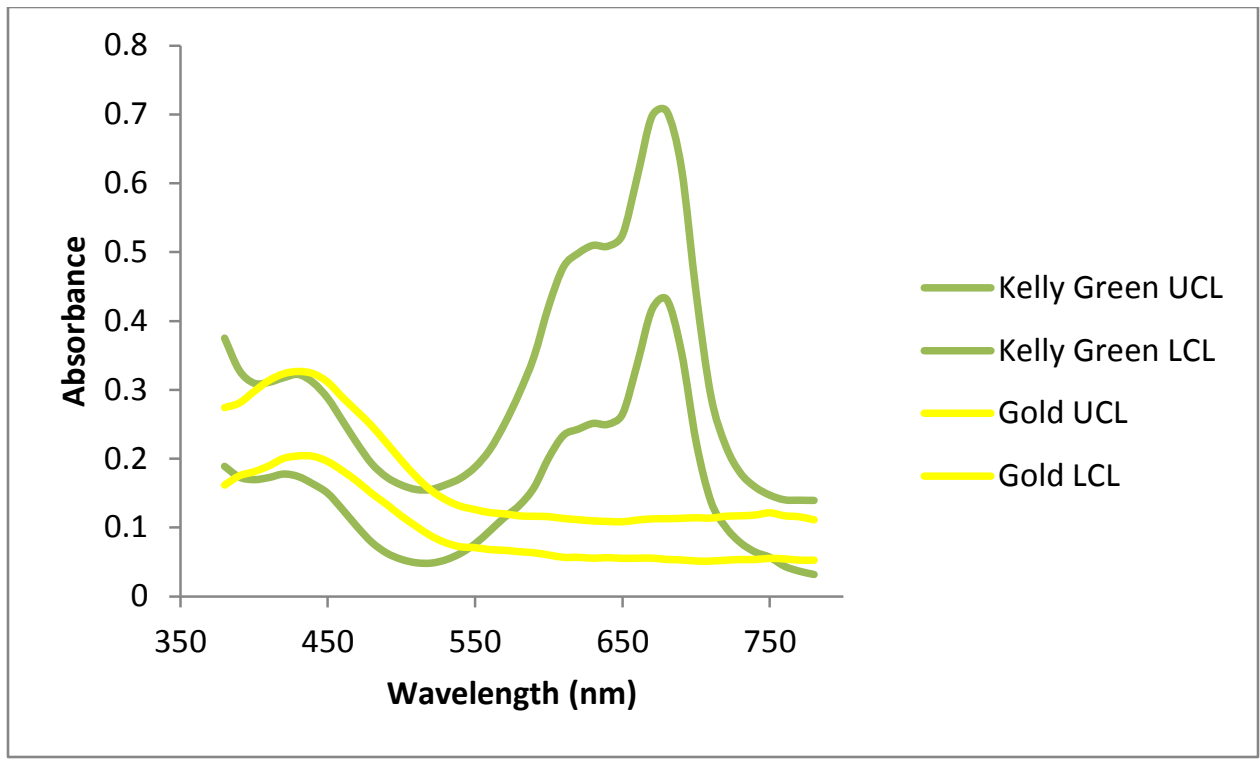

Figure146: The 95\% UCL and the 95\% LCL of all Kelly Green and Gold Shirts

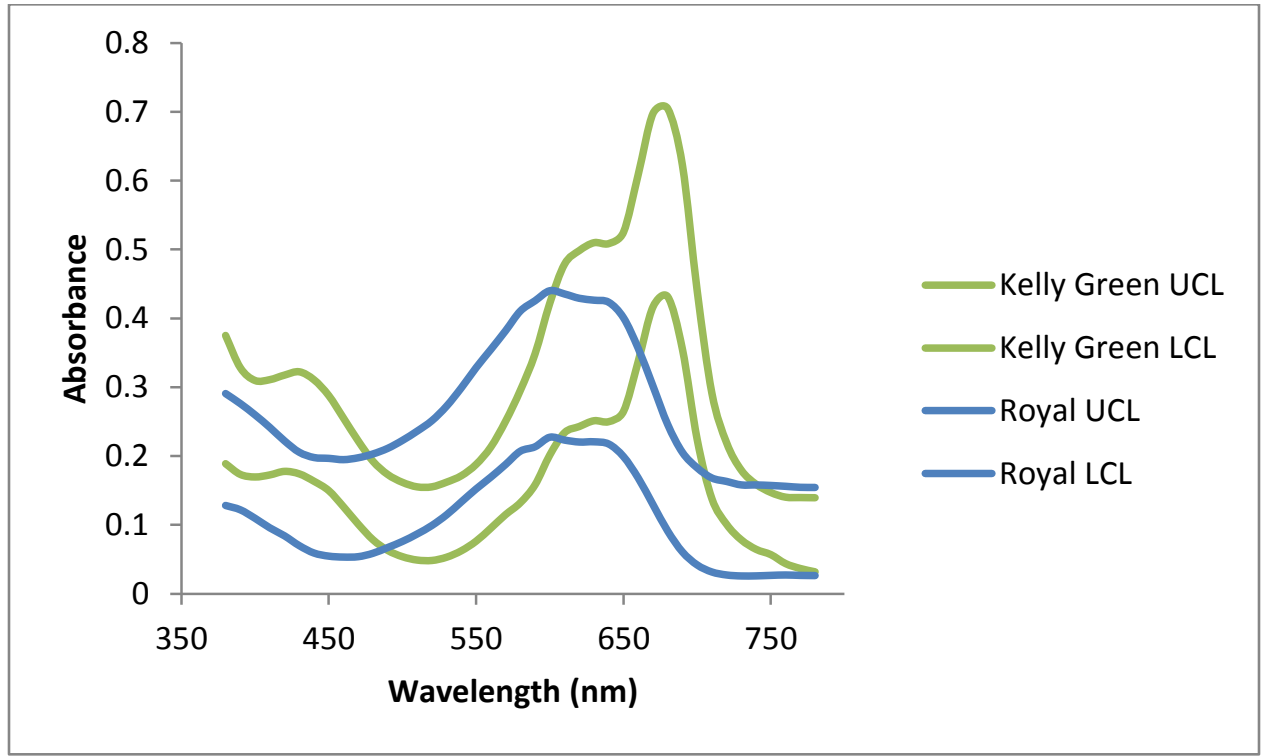

Figure 147: The 95\% UCL and the 95\% LCL of all Kelly Green and Royal Shirts 


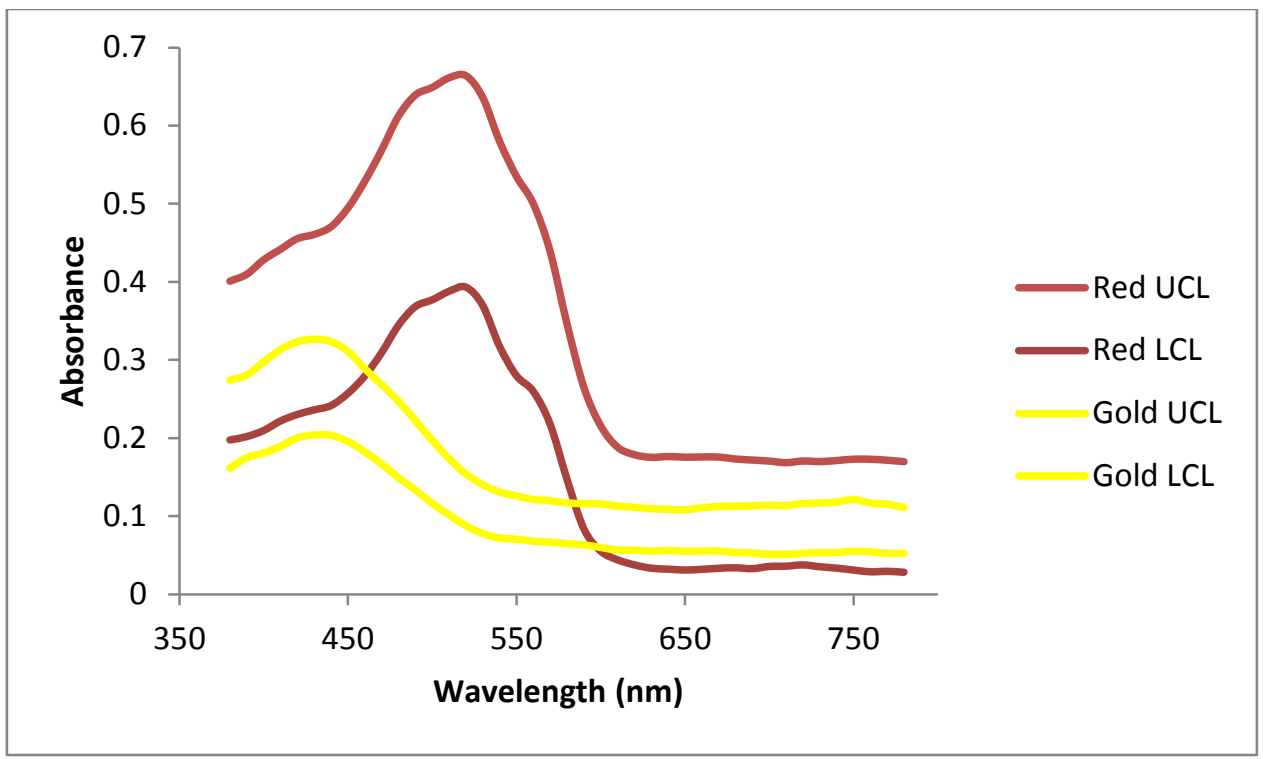

Figure 148: The 95\% UCL and the 95\% LCL of all Red and Gold Shirts

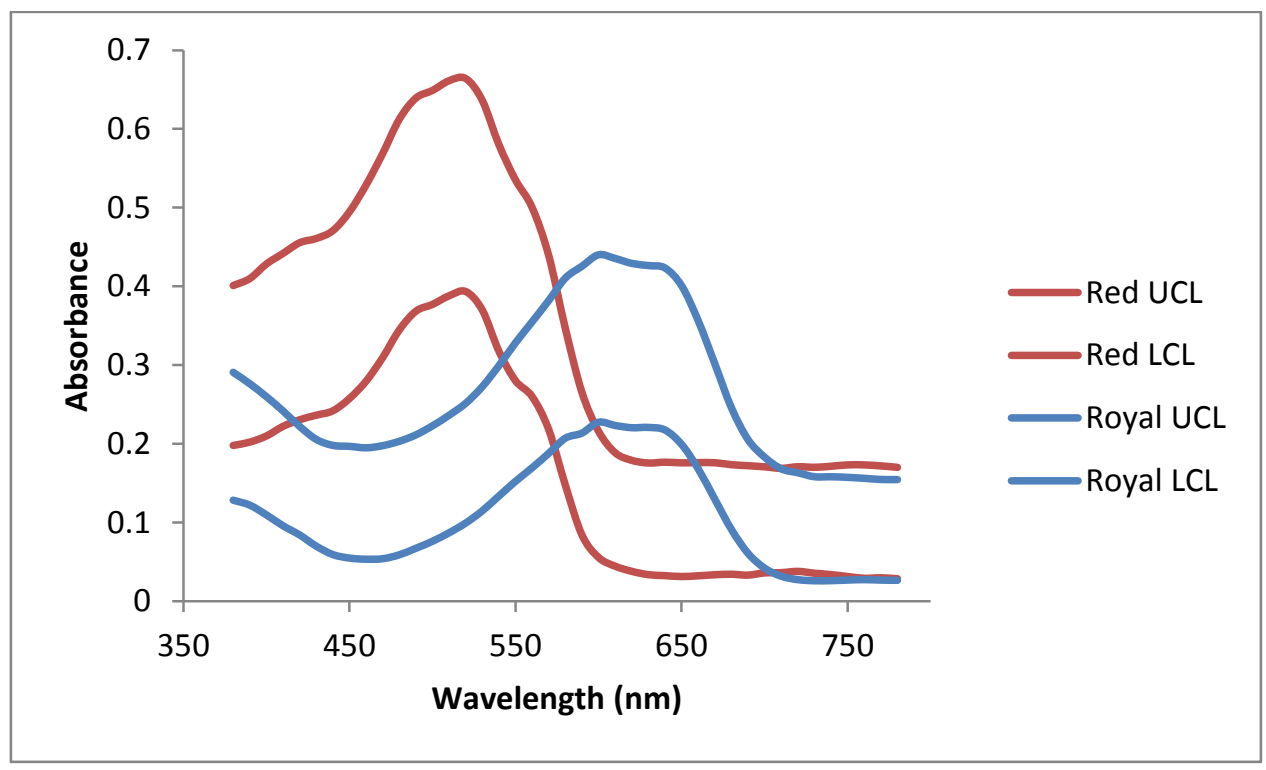

Figure 149: The 95\% UCL and the 95\% LCL of all Red and Royal Shirts 


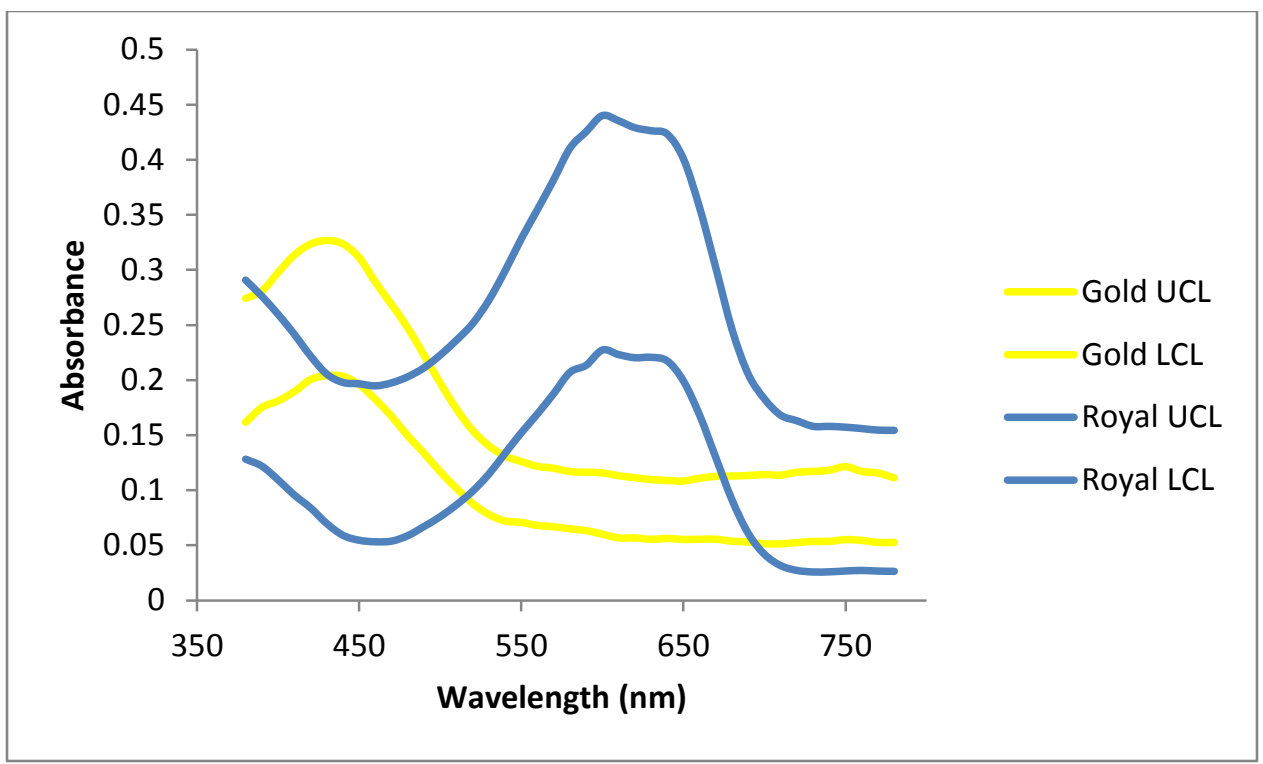

Figure 150: The 95\% UCL and the 95\% LCL of all Royal and Gold Shirts

Figures 141-150 represent the ten possible 95\% UCL and the 95\% LCL comparisons that can be done between all Gold, Kelly Green, Orange, Red and Royal shirts.

\section{IV: $\underline{\text { Discussion }}$}

\section{Phase I}

\section{Gold}

Table 7 shows the minimum and maximum wavelength values and their respective absorbance values for Gold A-Gold J and the Munsell chip that was chosen. When looking at the minimum wavelength values, every value falls outside the range that one would expect to observe for the color yellow or gold, which in the visible light spectrum is from $570 \mathrm{~nm}$ to 585 $\mathrm{nm}$. These minimum wavelength values fall from $620 \mathrm{~nm}$ to $662 \mathrm{~nm}$, which is in the red region of the visible light spectrum. The maximum wavelength values that are shown in Table 7 all fall into the violet range ( $400 \mathrm{~nm}$ to $420 \mathrm{~nm}$ ) and the indigo range (420 nm to $440 \mathrm{~nm}$ ) except for the 
t-shirt square Gold B and the t-shirt square Gold C, which fell just outside the range. Violet is the complementary color to yellow, so one would expect the maximum wavelength values to be in the violet and indigo range of the visible light spectrum.

\section{Kelly Green}

Table 8 shows the minimum and maximum wavelength values and their respective absorbance values for Kelly Green A-Kelly Green J and the Munsell chip that was chosen. When looking at the results in this table, the minimum wavelength values are within the range of the color green in the visible light spectrum for both the Munsell chip and the t-shirt squares, which is from $490 \mathrm{~nm}$ to $570 \mathrm{~nm}$. The maximum wavelength values that were recorded are within the red range of the visible light spectrum. Being that red is the complementary color to green these values would be expected to be within that range. There was a large differentiation observed in the maximum wavelength value of the Munsell chip 7.5G 4, 8 and that of the t-shirt squares, but the maximum wavelength value of $718 \mathrm{~nm}$ that was recorded is still in the red range of the visible light spectrum.

\section{Orange}

Table 9 shows the minimum and maximum wavelength values and their respective absorbance values for Orange A-Orange $J$ and the Munsell chip that was chosen. When looking at the results in this table, the minimum wavelength values for all ten t-shirt squares are in the orange region of the visible light spectrum, which is from $585 \mathrm{~nm}$ to $620 \mathrm{~nm}$, but the Munsell chip was not. The Munsell chip instead fell into the yellow region of the visible light spectrum with a minimum wavelength value of $580 \mathrm{~nm}$. Blue is the complementary color to orange, so it

expected that the maximum wavelength values of $t$-shirt squares would fall into that range of 440 $\mathrm{nm}$ to $490 \mathrm{~nm}$ in the visible light spectrum because all the minimum wavelength values that were recorded are in the orange region. However, as shown in Table 9 the values fall into the green range of the visible light spectrum (490 $\mathrm{nm}$ to $570 \mathrm{~nm}$ ) instead.

\section{Red}

Table 10 shows the minimum and maximum wavelength values and their respective absorbance values for Red A-Red J and the Munsell chip that was chosen. When looking at the 
results in this table, the wavelength values for the t-shirt squares and the Munsell chip are in the red region of the visible light spectrum, which is from $620 \mathrm{~nm}$ to $780 \mathrm{~nm}$. There was no more than a $5 \mathrm{~nm}$ difference between them. The maximum wavelength values that were recorded are within the green range of the visible light spectrum. One would expect these values to fall in this region being that green is the complementary color to red. There was a variation observed between the maximum wavelength value of the Munsell chip 5R 4, 14 and the ten t-shirt squares, but the value of $545 \mathrm{~nm}$ that was recorded is still the green range of the visible light spectrum.

\section{Royal}

Table 11 shows the minimum and maximum wavelength values and their respective absorbance values for Royal A-Royal $\mathrm{J}$ and the Munsell chip that was chosen. When looking at the results in this table, the minimum wavelength values for the $\mathrm{t}$-shirt squares and the Munsell chip are in the blue region of the visible light spectrum, which is from $440 \mathrm{~nm}$ to $490 \mathrm{~nm}$. The maximum wavelength values that were recorded fall within orange range of the visible light spectrum. Being that orange is the complementary color to blue is it expected that these values to fall in this region. There was a variation observed in the maximum wavelength value that was recorded for Royal $\mathrm{G}$ and the maximum values that were recorded for all the other $\mathrm{t}$-shirt squares and the Munsell chip. The maximum value that was recorded for Royal G was $627 \mathrm{~nm}$ is higher than all the other maximum values, but is still in the orange range in the visible light spectrum.

It can be noticed in the figures where the spectrum of the Munsell chip for each color and the ten t-shirts for every color are displayed that the spectra that was recorded for the Munsell Chip are different from the spectra of the t-shirts squares. This variation could be due to the cardboard surface that the Munsell chips are placed on in the Munsell Book of Color. A linear baseline correction was completed to get rid of the negative absorbance values that were recorded for gold, orange, red and royal. The negative absorbance values recorded can be explained due to the instrumental variations that can occur. Being that the CRAIC ${ }^{\circ}$ QDS QDI ${ }^{\mathrm{TM}}$ II was properly calibrated with the NIST® Traceable standards these negative absorbance values were corrected using the linear baseline correction method. 


\section{Phase II}

The absorbance measurements that are recorded in this phase of the experiment are subject to "noise" which can affect the reproducibility of the measurements, which is also known as the precision. When collecting spectra using the QDI 1000 Microspectrophotometer, it is important to have a good signal to noise ratio. The software that is used to collect and display the spectra can help to obtain a better signal to noise ratio. The software allows the user to change and set three different settings; the average scans, the boxcar width and the integration time. To further analysis the best average scans, the boxcar width and the integration times when colleting spectra on the QDI 1000 Microspectrophotometer the least squares model was used because it can help to minimize the sum of the squares of the errors that may have occurred in the experiment. This method was done by computing the sum of the squares (SSR) and then minimizing the SSR by adjusting $\mathrm{k}$ by using the solver add in on Microsoft@ Excel. If the model is used correctly and the noise is not too high, a set of coefficients can be found that closely fit the data.

\section{Average Scans}

When there is a higher number of average scans, the better the signal to noise ratio will be. The number of average scans is the number of spectral acquisitions that accumulate before the software receives the scan. In Figure 23, the absorbance spectra are displayed that were collected from varying the average scans of 10, 15 and 20 from $400 \mathrm{~nm}$ to $700 \mathrm{~nm}$. The spectrum that was obtained when the average scans were set to 20 displays less noise than when the average scan was set to 10 and 15 . The results obtained when the least squares method was applied to the absorbance values when varying the average scan values are displayed in Figure 24 and Figure 25. The absorbance values that were obtained when the least squares calculation was applied to the average scans are displayed in Figure 24. By using this method to minimize the absorbance values so that they are as close to zero as possible, it was determined that the average scans of 2,10,15 and 30 presented the highest variability in the absorbance values. This variation was observed between the wavelengths of $400 \mathrm{~nm}$ to $450 \mathrm{~nm}$ and $650 \mathrm{~nm}$ to $700 \mathrm{~nm}$. It was concluded that average scans of 5,20 and 50 displayed the most minimal absorbance values 
when this method was applied. For further analysis the sum of the squares of each average scan variation are displayed in Figure 25. The average scans of 5, 20 and 50 displayed the lowest sum of the squares values when using this method as well. The sum of the squares for the average scan of 5 was 0.212 , the sum of the squares for the average scan 20 was 0.228 and the sum of the squares for the average scan 50 was 0.116 . The assumption had already been made that the higher the average scans, the better the signal to noise ratio. With this being said, it was determined that the average scan of 5 would not be appropriate average scan value to use when collecting spectra. To determine if the average scan value of 20 or 50 should be used, the spectra that were collected from the QDI 1000 Microspectrophotometer were analyzed. The boxcar width was kept constant at 5 and the integration time was kept constant at $100 \mathrm{~ms}$ when collecting the spectra.

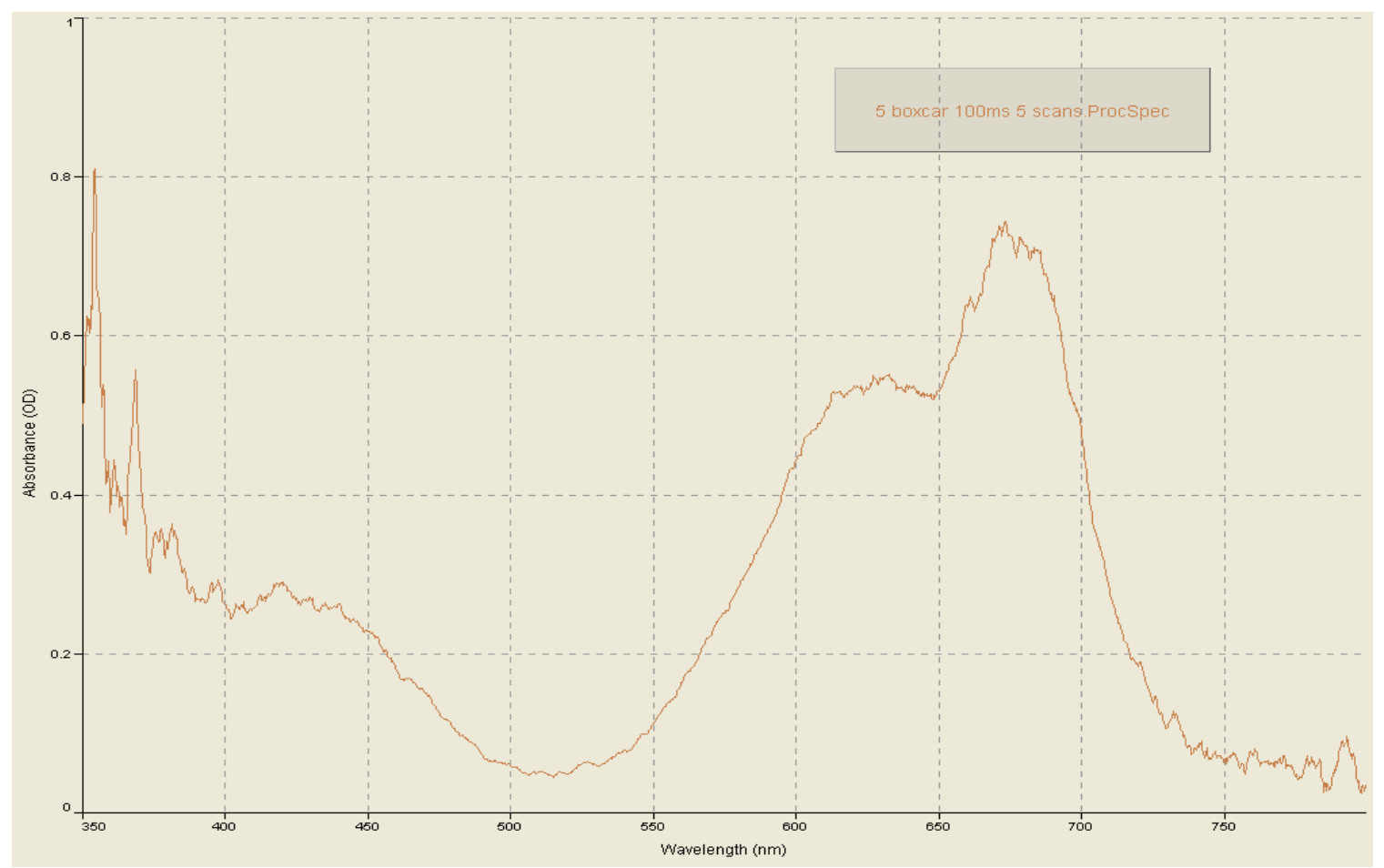

Figure151: Absorbance spectrum of Kelly Green A_F using average scan of 5 


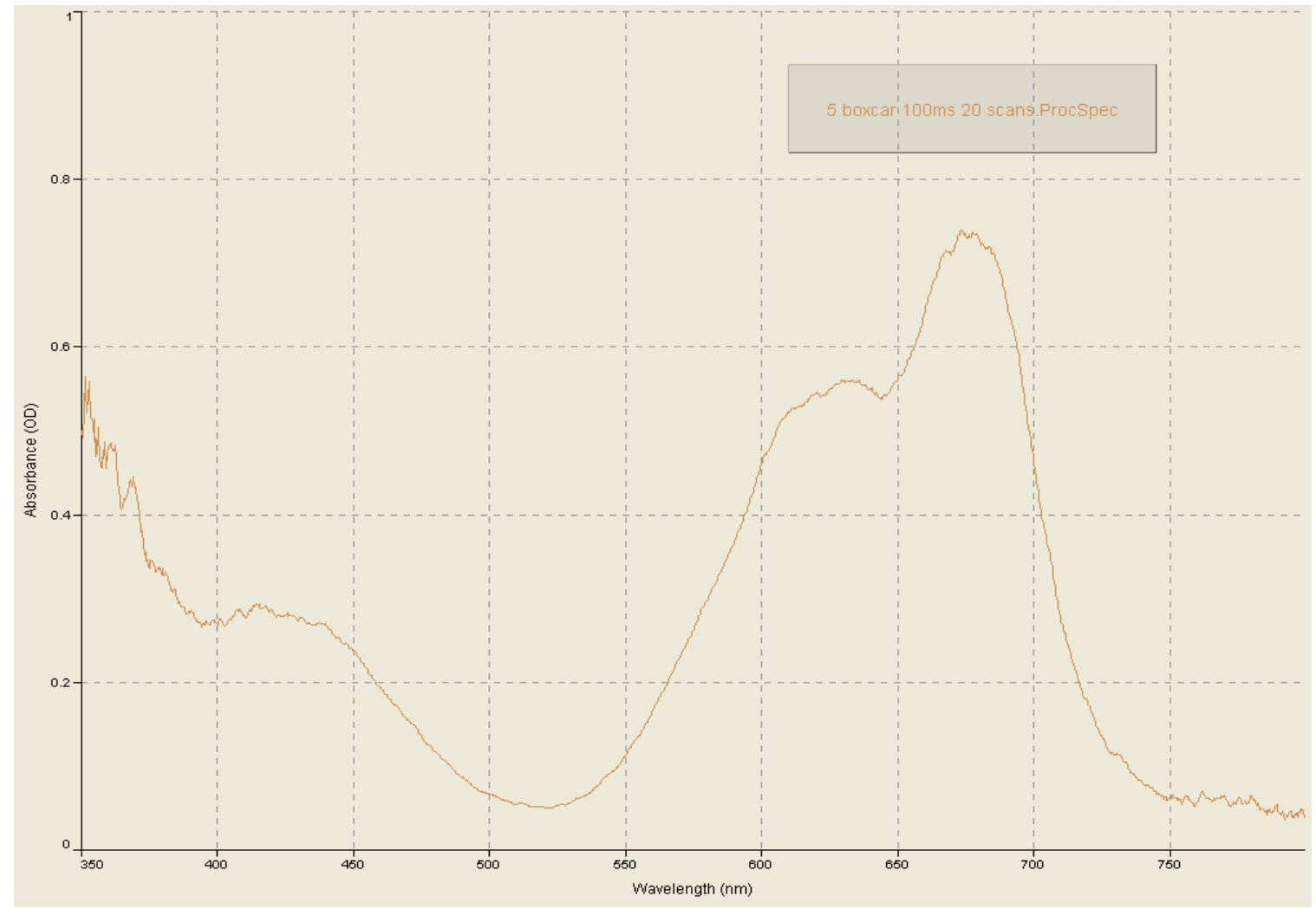

Figure 152: Absorbance spectrum of Kelly Green A_F using average scan of 20

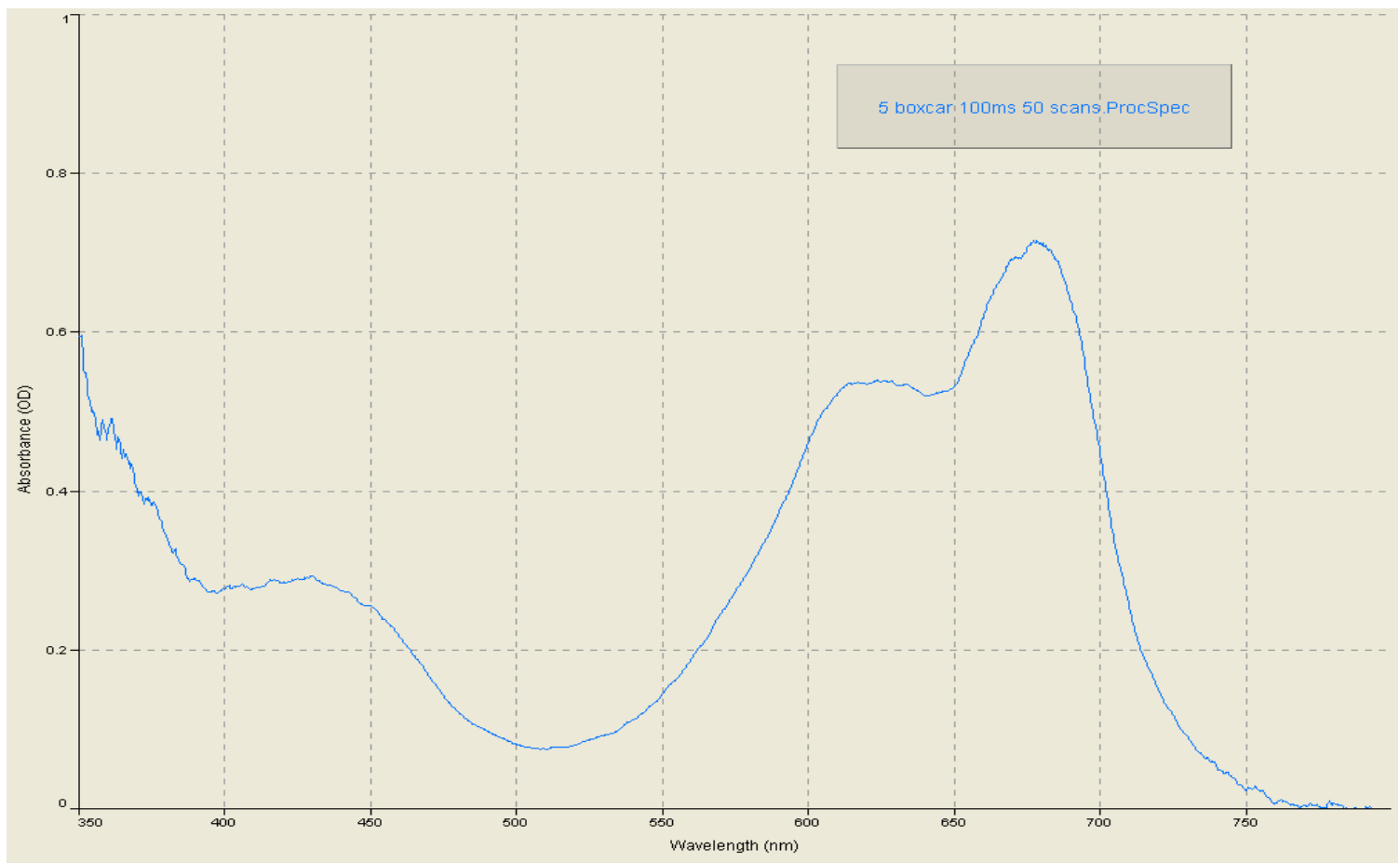

Figure 153: Absorbance spectrum of Kelly Green A_F using average scan of 50 
The absorbance spectrum that was collected when the average scan was set to 20 is displayed in Figure 107 and the absorbance spectrum that was collected when the average scan was set to 50 is displayed in Figure 108. It was determined that when collecting spectra using the QDI 1000 Microspectrophotometer, an average scan value of 20 should be used when collecting spectra.

\section{Boxcar Width}

The boxcar width parameter in the Ocean Optics ${ }^{\circledR}$ software is used as a smoothing to tool for the spectrum that are collected to help improve the signal to noise ratio. If the boxcar width is set to high, the spectrum will lose information, the signal to noise ratio will be effected and a loss of spectral resolution will be observed. In Figure 27, the absorbance spectra are displayed that were collected from varying the boxcar width of 1,3 and 5 from $400 \mathrm{~nm}$ to $700 \mathrm{~nm}$. The spectrum that was obtained when the average scans were set to 5 displays less noise than when the average scan was set to 1 and 3 . The results obtained when the least squares method was applied to the absorbance values when varying the boxcar width values are displayed in Figure 28 and Figure 29. The absorbance values that were obtained when the least squares calculation was applied to the boxcar width are displayed in Figure 28. By using this method is to minimize the absorbance values so that they are as close to zero as possible, it was determined that the boxcar width of 1 and 3 of presented the highest variability in the absorbance values. It was concluded that the boxcar width of 5, 9, 25 and 50 displayed the most minimal absorbance values when the sum of the squares method was applied in Figure 29. The sum for boxcar width 5 was 18.68, the sum for boxcar width 9 was 16.71, the sum for boxcar width of 25 was 15.80 and the sum for boxcar width 50 was 4.44 . The assumption had already been made that if the boxcar width was set too high, a loss of spectral resolution will be observed. It was concluded that even though a boxcar width of 50 has the lowest sum of squares value, it would not be an ideal boxcar width to use when collecting spectra. It was determined that the boxcar width of 5 should be used when collecting spectra by analyzing the spectra that were collected from the QDI 1000 Microspectrophotometer, which are shown in Figure 109-111. The average scans were kept constant at 20 based on the results of the previous section and the integration time was kept constant at $100 \mathrm{~ms}$ when collecting the spectra. 


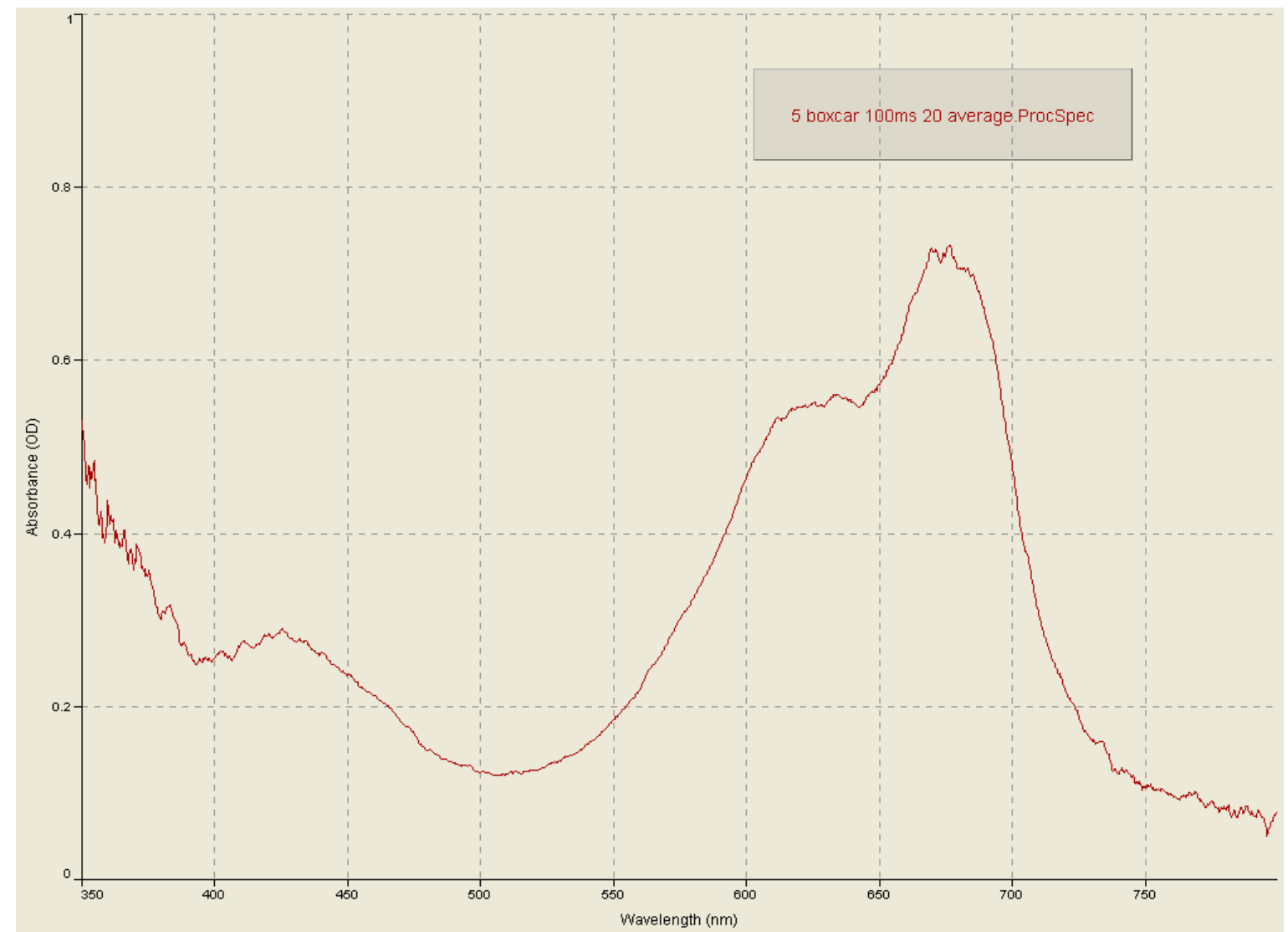

Figure 154: Absorbance spectrum of Kelly Green A_F using boxcar width of 5

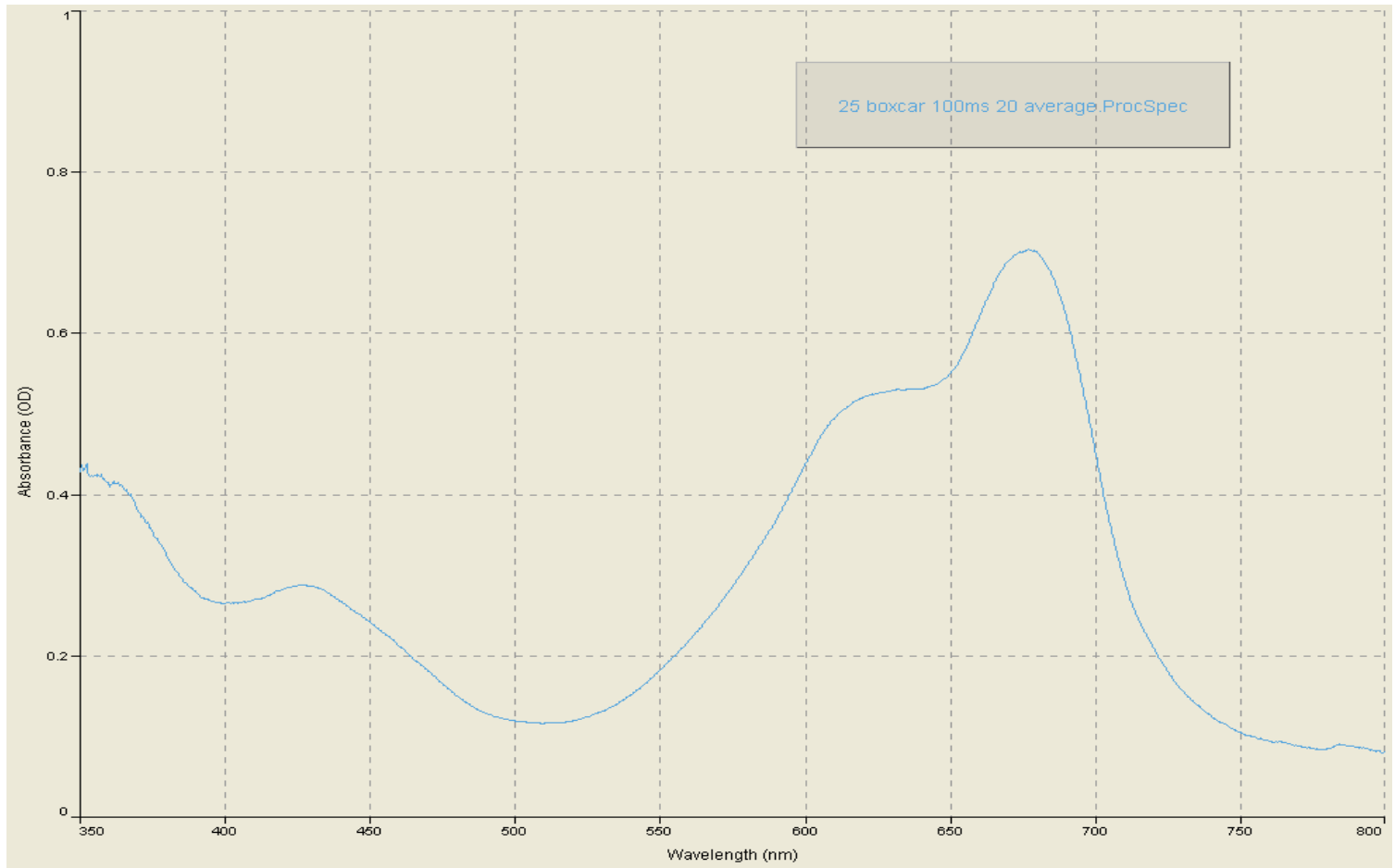

Figure 155: Absorbance spectrum of Kelly Green A_F using boxcar width of 25 


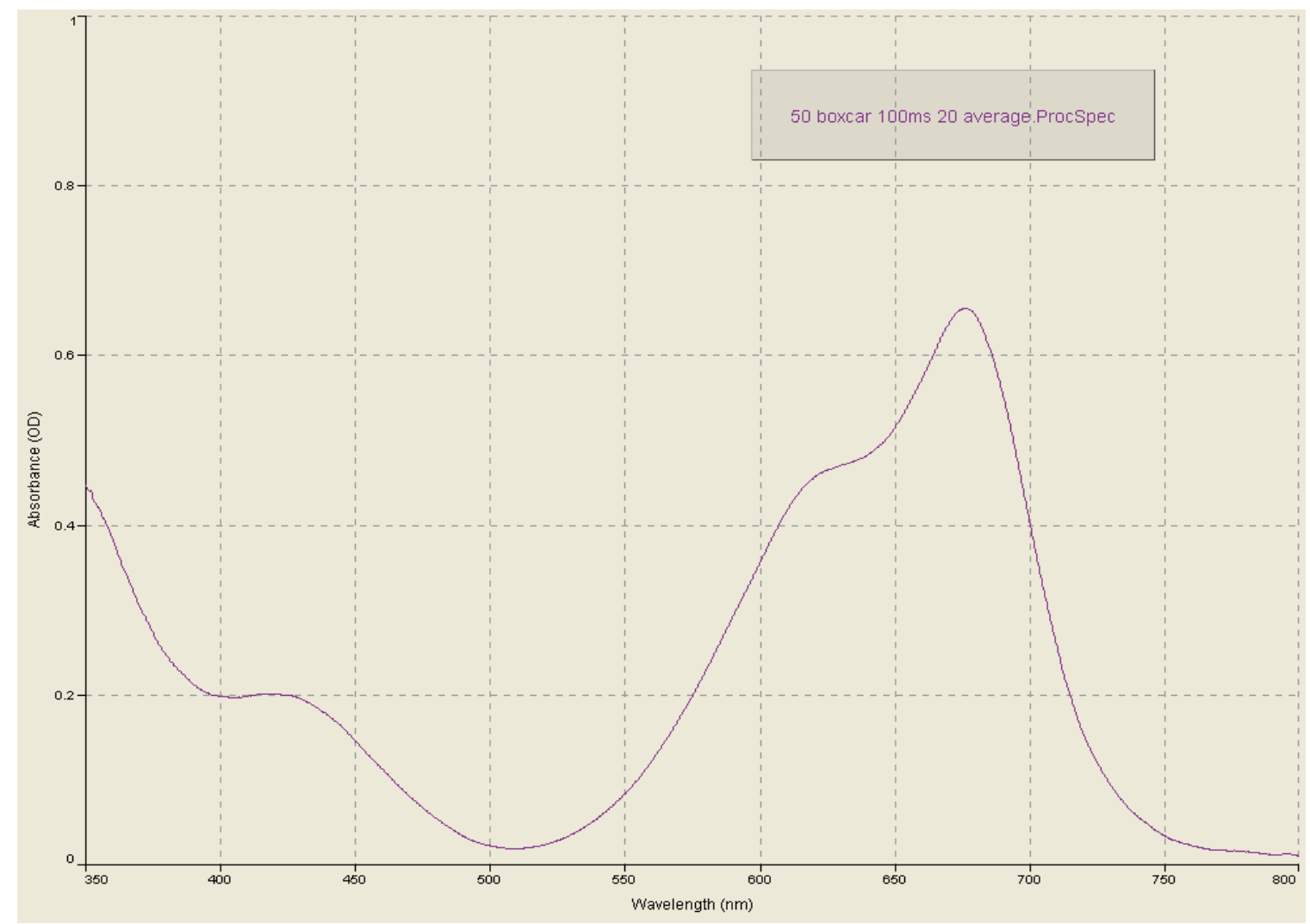

Figure 156: Absorbance spectrum of Kelly Green A_F using boxcar width of 50

\section{Integration Time}

When the integration time is set to a high value, the better the signal to noise ratio will be. A higher integration time means that the detector is monitoring for a longer period of time. In Figure 31, the absorbance spectra are displayed that were collected from varying the integration times of $20 \mathrm{~ms}, 30 \mathrm{~ms}, 50 \mathrm{~ms}$ and $100 \mathrm{~ms}$ from $400 \mathrm{~nm}$ to $700 \mathrm{~nm}$. The spectrum that was obtained when the integration time was set to $100 \mathrm{~ms}$ displayed less noise than when the integration time was set to $10 \mathrm{~ms}$ and $15 \mathrm{~ms}$. The results obtained when the least squares method was applied to the absorbance values when varying the average scan values are displayed in Figure 32 and Figure 33. The absorbance values that were obtained when the least squares calculation was applied to the integration time are displayed in Figure 32. By using this method is to minimize the absorbance values so that they are as close to zero as possible, it was 
determined that the integration time of $10 \mathrm{~ms}, 30 \mathrm{~ms}, 50 \mathrm{~ms}, 200 \mathrm{~ms}, 300 \mathrm{~ms}$ and $500 \mathrm{~ms}$ presented the highest variability in the absorbance values. It was concluded that integration time of $20 \mathrm{~ms}$ and $100 \mathrm{~ms}$ displayed the most minimal absorbance values when this method was applied. For further analysis, the sums of the squares of each average scan variation are displayed in Figure 33. The integration time of $20 \mathrm{~ms}$ and $100 \mathrm{~ms}$ displayed the lowest sum of the squares values when using this method as well. The sum of the squares for the integration time of $20 \mathrm{~ms}$ was 0.096 the sum of the squares for the average scan $100 \mathrm{~ms}$ was 4.99 . The assumption had already been made that the higher the integration time, the better the signal to noise ratio. With this being said, it was determined that the integration time of $20 \mathrm{~ms}$ would not be appropriate integration time value to use when collecting spectra even though it had a lesser sum of the squares value. An integration time of $100 \mathrm{~ms}$ would instead be the appropriate setting to use when collecting spectra. The spectra that were collected from the QDI 1000 Microspectrophotometer are shown in Figure 112 and Figure 113. With the results of the previous sections, the boxcar width was kept constant at 5 and the average scans were kept constant at 20 when collecting the spectra. 


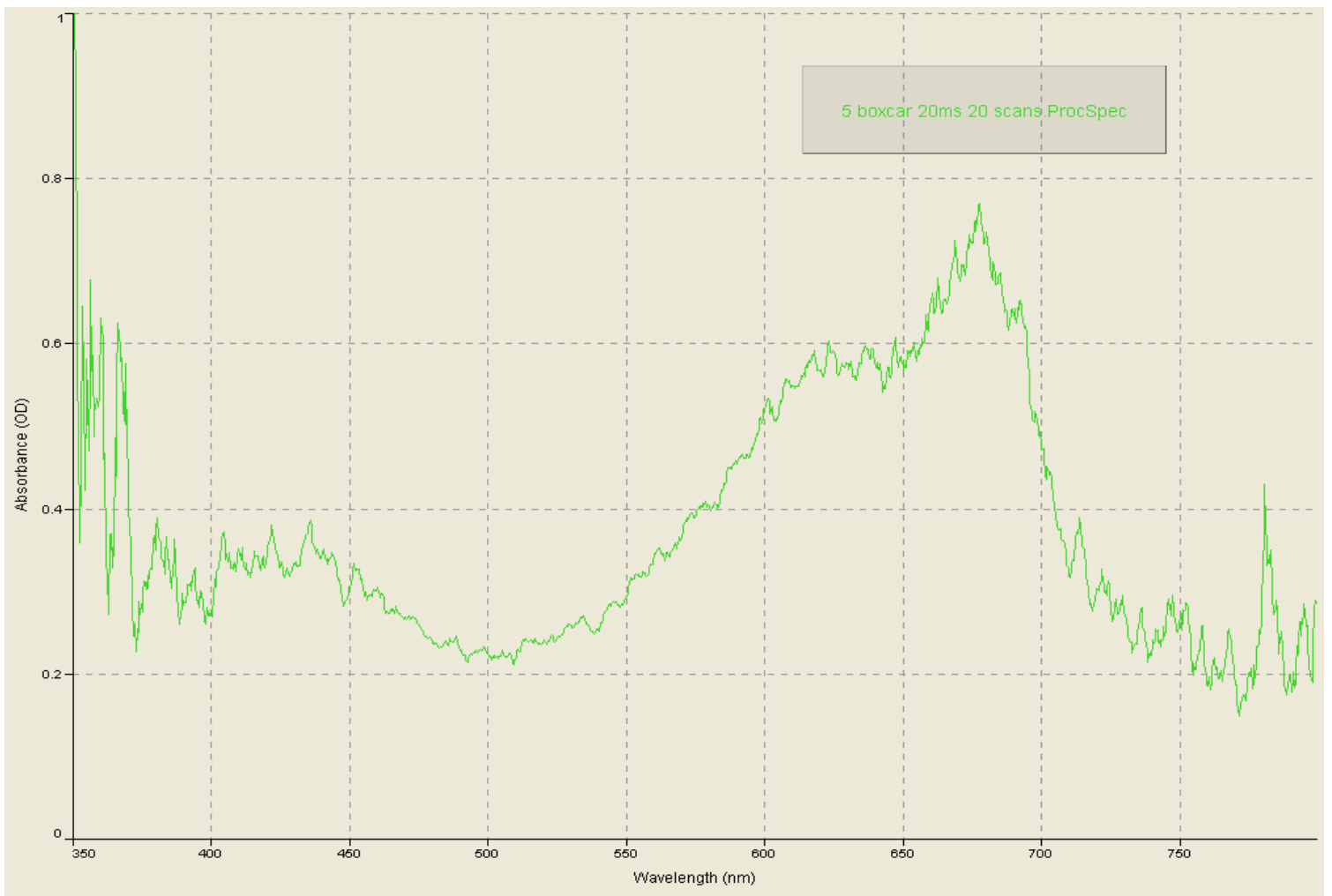

Figure 157: Absorbance spectrum of Kelly Green A_F using integration time of $20 \mathrm{~ms}$

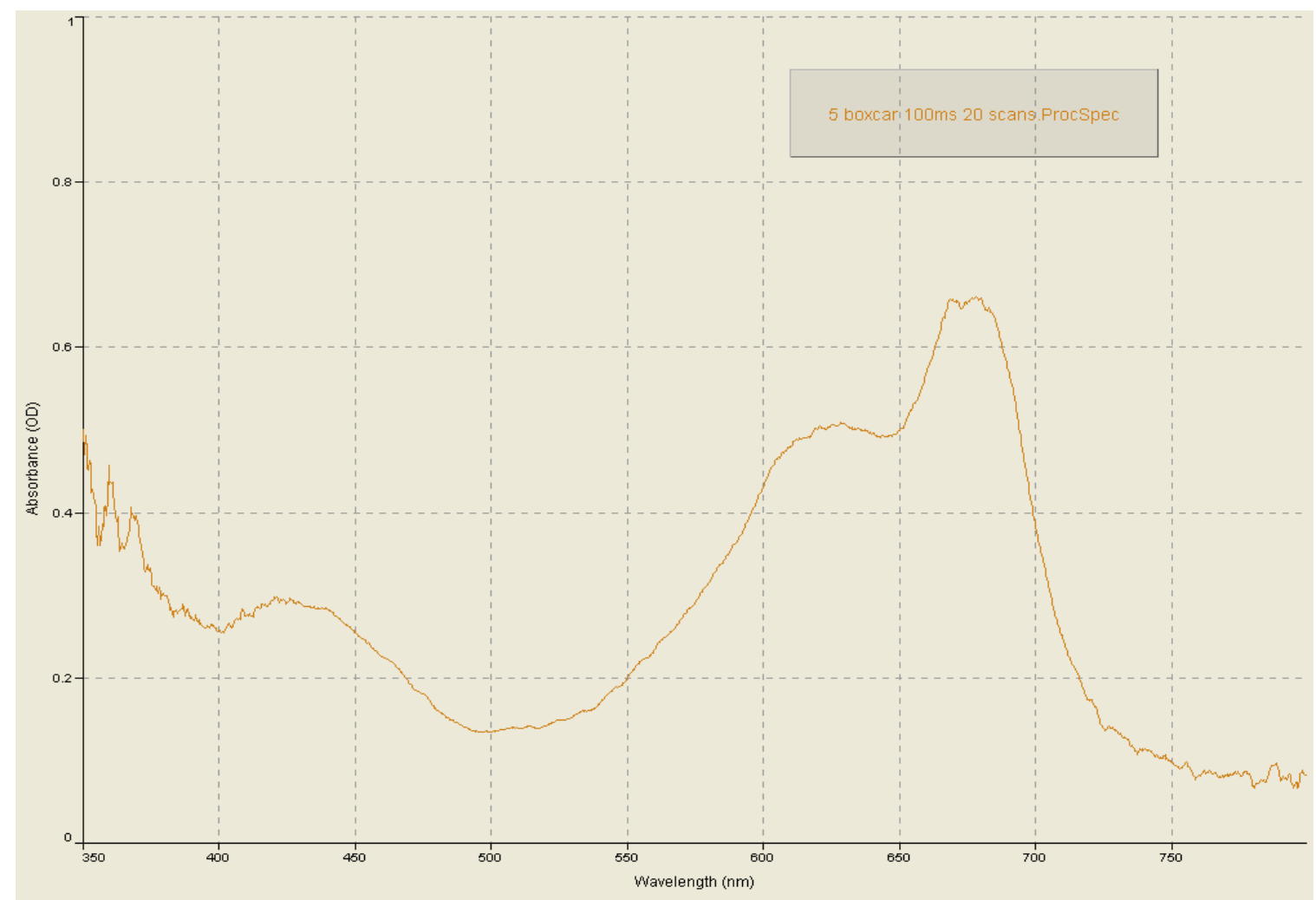

Figure 158: Absorbance spectrum of Kelly Green A_F using integration time of $100 \mathrm{~ms}$ 


\section{Phase III}

For this phase of the experiment, a time trial was completed to determine exactly how long the QDI 1000 Microspectrophotometer's 75W Xenon lamp source needed to warm up before use to obtain the best possible spectra. To help reduce the amount of noise in a spectrum, the parameters of average scans, boxcar width and integration time were determined in the previous phase, but it is also important to determine how much time the Xenon lamp and the detector needs to warm up to reach a constant operating temperature. A temperature change is another setting that can also affect the noise that may be present when a spectrum is being collected.

\section{Part 1}

In Part 1 of the phase, a sample spectrum was collected of Kelly Green A_F every minute for an hour using a wavelength from $380 \mathrm{~nm}$ to $700 \mathrm{~nm}$. A dark and reference scan was only collected before the first sample spectrum was collected. Figure 34 through Figure 38 displays the absorbance values that were obtained when certain wavelengths in the UV and Visible region were chosen. For every wavelength that was chosen $(425 \mathrm{~nm}, 520 \mathrm{~nm}, 575 \mathrm{~nm}, 620 \mathrm{~nm}$ and 700 $\mathrm{nm}$ ) after three minutes, there was little variation between the absorbance values that were recorded. At $425 \mathrm{~nm}$, the absorbance values after three minutes were between 0.347 and 0.394 . At $520 \mathrm{~nm}$, the absorbance values after three minutes were between 0.172 and 0.203 . At $625 \mathrm{~nm}$, the absorbance values after three minutes were between 0.579 and 0.597 . At $675 \mathrm{~nm}$, the absorbance values after three minutes were between 0.705 and 0.766 . At $700 \mathrm{~nm}$, the absorbance values after three minutes were between 0.495 and 0.541. In Figure 39, the absorbance spectra were plotted every ten minutes starting at one minute. The spectra that were collected after ten minutes had little differentiation between them. From these results, it can be conclude that the warm up time for the instrument is not as significant and influential on the spectra that were collected when only collecting a dark and reference scan before the first sample spectrum was collected. For this reason, part two of the phase was done to see the dark scan and reference scan affected the spectra over the span of an hour. 


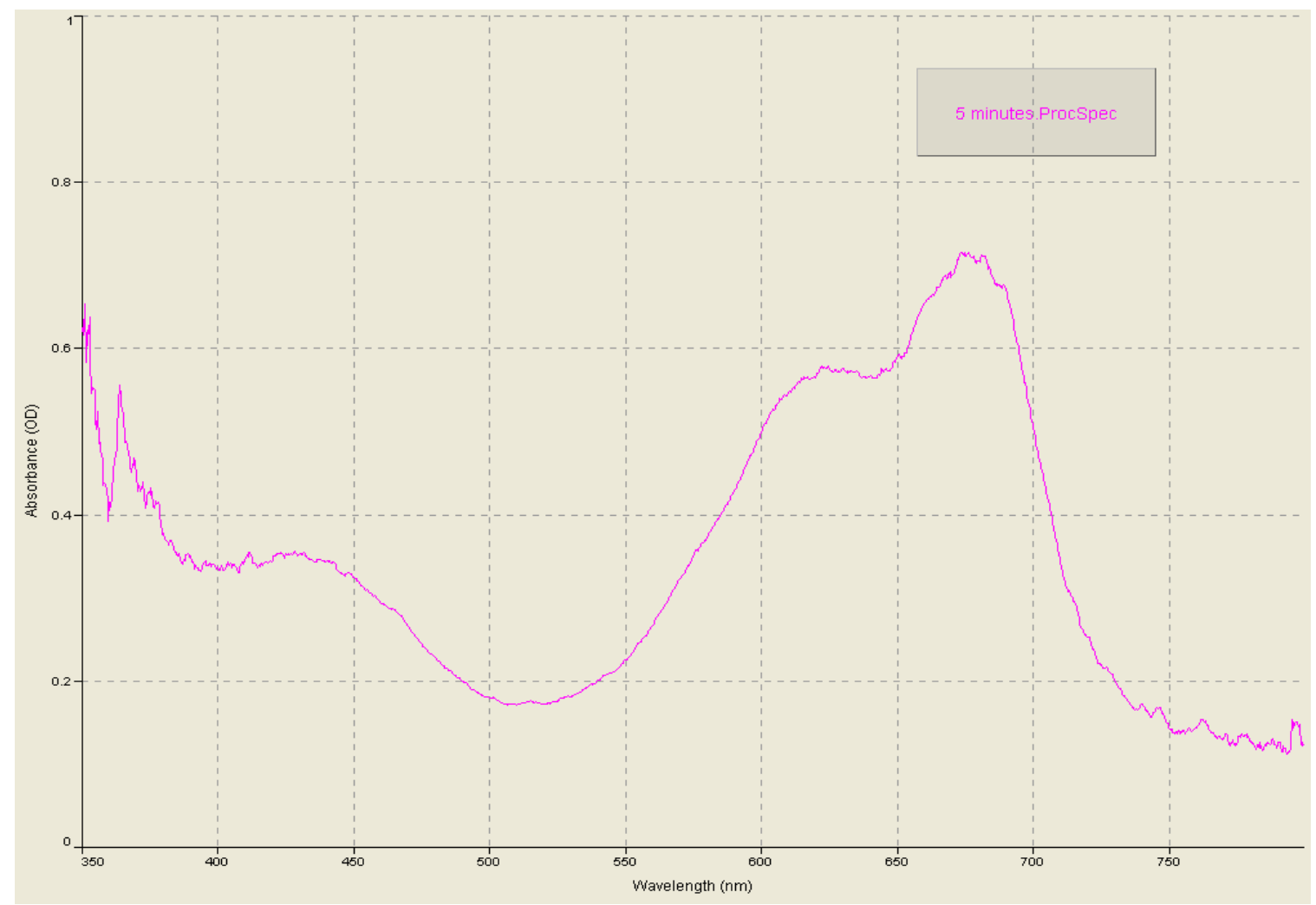

Figure 159: Absorbance spectrum of Kelly Green A_F after 5 minutes

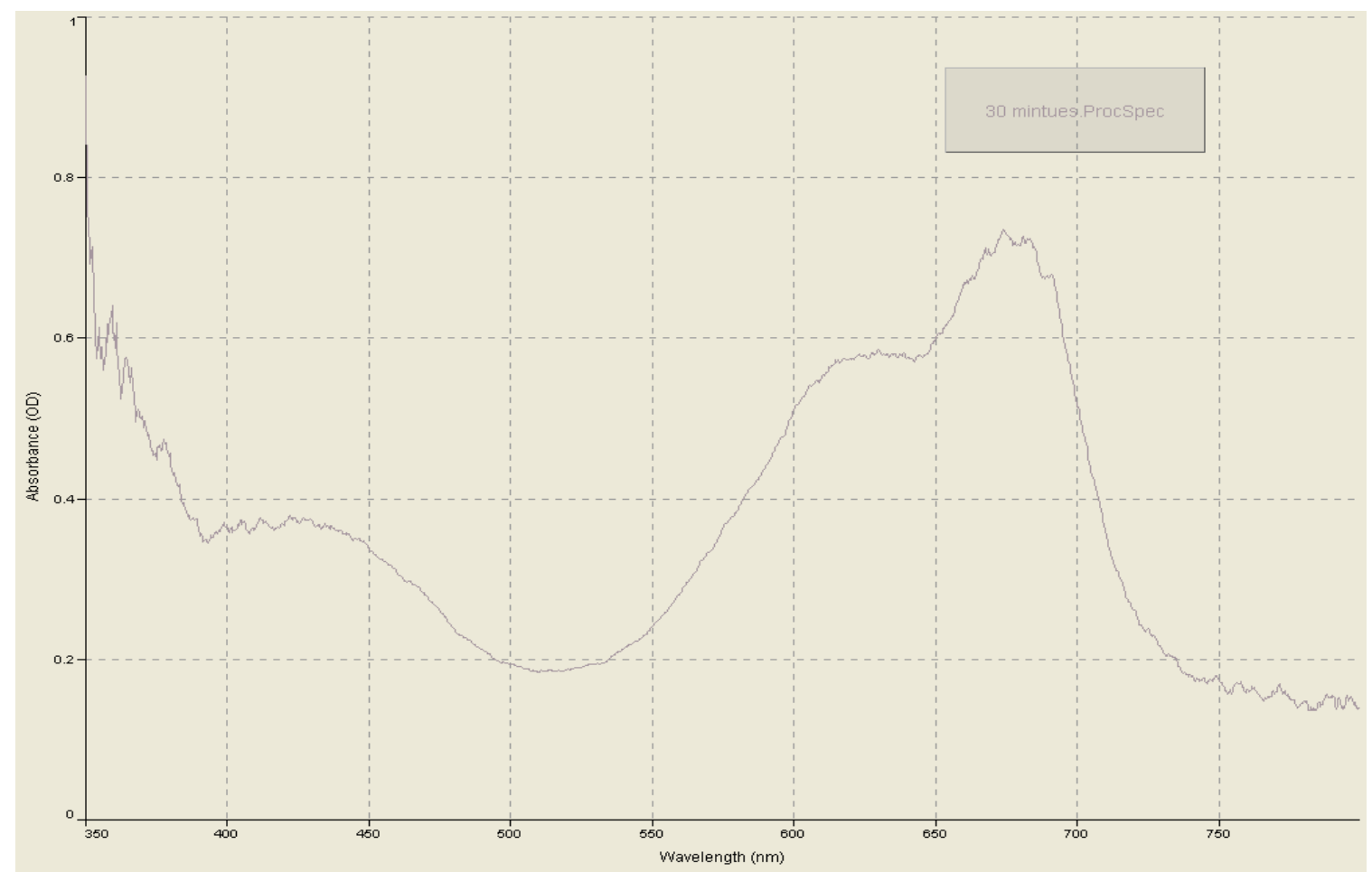

Figure 160: Absorbance spectrum of Kelly Green A_F after 30 minutes 


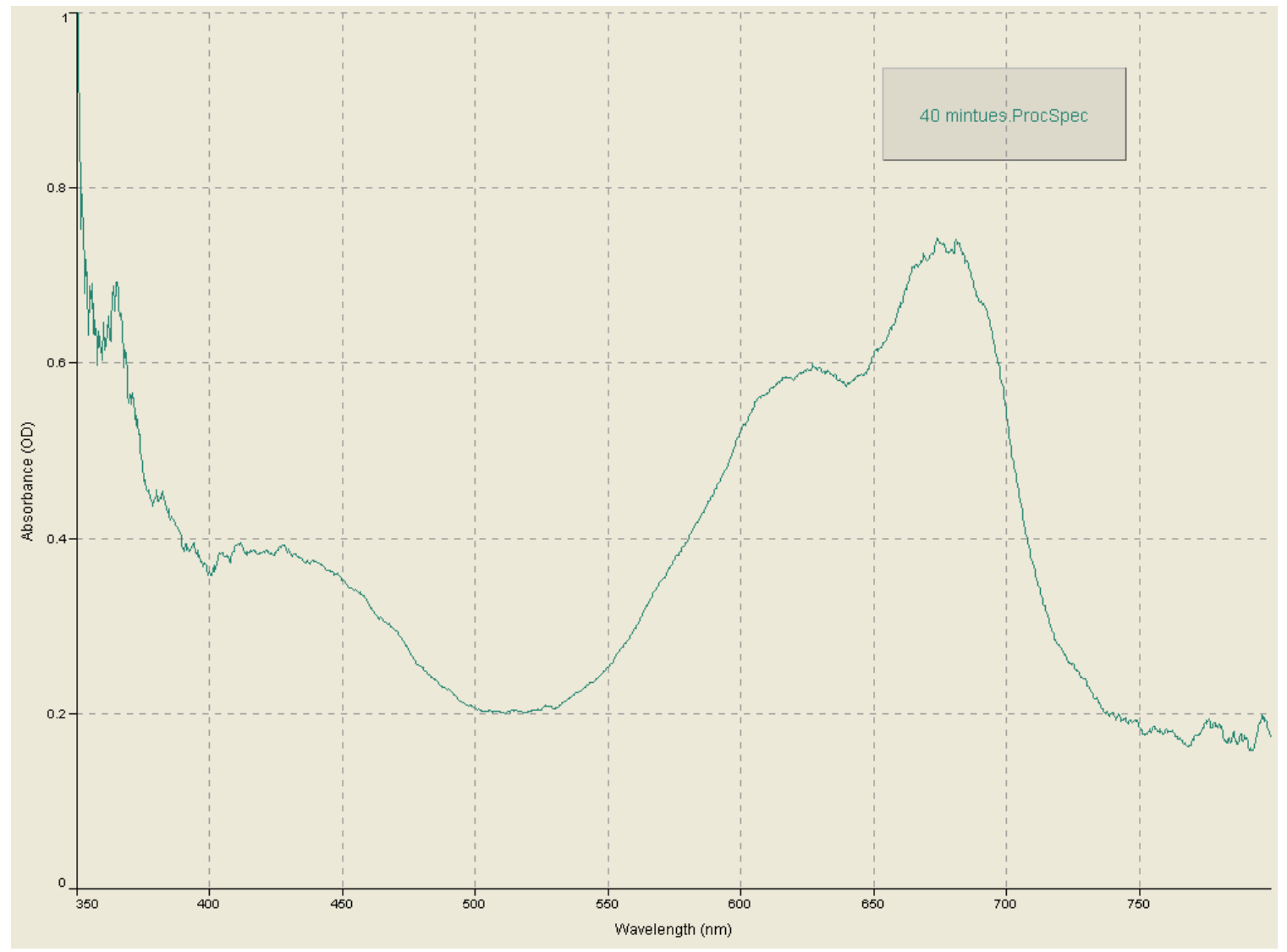

Figure 161: Absorbance spectrum of Kelly Green A_F after 40 minutes

\section{Part II}

In Part 11 of Phase III, a dark scan, a reference scan and a sample spectrum of Kelly Green A_F was collected every minute for an hour using a wavelength from $380 \mathrm{~nm}$ to $700 \mathrm{~nm}$. Figure 40 through Figure 44 displays the absorbance values that were obtained when certain wavelengths in the UV and Visible region were chosen. At every wavelength that was chosen, the maximum absorbance value was seen after 24 minutes of collecting the dark scan, reference scan and a sample spectrum. The lowest absorbance value was recorded at 52 minutes after collecting a dark scan, a reference scan and a sample spectrum at $425 \mathrm{~nm}, 520 \mathrm{~nm}, 625 \mathrm{~nm}$ and $700 \mathrm{~nm}$. At $627 \mathrm{~nm}$, the minimum absorbance value was seen at 42 minutes. In Figure 45, the absorbance spectra were plotted every ten minutes starting at one minute. In this figure, the sample spectrum that was collected after 30 minutes presented the highest absorbance value of 0.709 around $680 \mathrm{~nm}$. After 50 minutes, the absorbance values decreased to 0.559 around 680 $\mathrm{nm}$. At 10 minutes, the minimum absorbance value of 0.147 was observed around $515 \mathrm{~nm}$. At 20 
minutes, the minimum absorbance value of 0.057 was observed around $500 \mathrm{~nm}$. After 30 minutes the minimum absorbance value was recorded at $524 \mathrm{~nm}$ and after 40 minutes the minimum absorbance value was recorded at $525 \mathrm{~nm}$. Even after 50 minutes the minimum absorbance value was recorded at $522 \mathrm{~nm}$. It can be concluded from the spectra that were collected before 20 minutes that the wavelengths where the minimum absorbance values occurred show a higher variability. From the spectra that was collected at 30 minutes, 40 minutes and 50 minutes, it can be concluded that when collecting a dark scan, a reference scan and a sample spectrum there is little variation in the wavelengths where the minimum absorbance values occurred. The results of this part of Phase III demonstrate that the warm up time for the instrument is influential on the spectra that were collected when a dark scan and a reference scan were collected before the every sample spectrum was collected. The results also support the manual that was provided for the CRAIC@ QDI 1000 Microspectrophotometer that states to allow the instrument to warm up for at least 30 minutes.

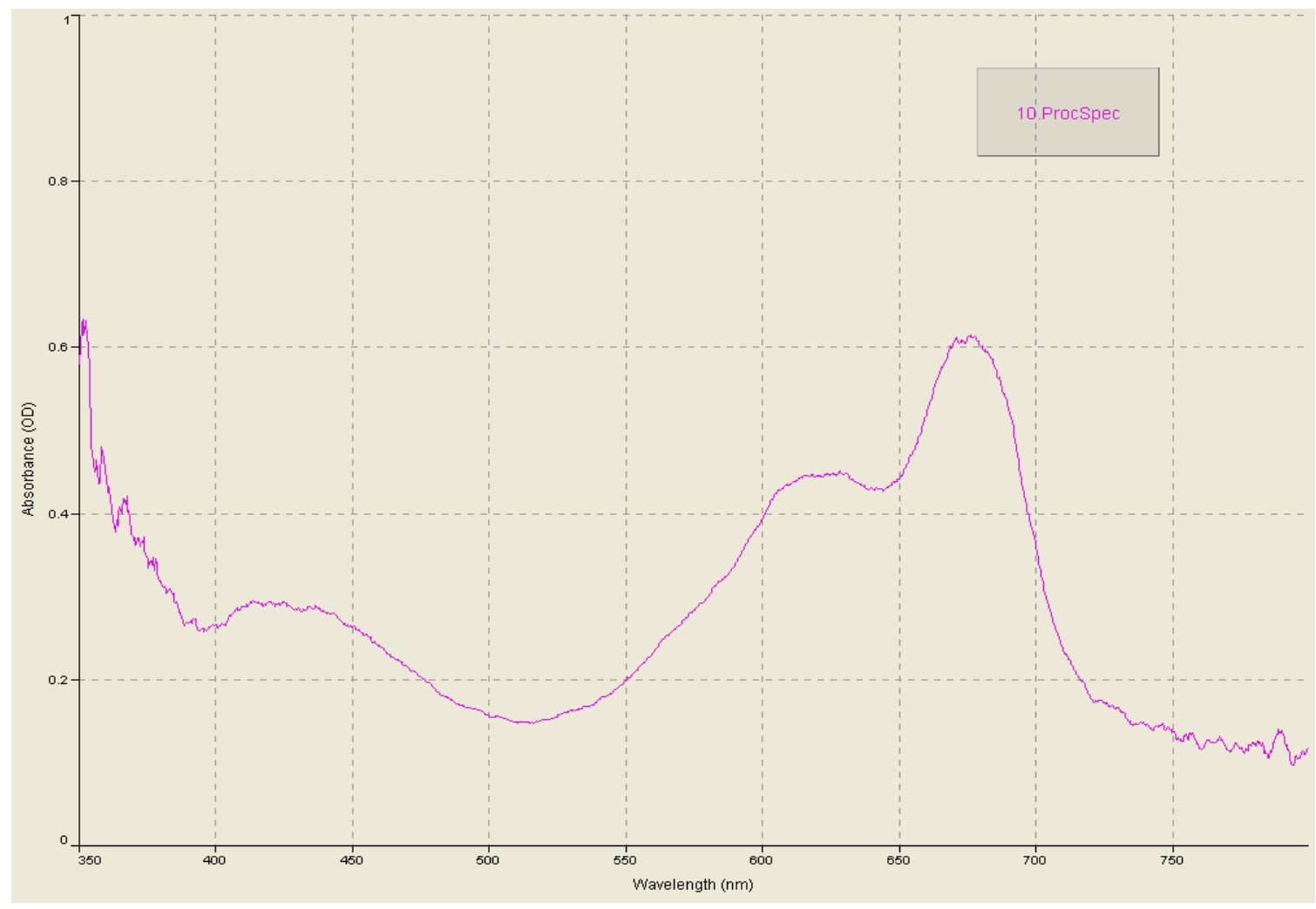

Figure 162: Absorbance spectrum of Kelly Green A_F after 10 minutes 


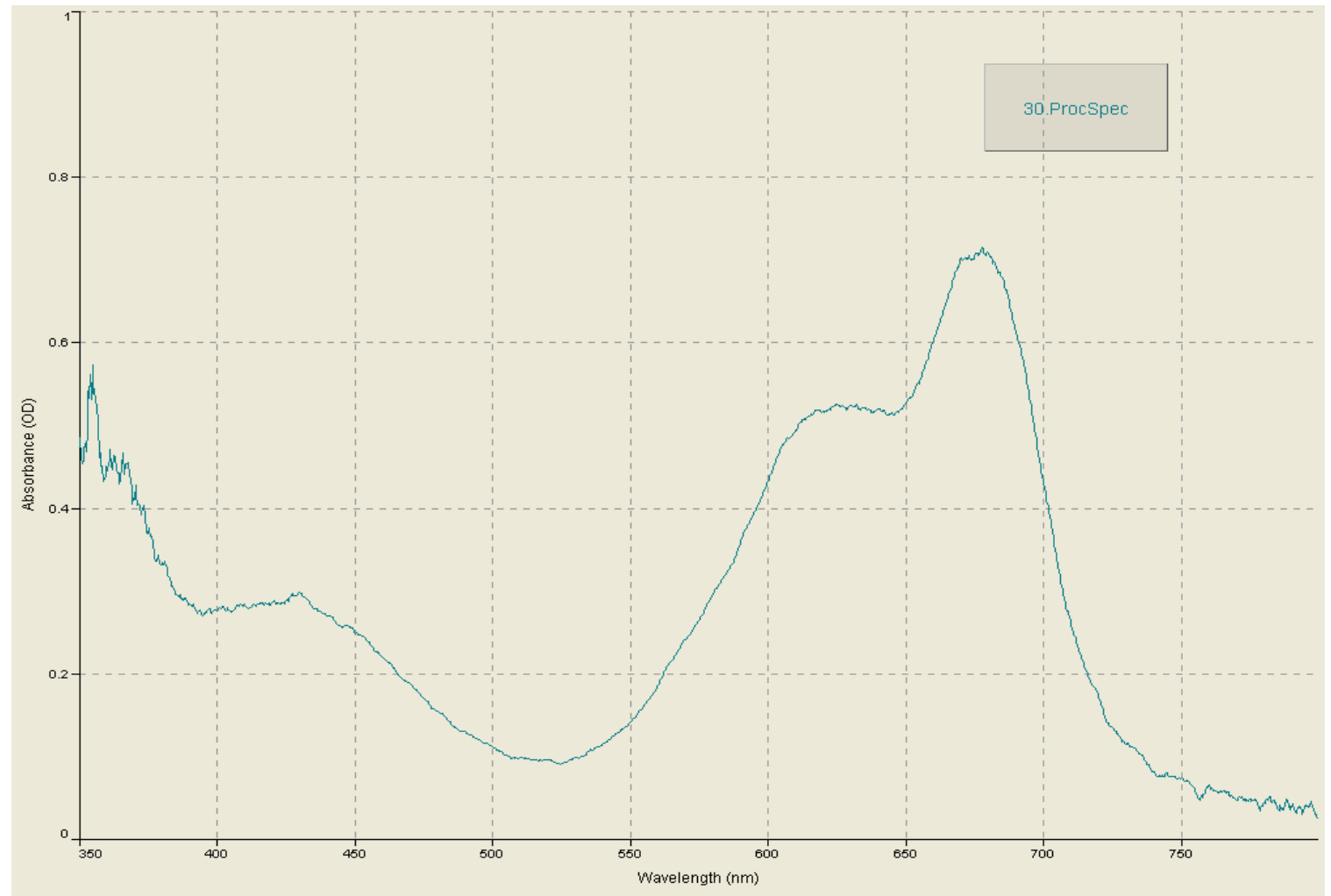

Figure 163: Absorbance spectrum of Kelly Green A_F after 30 minutes

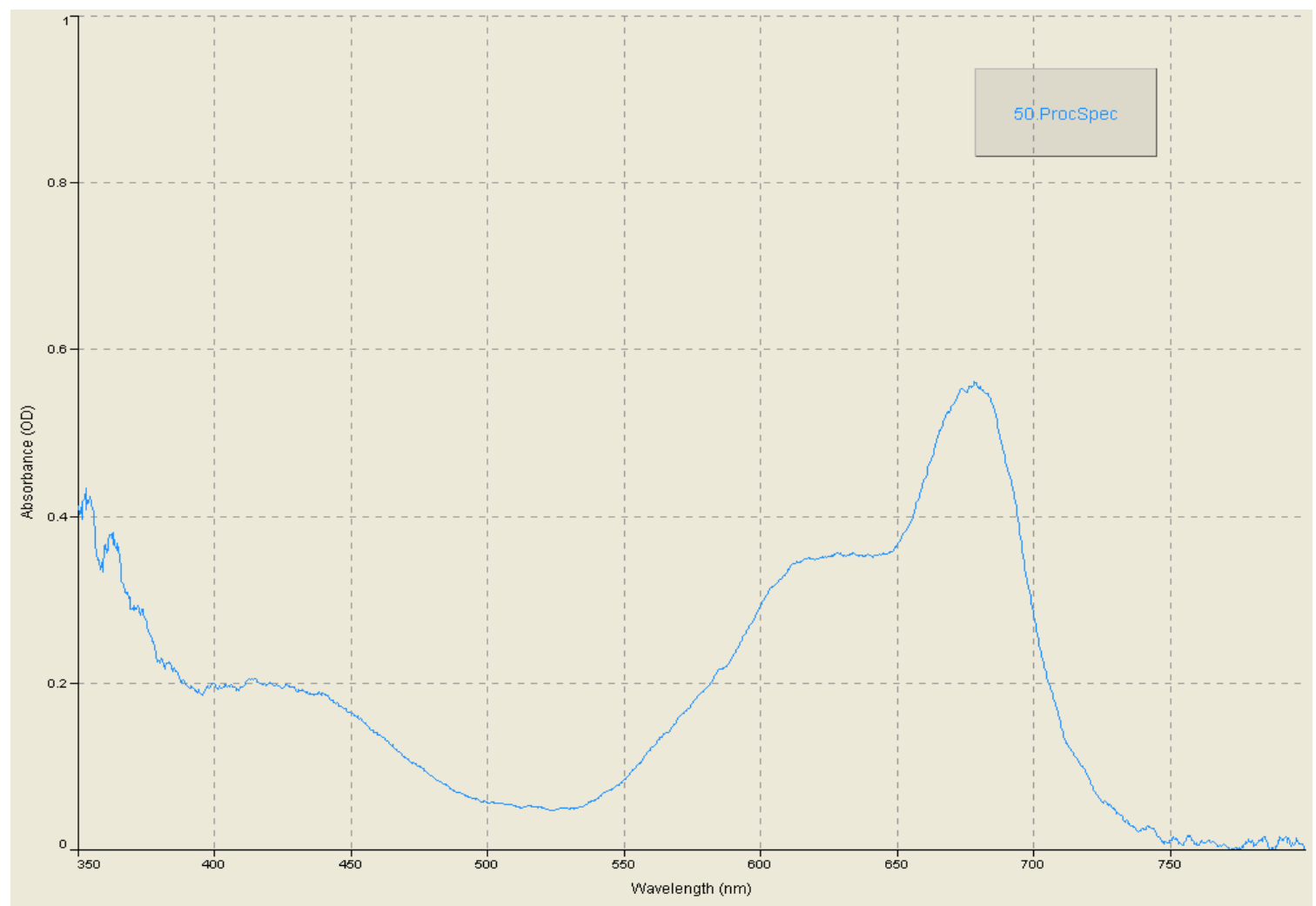

Figure 164: Absorbance spectrum of Kelly Green A_F after 50 minutes 
Phase IV

The $R \circledast$ program was used to display the results of the data that was obtained when the average absorbance, the UCL and the LCL was calculated at the $95 \%$ confidence level using the average of the average absorbance values and the average of the average standard deviations of all the front, back, sleeve and armpit sections of the Gold A and Gold B shirt, Kelly Green C and D shirt, Orange E and Orange F shirt, Red G and Red H shirt and Royal I and Royal J shirt. These results are shown in Figures 48 and 49, Figure 59 and 60, Figure 70 and 71, Figure 81 and 82 and Figure 92 and 93. If another fiber was analyzed from the front, back, sleeve or armpit of the Gold A or Gold B shirt given the operating conditions of the QDI 1000 Microspectrophotometer, it can be concluded that at the $95 \%$ confidence level that the absorbance spectrum would fall within the upper and lower limits that were determined in this study. The same conclusion can be made for the Kelly Green C and D shirt, the Orange E and F shirt, the Red G and H shirt and the Royal I and J shirt. Figure 50, 61, 72, 83 and 94 displays the average of the average absorbance values, the 95\% UCL and the 95\% LCL for every Gold, Kelly Green, Orange, Red and Royal shirt that was analyzed in this study. From Figure 50, it can be concluded at the $95 \%$ confidence level that if a fiber was analyzed from the front, back, sleeve or armpit of any Gold shirt used in this study given the operating conditions of the QDI 1000 Microspectrophotometer, its absorbance spectrum would fall within the upper and lower limits that were determined in this study. The same can be concluded for the Kelly Green, Orange, Red and Royal shirts.

An intra-garment comparison was carried out in this phase of the study as well to determine if gold, kelly green, orange, red, and royal cotton fibers can be differentiated depending on the part of the shirt that they come from. Fibers from the front, back, sleeve and armpit of ten different shirts were analyzed for each color. The UCL and the LCL at the 95\% confidence level of the front for all ten shirts of every color was determined using the average of the average absorbance values and the average of the standard deviations. This was repeated for the back, sleeve and armpit. For each color, there were six different comparisons that were done between the four different sections; front to back, front to sleeve, front to armpit, back to sleeve, back to armpit, sleeve to armpit. All these comparisons are displayed in Figure 51-56, Figure 6267, Figure 73-78, Figure 84-89 and Figure 95-100. The OR function in Microsoft® Excel was 
used to determine if the four different sections of each shirts that were displayed in the figures could or could not be differentiated from one another. If the front UCL was less than that of the back LCL or the front LCL was greater than that of the back UCL, the equation displayed "true" meaning that the front and back sections could be differentiated from one another. If the equation displayed a "false" than the front and back sections could not be differentiated from one another. If the front UCL was less than that of the sleeve LCL or the front LCL was greater than that of the sleeve UCL, the equation displayed "true" meaning that the front and sleeve sections could be differentiated from one another. If the equation displayed a "false" than the front and sleeve sections could not be differentiated from one another. If the front UCL was less than that of the armpit LCL or the front LCL was greater than that of the armpit UCL, the equation displayed "true" meaning that the front and armpit sections could be differentiated from one another. If the equation displayed a "false" than the front and armpit sections could not be differentiated from one another. If the back UCL was less than that of the sleeve LCL or the back LCL was greater than that of the sleeve UCL, the equation displayed "true" meaning that the back and sleeve sections could be differentiated from one another. If the equation displayed a "false" than the back and sleeve sections could not be differentiated from one another. If the back UCL was less than that of the armpit LCL or the back LCL was greater than that of the armpit UCL, the equation displayed "true" meaning that the back and armpit sections could be differentiated from one another. If the equation displayed a "false" than the back and armpit sections could not be differentiated from one another. If the sleeve UCL was less than that of the armpit LCL or the sleeve LCL was greater than that of the armpit UCL, the equation displayed "true" meaning that the sleeve and armpit sections could be differentiated from one another. If the equation displayed a "false" than the sleeve and armpit sections could not be differentiated from one another. For all six comparisons that were analyzed for every color, the equation displayed "false", which is displayed in Appendix B. When looking at the figures, the UCL of one section was never less than that of the LCL of another section and the LCL of one section was never greater than that of the UCL of another section. It can be concluded that given the operating conditions of the QDI 1000 Microspectrophotometer the front, back, sleeve and armpit of the ten gold, kelly green, orange, red and royal shirts cannot be differentiated from one another at the $95 \%$ confidence level. 


\section{Phase V}

The $R \AA$ program was used to display the results of the data that was obtained when the average absorbance, the UCL and the LCL was calculated at the $95 \%$ confidence level using the average of the average absorbance values and the average of the average standard deviations of section 1, section 2 and section 3 for the front square of the Gold I and Gold $\mathrm{J}$ shirt, for the front square of the Kelly Green G and H shirt, for the front square of the Orange E and Orange F shirt, for the front square of the Red C and Red D shirt and for the front square of the Royal A and Royal B shirt. These results are shown in Figures 103 and 104, Figure 111 and 112, Figure 119 and 120, Figure 127 and 128 and Figure 135 and 136. If another fiber was analyzed from the front square of the Gold I or Gold J shirt given the operating conditions of the QDI 1000 Microspectrophotometer, it can be concluded that at the 95\% confidence level that the absorbance spectrum would fall within the upper and lower limits that were determined in this study. The same conclusion can be made for the front square of the Kelly Green G and H shirts, for the front square of the Orange E and F shirts, for the front square of the Red C and D shirts and for the front square of the Royal A and B shirts. Figure 105, 113, 121, 129 and 137 displays the average of the average absorbance values, the 95\% UCL and the 95\% LCL for every Gold, Kelly Green, Orange, Red and Royal front square that was analyzed in this study. From Figure 105 , it can be concluded at the $95 \%$ confidence level that if a fiber was cut into three sections and analyzed from the front square of Gold shirt used in this study given the operating conditions of the QDI 1000 Microspectrophotometer, its absorbance spectrum would fall within the upper and lower limits that were determined in this study. The same can be concluded for the front square of the Kelly Green, Orange, Red and Royal shirts.

An inter-garment comparison was carried out in this phase of the study as well to determine if there was variability within the three sections of the same fiber. The UCL and the LCL at the $95 \%$ confidence level for section 1 for all ten front squares of the shirts of every color was determined using the average of the average absorbance values and the average of the standard deviations. This was repeated for section 2 and section 3. For each color, there were three different comparisons that were done between the three different sections; section 1 to section 2, section 1 to section 3 and section 2 to section 3. All these comparisons are displayed in Figure 106-108 Figure 114-116, Figure 122-124, Figure 130-132 and Figure 138-140. The OR 
function in Microsoft ${ }^{\circledR}$ Excel was used to determine if the three different sections of the front square of each shirt that were displayed in the figures could or could not be differentiated from one another. If the section1 UCL was less than that of the section 2 LCL or the section $1 \mathrm{LCL}$ was greater than that of the section 2 UCL, the equation displayed "true" meaning that section1 and section 2 could be differentiated from one another. If the equation displayed a "false" than section 1 and section 2 could not be differentiated from one another. If the section 1 UCL was less than that of the section 3 LCL or the section 1 LCL was greater than that of the section 3 UCL, the equation displayed "true" meaning that section 1 and section 3 could be differentiated from one another. If the equation displayed a "false" than section 1 and section 3 could not be differentiated from one another. If the section 2 UCL was less than that of the section 3 LCL or the section 2 LCL was greater than that of the section 3 UCL, the equation displayed "true" meaning section 2 and section 3 could be differentiated from one another. If the equation displayed a "false" than section 2 and section 3 could not be differentiated from one another. For all three comparisons that were analyzed for every front square of every color, the equation displayed "false", which is displayed in Appendix B. When looking at the figures, the UCL of one section was never less than that of the LCL of another section and the LCL of one section was never greater than that of the UCL of another section. It can be concluded that given the operating conditions of the QDI 1000 Microspectrophotometer section 1, section 2 and section 3 of the front squares of all five colors cannot be differentiated from one another at the $95 \%$ confidence level.

\section{V: $\underline{\text { Conclusion }}$}

To complete this study, it was conclude by comparing the five colors used in this study to each other. To determine if one can differentiate between Gold, Kelly Green, Orange, Red and Royal when comparing their absorbance spectra, the UCL and the LCL at the 95\% confidence level was determined for all Gold, Kelly Green, Orange, Red and Royal shirts using the average of the average absorbance values and the average of the standard deviations. There were ten different comparisons that were done between the five different color shirts; orange to red, orange to kelly green, orange to gold, orange to royal, kelly green to red, kelly green to gold, kelly green to royal, red to gold, red to royal and gold to royal. All these comparisons are 
displayed in Figure 141-150. The OR function in Microsoft@ Excel was used to determine if the five different color that are displayed in the figures could or could not be differentiated from one another. If the Red UCL was less than that of the Orange LCL or the Red LCL was greater than that of the Orange UCL, the equation displayed "true" meaning that red and orange could be differentiated from one another. If the equation displayed a "false" red and orange could not be differentiated from one another. If the Kelly Green UCL was less than that of the Orange LCL or the Kelly Green LCL was greater than that of the Orange UCL, the equation displayed "true" meaning that orange and kelly green could be differentiated from one another. If the equation displayed a "false" orange and kelly green could not be differentiated from one another. If the Gold UCL was less than that of the Orange LCL or the Gold LCL was greater than that of the Orange UCL, the equation displayed "true" meaning orange and gold could be differentiated from one another. If the equation displayed a "false" than orange and gold could not be differentiated from one another. If the Royal UCL was less than that of the Orange LCL or the Royal LCL was greater than that of the Orange UCL, the equation displayed "true" meaning that orange and royal could be differentiated from one another. If the equation displayed a "false" than orange and royal could be differentiated from one another. If the Red UCL was less than that of the Kelly Green LCL or the Red LCL was greater than that of the Kelly Green UCL, the equation displayed "true" meaning that red and kelly green could be differentiated from one another. If the equation displayed a "false" than red and kelly green could not be differentiated from one another. If the Gold UCL was less than that of the Kelly Green LCL or the Gold LCL was greater than that of the Kelly Green UCL, the equation displayed "true" meaning kelly green and gold could be differentiated from one another. If the equation displayed a "false" than kelly green and gold could not be differentiated from one another. If the Gold UCL was less than that of the Red LCL or the Gold LCL was greater than that of the Red UCL, the equation displayed "true" meaning red and gold could be differentiated from one another. If the equation displayed a "false" than red and gold could not be differentiated from one another. If the Royal UCL was less than that of the Red LCL or the Royal LCL was greater than that of the Red UCL, the equation displayed "true" meaning red and royal could be differentiated from one another. If the equation displayed a "false" than red and royal could not be differentiated from one another. If the Royal UCL was less than that of the Gold LCL or the Royal LCL was greater than that of the Gold UCL, the equation displayed "true" meaning gold and royal could be differentiated from 
one another. If the equation displayed a "false" than gold and royal could not be differentiated from one another. Table 12 indications the results when the OR function was applied to the data.

\begin{tabular}{|c|c|c|c|c|c|}
\hline $\begin{array}{l}\text { Orange - } \\
\text { Red }\end{array}$ & $\begin{array}{c}\text { Orange - Kelly } \\
\text { Green }\end{array}$ & $\begin{array}{c}\text { Orange- } \\
\text { Gold }\end{array}$ & $\begin{array}{c}\text { Orange- } \\
\text { Royal }\end{array}$ & $\begin{array}{l}\text { Kelly Green- } \\
\text { Red }\end{array}$ & $\begin{array}{l}\text { Kelly Green- } \\
\text { Gold }\end{array}$ \\
\hline FALSE & FALSE & FALSE & FALSE & FALSE & FALSE \\
\hline FALSE & FALSE & FALSE & FALSE & FALSE & FALSE \\
\hline FALSE & FALSE & FALSE & FALSE & FALSE & FALSE \\
\hline FALSE & FALSE & FALSE & FALSE & FALSE & FALSE \\
\hline FALSE & FALSE & FALSE & TRUE & FALSE & FALSE \\
\hline FALSE & FALSE & FALSE & TRUE & FALSE & FALSE \\
\hline FALSE & FALSE & FALSE & TRUE & FALSE & FALSE \\
\hline FALSE & FALSE & FALSE & TRUE & FALSE & FALSE \\
\hline FALSE & TRUE & FALSE & TRUE & TRUE & FALSE \\
\hline FALSE & TRUE & TRUE & TRUE & TRUE & FALSE \\
\hline FALSE & TRUE & TRUE & TRUE & TRUE & FALSE \\
\hline FALSE & TRUE & TRUE & TRUE & TRUE & FALSE \\
\hline FALSE & TRUE & TRUE & TRUE & TRUE & FALSE \\
\hline FALSE & TRUE & TRUE & TRUE & TRUE & FALSE \\
\hline FALSE & TRUE & TRUE & TRUE & TRUE & FALSE \\
\hline FALSE & TRUE & TRUE & TRUE & TRUE & FALSE \\
\hline FALSE & TRUE & TRUE & FALSE & TRUE & FALSE \\
\hline FALSE & TRUE & TRUE & FALSE & TRUE & FALSE \\
\hline TRUE & FALSE & TRUE & FALSE & TRUE & FALSE \\
\hline TRUE & FALSE & FALSE & TRUE & FALSE & FALSE \\
\hline TRUE & FALSE & FALSE & TRUE & FALSE & TRUE \\
\hline FALSE & TRUE & FALSE & TRUE & FALSE & TRUE \\
\hline FALSE & TRUE & FALSE & TRUE & FALSE & TRUE \\
\hline FALSE & TRUE & FALSE & TRUE & TRUE & TRUE \\
\hline FALSE & TRUE & FALSE & TRUE & TRUE & TRUE \\
\hline FALSE & TRUE & FALSE & TRUE & TRUE & TRUE \\
\hline FALSE & TRUE & FALSE & TRUE & TRUE & TRUE \\
\hline FALSE & TRUE & FALSE & TRUE & TRUE & TRUE \\
\hline FALSE & TRUE & FALSE & TRUE & TRUE & TRUE \\
\hline FALSE & TRUE & FALSE & TRUE & TRUE & TRUE \\
\hline FALSE & TRUE & FALSE & FALSE & TRUE & TRUE \\
\hline FALSE & TRUE & FALSE & FALSE & TRUE & TRUE \\
\hline FALSE & TRUE & FALSE & FALSE & TRUE & TRUE \\
\hline FALSE & TRUE & FALSE & FALSE & FALSE & TRUE \\
\hline
\end{tabular}




\begin{tabular}{|l|l|l|l|l|l|}
\hline FALSE & FALSE & FALSE & FALSE & FALSE & FALSE \\
\hline FALSE & FALSE & FALSE & FALSE & FALSE & FALSE \\
\hline FALSE & FALSE & FALSE & FALSE & FALSE & FALSE \\
\hline FALSE & FALSE & FALSE & FALSE & FALSE & FALSE \\
\hline FALSE & FALSE & FALSE & FALSE & FALSE & FALSE \\
\hline FALSE & FALSE & FALSE & FALSE & FALSE & FALSE \\
\hline FALSE & FALSE & FALSE & FALSE & FALSE & FALSE \\
\hline
\end{tabular}

\begin{tabular}{|c|c|c|c|}
\hline $\begin{array}{c}\text { Kelly Green- } \\
\text { Royal }\end{array}$ & $\begin{array}{c}\text { Red- } \\
\text { Gold }\end{array}$ & $\begin{array}{c}\text { Red- } \\
\text { Royal }\end{array}$ & $\begin{array}{c}\text { Gold- } \\
\text { Royal }\end{array}$ \\
\hline FALSE & FALSE & FALSE & FALSE \\
\hline FALSE & FALSE & FALSE & FALSE \\
\hline FALSE & FALSE & FALSE & FALSE \\
\hline FALSE & FALSE & FALSE & FALSE \\
\hline FALSE & FALSE & TRUE & FALSE \\
\hline FALSE & FALSE & TRUE & FALSE \\
\hline FALSE & FALSE & TRUE & TRUE \\
\hline FALSE & FALSE & TRUE & FALSE \\
\hline FALSE & FALSE & TRUE & FALSE \\
\hline FALSE & TRUE & TRUE & FALSE \\
\hline FALSE & TRUE & TRUE & FALSE \\
\hline FALSE & TRUE & TRUE & FALSE \\
\hline FALSE & TRUE & TRUE & FALSE \\
\hline FALSE & TRUE & TRUE & FALSE \\
\hline FALSE & TRUE & TRUE & FALSE \\
\hline FALSE & TRUE & TRUE & FALSE \\
\hline FALSE & TRUE & TRUE & TRUE \\
\hline FALSE & TRUE & FALSE & TRUE \\
\hline FALSE & TRUE & FALSE & TRUE \\
\hline FALSE & TRUE & FALSE & TRUE \\
\hline FALSE & TRUE & FALSE & TRUE \\
\hline FALSE & FALSE & FALSE & TRUE \\
\hline FALSE & FALSE & TRUE & TRUE \\
\hline FALSE & FALSE & TRUE & TRUE \\
\hline FALSE & FALSE & TRUE & TRUE \\
\hline FALSE & FALSE & TRUE & TRUE \\
\hline FALSE & FALSE & TRUE & TRUE \\
\hline FALSE & FALSE & TRUE & TRUE \\
\hline FALSE & FALSE & FALSE & TRUE \\
\hline TRUE & FALSE & FALSE & TRUE \\
\hline FRUE & FALSE & FALSE & FALSE \\
\hline & & \\
\hline
\end{tabular}




\begin{tabular}{|c|c|c|c|}
\hline TRUE & FALSE & FALSE & FALSE \\
\hline TRUE & FALSE & FALSE & FALSE \\
\hline FALSE & FALSE & FALSE & FALSE \\
\hline FALSE & FALSE & FALSE & FALSE \\
\hline FALSE & FALSE & FALSE & FALSE \\
\hline FALSE & FALSE & FALSE & FALSE \\
\hline FALSE & FALSE & FALSE & FALSE \\
\hline FALSE & FALSE & FALSE & FALSE \\
\hline FALSE & FALSE & FALSE & FALSE \\
\hline FALSE & FALSE & FALSE & FALSE \\
\hline
\end{tabular}

Table 12: The OR functions results of the ten comparisons of all five colors

Orange and kelly green can be differentiated between $460 \mathrm{~nm}$ and $550 \mathrm{~nm}$ and also between $590 \mathrm{~nm}$ and $710 \mathrm{~nm}$. Orange and gold can be differentiated between 470 and $560 \mathrm{~nm}$. Orange and royal can be differentiated between $420 \mathrm{~nm}$ and $530 \mathrm{~nm}$ as well as between $570 \mathrm{~nm}$ and $670 \mathrm{~nm}$. Kelly Green and red can be differentiated between $460 \mathrm{~nm}$ and $560 \mathrm{~nm}$ as well as between $610 \mathrm{~nm}$ and $700 \mathrm{~nm}$. Kelly green and gold can be differentiated between $580 \mathrm{~nm}$ and $710 \mathrm{~nm}$. Kelly Green and royal can be differentiated between $670 \mathrm{~nm}$ and $710 \mathrm{~nm}$. Red and gold can be differentiated between $470 \mathrm{~nm}$ and $580 \mathrm{~nm}$. Red and royal can be differentiated between $420 \mathrm{~nm}$ and $540 \mathrm{~nm}$ as well as between $580 \mathrm{~nm}$ and $710 \mathrm{~nm}$. Gold and royal can be differentiated between at $440 \mathrm{~nm}$ as well as between $540 \mathrm{~nm}$ and $670 \mathrm{~nm}$. When plotting the absorbance spectra of orange and red together, they both have the same general shape and had maximum absorbance values between $500 \mathrm{~nm}$ and $520 \mathrm{~nm}$. Although the red and orange absorbance spectra are very similar, they can still be differentiated between $560 \mathrm{~nm}$ and $580 \mathrm{~nm}$. It is also important to note that these colors can be differentiated given the operating conditions of the QDI 1000 Microspectrophotometer. When looking at the figures, the UCL of one color was less than that of the LCL of another color and the LCL of one color was greater than that of the UCL of another color at some point in the UV/ Visible region for all ten comparison that were analyzed. 


\section{VI: Future Research}

Phase IV and Phase V concluded that given the operating conditions on the QDI 1000 Microspectrophotometer, at the $95 \%$ confidence level the differentiation of cotton fibers cannot be achieved when conducting an intra- garment and inter-garment comparison. Although a negative conclusion was reached in this study, it would be interesting to analyze cotton fibers from other similar colored shirts or one could even use a different brand of shirt using the same analytical sequence. Using the absorbance value that was obtained, it could be determined if the average absorbance would fall within the upper confidence limit and the lower confidence limit that were determined in this study. 


\section{VII: $\underline{\text { References }}$}

1.Robertson, J., Classification of Textile Fibres, in Forensic Examination of Fibres, Second Edition1999, CRC Press.

2.Palenik, S., Microscopical Examination of Fibres, in Forensic Examination of Fibres, Second Edition1999, CRC Press.

3.Suzuki, S., et al., Guilty by his fibers: suspect confession versus textile fibers $r$ econstructed simulation. Forensic Science International, 2009. 189(1-3): p. e27-e32.

4.Robertson, J. and C. Roux, Classification of Textile Fibres, in Forensic Examination of Fibres, Second Edition1999, CRC Press.

5.Adolf, F.-P. and J. Dunlop, Microspectrophotometry/Colour Measurement, in Forensic Examination of Fibres, Second Edition1999, CRC Press.

6.Eng, M., P. Martin, and C. Bhagwandin, The Analysis of Metameric Blue Fibers and Their Forensic Significance. Journal of Forensic Sciences, 2009. 54(4): p. 841-845.

7.Berns, R.S., Billmeyer and Saltzman's Principles of Color Technology2000, New Jersey: John Wiley and Sons, INC.

8.Indow, T. and A.K. Romney, Reflectance spectra of Munsell standard chips and their appearance. Color Research and Application, 2008. 33(3): p. 229-237.

9.Wolken, J.J. and G.K. Strother, Microspectrophotometry. Appl. Opt., 1963. 2(9): p. 899-907.

10.Wiggins, K., et al., An investigation into the use of calculating the first derivative of absorbance spectra as a tool for forensic fibre analysis. Science \&amp; Justice, 2007. 47(1): p. 9-18.

11.Kopchick, K.A. and C.R. Bommarito, Color Analysis of Apparently Achromatic Automotive Paints by Visible Microspectrophotometry. Journal of Forensic Sciences, 2006. 51(2): p. 340343.

12.Barrett, J.A., J.A. Siegel, and J.V. Goodpaster, Forensic Discrimination of Dyed Hair Color: I. UV-Visible Microspectrophotometry*†. Journal of Forensic Sciences, 2010. 55(2): p. 323-333.

13.Max M, H., Inter-comparison of unrelated fiber evidence. Forensic Science International, 2003. 135(2): p. 146-149. 
14.Wiggins, K. and P. Drummond, The analysis and comparison of blue wool fibre populations found at random on clothing. Science \& Justice, 2005. 45(3): p. 157-162.

15.Grieve, M.C., T.W. Biermann, and K. Schaub, The individuality of fibres used to provide forensic evidence - not all blue polyesters are the same. Science \&amp; Justice, 2005. 45(1): p. $13-28$.

16.T.W, B., Blocks of colour IV: The evidential value of blue and red cotton fibres. Science \&amp; Justice, 2007. 47(2): p. 68-87.

17.Palmer, R., W. Hutchinson, and V. Fryer, The discrimination of (non-denim) blue cotton. Science \&amp; Justice, 2009. 49(1): p. 12-18. 


\section{VIII: Appendix A}

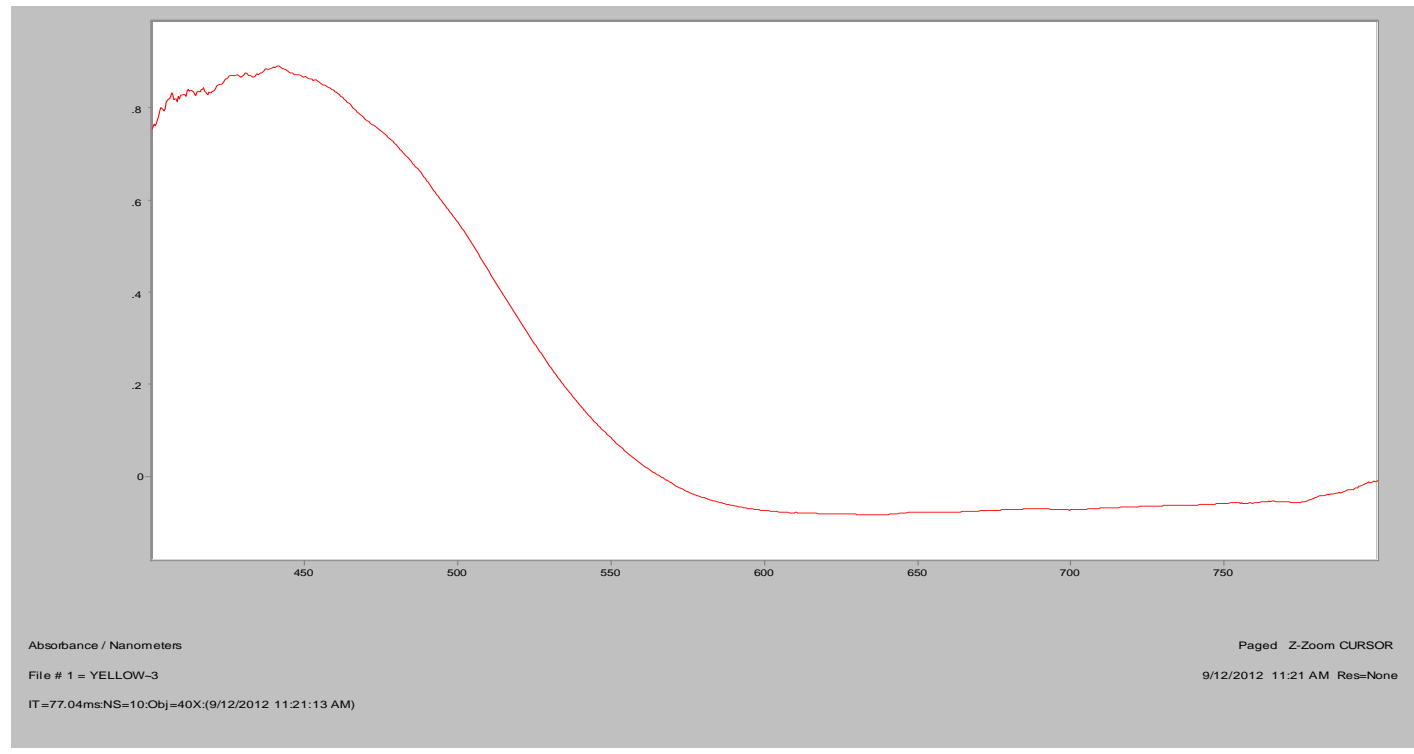

Absorbance spectrum of the front $t$-shirt square of Gold $A$

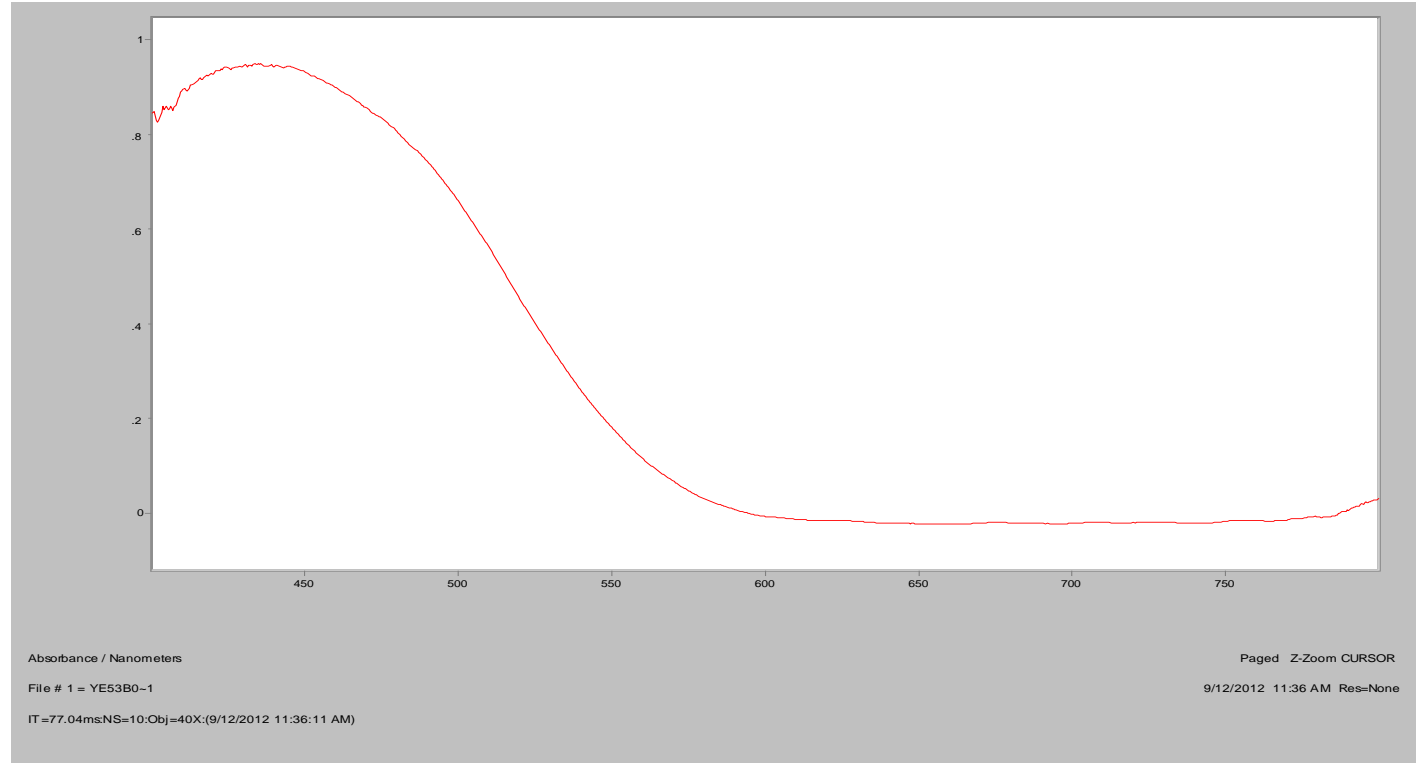

Absorbance spectrum of the back $t$-shirt square of Gold B 


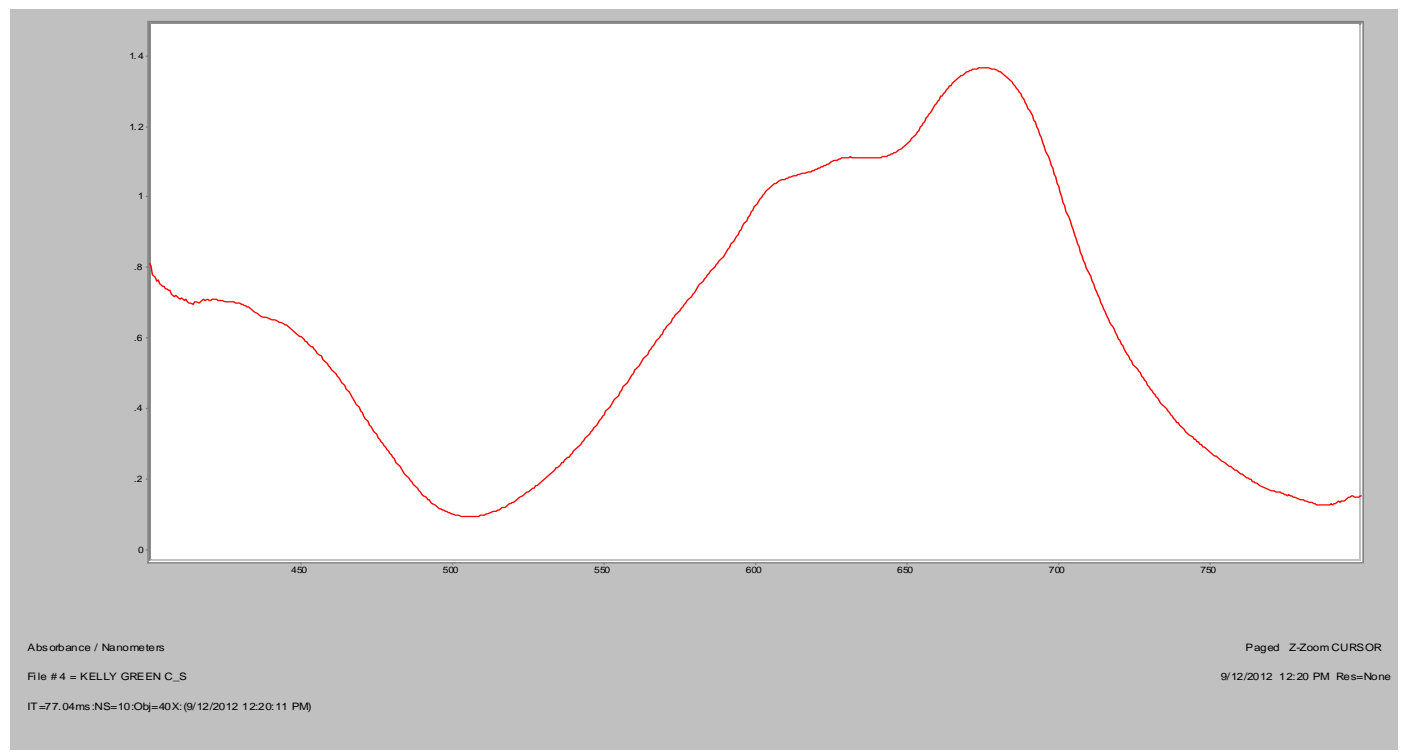

Absorbance spectrum of the sleeve t-shirt square of Kelly Green C

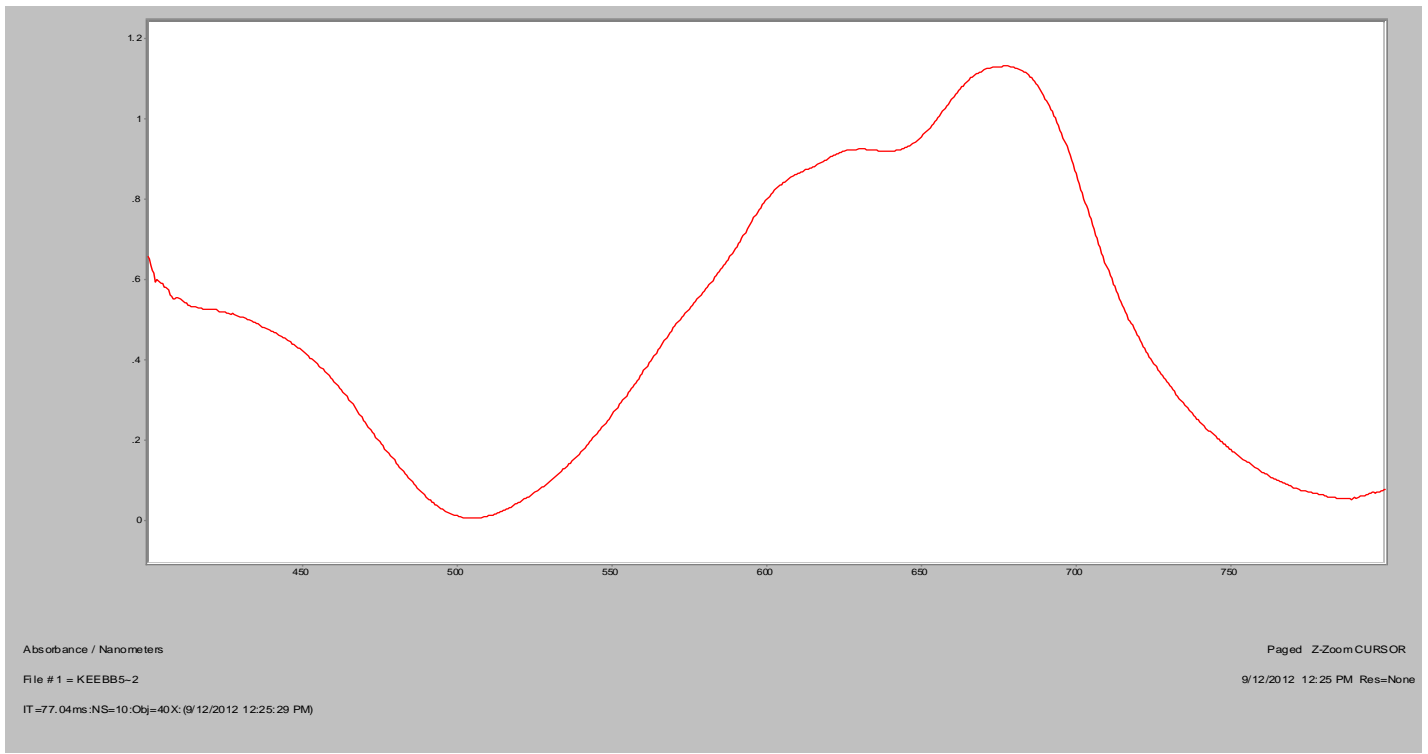

Absorbance spectrum of the armpit $t$-shirt square of Kelly Green D 


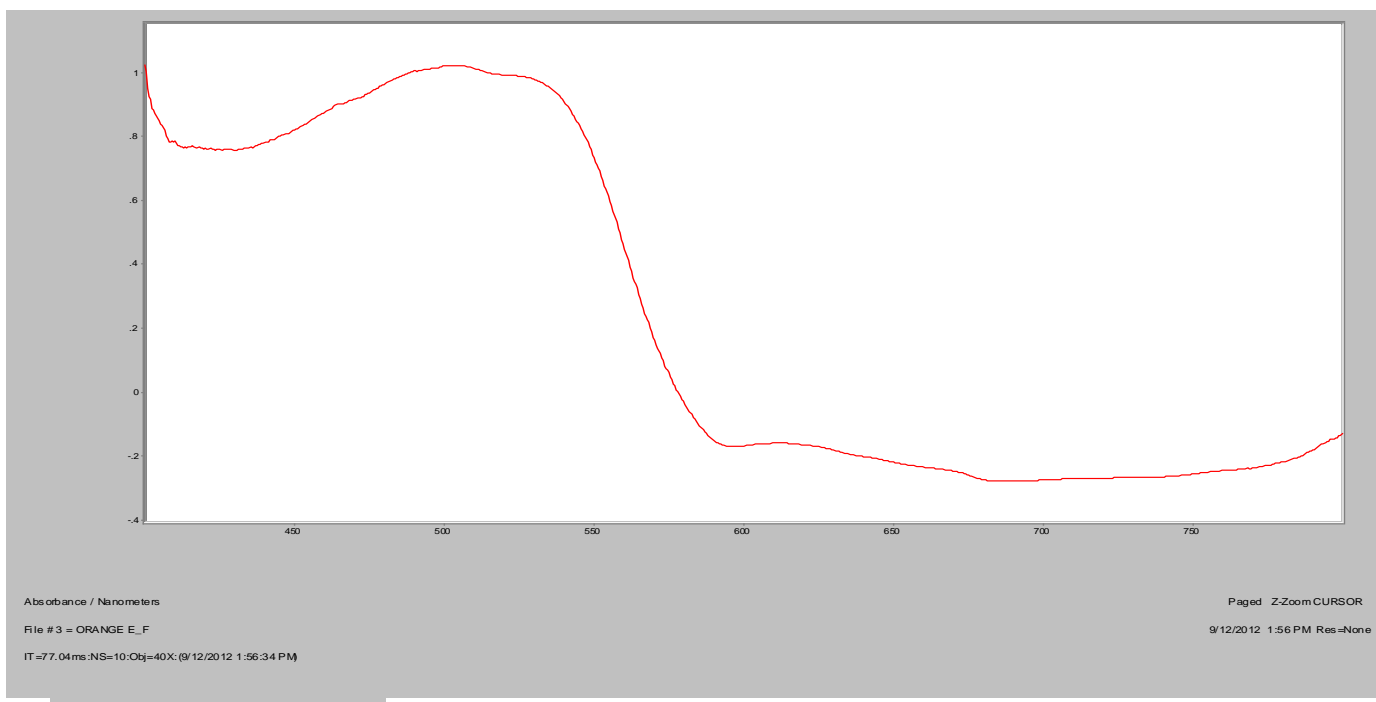

Absorbance spectrum of the front $t$-shirt square of Orange $E$

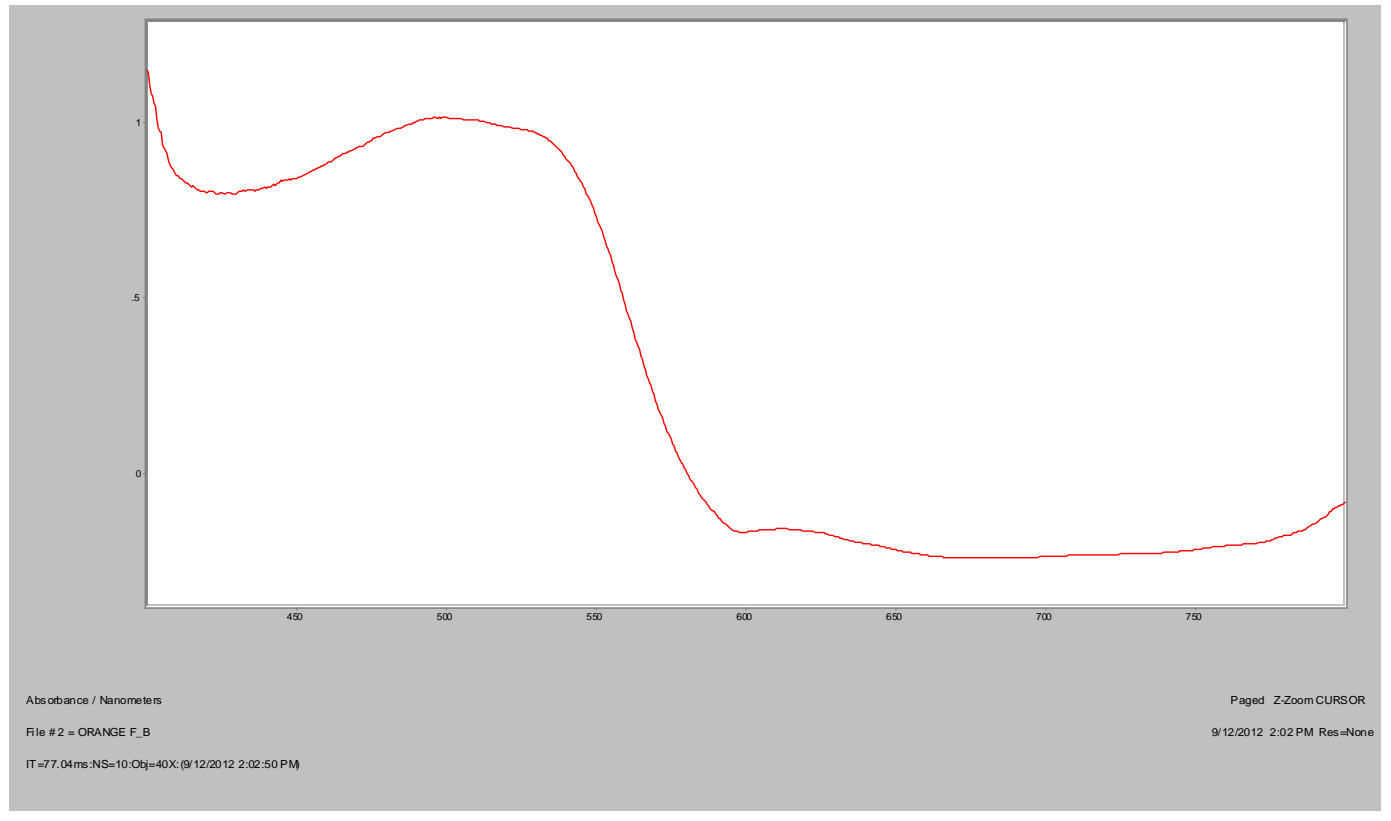

Absorbance spectrum of the back $t$-shirt square of Orange $F$ 


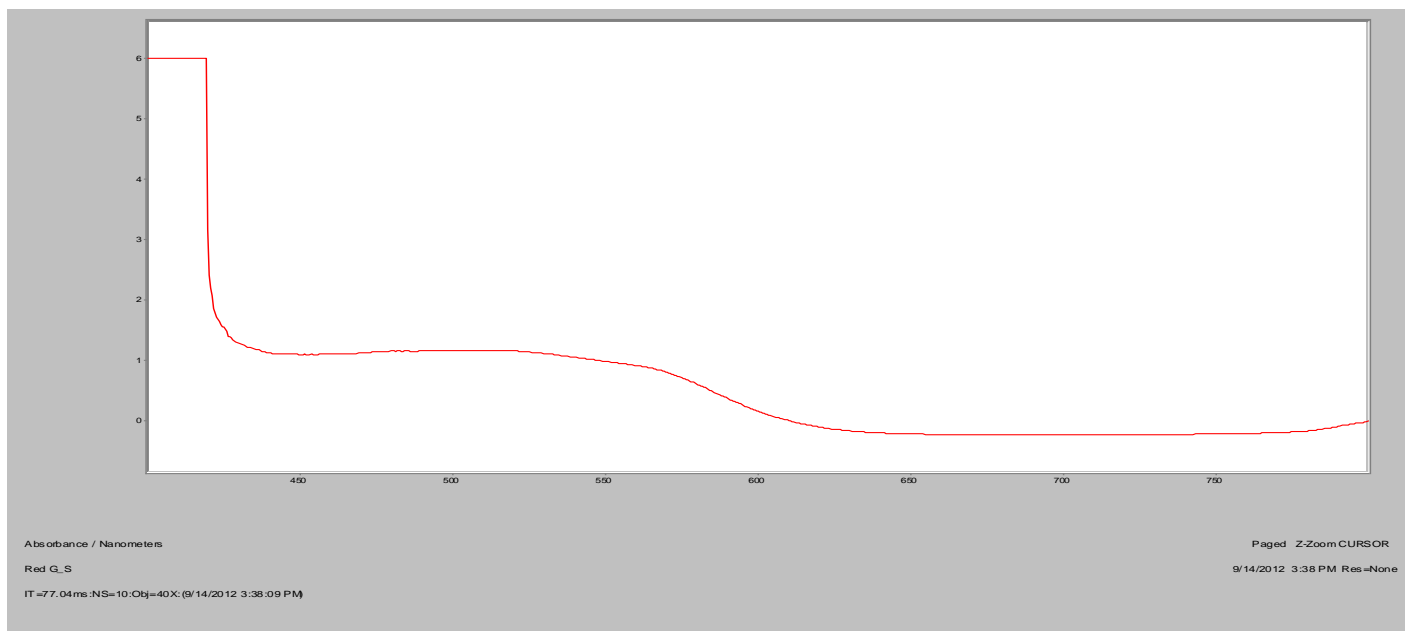

Absorbance spectrum of the sleeve t-shirt square of Red $G$

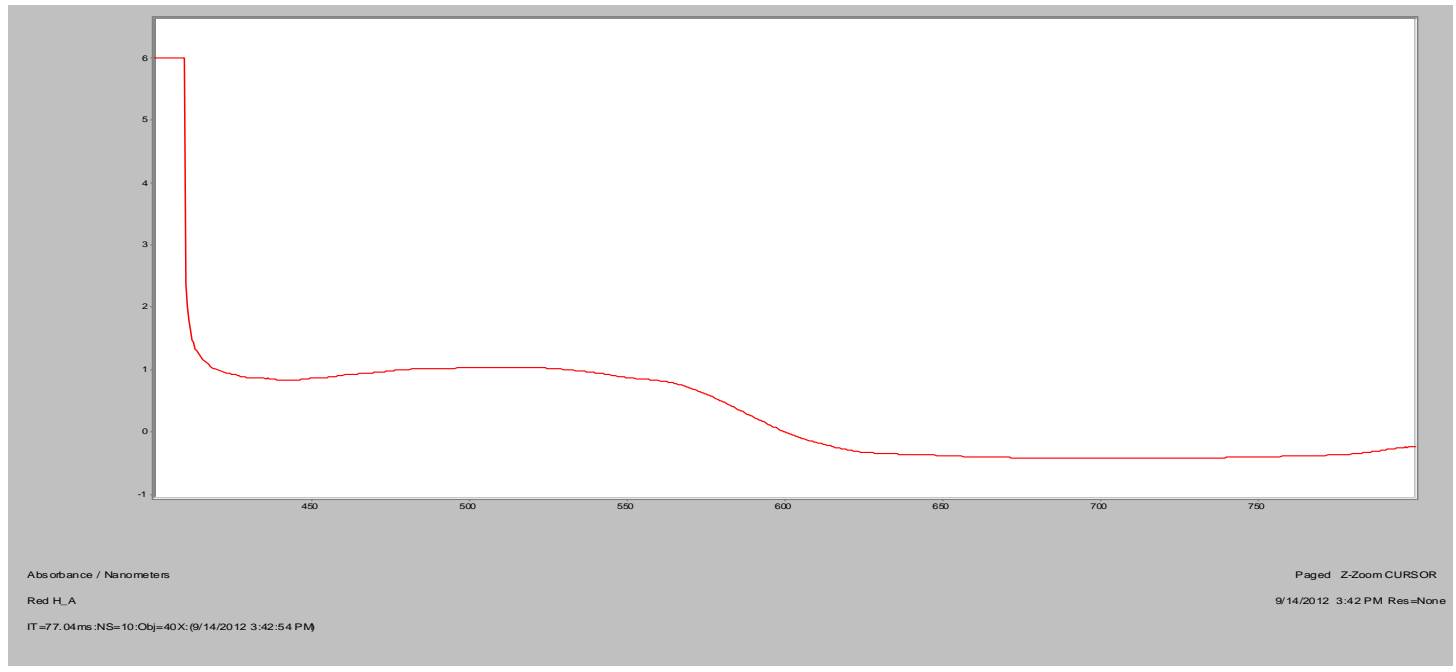

Absorbance spectrum of the armpit t-shirt square of Red $H$ 


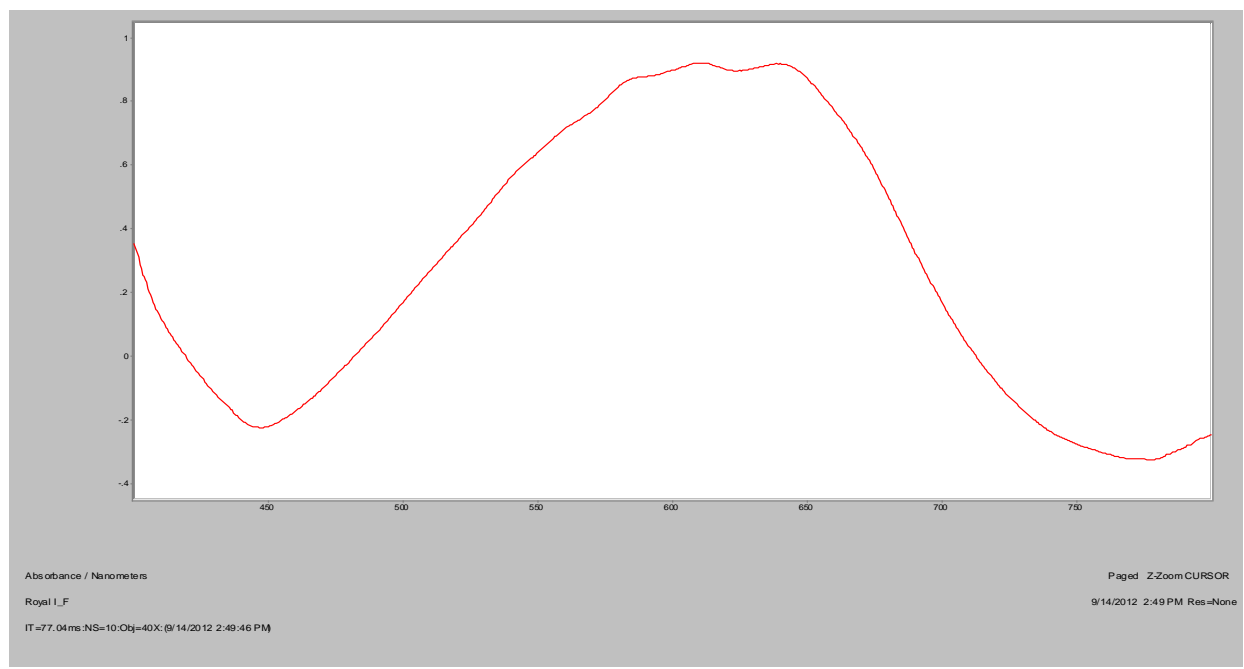

Absorbance spectrum of the front $t$-shirt square of Royal I

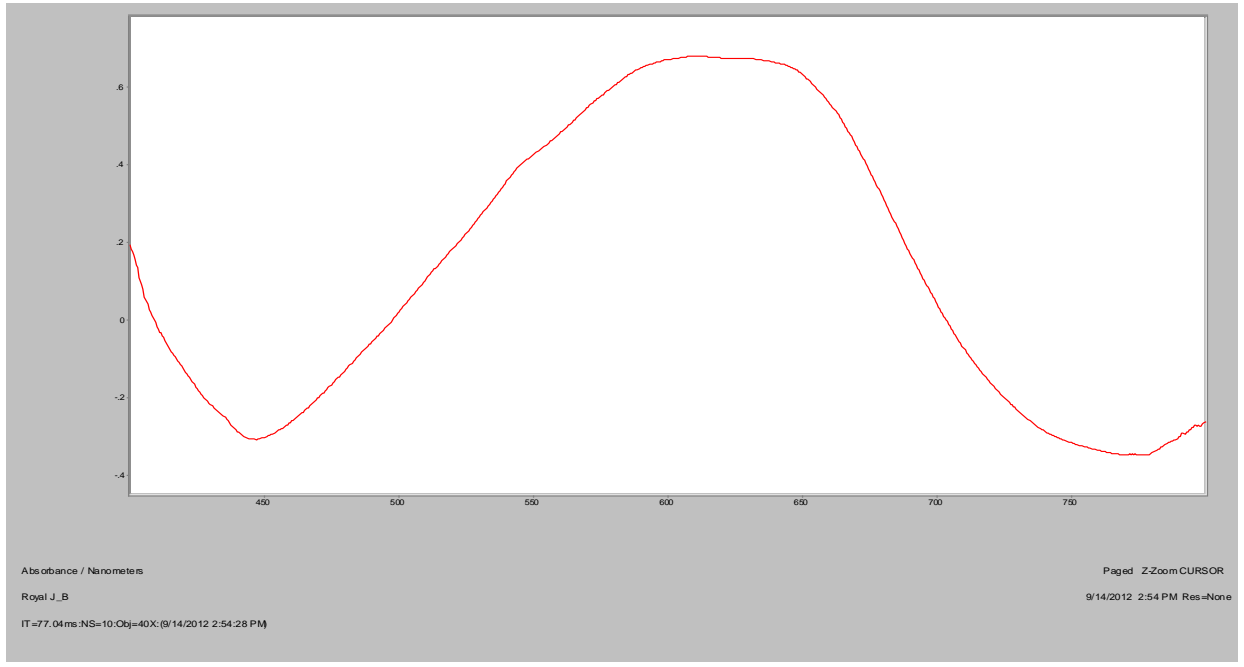

Absorbance spectrum of the back $t$-shirt square of Royal $J$ 


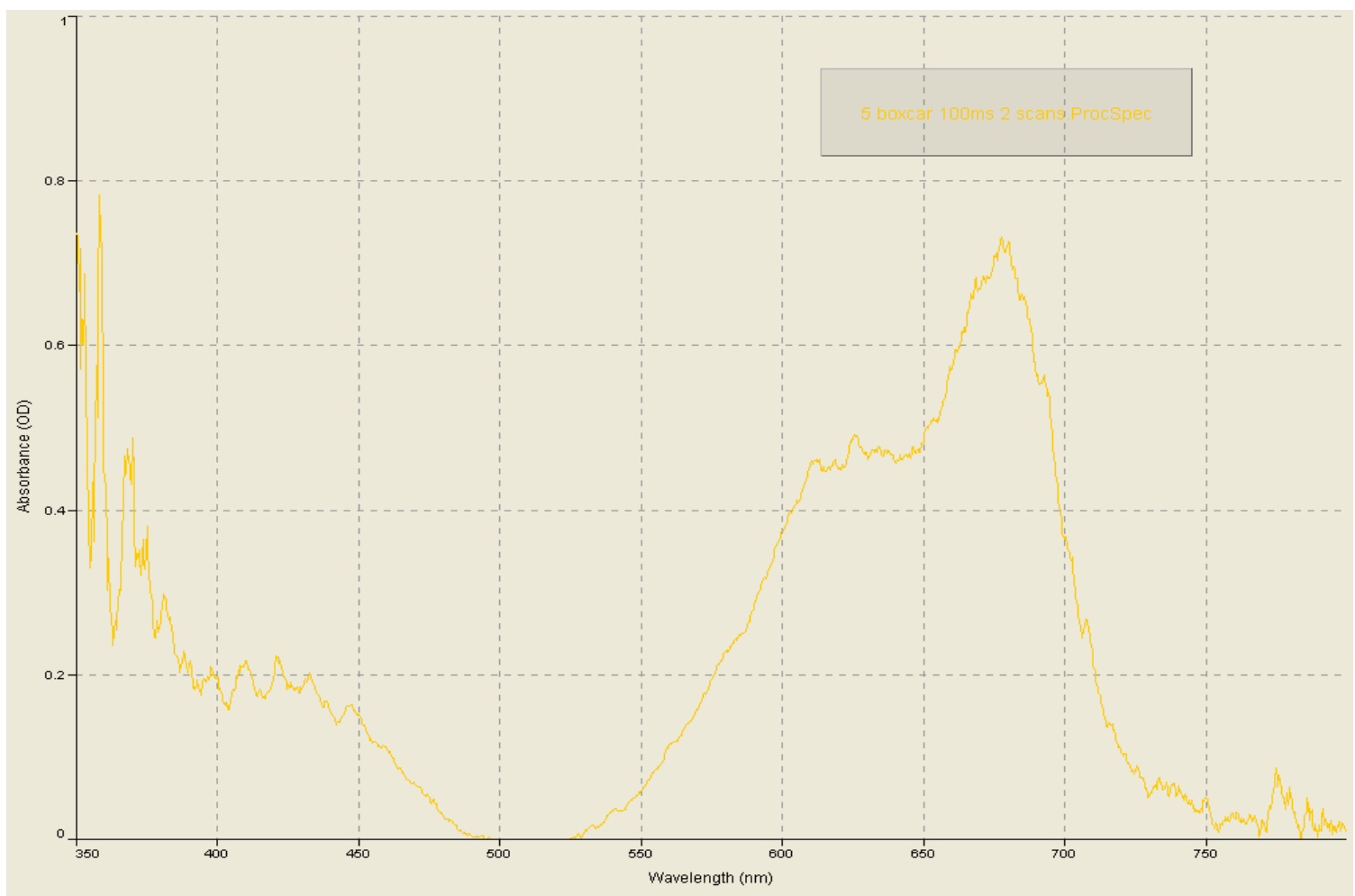

Absorbance spectrum of Kelly Green $A \_F$ when using an average scan of 2

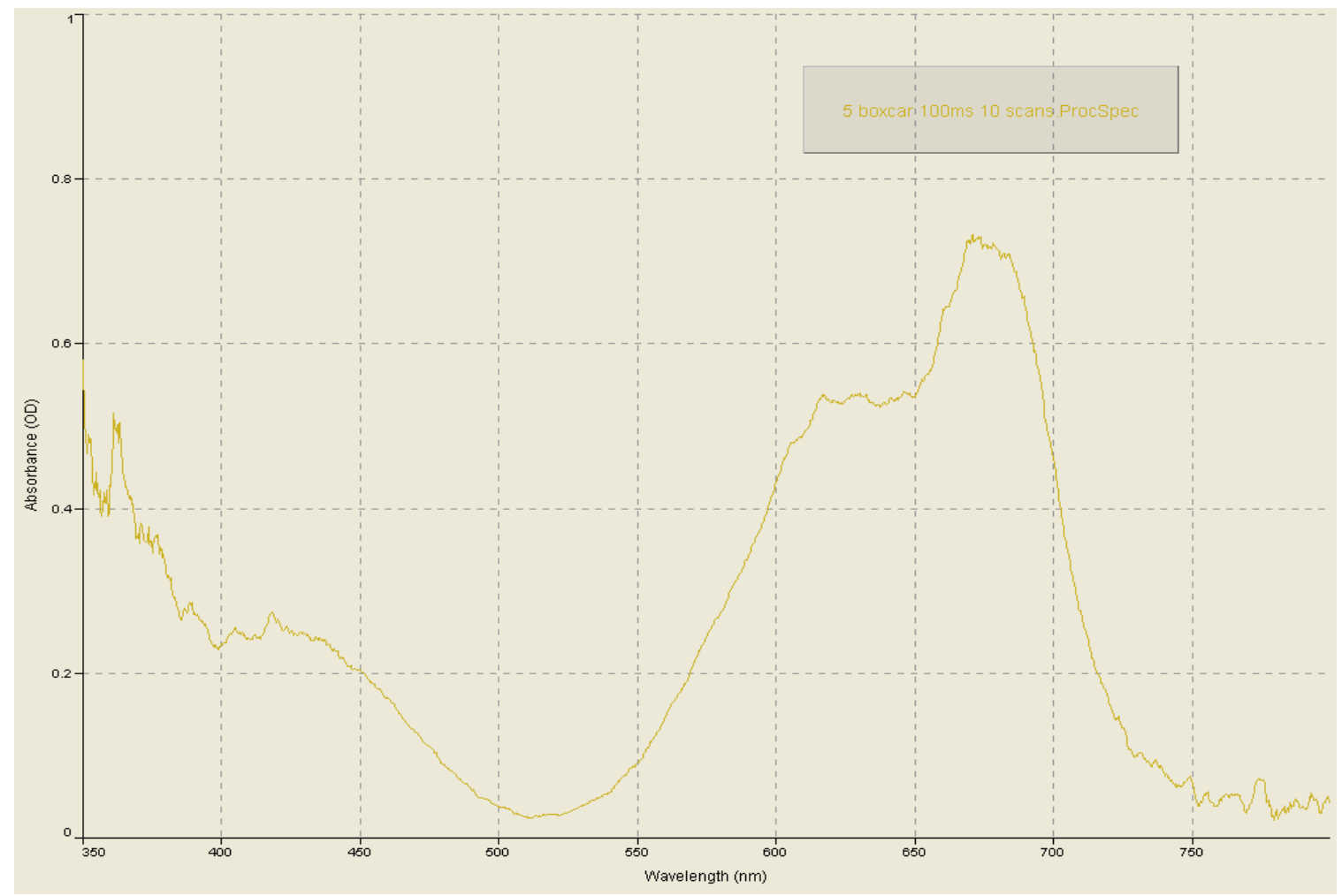

Absorbance spectrum of Kelly Green $A \_F$ when using an average scan of 10 


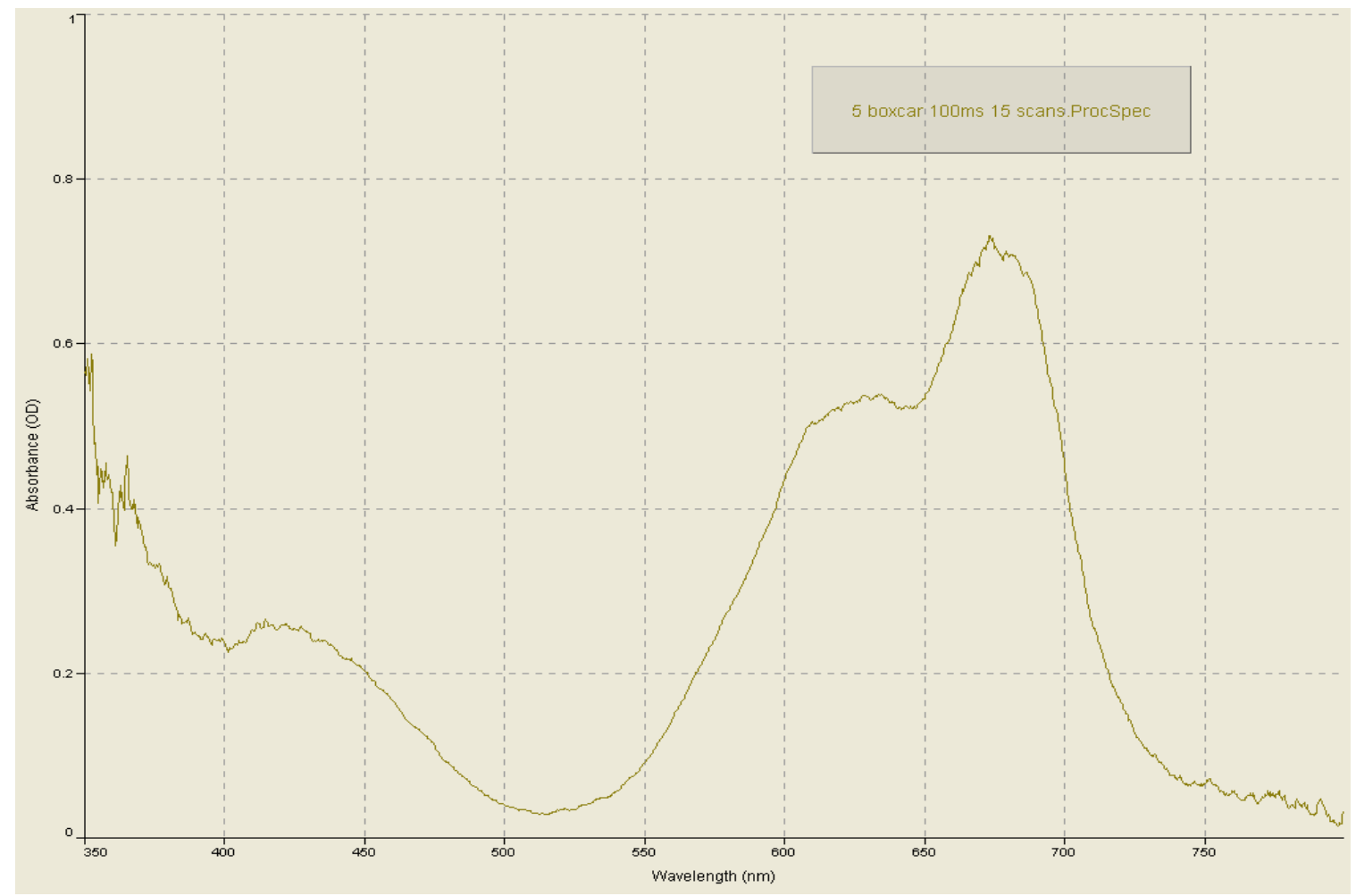

Absorbance spectrum of Kelly Green A_F when using an average scan of 15

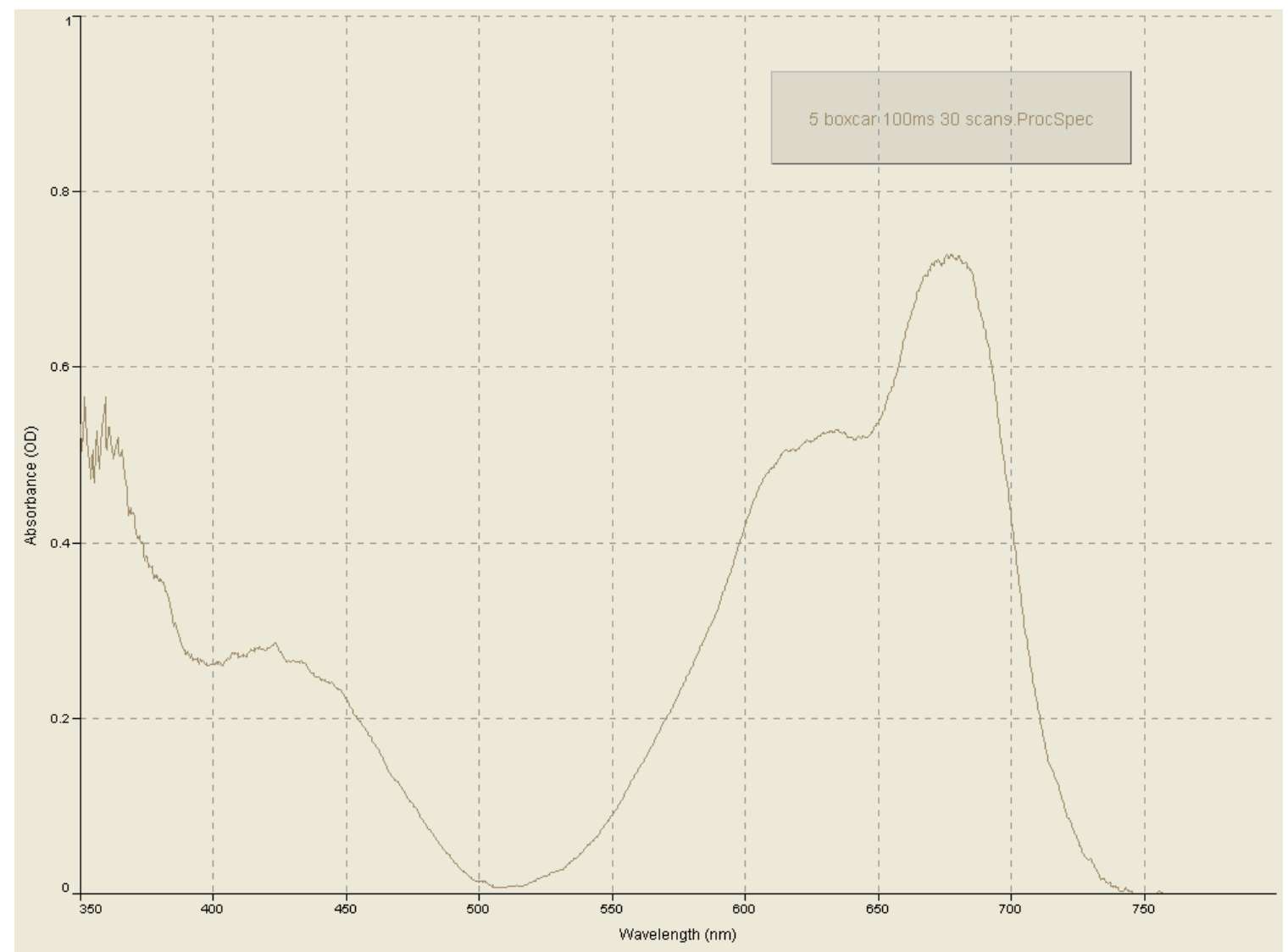

Absorbance spectrum of Kelly Green A_F when using an average scan of 30 


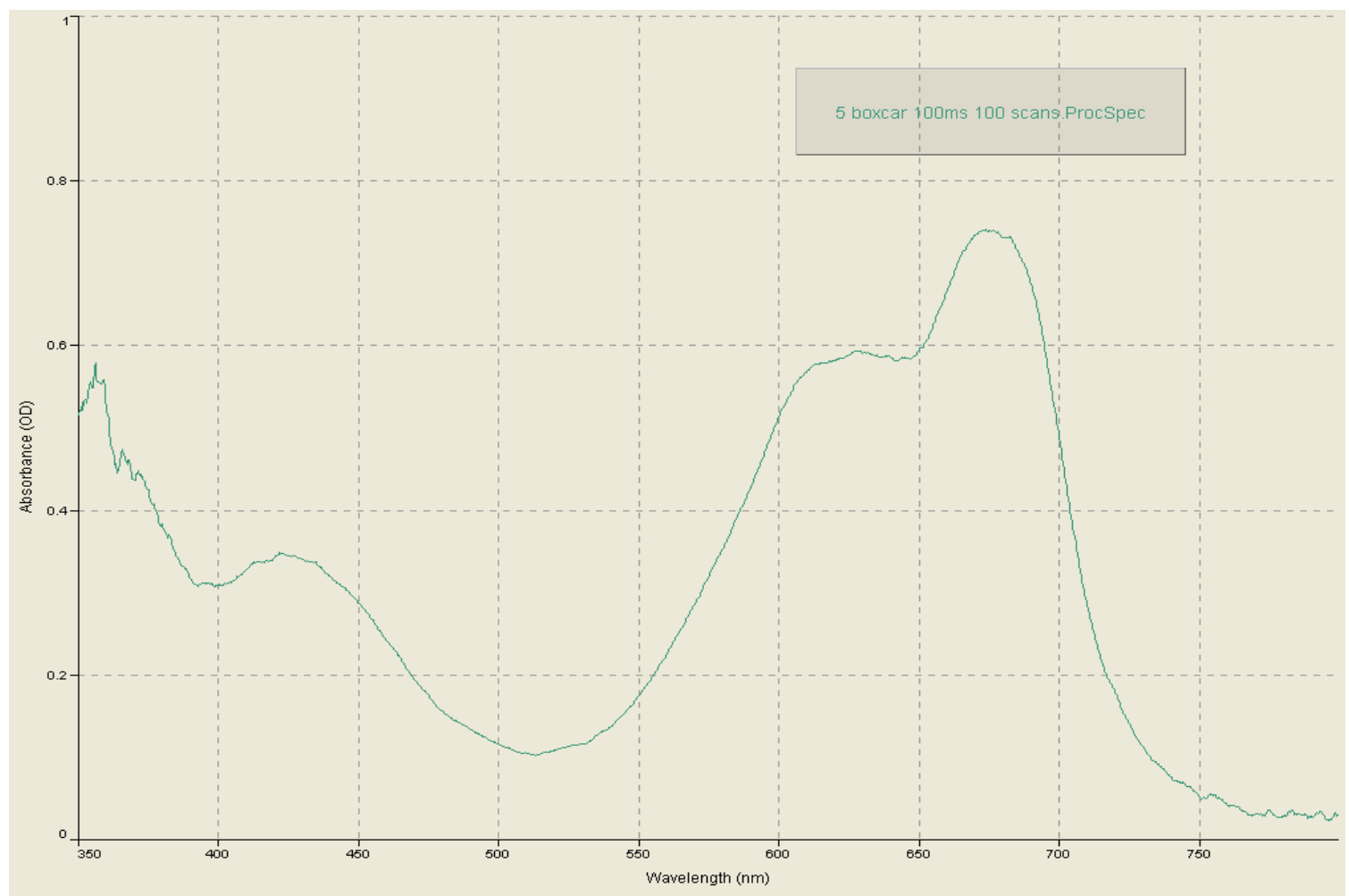

Absorbance spectrum of Kelly Green A_F when using an average scan of 100

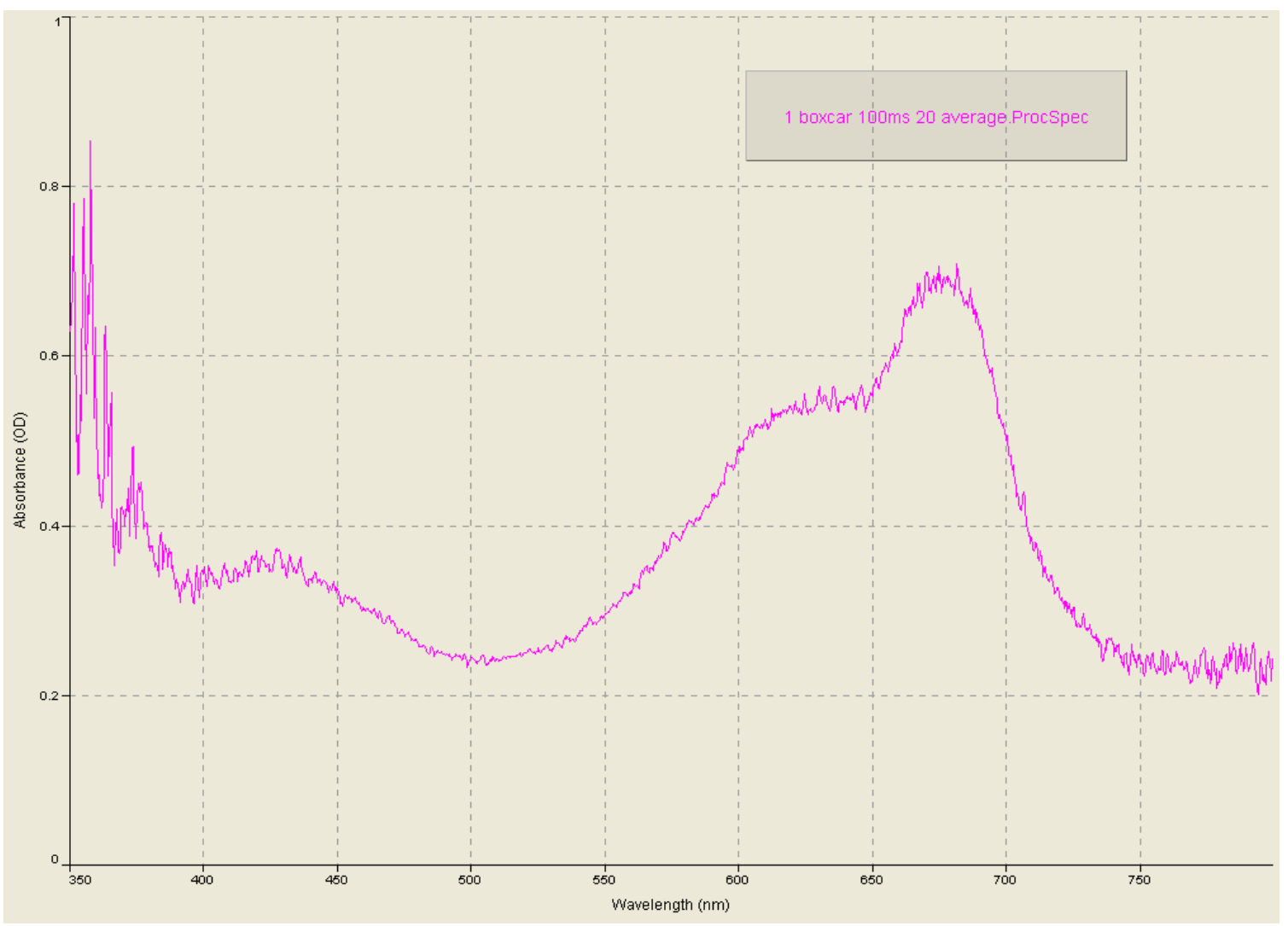

Absorbance spectrum of Kelly Green A_F when using a boxcar width of 1 


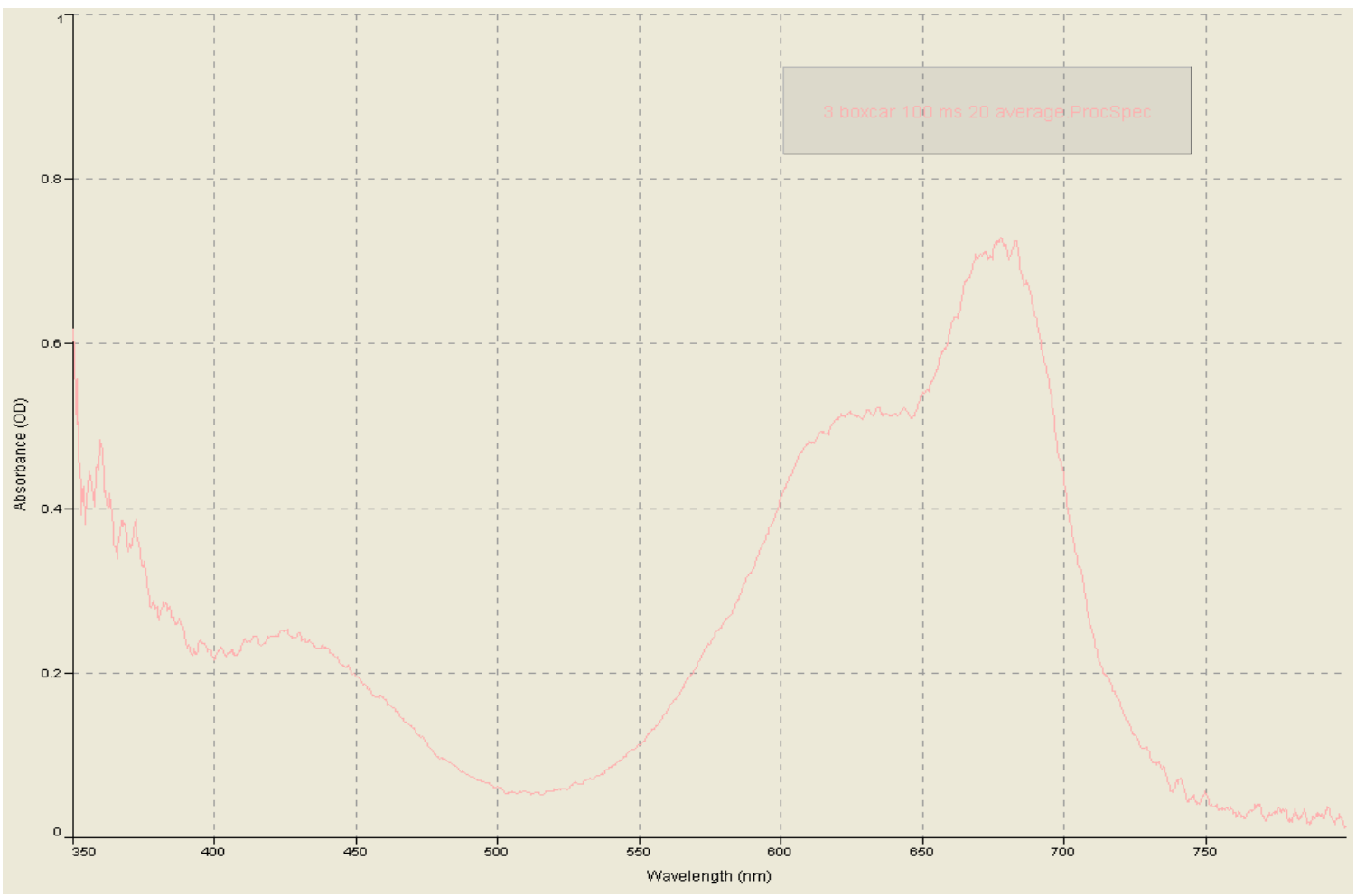

Absorbance spectrum of Kelly Green A_F when using a boxcar width of 3

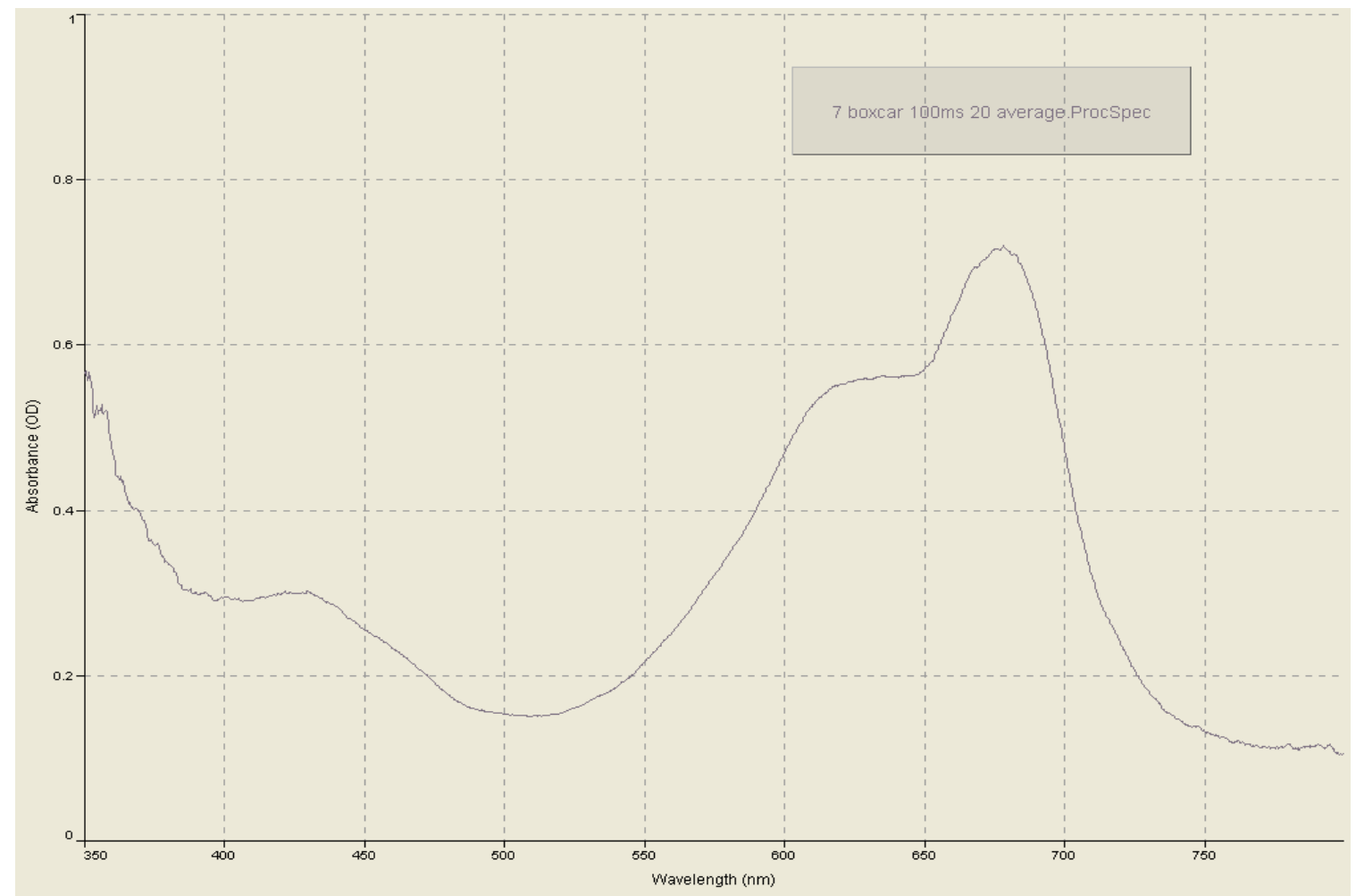

Absorbance spectrum of Kelly Green A_F when using a boxcar width of 7 


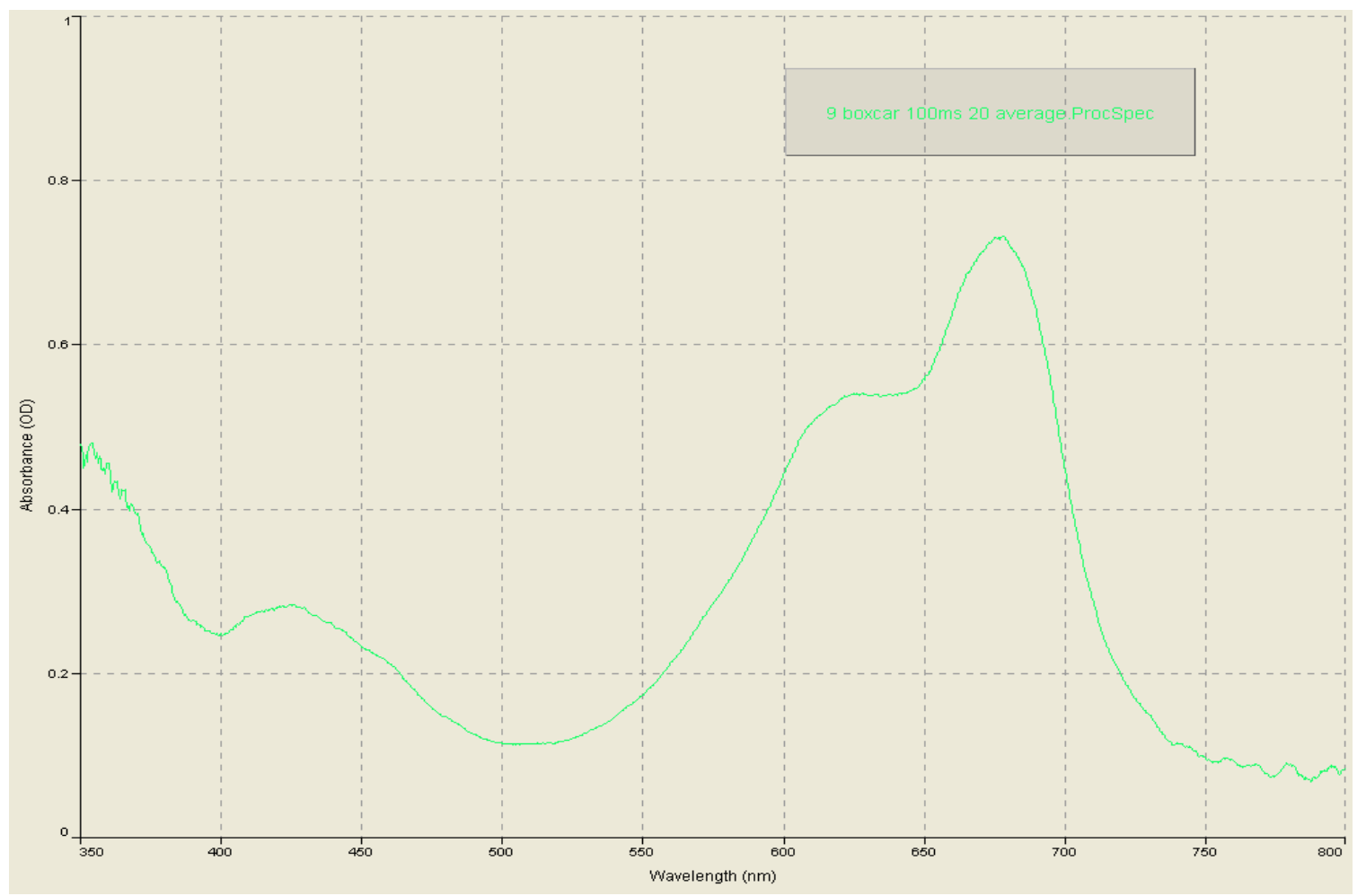

Absorbance spectrum of Kelly Green A_F when using a boxcar width of 9

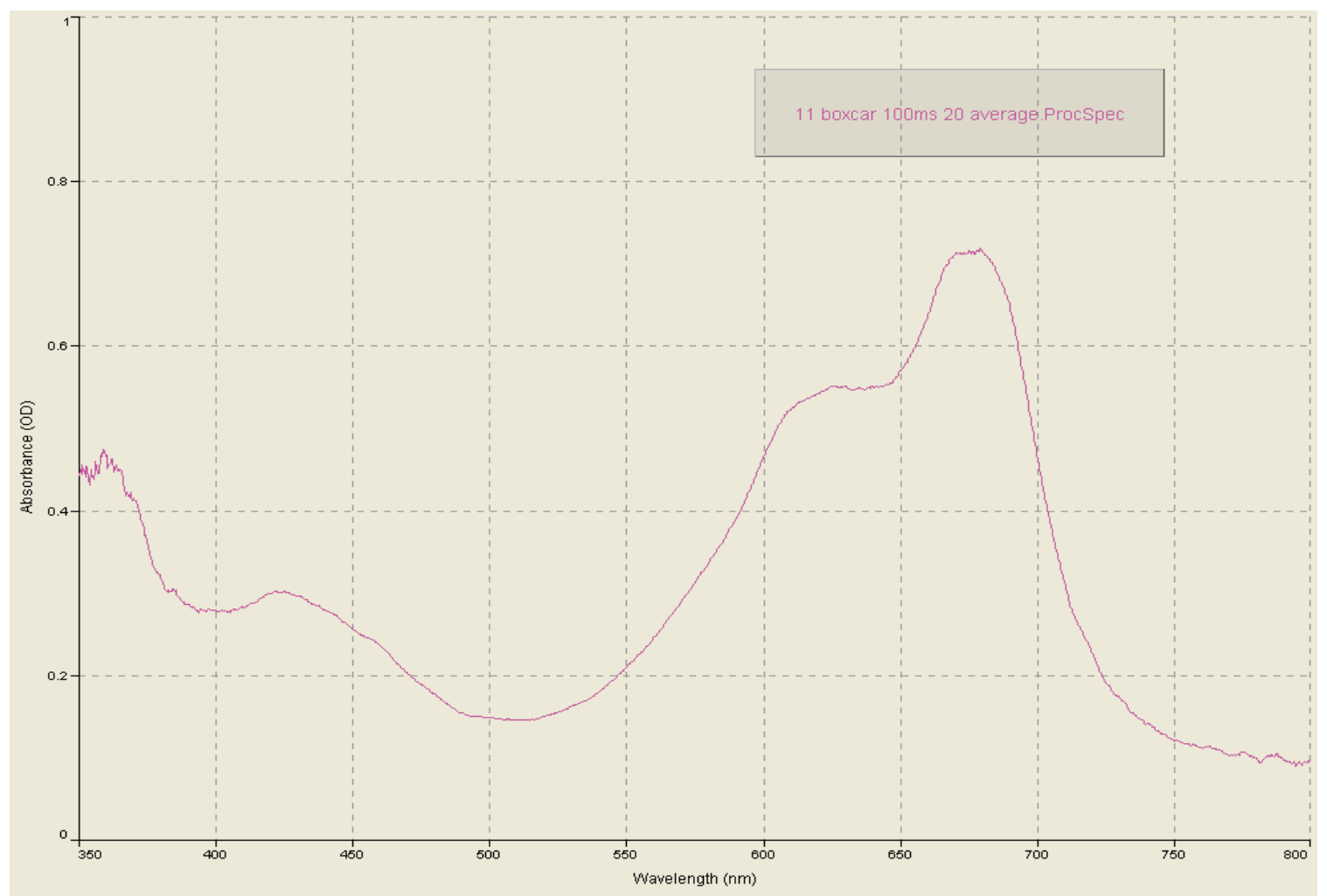

Absorbance spectrum of Kelly Green A_F when using a boxcar width of 11 


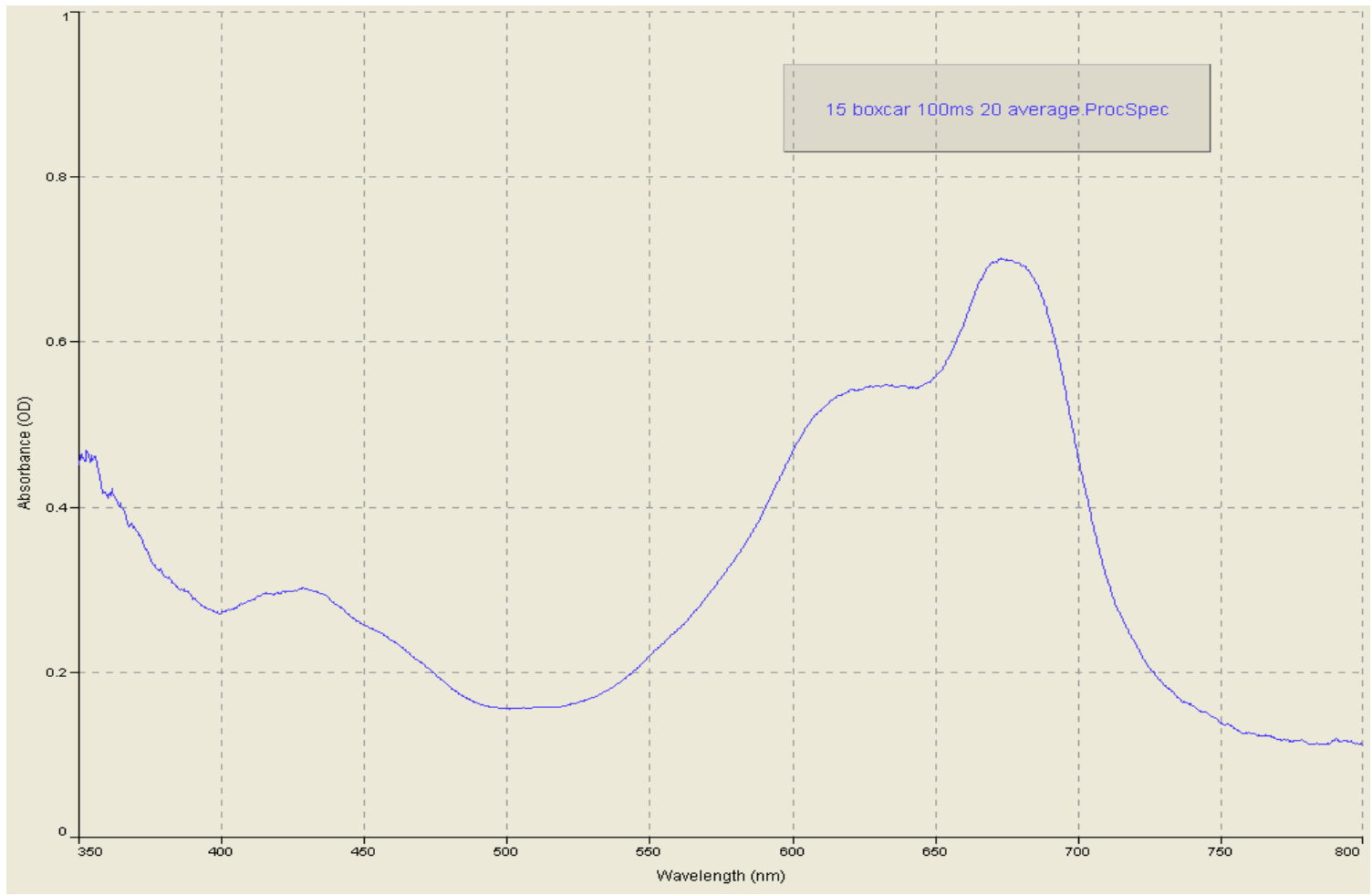

Absorbance spectrum of Kelly Green A_F when using a boxcar width of $15 \mathrm{~ms}$

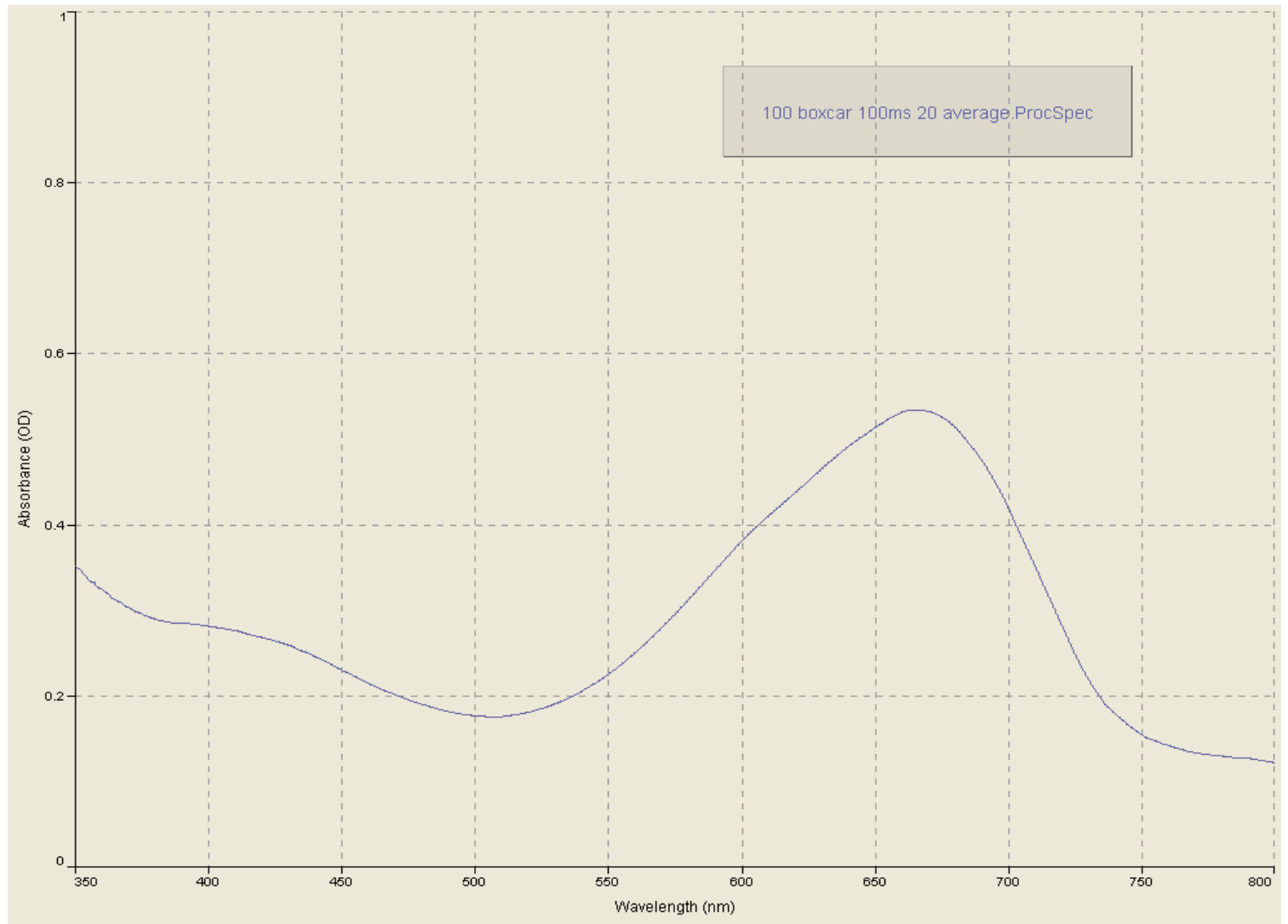

Absorbance spectrum of Kelly Green A_F when using a boxcar width of $100 \mathrm{~ms}$ 


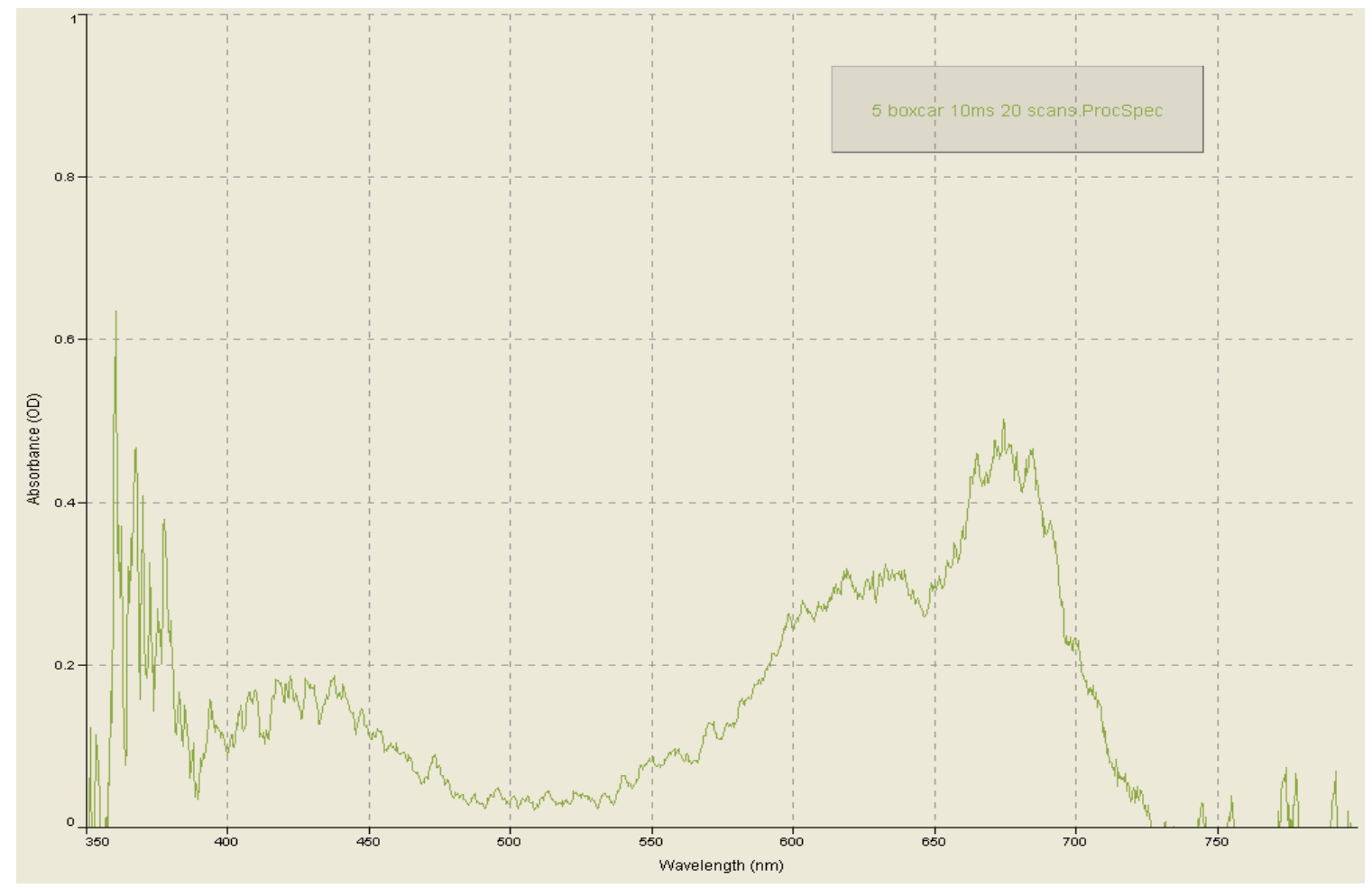

Absorbance spectrum of Kelly Green $A \_F$ when using an integration time of $10 \mathrm{~ms}$

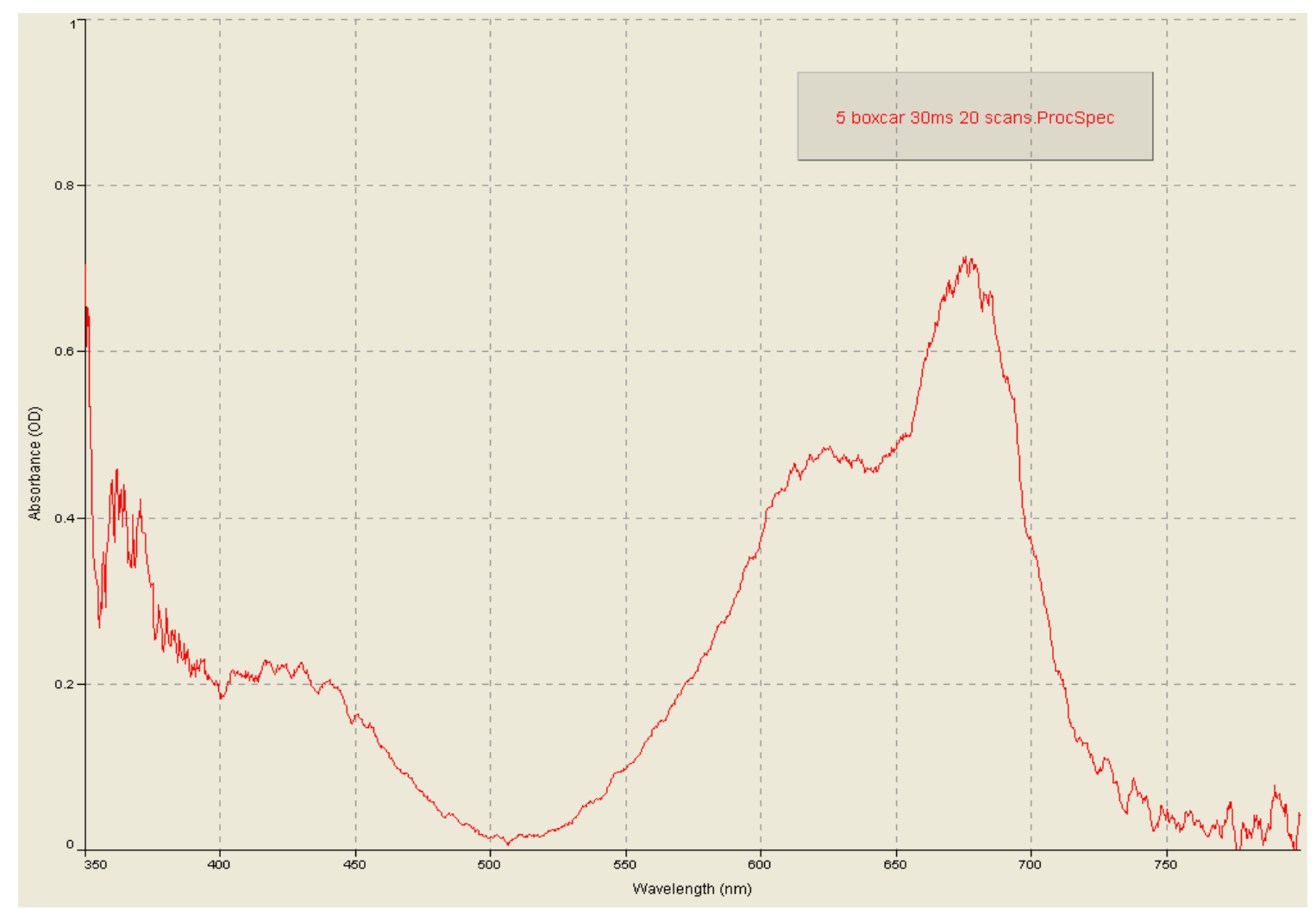

Absorbance spectrum of Kelly Green A_F when using an integration time of $30 \mathrm{~ms}$ 


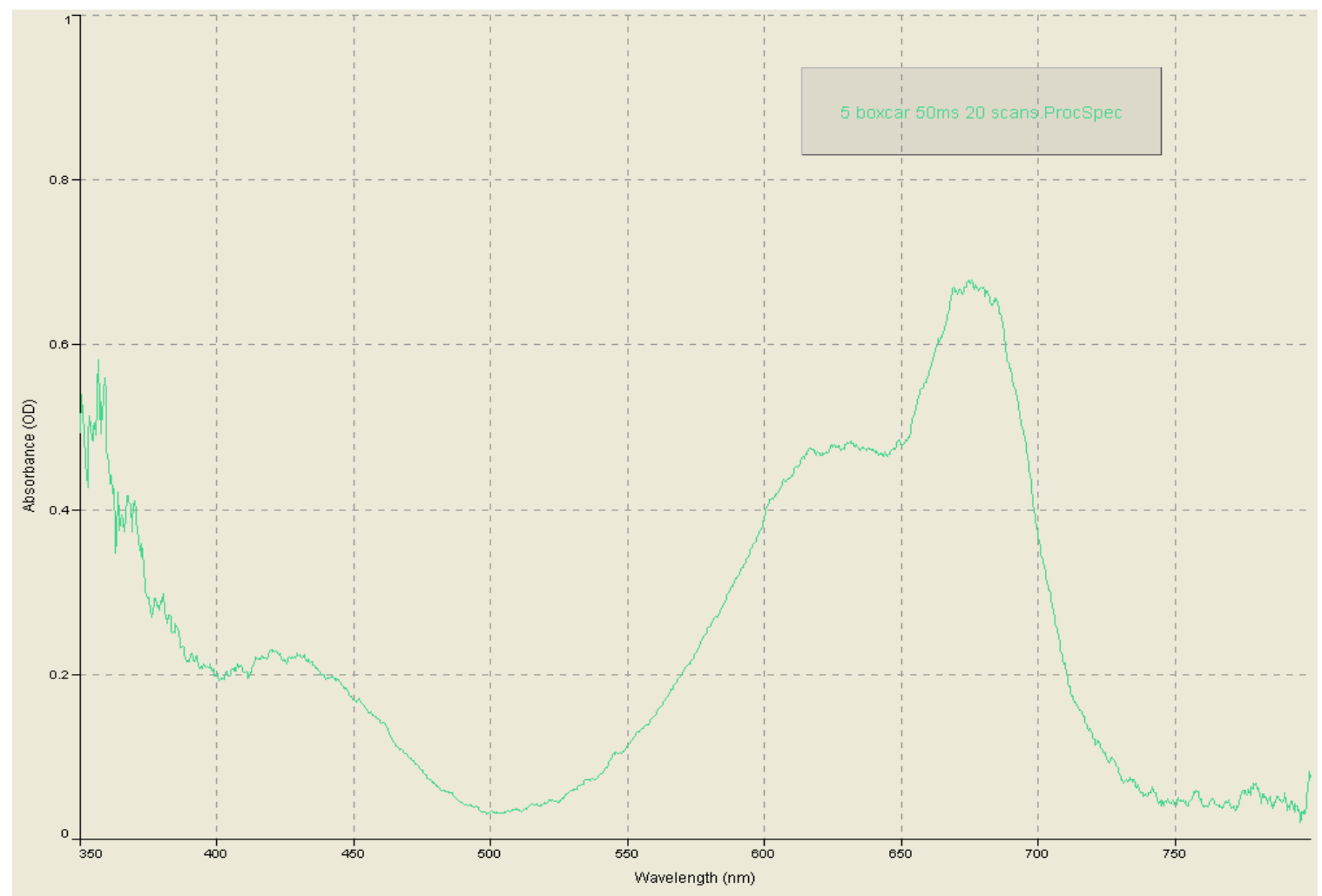

Absorbance spectrum of Kelly Green A_F when using an integration time of $50 \mathrm{~ms}$

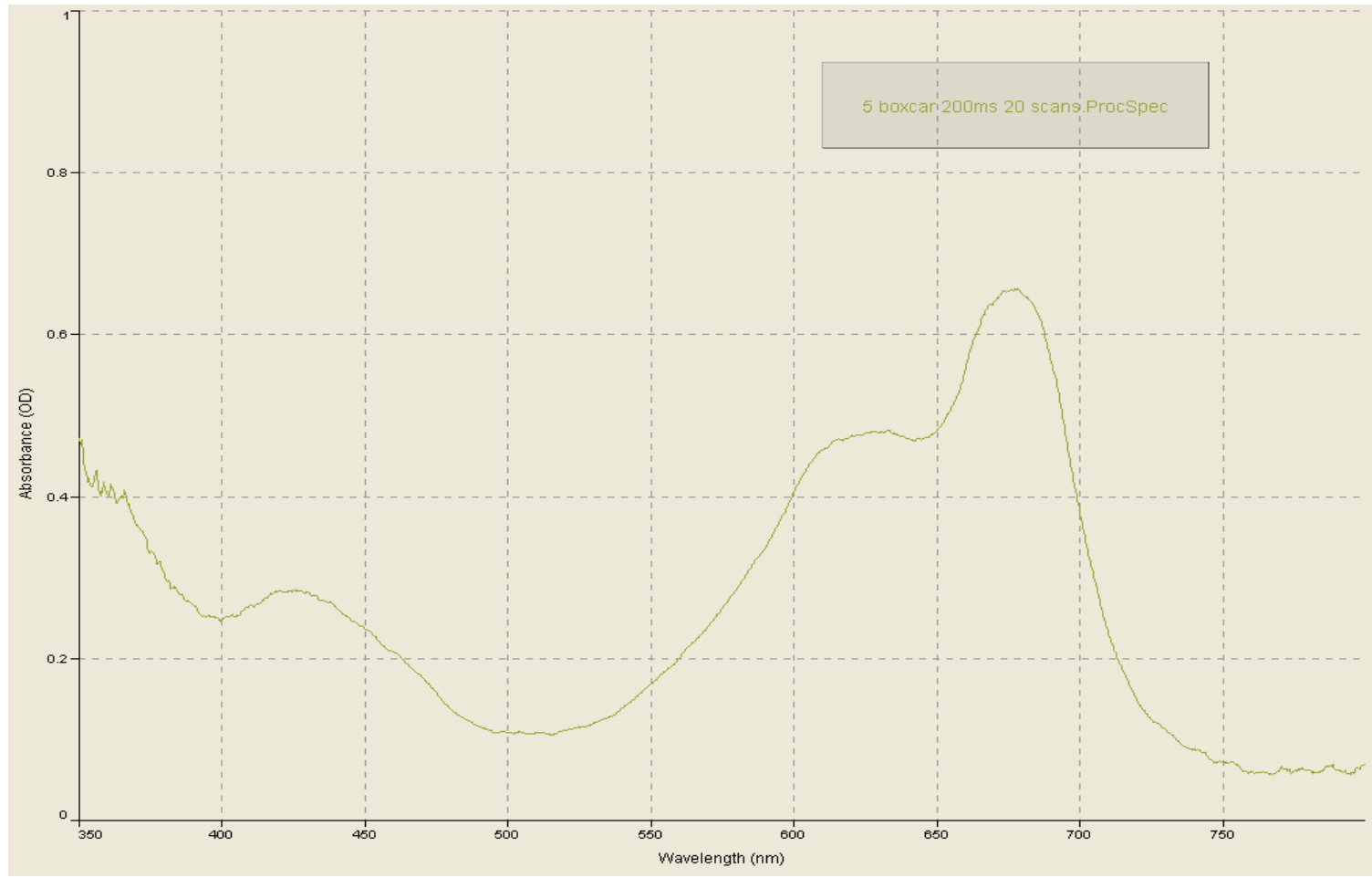

Absorbance spectrum of Kelly Green A_F when using an integration time of $200 \mathrm{~ms}$ 


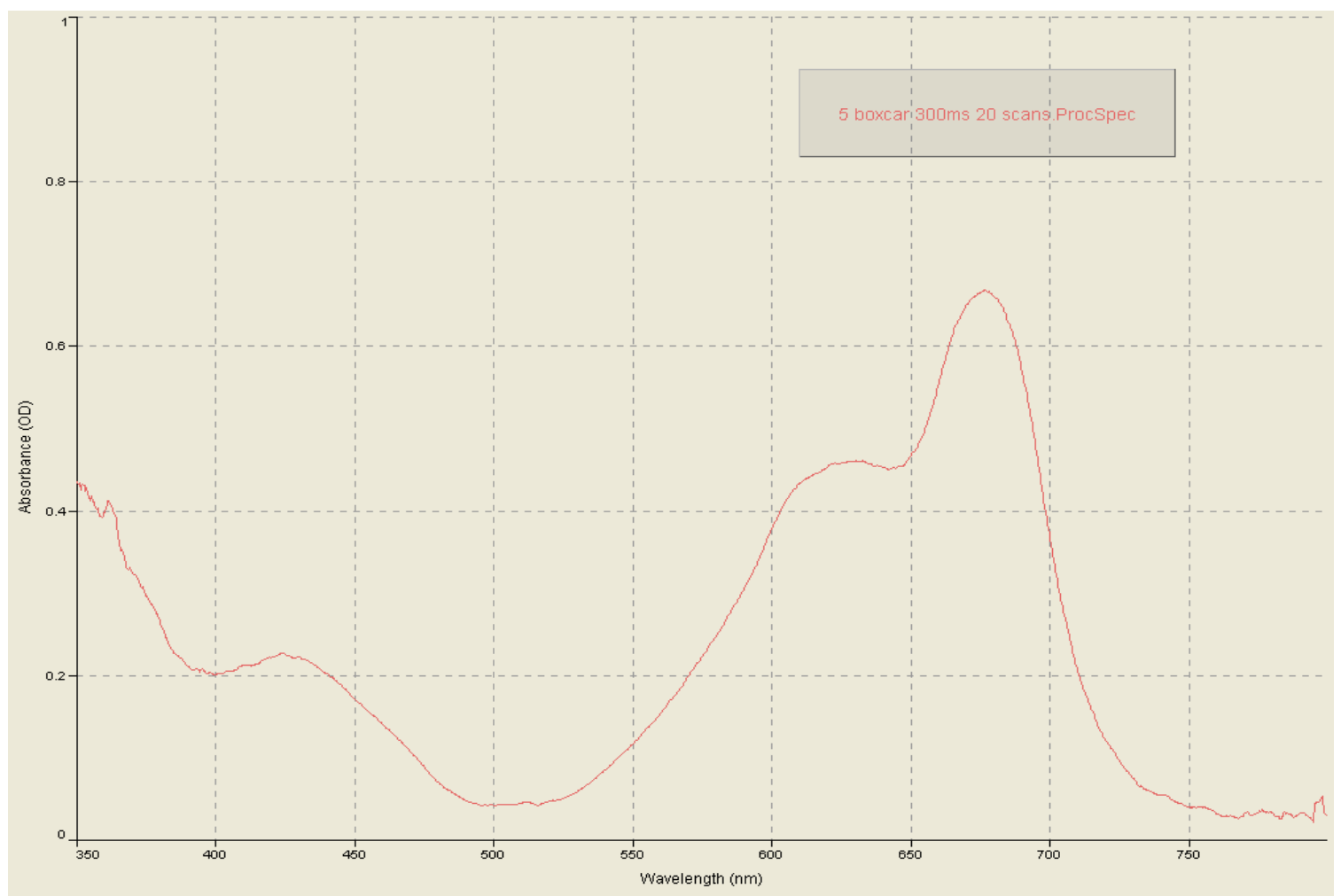

Absorbance spectrum of Kelly Green A_F when using an integration time of $300 \mathrm{~ms}$

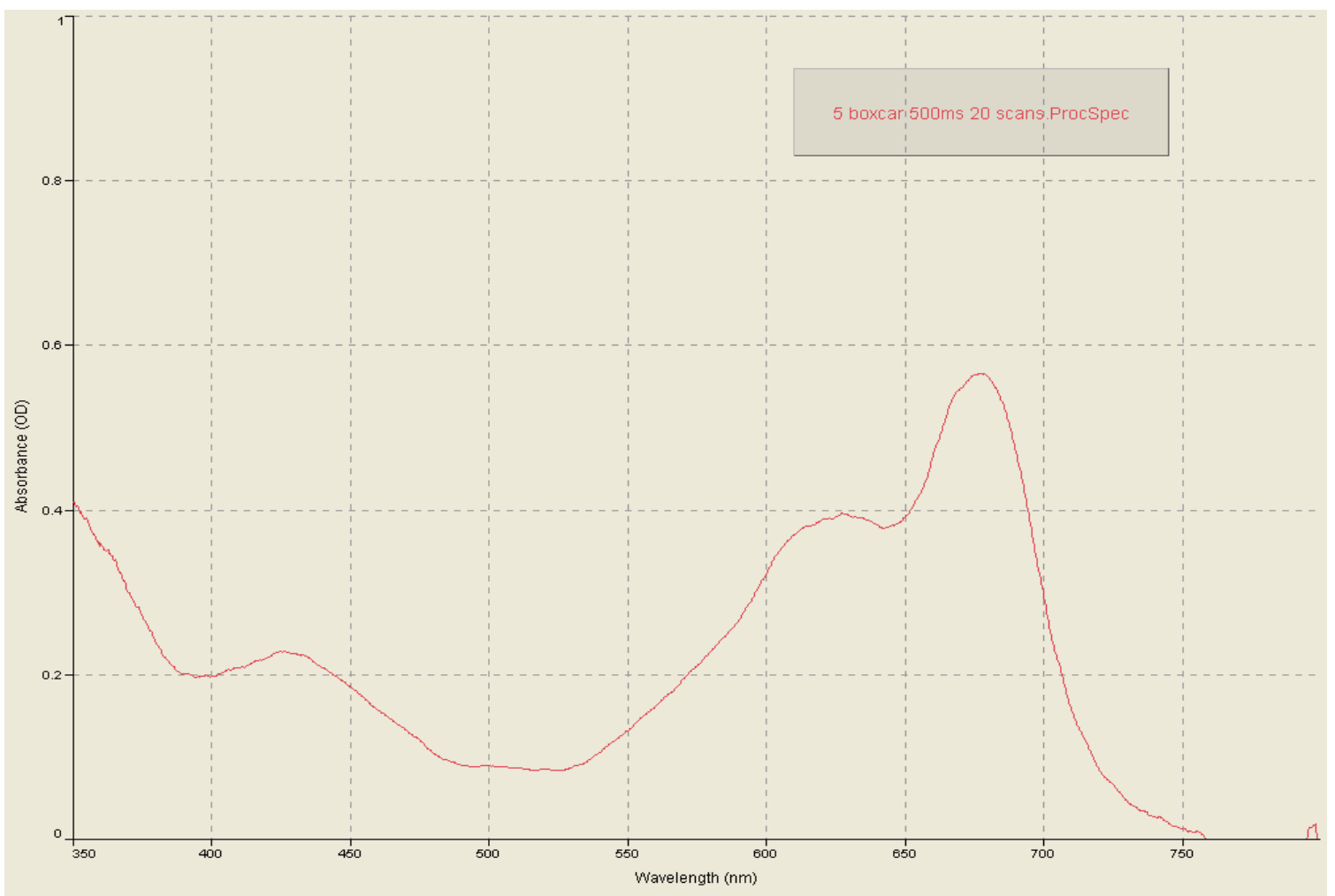

Absorbance spectrum of Kelly Green A_F when using an integration time of $500 \mathrm{~ms}$ 


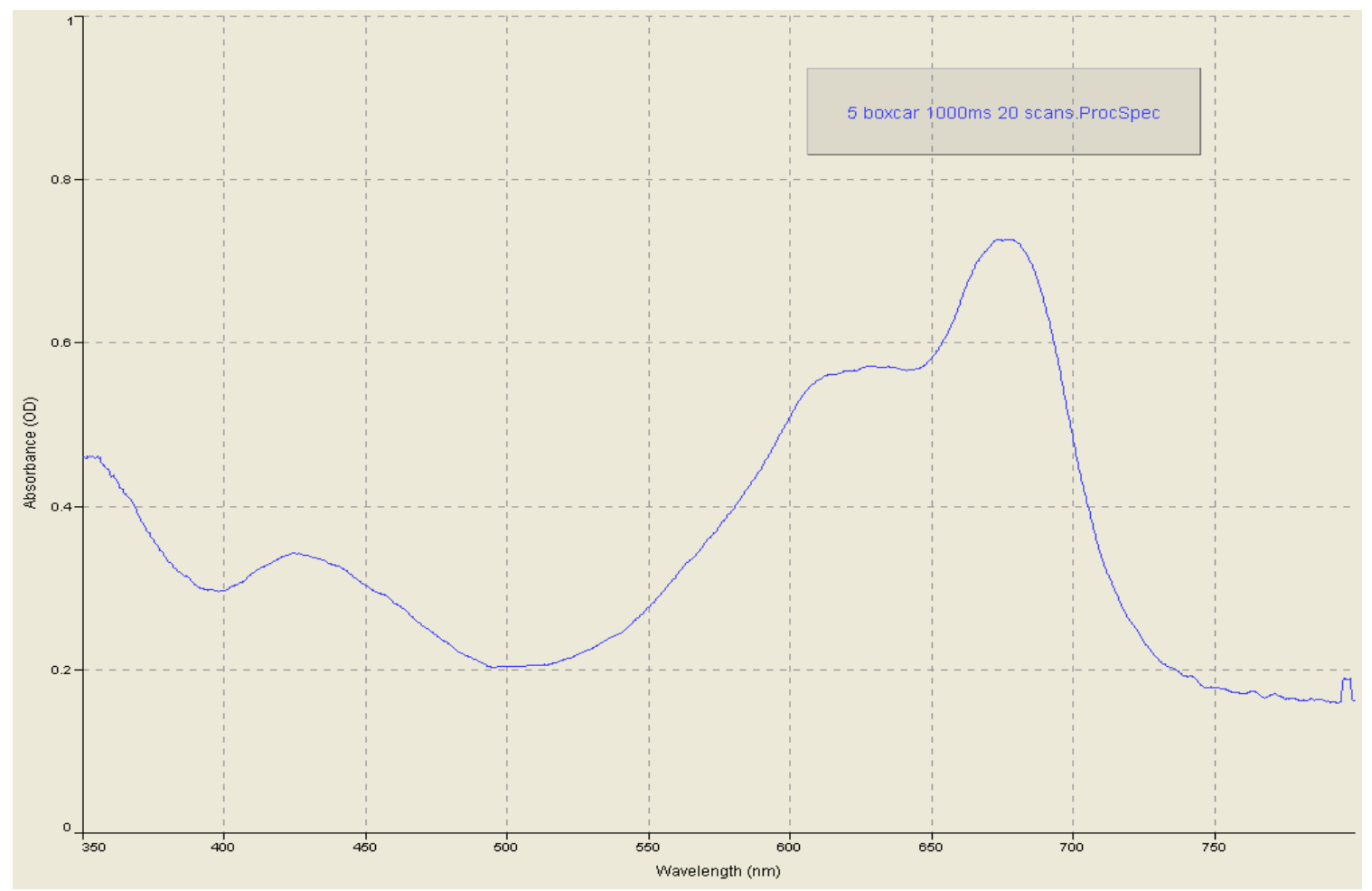

Absorbance spectrum of Kelly Green A_F when using an integration time of $1000 \mathrm{~ms}$

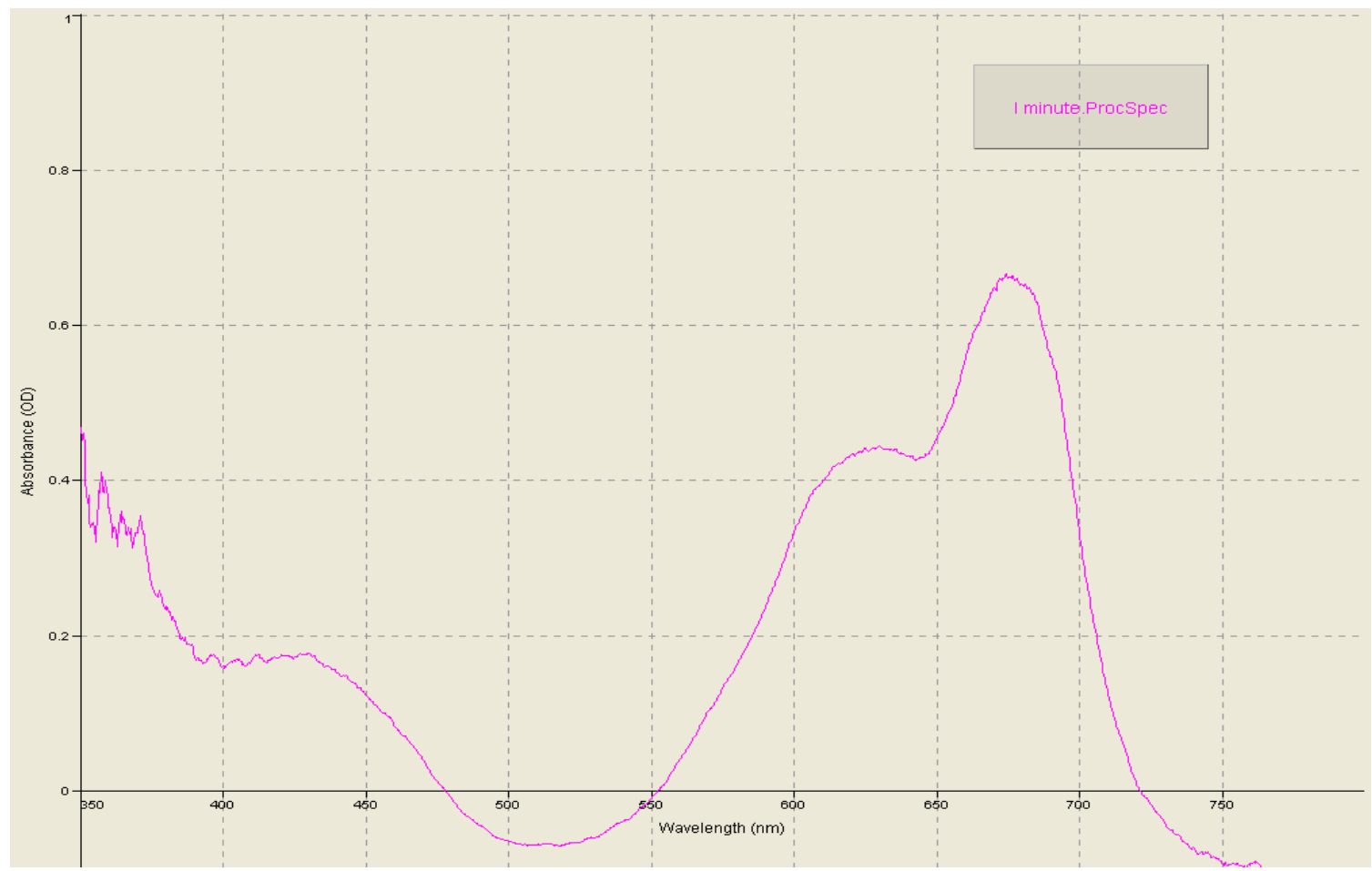

Absorbance spectrum of Kelly Green A_F after 1 minute in Part 1 of Phase III 


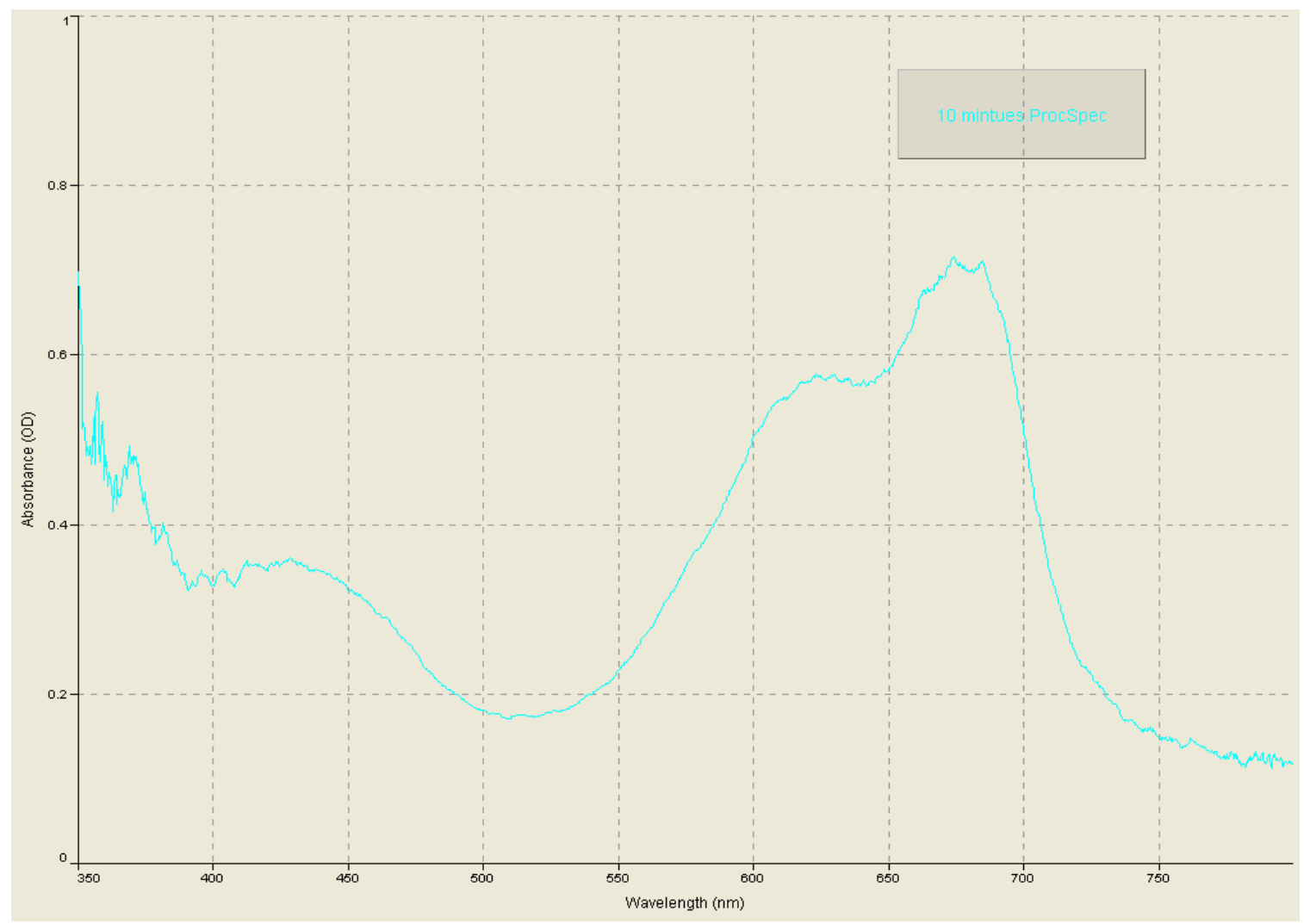

Absorbance spectrum of Kelly Green A_F after 10 minutes in Part 1 of Phase III

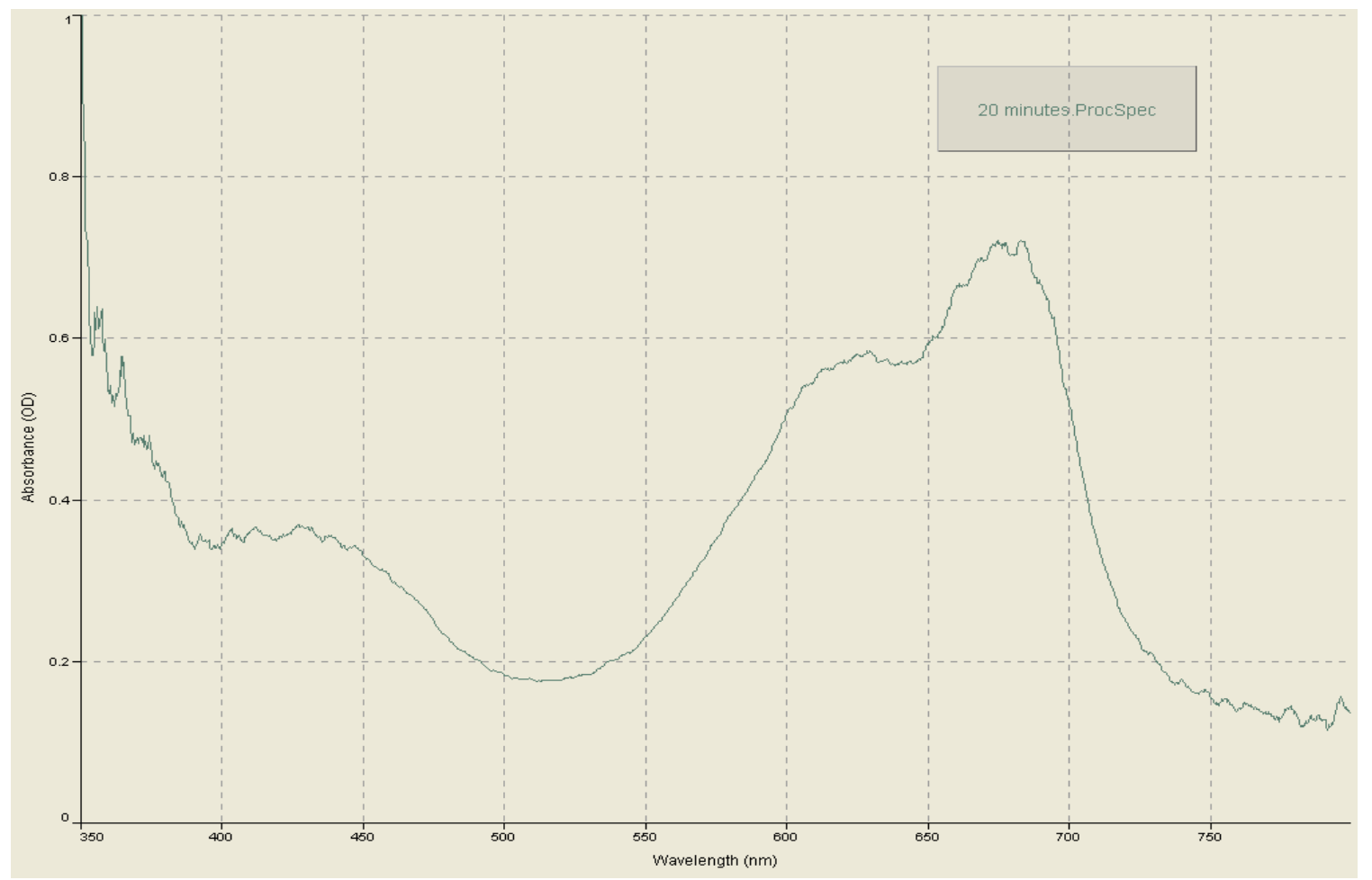

Absorbance spectrum of Kelly Green A_F after 20 minutes in Part 1 of Phase III 


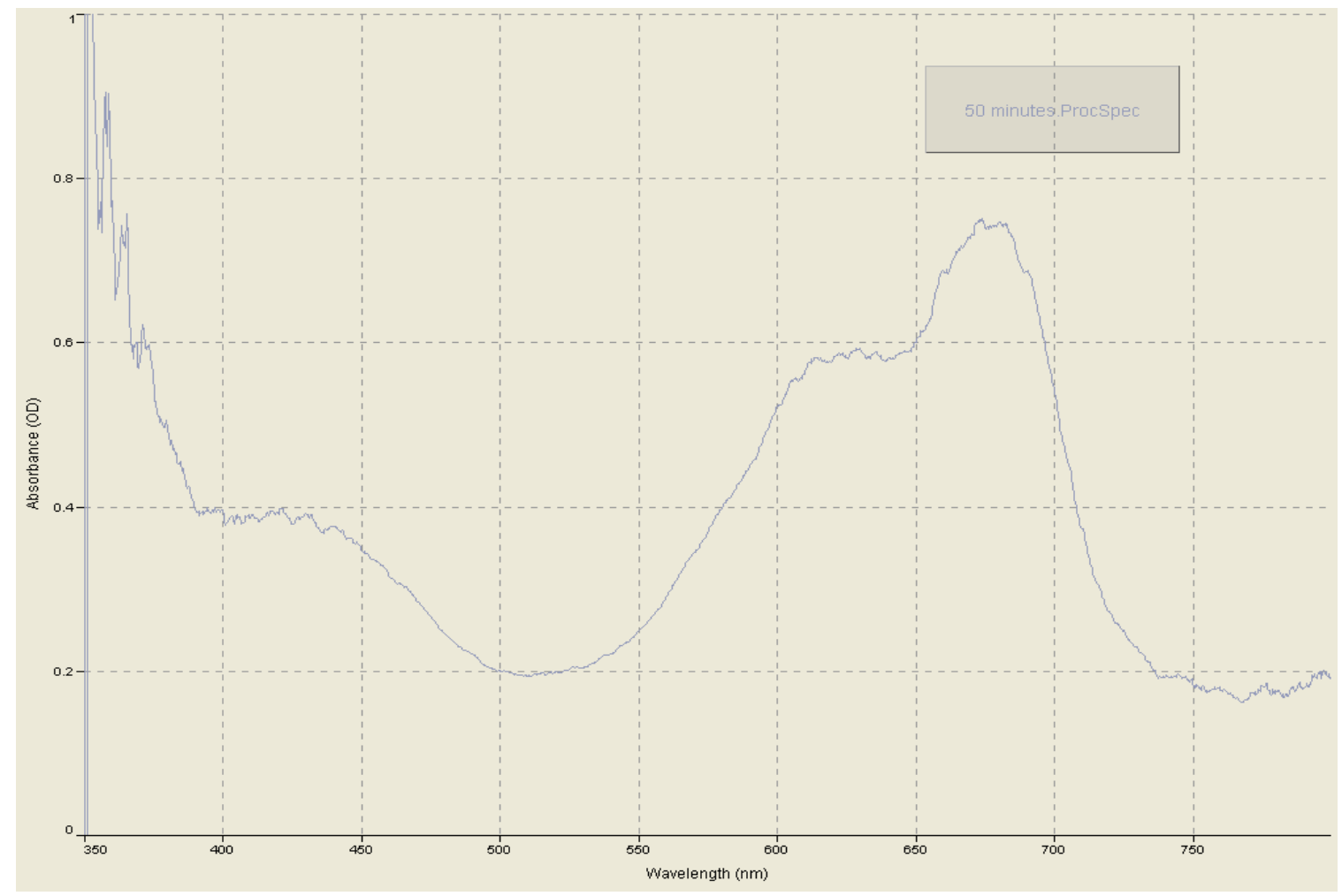

Absorbance spectrum of Kelly Green A_F after 50 minutes in Part 1 of Phase III

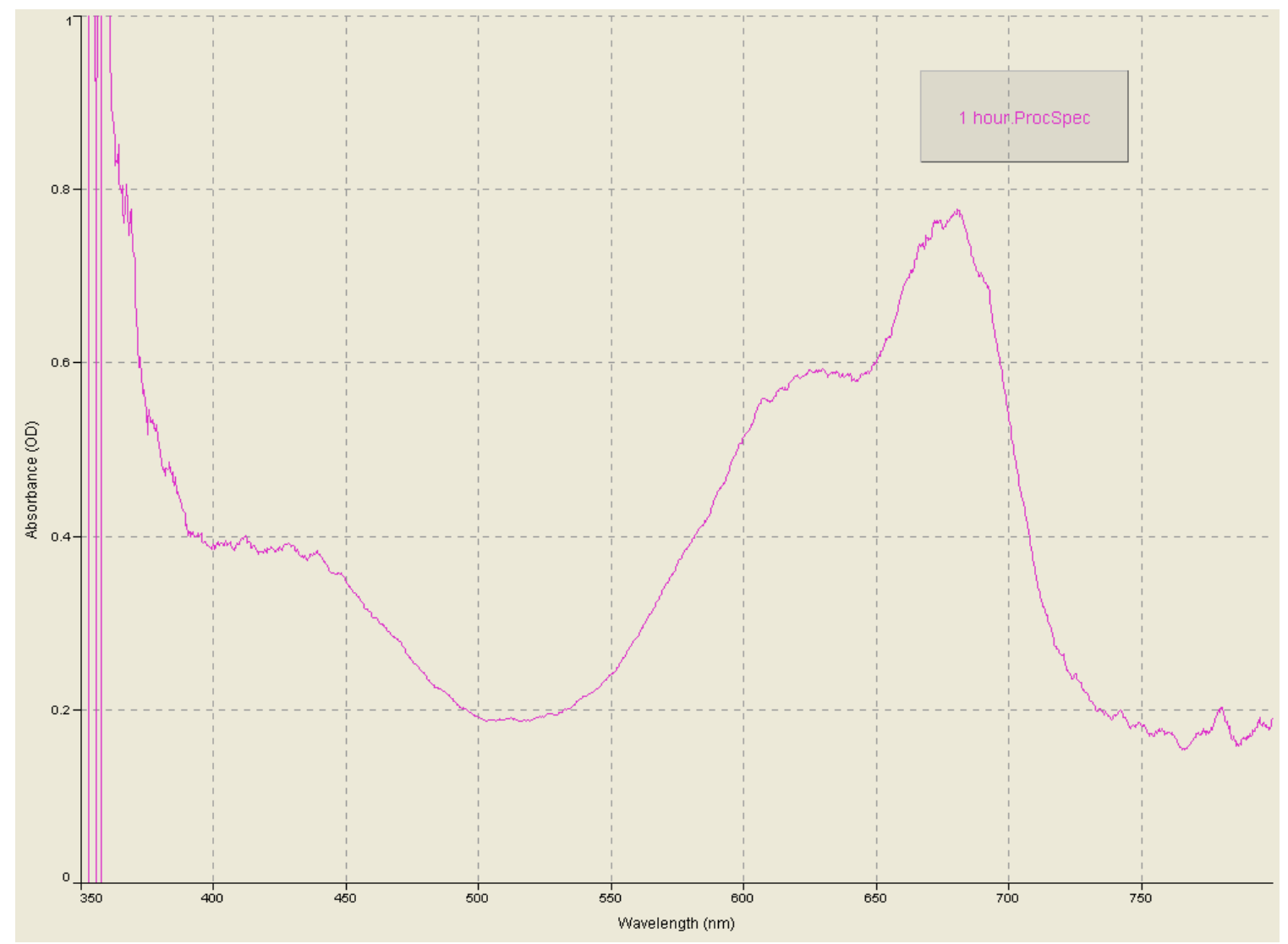

Absorbance spectrum of Kelly Green A_F after 1 hour in Part 1 of Phase III 


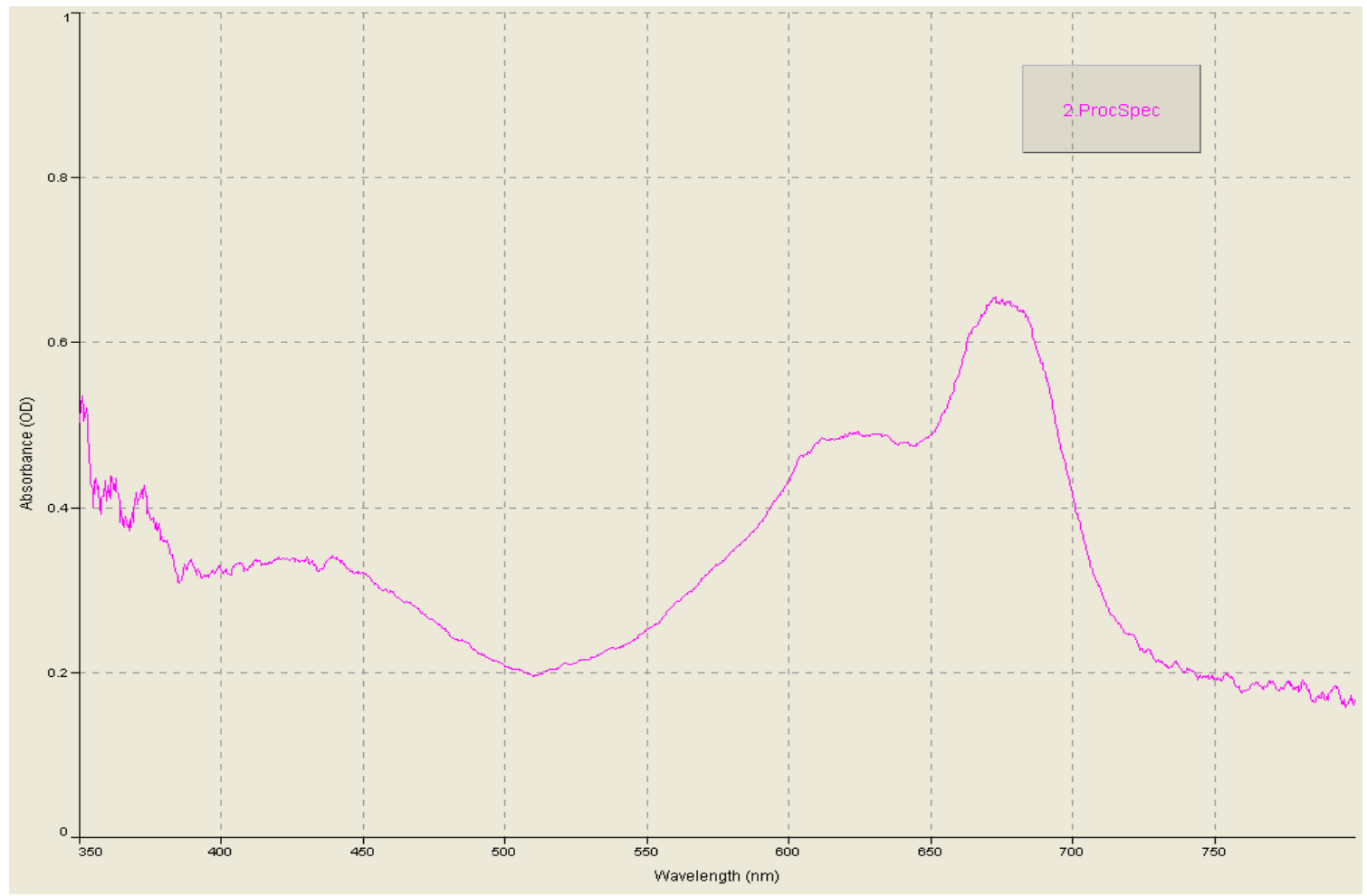

Absorbance spectrum of Kelly Green A_F after 2 minutes in Part II of Phase III

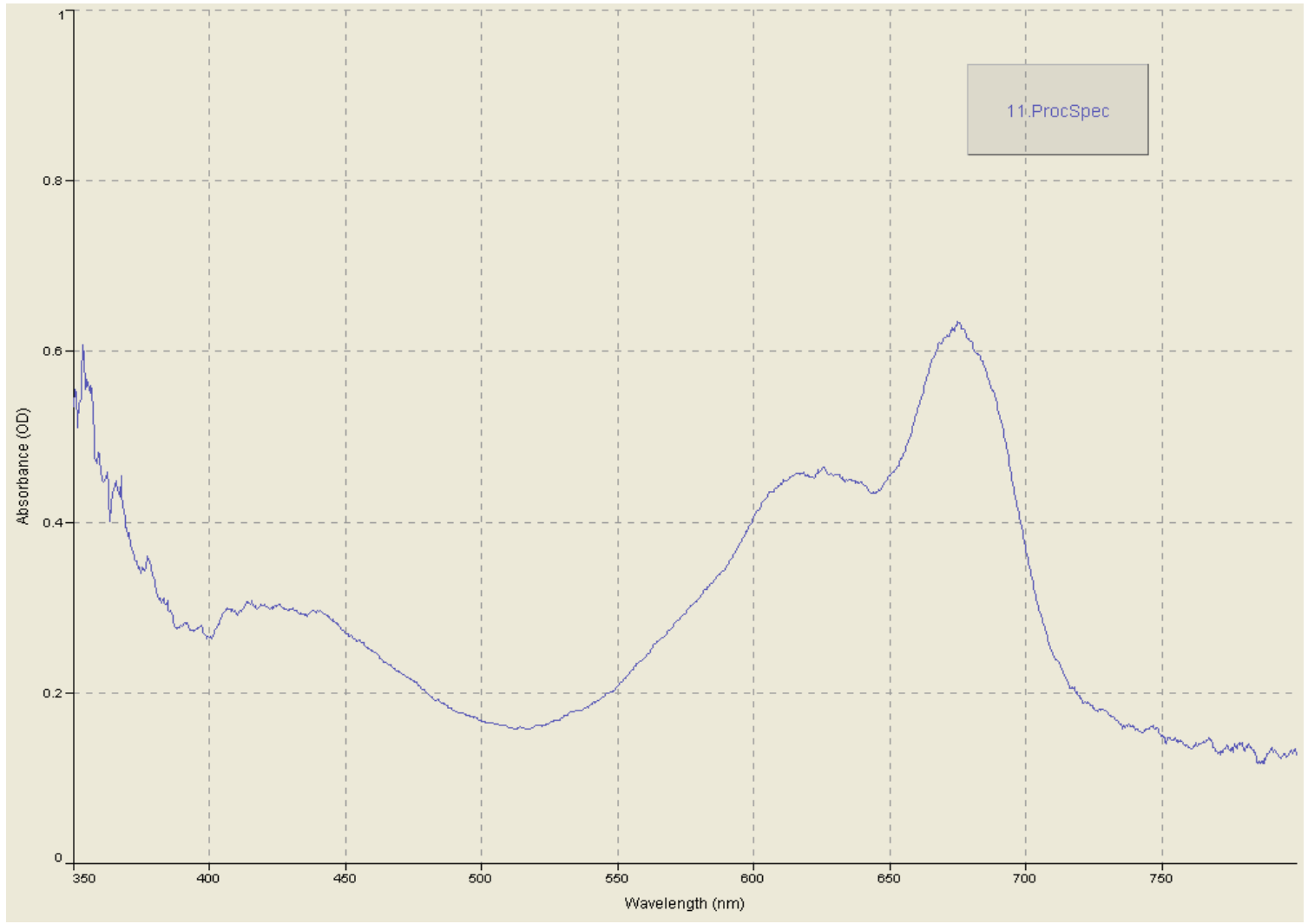

Absorbance spectrum of Kelly Green A_F after 11 minutes in Part II of Phase III 


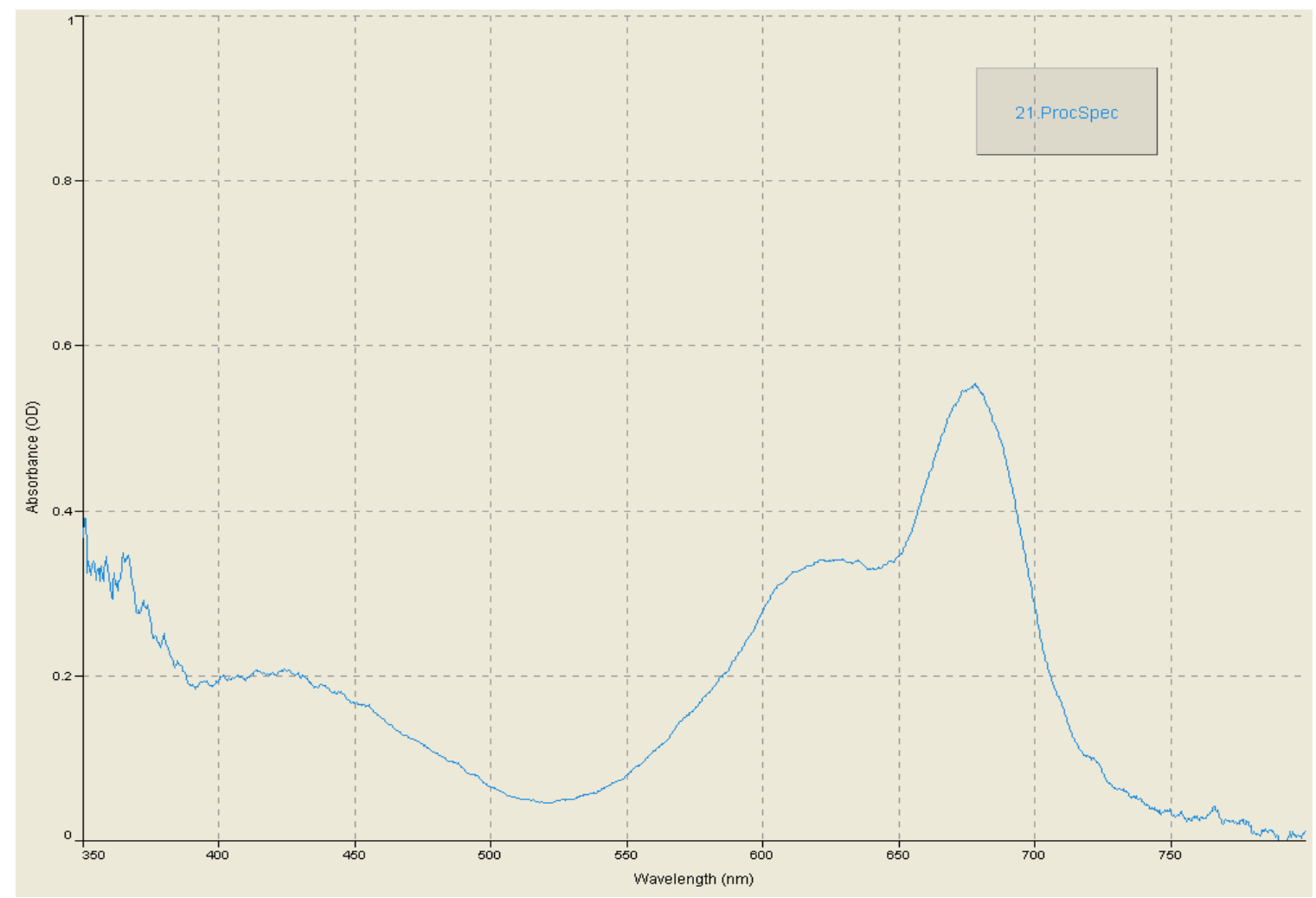

Absorbance spectrum of Kelly Green A_F after 21 minutes in Part II of Phase III

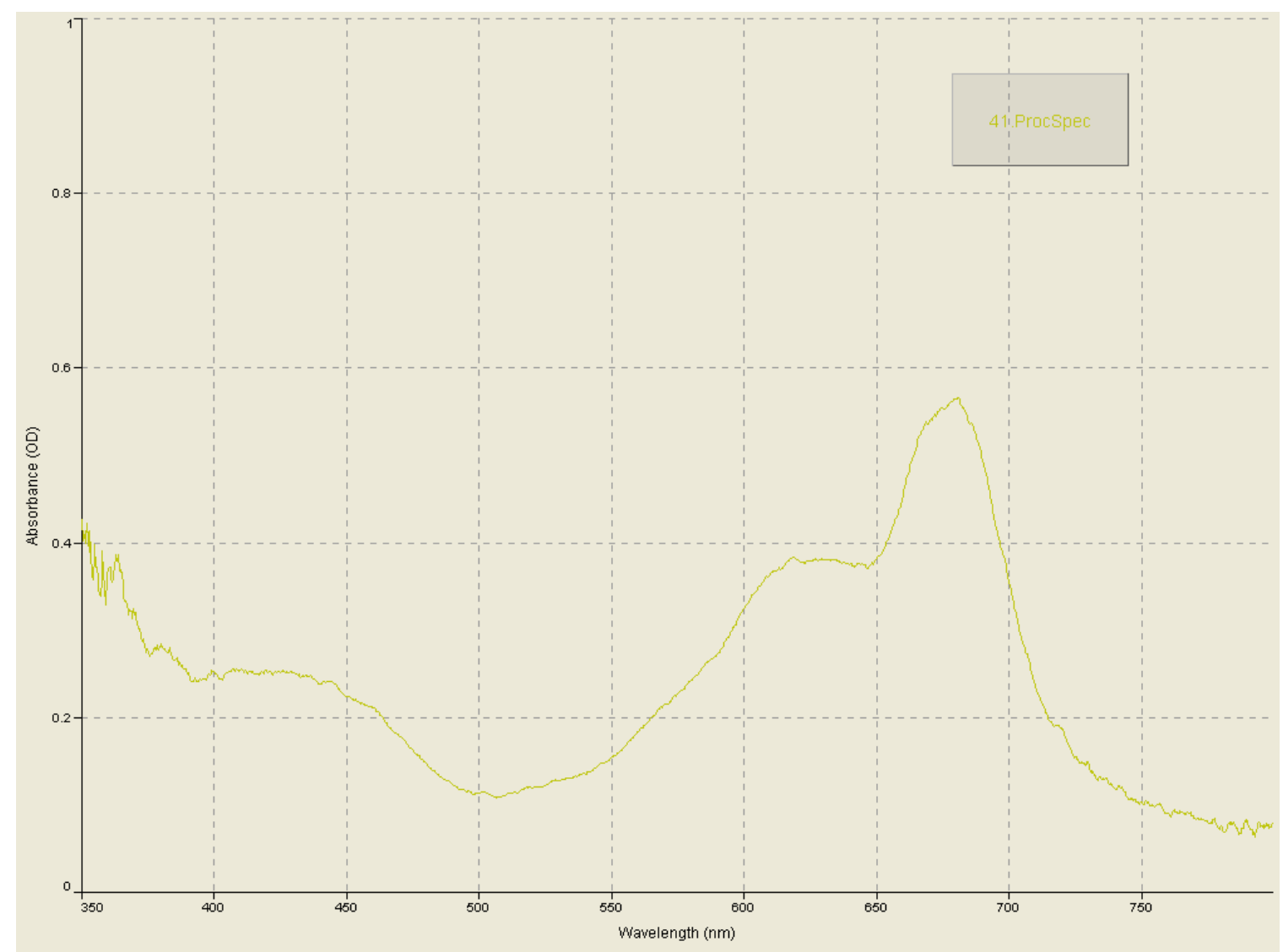

Absorbance spectrum of Kelly Green A_F after 41 minutes in Part II of Phase III 


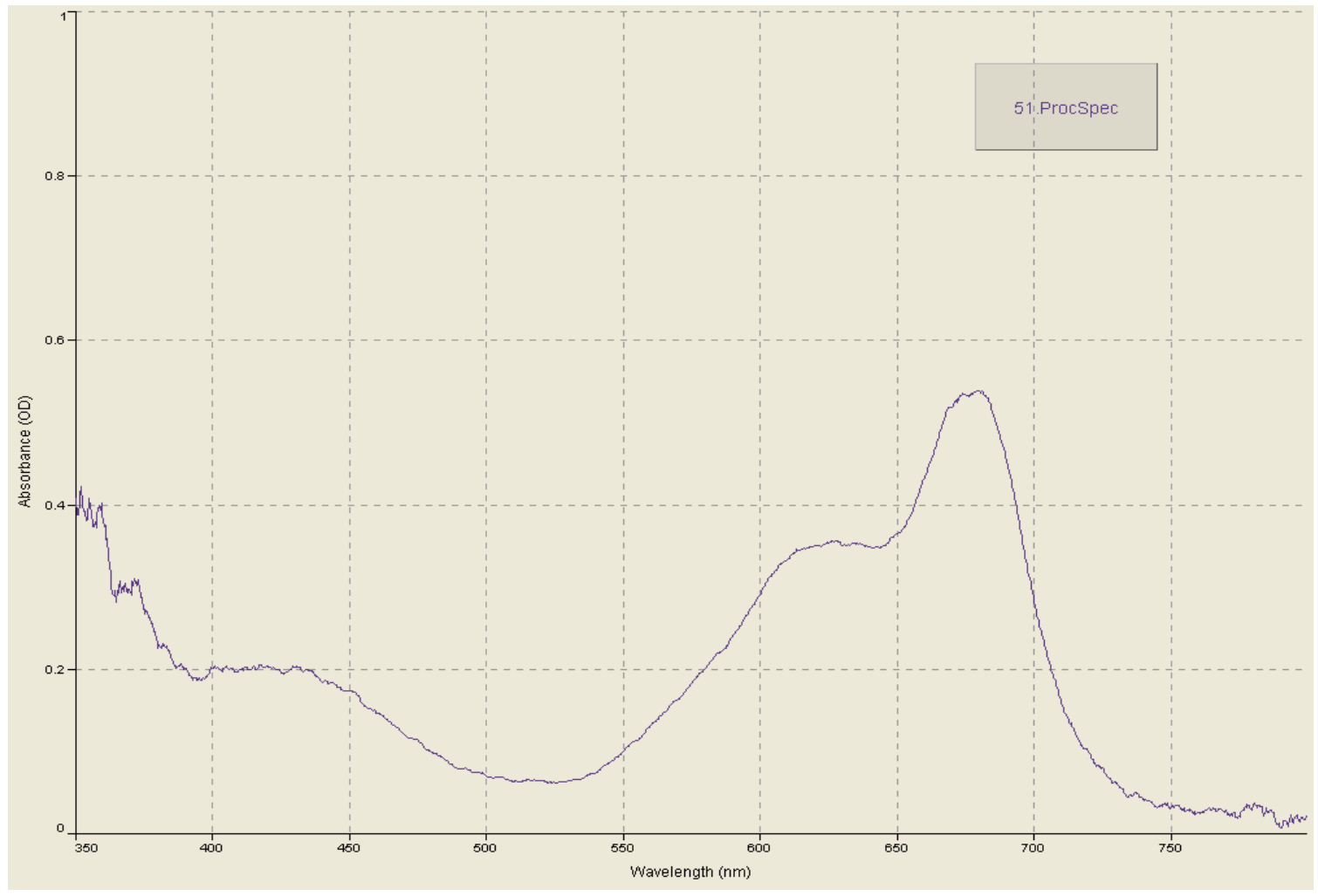

Absorbance spectrum of Kelly Green A_F after 51 minutes in Part II of Phase III

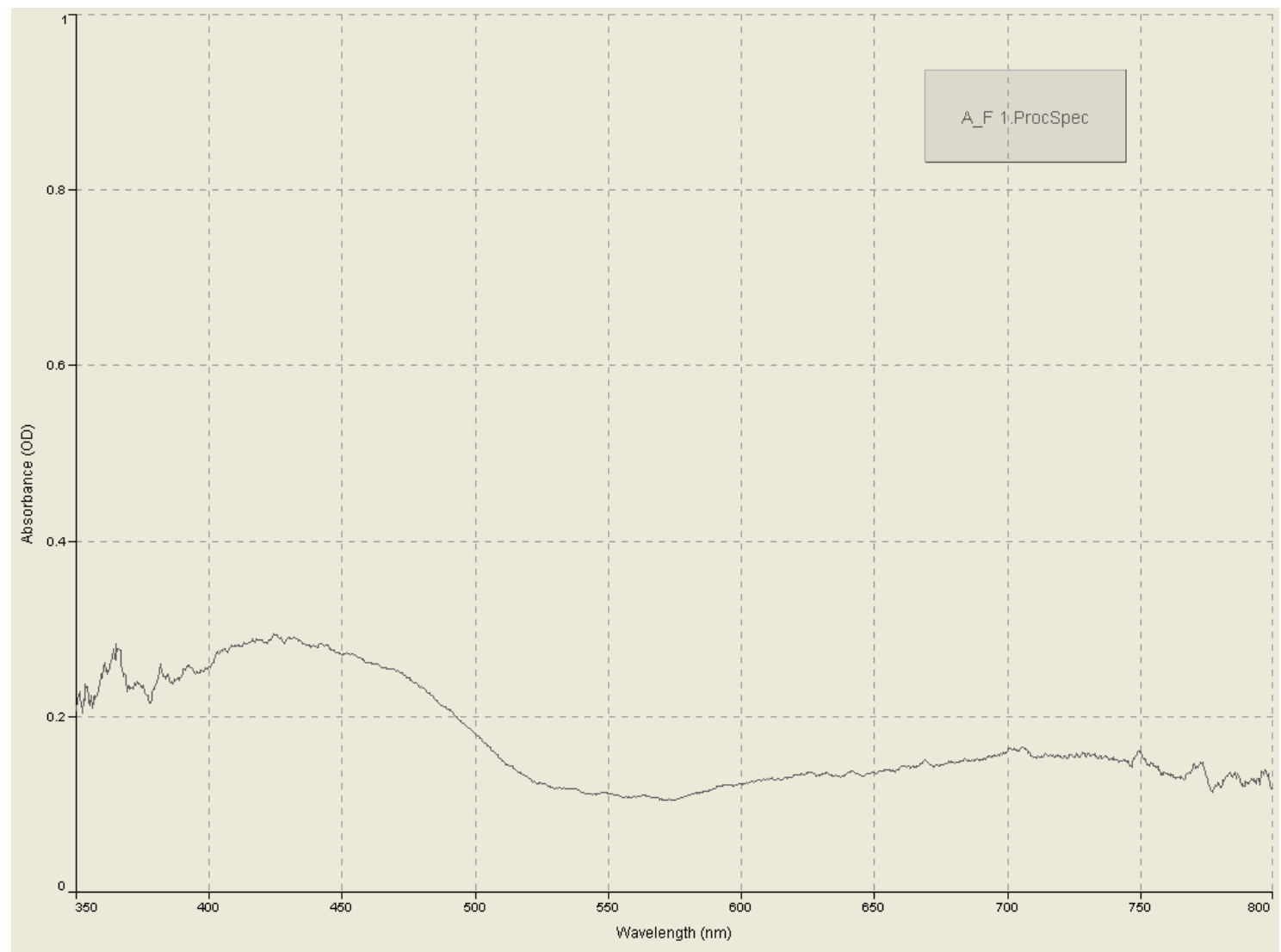

Run 1 absorbance spectrum of the front $t$-shirt square of Gold A 


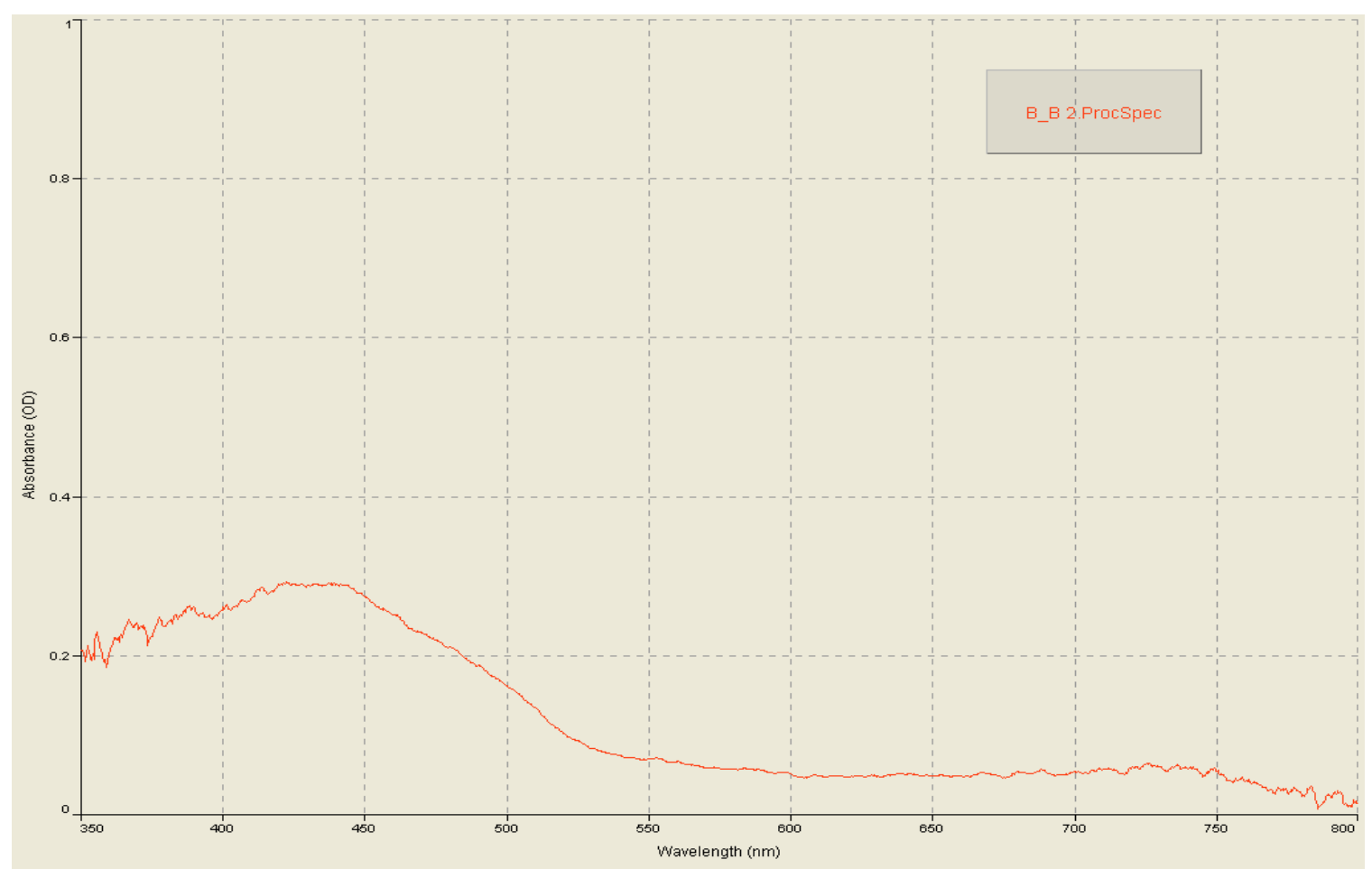

Run 2 absorbance spectrum of the back $t$-shirt square of Gold B

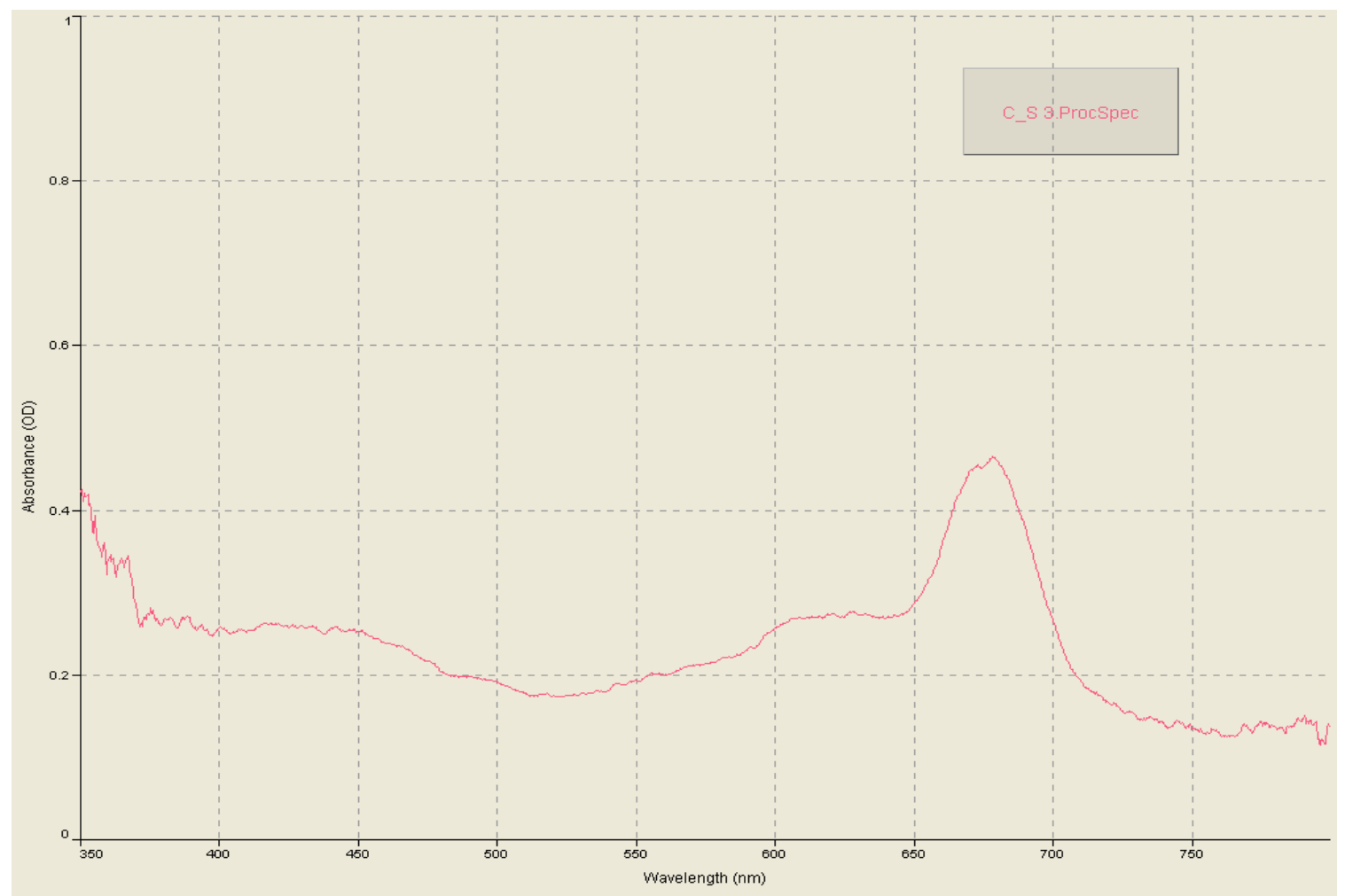

Run 3 absorbance spectrum of the sleeve t-shirt square of Kelly Green C 


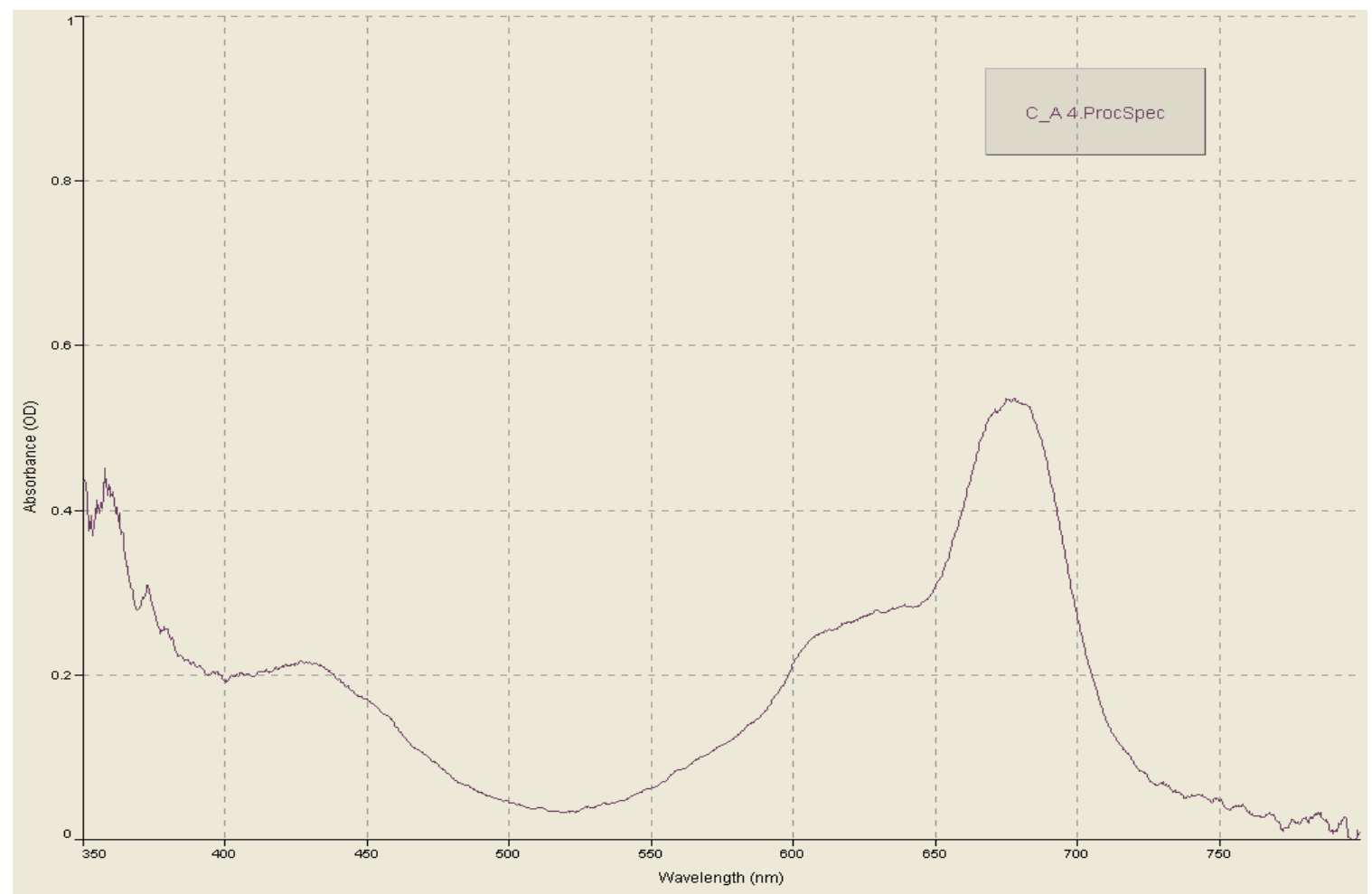

Run 4 absorbance spectrum of the armpit e t-shirt square of Kelly Green D

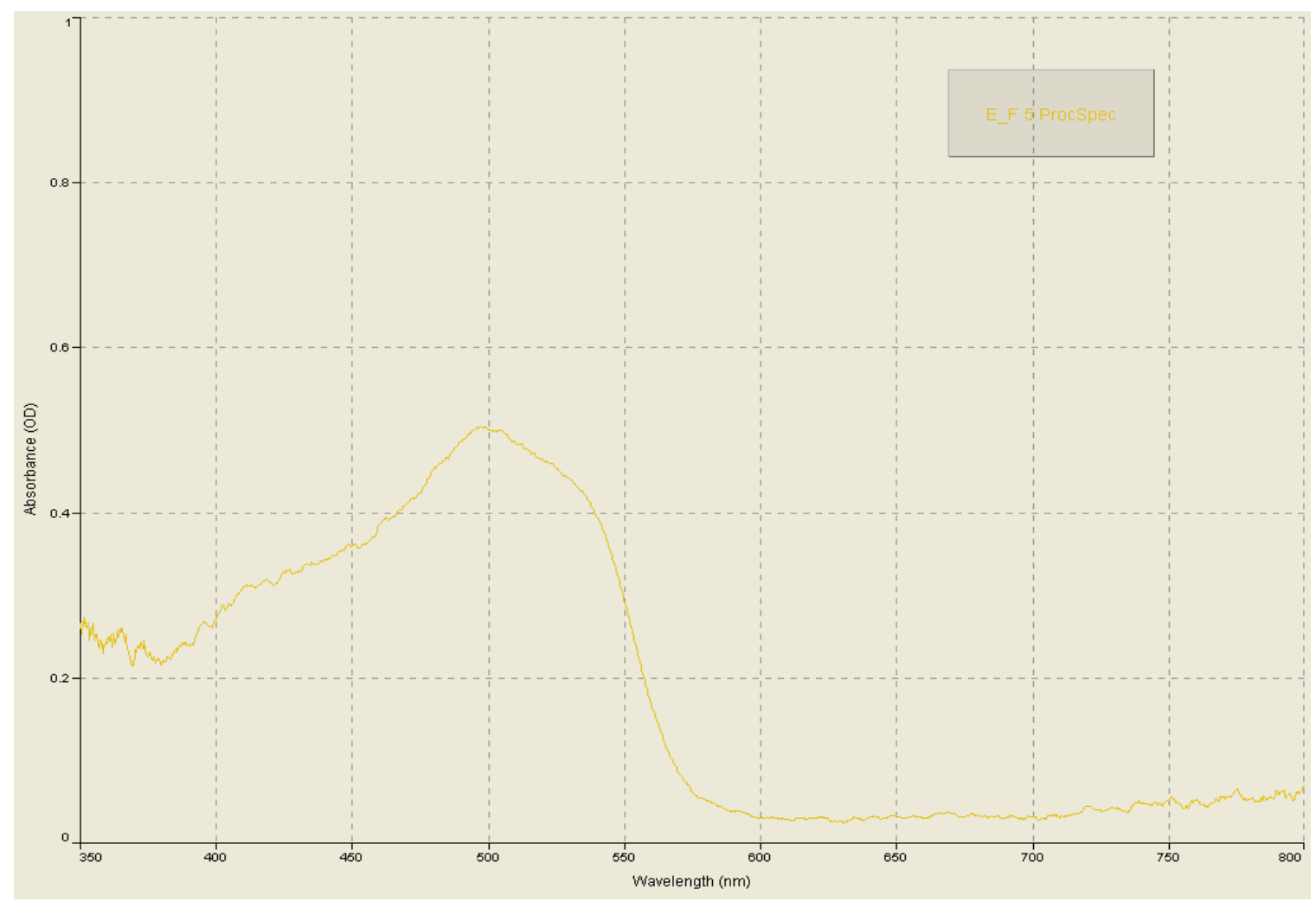

Run 5 absorbance spectrum of the front $t$-shirt square of Orange E 


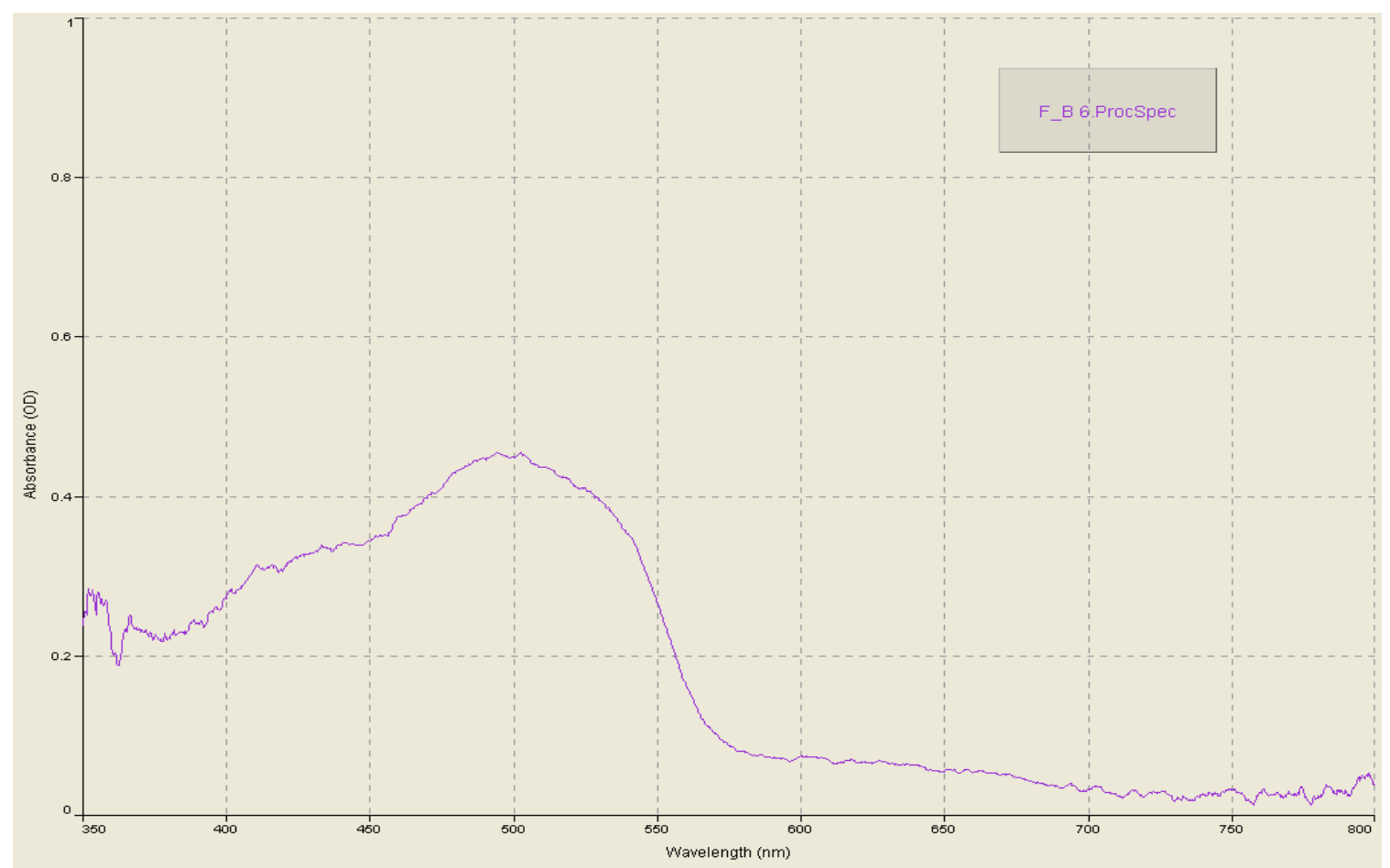

Run 6 absorbance spectrum of the back $t$-shirt square of Orange $F$

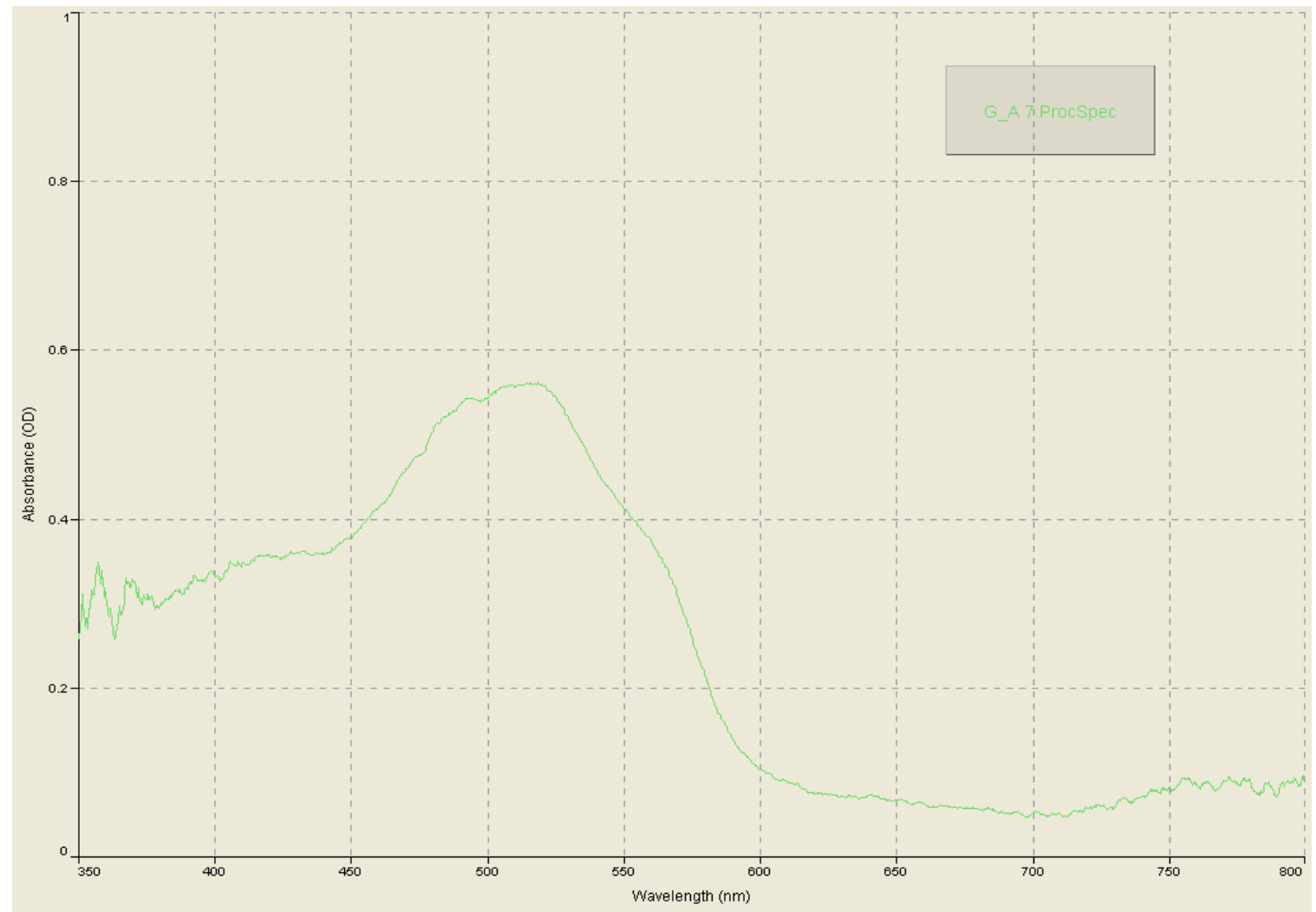

Run 7 absorbance spectrum of the sleeve t-shirt square of Red $G$ 


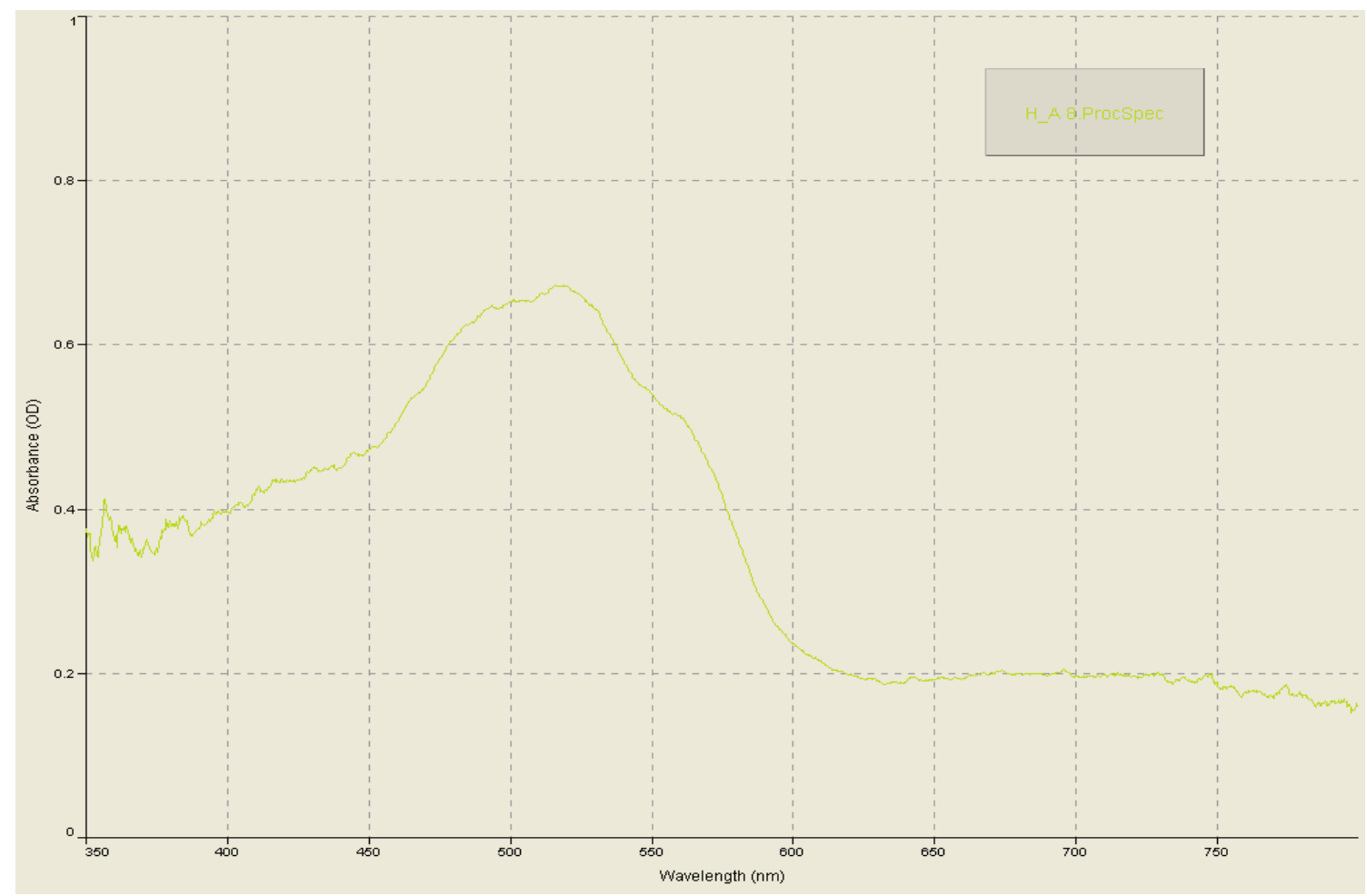

Run 8 absorbance spectrum of the armpit $t$-shirt square of Red $H$

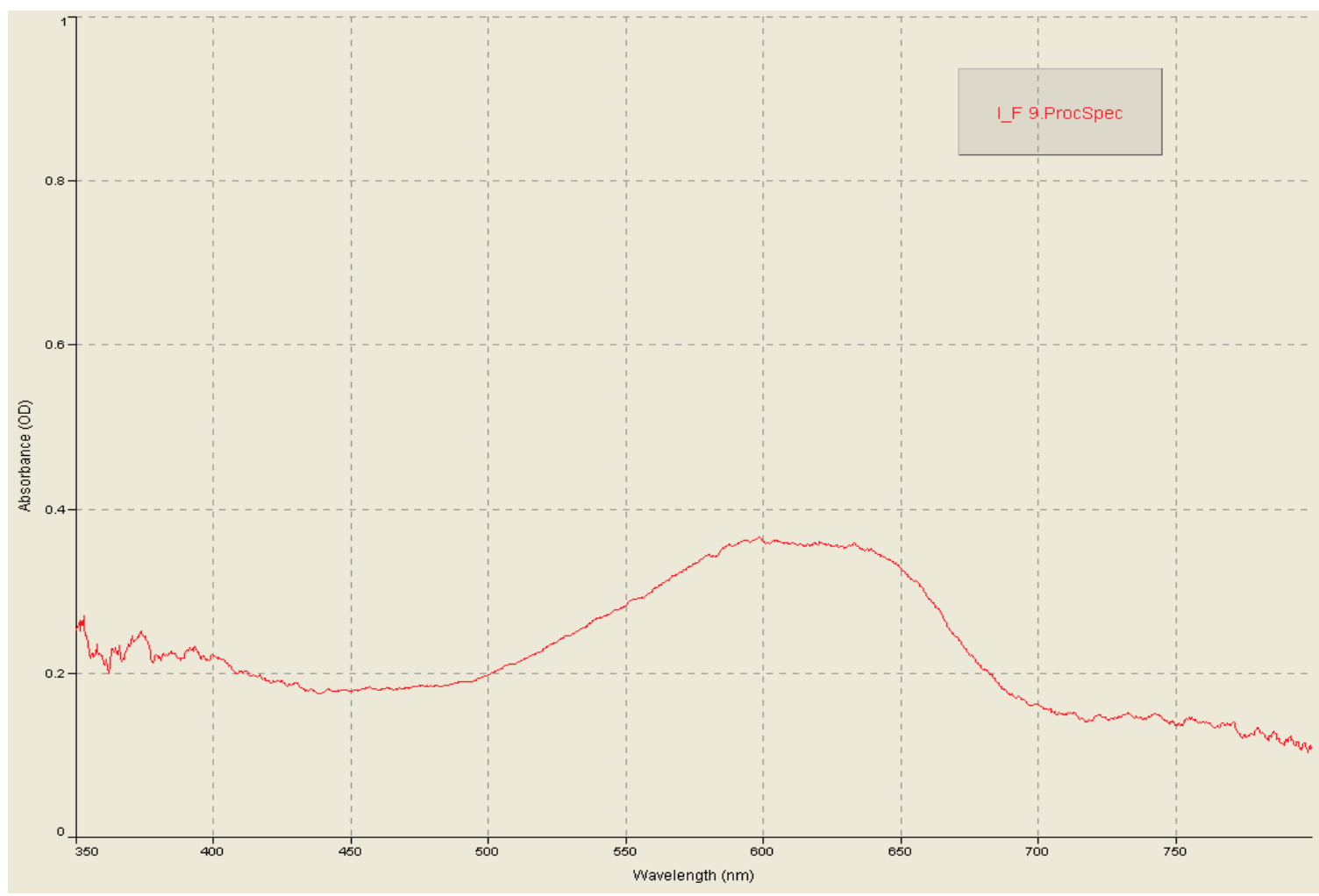

Run 9 absorbance spectrum of the front $t$-shirt square of Royal I 


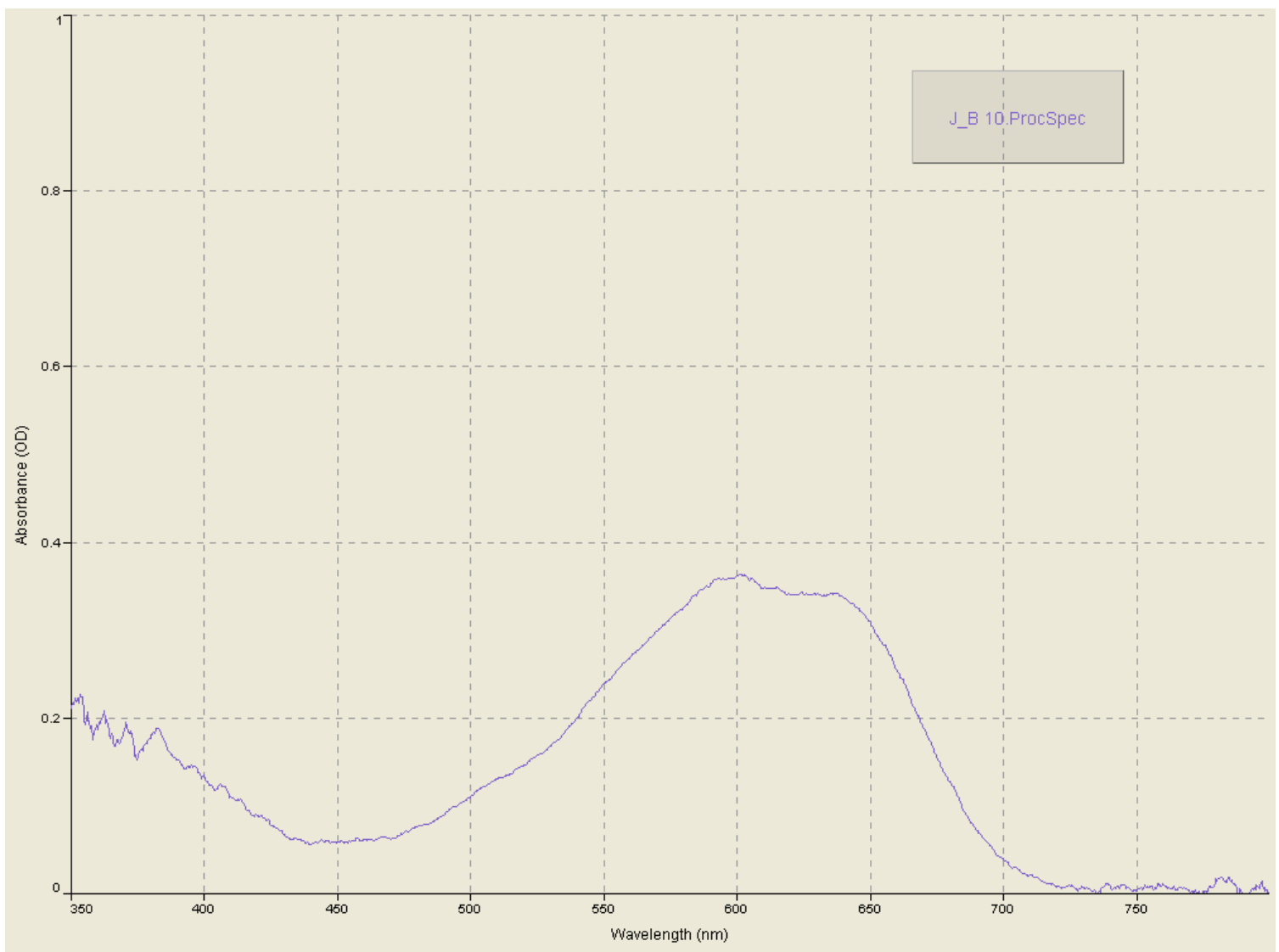

Run 10 absorbance spectrum of the back $t$ t-shirt square of Royal $J$

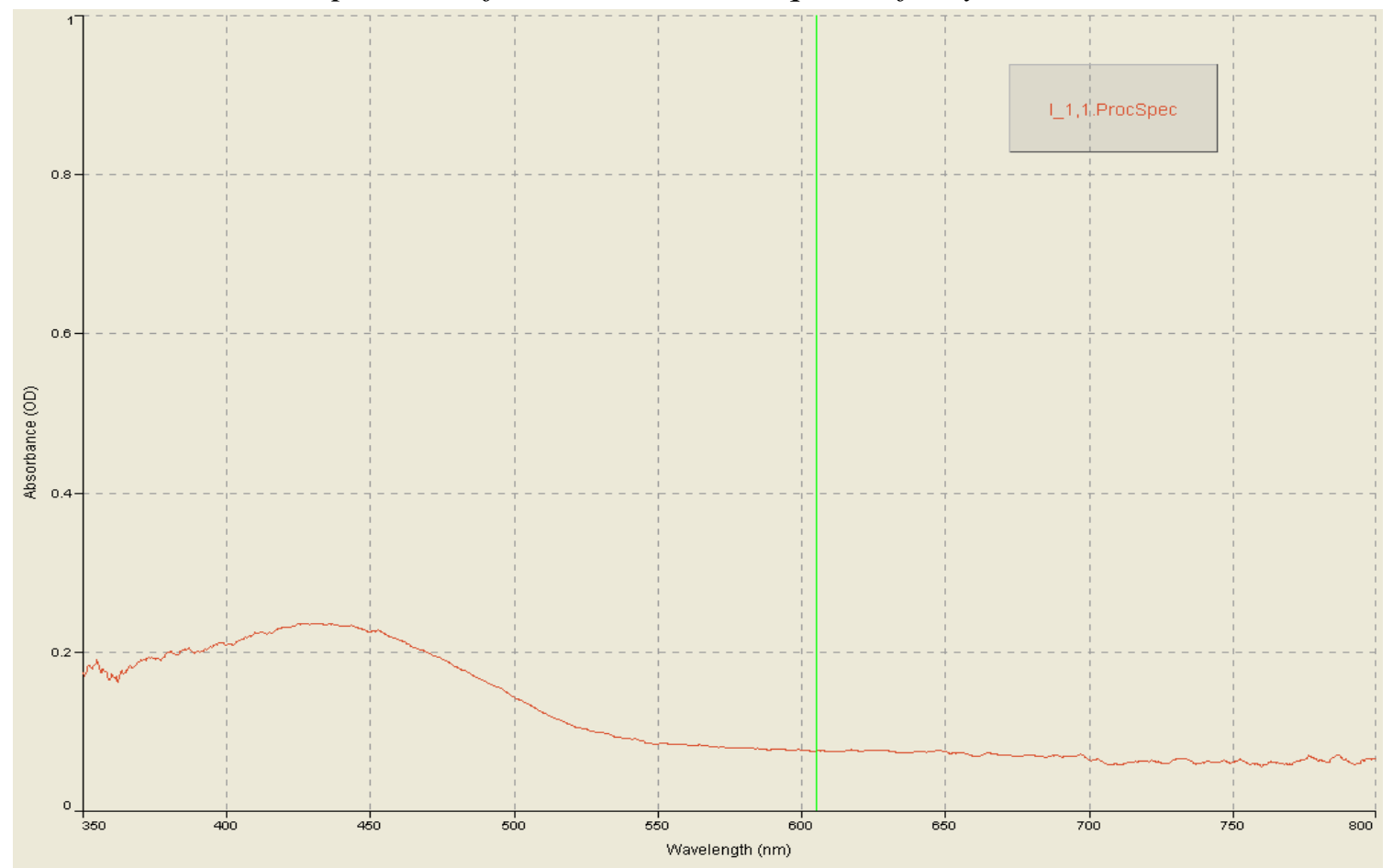

Run 1 absorbance spectrum of the Section 1 t-shirt square of Gold I 


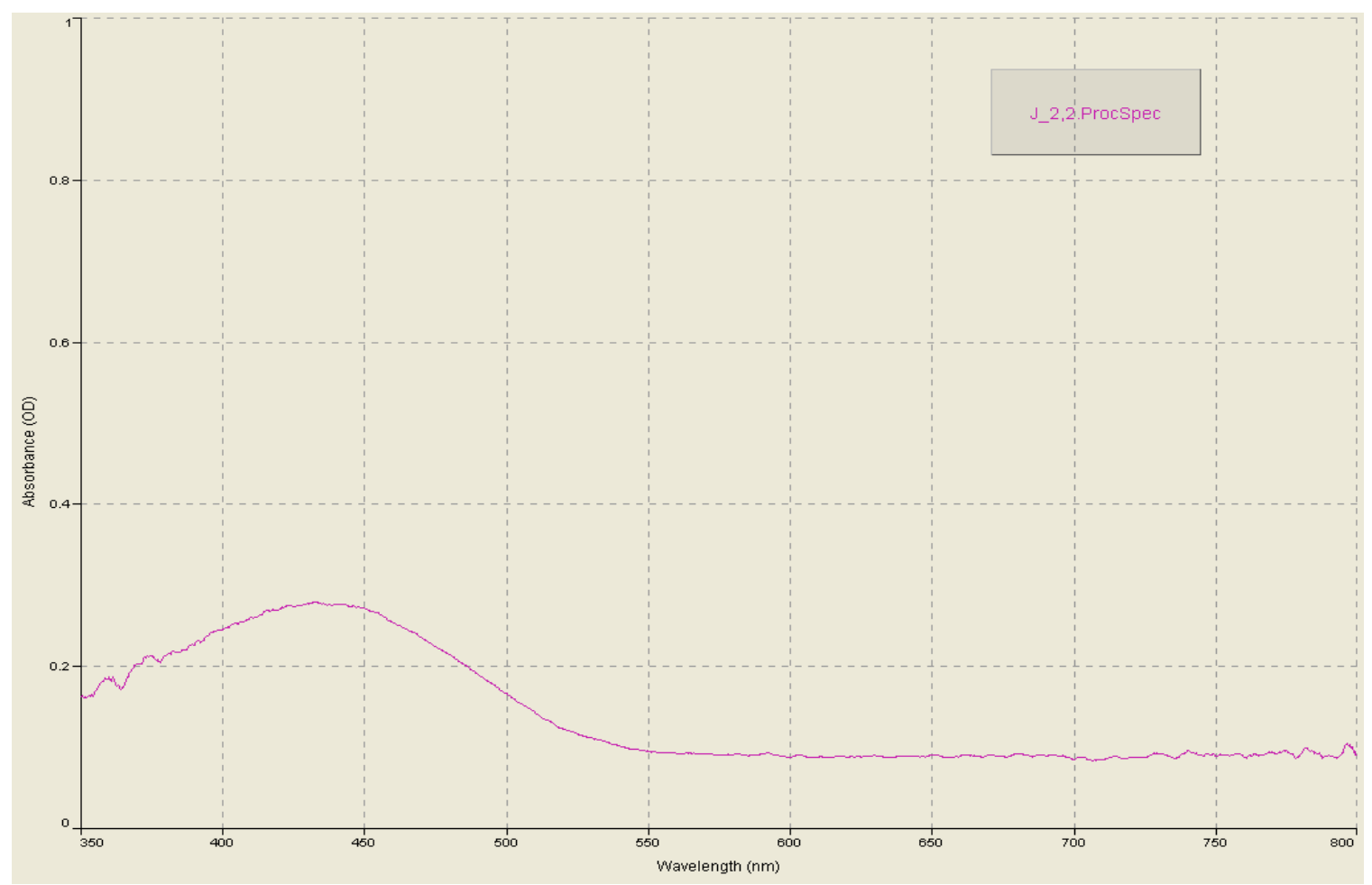

Run 2 absorbance spectrum of the Section $2 t$-shirt square of Gold $J$

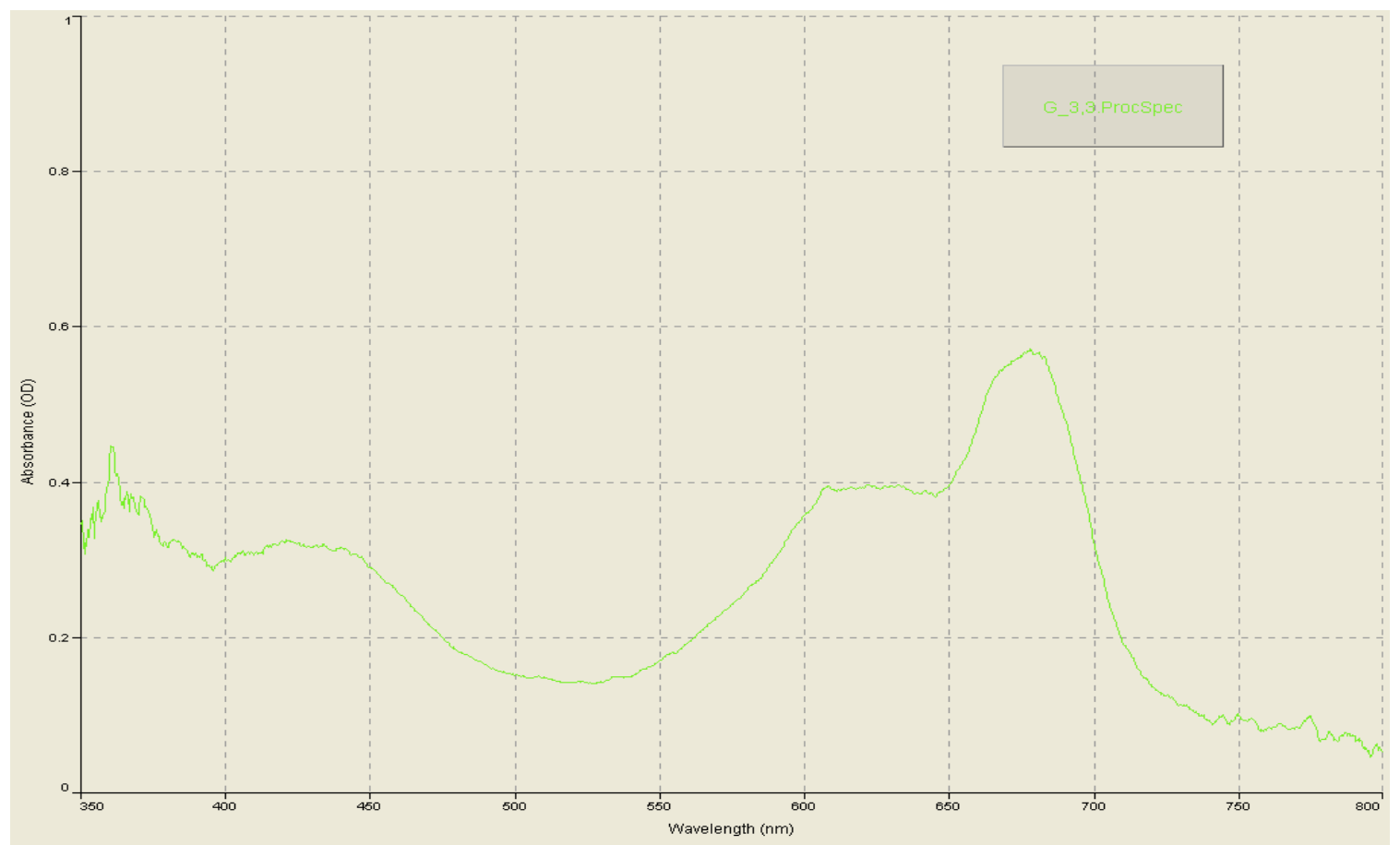

Run 3 absorbance spectrum of the Section 3 t-shirt square of Kelly Green $G$ 


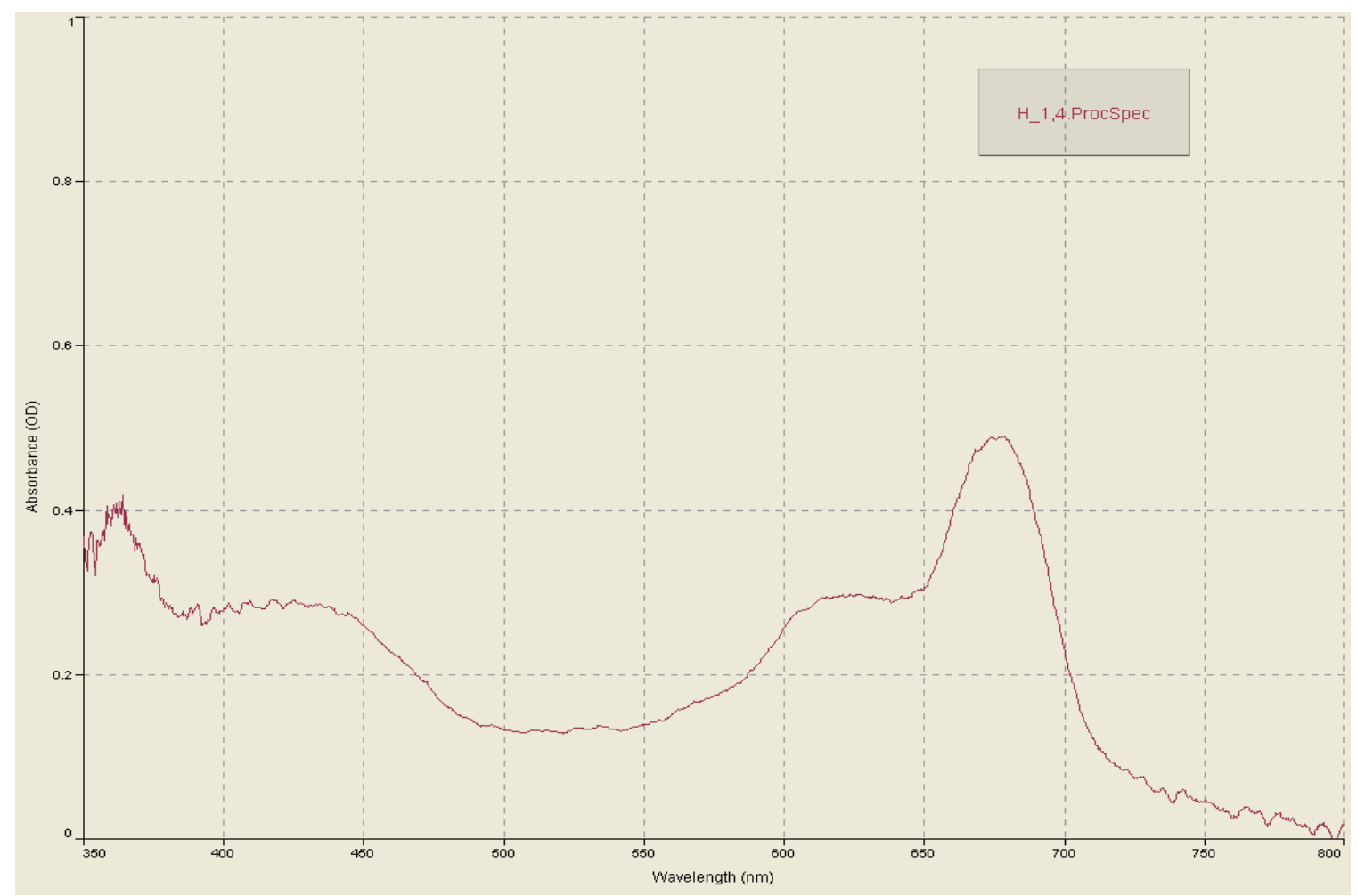

Run 4 absorbance spectrum of the Section 1 t-shirt square of Kelly Green H

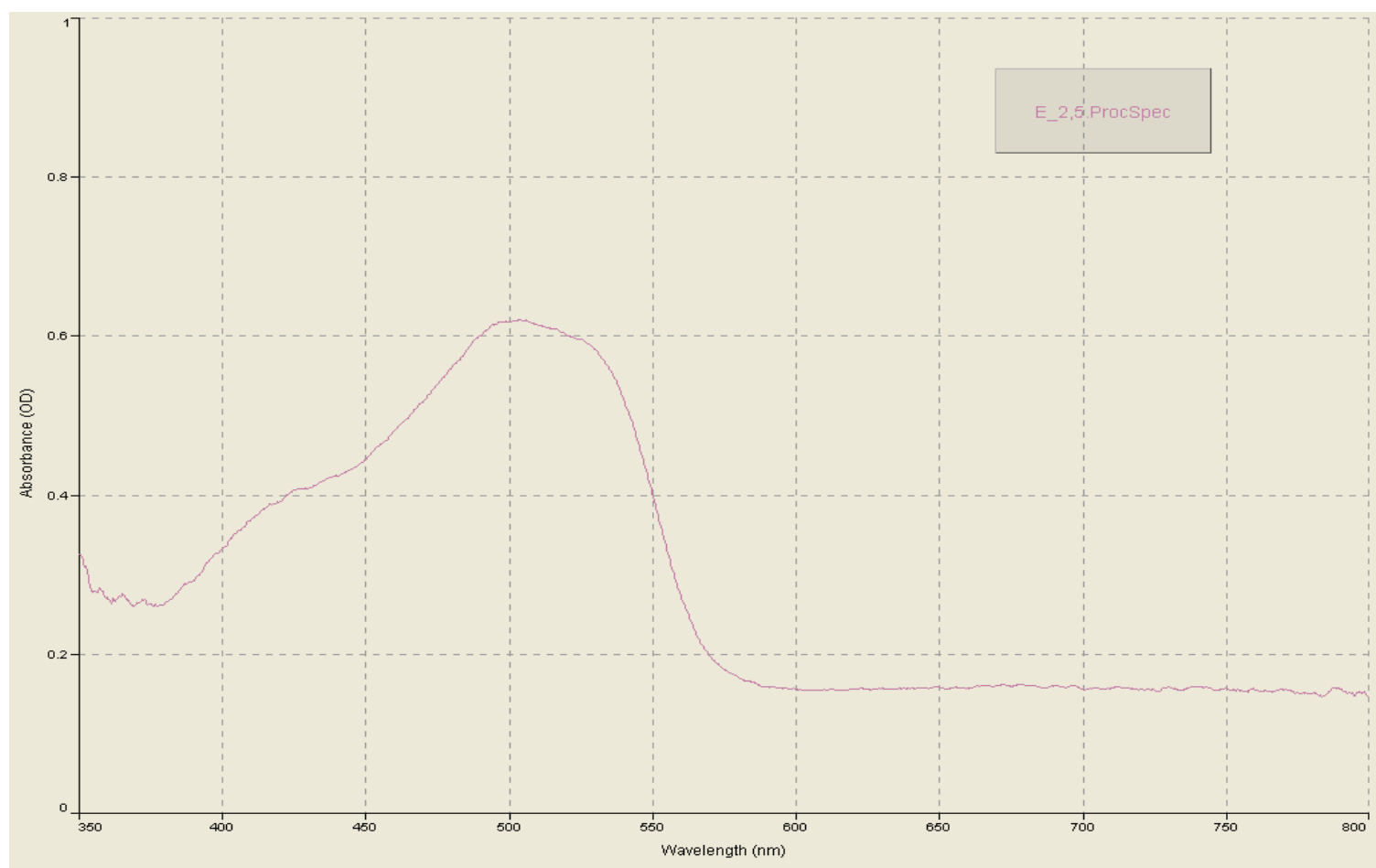

Run 5 absorbance spectrum of the Section $2 t$-shirt square of Orange $E$ 


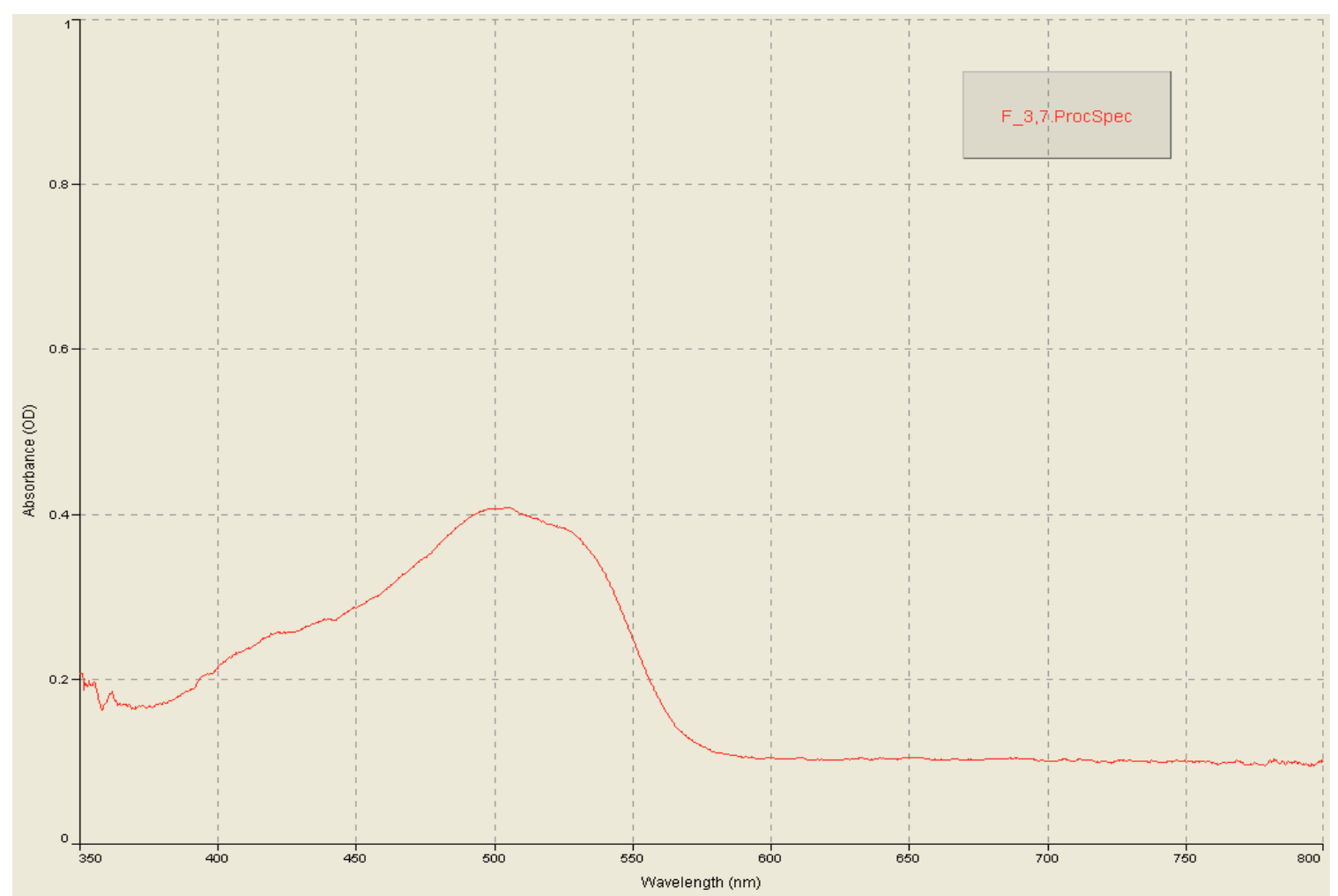

Run 6 absorbance spectrum of the Section $3 t$-shirt square of Orange $F$

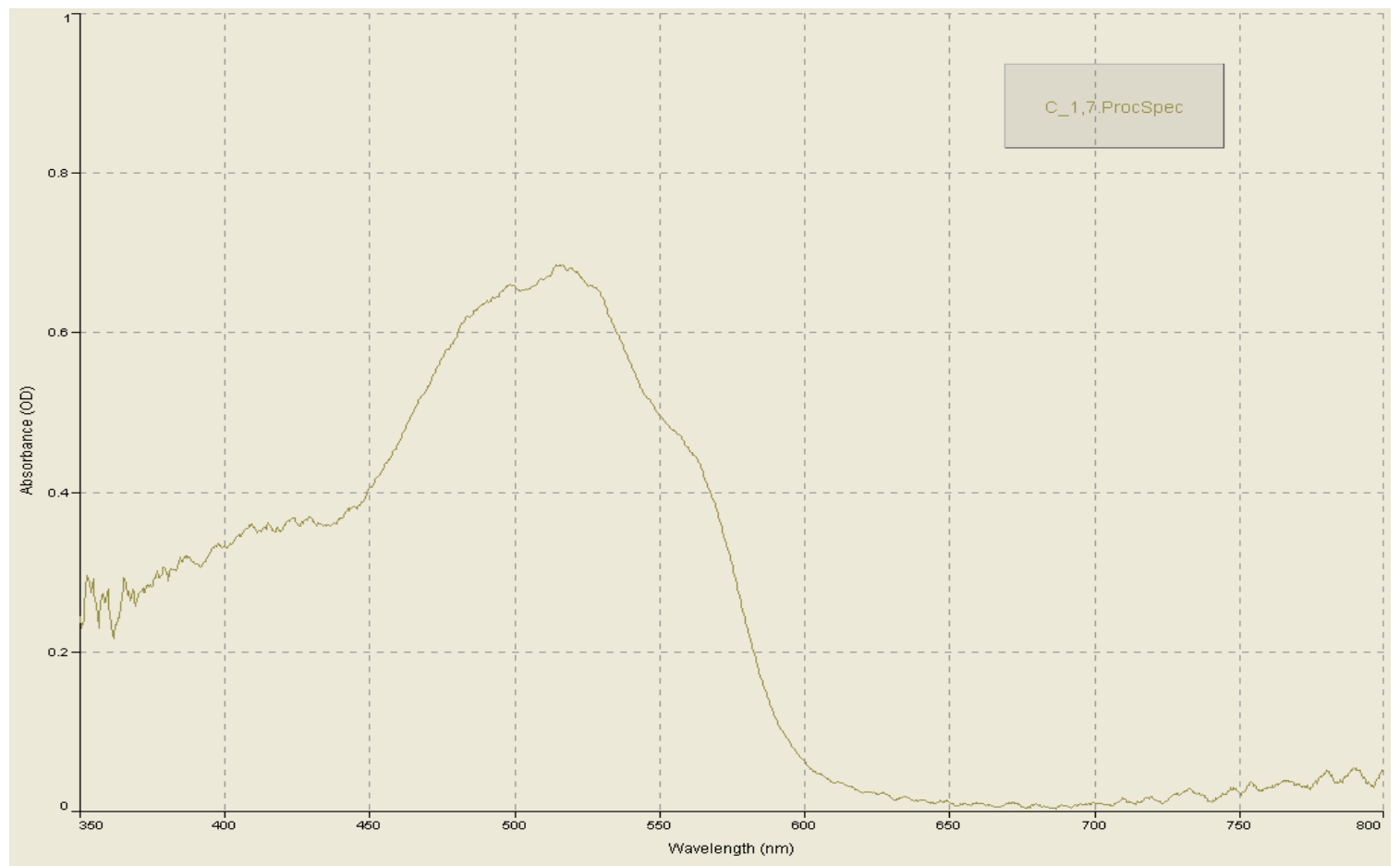

Run 7 absorbance spectrum of the Section1 t-shirt square of Red C 


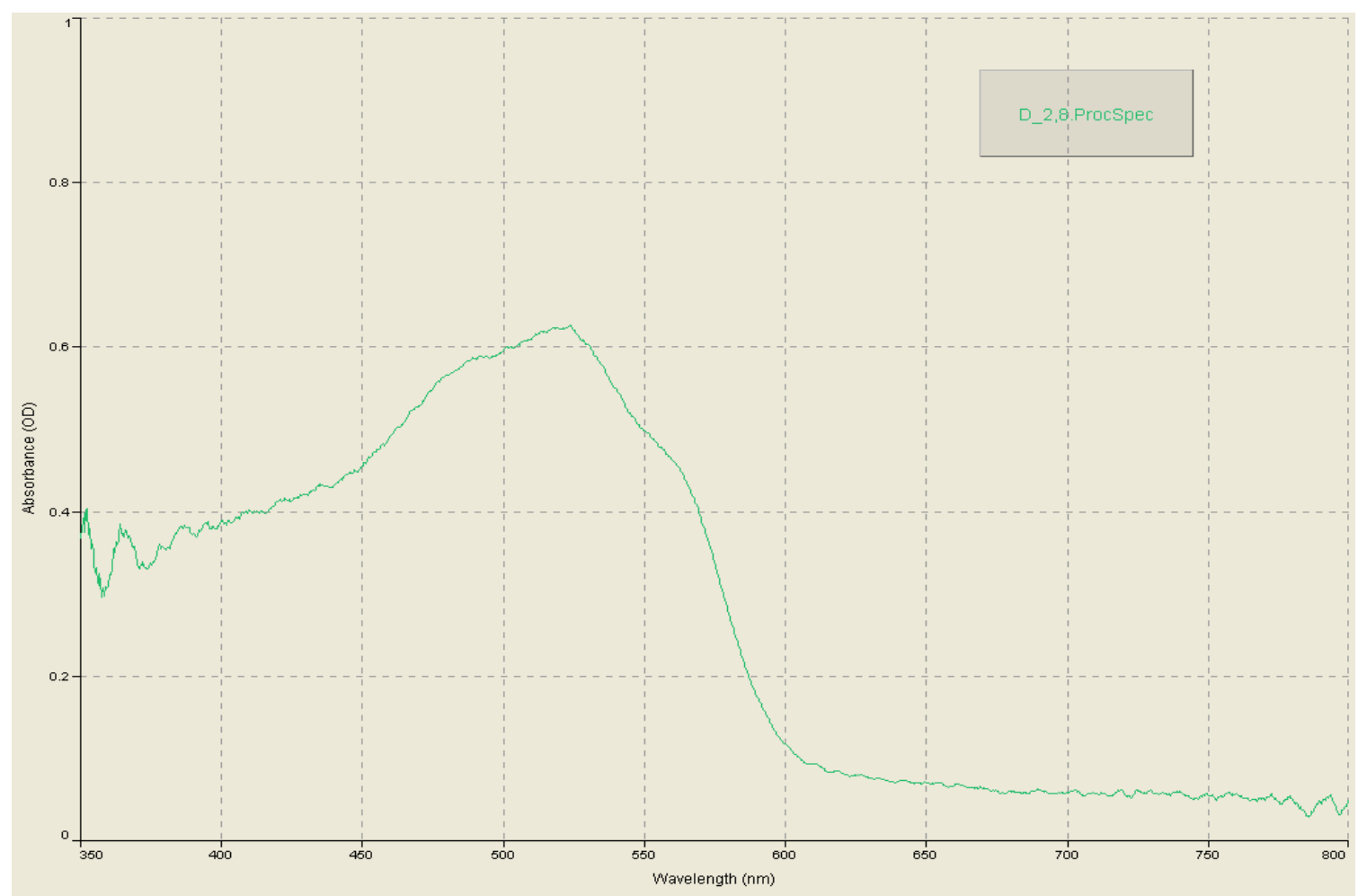

Run 8 absorbance spectrum of the Section $2 t$-shirt square of Red D

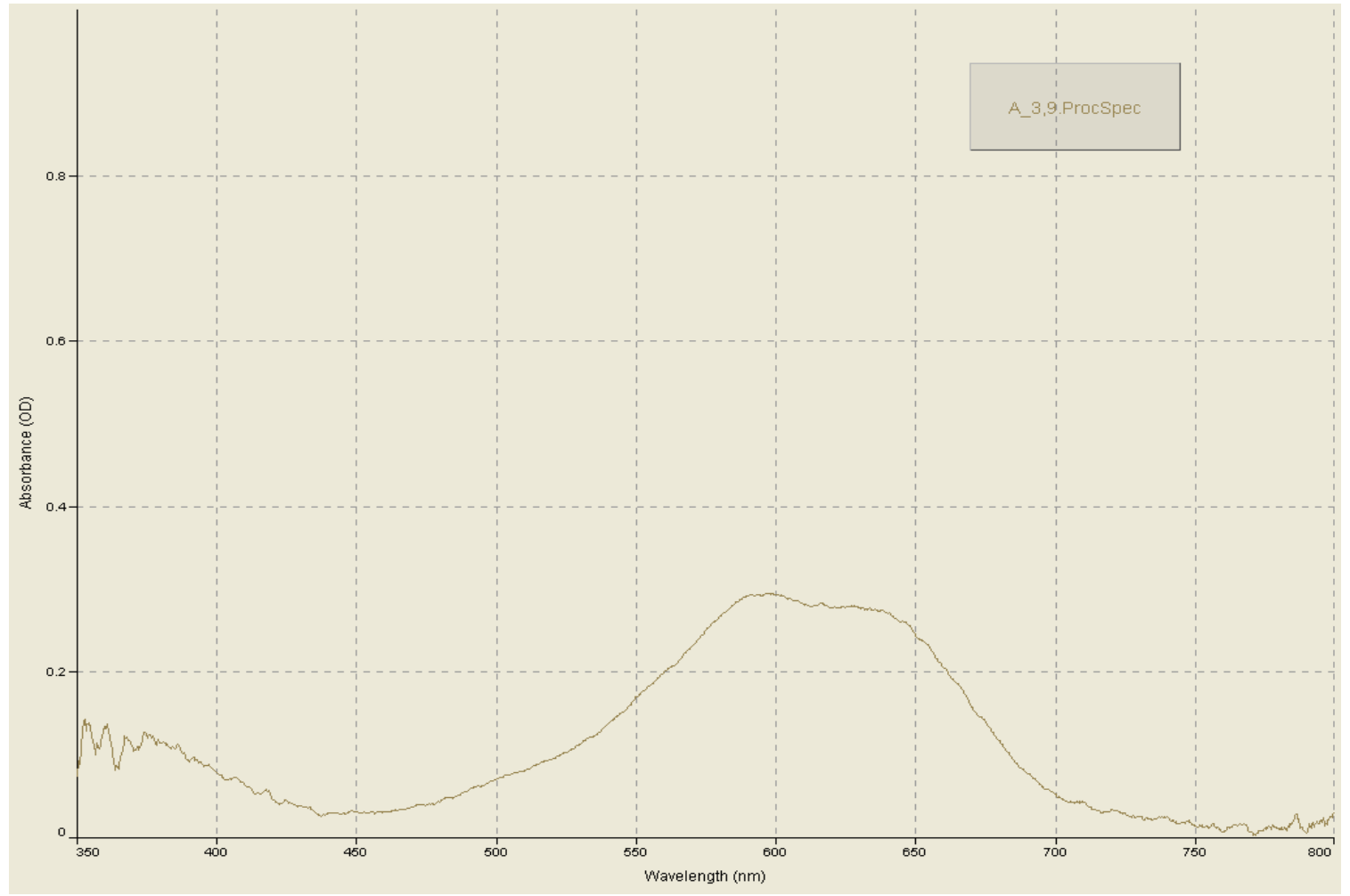

Run 9 absorbance spectrum of the Section 3 t-shirt square of Royal A 


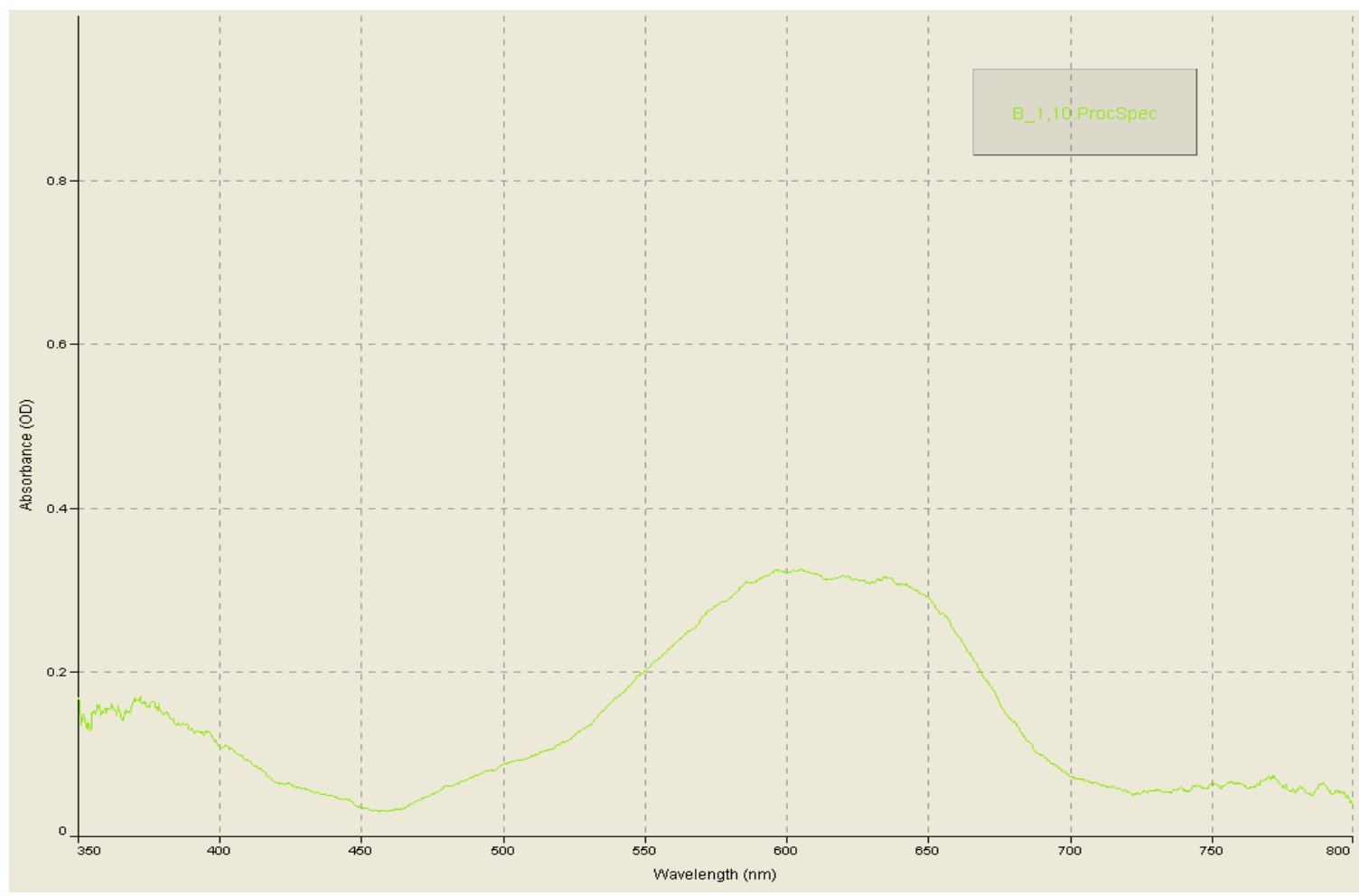

Run 10 absorbance spectrum of the Section 1 t-shirt square of Royal B 


\section{IX: $\underline{\text { Appendix B }}$}

\begin{tabular}{|c|c|c|c|c|c|}
\hline Wavelength & Armpit & Back & Front & Sleeve & Average \\
\hline 400.21 & 0.60978 & 0.975 & 0.75254 & 0.49203 & 0.707 \\
\hline 400.58 & 0.61376 & 0.99763 & 0.76506 & 0.49579 & 0.718 \\
\hline 400.95 & 0.6165 & 0.99443 & 0.76092 & 0.49347 & 0.716 \\
\hline 401.32 & 0.61975 & 1.02582 & 0.76662 & 0.4918 & 0.726 \\
\hline 401.69 & 0.6287 & 1.05518 & 0.77439 & 0.49732 & 0.739 \\
\hline 402.06 & 0.63185 & 1.05409 & 0.78163 & 0.49717 & 0.741 \\
\hline 402.43 & 0.63491 & 1.06503 & 0.79235 & 0.49559 & 0.747 \\
\hline 402.80 & 0.64733 & 1.08748 & 0.79932 & 0.50167 & 0.759 \\
\hline 403.18 & 0.66041 & 1.09818 & 0.80085 & 0.50313 & 0.766 \\
\hline 403.55 & 0.66399 & 1.09339 & 0.79633 & 0.50393 & 0.764 \\
\hline 403.92 & 0.66287 & 1.08779 & 0.79209 & 0.5033 & 0.762 \\
\hline 404.29 & 0.66027 & 1.10256 & 0.79926 & 0.50721 & 0.767 \\
\hline 404.66 & 0.66642 & 1.1328 & 0.81271 & 0.51111 & 0.781 \\
\hline 405.03 & 0.66918 & 1.13602 & 0.81477 & 0.50653 & 0.782 \\
\hline 405.40 & 0.67303 & 1.15118 & 0.81843 & 0.51395 & 0.789 \\
\hline 405.77 & 0.67292 & 1.1405 & 0.8191 & 0.5105 & 0.786 \\
\hline 406.14 & 0.67465 & 1.14248 & 0.8244 & 0.51371 & 0.789 \\
\hline 406.51 & 0.6779 & 1.16832 & 0.83132 & 0.51519 & 0.798 \\
\hline 406.88 & 0.67175 & 1.16239 & 0.82899 & 0.51867 & 0.795 \\
\hline 407.25 & 0.67209 & 1.16834 & 0.81703 & 0.51952 & 0.794 \\
\hline 407.62 & 0.67248 & 1.17869 & 0.8186 & 0.51944 & 0.797 \\
\hline 407.99 & 0.67241 & 1.16332 & 0.81884 & 0.52205 & 0.794 \\
\hline 408.36 & 0.67081 & 1.14253 & 0.8139 & 0.52123 & 0.787 \\
\hline 408.74 & 0.67992 & 1.1533 & 0.82459 & 0.52473 & 0.796 \\
\hline 409.11 & 0.6824 & 1.13112 & 0.81899 & 0.52921 & 0.790 \\
\hline 409.48 & 0.68733 & 1.13865 & 0.82657 & 0.53273 & 0.796 \\
\hline 409.85 & 0.6869 & 1.13588 & 0.82659 & 0.53285 & 0.796 \\
\hline 410.22 & 0.69338 & 1.13281 & 0.82774 & 0.53675 & 0.798 \\
\hline 410.59 & 0.69603 & 1.13537 & 0.82855 & 0.5381 & 0.800 \\
\hline 410.96 & 0.69133 & 1.12523 & 0.8247 & 0.53816 & 0.795 \\
\hline 411.33 & 0.69431 & 1.13639 & 0.83561 & 0.5412 & 0.802 \\
\hline 411.70 & 0.69843 & 1.13998 & 0.84019 & 0.54191 & 0.805 \\
\hline 412.07 & 0.70039 & 1.13212 & 0.83663 & 0.54085 & 0.802 \\
\hline & & & & & \\
\hline
\end{tabular}




\begin{tabular}{|c|c|c|c|c|c|}
\hline 412.44 & 0.6999 & 1.13997 & 0.83586 & 0.54281 & 0.805 \\
\hline 412.81 & 0.69823 & 1.14559 & 0.83871 & 0.54815 & 0.808 \\
\hline 413.18 & 0.70296 & 1.14779 & 0.83711 & 0.54981 & 0.809 \\
\hline 413.55 & 0.7013 & 1.14742 & 0.83388 & 0.55153 & 0.809 \\
\hline 413.92 & 0.70065 & 1.13481 & 0.82841 & 0.55039 & 0.804 \\
\hline 414.29 & 0.70009 & 1.12409 & 0.82679 & 0.55056 & 0.800 \\
\hline 414.66 & 0.70275 & 1.13143 & 0.83473 & 0.55223 & 0.805 \\
\hline 415.03 & 0.70348 & 1.13075 & 0.83599 & 0.55368 & 0.806 \\
\hline 415.40 & 0.7071 & 1.13491 & 0.83568 & 0.5553 & 0.808 \\
\hline 415.76 & 0.7082 & 1.13706 & 0.83426 & 0.55494 & 0.809 \\
\hline 416.13 & 0.71001 & 1.14126 & 0.83964 & 0.5571 & 0.812 \\
\hline 416.50 & 0.70827 & 1.13974 & 0.83909 & 0.55917 & 0.812 \\
\hline 416.87 & 0.7105 & 1.14917 & 0.84302 & 0.55804 & 0.815 \\
\hline 417.24 & 0.70826 & 1.14288 & 0.83655 & 0.55647 & 0.811 \\
\hline 417.61 & 0.70882 & 1.15089 & 0.83358 & 0.55862 & 0.813 \\
\hline 417.98 & 0.70395 & 1.15979 & 0.83091 & 0.55635 & 0.813 \\
\hline 418.35 & 0.70116 & 1.15761 & 0.82917 & 0.55474 & 0.811 \\
\hline 418.72 & 0.70507 & 1.1683 & 0.83377 & 0.55736 & 0.816 \\
\hline 419.09 & 0.70512 & 1.16195 & 0.83133 & 0.55743 & 0.814 \\
\hline 419.46 & 0.70367 & 1.15687 & 0.83285 & 0.55773 & 0.813 \\
\hline 419.83 & 0.70827 & 1.1581 & 0.83549 & 0.55994 & 0.815 \\
\hline 420.20 & 0.71077 & 1.15389 & 0.83654 & 0.5609 & 0.816 \\
\hline 420.57 & 0.7172 & 1.15498 & 0.8376 & 0.56182 & 0.818 \\
\hline 420.94 & 0.71877 & 1.15784 & 0.84251 & 0.56214 & 0.820 \\
\hline 421.31 & 0.72562 & 1.16981 & 0.84701 & 0.56469 & 0.827 \\
\hline 421.67 & 0.72758 & 1.17467 & 0.84889 & 0.56844 & 0.830 \\
\hline 422.04 & 0.73383 & 1.18191 & 0.85139 & 0.57256 & 0.835 \\
\hline 422.41 & 0.73476 & 1.18628 & 0.8511 & 0.57303 & 0.836 \\
\hline 422.78 & 0.73373 & 1.19239 & 0.85093 & 0.57248 & 0.837 \\
\hline 423.15 & 0.73424 & 1.19254 & 0.85188 & 0.57643 & 0.839 \\
\hline 423.52 & 0.73767 & 1.20422 & 0.85884 & 0.57957 & 0.845 \\
\hline 423.89 & 0.74151 & 1.21581 & 0.86212 & 0.58021 & 0.850 \\
\hline 424.26 & 0.74587 & 1.21826 & 0.86161 & 0.57895 & 0.851 \\
\hline 424.63 & 0.74966 & 1.2225 & 0.86464 & 0.58186 & 0.855 \\
\hline 424.99 & 0.75045 & 1.22576 & 0.86712 & 0.58518 & 0.857 \\
\hline 425.36 & 0.75499 & 1.22788 & 0.86957 & 0.58575 & 0.860 \\
\hline 425.73 & 0.75747 & 1.2277 & 0.86961 & 0.58954 & 0.861 \\
\hline 426.10 & 0.76033 & 1.22579 & 0.86895 & 0.59208 & 0.862 \\
\hline 426.47 & 0.76102 & 1.22914 & 0.87046 & 0.59146 & 0.863 \\
\hline
\end{tabular}




\begin{tabular}{|c|c|c|c|c|c|}
\hline 426.84 & 0.76168 & 1.22617 & 0.86931 & 0.59315 & 0.863 \\
\hline 427.21 & 0.76539 & 1.22957 & 0.8706 & 0.59511 & 0.865 \\
\hline 427.57 & 0.76425 & 1.23504 & 0.87001 & 0.59417 & 0.866 \\
\hline 427.94 & 0.7638 & 1.23478 & 0.87215 & 0.5938 & 0.866 \\
\hline 428.31 & 0.76134 & 1.23376 & 0.87101 & 0.59235 & 0.865 \\
\hline 428.68 & 0.76012 & 1.2362 & 0.86777 & 0.59224 & 0.864 \\
\hline 429.05 & 0.75648 & 1.23007 & 0.86695 & 0.5924 & 0.861 \\
\hline 429.42 & 0.75852 & 1.23786 & 0.86838 & 0.59299 & 0.864 \\
\hline 429.78 & 0.75891 & 1.23667 & 0.87015 & 0.59223 & 0.864 \\
\hline 430.15 & 0.75962 & 1.23791 & 0.87238 & 0.59334 & 0.866 \\
\hline 430.52 & 0.76134 & 1.2416 & 0.87571 & 0.59382 & 0.868 \\
\hline 430.89 & 0.76178 & 1.24315 & 0.87602 & 0.5933 & 0.869 \\
\hline 431.26 & 0.76052 & 1.23669 & 0.87443 & 0.59114 & 0.866 \\
\hline 431.62 & 0.75932 & 1.23067 & 0.87006 & 0.58888 & 0.862 \\
\hline 431.99 & 0.75619 & 1.23289 & 0.86964 & 0.58921 & 0.862 \\
\hline 432.36 & 0.75728 & 1.23674 & 0.86998 & 0.58966 & 0.863 \\
\hline 432.73 & 0.75844 & 1.23786 & 0.86915 & 0.5902 & 0.864 \\
\hline 433.10 & 0.7562 & 1.2338 & 0.86697 & 0.59054 & 0.862 \\
\hline 433.46 & 0.75684 & 1.22958 & 0.86869 & 0.58921 & 0.861 \\
\hline 433.83 & 0.75619 & 1.23275 & 0.87087 & 0.58979 & 0.862 \\
\hline 434.20 & 0.75836 & 1.23532 & 0.87339 & 0.59241 & 0.865 \\
\hline 434.57 & 0.75907 & 1.23955 & 0.87207 & 0.59176 & 0.866 \\
\hline 434.94 & 0.75993 & 1.2515 & 0.87399 & 0.5919 & 0.869 \\
\hline 435.30 & 0.76145 & 1.25952 & 0.8754 & 0.5944 & 0.873 \\
\hline 435.67 & 0.76406 & 1.26157 & 0.87531 & 0.59453 & 0.874 \\
\hline 436.04 & 0.76574 & 1.25889 & 0.87691 & 0.59654 & 0.875 \\
\hline 436.41 & 0.7678 & 1.26077 & 0.87966 & 0.59824 & 0.877 \\
\hline 436.77 & 0.76939 & 1.25999 & 0.88298 & 0.59894 & 0.878 \\
\hline 437.14 & 0.76975 & 1.25912 & 0.88503 & 0.59896 & 0.878 \\
\hline 437.51 & 0.76871 & 1.26165 & 0.88479 & 0.5986 & 0.878 \\
\hline 437.88 & 0.77062 & 1.26446 & 0.88328 & 0.59862 & 0.879 \\
\hline 438.24 & 0.77173 & 1.2601 & 0.88274 & 0.59855 & 0.878 \\
\hline 438.61 & 0.77185 & 1.26365 & 0.88415 & 0.59945 & 0.880 \\
\hline 438.98 & 0.77275 & 1.26808 & 0.88504 & 0.60102 & 0.882 \\
\hline 439.34 & 0.77438 & 1.26925 & 0.88687 & 0.60244 & 0.883 \\
\hline 439.71 & 0.77673 & 1.2676 & 0.88872 & 0.603 & 0.884 \\
\hline 440.08 & 0.77368 & 1.26029 & 0.88741 & 0.60466 & 0.882 \\
\hline 440.45 & 0.77475 & 1.26017 & 0.88771 & 0.60366 & 0.882 \\
\hline 440.81 & 0.77613 & 1.25855 & 0.89044 & 0.60453 & 0.882 \\
\hline
\end{tabular}




\begin{tabular}{|c|c|c|c|c|c|}
\hline 441.18 & 0.77555 & 1.25915 & 0.89089 & 0.60452 & 0.883 \\
\hline 441.55 & 0.77461 & 1.25729 & 0.89078 & 0.60318 & 0.881 \\
\hline 441.91 & 0.77315 & 1.25474 & 0.88883 & 0.60176 & 0.880 \\
\hline 442.28 & 0.77224 & 1.25072 & 0.88677 & 0.60228 & 0.878 \\
\hline 442.65 & 0.77177 & 1.24398 & 0.88512 & 0.60195 & 0.876 \\
\hline 443.01 & 0.77094 & 1.23977 & 0.88498 & 0.60079 & 0.874 \\
\hline 443.38 & 0.77059 & 1.23902 & 0.88382 & 0.60221 & 0.874 \\
\hline 443.75 & 0.7699 & 1.23682 & 0.88372 & 0.60225 & 0.873 \\
\hline 444.12 & 0.76722 & 1.22964 & 0.88141 & 0.60156 & 0.870 \\
\hline 444.48 & 0.76794 & 1.2302 & 0.88006 & 0.60043 & 0.870 \\
\hline 444.85 & 0.76821 & 1.23219 & 0.87937 & 0.59985 & 0.870 \\
\hline 445.22 & 0.76814 & 1.23008 & 0.87605 & 0.59956 & 0.868 \\
\hline 445.58 & 0.76698 & 1.22411 & 0.87521 & 0.59837 & 0.866 \\
\hline 445.95 & 0.76609 & 1.2236 & 0.87629 & 0.59913 & 0.866 \\
\hline 446.31 & 0.76623 & 1.22239 & 0.87438 & 0.5985 & 0.865 \\
\hline 446.68 & 0.76567 & 1.2225 & 0.87252 & 0.59769 & 0.865 \\
\hline 447.05 & 0.76423 & 1.22185 & 0.87153 & 0.59849 & 0.864 \\
\hline 447.41 & 0.76297 & 1.21949 & 0.87244 & 0.59825 & 0.863 \\
\hline 447.78 & 0.76312 & 1.21985 & 0.87226 & 0.59665 & 0.863 \\
\hline 448.15 & 0.76197 & 1.21805 & 0.87174 & 0.59638 & 0.862 \\
\hline 448.51 & 0.76302 & 1.218 & 0.87007 & 0.59617 & 0.862 \\
\hline 448.88 & 0.76452 & 1.21738 & 0.86804 & 0.59679 & 0.862 \\
\hline 449.25 & 0.76517 & 1.21556 & 0.86641 & 0.5961 & 0.861 \\
\hline 449.61 & 0.76538 & 1.2125 & 0.86563 & 0.59598 & 0.860 \\
\hline 449.98 & 0.76441 & 1.21579 & 0.86729 & 0.59585 & 0.861 \\
\hline 450.34 & 0.76359 & 1.21416 & 0.86715 & 0.59633 & 0.860 \\
\hline 450.71 & 0.76377 & 1.21007 & 0.86453 & 0.5959 & 0.859 \\
\hline 451.08 & 0.76347 & 1.20932 & 0.864 & 0.59523 & 0.858 \\
\hline 451.44 & 0.76344 & 1.21128 & 0.86262 & 0.59432 & 0.858 \\
\hline 451.81 & 0.76447 & 1.21241 & 0.86192 & 0.59378 & 0.858 \\
\hline 452.17 & 0.7635 & 1.21059 & 0.86232 & 0.59288 & 0.857 \\
\hline 452.54 & 0.76323 & 1.20596 & 0.85942 & 0.59216 & 0.855 \\
\hline 452.91 & 0.76133 & 1.20411 & 0.86031 & 0.59151 & 0.854 \\
\hline 453.27 & 0.76103 & 1.20548 & 0.86027 & 0.59203 & 0.855 \\
\hline 453.64 & 0.76074 & 1.20533 & 0.85949 & 0.59064 & 0.854 \\
\hline 454.00 & 0.76035 & 1.20339 & 0.85846 & 0.58918 & 0.853 \\
\hline 454.37 & 0.76011 & 1.19974 & 0.85645 & 0.58857 & 0.851 \\
\hline 454.73 & 0.7601 & 1.19928 & 0.85458 & 0.58805 & 0.851 \\
\hline 455.10 & 0.75981 & 1.19862 & 0.85311 & 0.58738 & 0.850 \\
\hline
\end{tabular}




\begin{tabular}{|c|c|c|c|c|c|}
\hline 455.47 & 0.75807 & 1.19387 & 0.85042 & 0.58652 & 0.847 \\
\hline 455.83 & 0.75779 & 1.19266 & 0.84955 & 0.58594 & 0.846 \\
\hline 456.20 & 0.75588 & 1.19192 & 0.84945 & 0.58433 & 0.845 \\
\hline 456.56 & 0.75477 & 1.19025 & 0.84931 & 0.58351 & 0.844 \\
\hline 456.93 & 0.75445 & 1.19035 & 0.84712 & 0.58411 & 0.844 \\
\hline 457.29 & 0.75212 & 1.18908 & 0.84597 & 0.58211 & 0.842 \\
\hline 457.66 & 0.75319 & 1.18641 & 0.84538 & 0.58056 & 0.841 \\
\hline 458.02 & 0.75249 & 1.18431 & 0.84348 & 0.57892 & 0.840 \\
\hline 458.39 & 0.75115 & 1.18386 & 0.84292 & 0.57803 & 0.839 \\
\hline 458.75 & 0.74843 & 1.17973 & 0.83983 & 0.576 & 0.836 \\
\hline 459.12 & 0.747 & 1.17813 & 0.83944 & 0.57565 & 0.835 \\
\hline 459.48 & 0.74559 & 1.17449 & 0.83829 & 0.57519 & 0.833 \\
\hline 459.85 & 0.7445 & 1.1725 & 0.83494 & 0.57541 & 0.832 \\
\hline 460.21 & 0.74253 & 1.17022 & 0.8341 & 0.57432 & 0.830 \\
\hline 460.58 & 0.74221 & 1.16755 & 0.83288 & 0.5731 & 0.829 \\
\hline 460.94 & 0.74057 & 1.16202 & 0.82951 & 0.5713 & 0.826 \\
\hline 461.31 & 0.73981 & 1.15882 & 0.8276 & 0.5706 & 0.824 \\
\hline 461.67 & 0.73848 & 1.15637 & 0.82541 & 0.57018 & 0.823 \\
\hline 462.04 & 0.73722 & 1.15375 & 0.82414 & 0.56971 & 0.821 \\
\hline 462.40 & 0.73684 & 1.15211 & 0.82221 & 0.56915 & 0.820 \\
\hline 462.77 & 0.7354 & 1.15073 & 0.8184 & 0.56801 & 0.818 \\
\hline 463.13 & 0.73485 & 1.14964 & 0.81689 & 0.56716 & 0.817 \\
\hline 463.50 & 0.73325 & 1.14597 & 0.81444 & 0.56563 & 0.815 \\
\hline 463.86 & 0.73162 & 1.14214 & 0.81186 & 0.56497 & 0.813 \\
\hline 464.23 & 0.72997 & 1.14224 & 0.80931 & 0.56376 & 0.811 \\
\hline 464.59 & 0.72868 & 1.13924 & 0.80684 & 0.56267 & 0.809 \\
\hline 464.96 & 0.72771 & 1.13702 & 0.80409 & 0.5624 & 0.808 \\
\hline 465.32 & 0.72544 & 1.13355 & 0.80035 & 0.56168 & 0.805 \\
\hline 465.69 & 0.72439 & 1.13421 & 0.79851 & 0.56129 & 0.805 \\
\hline 466.05 & 0.72259 & 1.1293 & 0.79592 & 0.55949 & 0.802 \\
\hline 466.41 & 0.72208 & 1.127 & 0.7944 & 0.55856 & 0.801 \\
\hline 466.78 & 0.71983 & 1.12236 & 0.79098 & 0.55726 & 0.798 \\
\hline 467.14 & 0.71858 & 1.12106 & 0.78851 & 0.55575 & 0.796 \\
\hline 467.51 & 0.71679 & 1.11986 & 0.78662 & 0.55431 & 0.794 \\
\hline 467.87 & 0.71522 & 1.11832 & 0.7854 & 0.5535 & 0.793 \\
\hline 468.24 & 0.71343 & 1.11869 & 0.78311 & 0.55265 & 0.792 \\
\hline 468.60 & 0.71253 & 1.11815 & 0.78182 & 0.55247 & 0.791 \\
\hline 468.96 & 0.71011 & 1.1151 & 0.77868 & 0.55055 & 0.789 \\
\hline 469.33 & 0.70804 & 1.11149 & 0.77618 & 0.5486 & 0.786 \\
\hline & & & & & \\
\hline
\end{tabular}




\begin{tabular}{|c|c|c|c|c|c|}
\hline 469.69 & 0.70658 & 1.10952 & 0.77453 & 0.54731 & 0.784 \\
\hline 470.06 & 0.70401 & 1.10797 & 0.77287 & 0.54509 & 0.782 \\
\hline 470.42 & 0.70269 & 1.10658 & 0.77137 & 0.54351 & 0.781 \\
\hline 470.78 & 0.70098 & 1.1049 & 0.76953 & 0.54175 & 0.779 \\
\hline 471.15 & 0.69835 & 1.10215 & 0.7667 & 0.54041 & 0.777 \\
\hline 471.51 & 0.69711 & 1.10124 & 0.76461 & 0.53948 & 0.776 \\
\hline 471.88 & 0.69474 & 1.09832 & 0.76297 & 0.53776 & 0.773 \\
\hline 472.24 & 0.69294 & 1.09834 & 0.76206 & 0.53647 & 0.772 \\
\hline 472.60 & 0.69109 & 1.0963 & 0.76063 & 0.5348 & 0.771 \\
\hline 472.97 & 0.68882 & 1.09477 & 0.75949 & 0.53268 & 0.769 \\
\hline 473.33 & 0.68718 & 1.09171 & 0.7574 & 0.53052 & 0.767 \\
\hline 473.69 & 0.68551 & 1.09151 & 0.7557 & 0.52953 & 0.766 \\
\hline 474.06 & 0.68398 & 1.09051 & 0.75425 & 0.52829 & 0.764 \\
\hline 474.42 & 0.68271 & 1.0887 & 0.75227 & 0.52606 & 0.762 \\
\hline 474.78 & 0.68085 & 1.08631 & 0.74982 & 0.52426 & 0.760 \\
\hline 475.15 & 0.67987 & 1.08288 & 0.74798 & 0.52271 & 0.758 \\
\hline 475.51 & 0.67784 & 1.08038 & 0.74533 & 0.52061 & 0.756 \\
\hline 475.87 & 0.67613 & 1.07667 & 0.74317 & 0.51878 & 0.754 \\
\hline 476.24 & 0.67383 & 1.07463 & 0.74117 & 0.51659 & 0.752 \\
\hline 476.60 & 0.6727 & 1.07136 & 0.73918 & 0.51551 & 0.750 \\
\hline 476.96 & 0.67122 & 1.06991 & 0.73721 & 0.51393 & 0.748 \\
\hline 477.33 & 0.66941 & 1.06829 & 0.73499 & 0.51187 & 0.746 \\
\hline 477.69 & 0.6674 & 1.06475 & 0.73269 & 0.5097 & 0.744 \\
\hline 478.05 & 0.66529 & 1.06109 & 0.73064 & 0.5075 & 0.741 \\
\hline 478.42 & 0.66344 & 1.0596 & 0.72801 & 0.50579 & 0.739 \\
\hline 478.78 & 0.6616 & 1.05751 & 0.72613 & 0.50379 & 0.737 \\
\hline 479.14 & 0.65905 & 1.05549 & 0.72401 & 0.50165 & 0.735 \\
\hline 479.51 & 0.65707 & 1.05274 & 0.72151 & 0.49997 & 0.733 \\
\hline 479.87 & 0.65483 & 1.04944 & 0.71876 & 0.49797 & 0.730 \\
\hline 480.23 & 0.65328 & 1.04688 & 0.71632 & 0.49628 & 0.728 \\
\hline 480.60 & 0.65131 & 1.04378 & 0.71368 & 0.49482 & 0.726 \\
\hline 480.96 & 0.64868 & 1.03913 & 0.71082 & 0.49304 & 0.723 \\
\hline 481.32 & 0.64659 & 1.03614 & 0.70834 & 0.49087 & 0.720 \\
\hline 481.68 & 0.64453 & 1.03421 & 0.7057 & 0.48879 & 0.718 \\
\hline 482.05 & 0.64251 & 1.03161 & 0.7031 & 0.48657 & 0.716 \\
\hline 482.41 & 0.64044 & 1.02913 & 0.70057 & 0.48438 & 0.714 \\
\hline 482.77 & 0.63738 & 1.02713 & 0.69754 & 0.48218 & 0.711 \\
\hline 483.13 & 0.63527 & 1.02482 & 0.69537 & 0.4805 & 0.709 \\
\hline 483.50 & 0.63326 & 1.02184 & 0.69287 & 0.47872 & 0.707 \\
\hline
\end{tabular}




\begin{tabular}{|c|c|c|c|c|c|}
\hline 483.86 & 0.63089 & 1.01807 & 0.69036 & 0.47638 & 0.704 \\
\hline 484.22 & 0.62837 & 1.0162 & 0.68769 & 0.47403 & 0.702 \\
\hline 484.58 & 0.62609 & 1.01334 & 0.68484 & 0.47227 & 0.699 \\
\hline 484.95 & 0.6236 & 1.01029 & 0.68191 & 0.4703 & 0.697 \\
\hline 485.31 & 0.62111 & 1.00677 & 0.67855 & 0.46836 & 0.694 \\
\hline 485.67 & 0.61846 & 1.00393 & 0.67534 & 0.46667 & 0.691 \\
\hline 486.03 & 0.61577 & 1.00032 & 0.67245 & 0.46443 & 0.688 \\
\hline 486.40 & 0.61318 & 0.99673 & 0.66947 & 0.46257 & 0.685 \\
\hline 486.76 & 0.611 & 0.99335 & 0.66626 & 0.46077 & 0.683 \\
\hline 487.12 & 0.6091 & 0.9907 & 0.66341 & 0.45868 & 0.680 \\
\hline 487.48 & 0.60668 & 0.98806 & 0.66034 & 0.45685 & 0.678 \\
\hline 487.85 & 0.60401 & 0.98482 & 0.65687 & 0.45497 & 0.675 \\
\hline 488.21 & 0.60169 & 0.98217 & 0.65364 & 0.45315 & 0.673 \\
\hline 488.57 & 0.59914 & 0.98007 & 0.6504 & 0.45128 & 0.670 \\
\hline 488.93 & 0.59653 & 0.97722 & 0.6474 & 0.44947 & 0.668 \\
\hline 489.29 & 0.59363 & 0.97354 & 0.6443 & 0.44731 & 0.665 \\
\hline 489.65 & 0.5912 & 0.97101 & 0.64122 & 0.44602 & 0.662 \\
\hline 490.02 & 0.58826 & 0.96737 & 0.63802 & 0.44403 & 0.659 \\
\hline 490.38 & 0.58592 & 0.96347 & 0.63475 & 0.44236 & 0.657 \\
\hline 490.74 & 0.58325 & 0.95917 & 0.63091 & 0.44011 & 0.653 \\
\hline 491.10 & 0.58033 & 0.95521 & 0.62747 & 0.43801 & 0.650 \\
\hline 491.46 & 0.57764 & 0.95196 & 0.62371 & 0.43611 & 0.647 \\
\hline 491.83 & 0.57463 & 0.94822 & 0.62034 & 0.43419 & 0.644 \\
\hline 492.19 & 0.57157 & 0.94457 & 0.61732 & 0.43202 & 0.641 \\
\hline 492.55 & 0.56894 & 0.94088 & 0.61388 & 0.42982 & 0.638 \\
\hline 492.91 & 0.56604 & 0.9375 & 0.61094 & 0.42776 & 0.636 \\
\hline 493.27 & 0.56313 & 0.93464 & 0.60791 & 0.4259 & 0.633 \\
\hline 493.63 & 0.56024 & 0.93078 & 0.60445 & 0.42386 & 0.630 \\
\hline 493.99 & 0.55765 & 0.92791 & 0.60153 & 0.42164 & 0.627 \\
\hline 494.36 & 0.55468 & 0.92372 & 0.59847 & 0.41908 & 0.624 \\
\hline 494.72 & 0.55171 & 0.91974 & 0.59504 & 0.41693 & 0.621 \\
\hline 495.08 & 0.54891 & 0.91542 & 0.59218 & 0.41449 & 0.618 \\
\hline 495.44 & 0.54595 & 0.91168 & 0.58924 & 0.41202 & 0.615 \\
\hline 495.80 & 0.54296 & 0.90773 & 0.58629 & 0.40954 & 0.612 \\
\hline 496.16 & 0.53977 & 0.90375 & 0.58295 & 0.4071 & 0.608 \\
\hline 496.52 & 0.53675 & 0.89945 & 0.57957 & 0.40482 & 0.605 \\
\hline 496.88 & 0.53378 & 0.89568 & 0.57617 & 0.40257 & 0.602 \\
\hline 497.25 & 0.53081 & 0.89153 & 0.57298 & 0.39989 & 0.599 \\
\hline 497.61 & 0.52813 & 0.88757 & 0.57013 & 0.39772 & 0.596 \\
\hline & & & & & \\
\hline
\end{tabular}




\begin{tabular}{|c|c|c|c|c|c|}
\hline 497.97 & 0.52528 & 0.88404 & 0.56705 & 0.39512 & 0.593 \\
\hline 498.33 & 0.52208 & 0.88017 & 0.56404 & 0.39248 & 0.590 \\
\hline 498.69 & 0.5189 & 0.87664 & 0.56065 & 0.38999 & 0.587 \\
\hline 499.05 & 0.51591 & 0.87302 & 0.55776 & 0.38765 & 0.584 \\
\hline 499.41 & 0.51283 & 0.86883 & 0.55471 & 0.38504 & 0.580 \\
\hline 499.77 & 0.51001 & 0.86468 & 0.55135 & 0.38254 & 0.577 \\
\hline 500.13 & 0.50682 & 0.86031 & 0.54806 & 0.38007 & 0.574 \\
\hline 500.49 & 0.50371 & 0.85567 & 0.5448 & 0.37749 & 0.570 \\
\hline 500.85 & 0.50059 & 0.8508 & 0.54129 & 0.37443 & 0.567 \\
\hline 501.22 & 0.49756 & 0.8466 & 0.53789 & 0.3719 & 0.563 \\
\hline 501.58 & 0.49461 & 0.84207 & 0.53429 & 0.36942 & 0.560 \\
\hline 501.94 & 0.49136 & 0.83779 & 0.53067 & 0.36701 & 0.557 \\
\hline 502.30 & 0.48798 & 0.83362 & 0.52722 & 0.36417 & 0.553 \\
\hline 502.66 & 0.48449 & 0.82906 & 0.52349 & 0.36129 & 0.550 \\
\hline 503.02 & 0.481 & 0.82459 & 0.5198 & 0.35849 & 0.546 \\
\hline 503.38 & 0.47755 & 0.82006 & 0.51595 & 0.35566 & 0.542 \\
\hline 503.74 & 0.47424 & 0.81537 & 0.51246 & 0.35287 & 0.539 \\
\hline 504.10 & 0.4711 & 0.81098 & 0.50897 & 0.34998 & 0.535 \\
\hline 504.46 & 0.46752 & 0.8059 & 0.50487 & 0.34687 & 0.531 \\
\hline 504.82 & 0.46375 & 0.80125 & 0.50113 & 0.34395 & 0.528 \\
\hline 505.18 & 0.46007 & 0.79652 & 0.49709 & 0.34104 & 0.524 \\
\hline 505.54 & 0.45628 & 0.7917 & 0.49329 & 0.3382 & 0.520 \\
\hline 505.90 & 0.45274 & 0.78712 & 0.48949 & 0.33549 & 0.516 \\
\hline 506.26 & 0.44921 & 0.7821 & 0.4857 & 0.33264 & 0.512 \\
\hline 506.62 & 0.44557 & 0.77702 & 0.48166 & 0.32937 & 0.508 \\
\hline 506.98 & 0.44213 & 0.7725 & 0.47784 & 0.32646 & 0.505 \\
\hline 507.34 & 0.43845 & 0.7674 & 0.4737 & 0.32315 & 0.501 \\
\hline 507.70 & 0.43442 & 0.76244 & 0.46976 & 0.31996 & 0.497 \\
\hline 508.06 & 0.43083 & 0.75785 & 0.46584 & 0.31692 & 0.493 \\
\hline 508.42 & 0.42733 & 0.75324 & 0.46201 & 0.31391 & 0.489 \\
\hline 508.78 & 0.42386 & 0.74827 & 0.45822 & 0.31078 & 0.485 \\
\hline 509.14 & 0.41987 & 0.74294 & 0.45444 & 0.30754 & 0.481 \\
\hline 509.50 & 0.41599 & 0.73756 & 0.45033 & 0.3042 & 0.477 \\
\hline 509.86 & 0.41223 & 0.73228 & 0.44616 & 0.30103 & 0.473 \\
\hline 510.22 & 0.40854 & 0.72721 & 0.4422 & 0.29792 & 0.469 \\
\hline 510.58 & 0.40471 & 0.72213 & 0.43814 & 0.29485 & 0.465 \\
\hline 510.94 & 0.40093 & 0.71722 & 0.4344 & 0.29157 & 0.461 \\
\hline 511.30 & 0.39729 & 0.71228 & 0.43046 & 0.28836 & 0.457 \\
\hline 511.66 & 0.39337 & 0.70714 & 0.42648 & 0.28522 & 0.453 \\
\hline & & & & & \\
\hline
\end{tabular}




\begin{tabular}{|c|c|c|c|c|c|}
\hline 512.02 & 0.38961 & 0.70242 & 0.42289 & 0.28223 & 0.449 \\
\hline 512.38 & 0.38622 & 0.69739 & 0.41925 & 0.27945 & 0.446 \\
\hline 512.74 & 0.38238 & 0.69257 & 0.41536 & 0.2763 & 0.442 \\
\hline 513.09 & 0.37862 & 0.68765 & 0.41148 & 0.27309 & 0.438 \\
\hline 513.45 & 0.37497 & 0.68288 & 0.40758 & 0.27006 & 0.434 \\
\hline 513.81 & 0.37118 & 0.6784 & 0.40384 & 0.267 & 0.430 \\
\hline 514.17 & 0.36734 & 0.67341 & 0.40009 & 0.26409 & 0.426 \\
\hline 514.53 & 0.36365 & 0.66866 & 0.39639 & 0.26096 & 0.422 \\
\hline 514.89 & 0.35986 & 0.66424 & 0.39271 & 0.2582 & 0.419 \\
\hline 515.25 & 0.35625 & 0.6594 & 0.38904 & 0.25557 & 0.415 \\
\hline 515.61 & 0.35253 & 0.65459 & 0.38533 & 0.25239 & 0.411 \\
\hline 515.97 & 0.34861 & 0.64933 & 0.38137 & 0.24943 & 0.407 \\
\hline 516.33 & 0.34466 & 0.64461 & 0.37747 & 0.24643 & 0.403 \\
\hline 516.69 & 0.34085 & 0.63981 & 0.37373 & 0.24351 & 0.399 \\
\hline 517.04 & 0.3373 & 0.63541 & 0.37007 & 0.2407 & 0.396 \\
\hline 517.40 & 0.33351 & 0.63038 & 0.36636 & 0.23784 & 0.392 \\
\hline 517.76 & 0.32966 & 0.62569 & 0.36253 & 0.23491 & 0.388 \\
\hline 518.12 & 0.32608 & 0.62091 & 0.3587 & 0.23193 & 0.384 \\
\hline 518.48 & 0.3223 & 0.616 & 0.35473 & 0.22893 & 0.380 \\
\hline 518.84 & 0.31839 & 0.61126 & 0.35081 & 0.22607 & 0.377 \\
\hline 519.20 & 0.31452 & 0.60642 & 0.34713 & 0.22302 & 0.373 \\
\hline 519.56 & 0.31091 & 0.60144 & 0.34328 & 0.22021 & 0.369 \\
\hline 519.91 & 0.30697 & 0.59663 & 0.33948 & 0.21711 & 0.365 \\
\hline 520.27 & 0.30332 & 0.59176 & 0.33579 & 0.21412 & 0.361 \\
\hline 520.63 & 0.29953 & 0.58661 & 0.33188 & 0.21113 & 0.357 \\
\hline 520.99 & 0.29572 & 0.58145 & 0.32821 & 0.20816 & 0.353 \\
\hline 521.35 & 0.2921 & 0.57612 & 0.3245 & 0.20512 & 0.349 \\
\hline 521.71 & 0.28865 & 0.57157 & 0.32075 & 0.2021 & 0.346 \\
\hline 522.06 & 0.28493 & 0.56623 & 0.31694 & 0.19903 & 0.342 \\
\hline 522.42 & 0.28141 & 0.56137 & 0.31355 & 0.19617 & 0.338 \\
\hline 522.78 & 0.2778 & 0.55686 & 0.30987 & 0.19293 & 0.334 \\
\hline 523.14 & 0.27415 & 0.55186 & 0.30609 & 0.18988 & 0.330 \\
\hline 523.50 & 0.27058 & 0.54719 & 0.30254 & 0.18698 & 0.327 \\
\hline 523.86 & 0.26669 & 0.54217 & 0.29891 & 0.18399 & 0.323 \\
\hline 524.21 & 0.26316 & 0.53728 & 0.29508 & 0.18094 & 0.319 \\
\hline 524.57 & 0.25944 & 0.53246 & 0.29152 & 0.17798 & 0.315 \\
\hline 524.93 & 0.25579 & 0.52763 & 0.28791 & 0.17501 & 0.312 \\
\hline 525.29 & 0.25233 & 0.52284 & 0.28447 & 0.17222 & 0.308 \\
\hline 525.65 & 0.24894 & 0.51789 & 0.28125 & 0.16951 & 0.304 \\
\hline
\end{tabular}




\begin{tabular}{|c|c|c|c|c|c|}
\hline 526.00 & 0.24533 & 0.51283 & 0.27771 & 0.16671 & 0.301 \\
\hline 526.36 & 0.24191 & 0.50798 & 0.27442 & 0.16384 & 0.297 \\
\hline 526.72 & 0.23846 & 0.50307 & 0.27066 & 0.16112 & 0.293 \\
\hline 527.08 & 0.23501 & 0.49821 & 0.26736 & 0.1584 & 0.290 \\
\hline 527.44 & 0.23156 & 0.49315 & 0.26391 & 0.15572 & 0.286 \\
\hline 527.79 & 0.22798 & 0.48821 & 0.26036 & 0.15284 & 0.282 \\
\hline 528.15 & 0.22448 & 0.48355 & 0.25697 & 0.14994 & 0.279 \\
\hline 528.51 & 0.22101 & 0.47876 & 0.25335 & 0.14718 & 0.275 \\
\hline 528.87 & 0.2174 & 0.47404 & 0.24978 & 0.14436 & 0.271 \\
\hline 529.22 & 0.21381 & 0.46891 & 0.24626 & 0.14166 & 0.268 \\
\hline 529.58 & 0.21057 & 0.46421 & 0.24264 & 0.13916 & 0.264 \\
\hline 529.94 & 0.20708 & 0.45929 & 0.23915 & 0.13639 & 0.260 \\
\hline 530.30 & 0.20367 & 0.45415 & 0.23561 & 0.13372 & 0.257 \\
\hline 530.65 & 0.20023 & 0.44931 & 0.23233 & 0.13134 & 0.253 \\
\hline 531.01 & 0.19679 & 0.44443 & 0.22892 & 0.12858 & 0.250 \\
\hline 531.37 & 0.19357 & 0.43976 & 0.22576 & 0.12612 & 0.246 \\
\hline 531.72 & 0.19019 & 0.43502 & 0.22268 & 0.1236 & 0.243 \\
\hline 532.08 & 0.18681 & 0.42989 & 0.21936 & 0.12093 & 0.239 \\
\hline 532.44 & 0.18362 & 0.42511 & 0.21628 & 0.11843 & 0.236 \\
\hline 532.80 & 0.18013 & 0.42001 & 0.21276 & 0.11559 & 0.232 \\
\hline 533.15 & 0.17674 & 0.41546 & 0.20948 & 0.1129 & 0.229 \\
\hline 533.51 & 0.17338 & 0.41078 & 0.2063 & 0.11041 & 0.225 \\
\hline 533.87 & 0.17012 & 0.40589 & 0.20308 & 0.10778 & 0.222 \\
\hline 534.22 & 0.16682 & 0.40133 & 0.20009 & 0.10532 & 0.218 \\
\hline 534.58 & 0.16362 & 0.3966 & 0.19691 & 0.10264 & 0.215 \\
\hline 534.94 & 0.16031 & 0.39207 & 0.1938 & 0.10001 & 0.212 \\
\hline 535.30 & 0.15723 & 0.38737 & 0.19084 & 0.09773 & 0.208 \\
\hline 535.65 & 0.15402 & 0.38268 & 0.1878 & 0.09541 & 0.205 \\
\hline 536.01 & 0.15088 & 0.37801 & 0.18491 & 0.09306 & 0.202 \\
\hline 536.37 & 0.14789 & 0.37338 & 0.18211 & 0.09074 & 0.199 \\
\hline 536.72 & 0.14459 & 0.36881 & 0.17917 & 0.08851 & 0.195 \\
\hline 537.08 & 0.14145 & 0.36435 & 0.1762 & 0.08602 & 0.192 \\
\hline 537.44 & 0.1383 & 0.35964 & 0.17313 & 0.08354 & 0.189 \\
\hline 537.79 & 0.13524 & 0.35506 & 0.17035 & 0.08116 & 0.185 \\
\hline 538.15 & 0.1321 & 0.35057 & 0.16748 & 0.0788 & 0.182 \\
\hline 538.51 & 0.12896 & 0.34608 & 0.16458 & 0.07656 & 0.179 \\
\hline 538.86 & 0.12587 & 0.34138 & 0.16157 & 0.07413 & 0.176 \\
\hline 539.22 & 0.12278 & 0.33693 & 0.15844 & 0.0717 & 0.172 \\
\hline 539.57 & 0.11966 & 0.33247 & 0.15565 & 0.0695 & 0.169 \\
\hline & & & & & \\
\hline
\end{tabular}




\begin{tabular}{|c|c|c|c|c|c|}
\hline 539.93 & 0.11643 & 0.32806 & 0.15283 & 0.06716 & 0.166 \\
\hline 540.29 & 0.11335 & 0.32365 & 0.15026 & 0.06502 & 0.163 \\
\hline 540.64 & 0.11007 & 0.31904 & 0.14735 & 0.0627 & 0.160 \\
\hline 541.00 & 0.10698 & 0.31448 & 0.14457 & 0.06035 & 0.157 \\
\hline 541.36 & 0.10402 & 0.31015 & 0.14189 & 0.05825 & 0.154 \\
\hline 541.71 & 0.10097 & 0.30563 & 0.13897 & 0.05591 & 0.150 \\
\hline 542.07 & 0.09771 & 0.30123 & 0.13616 & 0.05359 & 0.147 \\
\hline 542.42 & 0.09467 & 0.29676 & 0.1334 & 0.05132 & 0.144 \\
\hline 542.78 & 0.09159 & 0.29258 & 0.13093 & 0.04906 & 0.141 \\
\hline 543.14 & 0.08861 & 0.28816 & 0.12837 & 0.04691 & 0.138 \\
\hline 543.49 & 0.0858 & 0.28398 & 0.1258 & 0.04467 & 0.135 \\
\hline 543.85 & 0.08274 & 0.27978 & 0.12321 & 0.04246 & 0.132 \\
\hline 544.20 & 0.0799 & 0.27563 & 0.12069 & 0.04007 & 0.129 \\
\hline 544.56 & 0.0768 & 0.2712 & 0.11814 & 0.0379 & 0.126 \\
\hline 544.92 & 0.07391 & 0.26702 & 0.11582 & 0.03582 & 0.123 \\
\hline 545.27 & 0.07091 & 0.26277 & 0.11334 & 0.03375 & 0.120 \\
\hline 545.63 & 0.06812 & 0.25837 & 0.11082 & 0.03154 & 0.117 \\
\hline 545.98 & 0.0653 & 0.25407 & 0.10836 & 0.02937 & 0.114 \\
\hline 546.34 & 0.06245 & 0.24976 & 0.10596 & 0.02716 & 0.111 \\
\hline 546.69 & 0.05975 & 0.24552 & 0.10379 & 0.0252 & 0.109 \\
\hline 547.05 & 0.05709 & 0.24144 & 0.10154 & 0.02298 & 0.106 \\
\hline 547.40 & 0.05448 & 0.23726 & 0.09905 & 0.02089 & 0.103 \\
\hline 547.76 & 0.0519 & 0.23316 & 0.09662 & 0.01876 & 0.100 \\
\hline 548.12 & 0.04947 & 0.2291 & 0.09444 & 0.01677 & 0.097 \\
\hline 548.47 & 0.0469 & 0.2251 & 0.09208 & 0.01477 & 0.095 \\
\hline 548.83 & 0.04408 & 0.22092 & 0.08967 & 0.01257 & 0.092 \\
\hline 549.18 & 0.04159 & 0.217 & 0.08766 & 0.01063 & 0.089 \\
\hline 549.54 & 0.03916 & 0.21293 & 0.08547 & 0.0088 & 0.087 \\
\hline 549.89 & 0.03669 & 0.20891 & 0.08334 & 0.00685 & 0.084 \\
\hline 550.25 & 0.03413 & 0.20468 & 0.08074 & 0.00483 & 0.081 \\
\hline 550.60 & 0.03156 & 0.20065 & 0.07841 & 0.00273 & 0.078 \\
\hline 550.96 & 0.02887 & 0.19662 & 0.076 & 0.00068 & 0.076 \\
\hline 551.31 & 0.02631 & 0.1925 & 0.07357 & -0.0014 & 0.073 \\
\hline 551.67 & 0.02384 & 0.18843 & 0.07141 & -0.0033 & 0.070 \\
\hline 552.02 & 0.02144 & 0.18456 & 0.06938 & -0.0052 & 0.068 \\
\hline 552.38 & 0.01896 & 0.18051 & 0.06718 & -0.0071 & 0.065 \\
\hline 552.73 & 0.01672 & 0.1768 & 0.06514 & -0.0089 & 0.062 \\
\hline 553.09 & 0.01428 & 0.17305 & 0.06304 & -0.0108 & 0.060 \\
\hline 553.44 & 0.0119 & 0.16921 & 0.06093 & -0.0127 & 0.057 \\
\hline
\end{tabular}




\begin{tabular}{|c|c|c|c|c|c|}
\hline 553.80 & 0.00945 & 0.16551 & 0.05878 & -0.0145 & 0.055 \\
\hline 554.15 & 0.00704 & 0.16185 & 0.05672 & -0.0164 & 0.052 \\
\hline 554.51 & 0.00464 & 0.1581 & 0.05461 & -0.0181 & 0.050 \\
\hline 554.86 & 0.00228 & 0.15445 & 0.05268 & -0.0199 & 0.047 \\
\hline 555.21 & $2.8 \mathrm{E}-05$ & 0.15083 & 0.05079 & -0.0216 & 0.045 \\
\hline 555.57 & -0.0023 & 0.14722 & 0.04878 & -0.0233 & 0.043 \\
\hline 555.92 & -0.0045 & 0.14357 & 0.04693 & -0.025 & 0.040 \\
\hline 556.28 & -0.0068 & 0.13981 & 0.04492 & -0.0267 & 0.038 \\
\hline 556.63 & -0.0092 & 0.13605 & 0.04283 & -0.0286 & 0.035 \\
\hline 556.99 & -0.0116 & 0.13242 & 0.04078 & -0.0304 & 0.033 \\
\hline 557.34 & -0.0138 & 0.12893 & 0.03872 & -0.032 & 0.030 \\
\hline 557.70 & -0.016 & 0.12551 & 0.03687 & -0.0337 & 0.028 \\
\hline 558.05 & -0.0181 & 0.12225 & 0.03518 & -0.0352 & 0.026 \\
\hline 558.40 & -0.0202 & 0.1188 & 0.03344 & -0.0368 & 0.024 \\
\hline 558.76 & -0.0224 & 0.11541 & 0.03164 & -0.0384 & 0.022 \\
\hline 559.11 & -0.0246 & 0.11201 & 0.02977 & -0.0399 & 0.019 \\
\hline 559.47 & -0.0268 & 0.10864 & 0.02794 & -0.0414 & 0.017 \\
\hline 559.82 & -0.0288 & 0.1054 & 0.02607 & -0.043 & 0.015 \\
\hline 560.17 & -0.0311 & 0.10204 & 0.02413 & -0.0445 & 0.013 \\
\hline 560.53 & -0.0332 & 0.09881 & 0.02232 & -0.046 & 0.010 \\
\hline 560.88 & -0.0352 & 0.09565 & 0.02073 & -0.0475 & 0.008 \\
\hline 561.24 & -0.037 & 0.0926 & 0.01927 & -0.0489 & 0.007 \\
\hline 561.59 & -0.039 & 0.08927 & 0.01746 & -0.0504 & 0.004 \\
\hline 561.94 & -0.0408 & 0.08626 & 0.01579 & -0.0519 & 0.002 \\
\hline 562.30 & -0.0429 & 0.08304 & 0.01414 & -0.0534 & 0.000 \\
\hline 562.65 & -0.0449 & 0.07999 & 0.01254 & -0.0548 & -0.002 \\
\hline 563.00 & -0.0469 & 0.07701 & 0.01097 & -0.0563 & -0.004 \\
\hline 563.36 & -0.0487 & 0.07409 & 0.00938 & -0.0579 & -0.006 \\
\hline 563.71 & -0.0504 & 0.07138 & 0.00799 & -0.0592 & -0.008 \\
\hline 564.07 & -0.0522 & 0.06853 & 0.00653 & -0.0605 & -0.009 \\
\hline 564.42 & -0.054 & 0.06568 & 0.00519 & -0.0618 & -0.011 \\
\hline 564.77 & -0.0558 & 0.06288 & 0.00369 & -0.0632 & -0.013 \\
\hline 565.13 & -0.0574 & 0.06025 & 0.00244 & -0.0644 & -0.015 \\
\hline 565.48 & -0.0593 & 0.05739 & 0.00087 & -0.0659 & -0.017 \\
\hline 565.83 & -0.0611 & 0.05471 & -0.0005 & -0.0672 & -0.019 \\
\hline 566.19 & -0.0628 & 0.05215 & -0.0019 & -0.0685 & -0.020 \\
\hline 566.54 & -0.0643 & 0.04958 & -0.0031 & -0.0698 & -0.022 \\
\hline 566.89 & -0.0658 & 0.04709 & -0.0046 & -0.071 & -0.024 \\
\hline 567.25 & -0.0674 & 0.04459 & -0.0058 & -0.0722 & -0.025 \\
\hline & & & & & \\
\hline
\end{tabular}




\begin{tabular}{|c|c|c|c|c|c|}
\hline 567.60 & -0.0691 & 0.04199 & -0.0072 & -0.0736 & -0.027 \\
\hline 567.95 & -0.0706 & 0.03944 & -0.0084 & -0.0747 & -0.029 \\
\hline 568.31 & -0.0721 & 0.03697 & -0.0097 & -0.0759 & -0.030 \\
\hline 568.66 & -0.0739 & 0.03426 & -0.0113 & -0.0773 & -0.032 \\
\hline 569.01 & -0.0754 & 0.03172 & -0.0126 & -0.0785 & -0.034 \\
\hline 569.36 & -0.077 & 0.02923 & -0.0139 & -0.0797 & -0.035 \\
\hline 569.72 & -0.0785 & 0.02696 & -0.0153 & -0.0808 & -0.037 \\
\hline 570.07 & -0.0799 & 0.02458 & -0.0166 & -0.082 & -0.038 \\
\hline 570.42 & -0.0815 & 0.02213 & -0.0179 & -0.0832 & -0.040 \\
\hline 570.78 & -0.0829 & 0.01989 & -0.0192 & -0.0842 & -0.042 \\
\hline 571.13 & -0.0844 & 0.01758 & -0.0205 & -0.0853 & -0.043 \\
\hline 571.48 & -0.0857 & 0.01541 & -0.0217 & -0.0864 & -0.045 \\
\hline 571.83 & -0.0872 & 0.01322 & -0.0231 & -0.0876 & -0.046 \\
\hline 572.19 & -0.0884 & 0.01102 & -0.0241 & -0.0886 & -0.048 \\
\hline 572.54 & -0.0899 & 0.00868 & -0.0254 & -0.0897 & -0.049 \\
\hline 572.89 & -0.0911 & 0.00659 & -0.0266 & -0.0908 & -0.050 \\
\hline 573.24 & -0.0923 & 0.00444 & -0.0277 & -0.0918 & -0.052 \\
\hline 573.60 & -0.0934 & 0.00231 & -0.0288 & -0.0927 & -0.053 \\
\hline 573.95 & -0.0947 & 0.00028 & -0.03 & -0.0939 & -0.055 \\
\hline 574.30 & -0.0959 & -0.0018 & -0.031 & -0.0949 & -0.056 \\
\hline 574.65 & -0.0973 & -0.0039 & -0.0321 & -0.096 & -0.057 \\
\hline 575.01 & -0.0983 & -0.0057 & -0.0331 & -0.0969 & -0.059 \\
\hline 575.36 & -0.0997 & -0.0076 & -0.0343 & -0.0981 & -0.060 \\
\hline 575.71 & -0.1008 & -0.0094 & -0.0354 & -0.0989 & -0.061 \\
\hline 576.06 & -0.1019 & -0.0111 & -0.0363 & -0.0997 & -0.062 \\
\hline 576.41 & -0.103 & -0.0127 & -0.0372 & -0.1006 & -0.063 \\
\hline 576.77 & -0.1042 & -0.0145 & -0.0382 & -0.1017 & -0.065 \\
\hline 577.12 & -0.1052 & -0.0163 & -0.0392 & -0.1025 & -0.066 \\
\hline 577.47 & -0.1063 & -0.018 & -0.0402 & -0.1035 & -0.067 \\
\hline 577.82 & -0.1074 & -0.0196 & -0.0409 & -0.1043 & -0.068 \\
\hline 578.17 & -0.1084 & -0.0213 & -0.0418 & -0.1051 & -0.069 \\
\hline 578.53 & -0.1094 & -0.0229 & -0.0425 & -0.1059 & -0.070 \\
\hline 578.88 & -0.1104 & -0.0245 & -0.0433 & -0.1066 & -0.071 \\
\hline 579.23 & -0.1112 & -0.0259 & -0.0438 & -0.1073 & -0.072 \\
\hline 579.58 & -0.1124 & -0.0274 & -0.0448 & -0.1082 & -0.073 \\
\hline 579.93 & -0.1134 & -0.0288 & -0.0455 & -0.1089 & -0.074 \\
\hline 580.28 & -0.1145 & -0.0304 & -0.0465 & -0.1097 & -0.075 \\
\hline 580.64 & -0.1155 & -0.0319 & -0.0473 & -0.1105 & -0.076 \\
\hline 580.99 & -0.1165 & -0.0332 & -0.0481 & -0.1112 & -0.077 \\
\hline & & & & & \\
\hline
\end{tabular}




\begin{tabular}{|l|l|l|l|l|l|}
\hline 581.34 & -0.1175 & -0.0346 & -0.0489 & -0.1119 & -0.078 \\
\hline 581.69 & -0.1185 & -0.0359 & -0.0498 & -0.1126 & -0.079 \\
\hline 582.04 & -0.1194 & -0.0372 & -0.0505 & -0.1134 & -0.080 \\
\hline 582.39 & -0.1204 & -0.0386 & -0.0513 & -0.1141 & -0.081 \\
\hline 582.75 & -0.1211 & -0.0398 & -0.0519 & -0.1146 & -0.082 \\
\hline 583.10 & -0.122 & -0.0411 & -0.0526 & -0.1153 & -0.083 \\
\hline 583.45 & -0.1229 & -0.0423 & -0.0533 & -0.1158 & -0.084 \\
\hline 583.80 & -0.1237 & -0.0435 & -0.054 & -0.1163 & -0.084 \\
\hline 584.15 & -0.1244 & -0.0445 & -0.0545 & -0.1168 & -0.085 \\
\hline 584.50 & -0.1251 & -0.0456 & -0.055 & -0.1174 & -0.086 \\
\hline 584.85 & -0.1258 & -0.0466 & -0.0555 & -0.1178 & -0.086 \\
\hline 585.20 & -0.1265 & -0.0477 & -0.0563 & -0.1184 & -0.087 \\
\hline 585.55 & -0.1273 & -0.0487 & -0.057 & -0.119 & -0.088 \\
\hline 585.91 & -0.128 & -0.0496 & -0.0577 & -0.1195 & -0.089 \\
\hline 586.26 & -0.1289 & -0.0507 & -0.0583 & -0.1201 & -0.089 \\
\hline 586.61 & -0.1297 & -0.0519 & -0.059 & -0.1206 & -0.090 \\
\hline 586.96 & -0.1302 & -0.0528 & -0.0595 & -0.1211 & -0.091 \\
\hline 587.31 & -0.1307 & -0.0538 & -0.06 & -0.1215 & -0.091 \\
\hline 587.66 & -0.1314 & -0.0545 & -0.0603 & -0.1219 & -0.092 \\
\hline 588.01 & -0.132 & -0.0555 & -0.0608 & -0.1224 & -0.093 \\
\hline 588.36 & -0.1326 & -0.0564 & -0.0614 & -0.123 & -0.093 \\
\hline 588.71 & -0.1332 & -0.0574 & -0.062 & -0.1235 & -0.094 \\
\hline 589.06 & -0.1338 & -0.0583 & -0.0623 & -0.1239 & -0.095 \\
\hline 589.41 & -0.1344 & -0.0592 & -0.0628 & -0.1242 & -0.095 \\
\hline 589.76 & -0.135 & -0.0601 & -0.0631 & -0.1247 & -0.096 \\
\hline 590.11 & -0.1356 & -0.0608 & -0.0636 & -0.1251 & -0.096 \\
\hline 590.46 & -0.1362 & -0.0616 & -0.064 & -0.1255 & -0.097 \\
\hline 590.82 & -0.1367 & -0.0625 & -0.0645 & -0.126 & -0.097 \\
\hline 591.17 & -0.1373 & -0.0633 & -0.065 & -0.1265 & -0.098 \\
\hline 591.52 & -0.1379 & -0.0642 & -0.0654 & -0.1269 & -0.099 \\
\hline 591.87 & -0.1385 & -0.0651 & -0.0659 & -0.1272 & -0.099 \\
\hline 592.22 & -0.1391 & -0.0658 & -0.0664 & -0.1276 & -0.100 \\
\hline 592.57 & -0.1396 & -0.0665 & -0.0667 & -0.1279 & -0.100 \\
\hline 592.92 & -0.1399 & -0.0673 & -0.067 & -0.1282 & -0.101 \\
\hline 593.27 & -0.1405 & -0.0682 & -0.0675 & -0.1286 & -0.101 \\
\hline 593.62 & -0.141 & -0.0689 & -0.068 & -0.1289 & -0.102 \\
\hline 593.97 & -0.1414 & -0.0695 & -0.0684 & -0.1292 & -0.102 \\
\hline 594.32 & -0.1419 & -0.0702 & -0.0689 & -0.1295 & -0.103 \\
\hline 594.67 & -0.1424 & -0.071 & -0.0694 & -0.1299 & -0.103 \\
\hline & & & & & \\
\hline 5
\end{tabular}




\begin{tabular}{|c|c|c|c|c|c|}
\hline 595.02 & -0.1428 & -0.0716 & -0.0697 & -0.1302 & -0.104 \\
\hline 595.37 & -0.1433 & -0.0722 & -0.07 & -0.1305 & -0.104 \\
\hline 595.72 & -0.1438 & -0.0729 & -0.0704 & -0.1307 & -0.104 \\
\hline 596.07 & -0.1441 & -0.0735 & -0.0707 & -0.1309 & -0.105 \\
\hline 596.42 & -0.1444 & -0.074 & -0.0709 & -0.1311 & -0.105 \\
\hline 596.77 & -0.1448 & -0.0745 & -0.0714 & -0.1314 & -0.106 \\
\hline 597.12 & -0.145 & -0.075 & -0.0717 & -0.1316 & -0.106 \\
\hline 597.46 & -0.1454 & -0.0756 & -0.0721 & -0.1319 & -0.106 \\
\hline 597.81 & -0.1458 & -0.0763 & -0.0725 & -0.1322 & -0.107 \\
\hline 598.16 & -0.146 & -0.0765 & -0.0726 & -0.1324 & -0.107 \\
\hline 598.51 & -0.1464 & -0.077 & -0.0729 & -0.1326 & -0.107 \\
\hline 598.86 & -0.1466 & -0.0774 & -0.0731 & -0.1328 & -0.107 \\
\hline 599.21 & -0.1468 & -0.0778 & -0.0733 & -0.133 & -0.108 \\
\hline 599.56 & -0.1472 & -0.0784 & -0.0736 & -0.1334 & -0.108 \\
\hline 599.91 & -0.1476 & -0.0789 & -0.0738 & -0.1337 & -0.109 \\
\hline 600.26 & -0.1479 & -0.0794 & -0.074 & -0.134 & -0.109 \\
\hline 600.61 & -0.1482 & -0.0797 & -0.0743 & -0.1343 & -0.109 \\
\hline 600.96 & -0.1485 & -0.0802 & -0.0744 & -0.1345 & -0.109 \\
\hline 601.31 & -0.1489 & -0.0807 & -0.0747 & -0.1347 & -0.110 \\
\hline 601.66 & -0.1494 & -0.0813 & -0.075 & -0.1351 & -0.110 \\
\hline 602.01 & -0.1496 & -0.0816 & -0.0753 & -0.1353 & -0.110 \\
\hline 602.35 & -0.1501 & -0.0822 & -0.0757 & -0.1356 & -0.111 \\
\hline 602.70 & -0.1502 & -0.0825 & -0.076 & -0.1358 & -0.111 \\
\hline 603.05 & -0.1505 & -0.083 & -0.0762 & -0.1359 & -0.111 \\
\hline 603.40 & -0.1508 & -0.0833 & -0.0763 & -0.1361 & -0.112 \\
\hline 603.75 & -0.1511 & -0.0837 & -0.0766 & -0.1363 & -0.112 \\
\hline 604.10 & -0.1513 & -0.084 & -0.0765 & -0.1364 & -0.112 \\
\hline 604.45 & -0.1516 & -0.0843 & -0.0768 & -0.1367 & -0.112 \\
\hline 604.80 & -0.1519 & -0.0847 & -0.0769 & -0.137 & -0.113 \\
\hline 605.15 & -0.152 & -0.085 & -0.0771 & -0.1372 & -0.113 \\
\hline 605.49 & -0.1522 & -0.0854 & -0.0773 & -0.1373 & -0.113 \\
\hline 605.84 & -0.1525 & -0.0857 & -0.0774 & -0.1375 & -0.113 \\
\hline 606.19 & -0.1528 & -0.086 & -0.0778 & -0.1376 & -0.114 \\
\hline 606.54 & -0.1529 & -0.0862 & -0.0779 & -0.1377 & -0.114 \\
\hline 606.89 & -0.1529 & -0.0863 & -0.078 & -0.1377 & -0.114 \\
\hline 607.24 & -0.1531 & -0.0865 & -0.0782 & -0.1378 & -0.114 \\
\hline 607.59 & -0.1531 & -0.0866 & -0.0784 & -0.1378 & -0.114 \\
\hline 607.93 & -0.1533 & -0.0868 & -0.0785 & -0.1379 & -0.114 \\
\hline 608.28 & -0.1533 & -0.0869 & -0.0786 & -0.1379 & -0.114 \\
\hline
\end{tabular}




\begin{tabular}{|l|l|l|l|l|l|}
\hline 608.63 & -0.1533 & -0.0872 & -0.0787 & -0.138 & -0.114 \\
\hline 608.98 & -0.1533 & -0.0871 & -0.0787 & -0.1378 & -0.114 \\
\hline 609.33 & -0.1534 & -0.0873 & -0.0787 & -0.1378 & -0.114 \\
\hline 609.67 & -0.1533 & -0.0873 & -0.0786 & -0.1378 & -0.114 \\
\hline 610.02 & -0.1533 & -0.0873 & -0.0785 & -0.1377 & -0.114 \\
\hline 610.37 & -0.1532 & -0.0872 & -0.0785 & -0.1377 & -0.114 \\
\hline 610.72 & -0.1533 & -0.0874 & -0.0787 & -0.1378 & -0.114 \\
\hline 611.07 & -0.1533 & -0.0875 & -0.0788 & -0.1378 & -0.114 \\
\hline 611.41 & -0.1535 & -0.0877 & -0.0791 & -0.1379 & -0.115 \\
\hline 611.76 & -0.1536 & -0.0879 & -0.0793 & -0.138 & -0.115 \\
\hline 612.11 & -0.1535 & -0.0879 & -0.0793 & -0.1378 & -0.115 \\
\hline 612.46 & -0.1536 & -0.088 & -0.0793 & -0.1378 & -0.115 \\
\hline 612.81 & -0.1536 & -0.0881 & -0.0794 & -0.1379 & -0.115 \\
\hline 613.15 & -0.1535 & -0.0881 & -0.0794 & -0.1378 & -0.115 \\
\hline 613.50 & -0.1535 & -0.0882 & -0.0794 & -0.1377 & -0.115 \\
\hline 613.85 & -0.1535 & -0.0883 & -0.0795 & -0.1377 & -0.115 \\
\hline 614.20 & -0.1536 & -0.0885 & -0.0795 & -0.1378 & -0.115 \\
\hline 614.54 & -0.1537 & -0.0886 & -0.0796 & -0.1378 & -0.115 \\
\hline 614.89 & -0.1539 & -0.0886 & -0.0797 & -0.1378 & -0.115 \\
\hline 615.24 & -0.1539 & -0.0887 & -0.0797 & -0.1378 & -0.115 \\
\hline 615.59 & -0.154 & -0.0888 & -0.0798 & -0.1378 & -0.115 \\
\hline 615.93 & -0.154 & -0.0888 & -0.0798 & -0.1378 & -0.115 \\
\hline 616.28 & -0.1542 & -0.089 & -0.08 & -0.138 & -0.115 \\
\hline 616.63 & -0.1543 & -0.0892 & -0.0802 & -0.1381 & -0.115 \\
\hline 616.98 & -0.1545 & -0.0895 & -0.0804 & -0.1382 & -0.116 \\
\hline 617.32 & -0.1546 & -0.0898 & -0.0806 & -0.1384 & -0.116 \\
\hline 617.67 & -0.1548 & -0.09 & -0.0808 & -0.1384 & -0.116 \\
\hline 618.02 & -0.1548 & -0.09 & -0.0809 & -0.1383 & -0.116 \\
\hline 618.36 & -0.1549 & -0.0901 & -0.0808 & -0.1383 & -0.116 \\
\hline 618.71 & -0.1549 & -0.0902 & -0.0808 & -0.1382 & -0.116 \\
\hline 619.06 & -0.1549 & -0.0901 & -0.0807 & -0.1381 & -0.116 \\
\hline 619.41 & -0.155 & -0.0903 & -0.0808 & -0.1382 & -0.116 \\
\hline 619.75 & -0.1551 & -0.0903 & -0.081 & -0.1383 & -0.116 \\
\hline 620.10 & -0.1552 & -0.0904 & -0.081 & -0.1383 & -0.116 \\
\hline 620.45 & -0.1554 & -0.0906 & -0.0813 & -0.1385 & -0.116 \\
\hline 620.79 & -0.1555 & -0.0907 & -0.0813 & -0.1386 & -0.117 \\
\hline 621.14 & -0.1556 & -0.091 & -0.0815 & -0.1388 & -0.117 \\
\hline 621.49 & -0.1557 & -0.0912 & -0.0817 & -0.1389 & -0.117 \\
\hline 621.83 & -0.1558 & -0.0914 & -0.0818 & -0.1389 & -0.117 \\
\hline & & & & & \\
\hline 637 &
\end{tabular}




\begin{tabular}{|c|c|c|c|c|c|}
\hline 622.18 & -0.1558 & -0.0916 & -0.0818 & -0.1391 & -0.117 \\
\hline 622.53 & -0.1558 & -0.0917 & -0.0818 & -0.1392 & -0.117 \\
\hline 622.87 & -0.1559 & -0.0919 & -0.0819 & -0.1393 & -0.117 \\
\hline 623.22 & -0.156 & -0.092 & -0.0818 & -0.1393 & -0.117 \\
\hline 623.57 & -0.1559 & -0.0919 & -0.0817 & -0.1392 & -0.117 \\
\hline 623.91 & -0.1559 & -0.092 & -0.0817 & -0.1392 & -0.117 \\
\hline 624.26 & -0.1558 & -0.0919 & -0.0816 & -0.1392 & -0.117 \\
\hline 624.60 & -0.1558 & -0.0919 & -0.0816 & -0.1393 & -0.117 \\
\hline 624.95 & -0.1558 & -0.092 & -0.0816 & -0.1394 & -0.117 \\
\hline 625.30 & -0.1559 & -0.0921 & -0.0816 & -0.1395 & -0.117 \\
\hline 625.64 & -0.1559 & -0.0923 & -0.0817 & -0.1397 & -0.117 \\
\hline 625.99 & -0.1561 & -0.0924 & -0.0819 & -0.1399 & -0.118 \\
\hline 626.34 & -0.1562 & -0.0926 & -0.0819 & -0.14 & -0.118 \\
\hline 626.68 & -0.1562 & -0.0926 & -0.0819 & -0.1401 & -0.118 \\
\hline 627.03 & -0.1562 & -0.0927 & -0.0819 & -0.1401 & -0.118 \\
\hline 627.37 & -0.1563 & -0.0928 & -0.0821 & -0.1403 & -0.118 \\
\hline 627.72 & -0.1561 & -0.0928 & -0.0819 & -0.1403 & -0.118 \\
\hline 628.07 & -0.1561 & -0.0928 & -0.0819 & -0.1402 & -0.118 \\
\hline 628.41 & -0.1561 & -0.0927 & -0.0819 & -0.1402 & -0.118 \\
\hline 628.76 & -0.1561 & -0.0927 & -0.0819 & -0.1401 & -0.118 \\
\hline 629.10 & -0.156 & -0.0927 & -0.082 & -0.1402 & -0.118 \\
\hline 629.45 & -0.156 & -0.0927 & -0.0821 & -0.1401 & -0.118 \\
\hline 629.79 & -0.1561 & -0.0927 & -0.0822 & -0.1402 & -0.118 \\
\hline 630.14 & -0.1561 & -0.0927 & -0.0823 & -0.1402 & -0.118 \\
\hline 630.49 & -0.1561 & -0.0927 & -0.0823 & -0.1402 & -0.118 \\
\hline 630.83 & -0.1562 & -0.0928 & -0.0825 & -0.1402 & -0.118 \\
\hline 631.18 & -0.1562 & -0.0928 & -0.0824 & -0.1402 & -0.118 \\
\hline 631.52 & -0.1563 & -0.0928 & -0.0826 & -0.1402 & -0.118 \\
\hline 631.87 & -0.1563 & -0.0928 & -0.0827 & -0.1402 & -0.118 \\
\hline 632.21 & -0.1564 & -0.0929 & -0.0828 & -0.1403 & -0.118 \\
\hline 632.56 & -0.1565 & -0.0931 & -0.083 & -0.1404 & -0.118 \\
\hline 632.90 & -0.1564 & -0.093 & -0.0831 & -0.1403 & -0.118 \\
\hline 633.25 & -0.1563 & -0.0929 & -0.083 & -0.14 & -0.118 \\
\hline 633.59 & -0.1563 & -0.0928 & -0.0831 & -0.14 & -0.118 \\
\hline 633.94 & -0.1563 & -0.0929 & -0.0832 & -0.1399 & -0.118 \\
\hline 634.28 & -0.1564 & -0.0929 & -0.0832 & -0.14 & -0.118 \\
\hline 634.63 & -0.1564 & -0.0928 & -0.0832 & -0.1399 & -0.118 \\
\hline 634.97 & -0.1565 & -0.093 & -0.0834 & -0.1401 & -0.118 \\
\hline 635.32 & -0.1565 & -0.0929 & -0.0835 & -0.1401 & -0.118 \\
\hline
\end{tabular}




\begin{tabular}{|c|c|c|c|c|c|}
\hline 635.66 & -0.1565 & -0.0929 & -0.0834 & -0.14 & -0.118 \\
\hline 636.01 & -0.1566 & -0.0929 & -0.0834 & -0.14 & -0.118 \\
\hline 636.35 & -0.1566 & -0.0928 & -0.0833 & -0.1399 & -0.118 \\
\hline 636.70 & -0.1566 & -0.0928 & -0.0834 & -0.1399 & -0.118 \\
\hline 637.04 & -0.1565 & -0.0927 & -0.0832 & -0.1398 & -0.118 \\
\hline 637.39 & -0.1563 & -0.0926 & -0.0832 & -0.1398 & -0.118 \\
\hline 637.73 & -0.1563 & -0.0924 & -0.0833 & -0.1397 & -0.118 \\
\hline 638.08 & -0.1561 & -0.0922 & -0.0831 & -0.1396 & -0.118 \\
\hline 638.42 & -0.1561 & -0.0922 & -0.0831 & -0.1394 & -0.118 \\
\hline 638.77 & -0.1561 & -0.0921 & -0.083 & -0.1394 & -0.118 \\
\hline 639.11 & -0.156 & -0.092 & -0.083 & -0.1393 & -0.118 \\
\hline 639.46 & -0.1559 & -0.0919 & -0.0828 & -0.1393 & -0.117 \\
\hline 639.80 & -0.1558 & -0.0917 & -0.0827 & -0.1392 & -0.117 \\
\hline 640.14 & -0.1557 & -0.0915 & -0.0825 & -0.139 & -0.117 \\
\hline 640.49 & -0.1 & -0.0914 & -0.0824 & -0.1389 & -0.117 \\
\hline 640.83 & -0.1553 & -0.0911 & -0.0821 & -0.1388 & -0.117 \\
\hline 641.18 & -0.1552 & -0.091 & -0.0819 & -0.1387 & -0.117 \\
\hline 641.52 & -0.1549 & -0.0907 & -0.0817 & -0.1384 & -0.116 \\
\hline 641.87 & -0.1548 & -0.0907 & -0.0815 & -0.1383 & -0.116 \\
\hline 642.21 & -0.1546 & -0.0903 & -0.0812 & -0.138 & -0.116 \\
\hline 642.55 & -0.1545 & -0.0901 & -0.081 & -0.1378 & -0.116 \\
\hline 642.90 & -0.1541 & -0.0899 & -0.0808 & -0.1375 & -0.116 \\
\hline 643.24 & -0.154 & -0.0898 & -0.0807 & -0.1375 & -0.116 \\
\hline 643.59 & -0.1538 & -0.0896 & -0.0804 & -0.1373 & -0.115 \\
\hline 643.93 & -0.1535 & -0.0893 & -0.0801 & -0.137 & -0.115 \\
\hline 644.27 & -0.1532 & -0.0891 & -0.0799 & -0.1367 & -0.115 \\
\hline 644.62 & -0.153 & -0.089 & -0.0796 & -0.1364 & -0.115 \\
\hline 644.96 & -0.1528 & -0.0889 & -0.0793 & -0.1363 & -0.114 \\
\hline 645.31 & -0.1528 & -0.089 & -0.0794 & -0.1363 & -0.114 \\
\hline 645.65 & -0.1525 & -0.0888 & -0.0792 & -0.1361 & -0.114 \\
\hline 645.99 & -0.1522 & -0.0886 & -0.0789 & -0.1359 & -0.114 \\
\hline 646.34 & -0.1522 & -0.0884 & -0.0787 & -0.1357 & -0.114 \\
\hline 646.68 & -0.1518 & -0.0881 & -0.0784 & -0.1354 & -0.113 \\
\hline 647.02 & -0.1516 & -0.0879 & -0.078 & -0.1351 & -0.113 \\
\hline 647.37 & -0.1515 & -0.0878 & -0.0779 & -0.135 & -0.113 \\
\hline 647.71 & -0.1516 & -0.0878 & -0.0779 & -0.1351 & -0.113 \\
\hline 648.05 & -0.1515 & -0.0878 & -0.0778 & -0.1349 & -0.113 \\
\hline 648.40 & -0.1513 & -0.0877 & -0.0778 & -0.1348 & -0.113 \\
\hline 648.74 & -0.1512 & -0.0875 & -0.0776 & -0.1348 & -0.113 \\
\hline
\end{tabular}




\begin{tabular}{|c|c|c|c|c|c|}
\hline 649.08 & -0.1509 & -0.0872 & -0.0774 & -0.1344 & -0.112 \\
\hline 649.43 & -0.1508 & -0.0873 & -0.0774 & -0.1342 & -0.112 \\
\hline 649.77 & -0.1507 & -0.0872 & -0.0772 & -0.1342 & -0.112 \\
\hline 650.11 & -0.1507 & -0.0874 & -0.0772 & -0.1343 & -0.112 \\
\hline 650.46 & -0.1505 & -0.0871 & -0.0769 & -0.134 & -0.112 \\
\hline 650.80 & -0.1506 & -0.0871 & -0.0769 & -0.1339 & -0.112 \\
\hline 651.14 & -0.1506 & -0.087 & -0.077 & -0.1338 & -0.112 \\
\hline 651.49 & -0.1507 & -0.0871 & -0.0771 & -0.1339 & -0.112 \\
\hline 651.83 & -0.1508 & -0.0871 & -0.0772 & -0.134 & -0.112 \\
\hline 652.17 & -0.1507 & -0.0868 & -0.0772 & -0.1339 & -0.112 \\
\hline 652.51 & -0.1506 & -0.0865 & -0.077 & -0.1336 & -0.112 \\
\hline 652.86 & -0.1506 & -0.0866 & -0.0769 & -0.1336 & -0.112 \\
\hline 653.20 & -0.1505 & -0.0865 & -0.0769 & -0.1336 & -0.112 \\
\hline 653.54 & -0.1504 & -0.0865 & -0.077 & -0.1335 & -0.112 \\
\hline 653.88 & -0.1506 & -0.0865 & -0.0771 & -0.1336 & -0.112 \\
\hline 654.23 & -0.1507 & -0.0865 & -0.0771 & -0.1335 & -0.112 \\
\hline 654.57 & -0.1506 & -0.0864 & -0.077 & -0.1333 & -0.112 \\
\hline 654.91 & -0.1503 & -0.086 & -0.0768 & -0.1331 & -0.112 \\
\hline 655.26 & -0.1504 & -0.086 & -0.0769 & -0.1331 & -0.112 \\
\hline 655.60 & -0.1504 & -0.0861 & -0.077 & -0.1331 & -0.112 \\
\hline 655.94 & -0.1504 & -0.0862 & -0.0771 & -0.1331 & -0.112 \\
\hline 656.28 & -0.1504 & -0.0862 & -0.077 & -0.1332 & -0.112 \\
\hline 656.62 & -0.1504 & -0.0862 & -0.077 & -0.1332 & -0.112 \\
\hline 656.97 & -0.1503 & -0.0861 & -0.0771 & -0.1331 & -0.112 \\
\hline 657.31 & -0.1502 & -0.086 & -0.0772 & -0.133 & -0.112 \\
\hline 657.65 & -0.1502 & -0.086 & -0.0773 & -0.133 & -0.112 \\
\hline 657.99 & -0.1501 & -0.086 & -0.0774 & -0.1329 & -0.112 \\
\hline 658.34 & -0.1501 & -0.0861 & -0.0775 & -0.133 & -0.112 \\
\hline 658.68 & -0.15 & -0.0859 & -0.0773 & -0.1328 & -0.112 \\
\hline 659.02 & -0.1499 & -0.0857 & -0.0772 & -0.1326 & -0.111 \\
\hline 659.36 & -0.1498 & -0.0857 & -0.0771 & -0.1325 & -0.111 \\
\hline 659.70 & -0.1496 & -0.0856 & -0.0771 & -0.1326 & -0.111 \\
\hline 660.05 & -0.1497 & -0.0856 & -0.0772 & -0.1327 & -0.111 \\
\hline 660.39 & -0.1498 & -0.0856 & -0.0772 & -0.1328 & -0.111 \\
\hline 660.73 & -0.1496 & -0.0855 & -0.0769 & -0.1327 & -0.111 \\
\hline 661.07 & -0.1495 & -0.0855 & -0.077 & -0.1326 & -0.111 \\
\hline 661.41 & -0.1494 & -0.0855 & -0.0771 & -0.1328 & -0.111 \\
\hline 661.75 & -0.1493 & -0.0854 & -0.0771 & -0.1326 & -0.111 \\
\hline 662.10 & -0.1495 & -0.0856 & -0.0772 & -0.1329 & -0.111 \\
\hline
\end{tabular}




\begin{tabular}{|c|c|c|c|c|c|}
\hline 662.44 & -0.1494 & -0.0854 & -0.077 & -0.1328 & -0.111 \\
\hline 662.78 & -0.1494 & -0.0852 & -0.077 & -0.1328 & -0.111 \\
\hline 663.12 & -0.1493 & -0.0851 & -0.0769 & -0.1328 & -0.111 \\
\hline 663.46 & -0.1493 & -0.085 & -0.0768 & -0.1328 & -0.111 \\
\hline 663.80 & -0.1492 & -0.0848 & -0.0766 & -0.1328 & -0.111 \\
\hline 664.14 & -0.1494 & -0.0848 & -0.0766 & -0.1328 & -0.111 \\
\hline 664.49 & -0.1493 & -0.0845 & -0.0764 & -0.1327 & -0.111 \\
\hline 664.83 & -0.1493 & -0.0845 & -0.0763 & -0.1327 & -0.111 \\
\hline 665.17 & -0.1492 & -0.0844 & -0.0762 & -0.1327 & -0.111 \\
\hline 665.51 & -0.1491 & -0.0843 & -0.0759 & -0.1326 & -0.110 \\
\hline 665.85 & -0.149 & -0.0842 & -0.0759 & -0.1326 & -0.110 \\
\hline 666.19 & -0.1491 & -0.0841 & -0.0758 & -0.1327 & -0.110 \\
\hline 666.53 & -0.1491 & -0.0841 & -0.0757 & -0.1328 & -0.110 \\
\hline 666.87 & -0.1493 & -0.0842 & -0.0757 & -0.1327 & -0.110 \\
\hline 667.21 & -0.149 & -0.084 & -0.0755 & -0.1326 & -0.110 \\
\hline 667.56 & -0.1 & -0.0 & -0.0753 & 324 & -0.110 \\
\hline 667.90 & -0.1489 & -0.0837 & -0.0753 & -0.1324 & -0.110 \\
\hline 668.24 & -0.1489 & -0.0836 & -0.0752 & -0.1324 & -0.110 \\
\hline 668.58 & -0.1489 & -0.0835 & -0.075 & -0.1324 & -0.110 \\
\hline 668.92 & -0.149 & -0.0836 & -0.0751 & -0.1325 & -0.110 \\
\hline 669.26 & -0.1489 & -0.0835 & -0.075 & -0.1324 & -0.110 \\
\hline 669.60 & -0.1487 & -0.0834 & -0.075 & -0.1322 & -0.110 \\
\hline 669.94 & -0.1486 & -0.0833 & -0.0748 & -0.1322 & -0.110 \\
\hline 670.28 & -0.1486 & -0.0831 & -0.0746 & -0.132 & -0.110 \\
\hline 670.62 & -0.1485 & -0.083 & -0.0746 & -0.1318 & -0.109 \\
\hline 670.96 & -0.1485 & -0.0829 & -0.0745 & -0.1317 & -0.109 \\
\hline 671.30 & -0.1484 & -0.0828 & -0.0745 & -0.1317 & -0.109 \\
\hline 671.64 & -0.1482 & -0.0827 & -0.0744 & -0.1316 & -0.109 \\
\hline 671.98 & -0.1482 & -0.0826 & -0.0744 & -0.1316 & -0.109 \\
\hline 672.32 & -0.1481 & -0.0823 & -0.0741 & -0.1315 & -0.109 \\
\hline 672.66 & -0.148 & -0.0822 & -0.0741 & -0.1314 & -0.109 \\
\hline 673.00 & -0.148 & -0.0821 & -0.074 & -0.1313 & -0.109 \\
\hline 673.34 & -0.1477 & -0.082 & -0.0737 & -0.1311 & -0.109 \\
\hline 673.68 & -0.1476 & -0.0819 & -0.0736 & -0.1309 & -0.109 \\
\hline 674.02 & -0.1476 & -0.0818 & -0.0736 & -0.1309 & -0.108 \\
\hline 674.36 & -0.1477 & -0.0819 & -0.0738 & -0.131 & -0.109 \\
\hline 674.70 & -0.1477 & -0.082 & -0.0738 & -0.131 & -0.109 \\
\hline 675.04 & -0.1476 & -0.0819 & -0.0738 & -0.131 & -0.109 \\
\hline 675.38 & -0.1474 & -0.0819 & -0.0736 & -0.1309 & -0.108 \\
\hline
\end{tabular}




\begin{tabular}{|l|l|l|l|l|l|}
\hline 675.72 & -0.1473 & -0.0817 & -0.0735 & -0.1307 & -0.108 \\
\hline 676.06 & -0.1471 & -0.0816 & -0.0732 & -0.1306 & -0.108 \\
\hline 676.40 & -0.1469 & -0.0814 & -0.0729 & -0.1304 & -0.108 \\
\hline 676.74 & -0.147 & -0.0815 & -0.0729 & -0.1305 & -0.108 \\
\hline 677.08 & -0.1469 & -0.0813 & -0.0727 & -0.1304 & -0.108 \\
\hline 677.42 & -0.1467 & -0.0812 & -0.0726 & -0.1303 & -0.108 \\
\hline 677.76 & -0.1467 & -0.0812 & -0.0725 & -0.1302 & -0.108 \\
\hline 678.10 & -0.1466 & -0.0811 & -0.0724 & -0.1302 & -0.108 \\
\hline 678.44 & -0.1466 & -0.0812 & -0.0724 & -0.1302 & -0.108 \\
\hline 678.78 & -0.1466 & -0.0812 & -0.0724 & -0.1302 & -0.108 \\
\hline 679.12 & -0.1465 & -0.0811 & -0.0722 & -0.13 & -0.107 \\
\hline 679.46 & -0.1464 & -0.0812 & -0.0722 & -0.13 & -0.107 \\
\hline 679.80 & -0.1464 & -0.0813 & -0.0721 & -0.1302 & -0.108 \\
\hline 680.14 & -0.1463 & -0.0814 & -0.0721 & -0.1302 & -0.108 \\
\hline 680.48 & -0.1463 & -0.0812 & -0.0722 & -0.1302 & -0.107 \\
\hline 680.82 & -0.1464 & -0.0812 & -0.0722 & -0.1303 & -0.108 \\
\hline 681.16 & -0.1462 & -0.081 & -0.072 & -0.1301 & -0.107 \\
\hline 681.50 & -0.1459 & -0.0808 & -0.0716 & -0.1299 & -0.107 \\
\hline 681.83 & -0.1457 & -0.0807 & -0.0715 & -0.1298 & -0.107 \\
\hline 682.17 & -0.1455 & -0.0805 & -0.0714 & -0.1297 & -0.107 \\
\hline 682.51 & -0.1453 & -0.0802 & -0.0713 & -0.1295 & -0.107 \\
\hline 682.85 & -0.1451 & -0.08 & -0.0712 & -0.1293 & -0.106 \\
\hline 683.19 & -0.1452 & -0.0798 & -0.0712 & -0.1294 & -0.106 \\
\hline 683.53 & -0.145 & -0.0798 & -0.0711 & -0.1292 & -0.106 \\
\hline 683.87 & -0.1447 & -0.0794 & -0.071 & -0.129 & -0.106 \\
\hline 684.21 & -0.1446 & -0.0793 & -0.0709 & -0.1289 & -0.106 \\
\hline 684.55 & -0.1446 & -0.0794 & -0.0709 & -0.1289 & -0.106 \\
\hline 684.88 & -0.1444 & -0.0794 & -0.0709 & -0.1289 & -0.106 \\
\hline 685.22 & -0.1442 & -0.0793 & -0.0708 & -0.1288 & -0.106 \\
\hline 685.56 & -0.1441 & -0.0791 & -0.0705 & -0.1287 & -0.106 \\
\hline 685.90 & -0.144 & -0.0789 & -0.0703 & -0.1286 & -0.105 \\
\hline 686.24 & -0.144 & -0.0788 & -0.0702 & -0.1286 & -0.105 \\
\hline 686.58 & -0.1441 & -0.0786 & -0.0702 & -0.1286 & -0.105 \\
\hline 686.92 & -0.1439 & -0.0784 & -0.0702 & -0.1285 & -0.105 \\
\hline 687.25 & -0.1437 & -0.0782 & -0.07 & -0.1282 & -0.105 \\
\hline 687.59 & -0.1437 & -0.0783 & -0.0699 & -0.1283 & -0.105 \\
\hline 687.93 & -0.1436 & -0.0782 & -0.0699 & -0.1283 & -0.105 \\
\hline 688.27 & -0.1436 & -0.0781 & -0.0699 & -0.1283 & -0.105 \\
\hline 688.61 & -0.1437 & -0.0781 & -0.0699 & -0.1284 & -0.105 \\
\hline & & & & & \\
\hline 6
\end{tabular}




\begin{tabular}{|l|l|l|l|l|l|}
\hline 688.94 & -0.1438 & -0.078 & -0.0698 & -0.1283 & -0.105 \\
\hline 689.28 & -0.1437 & -0.078 & -0.0698 & -0.1283 & -0.105 \\
\hline 689.62 & -0.1438 & -0.0782 & -0.07 & -0.1285 & -0.105 \\
\hline 689.96 & -0.1439 & -0.0783 & -0.07 & -0.1287 & -0.105 \\
\hline 690.30 & -0.1438 & -0.0784 & -0.0701 & -0.1287 & -0.105 \\
\hline 690.64 & -0.144 & -0.0786 & -0.0703 & -0.1289 & -0.105 \\
\hline 690.97 & -0.1441 & -0.0788 & -0.0703 & -0.129 & -0.106 \\
\hline 691.31 & -0.1442 & -0.0789 & -0.0704 & -0.1291 & -0.106 \\
\hline 691.65 & -0.1443 & -0.0788 & -0.0704 & -0.1291 & -0.106 \\
\hline 691.99 & -0.1444 & -0.0787 & -0.0703 & -0.1292 & -0.106 \\
\hline 692.32 & -0.1446 & -0.0787 & -0.0704 & -0.1293 & -0.106 \\
\hline 692.66 & -0.1448 & -0.0789 & -0.0706 & -0.1295 & -0.106 \\
\hline 693.00 & -0.1449 & -0.0789 & -0.0708 & -0.1297 & -0.106 \\
\hline 693.34 & -0.145 & -0.0791 & -0.071 & -0.1298 & -0.106 \\
\hline 693.67 & -0.145 & -0.079 & -0.0712 & -0.1298 & -0.106 \\
\hline 694.01 & -0.145 & -0.0791 & -0.0712 & -0.1299 & -0.106 \\
\hline 694.35 & -0.1453 & -0.0791 & -0.0715 & -0.1302 & -0.107 \\
\hline 694.69 & -0.1454 & -0.0792 & -0.0716 & -0.1302 & -0.107 \\
\hline 695.02 & -0.1455 & -0.0792 & -0.0717 & -0.1302 & -0.107 \\
\hline 695.36 & -0.1456 & -0.0794 & -0.0718 & -0.1303 & -0.107 \\
\hline 695.70 & -0.1456 & -0.0796 & -0.0719 & -0.1304 & -0.107 \\
\hline 696.04 & -0.1456 & -0.0794 & -0.072 & -0.1304 & -0.107 \\
\hline 696.37 & -0.1458 & -0.0794 & -0.0722 & -0.1305 & -0.107 \\
\hline 696.71 & -0.1459 & -0.0795 & -0.0723 & -0.1306 & -0.107 \\
\hline 697.05 & -0.146 & -0.0796 & -0.0724 & -0.1307 & -0.107 \\
\hline 697.38 & -0.1463 & -0.0797 & -0.0726 & -0.1308 & -0.107 \\
\hline 697.72 & -0.1464 & -0.0795 & -0.0725 & -0.1308 & -0.107 \\
\hline 698.06 & -0.1464 & -0.0796 & -0.0725 & -0.1308 & -0.107 \\
\hline 698.40 & -0.1465 & -0.0797 & -0.0726 & -0.1307 & -0.107 \\
\hline 698.73 & -0.1467 & -0.0798 & -0.0728 & -0.1309 & -0.108 \\
\hline 699.07 & -0.1467 & -0.0799 & -0.0728 & -0.131 & -0.108 \\
\hline 699.41 & -0.1467 & -0.08 & -0.0729 & -0.1309 & -0.108 \\
\hline 699.74 & -0.1466 & -0.08 & -0.0728 & -0.1308 & -0.108 \\
\hline 700.08 & -0.1468 & -0.08 & -0.0728 & -0.1308 & -0.108 \\
\hline 700.42 & -0.1468 & -0.08 & -0.0728 & -0.1307 & -0.108 \\
\hline 700.75 & -0.1467 & -0.08 & -0.0727 & -0.1306 & -0.108 \\
\hline 701.09 & -0.1468 & -0.08 & -0.0725 & -0.1305 & -0.107 \\
\hline 701.43 & -0.1467 & -0.08 & -0.0726 & -0.1305 & -0.107 \\
\hline 701.76 & -0.1466 & -0.0799 & -0.0725 & -0.1305 & -0.107 \\
\hline & & & & & \\
\hline 6
\end{tabular}




\begin{tabular}{|l|l|l|l|l|l|}
\hline 702.10 & -0.1466 & -0.0799 & -0.0724 & -0.1305 & -0.107 \\
\hline 702.43 & -0.1466 & -0.0798 & -0.0724 & -0.1305 & -0.107 \\
\hline 702.77 & -0.1465 & -0.0795 & -0.0722 & -0.1303 & -0.107 \\
\hline 703.11 & -0.1463 & -0.0793 & -0.0719 & -0.13 & -0.107 \\
\hline 703.44 & -0.1463 & -0.0792 & -0.0717 & -0.1299 & -0.107 \\
\hline 703.78 & -0.1461 & -0.0789 & -0.0714 & -0.1296 & -0.106 \\
\hline 704.12 & -0.146 & -0.0788 & -0.0713 & -0.1294 & -0.106 \\
\hline 704.45 & -0.1459 & -0.0786 & -0.0712 & -0.1293 & -0.106 \\
\hline 704.79 & -0.1456 & -0.0784 & -0.0711 & -0.1291 & -0.106 \\
\hline 705.12 & -0.1455 & -0.0783 & -0.0712 & -0.129 & -0.106 \\
\hline 705.46 & -0.1453 & -0.0779 & -0.0709 & -0.129 & -0.106 \\
\hline 705.80 & -0.1452 & -0.0779 & -0.0707 & -0.1287 & -0.106 \\
\hline 706.13 & -0.1449 & -0.0775 & -0.0705 & -0.1285 & -0.105 \\
\hline 706.47 & -0.1447 & -0.0773 & -0.0704 & -0.1283 & -0.105 \\
\hline 706.80 & -0.1445 & -0.077 & -0.0703 & -0.1282 & -0.105 \\
\hline 707.14 & -0.1442 & -0.0767 & -0.0701 & -0.1279 & -0.105 \\
\hline 707.47 & -0.144 & -0.0767 & -0.0701 & -0.1277 & -0.105 \\
\hline 707.81 & -0.1438 & -0.0765 & -0.0699 & -0.1275 & -0.104 \\
\hline 708.15 & -0.1436 & -0.0762 & -0.0697 & -0.1272 & -0.104 \\
\hline 708.48 & -0.1433 & -0.0759 & -0.0694 & -0.1268 & -0.104 \\
\hline 708.82 & -0.1432 & -0.0757 & -0.0692 & -0.1266 & -0.104 \\
\hline 709.15 & -0.1429 & -0.0755 & -0.0692 & -0.1264 & -0.103 \\
\hline 709.49 & -0.1426 & -0.0753 & -0.0689 & -0.1262 & -0.103 \\
\hline 709.82 & -0.1424 & -0.0752 & -0.0689 & -0.1262 & -0.103 \\
\hline 710.16 & -0.1425 & -0.0753 & -0.069 & -0.1263 & -0.103 \\
\hline 710.49 & -0.1422 & -0.075 & -0.069 & -0.1261 & -0.103 \\
\hline 710.83 & -0.142 & -0.075 & -0.0689 & -0.1261 & -0.103 \\
\hline 711.16 & -0.1417 & -0.0746 & -0.0686 & -0.1258 & -0.103 \\
\hline 711.50 & -0.1414 & -0.0743 & -0.0683 & -0.1256 & -0.102 \\
\hline 711.83 & -0.1412 & -0.074 & -0.0682 & -0.1254 & -0.102 \\
\hline 712.17 & -0.1409 & -0.0738 & -0.0681 & -0.1252 & -0.102 \\
\hline 712.50 & -0.1408 & -0.0738 & -0.0682 & -0.125 & -0.102 \\
\hline 712.84 & -0.1404 & -0.0735 & -0.068 & -0.1247 & -0.102 \\
\hline 713.17 & -0.1404 & -0.0734 & -0.0681 & -0.1246 & -0.102 \\
\hline 713.51 & -0.1403 & -0.0734 & -0.0681 & -0.1244 & -0.102 \\
\hline 713.84 & -0.14 & -0.0732 & -0.0679 & -0.1242 & -0.101 \\
\hline 714.18 & -0.1399 & -0.0731 & -0.0678 & -0.1241 & -0.101 \\
\hline 714.51 & -0.1395 & -0.0725 & -0.0675 & -0.1237 & -0.101 \\
\hline 714.85 & -0.1393 & -0.0723 & -0.0674 & -0.1236 & -0.101 \\
\hline & & & & & \\
\hline
\end{tabular}




\begin{tabular}{|l|l|l|l|l|l|}
\hline 715.18 & -0.1393 & -0.0724 & -0.0676 & -0.1236 & -0.101 \\
\hline 715.52 & -0.1391 & -0.0723 & -0.0674 & -0.1235 & -0.101 \\
\hline 715.85 & -0.1389 & -0.0723 & -0.0674 & -0.1235 & -0.101 \\
\hline 716.19 & -0.1387 & -0.072 & -0.0672 & -0.1231 & -0.100 \\
\hline 716.52 & -0.1386 & -0.0717 & -0.0672 & -0.1228 & -0.100 \\
\hline 716.86 & -0.1385 & -0.0715 & -0.0671 & -0.1225 & -0.100 \\
\hline 717.19 & -0.1382 & -0.0713 & -0.067 & -0.1222 & -0.100 \\
\hline 717.52 & -0.1384 & -0.0715 & -0.067 & -0.1223 & -0.100 \\
\hline 717.86 & -0.1383 & -0.0713 & -0.067 & -0.1221 & -0.100 \\
\hline 718.19 & -0.1381 & -0.0711 & -0.0669 & -0.1218 & -0.099 \\
\hline 718.53 & -0.138 & -0.071 & -0.0669 & -0.1217 & -0.099 \\
\hline 718.86 & -0.1378 & -0.0707 & -0.0666 & -0.1215 & -0.099 \\
\hline 719.20 & -0.1379 & -0.0705 & -0.0665 & -0.1215 & -0.099 \\
\hline 719.53 & -0.1378 & -0.0705 & -0.0665 & -0.1215 & -0.099 \\
\hline 719.86 & -0.1378 & -0.0701 & -0.0662 & -0.1213 & -0.099 \\
\hline 720.20 & -0.1376 & -0.0701 & -0.0661 & -0.1211 & -0.099 \\
\hline 720.53 & -0.1376 & -0.07 & -0.0659 & -0.1211 & -0.099 \\
\hline 720.87 & -0.1374 & -0.0697 & -0.0656 & -0.1209 & -0.098 \\
\hline 721.20 & -0.1373 & -0.0697 & -0.0655 & -0.1209 & -0.098 \\
\hline 721.53 & -0.1373 & -0.0696 & -0.0654 & -0.1209 & -0.098 \\
\hline 721.87 & -0.1373 & -0.0697 & -0.0654 & -0.121 & -0.098 \\
\hline 722.20 & -0.1371 & -0.0694 & -0.0652 & -0.1206 & -0.098 \\
\hline 722.53 & -0.1371 & -0.0694 & -0.0652 & -0.1207 & -0.098 \\
\hline 722.87 & -0.1372 & -0.0692 & -0.065 & -0.1205 & -0.098 \\
\hline 723.20 & -0.1374 & -0.0692 & -0.065 & -0.1206 & -0.098 \\
\hline 723.54 & -0.1374 & -0.0692 & -0.065 & -0.1207 & -0.098 \\
\hline 723.87 & -0.1374 & -0.0691 & -0.0649 & -0.1206 & -0.098 \\
\hline 724.20 & -0.1374 & -0.0691 & -0.0648 & -0.1206 & -0.098 \\
\hline 724.54 & -0.1371 & -0.0688 & -0.0644 & -0.1204 & -0.098 \\
\hline 724.87 & -0.1372 & -0.0688 & -0.0644 & -0.1203 & -0.098 \\
\hline 725.20 & -0.1374 & -0.069 & -0.0645 & -0.1206 & -0.098 \\
\hline 725.54 & -0.1373 & -0.0687 & -0.0643 & -0.1204 & -0.098 \\
\hline 725.87 & -0.1373 & -0.069 & -0.0645 & -0.1204 & -0.098 \\
\hline 726.20 & -0.1371 & -0.0688 & -0.0643 & -0.1203 & -0.098 \\
\hline 726.54 & -0.137 & -0.0687 & -0.0641 & -0.12 & -0.097 \\
\hline 726.87 & -0.1371 & -0.0689 & -0.0643 & -0.1201 & -0.098 \\
\hline 727.20 & -0.1371 & -0.0686 & -0.0641 & -0.1201 & -0.097 \\
\hline 727.53 & -0.137 & -0.0685 & -0.0639 & -0.12 & -0.097 \\
\hline 727.87 & -0.1372 & -0.0688 & -0.0641 & -0.12 & -0.098 \\
\hline
\end{tabular}




\begin{tabular}{|l|c|c|c|c|c|}
\hline 728.20 & -0.1371 & -0.0686 & -0.064 & -0.1197 & -0.097 \\
\hline 728.53 & -0.1371 & -0.0686 & -0.0638 & -0.1197 & -0.097 \\
\hline 728.87 & -0.1369 & -0.0685 & -0.0637 & -0.1195 & -0.097 \\
\hline 729.20 & -0.137 & -0.0686 & -0.0638 & -0.1196 & -0.097 \\
\hline 729.53 & -0.1369 & -0.0683 & -0.0636 & -0.1194 & -0.097 \\
\hline 729.86 & -0.1367 & -0.0681 & -0.0635 & -0.1192 & -0.097 \\
\hline 730.20 & -0.1366 & -0.068 & -0.0633 & -0.1191 & -0.097 \\
\hline 730.53 & -0.1365 & -0.0677 & -0.0631 & -0.1189 & -0.097 \\
\hline 730.86 & -0.1365 & -0.0677 & -0.0631 & -0.1189 & -0.097 \\
\hline 731.19 & -0.1364 & -0.0673 & -0.063 & -0.1188 & -0.096 \\
\hline 731.53 & -0.1362 & -0.0672 & -0.0631 & -0.1186 & -0.096 \\
\hline 731.86 & -0.1361 & -0.0671 & -0.063 & -0.1187 & -0.096 \\
\hline 732.19 & -0.136 & -0.0669 & -0.0628 & -0.1184 & -0.096 \\
\hline 732.52 & -0.1362 & -0.067 & -0.0627 & -0.1185 & -0.096 \\
\hline 732.86 & -0.136 & -0.0666 & -0.0624 & -0.1183 & -0.096 \\
\hline 733.19 & -0.136 & -0.0665 & -0.0624 & -0.1182 & -0.096 \\
\hline 733.52 & -0.1359 & -0.0662 & -0.0622 & -0.1182 & -0.096 \\
\hline 733.85 & -0.1359 & -0.066 & -0.062 & -0.118 & -0.095 \\
\hline 734.18 & -0.136 & -0.0661 & -0.0622 & -0.1182 & -0.096 \\
\hline 734.52 & -0.136 & -0.0659 & -0.0624 & -0.1181 & -0.096 \\
\hline 734.85 & -0.1359 & -0.0657 & -0.0623 & -0.1181 & -0.096 \\
\hline 735.18 & -0.1358 & -0.0655 & -0.0621 & -0.1182 & -0.095 \\
\hline 735.51 & -0.1359 & -0.0654 & -0.0622 & -0.1182 & -0.095 \\
\hline 735.84 & -0.1361 & -0.0655 & -0.0624 & -0.1184 & -0.096 \\
\hline 736.18 & -0.1358 & -0.0652 & -0.0621 & -0.1182 & -0.095 \\
\hline 736.51 & -0.1359 & -0.0653 & -0.0622 & -0.1183 & -0.095 \\
\hline 736.84 & -0.1358 & -0.0653 & -0.0622 & -0.1182 & -0.095 \\
\hline 737.17 & -0.1359 & -0.0655 & -0.0624 & -0.1185 & -0.096 \\
\hline 737.50 & -0.1359 & -0.0655 & -0.0625 & -0.1186 & -0.096 \\
\hline 737.83 & -0.1358 & -0.0655 & -0.0625 & -0.1187 & -0.096 \\
\hline 738.17 & -0.1358 & -0.0654 & -0.0625 & -0.1187 & -0.096 \\
\hline 738.50 & -0.1361 & -0.0654 & -0.0625 & -0.1187 & -0.096 \\
\hline 738.83 & -0.1359 & -0.0653 & -0.0622 & -0.1185 & -0.095 \\
\hline 739.16 & -0.1359 & -0.0654 & -0.0623 & -0.1186 & -0.096 \\
\hline 739.49 & -0.1355 & -0.0649 & -0.062 & -0.1182 & -0.095 \\
\hline 739.82 & -0.1357 & -0.065 & -0.0621 & -0.1184 & -0.095 \\
\hline 740.15 & -0.1356 & -0.065 & -0.062 & -0.1183 & -0.095 \\
\hline 740.49 & -0.1356 & -0.0651 & -0.0623 & -0.1185 & -0.095 \\
\hline 740.82 & -0.1352 & -0.0649 & -0.062 & -0.1184 & -0.095 \\
\hline & & & & & \\
\hline
\end{tabular}




\begin{tabular}{|l|l|l|l|l|l|}
\hline 741.15 & -0.1351 & -0.0648 & -0.062 & -0.1183 & -0.095 \\
\hline 741.48 & -0.135 & -0.0648 & -0.0617 & -0.1182 & -0.095 \\
\hline 741.81 & -0.1349 & -0.0646 & -0.0614 & -0.1181 & -0.095 \\
\hline 742.14 & -0.135 & -0.0647 & -0.0614 & -0.1178 & -0.095 \\
\hline 742.47 & -0.135 & -0.0647 & -0.0614 & -0.1179 & -0.095 \\
\hline 742.80 & -0.1349 & -0.0644 & -0.0612 & -0.1178 & -0.095 \\
\hline 743.13 & -0.1349 & -0.0644 & -0.0613 & -0.1179 & -0.095 \\
\hline 743.46 & -0.1349 & -0.0641 & -0.0613 & -0.1178 & -0.095 \\
\hline 743.80 & -0.135 & -0.064 & -0.0613 & -0.1178 & -0.095 \\
\hline 744.13 & -0.1345 & -0.0636 & -0.0611 & -0.1175 & -0.094 \\
\hline 744.46 & -0.1347 & -0.0637 & -0.0611 & -0.1174 & -0.094 \\
\hline 744.79 & -0.1343 & -0.0634 & -0.0607 & -0.1172 & -0.094 \\
\hline 745.12 & -0.1344 & -0.0637 & -0.0607 & -0.1171 & -0.094 \\
\hline 745.45 & -0.1339 & -0.0633 & -0.0603 & -0.1169 & -0.094 \\
\hline 745.78 & -0.1338 & -0.063 & -0.0602 & -0.1169 & -0.093 \\
\hline 746.11 & -0.1332 & -0.0626 & -0.06 & -0.1165 & -0.093 \\
\hline 746.44 & -0.1335 & -0.0631 & -0.0604 & -0.1167 & -0.093 \\
\hline 746.77 & -0.1332 & -0.063 & -0.0601 & -0.1165 & -0.093 \\
\hline 747.10 & -0.1328 & -0.0628 & -0.0598 & -0.1162 & -0.093 \\
\hline 747.43 & -0.1327 & -0.0626 & -0.0596 & -0.1157 & -0.093 \\
\hline 747.76 & -0.1325 & -0.0626 & -0.0597 & -0.1159 & -0.093 \\
\hline 748.09 & -0.1323 & -0.0621 & -0.0595 & -0.1156 & -0.092 \\
\hline 748.42 & -0.1322 & -0.062 & -0.0596 & -0.1153 & -0.092 \\
\hline 748.75 & -0.1319 & -0.062 & -0.0597 & -0.1153 & -0.092 \\
\hline 749.08 & -0.1316 & -0.0618 & -0.0596 & -0.1154 & -0.092 \\
\hline 749.41 & -0.1311 & -0.0614 & -0.0591 & -0.1149 & -0.092 \\
\hline 749.74 & -0.1309 & -0.0615 & -0.0589 & -0.1147 & -0.092 \\
\hline 750.07 & -0.1308 & -0.0613 & -0.0588 & -0.1145 & -0.091 \\
\hline 750.40 & -0.1307 & -0.0612 & -0.0586 & -0.1144 & -0.091 \\
\hline 750.73 & -0.1307 & -0.0613 & -0.0587 & -0.1142 & -0.091 \\
\hline 751.06 & -0.1306 & -0.061 & -0.0585 & -0.114 & -0.091 \\
\hline 751.39 & -0.1301 & -0.0604 & -0.0579 & -0.1135 & -0.090 \\
\hline 751.72 & -0.1301 & -0.0606 & -0.058 & -0.1135 & -0.091 \\
\hline 752.05 & -0.1299 & -0.0603 & -0.0577 & -0.1134 & -0.090 \\
\hline 752.38 & -0.1298 & -0.0602 & -0.0577 & -0.1135 & -0.090 \\
\hline 752.71 & -0.1297 & -0.0602 & -0.0578 & -0.1133 & -0.090 \\
\hline 753.04 & -0.1301 & -0.0602 & -0.0577 & -0.1134 & -0.090 \\
\hline 753.37 & -0.1299 & -0.06 & -0.0574 & -0.1128 & -0.090 \\
\hline 753.70 & -0.1299 & -0.0598 & -0.0574 & -0.1127 & -0.090 \\
\hline
\end{tabular}




\begin{tabular}{|c|c|c|c|c|c|}
\hline 754.03 & -0.1301 & -0.0598 & -0.0576 & -0.1126 & -0.090 \\
\hline 754.36 & -0.13 & -0.0598 & -0.0578 & -0.1128 & -0.090 \\
\hline 754.68 & -0.13 & -0.0595 & -0.0578 & -0.1122 & -0.090 \\
\hline 755.01 & -0.1301 & -0.0598 & -0.0578 & -0.1121 & -0.090 \\
\hline 755.34 & -0.13 & -0.0599 & -0.0577 & -0.1121 & -0.090 \\
\hline 755.67 & -0.1305 & -0.0599 & -0.0578 & -0.1121 & -0.090 \\
\hline 756.00 & -0.1305 & -0.0599 & -0.0579 & -0.1121 & -0.090 \\
\hline 756.33 & -0.1305 & -0.06 & -0.0582 & -0.1122 & -0.090 \\
\hline 756.66 & -0.1305 & -0.0598 & -0.0581 & -0.1118 & -0.090 \\
\hline 756.99 & -0.1306 & -0.06 & -0.0584 & -0.1119 & -0.090 \\
\hline 757.32 & -0.1305 & -0.06 & -0.0583 & -0.1116 & -0.090 \\
\hline 757.65 & -0.1302 & -0.06 & -0.0579 & -0.1115 & -0.090 \\
\hline 757.97 & -0.1303 & -0.0602 & -0.0576 & -0.1116 & -0.090 \\
\hline 758.30 & -0.1305 & -0.0607 & -0.0577 & -0.1118 & -0.090 \\
\hline 758.63 & -0.1 & -0.0605 & -0.0578 & -0.1116 & -0.090 \\
\hline 758.96 & -0.1303 & -0.0605 & -0.0579 & -0.1113 & -0.090 \\
\hline 759.29 & -0.1307 & -0.0608 & -0.058 & -0.1112 & -0.090 \\
\hline 759.62 & -0.1306 & -0.0609 & -0.0577 & -0.1108 & -0.090 \\
\hline 759.95 & -0.1304 & -0.061 & -0.0576 & -0.111 & -0.090 \\
\hline 760.28 & -0.1304 & -0.0606 & -0.0575 & -0.1114 & -0.090 \\
\hline 760.60 & -0.1305 & -0.0604 & -0.0573 & -0.1115 & -0.090 \\
\hline 760.93 & -0.1308 & -0.0605 & -0.0573 & -0.1117 & -0.090 \\
\hline 761.26 & -0.1306 & -0.0599 & -0.0564 & -0.1111 & -0.090 \\
\hline 761.59 & -0.1308 & -0.0603 & -0.0563 & -0.1115 & -0.090 \\
\hline 761.92 & -0.1306 & -0.0601 & -0.0557 & -0.1116 & -0.090 \\
\hline 762.24 & -0.1306 & -0.0599 & -0.0555 & -0.1111 & -0.089 \\
\hline 762.57 & -0.1303 & -0.0598 & -0.0553 & -0.1108 & -0.089 \\
\hline 762.90 & -0.1302 & -0.0602 & -0.0554 & -0.1111 & -0.089 \\
\hline 763.23 & -0.1304 & -0.0601 & -0.0553 & -0.1113 & -0.089 \\
\hline 763.56 & -0.1301 & -0.0595 & -0.055 & -0.1113 & -0.089 \\
\hline 763.89 & -0.13 & -0.059 & -0.0545 & -0.1113 & -0.089 \\
\hline 764.21 & -0.1297 & -0.0584 & -0.054 & -0.1109 & -0.088 \\
\hline 764.54 & -0.1299 & -0.0581 & -0.0541 & -0.1108 & -0.088 \\
\hline 764.87 & -0.1299 & -0.0578 & -0.0543 & -0.111 & -0.088 \\
\hline 765.20 & -0.1296 & -0.0575 & -0.0542 & -0.1108 & -0.088 \\
\hline 765.52 & -0.1296 & -0.057 & -0.0537 & -0.1102 & -0.088 \\
\hline 765.85 & -0.1297 & -0.057 & -0.0536 & -0.1102 & -0.088 \\
\hline 766.18 & -0.1294 & -0.057 & -0.054 & -0.1101 & -0.088 \\
\hline 766.51 & -0.1298 & -0.057 & -0.0545 & -0.1106 & -0.088 \\
\hline
\end{tabular}




\begin{tabular}{|l|l|l|l|l|l|}
\hline 766.83 & -0.1299 & -0.057 & -0.0544 & -0.1104 & -0.088 \\
\hline 767.16 & -0.1298 & -0.0572 & -0.0545 & -0.1101 & -0.088 \\
\hline 767.49 & -0.1295 & -0.0576 & -0.0546 & -0.1099 & -0.088 \\
\hline 767.82 & -0.1292 & -0.0575 & -0.0542 & -0.1097 & -0.088 \\
\hline 768.14 & -0.1294 & -0.0577 & -0.0548 & -0.1098 & -0.088 \\
\hline 768.47 & -0.1297 & -0.0573 & -0.0548 & -0.1097 & -0.088 \\
\hline 768.80 & -0.1295 & -0.0567 & -0.0548 & -0.1097 & -0.088 \\
\hline 769.13 & -0.1295 & -0.0568 & -0.055 & -0.1101 & -0.088 \\
\hline 769.45 & -0.1293 & -0.0564 & -0.0551 & -0.1102 & -0.088 \\
\hline 769.78 & -0.1294 & -0.0558 & -0.0551 & -0.11 & -0.088 \\
\hline 770.11 & -0.1295 & -0.056 & -0.0549 & -0.1098 & -0.088 \\
\hline 770.43 & -0.13 & -0.0566 & -0.0557 & -0.1101 & -0.088 \\
\hline 770.76 & -0.1298 & -0.0563 & -0.0554 & -0.1093 & -0.088 \\
\hline 771.09 & -0.1303 & -0.0567 & -0.0558 & -0.11 & -0.088 \\
\hline 771.42 & -0.1306 & -0.0567 & -0.0564 & -0.1102 & -0.088 \\
\hline 771.74 & -0.1299 & -0.0561 & -0.056 & -0.1094 & -0.088 \\
\hline 772.07 & -0.1304 & -0.0562 & -0.0565 & -0.1096 & -0.088 \\
\hline 772.40 & -0.1302 & -0.0567 & -0.0565 & -0.1097 & -0.088 \\
\hline 772.72 & -0.1301 & -0.056 & -0.0565 & -0.1096 & -0.088 \\
\hline 773.05 & -0.1301 & -0.0551 & -0.0565 & -0.1094 & -0.088 \\
\hline 773.38 & -0.1297 & -0.0551 & -0.0562 & -0.1093 & -0.088 \\
\hline 773.70 & -0.1299 & -0.055 & -0.0567 & -0.1094 & -0.088 \\
\hline 774.03 & -0.1298 & -0.0551 & -0.0561 & -0.1091 & -0.088 \\
\hline 774.36 & -0.1298 & -0.0547 & -0.0566 & -0.1093 & -0.088 \\
\hline 774.68 & -0.1299 & -0.0541 & -0.0561 & -0.1084 & -0.087 \\
\hline 775.01 & -0.1292 & -0.0535 & -0.0556 & -0.1081 & -0.087 \\
\hline 775.33 & -0.1289 & -0.0531 & -0.0554 & -0.1078 & -0.086 \\
\hline 775.66 & -0.129 & -0.0537 & -0.0555 & -0.1075 & -0.086 \\
\hline 775.99 & -0.1286 & -0.053 & -0.0557 & -0.107 & -0.086 \\
\hline 776.31 & -0.1292 & -0.0529 & -0.0554 & -0.1072 & -0.086 \\
\hline 776.64 & -0.1285 & -0.0526 & -0.0545 & -0.1066 & -0.086 \\
\hline 776.97 & -0.1276 & -0.0522 & -0.054 & -0.1058 & -0.085 \\
\hline 777.29 & -0.1268 & -0.0508 & -0.052 & -0.1046 & -0.084 \\
\hline 777.62 & -0.1262 & -0.0505 & -0.0514 & -0.1045 & -0.083 \\
\hline 777.94 & -0.1256 & -0.0502 & -0.0509 & -0.1043 & -0.083 \\
\hline 778.27 & -0.1239 & -0.0492 & -0.0488 & -0.1035 & -0.081 \\
\hline 778.60 & -0.1243 & -0.0491 & -0.0485 & -0.1029 & -0.081 \\
\hline 778.92 & -0.123 & -0.0485 & -0.0478 & -0.1016 & -0.080 \\
\hline 779.25 & -0.1237 & -0.0477 & -0.0478 & -0.1018 & -0.080 \\
\hline & & & & & \\
\hline
\end{tabular}




\begin{tabular}{|l|l|l|l|l|l|}
\hline 779.57 & -0.1221 & -0.0471 & -0.0463 & -0.1009 & -0.079 \\
\hline 779.90 & -0.1219 & -0.048 & -0.0457 & -0.101 & -0.079 \\
\hline 780.22 & -0.1217 & -0.0475 & -0.0448 & -0.1003 & -0.079 \\
\hline 780.55 & -0.1209 & -0.0468 & -0.0435 & -0.0996 & -0.078 \\
\hline 780.88 & -0.1193 & -0.045 & -0.0424 & -0.0987 & -0.076 \\
\hline 781.20 & -0.1181 & -0.0446 & -0.0417 & -0.0975 & -0.075 \\
\hline 781.53 & -0.1174 & -0.0443 & -0.0421 & -0.0983 & -0.076 \\
\hline 781.85 & -0.1177 & -0.0445 & -0.0427 & -0.098 & -0.076 \\
\hline 782.18 & -0.1168 & -0.044 & -0.0428 & -0.0978 & -0.075 \\
\hline 782.50 & -0.1166 & -0.0432 & -0.0424 & -0.0973 & -0.075 \\
\hline 782.83 & -0.1149 & -0.0426 & -0.0407 & -0.0961 & -0.074 \\
\hline 783.15 & -0.1142 & -0.0425 & -0.0406 & -0.0949 & -0.073 \\
\hline 783.48 & -0.114 & -0.0418 & -0.0405 & -0.0946 & -0.073 \\
\hline 783.80 & -0.1134 & -0.0407 & -0.0389 & -0.0944 & -0.072 \\
\hline 784.13 & -0.1125 & -0.0407 & -0.0391 & -0.094 & -0.072 \\
\hline 784.45 & -0.1113 & -0.0403 & -0.0387 & -0.094 & -0.071 \\
\hline 784.78 & -0.1095 & -0.0393 & -0.0378 & -0.0919 & -0.070 \\
\hline 785.10 & -0.1095 & -0.0387 & -0.0381 & -0.0908 & -0.069 \\
\hline 785.43 & -0.1085 & -0.0387 & -0.038 & -0.0908 & -0.069 \\
\hline 785.75 & -0.1088 & -0.0383 & -0.0379 & -0.0917 & -0.069 \\
\hline 786.08 & -0.1061 & -0.0368 & -0.0359 & -0.0896 & -0.067 \\
\hline 786.40 & -0.1069 & -0.0361 & -0.0363 & -0.0897 & -0.067 \\
\hline 786.73 & -0.1067 & -0.036 & -0.0359 & -0.0883 & -0.067 \\
\hline 787.05 & -0.1049 & -0.0347 & -0.0361 & -0.0879 & -0.066 \\
\hline 787.38 & -0.1041 & -0.0342 & -0.0351 & -0.0856 & -0.065 \\
\hline 787.70 & -0.1052 & -0.0344 & -0.0358 & -0.0867 & -0.066 \\
\hline 788.03 & -0.1067 & -0.0355 & -0.0356 & -0.087 & -0.066 \\
\hline 788.35 & -0.1053 & -0.0345 & -0.034 & -0.085 & -0.065 \\
\hline 788.67 & -0.1052 & -0.0349 & -0.0337 & -0.0862 & -0.065 \\
\hline 789.00 & -0.1051 & -0.0338 & -0.0322 & -0.0849 & -0.064 \\
\hline 789.32 & -0.1039 & -0.0332 & -0.032 & -0.0842 & -0.063 \\
\hline 789.65 & -0.1039 & -0.0333 & -0.0313 & -0.0845 & -0.063 \\
\hline 789.97 & -0.1017 & -0.0305 & -0.0289 & -0.0834 & -0.061 \\
\hline 790.30 & -0.1005 & -0.0307 & -0.0288 & -0.0817 & -0.060 \\
\hline 790.62 & -0.1005 & -0.0313 & -0.0296 & -0.0834 & -0.061 \\
\hline 790.94 & -0.1005 & -0.0306 & -0.0292 & -0.0833 & -0.061 \\
\hline 791.27 & -0.0998 & -0.0304 & -0.0288 & -0.0827 & -0.060 \\
\hline 791.59 & -0.1006 & -0.0295 & -0.0281 & -0.0837 & -0.060 \\
\hline 791.92 & -0.1018 & -0.0304 & -0.0278 & -0.0842 & -0.061 \\
\hline
\end{tabular}




\begin{tabular}{|l|l|l|l|l|l|}
\hline 792.24 & -0.1012 & -0.0288 & -0.0268 & -0.0826 & -0.060 \\
\hline 792.56 & -0.0999 & -0.0271 & -0.0241 & -0.0812 & -0.058 \\
\hline 792.89 & -0.1006 & -0.027 & -0.0245 & -0.0828 & -0.059 \\
\hline 793.21 & -0.1008 & -0.0274 & -0.0246 & -0.0833 & -0.059 \\
\hline 793.53 & -0.0996 & -0.0261 & -0.0229 & -0.0823 & -0.058 \\
\hline 793.86 & -0.0995 & -0.0253 & -0.0206 & -0.0816 & -0.057 \\
\hline 794.18 & -0.0996 & -0.0246 & -0.0212 & -0.0844 & -0.057 \\
\hline 794.51 & -0.0989 & -0.0246 & -0.0203 & -0.0836 & -0.057 \\
\hline 794.83 & -0.0966 & -0.023 & -0.0189 & -0.0824 & -0.055 \\
\hline 795.15 & -0.0967 & -0.0234 & -0.0196 & -0.0838 & -0.056 \\
\hline 795.48 & -0.0956 & -0.0226 & -0.0181 & -0.0815 & -0.054 \\
\hline 795.80 & -0.0933 & -0.0223 & -0.0163 & -0.0803 & -0.053 \\
\hline 796.12 & -0.0922 & -0.0208 & -0.0152 & -0.0783 & -0.052 \\
\hline 796.45 & -0.0939 & -0.0195 & -0.0153 & -0.077 & -0.051 \\
\hline 796.77 & -0.0936 & -0.0193 & -0.0143 & -0.0766 & -0.051 \\
\hline 797.09 & -0.0929 & -0.0166 & -0.0119 & -0.0763 & -0.049 \\
\hline 797.42 & -0.0933 & -0.0174 & -0.0129 & -0.0737 & -0.049 \\
\hline 797.74 & -0.0936 & -0.0184 & -0.0132 & -0.0745 & -0.050 \\
\hline 798.06 & -0.0961 & -0.0175 & -0.0128 & -0.0738 & -0.050 \\
\hline 798.38 & -0.096 & -0.0164 & -0.0121 & -0.074 & -0.050 \\
\hline 798.71 & -0.0938 & -0.0147 & -0.0099 & -0.0724 & -0.048 \\
\hline 799.03 & -0.0943 & -0.0156 & -0.0111 & -0.0739 & -0.049 \\
\hline 799.35 & -0.0935 & -0.0141 & -0.0116 & -0.0729 & -0.048 \\
\hline 799.68 & -0.092 & -0.0132 & -0.0105 & -0.0712 & -0.047 \\
\hline 800.00 & -0.0898 & -0.011 & -0.0072 & -0.0676 & -0.044 \\
\hline & & & & & \\
\hline 79 &
\end{tabular}

Absorbance values for front, back, armpit and sleeve of the Gold A t-shirt square for Phase I

\begin{tabular}{|c|c|}
\hline Wavelength & Absorbance \\
\hline 400.21 & 0.6858 \\
\hline 400.58 & 0.6785 \\
\hline 400.95 & 0.6762 \\
\hline 401.32 & 0.6690 \\
\hline 401.69 & 0.6658 \\
\hline 402.06 & 0.6722 \\
\hline 402.43 & 0.6696 \\
\hline
\end{tabular}




\begin{tabular}{|l|l|}
\hline 402.80 & 0.6737 \\
\hline 403.18 & 0.6770 \\
\hline 403.55 & 0.6766 \\
\hline 403.92 & 0.6775 \\
\hline 404.29 & 0.6729 \\
\hline 404.66 & 0.6722 \\
\hline 405.03 & 0.6696 \\
\hline 405.40 & 0.6611 \\
\hline 405.77 & 0.6590 \\
\hline 406.14 & 0.6548 \\
\hline 406.51 & 0.6516 \\
\hline 406.88 & 0.6453 \\
\hline 407.25 & 0.6439 \\
\hline 407.62 & 0.6424 \\
\hline 407.99 & 0.6433 \\
\hline 408.36 & 0.6405 \\
\hline 408.74 & 0.6375 \\
\hline 409.11 & 0.6368 \\
\hline 409.48 & 0.6362 \\
\hline 409.85 & 0.6318 \\
\hline 410.22 & 0.6305 \\
\hline 410.59 & 0.6290 \\
\hline 410.96 & 0.6266 \\
\hline 411.33 & 0.6241 \\
\hline 411.70 & 0.6204 \\
\hline 412.07 & 0.6203 \\
\hline 412.44 & 0.6197 \\
\hline 412.81 & 0.6167 \\
\hline 413.18 & 0.6178 \\
\hline 413.55 & 0.6151 \\
\hline 413.92 & 0.6124 \\
\hline 414.29 & 0.6112 \\
\hline 414.66 & 0.6122 \\
\hline 415.03 & 0.6108 \\
\hline 415.40 & 0.6075 \\
\hline 415.76 & 0.6066 \\
\hline 416.13 & 0.6062 \\
\hline 416.50 & 0.6028 \\
\hline 416.87 & 0.5996 \\
\hline & \\
\hline
\end{tabular}




\begin{tabular}{|l|l|}
\hline 417.24 & 0.5977 \\
\hline 417.61 & 0.5956 \\
\hline 417.98 & 0.5919 \\
\hline 418.35 & 0.5912 \\
\hline 418.72 & 0.5898 \\
\hline 419.09 & 0.5883 \\
\hline 419.46 & 0.5885 \\
\hline 419.83 & 0.5875 \\
\hline 420.20 & 0.5851 \\
\hline 420.57 & 0.5836 \\
\hline 420.94 & 0.5812 \\
\hline 421.31 & 0.5806 \\
\hline 421.67 & 0.5805 \\
\hline 422.04 & 0.5814 \\
\hline 422.41 & 0.5799 \\
\hline 422.78 & 0.5790 \\
\hline 423.15 & 0.5783 \\
\hline 423.52 & 0.5767 \\
\hline 423.89 & 0.5768 \\
\hline 424.26 & 0.5773 \\
\hline 424.63 & 0.5780 \\
\hline 424.99 & 0.5790 \\
\hline 425.36 & 0.5798 \\
\hline 425.73 & 0.5798 \\
\hline 426.10 & 0.5791 \\
\hline 426.47 & 0.5778 \\
\hline 426.84 & 0.5778 \\
\hline 427.21 & 0.5768 \\
\hline 427.57 & 0.5765 \\
\hline 427.94 & 0.5773 \\
\hline 428.31 & 0.5771 \\
\hline 428.68 & 0.5782 \\
\hline 429.05 & 0.5780 \\
\hline 429.42 & 0.5791 \\
\hline 429.78 & 0.5776 \\
\hline 430.15 & 0.5778 \\
\hline 430.52 & 0.5782 \\
\hline 430.89 & 0.5788 \\
\hline 431.26 & 0.5799 \\
\hline & \\
\hline
\end{tabular}




\begin{tabular}{|l|l|}
\hline 431.62 & 0.5799 \\
\hline 431.99 & 0.5795 \\
\hline 432.36 & 0.5798 \\
\hline 432.73 & 0.5785 \\
\hline 433.10 & 0.5774 \\
\hline 433.46 & 0.5782 \\
\hline 433.83 & 0.5781 \\
\hline 434.20 & 0.5784 \\
\hline 434.57 & 0.5787 \\
\hline 434.94 & 0.5790 \\
\hline 435.30 & 0.5790 \\
\hline 435.67 & 0.5781 \\
\hline 436.04 & 0.5785 \\
\hline 436.41 & 0.5777 \\
\hline 436.77 & 0.5778 \\
\hline 437.14 & 0.5771 \\
\hline 437.51 & 0.5774 \\
\hline 437.88 & 0.5774 \\
\hline 438.24 & 0.5765 \\
\hline 438.61 & 0.5747 \\
\hline 438.98 & 0.5735 \\
\hline 439.34 & 0.5727 \\
\hline 439.71 & 0.5718 \\
\hline 440.08 & 0.5708 \\
\hline 440.45 & 0.5707 \\
\hline 440.81 & 0.5709 \\
\hline 441.18 & 0.5706 \\
\hline 441.55 & 0.5703 \\
\hline 441.91 & 0.5705 \\
\hline 442.28 & 0.5707 \\
\hline 442.65 & 0.5697 \\
\hline 443.01 & 0.5697 \\
\hline 443.38 & 0.5689 \\
\hline 443.75 & 0.5677 \\
\hline 444.12 & 0.5671 \\
\hline 444.48 & 0.5666 \\
\hline 444.85 & 0.5651 \\
\hline 445.22 & 0.5642 \\
\hline 445.58 & 0.5645 \\
\hline
\end{tabular}




\begin{tabular}{|l|l|}
\hline 445.95 & 0.5646 \\
\hline 446.31 & 0.5656 \\
\hline 446.68 & 0.5652 \\
\hline 447.05 & 0.5636 \\
\hline 447.41 & 0.5636 \\
\hline 447.78 & 0.5619 \\
\hline 448.15 & 0.5615 \\
\hline 448.51 & 0.5604 \\
\hline 448.88 & 0.5588 \\
\hline 449.25 & 0.5580 \\
\hline 449.61 & 0.5573 \\
\hline 449.98 & 0.5564 \\
\hline 450.34 & 0.5562 \\
\hline 450.71 & 0.5555 \\
\hline 451.08 & 0.5555 \\
\hline 451.44 & 0.5545 \\
\hline 451.81 & 0.5536 \\
\hline 452.17 & 0.5526 \\
\hline 452.54 & 0.5522 \\
\hline 452.91 & 0.5516 \\
\hline 453.27 & 0.5503 \\
\hline 453.64 & 0.5489 \\
\hline 454.00 & 0.5479 \\
\hline 454.37 & 0.5467 \\
\hline 454.73 & 0.5465 \\
\hline 455.10 & 0.5456 \\
\hline 455.47 & 0.5457 \\
\hline 455.83 & 0.5440 \\
\hline 456.20 & 0.5428 \\
\hline 456.56 & 0.5417 \\
\hline 456.93 & 0.5396 \\
\hline 457.29 & 0.5374 \\
\hline 457.66 & 0.5357 \\
\hline 458.02 & 0.5337 \\
\hline 458.39 & 0.5318 \\
\hline 458.75 & 0.5286 \\
\hline 459.12 & 0.5264 \\
\hline 459.48 & 0.5237 \\
\hline 459.85 & 0.5218 \\
\hline
\end{tabular}




\begin{tabular}{|l|l|}
\hline 460.21 & 0.5194 \\
\hline 460.58 & 0.5165 \\
\hline 460.94 & 0.5134 \\
\hline 461.31 & 0.5109 \\
\hline 461.67 & 0.5085 \\
\hline 462.04 & 0.5052 \\
\hline 462.40 & 0.5021 \\
\hline 462.77 & 0.4993 \\
\hline 463.13 & 0.4964 \\
\hline 463.50 & 0.4935 \\
\hline 463.86 & 0.4907 \\
\hline 464.23 & 0.4882 \\
\hline 464.59 & 0.4856 \\
\hline 464.96 & 0.4828 \\
\hline 465.32 & 0.4795 \\
\hline 465.69 & 0.4760 \\
\hline 466.05 & 0.4729 \\
\hline 466.41 & 0.4700 \\
\hline 466.78 & 0.4670 \\
\hline 467.14 & 0.4641 \\
\hline 467.51 & 0.4606 \\
\hline 467.87 & 0.4577 \\
\hline 468.24 & 0.4549 \\
\hline 468.60 & 0.4521 \\
\hline 468.96 & 0.4485 \\
\hline 469.33 & 0.4448 \\
\hline 469.69 & 0.4418 \\
\hline 470.06 & 0.4388 \\
\hline 470.42 & 0.4353 \\
\hline 470.78 & 0.4314 \\
\hline 471.15 & 0.4282 \\
\hline 471.51 & 0.4245 \\
\hline 471.88 & 0.4207 \\
\hline 472.24 & 0.4164 \\
\hline 472.60 & 0.4127 \\
\hline 472.97 & 0.4091 \\
\hline 473.33 & 0.4056 \\
\hline 473.69 & 0.4020 \\
\hline 474.06 & 0.3983 \\
\hline
\end{tabular}




\begin{tabular}{|l|l|}
\hline 474.42 & 0.3947 \\
\hline 474.78 & 0.3911 \\
\hline 475.15 & 0.3875 \\
\hline 475.51 & 0.3836 \\
\hline 475.87 & 0.3797 \\
\hline 476.24 & 0.3760 \\
\hline 476.60 & 0.3726 \\
\hline 476.96 & 0.3692 \\
\hline 477.33 & 0.3654 \\
\hline 477.69 & 0.3615 \\
\hline 478.05 & 0.3577 \\
\hline 478.42 & 0.3542 \\
\hline 478.78 & 0.3505 \\
\hline 479.14 & 0.3473 \\
\hline 479.51 & 0.3439 \\
\hline 479.87 & 0.3406 \\
\hline 480.23 & 0.3372 \\
\hline 480.60 & 0.3338 \\
\hline 480.96 & 0.3306 \\
\hline 481.32 & 0.3272 \\
\hline 481.68 & 0.3240 \\
\hline 482.05 & 0.3205 \\
\hline 482.41 & 0.3169 \\
\hline 482.77 & 0.3134 \\
\hline 483.13 & 0.3100 \\
\hline 483.50 & 0.3066 \\
\hline 483.86 & 0.3031 \\
\hline 484.22 & 0.2996 \\
\hline 484.58 & 0.2961 \\
\hline 484.95 & 0.2927 \\
\hline 485.31 & 0.2893 \\
\hline 485.67 & 0.2860 \\
\hline 486.03 & 0.2828 \\
\hline 486.40 & 0.2796 \\
\hline 486.76 & 0.2762 \\
\hline 487.12 & 0.2729 \\
\hline 487.48 & 0.2695 \\
\hline 487.85 & 0.2663 \\
\hline 488.21 & 0.2632 \\
\hline
\end{tabular}




\begin{tabular}{|l|l|}
\hline 488.57 & 0.2599 \\
\hline 488.93 & 0.2568 \\
\hline 489.29 & 0.2534 \\
\hline 489.65 & 0.2507 \\
\hline 490.02 & 0.2478 \\
\hline 490.38 & 0.2446 \\
\hline 490.74 & 0.2416 \\
\hline 491.10 & 0.2387 \\
\hline 491.46 & 0.2359 \\
\hline 491.83 & 0.2330 \\
\hline 492.19 & 0.2305 \\
\hline 492.55 & 0.2277 \\
\hline 492.91 & 0.2251 \\
\hline 493.27 & 0.2227 \\
\hline 493.63 & 0.2197 \\
\hline 493.99 & 0.2174 \\
\hline 494.36 & 0.2149 \\
\hline 494.72 & 0.2124 \\
\hline 495.08 & 0.2100 \\
\hline 495.44 & 0.2076 \\
\hline 495.80 & 0.2052 \\
\hline 496.16 & 0.2029 \\
\hline 496.52 & 0.2006 \\
\hline 496.88 & 0.1985 \\
\hline 497.25 & 0.1964 \\
\hline 497.61 & 0.1940 \\
\hline 497.97 & 0.1922 \\
\hline 498.33 & 0.1904 \\
\hline 498.69 & 0.1887 \\
\hline 499.05 & 0.1869 \\
\hline 499.41 & 0.1852 \\
\hline 499.77 & 0.1834 \\
\hline 500.13 & 0.1817 \\
\hline 500.49 & 0.1801 \\
\hline 500.85 & 0.1784 \\
\hline 501.22 & 0.1772 \\
\hline 501.58 & 0.1756 \\
\hline 501.94 & 0.1743 \\
\hline 502.30 & 0.1734 \\
\hline
\end{tabular}




\begin{tabular}{|l|l|}
\hline 502.66 & 0.1723 \\
\hline 503.02 & 0.1714 \\
\hline 503.38 & 0.1706 \\
\hline 503.74 & 0.1698 \\
\hline 504.10 & 0.1692 \\
\hline 504.46 & 0.1686 \\
\hline 504.82 & 0.1680 \\
\hline 505.18 & 0.1679 \\
\hline 505.54 & 0.1675 \\
\hline 505.90 & 0.1671 \\
\hline 506.26 & 0.1670 \\
\hline 506.62 & 0.1667 \\
\hline 506.98 & 0.1665 \\
\hline 507.34 & 0.1665 \\
\hline 507.70 & 0.1666 \\
\hline 508.06 & 0.1668 \\
\hline 508.42 & 0.1670 \\
\hline 508.78 & 0.1673 \\
\hline 509.14 & 0.1677 \\
\hline 509.50 & 0.1680 \\
\hline 509.86 & 0.1683 \\
\hline 510.22 & 0.1686 \\
\hline 510.58 & 0.1692 \\
\hline 510.94 & 0.1697 \\
\hline 511.30 & 0.1704 \\
\hline 511.66 & 0.1710 \\
\hline 512.02 & 0.1719 \\
\hline 512.38 & 0.1726 \\
\hline 512.74 & 0.1735 \\
\hline 513.09 & 0.1745 \\
\hline 513.45 & 0.1757 \\
\hline 513.81 & 0.1765 \\
\hline 514.17 & 0.1776 \\
\hline 514.53 & 0.1791 \\
\hline 514.89 & 0.1804 \\
\hline 515.25 & 0.1818 \\
\hline 515.61 & 0.1832 \\
\hline 515.97 & 0.1848 \\
\hline 516.33 & 0.1863 \\
\hline & \\
\hline
\end{tabular}




\begin{tabular}{|l|l|}
\hline 516.69 & 0.1879 \\
\hline 517.04 & 0.1898 \\
\hline 517.40 & 0.1915 \\
\hline 517.76 & 0.1934 \\
\hline 518.12 & 0.1951 \\
\hline 518.48 & 0.1972 \\
\hline 518.84 & 0.1990 \\
\hline 519.20 & 0.2010 \\
\hline 519.56 & 0.2031 \\
\hline 519.91 & 0.2053 \\
\hline 520.27 & 0.2073 \\
\hline 520.63 & 0.2091 \\
\hline 520.99 & 0.2112 \\
\hline 521.35 & 0.2135 \\
\hline 521.71 & 0.2158 \\
\hline 522.06 & 0.2180 \\
\hline 522.42 & 0.2202 \\
\hline 522.78 & 0.2224 \\
\hline 523.14 & 0.2247 \\
\hline 523.50 & 0.2270 \\
\hline 523.86 & 0.2292 \\
\hline 524.21 & 0.2316 \\
\hline 524.57 & 0.2337 \\
\hline 524.93 & 0.2360 \\
\hline 525.29 & 0.2384 \\
\hline 525.65 & 0.2409 \\
\hline 526.00 & 0.2434 \\
\hline 526.36 & 0.2459 \\
\hline 526.72 & 0.2484 \\
\hline 527.08 & 0.2509 \\
\hline 527.44 & 0.2534 \\
\hline 527.79 & 0.2561 \\
\hline 528.15 & 0.2588 \\
\hline 528.51 & 0.2615 \\
\hline 528.87 & 0.2643 \\
\hline 529.22 & 0.2670 \\
\hline 529.58 & 0.2697 \\
\hline 529.94 & 0.2725 \\
\hline 530.30 & 0.2754 \\
\hline
\end{tabular}




\begin{tabular}{|l|l|}
\hline 530.65 & 0.2782 \\
\hline 531.01 & 0.2812 \\
\hline 531.37 & 0.2841 \\
\hline 531.72 & 0.2871 \\
\hline 532.08 & 0.2904 \\
\hline 532.44 & 0.2937 \\
\hline 532.80 & 0.2968 \\
\hline 533.15 & 0.3000 \\
\hline 533.51 & 0.3031 \\
\hline 533.87 & 0.3063 \\
\hline 534.22 & 0.3096 \\
\hline 534.58 & 0.3130 \\
\hline 534.94 & 0.3163 \\
\hline 535.30 & 0.3198 \\
\hline 535.65 & 0.3235 \\
\hline 536.01 & 0.3269 \\
\hline 536.37 & 0.3305 \\
\hline 536.72 & 0.3341 \\
\hline 537.08 & 0.3377 \\
\hline 537.44 & 0.3414 \\
\hline 537.79 & 0.3453 \\
\hline 538.15 & 0.3490 \\
\hline 538.51 & 0.3529 \\
\hline 538.86 & 0.3568 \\
\hline 539.22 & 0.3609 \\
\hline 539.57 & 0.3648 \\
\hline 539.93 & 0.3686 \\
\hline 540.29 & 0.3725 \\
\hline 540.64 & 0.3766 \\
\hline 541.00 & 0.3807 \\
\hline 541.36 & 0.3847 \\
\hline 541.71 & 0.3887 \\
\hline 542.07 & 0.3927 \\
\hline 542.42 & 0.3967 \\
\hline 542.78 & 0.4006 \\
\hline 543.14 & 0.4044 \\
\hline 543.49 & 0.4083 \\
\hline 543.85 & 0.4123 \\
\hline 544.20 & 0.4164 \\
\hline
\end{tabular}




\begin{tabular}{|l|l|}
\hline 544.56 & 0.4203 \\
\hline 544.92 & 0.4241 \\
\hline 545.27 & 0.4277 \\
\hline 545.63 & 0.4315 \\
\hline 545.98 & 0.4352 \\
\hline 546.34 & 0.4391 \\
\hline 546.69 & 0.4426 \\
\hline 547.05 & 0.4463 \\
\hline 547.40 & 0.4499 \\
\hline 547.76 & 0.4534 \\
\hline 548.12 & 0.4572 \\
\hline 548.47 & 0.4607 \\
\hline 548.83 & 0.4644 \\
\hline 549.18 & 0.4680 \\
\hline 549.54 & 0.4716 \\
\hline 549.89 & 0.4754 \\
\hline 550.25 & 0.4789 \\
\hline 550.60 & 0.4823 \\
\hline 550.96 & 0.4861 \\
\hline 551.31 & 0.4897 \\
\hline 551.67 & 0.4930 \\
\hline 552.02 & 0.4965 \\
\hline 552.38 & 0.5001 \\
\hline 552.73 & 0.5036 \\
\hline 553.09 & 0.5072 \\
\hline 553.44 & 0.5108 \\
\hline 553.80 & 0.5142 \\
\hline 554.15 & 0.5176 \\
\hline 554.51 & 0.5208 \\
\hline 554.86 & 0.5242 \\
\hline 555.21 & 0.5277 \\
\hline 555.57 & 0.5309 \\
\hline 555.92 & 0.5341 \\
\hline 556.28 & 0.5372 \\
\hline 556.63 & 0.5402 \\
\hline 556.99 & 0.5434 \\
\hline 557.34 & 0.5465 \\
\hline 557.70 & 0.5495 \\
\hline 558.05 & 0.5530 \\
\hline
\end{tabular}




\begin{tabular}{|l|l|}
\hline 558.40 & 0.5560 \\
\hline 558.76 & 0.5589 \\
\hline 559.11 & 0.5620 \\
\hline 559.47 & 0.5650 \\
\hline 559.82 & 0.5680 \\
\hline 560.17 & 0.5710 \\
\hline 560.53 & 0.5739 \\
\hline 560.88 & 0.5766 \\
\hline 561.24 & 0.5796 \\
\hline 561.59 & 0.5826 \\
\hline 561.94 & 0.5857 \\
\hline 562.30 & 0.5886 \\
\hline 562.65 & 0.5916 \\
\hline 563.00 & 0.5945 \\
\hline 563.36 & 0.5975 \\
\hline 563.71 & 0.6005 \\
\hline 564.07 & 0.6035 \\
\hline 564.42 & 0.6067 \\
\hline 564.77 & 0.6097 \\
\hline 565.13 & 0.6126 \\
\hline 565.48 & 0.6154 \\
\hline 565.83 & 0.6185 \\
\hline 566.19 & 0.6216 \\
\hline 566.54 & 0.6246 \\
\hline 566.89 & 0.6277 \\
\hline 567.25 & 0.6307 \\
\hline 567.60 & 0.6335 \\
\hline 567.95 & 0.6367 \\
\hline 568.31 & 0.6399 \\
\hline 568.66 & 0.6428 \\
\hline 569.01 & 0.6459 \\
\hline 569.36 & 0.6489 \\
\hline 569.72 & 0.6521 \\
\hline 570.07 & 0.6549 \\
\hline 570.42 & 0.6579 \\
\hline 570.78 & 0.6608 \\
\hline 571.13 & 0.6638 \\
\hline 571.48 & 0.6668 \\
\hline 571.83 & 0.6696 \\
\hline & \\
\hline
\end{tabular}




\begin{tabular}{|l|l|}
\hline 572.19 & 0.6723 \\
\hline 572.54 & 0.6754 \\
\hline 572.89 & 0.6783 \\
\hline 573.24 & 0.6807 \\
\hline 573.60 & 0.6836 \\
\hline 573.95 & 0.6864 \\
\hline 574.30 & 0.6893 \\
\hline 574.65 & 0.6922 \\
\hline 575.01 & 0.6950 \\
\hline 575.36 & 0.6976 \\
\hline 575.71 & 0.7002 \\
\hline 576.06 & 0.7028 \\
\hline 576.41 & 0.7054 \\
\hline 576.77 & 0.7080 \\
\hline 577.12 & 0.7109 \\
\hline 577.47 & 0.7135 \\
\hline 577.82 & 0.7161 \\
\hline 578.17 & 0.7187 \\
\hline 578.53 & 0.7212 \\
\hline 578.88 & 0.7234 \\
\hline 579.23 & 0.7262 \\
\hline 579.58 & 0.7289 \\
\hline 579.93 & 0.7312 \\
\hline 580.28 & 0.7337 \\
\hline 580.64 & 0.7364 \\
\hline 580.99 & 0.7389 \\
\hline 581.34 & 0.7412 \\
\hline 581.69 & 0.7433 \\
\hline 582.04 & 0.7457 \\
\hline 582.39 & 0.7482 \\
\hline 582.75 & 0.7505 \\
\hline 583.10 & 0.7530 \\
\hline 583.45 & 0.7554 \\
\hline 583.80 & 0.7578 \\
\hline 584.15 & 0.7601 \\
\hline 584.50 & 0.7622 \\
\hline 584.85 & 0.7645 \\
\hline 585.20 & 0.7670 \\
\hline 585.55 & 0.7695 \\
\hline & \\
\hline
\end{tabular}




\begin{tabular}{|l|l|}
\hline 585.91 & 0.7717 \\
\hline 586.26 & 0.7740 \\
\hline 586.61 & 0.7763 \\
\hline 586.96 & 0.7784 \\
\hline 587.31 & 0.7806 \\
\hline 587.66 & 0.7825 \\
\hline 588.01 & 0.7845 \\
\hline 588.36 & 0.7861 \\
\hline 588.71 & 0.7882 \\
\hline 589.06 & 0.7901 \\
\hline 589.41 & 0.7918 \\
\hline 589.76 & 0.7936 \\
\hline 590.11 & 0.7956 \\
\hline 590.46 & 0.7973 \\
\hline 590.82 & 0.7995 \\
\hline 591.17 & 0.8015 \\
\hline 591.52 & 0.8034 \\
\hline 591.87 & 0.8048 \\
\hline 592.22 & 0.8065 \\
\hline 592.57 & 0.8078 \\
\hline 592.92 & 0.8093 \\
\hline 593.27 & 0.8108 \\
\hline 593.62 & 0.8127 \\
\hline 593.97 & 0.8140 \\
\hline 594.32 & 0.8156 \\
\hline 594.67 & 0.8172 \\
\hline 595.02 & 0.8190 \\
\hline 595.37 & 0.8207 \\
\hline 595.72 & 0.8226 \\
\hline 596.07 & 0.8243 \\
\hline 596.42 & 0.8258 \\
\hline 596.77 & 0.8276 \\
\hline 597.12 & 0.8290 \\
\hline 597.46 & 0.8304 \\
\hline 597.81 & 0.8320 \\
\hline 598.16 & 0.8334 \\
\hline 598.51 & 0.8343 \\
\hline 598.86 & 0.8359 \\
\hline 599.21 & 0.8377 \\
\hline & \\
\hline
\end{tabular}




\begin{tabular}{|l|l|}
\hline 599.56 & 0.8395 \\
\hline 599.91 & 0.8412 \\
\hline 600.26 & 0.8425 \\
\hline 600.61 & 0.8441 \\
\hline 600.96 & 0.8453 \\
\hline 601.31 & 0.8465 \\
\hline 601.66 & 0.8478 \\
\hline 602.01 & 0.8490 \\
\hline 602.35 & 0.8504 \\
\hline 602.70 & 0.8515 \\
\hline 603.05 & 0.8530 \\
\hline 603.40 & 0.8544 \\
\hline 603.75 & 0.8558 \\
\hline 604.10 & 0.8570 \\
\hline 604.45 & 0.8584 \\
\hline 604.80 & 0.8597 \\
\hline 605.15 & 0.8608 \\
\hline 605.49 & 0.8618 \\
\hline 605.84 & 0.8632 \\
\hline 606.19 & 0.8643 \\
\hline 606.54 & 0.8654 \\
\hline 606.89 & 0.8660 \\
\hline 607.24 & 0.8670 \\
\hline 607.59 & 0.8681 \\
\hline 607.93 & 0.8690 \\
\hline 608.28 & 0.8701 \\
\hline 608.63 & 0.8715 \\
\hline 608.98 & 0.8723 \\
\hline 609.33 & 0.8733 \\
\hline 609.67 & 0.8739 \\
\hline 610.02 & 0.8749 \\
\hline 610.37 & 0.8756 \\
\hline 610.72 & 0.8760 \\
\hline 611.07 & 0.8769 \\
\hline 611.41 & 0.8775 \\
\hline 611.76 & 0.8778 \\
\hline 612.11 & 0.8785 \\
\hline 612.46 & 0.8791 \\
\hline 612.81 & 0.8797 \\
\hline
\end{tabular}




\begin{tabular}{|l|l|}
\hline 613.15 & 0.8801 \\
\hline 613.50 & 0.8804 \\
\hline 613.85 & 0.8806 \\
\hline 614.20 & 0.8810 \\
\hline 614.54 & 0.8814 \\
\hline 614.89 & 0.8818 \\
\hline 615.24 & 0.8821 \\
\hline 615.59 & 0.8821 \\
\hline 615.93 & 0.8821 \\
\hline 616.28 & 0.8823 \\
\hline 616.63 & 0.8824 \\
\hline 616.98 & 0.8829 \\
\hline 617.32 & 0.8831 \\
\hline 617.67 & 0.8830 \\
\hline 618.02 & 0.8833 \\
\hline 618.36 & 0.8828 \\
\hline 618.71 & 0.8828 \\
\hline 619.06 & 0.8830 \\
\hline 619.41 & 0.8826 \\
\hline 619.75 & 0.8828 \\
\hline 620.10 & 0.8826 \\
\hline 620.45 & 0.8825 \\
\hline 620.79 & 0.8824 \\
\hline 621.14 & 0.8826 \\
\hline 621.49 & 0.8829 \\
\hline 621.83 & 0.8830 \\
\hline 622.18 & 0.8829 \\
\hline 622.53 & 0.8831 \\
\hline 622.87 & 0.8831 \\
\hline 623.22 & 0.8831 \\
\hline 623.57 & 0.8834 \\
\hline 623.91 & 0.8838 \\
\hline 624.26 & 0.8836 \\
\hline 624.60 & 0.8837 \\
\hline 624.95 & 0.8840 \\
\hline 625.30 & 0.8840 \\
\hline 625.64 & 0.8845 \\
\hline 625.99 & 0.8850 \\
\hline 626.34 & 0.8852 \\
\hline
\end{tabular}




\begin{tabular}{|l|l|}
\hline 626.68 & 0.8857 \\
\hline 627.03 & 0.8857 \\
\hline 627.37 & 0.8858 \\
\hline 627.72 & 0.8861 \\
\hline 628.07 & 0.8867 \\
\hline 628.41 & 0.8870 \\
\hline 628.76 & 0.8872 \\
\hline 629.10 & 0.8874 \\
\hline 629.45 & 0.8875 \\
\hline 629.79 & 0.8877 \\
\hline 630.14 & 0.8879 \\
\hline 630.49 & 0.8883 \\
\hline 630.83 & 0.8880 \\
\hline 631.18 & 0.8878 \\
\hline 631.52 & 0.8879 \\
\hline 631.87 & 0.8878 \\
\hline 632.21 & 0.8876 \\
\hline 632.56 & 0.8876 \\
\hline 632.90 & 0.8875 \\
\hline 633.25 & 0.8875 \\
\hline 633.59 & 0.8877 \\
\hline 633.94 & 0.8875 \\
\hline 634.28 & 0.8875 \\
\hline 634.63 & 0.8877 \\
\hline 634.97 & 0.8879 \\
\hline 635.32 & 0.8877 \\
\hline 635.66 & 0.8876 \\
\hline 636.01 & 0.8875 \\
\hline 636.35 & 0.8871 \\
\hline 636.70 & 0.8872 \\
\hline 637.04 & 0.8872 \\
\hline 637.39 & 0.8873 \\
\hline 637.73 & 0.8872 \\
\hline 638.08 & 0.8877 \\
\hline 638.42 & 0.8880 \\
\hline 638.77 & 0.8881 \\
\hline 639.11 & 0.8877 \\
\hline 639.46 & 0.8881 \\
\hline 639.80 & 0.8883 \\
\hline
\end{tabular}




\begin{tabular}{|l|l|}
\hline 640.14 & 0.8884 \\
\hline 640.49 & 0.8883 \\
\hline 640.83 & 0.8883 \\
\hline 641.18 & 0.8886 \\
\hline 641.52 & 0.8887 \\
\hline 641.87 & 0.8888 \\
\hline 642.21 & 0.8888 \\
\hline 642.55 & 0.8886 \\
\hline 642.90 & 0.8887 \\
\hline 643.24 & 0.8887 \\
\hline 643.59 & 0.8889 \\
\hline 643.93 & 0.8892 \\
\hline 644.27 & 0.8893 \\
\hline 644.62 & 0.8893 \\
\hline 644.96 & 0.8892 \\
\hline 645.31 & 0.8890 \\
\hline 645.65 & 0.8891 \\
\hline 645.99 & 0.8890 \\
\hline 646.34 & 0.8896 \\
\hline 646.68 & 0.8896 \\
\hline 647.02 & 0.8898 \\
\hline 647.37 & 0.8903 \\
\hline 647.71 & 0.8905 \\
\hline 648.05 & 0.8906 \\
\hline 648.40 & 0.8907 \\
\hline 648.74 & 0.8910 \\
\hline 649.08 & 0.8912 \\
\hline 649.43 & 0.8914 \\
\hline 649.77 & 0.8917 \\
\hline 650.11 & 0.8917 \\
\hline 650.46 & 0.8919 \\
\hline 650.80 & 0.8921 \\
\hline 651.14 & 0.8920 \\
\hline 651.49 & 0.8918 \\
\hline 651.83 & 0.8921 \\
\hline 652.17 & 0.8924 \\
\hline 652.51 & 0.8927 \\
\hline 652.86 & 0.8931 \\
\hline 653.20 & 0.8933 \\
\hline
\end{tabular}




\begin{tabular}{|l|l|}
\hline 653.54 & 0.8934 \\
\hline 653.88 & 0.8932 \\
\hline 654.23 & 0.8933 \\
\hline 654.57 & 0.8931 \\
\hline 654.91 & 0.8932 \\
\hline 655.26 & 0.8931 \\
\hline 655.60 & 0.8933 \\
\hline 655.94 & 0.8932 \\
\hline 656.28 & 0.8930 \\
\hline 656.62 & 0.8932 \\
\hline 656.97 & 0.8935 \\
\hline 657.31 & 0.8937 \\
\hline 657.65 & 0.8938 \\
\hline 657.99 & 0.8938 \\
\hline 658.34 & 0.8939 \\
\hline 658.68 & 0.8944 \\
\hline 659.02 & 0.8943 \\
\hline 659.36 & 0.8945 \\
\hline 659.70 & 0.8944 \\
\hline 660.05 & 0.8942 \\
\hline 660.39 & 0.8943 \\
\hline 660.73 & 0.8944 \\
\hline 661.07 & 0.8944 \\
\hline 661.41 & 0.8947 \\
\hline 661.75 & 0.8947 \\
\hline 662.10 & 0.8949 \\
\hline 662.44 & 0.8950 \\
\hline 662.78 & 0.8950 \\
\hline 663.12 & 0.8953 \\
\hline 663.46 & 0.8956 \\
\hline 663.80 & 0.8956 \\
\hline 664.14 & 0.8954 \\
\hline 664.49 & 0.8955 \\
\hline 664.83 & 0.8955 \\
\hline 665.17 & 0.8955 \\
\hline 665.51 & 0.8955 \\
\hline 665.85 & 0.8954 \\
\hline 666.19 & 0.8956 \\
\hline 666.53 & 0.8957 \\
\hline & \\
\hline &
\end{tabular}




\begin{tabular}{|l|l|}
\hline 666.87 & 0.8956 \\
\hline 667.21 & 0.8957 \\
\hline 667.56 & 0.8954 \\
\hline 667.90 & 0.8954 \\
\hline 668.24 & 0.8959 \\
\hline 668.58 & 0.8957 \\
\hline 668.92 & 0.8960 \\
\hline 669.26 & 0.8962 \\
\hline 669.60 & 0.8963 \\
\hline 669.94 & 0.8962 \\
\hline 670.28 & 0.8961 \\
\hline 670.62 & 0.8960 \\
\hline 670.96 & 0.8958 \\
\hline 671.30 & 0.8958 \\
\hline 671.64 & 0.8954 \\
\hline 671.98 & 0.8950 \\
\hline 672.32 & 0.8949 \\
\hline 672.66 & 0.8950 \\
\hline 673.00 & 0.8948 \\
\hline 673.34 & 0.8945 \\
\hline 673.68 & 0.8942 \\
\hline 674.02 & 0.8943 \\
\hline 674.36 & 0.8942 \\
\hline 674.70 & 0.8943 \\
\hline 675.04 & 0.8942 \\
\hline 675.38 & 0.8937 \\
\hline 675.72 & 0.8936 \\
\hline 676.06 & 0.8935 \\
\hline 676.40 & 0.8935 \\
\hline 676.74 & 0.8933 \\
\hline 677.08 & 0.8931 \\
\hline 677.42 & 0.8930 \\
\hline 677.76 & 0.8930 \\
\hline 678.10 & 0.8932 \\
\hline 678.44 & 0.8934 \\
\hline 678.78 & 0.8938 \\
\hline 679.12 & 0.8943 \\
\hline 679.46 & 0.8948 \\
\hline 679.80 & 0.8946 \\
\hline
\end{tabular}




\begin{tabular}{|l|l|}
\hline 680.14 & 0.8944 \\
\hline 680.48 & 0.8941 \\
\hline 680.82 & 0.8940 \\
\hline 681.16 & 0.8936 \\
\hline 681.50 & 0.8935 \\
\hline 681.83 & 0.8936 \\
\hline 682.17 & 0.8936 \\
\hline 682.51 & 0.8934 \\
\hline 682.85 & 0.8933 \\
\hline 683.19 & 0.8928 \\
\hline 683.53 & 0.8923 \\
\hline 683.87 & 0.8922 \\
\hline 684.21 & 0.8924 \\
\hline 684.55 & 0.8923 \\
\hline 684.88 & 0.8922 \\
\hline 685.22 & 0.8916 \\
\hline 685.56 & 0.8917 \\
\hline 685.90 & 0.8913 \\
\hline 686.24 & 0.8908 \\
\hline 686.58 & 0.8902 \\
\hline 686.92 & 0.8900 \\
\hline 687.25 & 0.8901 \\
\hline 687.59 & 0.8902 \\
\hline 687.93 & 0.8903 \\
\hline 688.27 & 0.8902 \\
\hline 688.61 & 0.8900 \\
\hline 688.94 & 0.8901 \\
\hline 689.28 & 0.8896 \\
\hline 689.62 & 0.8896 \\
\hline 689.96 & 0.8893 \\
\hline 690.30 & 0.8890 \\
\hline 690.64 & 0.8892 \\
\hline 690.97 & 0.8892 \\
\hline 691.31 & 0.8893 \\
\hline 691.65 & 0.8892 \\
\hline 691.99 & 0.8890 \\
\hline 692.32 & 0.8892 \\
\hline 692.66 & 0.8883 \\
\hline 693.00 & 0.8879 \\
\hline
\end{tabular}




\begin{tabular}{|l|l|}
\hline 693.34 & 0.8875 \\
\hline 693.67 & 0.8873 \\
\hline 694.01 & 0.8871 \\
\hline 694.35 & 0.8872 \\
\hline 694.69 & 0.8874 \\
\hline 695.02 & 0.8869 \\
\hline 695.36 & 0.8866 \\
\hline 695.70 & 0.8871 \\
\hline 696.04 & 0.8867 \\
\hline 696.37 & 0.8869 \\
\hline 696.71 & 0.8868 \\
\hline 697.05 & 0.8865 \\
\hline 697.38 & 0.8868 \\
\hline 697.72 & 0.8867 \\
\hline 698.06 & 0.8869 \\
\hline 698.40 & 0.8865 \\
\hline 698.73 & 0.8866 \\
\hline 699.07 & 0.8869 \\
\hline 699.41 & 0.8866 \\
\hline 699.74 & 0.8869 \\
\hline 700.08 & 0.8874 \\
\hline 700.42 & 0.8879 \\
\hline 700.75 & 0.8880 \\
\hline
\end{tabular}

Absorbance values of the Kelly Green Munsell Chip 7.5 4, 14 for Phase I

\begin{tabular}{|l|l|c|c|l|l|l|l|}
\hline $\mathbf{k}$ & 0.11893 & 0.05496 & 0.06776 & 0.06828 & 0.04487 & 0.07136 & 0.04100 \\
\hline sum & 0.67484 & 0.21180 & 0.38323 & 0.30863 & 0.22786 & 0.50314 & 0.11566 \\
\hline & 2 scans & $\mathbf{5}$ scans & $\mathbf{1 0}$ scans & $\begin{array}{l}\mathbf{1 5} \\
\text { scans }\end{array}$ & $\begin{array}{l}\mathbf{2 0} \\
\text { scans }\end{array}$ & $\begin{array}{l}\mathbf{3 0} \\
\text { scans }\end{array}$ & $\begin{array}{l}\mathbf{5 0} \\
\text { scans }\end{array}$ \\
\hline 400.33 & 0.00005 & 0.00005 & 0.00004 & 0.00002 & 0.00002 & 0.00059 & 0.00010 \\
\hline 400.69 & 0.00008 & 0.00000 & 0.00005 & 0.00006 & 0.00003 & 0.00055 & 0.00008 \\
\hline 401.06 & 0.00004 & 0.00000 & 0.00002 & 0.00009 & 0.00014 & 0.00059 & 0.00014 \\
\hline 401.42 & 0.00012 & 0.00000 & 0.00002 & 0.00022 & 0.00014 & 0.00070 & 0.00017 \\
\hline 401.78 & 0.00020 & 0.00005 & 0.00003 & 0.00009 & 0.00005 & 0.00055 & 0.00014 \\
\hline 402.14 & 0.00049 & 0.00012 & 0.00000 & 0.00019 & 0.00003 & 0.00064 & 0.00006 \\
\hline 402.5 & 0.00073 & 0.00017 & 0.00000 & 0.00016 & 0.00001 & 0.00059 & 0.00005 \\
\hline 402.87 & 0.00079 & 0.00005 & 0.00001 & 0.00016 & 0.00000 & 0.00055 & 0.00006 \\
\hline
\end{tabular}




\begin{tabular}{|c|c|c|c|c|c|c|c|}
\hline 403.23 & 0.00068 & 0.00003 & 0.00001 & 0.00014 & 0.00000 & 0.00038 & 0.00008 \\
\hline 403.59 & 0.00109 & 0.00002 & 0.00003 & 0.00005 & 0.00001 & 0.00046 & 0.00006 \\
\hline 403.95 & 0.00097 & 0.00004 & 0.00001 & 0.00005 & 0.00002 & 0.00046 & 0.00010 \\
\hline 404.31 & 0.00123 & 0.00001 & 0.00003 & 0.00008 & 0.00003 & 0.00050 & 0.00008 \\
\hline 404.68 & 0.00063 & 0.00000 & 0.00006 & 0.00009 & 0.00008 & 0.00055 & 0.00008 \\
\hline 405.04 & 0.00044 & 0.00002 & 0.00008 & 0.00009 & 0.00003 & 0.00059 & 0.00005 \\
\hline 405.4 & 0.00029 & 0.00000 & 0.00005 & 0.00003 & 0.00003 & 0.00050 & 0.00005 \\
\hline 405.76 & 0.00020 & 0.00000 & 0.00002 & 0.00009 & 0.00010 & 0.00059 & 0.00005 \\
\hline 406.12 & 0.00029 & 0.00002 & 0.00000 & 0.00014 & 0.00005 & 0.00046 & 0.00005 \\
\hline 406.49 & 0.00017 & 0.00001 & 0.00000 & 0.00014 & 0.00010 & 0.00050 & 0.00004 \\
\hline 406.85 & 0.00001 & 0.00006 & 0.00001 & 0.00016 & 0.00010 & 0.00050 & 0.00001 \\
\hline 407.21 & 0.00003 & 0.00006 & 0.00002 & 0.00016 & 0.00012 & 0.00070 & 0.00000 \\
\hline 407.57 & 0.00002 & 0.00023 & 0.00001 & 0.00025 & 0.00010 & 0.00064 & 0.00000 \\
\hline 407.93 & 0.00000 & 0.00020 & 0.00004 & 0.00022 & 0.00005 & 0.00055 & 0.00000 \\
\hline 408.29 & 0.00008 & 0.00023 & 0.00007 & 0.00019 & 0.00012 & 0.00059 & 0.00000 \\
\hline 408.65 & 0.00005 & 0.00017 & 0.00009 & 0.00014 & 0.00008 & 0.00050 & 0.00002 \\
\hline 409.02 & 0.00002 & 0.00012 & 0.00020 & 0.00008 & 0.00001 & 0.00041 & 0.00004 \\
\hline 409.38 & 0.00002 & 0.00023 & 0.00023 & 0.00008 & 0.00000 & 0.00038 & 0.00008 \\
\hline 409.74 & 0.00000 & 0.00029 & 0.00033 & 0.00006 & 0.00000 & 0.00021 & 0.00008 \\
\hline 410.1 & 0.00006 & 0.00023 & 0.00026 & 0.00005 & 0.00001 & 0.00021 & 0.00010 \\
\hline 410.46 & 0.00005 & 0.00023 & 0.00041 & 0.00008 & 0.00004 & 0.00015 & 0.00010 \\
\hline 410.82 & 0.00002 & 0.00023 & 0.00045 & 0.00011 & 0.00005 & 0.00018 & 0.00010 \\
\hline 411.18 & 0.00007 & 0.00029 & 0.00054 & 0.00009 & 0.00004 & 0.00007 & 0.00017 \\
\hline 411.54 & 0.00007 & 0.00017 & 0.00054 & 0.00005 & 0.00004 & 0.00011 & 0.00020 \\
\hline 411.91 & 0.00015 & 0.00008 & 0.00049 & 0.00002 & 0.00002 & 0.00007 & 0.00022 \\
\hline 412.27 & 0.00033 & 0.00003 & 0.00037 & 0.00003 & 0.00002 & 0.00005 & 0.00022 \\
\hline 412.63 & 0.00068 & 0.00008 & 0.00059 & 0.00008 & 0.00005 & 0.00003 & 0.00026 \\
\hline 412.99 & 0.00079 & 0.00017 & 0.00064 & 0.00009 & 0.00001 & 0.00009 & 0.00022 \\
\hline 413.35 & 0.00103 & 0.00020 & 0.00069 & 0.00019 & 0.00000 & 0.00009 & 0.00022 \\
\hline 413.71 & 0.00194 & 0.00012 & 0.00064 & 0.00022 & 0.00000 & 0.00007 & 0.00026 \\
\hline 414.07 & 0.00194 & 0.00010 & 0.00074 & 0.00019 & 0.00000 & 0.00013 & 0.00020 \\
\hline 414.43 & 0.00194 & 0.00029 & 0.00069 & 0.00006 & 0.00000 & 0.00013 & 0.00020 \\
\hline 414.79 & 0.00169 & 0.00012 & 0.00059 & 0.00001 & 0.00000 & 0.00013 & 0.00017 \\
\hline 415.15 & 0.00137 & 0.00006 & 0.00049 & 0.00003 & 0.00000 & 0.00011 & 0.00012 \\
\hline 415.51 & 0.00161 & 0.00008 & 0.00033 & 0.00005 & 0.00000 & 0.00013 & 0.00008 \\
\hline 415.88 & 0.00161 & 0.00008 & 0.00023 & 0.00011 & 0.00000 & 0.00021 & 0.00005 \\
\hline 416.24 & 0.00203 & 0.00005 & 0.00018 & 0.00011 & 0.00003 & 0.00018 & 0.00006 \\
\hline 416.6 & 0.00194 & 0.00002 & 0.00010 & 0.00011 & 0.00001 & 0.00021 & 0.00006 \\
\hline 416.96 & 0.00261 & 0.00000 & 0.00007 & 0.00016 & 0.00004 & 0.00011 & 0.00010 \\
\hline
\end{tabular}




\begin{tabular}{|c|c|c|c|c|c|c|c|}
\hline 417.32 & 0.00241 & 0.00001 & 0.00002 & 0.00016 & 0.00003 & 0.00013 & 0.00008 \\
\hline 417.68 & 0.00177 & 0.00000 & 0.00000 & 0.00016 & 0.00001 & 0.00021 & 0.00010 \\
\hline 418.04 & 0.00153 & 0.00004 & 0.00001 & 0.00019 & 0.00000 & 0.00015 & 0.00010 \\
\hline 418.4 & 0.00169 & 0.00002 & 0.00001 & 0.00031 & 0.00003 & 0.00011 & 0.00012 \\
\hline 418.76 & 0.00130 & 0.00001 & 0.00000 & 0.00031 & 0.00005 & 0.00013 & 0.00014 \\
\hline 419.12 & 0.00145 & 0.00000 & 0.00000 & 0.00031 & 0.00008 & 0.00011 & 0.00017 \\
\hline 419.48 & 0.00097 & 0.00001 & 0.00004 & 0.00028 & 0.00010 & 0.00009 & 0.00020 \\
\hline 419.84 & 0.00085 & 0.00001 & 0.00018 & 0.00031 & 0.00010 & 0.00004 & 0.00029 \\
\hline 420.2 & 0.00044 & 0.00000 & 0.00010 & 0.00025 & 0.00015 & 0.00005 & 0.00029 \\
\hline 420.56 & 0.00023 & 0.00000 & 0.00013 & 0.00031 & 0.00020 & 0.00004 & 0.00032 \\
\hline 420.92 & 0.00001 & 0.00000 & 0.00020 & 0.00035 & 0.00029 & 0.00004 & 0.00036 \\
\hline 421.28 & 0.00003 & 0.00003 & 0.00033 & 0.00031 & 0.00041 & 0.00004 & 0.00040 \\
\hline 421.64 & 0.00004 & 0.00005 & 0.00059 & 0.00039 & 0.00054 & 0.00003 & 0.00040 \\
\hline 422 & 0.00026 & 0.00023 & 0.00080 & 0.00043 & 0.00068 & 0.00002 & 0.00044 \\
\hline 422.36 & 0.00015 & 0.00020 & 0.00086 & 0.00043 & 0.00058 & 0.00003 & 0.00048 \\
\hline 422.72 & 0.00044 & 0.00020 & 0.00074 & 0.00047 & 0.00049 & 0.00004 & 0.00044 \\
\hline 423.08 & 0.00058 & 0.00023 & 0.00069 & 0.00047 & 0.00054 & 0.00009 & 0.00036 \\
\hline 423.44 & 0.00097 & 0.00026 & 0.00059 & 0.00061 & 0.00058 & 0.00009 & 0.00032 \\
\hline 423.8 & 0.00085 & 0.00044 & 0.00045 & 0.00056 & 0.00054 & 0.00013 & 0.00029 \\
\hline 424.16 & 0.00103 & 0.00033 & 0.00064 & 0.00061 & 0.00049 & 0.00007 & 0.00022 \\
\hline 424.52 & 0.00130 & 0.00049 & 0.00080 & 0.00061 & 0.00049 & 0.00004 & 0.00026 \\
\hline 424.88 & 0.00194 & 0.00040 & 0.00074 & 0.00056 & 0.00045 & 0.00002 & 0.00022 \\
\hline 425.24 & 0.00137 & 0.00040 & 0.00069 & 0.00047 & 0.00037 & 0.00001 & 0.00020 \\
\hline 425.6 & 0.00169 & 0.00068 & 0.00069 & 0.00052 & 0.00033 & 0.00001 & 0.00020 \\
\hline 425.96 & 0.00116 & 0.00058 & 0.00074 & 0.00052 & 0.00026 & 0.00000 & 0.00020 \\
\hline 426.32 & 0.00161 & 0.00053 & 0.00091 & 0.00052 & 0.00037 & 0.00000 & 0.00020 \\
\hline 426.68 & 0.00161 & 0.00079 & 0.00080 & 0.00061 & 0.00037 & 0.00001 & 0.00022 \\
\hline 427.04 & 0.00177 & 0.00058 & 0.00086 & 0.00052 & 0.00041 & 0.00003 & 0.00022 \\
\hline 427.4 & 0.00203 & 0.00049 & 0.00091 & 0.00035 & 0.00045 & 0.00006 & 0.00020 \\
\hline 427.76 & 0.00177 & 0.00044 & 0.00098 & 0.00043 & 0.00045 & 0.00007 & 0.00014 \\
\hline 428.12 & 0.00169 & 0.00049 & 0.00086 & 0.00043 & 0.00037 & 0.00006 & 0.00014 \\
\hline 428.47 & 0.00153 & 0.00040 & 0.00064 & 0.00047 & 0.00037 & 0.00003 & 0.00012 \\
\hline 428.83 & 0.00194 & 0.00044 & 0.00064 & 0.00047 & 0.00037 & 0.00003 & 0.00010 \\
\hline 429.19 & 0.00203 & 0.00040 & 0.00059 & 0.00047 & 0.00041 & 0.00004 & 0.00008 \\
\hline 429.55 & 0.00137 & 0.00026 & 0.00069 & 0.00052 & 0.00041 & 0.00004 & 0.00005 \\
\hline 429.91 & 0.00153 & 0.00020 & 0.00069 & 0.00052 & 0.00049 & 0.00001 & 0.00005 \\
\hline 430.27 & 0.00123 & 0.00036 & 0.00064 & 0.00056 & 0.00037 & 0.00001 & 0.00005 \\
\hline 430.63 & 0.00097 & 0.00017 & 0.00054 & 0.00047 & 0.00029 & 0.00001 & 0.00004 \\
\hline 430.99 & 0.00079 & 0.00036 & 0.00064 & 0.00056 & 0.00029 & 0.00001 & 0.00004 \\
\hline
\end{tabular}




\begin{tabular}{|c|c|c|c|c|c|c|c|}
\hline 431.35 & 0.00058 & 0.00068 & 0.00054 & 0.00094 & 0.00029 & 0.00001 & 0.00005 \\
\hline 431.71 & 0.00079 & 0.00079 & 0.00074 & 0.00101 & 0.00033 & 0.00001 & 0.00010 \\
\hline 432.07 & 0.00068 & 0.00084 & 0.00074 & 0.00094 & 0.00041 & 0.00001 & 0.00012 \\
\hline 432.42 & 0.00063 & 0.00079 & 0.00086 & 0.00088 & 0.00041 & 0.00000 & 0.00010 \\
\hline 432.78 & 0.00026 & 0.00073 & 0.00091 & 0.00094 & 0.00045 & 0.00001 & 0.00010 \\
\hline 433.14 & 0.00040 & 0.00079 & 0.00086 & 0.00082 & 0.00045 & 0.00000 & 0.00012 \\
\hline 433.5 & 0.00068 & 0.00068 & 0.00091 & 0.00082 & 0.00054 & 0.00000 & 0.00017 \\
\hline 433.86 & 0.00073 & 0.00068 & 0.00074 & 0.00088 & 0.00045 & 0.00001 & 0.00017 \\
\hline 434.22 & 0.00073 & 0.00040 & 0.00064 & 0.00088 & 0.00049 & 0.00001 & 0.00017 \\
\hline 434.58 & 0.00103 & 0.00053 & 0.00080 & 0.00088 & 0.00058 & 0.00001 & 0.00020 \\
\hline 434.94 & 0.00153 & 0.00040 & 0.00069 & 0.00088 & 0.00054 & 0.00001 & 0.00020 \\
\hline 435.29 & 0.00153 & 0.00029 & 0.00086 & 0.00077 & 0.00054 & 0.00003 & 0.00020 \\
\hline 435.65 & 0.00145 & 0.00036 & 0.00054 & 0.00061 & 0.00041 & 0.00002 & 0.00012 \\
\hline 436.01 & 0.00153 & 0.00036 & 0.00059 & 0.00056 & 0.00041 & 0.00003 & 0.00010 \\
\hline 436.37 & 0.00177 & 0.00049 & 0.00054 & 0.00071 & 0.00033 & 0.00004 & 0.00012 \\
\hline 436.73 & 0.00186 & 0.00040 & 0.00054 & 0.00056 & 0.00023 & 0.00002 & 0.00008 \\
\hline 437.09 & 0.00212 & 0.00026 & 0.00054 & 0.00061 & 0.00020 & 0.00004 & 0.00008 \\
\hline 437.44 & 0.00231 & 0.00023 & 0.00037 & 0.00061 & 0.00012 & 0.00002 & 0.00005 \\
\hline 437.8 & 0.00212 & 0.00014 & 0.00049 & 0.00056 & 0.00012 & 0.00002 & 0.00005 \\
\hline 438.16 & 0.00145 & 0.00012 & 0.00049 & 0.00043 & 0.00010 & 0.00002 & 0.00002 \\
\hline 438.52 & 0.00145 & 0.00008 & 0.00049 & 0.00047 & 0.00007 & 0.00002 & 0.00002 \\
\hline 438.88 & 0.00130 & 0.00010 & 0.00037 & 0.00047 & 0.00007 & 0.00001 & 0.00002 \\
\hline 439.24 & 0.00145 & 0.00006 & 0.00037 & 0.00047 & 0.00007 & 0.00001 & 0.00002 \\
\hline 439.59 & 0.00161 & 0.00002 & 0.00045 & 0.00047 & 0.00005 & 0.00001 & 0.00002 \\
\hline 439.95 & 0.00203 & 0.00001 & 0.00059 & 0.00061 & 0.00004 & 0.00000 & 0.00004 \\
\hline 440.31 & 0.00203 & 0.00000 & 0.00054 & 0.00052 & 0.00005 & 0.00001 & 0.00001 \\
\hline 440.67 & 0.00212 & 0.00001 & 0.00041 & 0.00056 & 0.00005 & 0.00001 & 0.00001 \\
\hline 441.03 & 0.00203 & 0.00005 & 0.00041 & 0.00061 & 0.00005 & 0.00000 & 0.00000 \\
\hline 441.38 & 0.00222 & 0.00005 & 0.00037 & 0.00061 & 0.00004 & 0.00000 & 0.00000 \\
\hline 441.74 & 0.00231 & 0.00014 & 0.00041 & 0.00066 & 0.00005 & 0.00000 & 0.00000 \\
\hline 442.1 & 0.00314 & 0.00014 & 0.00037 & 0.00066 & 0.00007 & 0.00000 & 0.00000 \\
\hline 442.46 & 0.00282 & 0.00017 & 0.00045 & 0.00066 & 0.00008 & 0.00000 & 0.00000 \\
\hline 442.81 & 0.00231 & 0.00010 & 0.00059 & 0.00061 & 0.00008 & 0.00001 & 0.00002 \\
\hline 443.17 & 0.00251 & 0.00012 & 0.00059 & 0.00061 & 0.00010 & 0.00001 & 0.00000 \\
\hline 443.53 & 0.00212 & 0.00010 & 0.00049 & 0.00052 & 0.00010 & 0.00001 & 0.00000 \\
\hline 443.89 & 0.00212 & 0.00014 & 0.00049 & 0.00052 & 0.00012 & 0.00001 & 0.00000 \\
\hline 444.24 & 0.00161 & 0.00014 & 0.00049 & 0.00052 & 0.00008 & 0.00001 & 0.00000 \\
\hline 444.6 & 0.00145 & 0.00008 & 0.00064 & 0.00047 & 0.00007 & 0.00003 & 0.00000 \\
\hline 444.96 & 0.00123 & 0.00004 & 0.00064 & 0.00047 & 0.00008 & 0.00003 & 0.00000 \\
\hline
\end{tabular}




\begin{tabular}{|c|c|c|c|c|c|c|c|}
\hline 445.32 & 0.00079 & 0.00008 & 0.00074 & 0.00035 & 0.00012 & 0.00002 & 0.00000 \\
\hline 445.67 & 0.00058 & 0.00005 & 0.00074 & 0.00031 & 0.00012 & 0.00002 & 0.00000 \\
\hline 446.03 & 0.00049 & 0.00006 & 0.00069 & 0.00043 & 0.00012 & 0.00002 & 0.00000 \\
\hline 446.39 & 0.00044 & 0.00004 & 0.00069 & 0.00043 & 0.00010 & 0.00002 & 0.00000 \\
\hline 446.75 & 0.00033 & 0.00006 & 0.00049 & 0.00039 & 0.00007 & 0.00003 & 0.00000 \\
\hline 447.1 & 0.00029 & 0.00006 & 0.00054 & 0.00039 & 0.00007 & 0.00005 & 0.00000 \\
\hline 447.46 & 0.00023 & 0.00006 & 0.00069 & 0.00039 & 0.00004 & 0.00004 & 0.00000 \\
\hline 447.82 & 0.00023 & 0.00006 & 0.00054 & 0.00035 & 0.00004 & 0.00004 & 0.00000 \\
\hline 448.18 & 0.00040 & 0.00005 & 0.00049 & 0.00025 & 0.00004 & 0.00007 & 0.00000 \\
\hline 448.53 & 0.00033 & 0.00008 & 0.00045 & 0.00031 & 0.00007 & 0.00004 & 0.00000 \\
\hline 448.89 & 0.00029 & 0.00012 & 0.00041 & 0.00028 & 0.00005 & 0.00004 & 0.00002 \\
\hline 449.25 & 0.00036 & 0.00005 & 0.00037 & 0.00031 & 0.00003 & 0.00004 & 0.00003 \\
\hline 449.6 & 0.00040 & 0.00003 & 0.00030 & 0.00031 & 0.00002 & 0.00004 & 0.00005 \\
\hline 449.96 & 0.00026 & 0.00002 & 0.00026 & 0.00025 & 0.00002 & 0.00004 & 0.00010 \\
\hline 450.32 & 0.00026 & 0.00001 & 0.00023 & 0.00025 & 0.00002 & 0.00003 & 0.00010 \\
\hline 450.67 & 0.00036 & 0.00000 & 0.00030 & 0.00025 & 0.00002 & 0.00002 & 0.00008 \\
\hline 451.03 & 0.00036 & 0.00000 & 0.00023 & 0.00022 & 0.00001 & 0.00002 & 0.00010 \\
\hline 451.39 & 0.00058 & 0.00000 & 0.00020 & 0.00025 & 0.00003 & 0.00001 & 0.00012 \\
\hline 451.74 & 0.00053 & 0.00000 & 0.00020 & 0.00028 & 0.00003 & 0.00001 & 0.00012 \\
\hline 452.1 & 0.00049 & 0.00000 & 0.00020 & 0.00028 & 0.00003 & 0.00001 & 0.00014 \\
\hline 452.46 & 0.00063 & 0.00000 & 0.00020 & 0.00028 & 0.00002 & 0.00001 & 0.00014 \\
\hline 452.81 & 0.00068 & 0.00000 & 0.00023 & 0.00031 & 0.00004 & 0.00000 & 0.00012 \\
\hline 453.17 & 0.00073 & 0.00000 & 0.00023 & 0.00025 & 0.00005 & 0.00000 & 0.00012 \\
\hline 453.53 & 0.00079 & 0.00000 & 0.00026 & 0.00022 & 0.00005 & 0.00000 & 0.00008 \\
\hline 453.88 & 0.00090 & 0.00000 & 0.00026 & 0.00025 & 0.00003 & 0.00000 & 0.00008 \\
\hline 454.24 & 0.00103 & 0.00002 & 0.00020 & 0.00025 & 0.00003 & 0.00000 & 0.00006 \\
\hline 454.6 & 0.00085 & 0.00000 & 0.00015 & 0.00025 & 0.00003 & 0.00001 & 0.00012 \\
\hline 454.95 & 0.00097 & 0.00002 & 0.00015 & 0.00028 & 0.00004 & 0.00000 & 0.00012 \\
\hline 455.31 & 0.00085 & 0.00004 & 0.00015 & 0.00028 & 0.00004 & 0.00000 & 0.00012 \\
\hline 455.66 & 0.00073 & 0.00004 & 0.00013 & 0.00022 & 0.00004 & 0.00001 & 0.00014 \\
\hline 456.02 & 0.00063 & 0.00002 & 0.00018 & 0.00019 & 0.00007 & 0.00001 & 0.00014 \\
\hline 456.38 & 0.00058 & 0.00000 & 0.00010 & 0.00014 & 0.00004 & 0.00001 & 0.00020 \\
\hline 456.73 & 0.00053 & 0.00000 & 0.00007 & 0.00011 & 0.00002 & 0.00002 & 0.00023 \\
\hline 457.09 & 0.00058 & 0.00002 & 0.00007 & 0.00011 & 0.00003 & 0.00002 & 0.00023 \\
\hline 457.45 & 0.00044 & 0.00003 & 0.00004 & 0.00009 & 0.00004 & 0.00002 & 0.00026 \\
\hline 457.8 & 0.00058 & 0.00002 & 0.00007 & 0.00011 & 0.00004 & 0.00001 & 0.00023 \\
\hline 458.16 & 0.00049 & 0.00000 & 0.00007 & 0.00008 & 0.00004 & 0.00001 & 0.00026 \\
\hline 458.51 & 0.00040 & 0.00004 & 0.00009 & 0.00008 & 0.00005 & 0.00001 & 0.00023 \\
\hline 458.87 & 0.00026 & 0.00001 & 0.00009 & 0.00008 & 0.00005 & 0.00001 & 0.00023 \\
\hline
\end{tabular}




\begin{tabular}{|c|c|c|c|c|c|c|c|}
\hline 459.23 & 0.00023 & 0.00000 & 0.00007 & 0.00005 & 0.00003 & 0.00002 & 0.00026 \\
\hline 459.58 & 0.00015 & 0.00002 & 0.00005 & 0.00006 & 0.00003 & 0.00002 & 0.00026 \\
\hline 459.94 & 0.00015 & 0.00003 & 0.00002 & 0.00003 & 0.00001 & 0.00001 & 0.00026 \\
\hline 460.29 & 0.00017 & 0.00006 & 0.00002 & 0.00005 & 0.00001 & 0.00001 & 0.00023 \\
\hline 460.65 & 0.00023 & 0.00008 & 0.00002 & 0.00005 & 0.00001 & 0.00001 & 0.00026 \\
\hline 461 & 0.00015 & 0.00010 & 0.00002 & 0.00005 & 0.00001 & 0.00000 & 0.00026 \\
\hline 461.36 & 0.00023 & 0.00014 & 0.00002 & 0.00006 & 0.00002 & 0.00000 & 0.00020 \\
\hline 461.71 & 0.00017 & 0.00017 & 0.00002 & 0.00005 & 0.00001 & 0.00001 & 0.00020 \\
\hline 462.07 & 0.00029 & 0.00010 & 0.00001 & 0.00006 & 0.00002 & 0.00001 & 0.00023 \\
\hline 462.43 & 0.00026 & 0.00010 & 0.00001 & 0.00008 & 0.00001 & 0.00001 & 0.00020 \\
\hline 462.78 & 0.00036 & 0.00010 & 0.00002 & 0.00006 & 0.00001 & 0.00000 & 0.00020 \\
\hline 463.14 & 0.00036 & 0.00008 & 0.00001 & 0.00006 & 0.00002 & 0.00000 & 0.00020 \\
\hline 463.49 & 0.00040 & 0.00003 & 0.00004 & 0.00008 & 0.00002 & 0.00000 & 0.00017 \\
\hline 463.85 & 0.00044 & 0.00002 & 0.00004 & 0.00008 & 0.00001 & 0.00000 & 0.00020 \\
\hline 464.2 & 0.00040 & 0.00000 & 0.00003 & 0.00008 & 0.00000 & 0.00000 & 0.00020 \\
\hline 464.56 & 0.00029 & 0.00000 & 0.00004 & 0.00008 & 0.00001 & 0.00000 & 0.00017 \\
\hline 464.91 & 0.00026 & 0.00000 & 0.00003 & 0.00009 & 0.00001 & 0.00001 & 0.00014 \\
\hline 465.27 & 0.00029 & 0.00001 & 0.00003 & 0.00009 & 0.00000 & 0.00001 & 0.00017 \\
\hline 465.62 & 0.00033 & 0.00002 & 0.00002 & 0.00006 & 0.00000 & 0.00001 & 0.00020 \\
\hline 465.98 & 0.00026 & 0.00002 & 0.00002 & 0.00005 & 0.00000 & 0.00001 & 0.00020 \\
\hline 466.33 & 0.00033 & 0.00004 & 0.00002 & 0.00003 & 0.00000 & 0.00001 & 0.00023 \\
\hline 466.69 & 0.00029 & 0.00002 & 0.00002 & 0.00003 & 0.00001 & 0.00001 & 0.00023 \\
\hline 467.04 & 0.00026 & 0.00005 & 0.00001 & 0.00001 & 0.00001 & 0.00001 & 0.00026 \\
\hline 467.4 & 0.00026 & 0.00005 & 0.00001 & 0.00001 & 0.00001 & 0.00001 & 0.00026 \\
\hline 467.75 & 0.00023 & 0.00004 & 0.00001 & 0.00001 & 0.00001 & 0.00001 & 0.00026 \\
\hline 468.11 & 0.00023 & 0.00005 & 0.00001 & 0.00000 & 0.00001 & 0.00001 & 0.00026 \\
\hline 468.46 & 0.00020 & 0.00006 & 0.00000 & 0.00000 & 0.00001 & 0.00000 & 0.00023 \\
\hline 468.82 & 0.00020 & 0.00010 & 0.00000 & 0.00000 & 0.00001 & 0.00000 & 0.00023 \\
\hline 469.17 & 0.00012 & 0.00014 & 0.00000 & 0.00000 & 0.00002 & 0.00000 & 0.00023 \\
\hline 469.53 & 0.00007 & 0.00014 & 0.00000 & 0.00001 & 0.00003 & 0.00001 & 0.00023 \\
\hline 469.88 & 0.00008 & 0.00014 & 0.00000 & 0.00001 & 0.00002 & 0.00001 & 0.00020 \\
\hline 470.24 & 0.00008 & 0.00012 & 0.00000 & 0.00001 & 0.00002 & 0.00000 & 0.00017 \\
\hline 470.59 & 0.00008 & 0.00017 & 0.00001 & 0.00001 & 0.00005 & 0.00000 & 0.00017 \\
\hline 470.94 & 0.00005 & 0.00017 & 0.00001 & 0.00002 & 0.00005 & 0.00000 & 0.00017 \\
\hline 471.3 & 0.00005 & 0.00014 & 0.00001 & 0.00002 & 0.00005 & 0.00000 & 0.00014 \\
\hline 471.65 & 0.00003 & 0.00022 & 0.00002 & 0.00003 & 0.00006 & 0.00000 & 0.00017 \\
\hline 472.01 & 0.00002 & 0.00017 & 0.00001 & 0.00003 & 0.00006 & 0.00000 & 0.00014 \\
\hline 472.36 & 0.00007 & 0.00012 & 0.00000 & 0.00004 & 0.00008 & 0.00000 & 0.00014 \\
\hline 472.72 & 0.00008 & 0.00008 & 0.00000 & 0.00003 & 0.00010 & 0.00000 & 0.00012 \\
\hline
\end{tabular}




\begin{tabular}{|c|c|c|c|c|c|c|c|}
\hline 473.07 & 0.00008 & 0.00006 & 0.00000 & 0.00004 & 0.00010 & 0.00000 & 0.00012 \\
\hline 473.43 & 0.00008 & 0.00006 & 0.00000 & 0.00003 & 0.00006 & 0.00000 & 0.00012 \\
\hline 473.78 & 0.00010 & 0.00005 & 0.00000 & 0.00003 & 0.00006 & 0.00001 & 0.00010 \\
\hline 474.13 & 0.00005 & 0.00006 & 0.00001 & 0.00004 & 0.00005 & 0.00000 & 0.00010 \\
\hline 474.49 & 0.00002 & 0.00005 & 0.00001 & 0.00005 & 0.00003 & 0.00000 & 0.00008 \\
\hline 474.84 & 0.00002 & 0.00005 & 0.00002 & 0.00005 & 0.00005 & 0.00000 & 0.00008 \\
\hline 475.2 & 0.00003 & 0.00002 & 0.00001 & 0.00002 & 0.00005 & 0.00000 & 0.00006 \\
\hline 475.55 & 0.00008 & 0.00002 & 0.00003 & 0.00003 & 0.00005 & 0.00000 & 0.00006 \\
\hline 475.9 & 0.00007 & 0.00002 & 0.00005 & 0.00002 & 0.00005 & 0.00000 & 0.00008 \\
\hline 476.26 & 0.00002 & 0.00002 & 0.00005 & 0.00001 & 0.00002 & 0.00000 & 0.00006 \\
\hline 476.61 & 0.00000 & 0.00002 & 0.00002 & 0.00001 & 0.00001 & 0.00000 & 0.00006 \\
\hline 476.96 & 0.00000 & 0.00002 & 0.00001 & 0.00001 & 0.00001 & 0.00000 & 0.00005 \\
\hline 477.32 & 0.00001 & 0.00006 & 0.00002 & 0.00001 & 0.00002 & 0.00000 & 0.00006 \\
\hline 477.67 & 0.00000 & 0.00006 & 0.00005 & 0.00001 & 0.00003 & 0.00000 & 0.00008 \\
\hline 478.03 & 0.00001 & 0.00010 & 0.00005 & 0.00001 & 0.00006 & 0.00001 & 0.00008 \\
\hline 478.38 & 0.00003 & 0.00012 & 0.00002 & 0.00001 & 0.00008 & 0.00001 & 0.00006 \\
\hline 478.73 & 0.00007 & 0.00012 & 0.00001 & 0.00001 & 0.00008 & 0.00001 & 0.00006 \\
\hline 479.09 & 0.00007 & 0.00010 & 0.00001 & 0.00001 & 0.00006 & 0.00001 & 0.00006 \\
\hline 479.44 & 0.00003 & 0.00008 & 0.00000 & 0.00001 & 0.00003 & 0.00001 & 0.00006 \\
\hline 479.79 & 0.00005 & 0.00005 & 0.00000 & 0.00001 & 0.00002 & 0.00003 & 0.00005 \\
\hline 480.15 & 0.00007 & 0.00004 & 0.00000 & 0.00002 & 0.00005 & 0.00003 & 0.00005 \\
\hline 480.5 & 0.00015 & 0.00004 & 0.00000 & 0.00001 & 0.00005 & 0.00004 & 0.00005 \\
\hline 480.85 & 0.00012 & 0.00004 & 0.00000 & 0.00001 & 0.00005 & 0.00006 & 0.00005 \\
\hline 481.21 & 0.00008 & 0.00002 & 0.00000 & 0.00001 & 0.00005 & 0.00006 & 0.00005 \\
\hline 481.56 & 0.00007 & 0.00002 & 0.00000 & 0.00001 & 0.00003 & 0.00006 & 0.00005 \\
\hline 481.91 & 0.00008 & 0.00002 & 0.00000 & 0.00001 & 0.00002 & 0.00007 & 0.00004 \\
\hline 482.27 & 0.00010 & 0.00001 & 0.00000 & 0.00001 & 0.00002 & 0.00009 & 0.00004 \\
\hline 482.62 & 0.00007 & 0.00000 & 0.00000 & 0.00000 & 0.00001 & 0.00009 & 0.00003 \\
\hline 482.97 & 0.00005 & 0.00002 & 0.00000 & 0.00000 & 0.00001 & 0.00011 & 0.00003 \\
\hline 483.33 & 0.00005 & 0.00002 & 0.00000 & 0.00000 & 0.00002 & 0.00011 & 0.00004 \\
\hline 483.68 & 0.00004 & 0.00002 & 0.00000 & 0.00000 & 0.00002 & 0.00011 & 0.00003 \\
\hline 484.03 & 0.00007 & 0.00002 & 0.00000 & 0.00000 & 0.00002 & 0.00014 & 0.00004 \\
\hline 484.38 & 0.00008 & 0.00001 & 0.00000 & 0.00000 & 0.00002 & 0.00019 & 0.00003 \\
\hline 484.74 & 0.00008 & 0.00001 & 0.00000 & 0.00000 & 0.00001 & 0.00021 & 0.00003 \\
\hline 485.09 & 0.00012 & 0.00002 & 0.00000 & 0.00000 & 0.00001 & 0.00021 & 0.00002 \\
\hline 485.44 & 0.00017 & 0.00002 & 0.00000 & 0.00000 & 0.00001 & 0.00024 & 0.00003 \\
\hline 485.8 & 0.00020 & 0.00000 & 0.00001 & 0.00000 & 0.00000 & 0.00028 & 0.00002 \\
\hline 486.15 & 0.00017 & 0.00000 & 0.00001 & 0.00000 & 0.00000 & 0.00031 & 0.00001 \\
\hline 486.5 & 0.00015 & 0.00000 & 0.00001 & 0.00000 & 0.00000 & 0.00035 & 0.00002 \\
\hline
\end{tabular}




\begin{tabular}{|c|c|c|c|c|c|c|c|}
\hline 486.85 & 0.00017 & 0.00000 & 0.00002 & 0.00000 & 0.00000 & 0.00035 & 0.00002 \\
\hline 487.21 & 0.00017 & 0.00000 & 0.00002 & 0.00000 & 0.00000 & 0.00039 & 0.00002 \\
\hline 487.56 & 0.00017 & 0.00001 & 0.00003 & 0.00000 & 0.00000 & 0.00039 & 0.00003 \\
\hline 487.91 & 0.00012 & 0.00000 & 0.00002 & 0.00001 & 0.00000 & 0.00043 & 0.00002 \\
\hline 488.26 & 0.00015 & 0.00000 & 0.00002 & 0.00001 & 0.00000 & 0.00043 & 0.00003 \\
\hline 488.62 & 0.00020 & 0.00000 & 0.00002 & 0.00003 & 0.00001 & 0.00047 & 0.00002 \\
\hline 488.97 & 0.00015 & 0.00002 & 0.00003 & 0.00003 & 0.00001 & 0.00047 & 0.00002 \\
\hline 489.32 & 0.00012 & 0.00002 & 0.00004 & 0.00003 & 0.00001 & 0.00056 & 0.00002 \\
\hline 489.67 & 0.00012 & 0.00003 & 0.00004 & 0.00002 & 0.00001 & 0.00056 & 0.00002 \\
\hline 490.02 & 0.00012 & 0.00005 & 0.00004 & 0.00002 & 0.00001 & 0.00056 & 0.00002 \\
\hline 490.38 & 0.00012 & 0.00006 & 0.00007 & 0.00002 & 0.00000 & 0.00061 & 0.00003 \\
\hline 490.73 & 0.00015 & 0.00010 & 0.00007 & 0.00005 & 0.00001 & 0.00066 & 0.00002 \\
\hline 491.08 & 0.00010 & 0.00010 & 0.00005 & 0.00005 & 0.00001 & 0.00071 & 0.00002 \\
\hline 491.43 & 0.00010 & 0.00010 & 0.00007 & 0.00003 & 0.00001 & 0.00071 & 0.00002 \\
\hline 491.79 & 0.00008 & 0.00008 & 0.00010 & 0.00002 & 0.00000 & 0.00071 & 0.00003 \\
\hline 492.14 & 0.00005 & 0.00008 & 0.00010 & 0.00003 & 0.00000 & 0.00071 & 0.00003 \\
\hline 492.49 & 0.00005 & 0.00008 & 0.00013 & 0.00005 & 0.00002 & 0.00076 & 0.00003 \\
\hline 492.84 & 0.00005 & 0.00006 & 0.00013 & 0.00005 & 0.00002 & 0.00082 & 0.00003 \\
\hline 493.19 & 0.00004 & 0.00006 & 0.00010 & 0.00006 & 0.00002 & 0.00082 & 0.00004 \\
\hline 493.54 & 0.00003 & 0.00005 & 0.00010 & 0.00006 & 0.00002 & 0.00088 & 0.00003 \\
\hline 493.9 & 0.00001 & 0.00004 & 0.00010 & 0.00005 & 0.00003 & 0.00082 & 0.00003 \\
\hline 494.25 & 0.00001 & 0.00006 & 0.00009 & 0.00005 & 0.00004 & 0.00088 & 0.00002 \\
\hline 494.6 & 0.00003 & 0.00003 & 0.00009 & 0.00005 & 0.00004 & 0.00088 & 0.00003 \\
\hline 494.95 & 0.00004 & 0.00002 & 0.00010 & 0.00005 & 0.00003 & 0.00088 & 0.00004 \\
\hline 495.3 & 0.00003 & 0.00004 & 0.00010 & 0.00008 & 0.00004 & 0.00088 & 0.00003 \\
\hline 495.65 & 0.00001 & 0.00004 & 0.00007 & 0.00009 & 0.00004 & 0.00088 & 0.00004 \\
\hline 496.01 & 0.00001 & 0.00003 & 0.00007 & 0.00006 & 0.00004 & 0.00094 & 0.00004 \\
\hline 496.36 & 0.00000 & 0.00001 & 0.00007 & 0.00005 & 0.00004 & 0.00094 & 0.00005 \\
\hline 496.71 & 0.00000 & 0.00001 & 0.00007 & 0.00005 & 0.00004 & 0.00094 & 0.00004 \\
\hline 497.06 & 0.00000 & 0.00000 & 0.00009 & 0.00003 & 0.00004 & 0.00100 & 0.00004 \\
\hline 497.41 & 0.00000 & 0.00000 & 0.00009 & 0.00003 & 0.00003 & 0.00094 & 0.00005 \\
\hline 497.76 & 0.00001 & 0.00000 & 0.00010 & 0.00006 & 0.00003 & 0.00100 & 0.00004 \\
\hline 498.11 & 0.00002 & 0.00000 & 0.00010 & 0.00008 & 0.00003 & 0.00100 & 0.00005 \\
\hline 498.47 & 0.00003 & 0.00001 & 0.00013 & 0.00008 & 0.00004 & 0.00107 & 0.00003 \\
\hline 498.82 & 0.00001 & 0.00000 & 0.00010 & 0.00006 & 0.00002 & 0.00107 & 0.00004 \\
\hline 499.17 & 0.00002 & 0.00000 & 0.00009 & 0.00006 & 0.00003 & 0.00107 & 0.00003 \\
\hline 499.52 & 0.00004 & 0.00000 & 0.00010 & 0.00005 & 0.00003 & 0.00100 & 0.00004 \\
\hline 499.87 & 0.00008 & 0.00000 & 0.00013 & 0.00006 & 0.00002 & 0.00100 & 0.00004 \\
\hline 500.22 & 0.00007 & 0.00001 & 0.00010 & 0.00006 & 0.00002 & 0.00094 & 0.00004 \\
\hline
\end{tabular}




\begin{tabular}{|c|c|c|c|c|c|c|c|}
\hline 500.57 & 0.00008 & 0.00001 & 0.00010 & 0.00008 & 0.00002 & 0.00094 & 0.00004 \\
\hline 500.92 & 0.00005 & 0.00000 & 0.00009 & 0.00006 & 0.00001 & 0.00088 & 0.00005 \\
\hline 501.27 & 0.00007 & 0.00000 & 0.00007 & 0.00005 & 0.00001 & 0.00088 & 0.00005 \\
\hline 501.62 & 0.00005 & 0.00000 & 0.00007 & 0.00005 & 0.00002 & 0.00082 & 0.00005 \\
\hline 501.97 & 0.00005 & 0.00000 & 0.00005 & 0.00005 & 0.00001 & 0.00071 & 0.00005 \\
\hline 502.33 & 0.00004 & 0.00000 & 0.00007 & 0.00005 & 0.00001 & 0.00076 & 0.00005 \\
\hline 502.68 & 0.00003 & 0.00000 & 0.00007 & 0.00003 & 0.00001 & 0.00076 & 0.00006 \\
\hline 503.03 & 0.00003 & 0.00000 & 0.00007 & 0.00003 & 0.00002 & 0.00076 & 0.00005 \\
\hline 503.38 & 0.00002 & 0.00000 & 0.00007 & 0.00005 & 0.00002 & 0.00088 & 0.00005 \\
\hline 503.73 & 0.00000 & 0.00002 & 0.00009 & 0.00005 & 0.00003 & 0.00088 & 0.00005 \\
\hline 504.08 & 0.00001 & 0.00002 & 0.00005 & 0.00003 & 0.00002 & 0.00082 & 0.00006 \\
\hline 504.43 & 0.00000 & 0.00003 & 0.00009 & 0.00005 & 0.00003 & 0.00088 & 0.00005 \\
\hline 504.78 & 0.00000 & 0.00003 & 0.00009 & 0.00005 & 0.00003 & 0.00082 & 0.00006 \\
\hline 505.13 & 0.00000 & 0.00002 & 0.00013 & 0.00005 & 0.00002 & 0.00082 & 0.00006 \\
\hline 505.48 & 0.00000 & 0.00002 & 0.00013 & 0.00005 & 0.00002 & 0.00088 & 0.00006 \\
\hline 505.83 & 0.00000 & 0.00002 & 0.00013 & 0.00002 & 0.00001 & 0.00082 & 0.00008 \\
\hline 506.18 & 0.00000 & 0.00003 & 0.00010 & 0.00002 & 0.00002 & 0.00088 & 0.00008 \\
\hline 506.53 & 0.00000 & 0.00002 & 0.00009 & 0.00001 & 0.00001 & 0.00076 & 0.00012 \\
\hline 506.88 & 0.00001 & 0.00000 & 0.00009 & 0.00001 & 0.00000 & 0.00071 & 0.00014 \\
\hline 507.23 & 0.00001 & 0.00000 & 0.00007 & 0.00001 & 0.00001 & 0.00071 & 0.00014 \\
\hline 507.58 & 0.00002 & 0.00000 & 0.00009 & 0.00001 & 0.00001 & 0.00071 & 0.00012 \\
\hline 507.93 & 0.00004 & 0.00000 & 0.00010 & 0.00001 & 0.00002 & 0.00076 & 0.00012 \\
\hline 508.28 & 0.00002 & 0.00001 & 0.00009 & 0.00001 & 0.00002 & 0.00071 & 0.00012 \\
\hline 508.63 & 0.00004 & 0.00000 & 0.00010 & 0.00001 & 0.00002 & 0.00066 & 0.00012 \\
\hline 508.98 & 0.00002 & 0.00000 & 0.00015 & 0.00002 & 0.00004 & 0.00071 & 0.00012 \\
\hline 509.33 & 0.00002 & 0.00000 & 0.00015 & 0.00002 & 0.00004 & 0.00071 & 0.00012 \\
\hline 509.68 & 0.00005 & 0.00000 & 0.00015 & 0.00003 & 0.00004 & 0.00071 & 0.00012 \\
\hline 510.03 & 0.00005 & 0.00000 & 0.00015 & 0.00005 & 0.00005 & 0.00071 & 0.00012 \\
\hline 510.38 & 0.00005 & 0.00000 & 0.00013 & 0.00003 & 0.00003 & 0.00066 & 0.00012 \\
\hline 510.73 & 0.00008 & 0.00000 & 0.00015 & 0.00005 & 0.00004 & 0.00066 & 0.00012 \\
\hline 511.08 & 0.00008 & 0.00000 & 0.00015 & 0.00003 & 0.00002 & 0.00066 & 0.00012 \\
\hline 511.43 & 0.00014 & 0.00001 & 0.00013 & 0.00003 & 0.00001 & 0.00056 & 0.00017 \\
\hline 511.78 & 0.00014 & 0.00001 & 0.00010 & 0.00001 & 0.00000 & 0.00051 & 0.00020 \\
\hline 512.13 & 0.00017 & 0.00000 & 0.00010 & 0.00002 & 0.00000 & 0.00051 & 0.00023 \\
\hline 512.48 & 0.00019 & 0.00000 & 0.00010 & 0.00003 & 0.00000 & 0.00051 & 0.00026 \\
\hline 512.83 & 0.00022 & 0.00000 & 0.00009 & 0.00002 & 0.00000 & 0.00051 & 0.00023 \\
\hline 513.18 & 0.00025 & 0.00000 & 0.00007 & 0.00002 & 0.00001 & 0.00051 & 0.00026 \\
\hline 513.53 & 0.00029 & 0.00000 & 0.00007 & 0.00002 & 0.00001 & 0.00051 & 0.00026 \\
\hline 513.88 & 0.00025 & 0.00000 & 0.00007 & 0.00002 & 0.00002 & 0.00051 & 0.00026 \\
\hline
\end{tabular}




\begin{tabular}{|c|c|c|c|c|c|c|c|}
\hline 514.22 & 0.00019 & 0.00000 & 0.00009 & 0.00005 & 0.00004 & 0.00051 & 0.00023 \\
\hline 514.57 & 0.00017 & 0.00000 & 0.00009 & 0.00005 & 0.00004 & 0.00056 & 0.00023 \\
\hline 514.92 & 0.00019 & 0.00001 & 0.00009 & 0.00005 & 0.00005 & 0.00056 & 0.00023 \\
\hline 515.27 & 0.00012 & 0.00003 & 0.00010 & 0.00006 & 0.00007 & 0.00066 & 0.00020 \\
\hline 515.62 & 0.00010 & 0.00001 & 0.00010 & 0.00008 & 0.00008 & 0.00071 & 0.00017 \\
\hline 515.97 & 0.00010 & 0.00002 & 0.00009 & 0.00006 & 0.00008 & 0.00066 & 0.00017 \\
\hline 516.32 & 0.00006 & 0.00000 & 0.00013 & 0.00008 & 0.00010 & 0.00071 & 0.00014 \\
\hline 516.67 & 0.00010 & 0.00000 & 0.00009 & 0.00006 & 0.00008 & 0.00061 & 0.00017 \\
\hline 517.02 & 0.00010 & 0.00000 & 0.00010 & 0.00006 & 0.00007 & 0.00061 & 0.00017 \\
\hline 517.37 & 0.00006 & 0.00000 & 0.00010 & 0.00005 & 0.00008 & 0.00061 & 0.00017 \\
\hline 517.72 & 0.00004 & 0.00000 & 0.00010 & 0.00005 & 0.00008 & 0.00066 & 0.00014 \\
\hline 518.06 & 0.00005 & 0.00000 & 0.00010 & 0.00005 & 0.00010 & 0.00061 & 0.00014 \\
\hline 518.41 & 0.00005 & 0.00000 & 0.00010 & 0.00005 & 0.00010 & 0.00061 & 0.00017 \\
\hline 518.76 & 0.00004 & 0.00000 & 0.00009 & 0.00003 & 0.00010 & 0.00056 & 0.00017 \\
\hline 519.11 & 0.00002 & 0.00001 & 0.00013 & 0.00003 & 0.00012 & 0.00056 & 0.00014 \\
\hline 519.46 & 0.00002 & 0.00002 & 0.00013 & 0.00003 & 0.00012 & 0.00056 & 0.00017 \\
\hline 519.81 & 0.00001 & 0.00003 & 0.00018 & 0.00003 & 0.00015 & 0.00061 & 0.00014 \\
\hline 520.16 & 0.00000 & 0.00003 & 0.00013 & 0.00005 & 0.00017 & 0.00056 & 0.00014 \\
\hline 520.5 & 0.00000 & 0.00002 & 0.00015 & 0.00003 & 0.00017 & 0.00056 & 0.00014 \\
\hline 520.85 & 0.00000 & 0.00003 & 0.00018 & 0.00005 & 0.00020 & 0.00056 & 0.00014 \\
\hline 521.2 & 0.00000 & 0.00003 & 0.00020 & 0.00006 & 0.00023 & 0.00061 & 0.00014 \\
\hline 521.55 & 0.00002 & 0.00002 & 0.00023 & 0.00005 & 0.00023 & 0.00056 & 0.00014 \\
\hline 521.9 & 0.00002 & 0.00002 & 0.00023 & 0.00005 & 0.00023 & 0.00051 & 0.00017 \\
\hline 522.25 & 0.00002 & 0.00000 & 0.00023 & 0.00006 & 0.00023 & 0.00051 & 0.00017 \\
\hline 522.59 & 0.00001 & 0.00000 & 0.00026 & 0.00009 & 0.00026 & 0.00051 & 0.00017 \\
\hline 522.94 & 0.00001 & 0.00000 & 0.00023 & 0.00009 & 0.00026 & 0.00051 & 0.00017 \\
\hline 523.29 & 0.00002 & 0.00000 & 0.00023 & 0.00009 & 0.00026 & 0.00047 & 0.00020 \\
\hline 523.64 & 0.00002 & 0.00000 & 0.00020 & 0.00009 & 0.00026 & 0.00047 & 0.00020 \\
\hline 523.99 & 0.00001 & 0.00001 & 0.00023 & 0.00011 & 0.00026 & 0.00047 & 0.00017 \\
\hline 524.33 & 0.00002 & 0.00000 & 0.00020 & 0.00009 & 0.00026 & 0.00047 & 0.00020 \\
\hline 524.68 & 0.00005 & 0.00001 & 0.00020 & 0.00009 & 0.00023 & 0.00043 & 0.00020 \\
\hline 525.03 & 0.00005 & 0.00001 & 0.00020 & 0.00014 & 0.00026 & 0.00051 & 0.00017 \\
\hline 525.38 & 0.00005 & 0.00002 & 0.00020 & 0.00011 & 0.00026 & 0.00047 & 0.00020 \\
\hline 525.73 & 0.00004 & 0.00002 & 0.00020 & 0.00011 & 0.00026 & 0.00047 & 0.00023 \\
\hline 526.07 & 0.00005 & 0.00002 & 0.00020 & 0.00009 & 0.00026 & 0.00047 & 0.00020 \\
\hline 526.42 & 0.00004 & 0.00001 & 0.00020 & 0.00009 & 0.00029 & 0.00051 & 0.00017 \\
\hline 526.77 & 0.00004 & 0.00002 & 0.00020 & 0.00008 & 0.00023 & 0.00043 & 0.00023 \\
\hline 527.12 & 0.00005 & 0.00002 & 0.00018 & 0.00005 & 0.00023 & 0.00039 & 0.00023 \\
\hline 527.46 & 0.00005 & 0.00001 & 0.00018 & 0.00008 & 0.00029 & 0.00039 & 0.00023 \\
\hline
\end{tabular}




\begin{tabular}{|c|c|c|c|c|c|c|c|}
\hline 527.81 & 0.00006 & 0.00001 & 0.00018 & 0.00006 & 0.00029 & 0.00039 & 0.00023 \\
\hline 528.16 & 0.00010 & 0.00001 & 0.00015 & 0.00006 & 0.00029 & 0.00039 & 0.00026 \\
\hline 528.51 & 0.00006 & 0.00000 & 0.00015 & 0.00006 & 0.00029 & 0.00039 & 0.00026 \\
\hline 528.85 & 0.00002 & 0.00000 & 0.00013 & 0.00006 & 0.00023 & 0.00035 & 0.00029 \\
\hline 529.2 & 0.00002 & 0.00000 & 0.00010 & 0.00005 & 0.00020 & 0.00035 & 0.00029 \\
\hline 529.55 & 0.00006 & 0.00000 & 0.00009 & 0.00005 & 0.00020 & 0.00035 & 0.00029 \\
\hline 529.9 & 0.00010 & 0.00000 & 0.00009 & 0.00005 & 0.00020 & 0.00031 & 0.00029 \\
\hline 530.24 & 0.00017 & 0.00000 & 0.00009 & 0.00003 & 0.00017 & 0.00028 & 0.00032 \\
\hline 530.59 & 0.00022 & 0.00000 & 0.00005 & 0.00003 & 0.00017 & 0.00031 & 0.00032 \\
\hline 530.94 & 0.00017 & 0.00001 & 0.00007 & 0.00005 & 0.00017 & 0.00035 & 0.00032 \\
\hline 531.29 & 0.00022 & 0.00001 & 0.00007 & 0.00003 & 0.00017 & 0.00031 & 0.00032 \\
\hline 531.63 & 0.00022 & 0.00001 & 0.00007 & 0.00003 & 0.00017 & 0.00035 & 0.00029 \\
\hline 531.98 & 0.00022 & 0.00001 & 0.00009 & 0.00005 & 0.00015 & 0.00035 & 0.00026 \\
\hline 532.33 & 0.00025 & 0.00001 & 0.00007 & 0.00002 & 0.00015 & 0.00031 & 0.00029 \\
\hline 532.67 & 0.00025 & 0.00001 & 0.00007 & 0.00002 & 0.00017 & 0.00031 & 0.00026 \\
\hline 533.02 & 0.00029 & 0.00001 & 0.00005 & 0.00002 & 0.00017 & 0.00028 & 0.00026 \\
\hline 533.37 & 0.00022 & 0.00002 & 0.00007 & 0.00002 & 0.00017 & 0.00028 & 0.00023 \\
\hline 533.71 & 0.00014 & 0.00001 & 0.00009 & 0.00005 & 0.00023 & 0.00031 & 0.00020 \\
\hline 534.06 & 0.00008 & 0.00002 & 0.00010 & 0.00006 & 0.00026 & 0.00031 & 0.00020 \\
\hline 534.41 & 0.00005 & 0.00002 & 0.00013 & 0.00008 & 0.00026 & 0.00031 & 0.00020 \\
\hline 534.75 & 0.00008 & 0.00001 & 0.00013 & 0.00008 & 0.00029 & 0.00031 & 0.00017 \\
\hline 535.1 & 0.00005 & 0.00001 & 0.00015 & 0.00009 & 0.00029 & 0.00031 & 0.00020 \\
\hline 535.45 & 0.00006 & 0.00003 & 0.00015 & 0.00011 & 0.00033 & 0.00031 & 0.00017 \\
\hline 535.79 & 0.00005 & 0.00002 & 0.00015 & 0.00014 & 0.00033 & 0.00031 & 0.00017 \\
\hline 536.14 & 0.00004 & 0.00003 & 0.00018 & 0.00016 & 0.00033 & 0.00035 & 0.00020 \\
\hline 536.49 & 0.00008 & 0.00003 & 0.00015 & 0.00016 & 0.00029 & 0.00031 & 0.00023 \\
\hline 536.83 & 0.00006 & 0.00002 & 0.00018 & 0.00019 & 0.00033 & 0.00035 & 0.00023 \\
\hline 537.18 & 0.00008 & 0.00001 & 0.00020 & 0.00019 & 0.00033 & 0.00031 & 0.00023 \\
\hline 537.53 & 0.00010 & 0.00001 & 0.00018 & 0.00016 & 0.00029 & 0.00028 & 0.00026 \\
\hline 537.87 & 0.00017 & 0.00001 & 0.00015 & 0.00019 & 0.00029 & 0.00028 & 0.00026 \\
\hline 538.22 & 0.00022 & 0.00000 & 0.00015 & 0.00016 & 0.00026 & 0.00021 & 0.00029 \\
\hline 538.57 & 0.00019 & 0.00001 & 0.00018 & 0.00016 & 0.00026 & 0.00024 & 0.00029 \\
\hline 538.91 & 0.00029 & 0.00000 & 0.00015 & 0.00014 & 0.00020 & 0.00021 & 0.00029 \\
\hline 539.26 & 0.00025 & 0.00001 & 0.00018 & 0.00011 & 0.00020 & 0.00021 & 0.00029 \\
\hline 539.6 & 0.00025 & 0.00004 & 0.00020 & 0.00019 & 0.00026 & 0.00021 & 0.00026 \\
\hline 539.95 & 0.00029 & 0.00001 & 0.00020 & 0.00016 & 0.00023 & 0.00016 & 0.00026 \\
\hline 540.3 & 0.00025 & 0.00002 & 0.00020 & 0.00016 & 0.00023 & 0.00019 & 0.00026 \\
\hline 540.64 & 0.00032 & 0.00004 & 0.00020 & 0.00016 & 0.00023 & 0.00016 & 0.00023 \\
\hline 540.99 & 0.00029 & 0.00006 & 0.00020 & 0.00019 & 0.00020 & 0.00016 & 0.00023 \\
\hline
\end{tabular}




\begin{tabular}{|c|c|c|c|c|c|c|c|}
\hline 541.33 & 0.00025 & 0.00008 & 0.00015 & 0.00019 & 0.00017 & 0.00019 & 0.00020 \\
\hline 541.68 & 0.00017 & 0.00006 & 0.00018 & 0.00019 & 0.00017 & 0.00019 & 0.00020 \\
\hline 542.02 & 0.00010 & 0.00006 & 0.00018 & 0.00016 & 0.00017 & 0.00019 & 0.00020 \\
\hline 542.37 & 0.00010 & 0.00010 & 0.00015 & 0.00014 & 0.00017 & 0.00021 & 0.00017 \\
\hline 542.72 & 0.00006 & 0.00010 & 0.00015 & 0.00016 & 0.00020 & 0.00021 & 0.00020 \\
\hline 543.06 & 0.00004 & 0.00010 & 0.00015 & 0.00019 & 0.00020 & 0.00024 & 0.00017 \\
\hline 543.41 & 0.00002 & 0.00008 & 0.00015 & 0.00016 & 0.00023 & 0.00024 & 0.00017 \\
\hline 543.75 & 0.00002 & 0.00006 & 0.00015 & 0.00016 & 0.00023 & 0.00024 & 0.00017 \\
\hline 544.1 & 0.00002 & 0.00006 & 0.00013 & 0.00016 & 0.00023 & 0.00021 & 0.00017 \\
\hline 544.44 & 0.00002 & 0.00004 & 0.00013 & 0.00014 & 0.00023 & 0.00021 & 0.00017 \\
\hline 544.79 & 0.00002 & 0.00003 & 0.00013 & 0.00011 & 0.00023 & 0.00021 & 0.00020 \\
\hline 545.13 & 0.00002 & 0.00005 & 0.00018 & 0.00016 & 0.00029 & 0.00021 & 0.00017 \\
\hline 545.48 & 0.00005 & 0.00004 & 0.00015 & 0.00019 & 0.00033 & 0.00021 & 0.00017 \\
\hline 545.82 & 0.00005 & 0.00003 & 0.00015 & 0.00019 & 0.00029 & 0.00021 & 0.00020 \\
\hline 546.17 & 0.00006 & 0.00002 & 0.00018 & 0.00022 & 0.00033 & 0.00019 & 0.00017 \\
\hline 546.52 & 0.00005 & 0.00003 & 0.00015 & 0.00022 & 0.00033 & 0.00019 & 0.00014 \\
\hline 546.86 & 0.00004 & 0.00004 & 0.00015 & 0.00022 & 0.00033 & 0.00019 & 0.00014 \\
\hline 547.21 & 0.00002 & 0.00005 & 0.00015 & 0.00022 & 0.00033 & 0.00016 & 0.00014 \\
\hline 547.55 & 44 & 0.0 & 0.00010 & 0.00022 & 0.00033 & 0.00016 & 0.00014 \\
\hline 54 & 0 & 0 & 3 & 22 & 29 & 6 & 0.00014 \\
\hline 548.24 & 02 & 0. & 13 & 0.00025 & 0.00029 & 0.00016 & 0.00012 \\
\hline 548.59 & 0.00002 & 0.00008 & 0.00015 & 0.00025 & 0.00029 & 0.00016 & 0.00010 \\
\hline 548.93 & 0.00002 & 0.00008 & 0.00020 & 0.00022 & 0.00029 & 0.00019 & 0.00010 \\
\hline 549.28 & 0.00002 & 0.00008 & 0.00026 & 0.00022 & 0.00029 & 0.00019 & 0.00010 \\
\hline 549.62 & 0.00002 & 0.00008 & 0.00026 & 0.00022 & 0.00029 & 0.00019 & 0.00010 \\
\hline 549.96 & 0.00000 & 0.00006 & 0.00026 & 0.00022 & 0.00029 & 0.00019 & 0.00010 \\
\hline 550.31 & 0.00001 & 0.00006 & 0.00023 & 0.00022 & 0.00029 & 0.00016 & 0.00012 \\
\hline 550.65 & 0.00002 & 0.00005 & 0.00030 & 0.00025 & 0.00029 & 0.00019 & 0.00010 \\
\hline 551 & 0.00002 & 0.00006 & 033 & 0.00025 & 0.00026 & 0.00019 & 0.00010 \\
\hline 551.34 & 0.00001 & 0.00005 & 030 & 0.00022 & 0.00023 & 0.00019 & 0.00012 \\
\hline 551.69 & 0.00000 & 0.00004 & 0.00033 & 0.00025 & 0.00023 & 0.00021 & 0.00012 \\
\hline 552.03 & 0.00001 & 0.00003 & 0.00030 & 0.00025 & 0.00026 & 0.00021 & 0.00010 \\
\hline 552.38 & 0.00001 & 0.00004 & 0.00026 & 0.00025 & 0.00026 & 0.00019 & 0.00012 \\
\hline 552.72 & 0.00002 & 0.00004 & 0.00023 & 0.00025 & 0.00026 & 0.00019 & 0.00012 \\
\hline 553.07 & 0.00000 & 0.00004 & 0.00023 & 0.00028 & 0.00026 & 0.00021 & 0.00008 \\
\hline 553.41 & 0.00000 & 0.00004 & 0.00023 & 0.00025 & 0.00026 & 0.00019 & 0.00008 \\
\hline 553.75 & 0.00002 & 0.00003 & 0.00018 & 0.00022 & 0.00023 & 0.00019 & 0.00008 \\
\hline 554.1 & 0.00004 & 0.00002 & 0.00023 & 0.00022 & 0.00023 & 0.00019 & 0.00008 \\
\hline 554.44 & 0.00005 & 0.00003 & 0.00018 & 0.00019 & 0.00023 & 0.00014 & 0.00008 \\
\hline
\end{tabular}




\begin{tabular}{|c|c|c|c|c|c|c|c|}
\hline 554.79 & 0.00002 & 0.00003 & 0.00013 & 0.00019 & 0.00023 & 0.00014 & 0.00006 \\
\hline 555.13 & 0.00002 & 0.00003 & 0.00018 & 0.00016 & 0.00026 & 0.00014 & 0.00005 \\
\hline 555.47 & 0.00002 & 0.00003 & 0.00018 & 0.00019 & 0.00033 & 0.00014 & 0.00004 \\
\hline 555.82 & 0.00001 & 0.00006 & 0.00018 & 0.00019 & 0.00029 & 0.00014 & 0.00004 \\
\hline 556.16 & 0.00001 & 0.00008 & 0.00020 & 0.00022 & 0.00029 & 0.00014 & 0.00004 \\
\hline 556.51 & 0.00000 & 0.00006 & 0.00020 & 0.00022 & 0.00029 & 0.00014 & 0.00003 \\
\hline 556.85 & 0.00000 & 0.00012 & 0.00020 & 0.00022 & 0.00029 & 0.00014 & 0.00003 \\
\hline 557.19 & 0.00000 & 0.00012 & 0.00018 & 0.00025 & 0.00029 & 0.00011 & 0.00002 \\
\hline 557.54 & 0.00000 & 0.00014 & 0.00023 & 0.00025 & 0.00029 & 0.00014 & 0.00002 \\
\hline 557.88 & 0.00000 & 0.00017 & 0.00023 & 0.00025 & 0.00029 & 0.00014 & 0.00002 \\
\hline 558.22 & 0.00000 & 0.00017 & 0.00023 & 0.00025 & 0.00033 & 0.00014 & 0.00001 \\
\hline 558.57 & 0.00002 & 0.00012 & 0.00026 & 0.00025 & 0.00029 & 0.00014 & 0.00001 \\
\hline 558.91 & 0.00004 & 0.00008 & 0.00020 & 0.00022 & 0.00029 & 0.00011 & 0.00002 \\
\hline 559.26 & 0.00008 & 0.00008 & 0.00018 & 0.00019 & 0.00026 & 0.00014 & 0.00002 \\
\hline 559.6 & 0.00008 & 0.00004 & 0.00013 & 0.00016 & 0.00023 & 0.00011 & 0.00003 \\
\hline 559.94 & 0.00008 & 0.00003 & 0.00015 & 0.00016 & 0.00023 & 0.00011 & 0.00003 \\
\hline 560.29 & 0.00010 & 0.00004 & 0.00013 & 0.00014 & 0.00020 & 0.00014 & 0.00003 \\
\hline 560.63 & 0.00006 & 0.00004 & 0.00009 & 0.00011 & 0.00017 & 0.00014 & 0.00002 \\
\hline 560.97 & 0.00005 & 0.00003 & 0.00009 & 0.00011 & 0.00017 & 0.00016 & 0.00002 \\
\hline 561.32 & 0.00002 & 0.00002 & 0.00010 & 0.00011 & 0.00017 & 0.00016 & 0.00002 \\
\hline 561.66 & 0.00002 & 0.00001 & 0.00009 & 0.00011 & 0.00017 & 0.00016 & 0.00002 \\
\hline 562 & 0.00000 & 0.00003 & 0.00009 & 0.00014 & 0.00017 & 0.00019 & 0.00002 \\
\hline 562.34 & 0.00000 & 0.00004 & 0.00009 & 0.00014 & 0.00020 & 0.00021 & 0.00001 \\
\hline 562.69 & 0.00001 & 0.00008 & 0.00009 & 0.00016 & 0.00020 & 0.00024 & 0.00000 \\
\hline 563.03 & 0.00003 & 0.00008 & 0.00009 & 0.00014 & 0.00020 & 0.00021 & 0.00000 \\
\hline 563.37 & 0.00003 & 0.00010 & 0.00015 & 0.00019 & 0.00023 & 0.00024 & 0.00000 \\
\hline 563.72 & 0.00003 & 0.00010 & 0.00013 & 0.00014 & 0.00017 & 0.00024 & 0.00000 \\
\hline 564.06 & 0.00002 & 0.00010 & 0.00013 & 0.00014 & 0.00020 & 0.00021 & 0.00000 \\
\hline 564.4 & 0.00003 & 0.00010 & 0.00015 & 0.00016 & 0.00023 & 0.00021 & 0.00000 \\
\hline 564.75 & 0.00002 & 0.00010 & 0.00013 & 0.00016 & 0.00023 & 0.00021 & 0.00000 \\
\hline 565.09 & 0.00001 & 0.00012 & 0.00015 & 0.00014 & 0.00020 & 0.00021 & 0.00000 \\
\hline 565.43 & 0.00001 & 0.00012 & 0.00015 & 0.00011 & 0.00020 & 0.00024 & 0.00000 \\
\hline 565.77 & 0.00001 & 0.00008 & 0.00018 & 0.00009 & 0.00020 & 0.00024 & 0.00000 \\
\hline 566.12 & 0.00002 & 0.00008 & 0.00015 & 0.00008 & 0.00017 & 0.00024 & 0.00000 \\
\hline 566.46 & 0.00000 & 0.00004 & 0.00018 & 0.00006 & 0.00012 & 0.00019 & 0.00000 \\
\hline 566.8 & 0.00003 & 0.00005 & 0.00018 & 0.00006 & 0.00015 & 0.00021 & 0.00000 \\
\hline 567.14 & 0.00004 & 0.00004 & 0.00015 & 0.00006 & 0.00015 & 0.00021 & 0.00000 \\
\hline 567.49 & 0.00005 & 0.00005 & 0.00020 & 0.00008 & 0.00017 & 0.00024 & 0.00000 \\
\hline 567.83 & 0.00010 & 0.00005 & 0.00026 & 0.00008 & 0.00017 & 0.00028 & 0.00000 \\
\hline
\end{tabular}




\begin{tabular}{|c|c|c|c|c|c|c|c|}
\hline 568.17 & 0.00007 & 0.00005 & 0.00026 & 0.00008 & 0.00015 & 0.00028 & 0.00000 \\
\hline 568.51 & 0.00012 & 0.00004 & 0.00026 & 0.00006 & 0.00012 & 0.00028 & 0.00000 \\
\hline 568.85 & 0.00012 & 0.00002 & 0.00020 & 0.00008 & 0.00010 & 0.00028 & 0.00000 \\
\hline 569.2 & 0.00007 & 0.00003 & 0.00015 & 0.00008 & 0.00010 & 0.00021 & 0.00000 \\
\hline 569.54 & 0.00008 & 0.00005 & 0.00010 & 0.00008 & 0.00012 & 0.00024 & 0.00000 \\
\hline 569.88 & 0.00005 & 0.00006 & 0.00010 & 0.00008 & 0.00012 & 0.00024 & 0.00000 \\
\hline 570.22 & 0.00008 & 0.00008 & 0.00007 & 0.00008 & 0.00012 & 0.00024 & 0.00000 \\
\hline 570.57 & 0.00007 & 0.00006 & 0.00007 & 0.00008 & 0.00010 & 0.00028 & 0.00000 \\
\hline 570.91 & 0.00008 & 0.00008 & 0.00009 & 0.00009 & 0.00012 & 0.00031 & 0.00000 \\
\hline 571.25 & 0.00008 & 0.00005 & 0.00004 & 0.00008 & 0.00010 & 0.00031 & 0.00000 \\
\hline 571.59 & 0.00005 & 0.00003 & 0.00004 & 0.00008 & 0.00010 & 0.00031 & 0.00000 \\
\hline 571.93 & 0.00004 & 0.00002 & 0.00004 & 0.00006 & 0.00010 & 0.00031 & 0.00000 \\
\hline 572.27 & 0.00003 & 0.00002 & 0.00002 & 0.00005 & 0.00010 & 0.00035 & 0.00001 \\
\hline 572.62 & 0.00003 & 0.00004 & 0.00004 & 0.00006 & 0.00015 & 0.00039 & 0.00001 \\
\hline 572.96 & 0.00010 & 0.00004 & 0.00004 & 0.00008 & 0.00015 & 0.00043 & 0.00002 \\
\hline 573.3 & 0.00010 & 0.00005 & 0.00005 & 0.00008 & 0.00012 & 0.00039 & 0.00002 \\
\hline 573.64 & 0.00015 & 0.00008 & 0.00007 & 0.00008 & 0.00015 & 0.00043 & 0.00002 \\
\hline 573.98 & 0.00012 & 0.00012 & 0.00009 & 0.00011 & 0.00017 & 0.00047 & 0.00004 \\
\hline 574.32 & 0.00007 & 0.00008 & 0.00007 & 0.00011 & 0.00020 & 0.00047 & 0.00004 \\
\hline 574.67 & 0.00005 & 0.00012 & 0.00007 & 0.00011 & 0.00020 & 0.00047 & 0.00004 \\
\hline 575.01 & 0.00003 & 0.00012 & 0.00005 & 0.00009 & 0.00015 & 0.00043 & 0.00004 \\
\hline 575.35 & 0.00004 & 0.00020 & 0.00005 & 0.00011 & 0.00015 & 0.00047 & 0.00004 \\
\hline 575.69 & 0.00010 & 0.00020 & 0.00005 & 0.00014 & 0.00015 & 0.00047 & 0.00005 \\
\hline 576.03 & 0.00008 & 0.00029 & 0.00009 & 0.00016 & 0.00017 & 0.00047 & 0.00006 \\
\hline 576.37 & 0.00007 & 0.00036 & 0.00007 & 0.00011 & 0.00012 & 0.00051 & 0.00006 \\
\hline 576.71 & 0.00004 & 0.00033 & 0.00009 & 0.00009 & 0.00015 & 0.00051 & 0.00008 \\
\hline 577.05 & 0.00004 & 0.00026 & 0.00010 & 0.00009 & 0.00012 & 0.00056 & 0.00008 \\
\hline 577.4 & 0.00002 & 0.00023 & 0.00009 & 0.00009 & 0.00012 & 0.00051 & 0.00008 \\
\hline 577.74 & 0.00004 & 0.00017 & 0.00009 & 0.00008 & 0.00012 & 0.00051 & 0.00008 \\
\hline 578.08 & 0.00007 & 0.00026 & 0.00009 & 0.00008 & 0.00008 & 0.00051 & 0.00008 \\
\hline 578.42 & 0.00005 & 0.00026 & 0.00009 & 0.00008 & 0.00008 & 0.00051 & 0.00010 \\
\hline 578.76 & 0.00008 & 0.00023 & 0.00010 & 0.00008 & 0.00010 & 0.00056 & 0.00010 \\
\hline 579.1 & 0.00007 & 0.00023 & 0.00013 & 0.00006 & 0.00008 & 0.00056 & 0.00010 \\
\hline 579.44 & 0.00002 & 0.00026 & 0.00015 & 0.00006 & 0.00010 & 0.00051 & 0.00010 \\
\hline 579.78 & 0.00003 & 0.00020 & 0.00015 & 0.00006 & 0.00008 & 0.00056 & 0.00010 \\
\hline 580.12 & 0.00003 & 0.00012 & 0.00015 & 0.00006 & 0.00010 & 0.00051 & 0.00010 \\
\hline 580.46 & 0.00008 & 0.00017 & 0.00015 & 0.00009 & 0.00015 & 0.00056 & 0.00012 \\
\hline 580.8 & 0.00008 & 0.00014 & 0.00020 & 0.00008 & 0.00012 & 0.00051 & 0.00010 \\
\hline 581.14 & 0.00015 & 0.00012 & 0.00020 & 0.00008 & 0.00010 & 0.00051 & 0.00010 \\
\hline
\end{tabular}




\begin{tabular}{|c|c|c|c|c|c|c|c|}
\hline 581.49 & 0.00010 & 0.00020 & 0.00020 & 0.00008 & 0.00008 & 0.00051 & 0.00008 \\
\hline 581.83 & 0.00010 & 0.00014 & 0.00020 & 0.00006 & 0.00008 & 0.00047 & 0.00008 \\
\hline 582.17 & 0.00017 & 0.00020 & 0.00018 & 0.00008 & 0.00008 & 0.00051 & 0.00010 \\
\hline 582.51 & 0.00015 & 0.00017 & 0.00015 & 0.00009 & 0.00010 & 0.00056 & 0.00010 \\
\hline 582.85 & 0.00023 & 0.00020 & 0.00018 & 0.00014 & 0.00015 & 0.00056 & 0.00014 \\
\hline 583.19 & 0.00026 & 0.00023 & 0.00013 & 0.00014 & 0.00015 & 0.00056 & 0.00012 \\
\hline 583.53 & 0.00026 & 0.00029 & 0.00013 & 0.00016 & 0.00017 & 0.00056 & 0.00014 \\
\hline 583.87 & 0.00040 & 0.00026 & 0.00013 & 0.00019 & 0.00020 & 0.00066 & 0.00014 \\
\hline 584.21 & 0.00044 & 0.00026 & 0.00020 & 0.00019 & 0.00020 & 0.00066 & 0.00017 \\
\hline 584.55 & 0.00053 & 0.00040 & 0.00018 & 0.00022 & 0.00020 & 0.00076 & 0.00017 \\
\hline 584.89 & 0.00040 & 0.00036 & 0.00018 & 0.00022 & 0.00017 & 0.00071 & 0.00017 \\
\hline 585.23 & 0.00063 & 0.00029 & 0.00020 & 0.00022 & 0.00020 & 0.00071 & 0.00020 \\
\hline 585.57 & 0.00068 & 0.00029 & 0.00020 & 0.00025 & 0.00026 & 0.00076 & 0.00022 \\
\hline 585.91 & 0.00079 & 0.00026 & 0.00023 & 0.00022 & 0.00023 & 0.00076 & 0.00022 \\
\hline 586.25 & 0.00085 & 0.00033 & 0.00026 & 0.00025 & 0.00023 & 0.00076 & 0.00022 \\
\hline 586.59 & 0.00109 & 0.00036 & 0.00026 & 0.00025 & 0.00023 & 0.00076 & 0.00026 \\
\hline 586.93 & 0.00109 & 0.00029 & 0.00026 & 0.00025 & 0.00023 & 0.00082 & 0.00026 \\
\hline 587.27 & 0.00109 & 0.00029 & 0.00026 & 0.00019 & 0.00020 & 0.00082 & 0.00020 \\
\hline 587.61 & 0.00090 & 0.00033 & 0.00026 & 0.00019 & 0.00017 & 0.00076 & 0.00020 \\
\hline 587.95 & 0.00097 & 0.00029 & 0.00026 & 0.00019 & 0.00015 & 0.00076 & 0.00020 \\
\hline 588.29 & 0.00079 & 0.00026 & 0.00023 & 0.00016 & 0.00017 & 0.00076 & 0.00017 \\
\hline 588.63 & 0.00097 & 0.00029 & 0.00026 & 0.00016 & 0.00020 & 0.00082 & 0.00017 \\
\hline 588.97 & 0.00073 & 0.00033 & 0.00023 & 0.00019 & 0.00020 & 0.00088 & 0.00017 \\
\hline 589.3 & 0.00068 & 0.00036 & 0.00026 & 0.00016 & 0.00023 & 0.00094 & 0.00020 \\
\hline 589.64 & 0.00073 & 0.00036 & 0.00033 & 0.00019 & 0.00023 & 0.00094 & 0.00020 \\
\hline 589.98 & 0.00079 & 0.00033 & 0.00030 & 0.00014 & 0.00023 & 0.00082 & 0.00017 \\
\hline 590.32 & 0.00068 & 0.00036 & 0.00033 & 0.00016 & 0.00026 & 0.00088 & 0.00017 \\
\hline 590.66 & 0.00053 & 0.00036 & 0.00033 & 0.00014 & 0.00023 & 0.00082 & 0.00017 \\
\hline 591 & 0.00044 & 0.00040 & 0.00033 & 0.00016 & 0.00023 & 0.00082 & 0.00017 \\
\hline 591.34 & 0.00053 & 0.00049 & 0.00030 & 0.00014 & 0.00026 & 0.00082 & 0.00022 \\
\hline 591.68 & 0.00053 & 0.00049 & 0.00026 & 0.00014 & 0.00029 & 0.00076 & 0.00022 \\
\hline 592.02 & 0.00058 & 0.00053 & 0.00030 & 0.00019 & 0.00026 & 0.00082 & 0.00022 \\
\hline 592.36 & 0.00044 & 0.00049 & 0.00033 & 0.00016 & 0.00029 & 0.00076 & 0.00022 \\
\hline 592.7 & 0.00044 & 0.00053 & 0.00033 & 0.00019 & 0.00029 & 0.00082 & 0.00026 \\
\hline 593.04 & 0.00044 & 0.00053 & 0.00037 & 0.00019 & 0.00029 & 0.00082 & 0.00026 \\
\hline 593.37 & 0.00040 & 0.00058 & 0.00037 & 0.00022 & 0.00029 & 0.00082 & 0.00029 \\
\hline 593.71 & 0.00058 & 0.00049 & 0.00041 & 0.00025 & 0.00029 & 0.00082 & 0.00029 \\
\hline 594.05 & 0.00053 & 0.00044 & 0.00049 & 0.00025 & 0.00026 & 0.00082 & 0.00029 \\
\hline 594.39 & 0.00073 & 0.00049 & 0.00049 & 0.00025 & 0.00029 & 0.00082 & 0.00026 \\
\hline
\end{tabular}




\begin{tabular}{|c|c|c|c|c|c|c|c|}
\hline 594.73 & 0.00073 & 0.00033 & 0.00045 & 0.00022 & 0.00029 & 0.00082 & 0.00022 \\
\hline 595.07 & 0.00079 & 0.00029 & 0.00054 & 0.00031 & 0.00029 & 0.00082 & 0.00022 \\
\hline 595.41 & 0.00063 & 0.00023 & 0.00054 & 0.00031 & 0.00026 & 0.00082 & 0.00026 \\
\hline 595.75 & 0.00079 & 0.00026 & 0.00059 & 0.00035 & 0.00029 & 0.00082 & 0.00029 \\
\hline 596.08 & 0.00079 & 0.00026 & 0.00049 & 0.00031 & 0.00026 & 0.00076 & 0.00029 \\
\hline 596.42 & 0.00085 & 0.00020 & 0.00054 & 0.00031 & 0.00023 & 0.00071 & 0.00022 \\
\hline 596.76 & 0.00079 & 0.00014 & 0.00049 & 0.00031 & 0.00023 & 0.00066 & 0.00022 \\
\hline 597.1 & 0.00058 & 0.00014 & 0.00049 & 0.00028 & 0.00023 & 0.00071 & 0.00022 \\
\hline 597.44 & 0.00036 & 0.00012 & 0.00045 & 0.00028 & 0.00023 & 0.00066 & 0.00022 \\
\hline 597.78 & 0.00040 & 0.00008 & 0.00037 & 0.00022 & 0.00020 & 0.00066 & 0.00022 \\
\hline 598.11 & 0.00033 & 0.00006 & 0.00033 & 0.00019 & 0.00010 & 0.00066 & 0.00020 \\
\hline 598.45 & 0.00049 & 0.00014 & 0.00033 & $\begin{array}{l}0.00019 \\
\end{array}$ & 0.00012 & 0.00066 & 0.00020 \\
\hline 598.79 & 0.00049 & 0.00020 & 0.00033 & 0.00011 & 0.00012 & 0.00061 & 0.00017 \\
\hline 599.13 & 0.00049 & 0.00029 & 0.00033 & 0.00014 & 0.00010 & 0.00061 & 0.00017 \\
\hline 599.47 & 0.00040 & 0.00026 & 0.00023 & 0.00011 & 0.00008 & 0.00051 & 0.00012 \\
\hline 599.81 & 0.00040 & 0.00023 & 0.00020 & 0.00011 & 0.00007 & 0.00056 & 0.00014 \\
\hline 600.14 & 0.00036 & 0.00026 & 0.00015 & 0.00011 & 0.00007 & 0.00047 & 0.00014 \\
\hline 600.48 & 0.00053 & 0.00029 & 0.00023 & 0.00009 & 0.00008 & 0.00051 & 0.00014 \\
\hline 600.82 & 0.00063 & 0.00026 & 0.00020 & 0.00008 & 0.00005 & 0.00043 & 0.00012 \\
\hline 601.16 & 0.00058 & 0.00029 & 0.00018 & 0.00006 & 0.00004 & 0.00047 & 0.00014 \\
\hline 601.49 & 0.00049 & 0.00036 & 0.00018 & 0.00008 & 0.00004 & 0.00043 & 0.00014 \\
\hline 601.83 & 0.00044 & 0.00040 & 0.00010 & 0.00006 & 0.00008 & 0.00039 & 0.00012 \\
\hline 602.17 & 0.00036 & 0.00026 & 0.00007 & 0.00008 & 0.00008 & 0.00035 & 0.00012 \\
\hline 602.51 & 0.00023 & 0.00014 & 0.00005 & 0.00008 & 0.00008 & 0.00031 & 0.00008 \\
\hline 602.85 & 0.00023 & 0.00012 & 0.00003 & 0.00006 & 0.00010 & 0.00028 & 0.00006 \\
\hline 603.18 & 0.00026 & 0.00005 & 0.00003 & 0.00006 & 0.00010 & 0.00028 & 0.00005 \\
\hline 603.52 & 0.00044 & 0.00006 & 0.00004 & 0.00005 & 0.00012 & 0.00024 & 0.00004 \\
\hline 603.86 & 0.00040 & 0.00010 & 0.00004 & 0.00006 & 0.00010 & 0.00028 & 0.00005 \\
\hline 604.2 & 0.00026 & 0.00008 & 0.00001 & 0.00005 & 0.00007 & 0.00024 & 0.00004 \\
\hline 604.53 & 0.00033 & 0.00012 & 0.00000 & 0.00002 & 0.00007 & 0.00021 & 0.00004 \\
\hline 604.87 & 0.00036 & 0.00017 & 0.00001 & 0.00002 & 0.00008 & 0.00021 & 0.00004 \\
\hline 605.21 & 0.00040 & 0.00023 & 0.00000 & 0.00001 & 0.00007 & 0.00016 & 0.00004 \\
\hline 605.54 & 0.00033 & 0.00020 & 0.00001 & 0.00002 & 0.00005 & 0.00021 & 0.00005 \\
\hline 605.88 & 0.00058 & 0.00029 & 0.00003 & 0.00002 & 0.00005 & 0.00024 & 0.00006 \\
\hline 606.22 & 0.00063 & 0.00033 & 0.00005 & 0.00003 & 0.00004 & 0.00024 & 0.00010 \\
\hline 606.56 & 0.00053 & 0.00036 & 0.00009 & 0.00002 & 0.00002 & 0.00024 & 0.00012 \\
\hline 606.89 & 0.00044 & 0.00040 & 0.00007 & 0.00000 & 0.00001 & 0.00021 & 0.00012 \\
\hline 607.23 & 0.00020 & 0.00040 & 0.00007 & 0.00000 & 0.00001 & 0.00019 & 0.00010 \\
\hline 607.57 & 0.00017 & 0.00029 & 0.00009 & 0.00001 & 0.00000 & 0.00019 & 0.00006 \\
\hline
\end{tabular}




\begin{tabular}{|c|c|c|c|c|c|c|c|}
\hline 607.9 & 0.00020 & 0.00029 & 0.00010 & 0.00002 & 0.00001 & 0.00019 & 0.00008 \\
\hline 608.24 & 0.00010 & 0.00029 & 0.00009 & 0.00002 & 0.00001 & 0.00016 & 0.00006 \\
\hline 608.58 & 0.00005 & 0.00029 & 0.00007 & 0.00003 & 0.00000 & 0.00014 & 0.00004 \\
\hline 608.91 & 0.00002 & 0.00029 & 0.00013 & 0.00002 & 0.00000 & 0.00016 & 0.00004 \\
\hline 609.25 & 0.00000 & 0.00023 & 0.00013 & 0.00002 & 0.00001 & 0.00016 & 0.00004 \\
\hline 609.59 & 0.00000 & 0.00023 & 0.00013 & 0.00002 & 0.00000 & 0.00014 & 0.00004 \\
\hline 609.92 & 0.00002 & 0.00020 & 0.00010 & 0.00004 & 0.00000 & 0.00014 & 0.00004 \\
\hline 610.26 & 0.00004 & 0.00010 & 0.00009 & 0.00003 & 0.00000 & 0.00014 & 0.00002 \\
\hline 610.6 & 0.00008 & 0.00010 & 0.00010 & 0.00000 & 0.00001 & 0.00019 & 0.00002 \\
\hline 610.93 & 0.00002 & 0.00004 & 0.00007 & 0.00000 & 0.00000 & 0.00019 & 0.00002 \\
\hline 611.27 & 0.00002 & 0.00005 & 0.00009 & 0.00000 & 0.00000 & 0.00016 & 0.00002 \\
\hline 611.61 & 0.00004 & 0.00012 & 0.00007 & 0.00001 & 0.00000 & 0.00016 & 0.00002 \\
\hline 611.94 & 0.00002 & 0.00005 & 0.00007 & 0.00000 & 0.00000 & 0.00016 & 0.00002 \\
\hline 612.28 & 0.00002 & 0.00000 & 0.00004 & 0.00001 & 0.00002 & 0.00014 & 0.00002 \\
\hline 612.61 & 0.00000 & 0.00002 & 0.00001 & 0.00000 & 0.00003 & 0.00011 & 0.00002 \\
\hline 612.95 & 0.00000 & 0.00005 & 0.00001 & 0.00000 & 0.00002 & 0.00009 & 0.00000 \\
\hline 613.29 & 0.00001 & 0.00008 & 0.00000 & 0.00000 & 0.00002 & 0.00007 & 0.00000 \\
\hline 613.62 & 0.00000 & 0.00005 & 0.00001 & 0.00000 & 0.00003 & 0.00004 & 0.00000 \\
\hline 613.96 & 0.00008 & 0.00002 & 0.00005 & 0.00000 & 0.00002 & 0.00003 & 0.00000 \\
\hline 614.3 & 0.00012 & 0.00005 & 0.00008 & 0.00000 & 0.00001 & 0.00001 & 0.00000 \\
\hline 614.63 & 0.00004 & 0.00004 & 0.00019 & 0.00001 & 0.00001 & 0.00000 & 0.00000 \\
\hline 614.97 & 0.00012 & 0.00002 & 0.00028 & 0.00000 & 0.00003 & 0.00001 & 0.00001 \\
\hline 615.3 & 0.00017 & 0.00005 & 0.00032 & 0.00002 & 0.00001 & 0.00000 & 0.00001 \\
\hline 615.64 & 0.00012 & 0.00004 & 0.00043 & 0.00001 & 0.00001 & 0.00000 & 0.00000 \\
\hline 615.97 & 0.00015 & 0.00001 & 0.00047 & 0.00002 & 0.00000 & 0.00000 & 0.00000 \\
\hline 616.31 & 0.00007 & 0.00001 & 0.00052 & 0.00004 & 0.00000 & 0.00001 & 0.00000 \\
\hline 616.65 & 0.00007 & 0.00000 & 0.00066 & 0.00005 & 0.00000 & 0.00001 & 0.00000 \\
\hline 616.98 & 0.00012 & 0.00000 & 0.00077 & 0.00009 & 0.00000 & 0.00000 & 0.00000 \\
\hline 617.32 & 0.00008 & 0.00000 & 0.00066 & 0.00009 & 0.00001 & 0.00001 & 0.00000 \\
\hline 617.65 & 0.00003 & 0.00001 & 0.00072 & 0.00009 & 0.00001 & 0.00000 & 0.00000 \\
\hline 617.99 & 0.00005 & 0.00000 & 0.00056 & 0.00005 & 0.00001 & 0.00001 & 0.00002 \\
\hline 618.32 & 0.00001 & 0.00000 & 0.00039 & 0.00004 & 0.00001 & 0.00001 & 0.00002 \\
\hline 618.66 & 0.00000 & 0.00002 & 0.00035 & 0.00007 & 0.00005 & 0.00001 & 0.00002 \\
\hline 618.99 & 0.00000 & 0.00002 & 0.00043 & 0.00009 & 0.00003 & 0.00003 & 0.00002 \\
\hline 619.33 & 0.00003 & 0.00001 & 0.00032 & 0.00009 & 0.00002 & 0.00002 & 0.00002 \\
\hline 619.66 & 0.00007 & 0.00002 & 0.00028 & 0.00009 & 0.00005 & 0.00002 & 0.00002 \\
\hline 620 & 0.00015 & 0.00005 & 0.00022 & 0.00005 & 0.00006 & 0.00001 & 0.00004 \\
\hline 620.33 & 0.00017 & 0.00008 & 0.00022 & 0.00004 & 0.00006 & 0.00001 & 0.00004 \\
\hline 620.67 & 0.00017 & 0.00008 & 0.00028 & 0.00004 & 0.00006 & 0.00001 & 0.00005 \\
\hline
\end{tabular}




\begin{tabular}{|c|c|c|c|c|c|c|c|}
\hline 621 & 0.00026 & 0.00004 & 0.00025 & 0.00005 & 0.00003 & 0.00000 & 0.00005 \\
\hline 621.34 & 0.00010 & 0.00004 & 0.00019 & 0.00009 & 0.00001 & 0.00000 & 0.00006 \\
\hline 621.67 & 0.00008 & 0.00008 & 0.00019 & 0.00013 & 0.00001 & 0.00000 & 0.00005 \\
\hline 622.01 & 0.00020 & 0.00005 & 0.00014 & 0.00013 & 0.00000 & 0.00000 & 0.00006 \\
\hline 622.34 & 0.00020 & 0.00002 & 0.00014 & 0.00013 & 0.00000 & 0.00000 & 0.00005 \\
\hline 622.68 & 0.00012 & 0.00000 & 0.00010 & 0.00009 & 0.00000 & 0.00000 & 0.00005 \\
\hline 623.01 & 0.00003 & 0.00006 & 0.00016 & 0.00013 & 0.00001 & 0.00001 & 0.00002 \\
\hline 623.35 & 0.00001 & 0.00002 & 0.00012 & 0.00015 & 0.00000 & 0.00001 & 0.00002 \\
\hline 623.68 & 0.00004 & 0.00002 & 0.00012 & 0.00018 & 0.00000 & 0.00000 & 0.00004 \\
\hline 624.02 & 0.00010 & 0.00002 & 0.00006 & 0.00015 & 0.00001 & 0.00000 & 0.00004 \\
\hline 624.35 & 0.00017 & 0.00000 & 0.00008 & 0.00011 & 0.00001 & 0.00000 & 0.00005 \\
\hline 624.69 & 0.00029 & 0.00000 & 0.00008 & 0.00007 & 0.00002 & 0.00000 & 0.00008 \\
\hline 625.02 & 0.00019 & 0.00001 & 0.00008 & 0.00007 & 0.00005 & 0.00000 & 0.00008 \\
\hline 625.36 & 0.00032 & 0.00000 & 0.00010 & 0.00005 & 0.00003 & 0.00000 & 0.00010 \\
\hline 625.69 & 0.00048 & 0.00000 & 0.00014 & 0.00009 & 0.00003 & 0.00001 & 0.00010 \\
\hline 626.02 & 0.00044 & 0.00000 & 0.00016 & 0.00005 & 0.00003 & 0.00000 & 0.00010 \\
\hline 626.36 & 0.00025 & 0.00001 & 0.00010 & 0.00005 & 0.00001 & 0.00000 & 0.00014 \\
\hline 626.69 & 0.00029 & 0.00000 & 0.00008 & 0.00005 & 0.00003 & 0.00001 & 0.00017 \\
\hline 627.03 & 0.00017 & 0.00002 & 0.00014 & 0.00003 & 0.00003 & 0.00001 & 0.00022 \\
\hline 627.36 & 0.00005 & 0.00014 & 0.00010 & 0.00005 & 0.00005 & 0.00000 & 0.00020 \\
\hline 627.7 & 0.00000 & 0.00008 & 0.00016 & 0.00009 & 0.00005 & 0.00000 & 0.00022 \\
\hline 628.03 & 0.00001 & 0.00006 & 0.00012 & 0.00015 & 0.00006 & 0.00000 & 0.00020 \\
\hline 628.36 & 0.00000 & 0.00004 & 0.00016 & 0.00015 & 0.00006 & 0.00000 & 0.00017 \\
\hline 628.7 & 0.00000 & 0.00000 & 0.00016 & 0.00015 & 0.00005 & 0.00000 & 0.00020 \\
\hline 629.03 & 0.00003 & 0.00000 & 0.00016 & 0.00015 & 0.00008 & 0.00001 & 0.00017 \\
\hline 629.36 & 0.00004 & 0.00005 & 0.00016 & 0.00013 & 0.00012 & 0.00002 & 0.00022 \\
\hline 629.7 & 0.00001 & 0.00010 & 0.00019 & 0.00013 & 0.00017 & 0.00001 & 0.00026 \\
\hline 630.03 & 0.00000 & 0.00008 & 0.00022 & 0.00015 & 0.00019 & 0.00002 & 0.00029 \\
\hline 630.37 & 0.00007 & 0.00012 & 0.00028 & 0.00013 & 0.00014 & 0.00001 & 0.00032 \\
\hline 630.7 & 0.00012 & 0.00006 & 0.00016 & 0.00013 & 0.00017 & 0.00002 & 0.00032 \\
\hline 631.03 & 0.00007 & 0.00008 & 0.00019 & 0.00007 & 0.00012 & 0.00003 & 0.00029 \\
\hline 631.37 & 0.00000 & 0.00017 & 0.00019 & 0.00013 & 0.00019 & 0.00004 & 0.00029 \\
\hline 631.7 & 0.00000 & 0.00019 & 0.00014 & 0.00011 & 0.00017 & 0.00003 & 0.00029 \\
\hline 632.03 & 0.00000 & 0.00019 & 0.00014 & 0.00013 & 0.00014 & 0.00004 & 0.00029 \\
\hline 632.37 & 0.00000 & 0.00022 & 0.00019 & 0.00018 & 0.00017 & 0.00007 & 0.00029 \\
\hline 632.7 & 0.00001 & 0.00029 & 0.00028 & 0.00018 & 0.00022 & 0.00007 & 0.00022 \\
\hline 633.03 & 0.00000 & 0.00025 & 0.00019 & 0.00023 & 0.00025 & 0.00007 & 0.00022 \\
\hline 633.37 & 0.00001 & 0.00014 & 0.00008 & 0.00023 & 0.00025 & 0.00007 & 0.00022 \\
\hline 633.7 & 0.00000 & 0.00014 & 0.00008 & 0.00033 & 0.00025 & 0.00007 & 0.00020 \\
\hline
\end{tabular}




\begin{tabular}{|c|c|c|c|c|c|c|c|}
\hline 634.03 & 0.00000 & 0.00006 & 0.00005 & 0.00033 & 0.00019 & 0.00009 & 0.00020 \\
\hline 634.37 & 0.00008 & 0.00014 & 0.00008 & 0.00030 & 0.00022 & 0.00013 & 0.00014 \\
\hline 634.7 & 0.00005 & 0.00012 & 0.00008 & 0.00037 & 0.00025 & 0.00015 & 0.00020 \\
\hline 635.03 & 0.00000 & 0.00004 & 0.00005 & 0.00030 & 0.00017 & 0.00015 & 0.00026 \\
\hline 635.37 & 0.00000 & 0.00000 & 0.00005 & 0.00030 & 0.00025 & 0.00015 & 0.00026 \\
\hline 635.7 & 0.00000 & 0.00002 & 0.00005 & 0.00030 & 0.00036 & 0.00013 & 0.00022 \\
\hline 636.03 & 0.00000 & 0.00001 & 0.00003 & 0.00020 & 0.00036 & 0.00011 & 0.00026 \\
\hline 636.36 & 0.00002 & 0.00001 & 0.00005 & 0.00027 & 0.00025 & 0.00011 & 0.00026 \\
\hline 636.7 & 0.00002 & 0.00001 & 0.00005 & 0.00027 & 0.00019 & 0.00009 & 0.00029 \\
\hline 637.03 & 0.00000 & 0.00002 & 0.00006 & 0.00020 & 0.00014 & 0.00011 & 0.00032 \\
\hline 637.36 & 0.00001 & 0.00000 & 0.00005 & 0.00015 & 0.00017 & 0.00011 & 0.00036 \\
\hline 637.69 & 0.00004 & 0.00000 & 0.00001 & 0.00011 & 0.00019 & 0.00009 & 0.00040 \\
\hline 638.03 & 0.00001 & 0.00000 & 0.00002 & 0.00011 & 0.00014 & 0.00005 & 0.00044 \\
\hline 638.36 & 0.00000 & 0.00001 & 0.00006 & 0.00015 & 0.00017 & 0.00004 & 0.00044 \\
\hline 638.69 & 0.00000 & 0.00000 & 0.00006 & 0.00011 & 0.00014 & 0.00002 & 0.00048 \\
\hline 639.02 & 0.00000 & 0.00004 & 0.00008 & 0.00009 & 0.00008 & 0.00001 & 0.00058 \\
\hline 639.36 & 0.00000 & 0.00000 & 0.00006 & 0.00007 & 0.00003 & 0.00002 & 0.00062 \\
\hline 639.69 & 0.00002 & 0.00001 & 0.00006 & 0.00007 & 0.00005 & 0.00002 & 0.00058 \\
\hline 640.02 & 0.00008 & 0.00004 & 0.00006 & 0.00004 & 0.00010 & 0.00004 & 0.00058 \\
\hline 640.35 & 0.00007 & 0.00002 & 0.00010 & 0.00003 & 0.00010 & 0.00004 & 0.00053 \\
\hline 640.69 & 0.00002 & 0.00002 & 0.00016 & 0.00003 & 0.00014 & 0.00004 & 0.00048 \\
\hline 641.02 & 0.00001 & 0.00004 & 0.00025 & 0.00007 & 0.00008 & 0.00003 & 0.00053 \\
\hline 641.35 & 0.00001 & 0.00012 & 0.00039 & 0.00009 & 0.00008 & 0.00005 & 0.00044 \\
\hline 641.68 & 0.00000 & 0.00005 & 0.00043 & 0.00007 & 0.00006 & 0.00005 & 0.00044 \\
\hline 642.01 & 0.00002 & 0.00006 & 0.00035 & 0.00005 & 0.00005 & 0.00009 & 0.00040 \\
\hline 642.35 & 0.00000 & 0.00002 & 0.00028 & 0.00007 & 0.00008 & 0.00011 & 0.00040 \\
\hline 642.68 & 0.00000 & 0.00000 & 0.00028 & 0.00007 & 0.00005 & 0.00013 & 0.00040 \\
\hline 643.01 & 0.00000 & 0.00001 & 0.00028 & 0.00007 & 0.00003 & 0.00013 & 0.00040 \\
\hline 643.34 & 0.00000 & 0.00000 & 0.00028 & 0.00011 & 0.00001 & 0.00009 & 0.00040 \\
\hline 643.67 & 0.00000 & 0.00004 & 0.00032 & 0.00007 & 0.00000 & 0.00004 & 0.00044 \\
\hline 644 & 0.00000 & 0.00003 & 0.00022 & 0.00004 & 0.00000 & 0.00005 & 0.00040 \\
\hline 644.34 & 0.00000 & 0.00002 & 0.00028 & 0.00003 & 0.00002 & 0.00004 & 0.00044 \\
\hline 644.67 & 0.00001 & 0.00000 & 0.00028 & 0.00002 & 0.00000 & 0.00004 & 0.00044 \\
\hline 645 & 0.00000 & 0.00005 & 0.00032 & 0.00003 & 0.00000 & 0.00003 & 0.00040 \\
\hline 645.33 & 0.00001 & 0.00001 & 0.00032 & 0.00004 & 0.00001 & 0.00003 & 0.00044 \\
\hline 645.66 & 0.00000 & 0.00002 & 0.00043 & 0.00003 & 0.00001 & 0.00005 & 0.00040 \\
\hline 645.99 & 0.00001 & 0.00004 & 0.00052 & 0.00002 & 0.00000 & 0.00004 & 0.00040 \\
\hline 646.32 & 0.00008 & 0.00001 & 0.00061 & 0.00004 & 0.00001 & 0.00005 & 0.00032 \\
\hline 646.66 & 0.00017 & 0.00002 & 0.00066 & 0.00007 & 0.00003 & 0.00009 & 0.00029 \\
\hline
\end{tabular}




\begin{tabular}{|c|c|c|c|c|c|c|c|}
\hline 646.99 & 0.00004 & 0.00002 & 0.00056 & 0.00004 & \begin{tabular}{|l|}
0.00005 \\
\end{tabular} & 0.00013 & 0.00029 \\
\hline 647.32 & 0.00001 & 0.00001 & 0.00043 & 0.00004 & 0.00006 & 0.00013 & 0.00032 \\
\hline 647.65 & 0.00004 & 0.00006 & 0.00052 & 0.00005 & 0.00008 & 0.00015 & 0.00032 \\
\hline 647.98 & 0.00004 & 0.00010 & 0.00047 & 0.00011 & 0.00012 & 0.00015 & 0.00036 \\
\hline 648.31 & 0.00004 & 0.00012 & 0.00043 & 0.00011 & 0.00017 & 0.00018 & 0.00036 \\
\hline 648.64 & 0.00001 & 0.00004 & 0.00035 & 0.00013 & 0.00019 & 0.00024 & 0.00032 \\
\hline 648.97 & 0.00000 & 0.00010 & 0.00025 & 0.00013 & 0.00022 & 0.00027 & 0.00036 \\
\hline 649.3 & 0.00008 & 0.00005 & 0.00019 & 0.00013 & 0.00022 & 0.00027 & 0.00036 \\
\hline 649.64 & 0.00010 & 0.00004 & 0.00016 & 0.00011 & 0.00019 & 0.00027 & 0.00044 \\
\hline 649.97 & 0.00004 & 0.00004 & 0.00012 & 0.00007 & 0.00012 & 0.00021 & 0.00044 \\
\hline 650.3 & 0.00005 & 0.00006 & 0.00010 & 0.00004 & 0.00012 & 0.00015 & 0.00048 \\
\hline 650.63 & 0.00017 & 0.00012 & 0.00012 & 0.00011 & 0.00014 & 0.00015 & 0.00048 \\
\hline 650.96 & 0.00040 & 0.00010 & 0.00008 & 0.00011 & 0.00017 & 0.00018 & 0.00053 \\
\hline 651.29 & 0.00048 & 0.00004 & 0.00019 & 0.00013 & 0.00019 & 0.00027 & 0.00040 \\
\hline 651.62 & 0.00044 & 0.00000 & 0.00025 & 0.00018 & 0.00022 & 0.00038 & 0.00029 \\
\hline 651.95 & 0.00044 & 0.00002 & 0.00022 & 0.00018 & 0.00012 & 0.00038 & 0.00032 \\
\hline 652.28 & 0.00032 & 0.00000 & 0.00022 & 0.00018 & 0.00010 & 0.00034 & 0.00036 \\
\hline 652.61 & 0.00040 & 0.00000 & 0.00035 & 0.00020 & 0.00012 & 0.00038 & 0.00029 \\
\hline 652.94 & 0.00040 & 0.00001 & 0.00032 & 0.00023 & 0.00012 & 0.00041 & 0.00022 \\
\hline 653.27 & 0.00036 & 0.00000 & 0.00022 & 0.00030 & 0.00017 & 0.00050 & 0.00014 \\
\hline 653.6 & 0.00048 & 0.00001 & 0.00019 & 0.00037 & 0.00022 & 0.00070 & 0.00012 \\
\hline 653.93 & 0.00022 & 0.00004 & 0.00022 & 0.00030 & 0.00032 & 0.00064 & 0.00010 \\
\hline 654.26 & 0.00019 & 0.00005 & 0.00022 & 0.00030 & 0.00025 & 0.00075 & 0.00008 \\
\hline 654.59 & 0.00008 & 0.00005 & 0.00019 & 0.00033 & 0.00017 & 0.00064 & 0.00008 \\
\hline 654.92 & 0.00006 & 0.00002 & 0.00008 & 0.00050 & 0.00017 & 0.00055 & 0.00008 \\
\hline 655.25 & 0.00000 & 0.00000 & 0.00005 & 0.00037 & 0.00019 & 0.00050 & 0.00012 \\
\hline 655.58 & 0.00000 & 0.00000 & 0.00003 & 0.00030 & 0.00017 & 0.00046 & 0.00010 \\
\hline 655.91 & 0.00004 & 0.00000 & 0.00005 & 0.00030 & 0.00012 & 0.00046 & 0.00008 \\
\hline 656.24 & 0.00004 & 0.00000 & 0.00000 & 0.00030 & 0.00010 & 0.00046 & 0.00012 \\
\hline 656.57 & 0.00002 & 0.00000 & 0.00001 & 0.00030 & 0.00010 & 0.00055 & 0.00012 \\
\hline 656.9 & 0.00008 & 0.00006 & 0.00003 & 0.00033 & 0.00014 & 0.00055 & 0.00012 \\
\hline 657.23 & 0.00008 & 0.00002 & 0.00008 & 0.00041 & 0.00017 & 0.00041 & 0.00012 \\
\hline 657.56 & 0.00008 & 0.00002 & 0.00012 & 0.00050 & 0.00014 & 0.00064 & 0.00012 \\
\hline 657.89 & 0.00012 & 0.00006 & 0.00028 & 0.00050 & 0.00014 & 0.00075 & 0.00014 \\
\hline 658.22 & 0.00019 & 0.00017 & 0.00032 & 0.00037 & 0.00019 & 0.00086 & 0.00012 \\
\hline 658.55 & 0.00040 & 0.00044 & 0.00052 & 0.00030 & 0.00025 & 0.00092 & 0.00010 \\
\hline 658.88 & 0.00072 & 0.00073 & 0.00061 & 0.00033 & 0.00025 & 0.00118 & 0.00005 \\
\hline 659.21 & 0.00090 & 0.00102 & 0.00083 & 0.00033 & 0.00028 & 0.00132 & 0.00005 \\
\hline 659.54 & 0.00102 & 0.00096 & 0.00089 & 0.00030 & 0.00028 & 0.00155 & 0.00005 \\
\hline
\end{tabular}




\begin{tabular}{|c|c|c|c|c|c|c|c|}
\hline 659.87 & 0.00062 & 0.00096 & 0.00121 & 0.00037 & 0.00036 & 0.00155 & 0.00002 \\
\hline 660.2 & 0.00053 & 0.00078 & 0.00135 & 0.00033 & 0.00044 & 0.00147 & 0.00005 \\
\hline 660.53 & 0.00053 & 0.00044 & 0.00150 & 0.00045 & 0.00048 & 0.00155 & 0.00006 \\
\hline 660.85 & 0.00067 & 0.00053 & 0.00135 & 0.00041 & 0.00062 & 0.00188 & 0.00004 \\
\hline 661.18 & 0.00108 & 0.00096 & 0.00150 & 0.00069 & 0.00062 & 0.00179 & 0.00002 \\
\hline 661.51 & 0.00136 & 0.00053 & 0.00121 & 0.00059 & 0.00067 & 0.00197 & 0.00000 \\
\hline 661.84 & 0.00122 & 0.00036 & 0.00121 & 0.00074 & 0.00072 & 0.00206 & 0.00000 \\
\hline 662.17 & 0.00084 & 0.00019 & 0.00095 & 0.00092 & 0.00078 & 0.00206 & 0.00000 \\
\hline 662.5 & 0.00108 & 0.00000 & 0.00083 & 0.00104 & 0.00078 & 0.00224 & 0.00000 \\
\hline 662.83 & 0.00084 & 0.00000 & 0.00066 & 0.00111 & 0.00067 & 0.00188 & 0.00002 \\
\hline 663.16 & 0.00062 & 0.00000 & 0.00066 & 0.00124 & 0.00083 & 0.00197 & 0.00002 \\
\hline 663.49 & 0.00115 & 0.00000 & 0.00089 & 0.00124 & 0.00089 & 0.00206 & 0.00002 \\
\hline 663.81 & 0.00144 & 0.00001 & 0.00077 & 0.00132 & 0.00067 & 0.00215 & 0.00002 \\
\hline 664.14 & 0.00115 & 0.00000 & 0.00077 & 0.00098 & 0.00067 & 0.00197 & 0.00002 \\
\hline 664.47 & 0.00144 & 0.00004 & 0.00089 & 0.00124 & 0.00067 & 0.00215 & 0.00005 \\
\hline 664.8 & 0.00084 & 0.00000 & 0.00061 & 0.00098 & 0.00078 & 0.00244 & 0.00005 \\
\hline 665.13 & 0.00072 & 0.00000 & 0.00066 & 0.00132 & 0.00078 & 0.00254 & 0.00004 \\
\hline 665.46 & 0.00122 & 0.00001 & 0.00052 & 0.00139 & 0.00072 & 0.00215 & 0.00008 \\
\hline 665.79 & 0.00202 & 0.00000 & 0.00072 & 0.00132 & 0.00083 & 0.00224 & 0.00010 \\
\hline 666.11 & 0.00184 & 0.00025 & 0.00114 & 0.00162 & 0.00115 & 0.00224 & 0.00008 \\
\hline 666.44 & 0.00280 & 0.00044 & 0.00128 & 0.00139 & 0.00136 & 0.00285 & 0.00005 \\
\hline 666.77 & 0.00371 & 0.00053 & 0.00166 & 0.00111 & 0.00129 & 0.00274 & 0.00002 \\
\hline 667.1 & 0.00383 & 0.00040 & 0.00135 & 0.00111 & 0.00115 & 0.00274 & 0.00004 \\
\hline 667.43 & 0.00302 & 0.00044 & 0.00174 & 0.00132 & 0.00129 & 0.00296 & 0.00004 \\
\hline 667.76 & 0.00302 & 0.00032 & 0.00209 & 0.00162 & 0.00122 & 0.00274 & 0.00004 \\
\hline 668.08 & 0.00270 & 0.00036 & 0.00278 & 0.00162 & 0.00129 & 0.00264 & 0.00000 \\
\hline 668.41 & 0.00503 & 0.00096 & 0.00268 & 0.00170 & 0.00108 & 0.00244 & 0.00001 \\
\hline 668.74 & 0.00517 & 0.00185 & 0.00311 & 0.00132 & 0.00083 & 0.00215 & 0.00001 \\
\hline 669.07 & 0.00422 & 0.00230 & 0.00369 & 0.00118 & 0.00078 & 0.00244 & 0.00000 \\
\hline 669.4 & 0.00280 & 0.00211 & 0.00381 & 0.00118 & 0.00083 & 0.00274 & 0.00000 \\
\hline 669.72 & 0.00291 & 0.00160 & 0.00311 & 0.00080 & 0.00048 & 0.00234 & 0.00000 \\
\hline 670.05 & 0.00291 & 0.00176 & 0.00357 & 0.00139 & 0.00048 & 0.00264 & 0.00000 \\
\hline 670.38 & 0.00291 & 0.00202 & 0.00311 & 0.00196 & 0.00062 & 0.00307 & 0.00000 \\
\hline 670.71 & 0.00336 & 0.00240 & 0.00381 & 0.00214 & 0.00057 & 0.00285 & 0.00000 \\
\hline 671.03 & 0.00461 & 0.00302 & 0.00406 & 0.00224 & 0.00083 & 0.00274 & 0.00000 \\
\hline 671.36 & 0.00422 & 0.00336 & 0.00322 & 0.00243 & 0.00095 & 0.00318 & 0.00000 \\
\hline 671.69 & 0.00409 & 0.00302 & 0.00289 & 0.00224 & 0.00102 & 0.00296 & 0.00001 \\
\hline 672.02 & 0.00313 & 0.00202 & 0.00322 & 0.00187 & 0.00122 & 0.00274 & 0.00002 \\
\hline 672.34 & 0.00324 & 0.00176 & 0.00300 & 0.00205 & 0.00143 & 0.00274 & 0.00002 \\
\hline
\end{tabular}




\begin{tabular}{|c|c|c|c|c|c|c|c|}
\hline 672.67 & 0.00409 & 0.00250 & 0.00322 & 0.00284 & 0.00151 & 0.00285 & 0.00002 \\
\hline 673 & 0.00359 & 0.00291 & 0.00289 & 0.00263 & 0.00151 & 0.00274 & 0.00006 \\
\hline 673.33 & 0.00383 & 0.00324 & 0.00334 & 0.00363 & 0.00192 & 0.00274 & 0.00002 \\
\hline 673.65 & 0.00371 & 0.00348 & 0.00322 & 0.00363 & 0.00201 & 0.00234 & 0.00001 \\
\hline 673.98 & 0.00409 & 0.00270 & 0.00311 & 0.00273 & 0.00167 & 0.00206 & 0.00000 \\
\hline 674.31 & 0.00422 & 0.00230 & 0.00278 & 0.00295 & 0.00175 & 0.00234 & 0.00000 \\
\hline 674.63 & 0.00517 & 0.00176 & 0.00200 & 0.00340 & 0.00167 & 0.00264 & 0.00000 \\
\hline 674.96 & 0.00592 & 0.00176 & 0.00219 & 0.00233 & 0.00167 & 0.00244 & 0.00001 \\
\hline 675.29 & 0.00809 & 0.00185 & 0.00238 & 0.00196 & 0.00159 & 0.00307 & 0.00006 \\
\hline 675.61 & 0.00756 & 0.00160 & 0.00238 & 0.00214 & 0.00122 & 0.00318 & 0.00008 \\
\hline 675.94 & 0.00845 & 0.00160 & 0.00248 & 0.00196 & 0.00129 & 0.00377 & 0.00014 \\
\hline 676.27 & 0.00688 & 0.00096 & 0.00191 & 0.00170 & 0.00159 & 0.00352 & 0.00010 \\
\hline 676.6 & 0.00773 & 0.00057 & 0.00219 & 0.00170 & 0.00122 & 0.00307 & 0.00014 \\
\hline 676.92 & 0.00979 & 0.00053 & 0.00200 & 0.00132 & 0.00136 & 0.00307 & 0.00020 \\
\hline 677.25 & 0.01080 & 0.00022 & 0.00209 & 0.00139 & 0.00136 & 0.00352 & 0.00026 \\
\hline 677.58 & 0.01143 & 0.00040 & 0.00238 & 0.00154 & 0.00175 & 0.00377 & 0.00032 \\
\hline 677.9 & 0.01298 & 0.00062 & 0.00219 & 0.00124 & 0.00210 & 0.00402 & 0.00029 \\
\hline 678.23 & 0.01101 & 0.00122 & 0.00238 & 0.00098 & 0.00167 & 0.00341 & 0.00023 \\
\hline 678.55 & 0.01080 & 0.00176 & 0.00278 & 0.00132 & 0.00175 & 0.00364 & 0.00036 \\
\hline 678.88 & 0.00901 & 0.00176 & 0.00258 & 0.00170 & 0.00175 & 0.00352 & 0.00032 \\
\hline 679.21 & 0.00959 & 0.00152 & 0.00268 & 0.00196 & 0.00201 & 0.00389 & 0.00040 \\
\hline 679.53 & 0.01039 & 0.00185 & 0.00258 & 0.00224 & 0.00220 & 0.00352 & 0.00036 \\
\hline 679.86 & 0.01143 & 0.00168 & 0.00268 & 0.00214 & 0.00192 & 0.00389 & 0.00048 \\
\hline 680.19 & 0.01275 & 0.00144 & 0.00248 & 0.00179 & 0.00167 & 0.00440 & 0.00036 \\
\hline 680.51 & 0.01275 & 0.00144 & 0.00228 & 0.00205 & 0.00129 & 0.00440 & 0.00048 \\
\hline 680.84 & 0.01059 & 0.00129 & 0.00209 & 0.00196 & 0.00122 & 0.00402 & 0.00032 \\
\hline 681.16 & 0.00809 & 0.00129 & 0.00158 & 0.00196 & 0.00136 & 0.00389 & 0.00029 \\
\hline 681.49 & 0.00623 & 0.00067 & 0.00191 & 0.00196 & 0.00122 & 0.00341 & 0.00036 \\
\hline 681.82 & 0.00655 & 0.00053 & 0.00191 & 0.00196 & 0.00129 & 0.00377 & 0.00036 \\
\hline 682.14 & 0.00688 & 0.00032 & 0.00209 & 0.00187 & 0.00102 & 0.00341 & 0.00020 \\
\hline 682.47 & 0.00607 & 0.00044 & 0.00200 & 0.00196 & 0.00089 & 0.00341 & 0.00017 \\
\hline 682.79 & 0.00475 & 0.00090 & 0.00183 & 0.00170 & 0.00095 & 0.00329 & 0.00014 \\
\hline 683.12 & 0.00475 & 0.00096 & 0.00166 & 0.00162 & 0.00102 & 0.00364 & 0.00020 \\
\hline 683.45 & 0.00503 & 0.00096 & 0.00209 & 0.00170 & 0.00108 & 0.00364 & 0.00023 \\
\hline 683.77 & 0.00435 & 0.00160 & 0.00248 & 0.00187 & 0.00151 & 0.00341 & 0.00032 \\
\hline 684.1 & 0.00249 & 0.00152 & 0.00289 & 0.00187 & 0.00167 & 0.00389 & 0.00020 \\
\hline 684.42 & 0.00270 & 0.00168 & 0.00278 & 0.00132 & 0.00159 & 0.00377 & 0.00017 \\
\hline 684.75 & 0.00324 & 0.00185 & 0.00322 & 0.00170 & 0.00143 & 0.00402 & 0.00023 \\
\hline 685.07 & 0.00422 & 0.00221 & 0.00258 & 0.00154 & 0.00159 & 0.00427 & 0.00026 \\
\hline
\end{tabular}




\begin{tabular}{|c|c|c|c|c|c|c|c|}
\hline 685.4 & 0.00347 & 0.00185 & 0.00248 & 0.00124 & 0.00175 & 0.00402 & 0.00023 \\
\hline 685.72 & 0.00448 & 0.00240 & 0.00200 & 0.00162 & 0.00167 & 0.00414 & 0.00032 \\
\hline 686.05 & 0.00422 & 0.00168 & 0.00219 & 0.00187 & 0.00175 & 0.00377 & 0.00036 \\
\hline 686.37 & 0.00422 & 0.00122 & 0.00219 & 0.00224 & 0.00175 & 0.00352 & 0.00029 \\
\hline 686.7 & 0.00347 & 0.00102 & 0.00258 & 0.00243 & 0.00151 & 0.00285 & 0.00020 \\
\hline 687.02 & 0.00259 & 0.00078 & 0.00219 & 0.00243 & 0.00151 & 0.00234 & 0.00014 \\
\hline 687.35 & 0.00239 & 0.00096 & 0.00191 & 0.00243 & 0.00151 & 0.00234 & 0.00010 \\
\hline 687.67 & 0.00270 & 0.00102 & 0.00158 & 0.00214 & 0.00129 & 0.00206 & 0.00008 \\
\hline 688 & 0.00239 & 0.00129 & 0.00143 & 0.00263 & 0.00143 & 0.00188 & 0.00012 \\
\hline 688.32 & 0.00211 & 0.00096 & 0.00143 & 0.00224 & 0.00136 & 0.00163 & 0.00008 \\
\hline 688.65 & 0.00129 & 0.00067 & 0.00135 & 0.00224 & 0.00115 & 0.00171 & 0.00005 \\
\hline 688.97 & 0.00115 & 0.00073 & 0.00121 & 0.00263 & 0.00095 & 0.00179 & 0.00004 \\
\hline 689.3 & 0.00019 & 0.00048 & 0.00150 & 0.00214 & 0.00072 & 0.00179 & 0.00004 \\
\hline 689.62 & 0.00029 & 0.00067 & 0.00183 & 0.00179 & 0.00078 & 0.00147 & 0.00001 \\
\hline 689.95 & 0.00025 & 0.00073 & 0.00158 & 0.00154 & 0.00078 & 0.00147 & 0.00003 \\
\hline 690.27 & 0.00006 & 0.00057 & 0.00135 & 0.00162 & 0.00062 & 0.00163 & 0.00004 \\
\hline 690.6 & 0.00014 & 0.00122 & 0.00121 & 0.00147 & 0.00057 & 0.00179 & 0.00003 \\
\hline 690.92 & 0.00012 & 0.00084 & 0.00107 & 0.00124 & 0.00039 & 0.00163 & 0.00002 \\
\hline 691.25 & 0.00008 & 0.00062 & 0.00095 & 0.00111 & 0.00048 & 0.00132 & 0.00003 \\
\hline 691.57 & 0.00019 & 0.00053 & 0.00077 & 0.00098 & 0.00048 & 0.00155 & 0.00004 \\
\hline 691.89 & 0.00040 & 0.00073 & 0.00072 & 0.00074 & 0.00052 & 0.00140 & 0.00005 \\
\hline 692.22 & 0.00072 & 0.00084 & 0.00083 & 0.00080 & 0.00067 & 0.00118 & 0.00005 \\
\hline 692.54 & 0.00108 & 0.00053 & 0.00089 & 0.00059 & 0.00062 & 0.00118 & 0.00005 \\
\hline 692.87 & 0.00211 & 0.00040 & 0.00089 & 0.00074 & 0.00078 & 0.00118 & 0.00003 \\
\hline 693.19 & 0.00184 & 0.00044 & 0.00072 & 0.00069 & 0.00083 & 0.00125 & 0.00008 \\
\hline 693.52 & 0.00202 & 0.00025 & 0.00101 & 0.00059 & 0.00078 & 0.00105 & 0.00005 \\
\hline 693.84 & 0.00176 & 0.00029 & 0.00101 & 0.00027 & 0.00078 & 0.00086 & 0.00004 \\
\hline 694.16 & 0.00167 & 0.00012 & 0.00077 & 0.00030 & 0.00083 & 0.00075 & 0.00004 \\
\hline 694.49 & 0.00270 & 0.00002 & 0.00089 & 0.00037 & 0.00102 & 0.00070 & 0.00004 \\
\hline 694.81 & 0.00259 & 0.00005 & 0.00114 & 0.00059 & 0.00102 & 0.00075 & 0.00002 \\
\hline 695.14 & 0.00176 & 0.00002 & 0.00150 & 0.00069 & 0.00095 & 0.00055 & 0.00000 \\
\hline 695.46 & 0.00122 & 0.00002 & 0.00135 & 0.00104 & 0.00083 & 0.00050 & 0.00000 \\
\hline 695.78 & 0.00078 & 0.00000 & 0.00128 & 0.00086 & 0.00062 & 0.00041 & 0.00000 \\
\hline 696.11 & 0.00032 & 0.00004 & 0.00166 & 0.00086 & 0.00057 & 0.00038 & 0.00000 \\
\hline 696.43 & 0.00029 & 0.00022 & 0.00150 & 0.00074 & 0.00067 & 0.00034 & 0.00000 \\
\hline 696.75 & 0.00014 & 0.00019 & 0.00101 & 0.00074 & 0.00052 & 0.00034 & 0.00000 \\
\hline 697.08 & 0.00002 & 0.00036 & 0.00072 & 0.00111 & 0.00057 & 0.00038 & 0.00000 \\
\hline 697.4 & 0.00008 & 0.00053 & 0.00056 & 0.00147 & 0.00052 & 0.00038 & 0.00000 \\
\hline 697.72 & 0.00004 & 0.00062 & 0.00077 & 0.00170 & 0.00057 & 0.00041 & 0.00000 \\
\hline
\end{tabular}




\begin{tabular}{|l|l|l|l|l|l|l|l|}
\hline 698.05 & 0.00003 & 0.00122 & 0.00077 & 0.00179 & 0.00048 & 0.00030 & 0.00000 \\
\hline 698.37 & 0.00003 & 0.00102 & 0.00101 & 0.00154 & 0.00044 & 0.00030 & 0.00000 \\
\hline 698.69 & 0.00003 & 0.00152 & 0.00089 & 0.00118 & 0.00039 & 0.00027 & 0.00000 \\
\hline 699.02 & 0.00007 & 0.00152 & 0.00121 & 0.00111 & 0.00039 & 0.00027 & 0.00000 \\
\hline 699.34 & 0.00033 & 0.00230 & 0.00150 & 0.00111 & 0.00039 & 0.00027 & 0.00000 \\
\hline 699.66 & 0.00015 & 0.00260 & 0.00135 & 0.00118 & 0.00044 & 0.00015 & 0.00000 \\
\hline 699.99 & 0.00015 & 0.00160 & 0.00114 & 0.00098 & 0.00036 & 0.00011 & 0.00000 \\
\hline 700.31 & 0.00001 & 0.00168 & 0.00158 & 0.00080 & 0.00032 & 0.00007 & 0.00001 \\
\hline & & & & & & & \\
\hline
\end{tabular}

Absorbance values of Kelly Green $A_{-} F$ when the least squares method was applied when varying the average scans for Phase II

\begin{tabular}{|c|c|c|c|c|c|c|c|c|c|c|}
\hline$\lambda$ & $1 \mathrm{~m}$ & $2 \mathrm{~m}$ & $3 m$ & $4 m$ & $5 \mathrm{~m}$ & $6 \mathrm{~m}$ & $7 \mathrm{~m}$ & $8 \mathrm{~m}$ & $9 \mathrm{~m}$ & $10 \mathrm{~m}$ \\
\hline 380.34 & 0.236 & 0.25 & 0.383 & 0.387 & 0.377 & 0.371 & 0.391 & 0.386 & 0.388 & 0.386 \\
\hline 380.71 & 0.235 & 0.243 & 0.368 & 0.382 & 0.369 & 0.374 & 0.38 & 0.377 & 0.395 & 0.384 \\
\hline 381.07 & 0.229 & 0.236 & 0.359 & 0.368 & 0.369 & 0.379 & 0.368 & 0.371 & 0.395 & 0.387 \\
\hline 381.44 & 0.228 & 0.237 & 0.359 & 0.368 & 0.368 & 0.383 & 0.369 & 0.373 & 0.397 & 0.402 \\
\hline 381.8 & 0.219 & 0.23 & 0.35 & 0.357 & 0.366 & 0.388 & 0.36 & 0.369 & 0.389 & 0.395 \\
\hline 382.17 & 0.221 & 0.229 & 0.348 & 0.357 & 0.363 & 0.385 & 0.364 & 0.369 & 0.393 & 0.394 \\
\hline 382.53 & 0.222 & 0.23 & 0.346 & 0.363 & 0.368 & 0.379 & 0.361 & 0.368 & 0.39 & 0.389 \\
\hline 382.89 & 0.217 & 0.228 & 0.349 & 0.364 & 0.368 & 0.38 & 0.359 & 0.367 & 0.383 & 0.39 \\
\hline 383.26 & 0.217 & 0.222 & 0.354 & 0.358 & 0.366 & 0.378 & 0.362 & 0.357 & 0.383 & 0.386 \\
\hline 383.62 & 0.213 & 0.22 & 0.356 & 0.358 & 0.366 & 0.385 & 0.352 & 0.358 & 0.378 & 0.379 \\
\hline 383.99 & 0.209 & 0.215 & 0.354 & 0.35 & 0.36 & 0.385 & 0.35 & 0.358 & 0.372 & 0.374 \\
\hline 384.35 & 0.203 & 0.211 & 0.352 & 51 & 5 & 37 & 0.341 & 59 & 0.361 & 0.371 \\
\hline 384.72 & 0.2 & 0.208 & 0.357 & 0.359 & 0.354 & 0.38 & 0.343 & 0.361 & 0.354 & 0.364 \\
\hline 385.08 & 0.196 & 0.206 & 0.361 & 0.361 & 0.353 & 0.378 & 0.346 & 0.364 & 0.35 & 0.361 \\
\hline 385.44 & 0.194 & 0.202 & 0.36 & 0.356 & 0.35 & 0.374 & 0.343 & 0.369 & 0.344 & 0.351 \\
\hline 385.81 & 0.196 & 0.205 & 0.366 & 0.364 & 0.353 & 0.371 & 0.349 & 0.375 & 0.348 & 0.354 \\
\hline 386.17 & 0.192 & 0.201 & 0.366 & 0.358 & 0.345 & 0.365 & 0.338 & 0.367 & 0.343 & 0.351 \\
\hline 386.53 & 0.193 & 0.203 & 0.368 & 0.357 & 0.342 & 0.366 & 0.336 & 0.373 & 0.35 & 0.355 \\
\hline 386.9 & 0.196 & 0.204 & 0.367 & 0.35 & 0.342 & 0.363 & 0.342 & 0.375 & 0.352 & 0.357 \\
\hline 387.26 & 0.188 & 0.201 & 0.365 & 0.35 & 0.341 & 0.357 & 0.334 & 0.369 & 0.348 & 0.349 \\
\hline 387.63 & 0.187 & 0.198 & 0.362 & 0.349 & 0.346 & 0.355 & 0.335 & 0.371 & 0.352 & 0.348 \\
\hline 387.99 & 0.188 & 0.195 & 0.357 & 0.351 & 0.344 & 0.346 & 0.33 & 0.364 & 0.349 & 0.341 \\
\hline 388.35 & 0.188 & 0.195 & 0.355 & 0.355 & 0.351 & 0.348 & 0.336 & 0.365 & 0.356 & 0.34 \\
\hline 388.72 & 0.186 & 0.197 & 0.353 & 0.349 & 0.351 & 0.351 & 0.337 & 0.362 & 0.357 & 0.342 \\
\hline
\end{tabular}




\begin{tabular}{|c|c|c|c|c|c|c|c|c|c|c|}
\hline 389.08 & 0.187 & 0.192 & 0.347 & 0.346 & 0.353 & 0.342 & 0.329 & 0.355 & 0.355 & 0.342 \\
\hline 389.44 & 0.184 & 0.188 & 0.346 & 0.351 & 0.351 & 0.339 & 0.325 & 0.349 & 0.355 & 0.341 \\
\hline 389.81 & 0.175 & 0.181 & 0.337 & 0.345 & 0.349 & 0.335 & 0.317 & 0.346 & 0.349 & 0.337 \\
\hline 390.17 & 0.173 & 0.177 & 0.331 & 0.343 & 0.349 & 0.334 & 0.317 & 0.343 & 0.345 & 0.331 \\
\hline 390.53 & 0.167 & 0.171 & 0.327 & 0.336 & 0.343 & 0.328 & 0.317 & 0.33 & 0.334 & 0.324 \\
\hline 390.9 & 0.169 & 0.172 & 0.329 & 0.344 & 0.342 & 0.334 & 0.317 & 0.334 & 0.336 & 0.321 \\
\hline 391.26 & 0.17 & 0.171 & 0.327 & 0.341 & 0.338 & 0.338 & 0.316 & 0.342 & 0.338 & 0.325 \\
\hline 391.62 & 0.167 & 0.167 & 0.324 & 0.335 & 0.334 & 0.335 & 0.317 & 0.337 & 0.337 & 0.323 \\
\hline 391.99 & 0.167 & 0.167 & 0.324 & 0.338 & 0.338 & 0.337 & 0.321 & 0.339 & 0.345 & 0.327 \\
\hline 392.35 & 0.166 & 0.167 & 0.327 & 0.333 & 0.336 & 0.338 & 0.32 & 0.341 & 0.34 & 0.333 \\
\hline 392.71 & 0.164 & 0.164 & 0.328 & 0.325 & 0.335 & 0.333 & 0.317 & 0.341 & 0.338 & 0.33 \\
\hline 393.08 & 0.163 & 0.167 & 0.329 & 0.322 & 0.333 & 0.335 & 0.318 & 0.343 & 0.338 & 0.328 \\
\hline 393.44 & 0.163 & 0.167 & 0.329 & 0.315 & 0.333 & 0.333 & 0.317 & 0.342 & 0.337 & 0.327 \\
\hline 393.8 & 0.165 & 0.169 & 0.332 & 0.316 & 0.332 & 0.329 & 0.316 & 0.337 & 0.34 & 0.327 \\
\hline 394.17 & 0.165 & 0.171 & 0.335 & 0.316 & 0.334 & 0.331 & 0.318 & 0.34 & 0.341 & 0.331 \\
\hline 394.53 & 0.169 & 0.171 & 0.337 & 0.321 & 0.339 & 0.333 & 0.315 & 0.345 & 0.344 & 0.337 \\
\hline 394.89 & 0.169 & 0.169 & 0.334 & 0.318 & 0.34 & 0.329 & 0.317 & 0.339 & 0.343 & 0.338 \\
\hline 395.25 & 0.172 & 0.168 & 0.334 & 0.319 & 0.342 & 0.328 & 0.318 & 0.338 & 0.344 & 0.339 \\
\hline 395.62 & 0.174 & 0.171 & 0.336 & 0.322 & 0.344 & 0.329 & 0.315 & 0.34 & 0.35 & 0.345 \\
\hline 395.98 & 0.173 & 0.172 & 0.336 & 0.32 & 0.339 & 0.329 & 0.312 & 0.336 & 0.346 & 0.345 \\
\hline 396.34 & 0.172 & 0.172 & 0.339 & 0.32 & 0.339 & 0.328 & 0.315 & 0.334 & 0.347 & 0.34 \\
\hline 396.71 & 0.175 & 0.172 & 0.333 & 0.322 & 0.338 & 0.329 & 0.314 & 0.329 & 0.346 & 0.338 \\
\hline 397.07 & 0.174 & 0.171 & 0.336 & 0.328 & 0.341 & 0.33 & 0.319 & 0.333 & 0.348 & 0.34 \\
\hline 397.43 & 0.17 & 0.168 & 0.335 & 0.329 & 0.337 & 0.331 & 0.319 & 0.329 & 0.347 & 0.337 \\
\hline 397.79 & 0.17 & 0.168 & 0.335 & 0.328 & 0.34 & 0.333 & 0.326 & 0.332 & 0.344 & 0.337 \\
\hline 398.16 & 0.17 & 0.169 & 0.339 & 0.331 & 0.34 & 0.335 & 0.327 & 0.335 & 0.345 & 0.338 \\
\hline 398.52 & 0.165 & 0.17 & 0.34 & 0.331 & 0.34 & 0.336 & 0.328 & 0.336 & 0.344 & 0.334 \\
\hline 398.88 & 0.161 & 0.171 & 0.339 & 0.33 & 0.338 & 0.337 & 0.329 & 0.339 & 0.344 & 0.332 \\
\hline 399.24 & 0.161 & 0.173 & 0.337 & 0.331 & 0.338 & 0.338 & 0.329 & 0.336 & 0.339 & 0.33 \\
\hline 399.61 & 0.158 & 0.171 & 0.335 & 0.329 & 0.333 & 0.337 & 0.33 & 0.333 & 0.33 & 0.327 \\
\hline 399.97 & 0.16 & 0.173 & 0.336 & 0.332 & 0.337 & 0.337 & 0.333 & 0.339 & 0.33 & 0.328 \\
\hline 400.33 & 0.159 & 0.17 & 0.329 & 0.334 & 0.334 & 0.334 & 0.329 & 0.334 & 0.33 & 0.327 \\
\hline 400.69 & 0.156 & 0.169 & 0.329 & 0.337 & 0.333 & 0.333 & 0.327 & 0.338 & 0.325 & 0.328 \\
\hline 401.06 & 0.158 & 0.17 & 0.329 & 0.334 & 0.332 & 0.334 & 0.327 & 0.337 & 0.324 & 0.329 \\
\hline 401.42 & 0.161 & 0.175 & 0.332 & 0.338 & 0.339 & 0.336 & 0.336 & 0.342 & 0.326 & 0.339 \\
\hline 401.78 & 0.162 & 0.174 & 0.33 & 0.337 & 0.335 & 0.338 & 0.329 & 0.342 & 0.331 & 0.34 \\
\hline 402.14 & 0.162 & 0.174 & 0.326 & 0.337 & 0.333 & 0.335 & 0.328 & 0.341 & 0.331 & 0.34 \\
\hline 402.5 & 0.163 & 0.172 & 0.325 & 0.335 & 0.333 & 0.338 & 0.332 & 0.345 & 0.334 & 0.342 \\
\hline 402.87 & 0.166 & 0.171 & 0.327 & 0.337 & 0.335 & 0.338 & 0.331 & 0.345 & 0.334 & 0.344 \\
\hline
\end{tabular}




\begin{tabular}{|c|c|c|c|c|c|c|c|c|c|c|}
\hline 403.23 & 0.164 & 0.172 & 0.329 & 0.338 & 0.337 & 0.336 & 0.333 & 0.347 & 0.335 & 0.344 \\
\hline 403.59 & 0.167 & 0.171 & 0.332 & 0.344 & 0.343 & 0.336 & 0.335 & 0.351 & 0.337 & 0.345 \\
\hline 403.95 & 0.166 & 0.169 & 0.335 & 0.341 & 0.342 & 0.337 & 0.337 & 0.35 & 0.336 & 0.347 \\
\hline 404.31 & 0.165 & 0.168 & 0.333 & 0.334 & 0.339 & 0.337 & 0.333 & 0.35 & 0.331 & 0.343 \\
\hline 404.68 & 0.168 & 0.168 & 0.338 & 0.338 & 0.342 & 0.339 & 0.342 & 0.355 & 0.338 & 0.345 \\
\hline 405.04 & 0.167 & 0.168 & 0.336 & 0.338 & 0.34 & 0.339 & 0.343 & 0.353 & 0.339 & 0.341 \\
\hline 405.4 & 0.168 & 0.165 & 0.333 & 0.334 & 0.336 & 0.335 & 0.336 & 0.349 & 0.337 & 0.333 \\
\hline 405.76 & 0.168 & 0.164 & 0.338 & 0.338 & 0.34 & 0.336 & 0.343 & 0.35 & 0.335 & 0.336 \\
\hline 406.12 & 0.166 & 0.163 & 0.337 & 0.333 & 0.339 & 0.336 & 0.343 & 0.347 & 0.332 & 0.333 \\
\hline 406.49 & 0.165 & 0.163 & 0.337 & 0.331 & 0.337 & 0.334 & 0.336 & 0.339 & 0.33 & 0.331 \\
\hline 406.85 & 0.161 & 0.164 & 0.335 & 0.333 & 0.337 & 0.333 & 0.335 & 0.334 & 0.328 & 0.331 \\
\hline 407.21 & 0.163 & 0.162 & 0.335 & 0.332 & 0.336 & 0.333 & 0.335 & 0.333 & 0.331 & 0.331 \\
\hline 407.57 & 0.16 & 0.161 & 0.331 & 0.328 & 0.33 & 0.333 & 0.331 & 0.327 & 0.33 & 0.327 \\
\hline 407.93 & 0.159 & 0.162 & 0.327 & 0.331 & 0.329 & 0.333 & 0.334 & 0.327 & 0.33 & 0.326 \\
\hline 408.29 & 0.16 & 0.164 & 0.335 & 0.336 & 0.336 & 0.336 & 0.338 & 0.332 & 0.333 & 0.328 \\
\hline 408.65 & 0.16 & 0.163 & 0.336 & 0.331 & 0.338 & 0.339 & 0.335 & 0.334 & 0.332 & 0.33 \\
\hline 409.02 & 0.164 & 0.164 & 0.339 & 0.331 & 0.343 & 0.342 & 0.339 & 0.337 & 0.334 & 0.332 \\
\hline 409.38 & 0.163 & 0.167 & 0.341 & 0.333 & 0.343 & 0.342 & 0.342 & 0.338 & 0.335 & 0.336 \\
\hline 409.74 & 0.162 & 0.17 & 0.339 & 0.335 & 0.34 & 0.34 & 0.34 & 0.341 & 0.338 & 0.334 \\
\hline 410.1 & 0.167 & 0.171 & 0.341 & 0.342 & 0.344 & 0.346 & 0.342 & 0.346 & 0.339 & 0.338 \\
\hline 410.46 & 0.169 & 0.174 & 0.345 & 0.346 & 0.346 & 0.351 & 0.349 & 0.351 & 0.342 & 0.344 \\
\hline 410.82 & 0.172 & 0.175 & 0.349 & 0.345 & 0.351 & 0.353 & 0.352 & 0.357 & 0.345 & 0.347 \\
\hline 411.18 & 0.172 & 0.176 & 0.353 & 0.343 & 0.349 & 0.353 & 0.354 & 0.359 & 0.345 & 0.348 \\
\hline 411.54 & 0.174 & 0.175 & 0.357 & 0.346 & 0.354 & 0.352 & 0.356 & 0.362 & 0.347 & 0.352 \\
\hline 411.91 & 0.173 & 0.176 & 0.357 & 0.344 & 0.354 & 0.353 & 0.353 & 0.36 & 0.35 & 0.351 \\
\hline 412.27 & 0.174 & 0.177 & 0.356 & 0.344 & 0.35 & 0.351 & 0.355 & 0.356 & 0.352 & 0.356 \\
\hline 412.63 & 0.174 & 0.18 & 0.355 & 0.347 & 0.349 & 0.348 & 0.354 & 0.353 & 0.351 & 0.357 \\
\hline 412.99 & 0.169 & 0.177 & 0.35 & 0.35 & 0.342 & 0.344 & 0.349 & 0.347 & 0.348 & 0.351 \\
\hline 413.35 & 0.17 & 0.175 & 0.351 & 0.348 & 0.342 & 0.347 & 0.346 & 0.347 & 0.348 & 0.35 \\
\hline 413.71 & 0.17 & 0.174 & 0.35 & 0.348 & 0.341 & 0.35 & 0.343 & 0.346 & 0.346 & 0.352 \\
\hline 414.07 & 0.167 & 0.174 & 0.35 & 0.345 & 0.34 & 0.35 & 0.344 & 0.347 & 0.347 & 0.351 \\
\hline 414.43 & 0.166 & 0.174 & 0.348 & 0.344 & 0.339 & 0.347 & 0.341 & 0.347 & 0.347 & 0.351 \\
\hline 414.79 & 0.165 & 0.173 & 0.347 & 0.345 & 0.336 & 0.346 & 0.34 & 0.346 & 0.345 & 0.351 \\
\hline 415.15 & 0.165 & 0.172 & 0.346 & 0.35 & 0.339 & 0.346 & 0.34 & 0.346 & 0.346 & 0.351 \\
\hline 415.51 & 0.165 & 0.174 & 0.347 & 0.349 & 0.336 & 0.345 & 0.341 & 0.347 & 0.345 & 0.352 \\
\hline 415.88 & 0.167 & 0.176 & 0.349 & 0.35 & 0.338 & 0.345 & 0.343 & 0.351 & 0.346 & 0.353 \\
\hline 416.24 & 0.166 & 0.177 & 0.348 & 0.351 & 0.341 & 0.348 & 0.344 & 0.353 & 0.349 & 0.353 \\
\hline 416.6 & 0.166 & 0.176 & 0.347 & 0.35 & 0.339 & 0.348 & 0.342 & 0.351 & 0.348 & 0.351 \\
\hline 416.96 & 0.169 & 0.179 & 0.35 & 0.349 & 0.341 & 0.35 & 0.344 & 0.356 & 0.35 & 0.353 \\
\hline
\end{tabular}




\begin{tabular}{|c|c|c|c|c|c|c|c|c|c|c|}
\hline 417.32 & 0.169 & 0.179 & 0.348 & 0.347 & 0.342 & 0.349 & 0.344 & 0.355 & 0.347 & 0.352 \\
\hline 417.68 & 0.17 & 0.179 & 0.35 & 0.344 & 0.342 & 0.346 & 0.347 & 0.355 & 0.348 & 0.351 \\
\hline 418.04 & 0.171 & 0.179 & 0.347 & 0.347 & 0.342 & 0.344 & 0.346 & 0.354 & 0.349 & 0.349 \\
\hline 418.4 & 0.171 & 0.179 & 0.349 & 0.345 & 0.342 & 0.343 & 0.345 & 0.352 & 0.349 & 0.349 \\
\hline 418.76 & 0.17 & 0.179 & 0.346 & 0.344 & 0.342 & 0.343 & 0.347 & 0.351 & 0.349 & 0.347 \\
\hline 419.12 & 0.17 & 0.179 & 0.345 & 0.344 & 0.342 & 0.344 & 0.347 & 0.35 & 0.348 & 0.348 \\
\hline 419.48 & 0.17 & 0.179 & 0.346 & 0.344 & 0.344 & 0.347 & 0.346 & 0.35 & 0.349 & 0.345 \\
\hline 419.84 & 0.171 & 0.177 & 0.344 & 0.345 & 0.343 & 0.347 & 0.345 & 0.352 & 0.348 & 0.347 \\
\hline 420.2 & 0.172 & 0.176 & 0.344 & 0.343 & 0.344 & 0.347 & 0.343 & 0.352 & 0.345 & 0.344 \\
\hline 420.56 & 0.174 & 0.176 & 0.347 & 0.345 & 0.349 & 0.349 & 0.347 & 0.357 & 0.347 & 0.349 \\
\hline 420.92 & 0.172 & 0.175 & 0.346 & 0.345 & 0.351 & 0.35 & 0.345 & 0.354 & 0.348 & 0.35 \\
\hline 421.28 & 0.172 & 0.175 & 0.347 & 0.346 & 0.35 & 0.35 & 0.347 & 0.353 & 0.35 & 0.352 \\
\hline 421.64 & 0.173 & 0.174 & 0.35 & 0.35 & 0.353 & 0.354 & 0.346 & 0.353 & 0.35 & 0.353 \\
\hline 422 & 0.173 & 0.173 & 0.351 & 0.348 & 0.351 & 0.354 & 0.347 & 0.349 & 0.349 & 0.354 \\
\hline 422.36 & 0.173 & 0.172 & 0.349 & 0.351 & 0.353 & 0.354 & 0.347 & 0.351 & 0.348 & 0.352 \\
\hline 422.72 & 0.173 & 0.174 & 0.351 & 0.35 & 0.353 & 0.353 & 0.346 & 0.351 & 0.35 & 0.351 \\
\hline 423.08 & 0.171 & 0.173 & 0.35 & 0.349 & 0.352 & 0.352 & 0.345 & 0.35 & 0.35 & 0.352 \\
\hline 423.44 & 0.173 & 0.175 & 0.351 & 0.348 & 0.354 & 0.353 & 0.347 & 0.353 & 0.35 & 0.356 \\
\hline 423.8 & 0.171 & 0.175 & 0.353 & 0.348 & 0.352 & 0.353 & 0.349 & 0.35 & 0.349 & 0.354 \\
\hline 424.16 & 0.171 & 0.176 & 0.352 & 0.351 & 0.351 & 0.352 & 0.347 & 0.351 & 0.349 & 0.355 \\
\hline 424.52 & 0.169 & 0.174 & 0.349 & 0.35 & 0.347 & 0.351 & 0.345 & 0.348 & 0.348 & 0.35 \\
\hline 424.88 & 0.17 & 0.174 & 0.35 & 0.351 & 0.347 & 0.352 & 0.344 & 0.348 & 0.346 & 0.35 \\
\hline 425.24 & 0.171 & 0.177 & 0.35 & 0.356 & 0.352 & 0.352 & 0.346 & 0.352 & 0.347 & 0.352 \\
\hline 425.6 & 0.171 & 0.178 & 0.349 & 0.355 & 0.352 & 0.35 & 0.346 & 0.352 & 0.347 & 0.353 \\
\hline 425.96 & 0.171 & 0.179 & 0.349 & 0.357 & 0.353 & 0.349 & 0.348 & 0.353 & 0.347 & 0.354 \\
\hline 426.32 & 0.172 & 0.179 & 0.352 & 0.356 & 0.351 & 0.349 & 0.348 & 0.356 & 0.35 & 0.355 \\
\hline 426.68 & 0.174 & 0.18 & 0.353 & 0.358 & 0.352 & 0.35 & 0.35 & 0.356 & 0.349 & 0.356 \\
\hline 427.04 & 0.176 & 0.18 & 0.354 & 0.36 & 0.353 & 0.351 & 0.35 & 0.357 & 0.35 & 0.357 \\
\hline 427.4 & 0.174 & 0.178 & 0.351 & 0.362 & 0.351 & 0.349 & 0.348 & 0.356 & 0.353 & 0.356 \\
\hline 427.76 & 0.174 & 0.178 & 0.351 & 0.359 & 0.352 & 0.347 & 0.346 & 0.356 & 0.353 & 0.357 \\
\hline 428.12 & 0.174 & 0.178 & 0.35 & 0.358 & 0.352 & 0.347 & 0.346 & 0.356 & 0.353 & 0.357 \\
\hline 428.47 & 0.175 & 0.18 & 0.351 & 0.358 & 0.356 & 0.348 & 0.348 & 0.357 & 0.355 & 0.359 \\
\hline 428.83 & 0.175 & 0.178 & 0.35 & 0.357 & 0.353 & 0.346 & 0.347 & 0.357 & 0.356 & 0.359 \\
\hline 429.19 & 0.176 & 0.178 & 0.35 & 0.354 & 0.351 & 0.347 & 0.346 & 0.357 & 0.355 & 0.359 \\
\hline 429.55 & 0.175 & 0.178 & 0.349 & 0.355 & 0.351 & 0.348 & 0.346 & 0.359 & 0.355 & 0.357 \\
\hline 429.91 & 0.175 & 0.177 & 0.349 & 0.354 & 0.352 & 0.349 & 0.346 & 0.359 & 0.356 & 0.356 \\
\hline 430.27 & 0.175 & 0.178 & 0.347 & 0.355 & 0.353 & 0.35 & 0.346 & 0.359 & 0.355 & 0.355 \\
\hline 430.63 & 0.174 & 0.174 & 0.346 & 0.353 & 0.353 & 0.35 & 0.345 & 0.356 & 0.352 & 0.355 \\
\hline 430.99 & 0.172 & 0.175 & 0.347 & 0.352 & 0.351 & 0.347 & 0.346 & 0.356 & 0.351 & 0.352 \\
\hline
\end{tabular}




\begin{tabular}{|c|c|c|c|c|c|c|c|c|c|c|}
\hline 431.35 & 0.171 & 0.176 & 0.348 & 0.35 & 0.351 & 0.349 & 0.345 & 0.354 & 0.348 & 0.351 \\
\hline 431.71 & 0.172 & 0.176 & 0.347 & 0.353 & 0.354 & 0.351 & 0.345 & 0.354 & 0.351 & 0.354 \\
\hline 432.07 & 0.172 & 0.173 & 0.347 & 0.354 & 0.353 & 0.351 & 0.346 & 0.354 & 0.352 & 0.353 \\
\hline 432.42 & 0.17 & 0.171 & 0.345 & 0.351 & 0.35 & 0.349 & 0.345 & 0.352 & 0.348 & 0.354 \\
\hline 432.78 & 0.168 & 0.17 & 0.345 & 0.351 & 0.35 & 0.347 & 0.346 & 0.349 & 0.346 & 0.355 \\
\hline 433.14 & 0.165 & 0.165 & 0.342 & 0.348 & 0.347 & 0.345 & 0.345 & 0.348 & 0.345 & 0.351 \\
\hline 433.5 & 0.166 & 0.167 & 0.343 & 0.347 & 0.346 & 0.345 & 0.347 & 0.349 & 0.345 & 0.352 \\
\hline 433.86 & 0.164 & 0.165 & 0.343 & 0.345 & 0.346 & 0.346 & 0.346 & 0.35 & 0.345 & 0.35 \\
\hline 434.22 & 0.163 & 0.164 & 0.345 & 0.346 & 0.346 & 0.347 & 0.345 & 0.349 & 0.343 & 0.35 \\
\hline 434.58 & 0.162 & 0.165 & 0.346 & 0.349 & 0.345 & 0.348 & 0.344 & 0.348 & 0.345 & 0.351 \\
\hline 434.94 & 0.163 & 0.164 & 0.345 & 0.346 & 0.344 & 0.349 & 0.343 & 0.348 & 0.345 & 0.35 \\
\hline 435.29 & 0.161 & 0.164 & 0.343 & 0.345 & 0.344 & 0.346 & 0.342 & 0.346 & 0.345 & 0.349 \\
\hline 435.65 & 0.159 & 0.162 & 0.343 & 0.343 & 0.343 & 0.344 & 0.34 & 0.344 & 0.341 & 0.345 \\
\hline 436.01 & 0.16 & 0.163 & 0.346 & 0.343 & 0.343 & 0.346 & 0.341 & 0.344 & 0.341 & 0.346 \\
\hline 436.37 & 0.16 & 0.163 & 0.345 & 0.344 & 0.342 & 0.344 & 0.342 & 0.341 & 0.341 & 0.344 \\
\hline 436.73 & 0.16 & 0.164 & 0.346 & 0.346 & 0.345 & 0.348 & 0.343 & 0.346 & 0.342 & 0.345 \\
\hline 437.09 & 0.16 & 0.165 & 0.347 & 0.347 & 0.345 & 0.347 & 0.343 & 0.344 & 0.343 & 0.346 \\
\hline 437.44 & 0.157 & 0.161 & 0.344 & 0.343 & 0.346 & 0.342 & 0.339 & 0.341 & 0.339 & 0.345 \\
\hline 437.8 & 0.157 & 0.16 & 0.344 & 0.346 & 0.345 & 0.343 & 0.339 & 0.34 & 0.339 & 0.347 \\
\hline 438.16 & 0.155 & 0.16 & 0.343 & 0.346 & 0.345 & 0.342 & 0.34 & 0.341 & 0.34 & 0.348 \\
\hline 438.52 & 0.155 & 0.158 & 0.339 & 0.343 & 0.344 & 0.34 & 0.342 & 0.342 & 0.339 & 0.345 \\
\hline 438.88 & 0.155 & 0.157 & 0.339 & 0.345 & 0.344 & 0.343 & 0.342 & 0.342 & 0.339 & 0.346 \\
\hline 439.24 & 0.154 & 0.155 & 0.34 & 0.344 & 0.344 & 0.343 & 0.342 & 0.343 & 0.34 & 0.345 \\
\hline 439.59 & 0.154 & 0.154 & 0.34 & 0.344 & 0.343 & 0.343 & 0.343 & 0.343 & 0.341 & 0.345 \\
\hline 439.95 & 0.151 & 0.154 & 0.338 & 0.344 & 0.343 & 0.34 & 0.344 & 0.344 & 0.341 & 0.344 \\
\hline 440.31 & 0.15 & 0.154 & 0.339 & 0.344 & 0.344 & 0.343 & 0.343 & 0.346 & 0.341 & 0.344 \\
\hline 440.67 & 0.149 & 0.151 & 0.337 & 0.341 & 0.343 & 0.341 & 0.342 & 0.342 & 0.341 & 0.344 \\
\hline 441.03 & 0.148 & 0.151 & 0.336 & 0.342 & 0.344 & 0.34 & 0.342 & 0.343 & 0.341 & 0.344 \\
\hline 441.38 & 0.147 & 0.151 & 0.336 & 0.344 & 0.344 & 0.339 & 0.343 & 0.342 & 0.341 & 0.342 \\
\hline 441.74 & 0.146 & 0.15 & 0.334 & 0.339 & 0.341 & 0.335 & 0.342 & 0.341 & 0.34 & 0.341 \\
\hline 442.1 & 0.147 & 0.15 & 0.334 & 0.338 & 0.342 & 0.336 & 0.342 & 0.341 & 0.34 & 0.34 \\
\hline 442.46 & 0.147 & 0.15 & 0.337 & 0.338 & 0.342 & 0.335 & 0.341 & 0.338 & 0.338 & 0.341 \\
\hline 442.81 & 0.146 & 0.149 & 0.336 & 0.339 & 0.343 & 0.332 & 0.341 & 0.338 & 0.339 & 0.341 \\
\hline 443.17 & 0.148 & 0.148 & 0.337 & 0.339 & 0.342 & 0.332 & 0.342 & 0.338 & 0.338 & 0.341 \\
\hline 443.53 & 0.147 & 0.146 & 0.335 & 0.338 & 0.339 & 0.332 & 0.34 & 0.336 & 0.335 & 0.34 \\
\hline 443.89 & 0.144 & 0.143 & 0.336 & 0.335 & 0.336 & 0.33 & 0.337 & 0.334 & 0.334 & 0.339 \\
\hline 444.24 & 0.142 & 0.142 & 0.334 & 0.333 & 0.333 & 0.327 & 0.337 & 0.332 & 0.332 & 0.337 \\
\hline 444.6 & 0.141 & 0.142 & 0.334 & 0.334 & 0.331 & 0.327 & 0.336 & 0.331 & 0.33 & 0.336 \\
\hline 444.96 & 0.139 & 0.141 & 0.336 & 0.335 & 0.33 & 0.328 & 0.337 & 0.332 & 0.331 & 0.336 \\
\hline
\end{tabular}




\begin{tabular}{|c|c|c|c|c|c|c|c|c|c|c|}
\hline 445.32 & 0.139 & 0.141 & 0.335 & 0.332 & 0.328 & 0.33 & 0.337 & 0.331 & 0.33 & 0.335 \\
\hline 445.67 & 0.138 & 0.14 & 0.337 & 0.334 & 0.33 & 0.333 & 0.335 & 0.331 & 0.333 & 0.336 \\
\hline 446.03 & 0.137 & 0.138 & 0.337 & 0.334 & 0.329 & 0.332 & 0.337 & 0.331 & 0.332 & 0.336 \\
\hline 446.39 & 0.137 & 0.137 & 0.335 & 0.335 & 0.328 & 0.331 & 0.336 & 0.332 & 0.333 & 0.335 \\
\hline 446.75 & 0.135 & 0.136 & 0.337 & 0.333 & 0.328 & 0.332 & 0.333 & 0.332 & 0.332 & 0.333 \\
\hline 447.1 & 0.132 & 0.136 & 0.335 & 0.332 & 0.326 & 0.331 & 0.329 & 0.329 & 0.33 & 0.331 \\
\hline 447.46 & 0.131 & 0.136 & 0.336 & 0.331 & 0.328 & 0.331 & 0.329 & 0.331 & 0.333 & 0.332 \\
\hline 447.82 & 0.131 & 0.134 & 0.334 & 0.331 & 0.329 & 0.332 & 0.329 & 0.329 & 0.332 & 0.331 \\
\hline 448.18 & 0.131 & 0.133 & 0.334 & 0.332 & 0.33 & 0.332 & 0.327 & 0.331 & 0.333 & 0.331 \\
\hline 448.53 & 0.129 & 0.131 & 0.335 & 0.331 & 0.33 & 0.331 & 0.326 & 0.331 & 0.333 & 0.331 \\
\hline 448.89 & 0.127 & 0.13 & 0.331 & 0.329 & 0.329 & 0.329 & 0.324 & 0.327 & 0.331 & 0.328 \\
\hline 449.25 & 0.127 & 0.128 & 0.331 & 0.329 & 0.328 & 0.33 & 0.323 & 0.326 & 0.332 & 0.328 \\
\hline 449.6 & 0.124 & 0.126 & 0.329 & 0.326 & 0.325 & 0.328 & 0.324 & 0.324 & 0.327 & 0.326 \\
\hline 449.96 & 0.122 & 0.125 & 0.326 & 0.323 & 0.323 & 0.325 & 0.321 & 0.32 & 0.326 & 0.322 \\
\hline 450.32 & 0.12 & 0.123 & 0.326 & 0.321 & 0.324 & 0.324 & 0.322 & 0.319 & 0.325 & 0.322 \\
\hline 450.67 & 0.119 & 0.123 & 0.323 & 0.321 & 0.323 & 0.323 & 0.323 & 0.318 & 0.324 & 0.321 \\
\hline 451.03 & 0.118 & 0.122 & 0.322 & 0.32 & 0.322 & 0.322 & 0.325 & 0.317 & 0.326 & 0.323 \\
\hline 451.39 & 0.117 & 0.12 & 0.32 & 0.318 & 0.32 & 0.321 & 0.325 & 0.316 & 0.324 & 0.321 \\
\hline 451.74 & 0.117 & 0.119 & 0.319 & 0.317 & 0.319 & 0.321 & 0.324 & 0.317 & 0.322 & 0.32 \\
\hline 452.1 & 0.114 & 0.117 & 0.317 & 0.315 & 0.318 & 0.32 & 0.323 & 0.315 & 0.321 & 0.319 \\
\hline 452.46 & 0.114 & 0.117 & 0.315 & 0.312 & 0.316 & 0.319 & 0.324 & 0.315 & 0.32 & 0.317 \\
\hline 452.81 & 0.113 & 0.115 & 0.316 & 0.312 & 0.314 & 0.318 & 0.324 & 0.316 & 0.319 & 0.318 \\
\hline 453.17 & 0.11 & 0.114 & 0.317 & 0.313 & 0.313 & 0.318 & 0.323 & 0.318 & 0.317 & 0.318 \\
\hline 453.53 & 0.11 & 0.112 & 0.315 & 0.312 & 0.313 & 0.316 & 0.32 & 0.317 & 0.316 & 0.316 \\
\hline 453.88 & 0.107 & 0.11 & 0.314 & 0.31 & 0.311 & 0.314 & 0.317 & 0.316 & 0.315 & 0.316 \\
\hline 454.24 & 0.106 & 0.11 & 0.313 & 0.31 & 0.31 & 0.316 & 0.316 & 0.317 & 0.316 & 0.317 \\
\hline 454.6 & 0.105 & 0.107 & 0.312 & 0.309 & 0.309 & 0.315 & 0.316 & 0.316 & 0.315 & 0.316 \\
\hline 454.95 & 0.103 & 0.106 & 0.312 & 0.308 & 0.309 & 0.313 & 0.314 & 0.317 & 0.313 & 0.315 \\
\hline 455.31 & 0.101 & 0.105 & 0.309 & 0.308 & 0.308 & 0.313 & 0.312 & 0.317 & 0.311 & 0.313 \\
\hline 455.66 & 0.1 & 0.105 & 0.31 & 0.307 & 0.306 & 0.311 & 0.311 & 0.315 & 0.31 & 0.312 \\
\hline 456.02 & 0.099 & 0.104 & 0.308 & 0.307 & 0.305 & 0.31 & 0.311 & 0.313 & 0.308 & 0.31 \\
\hline 456.38 & 0.1 & 0.102 & 0.307 & 0.307 & 0.305 & 0.307 & 0.309 & 0.311 & 0.307 & 0.311 \\
\hline 456.73 & 0.099 & 0.101 & 0.306 & 0.307 & 0.305 & 0.308 & 0.309 & 0.309 & 0.307 & 0.309 \\
\hline 457.09 & 0.098 & 0.098 & 0.304 & 0.305 & 0.303 & 0.306 & 0.307 & 0.304 & 0.307 & 0.308 \\
\hline 457.45 & 0.097 & 0.097 & 0.304 & 0.304 & 0.302 & 0.306 & 0.307 & 0.303 & 0.306 & 0.308 \\
\hline 457.8 & 0.095 & 0.096 & 0.303 & 0.305 & 0.302 & 0.306 & 0.307 & 0.302 & 0.305 & 0.306 \\
\hline 458.16 & 0.094 & 0.094 & 0.302 & 0.305 & 0.301 & 0.304 & 0.304 & 0.301 & 0.303 & 0.305 \\
\hline 458.51 & 0.093 & 0.093 & 0.302 & 0.304 & 0.3 & 0.304 & 0.303 & 0.3 & 0.301 & 0.305 \\
\hline 458.87 & 0.093 & 0.091 & 0.301 & 0.303 & 0.298 & 0.303 & 0.3 & 0.299 & 0.3 & 0.302 \\
\hline
\end{tabular}




\begin{tabular}{|c|c|c|c|c|c|c|c|c|c|c|}
\hline 459.23 & 0.09 & 0.089 & 0.3 & 0.303 & 0.297 & 0.3 & 0.298 & 0.296 & 0.298 & 0.301 \\
\hline 459.58 & 0.086 & 0.087 & 0.298 & 0.302 & 0.296 & 0.299 & 0.296 & 0.295 & 0.298 & 0.299 \\
\hline 459.94 & 0.084 & 0.085 & 0.297 & 0.3 & 0.295 & 0.298 & 0.293 & 0.293 & 0.297 & 0.298 \\
\hline 460.29 & 0.081 & 0.083 & 0.296 & 0.296 & 0.293 & 0.297 & 0.291 & 0.291 & 0.294 & 0.295 \\
\hline 460.65 & 0.079 & 0.081 & 0.294 & 0.295 & 0.292 & 0.293 & 0.288 & 0.291 & 0.293 & 0.294 \\
\hline 461 & 0.078 & 0.08 & 0.293 & 0.294 & 0.292 & 0.291 & 0.289 & 0.292 & 0.293 & 0.293 \\
\hline 461.36 & 0.077 & 0.079 & 0.292 & 0.293 & 0.292 & 0.29 & 0.289 & 0.292 & 0.294 & 0.294 \\
\hline 461.71 & 0.077 & 0.078 & 0.292 & 0.293 & 0.291 & 0.291 & 0.289 & 0.292 & 0.294 & 0.294 \\
\hline 462.07 & 0.073 & 0.076 & 0.29 & 0.29 & 0.289 & 0.29 & 0.288 & 0.29 & 0.293 & 0.293 \\
\hline 462.43 & 0.071 & 0.074 & 0.288 & 0.289 & 0.288 & 0.287 & 0.288 & 0.289 & 0.291 & 0.29 \\
\hline 462.78 & 0.07 & 0.072 & 0.286 & 0.289 & 0.288 & 0.286 & 0.288 & 0.288 & 0.29 & 0.29 \\
\hline 463.14 & 0.069 & 0.07 & 0.285 & 0.287 & 0.287 & 0.286 & 0.289 & 0.29 & 0.288 & 0.29 \\
\hline 463.49 & 0.069 & 0.069 & 0.285 & 0.286 & 0.287 & 0.285 & 0.288 & 0.289 & 0.286 & 0.29 \\
\hline 463.85 & 0.069 & 0.068 & 0.285 & 0.286 & 0.286 & 0.284 & 0.287 & 0.288 & 0.286 & 0.289 \\
\hline 464.2 & 0.067 & 0.069 & 0.284 & 0.287 & 0.287 & 0.284 & 0.288 & 0.288 & 0.286 & 0.29 \\
\hline 464.56 & 0.066 & 0.067 & 0.284 & 0.286 & 0.286 & 0.283 & 0.288 & 0.286 & 0.284 & 0.289 \\
\hline 464.91 & 0.064 & 0.065 & 0.282 & 0.283 & 0.286 & 0.282 & 0.286 & 0.284 & 0.282 & 0.288 \\
\hline 465.27 & 0.062 & 0.064 & 0.28 & 0.282 & 0.285 & 0.281 & 0.285 & 0.283 & 0.28 & 0.287 \\
\hline 465.62 & 0.06 & 0.063 & 0.279 & 0.28 & 0.284 & 0.28 & 0.282 & 0.282 & 0.279 & 0.284 \\
\hline 465.98 & 0.059 & 0.061 & 0.278 & 0.279 & 0.283 & 0.278 & 0.281 & 0.281 & 0.277 & 0.282 \\
\hline 466.33 & 0.057 & 0.059 & 0.275 & 0.279 & 0.281 & 0.276 & 0.278 & 0.28 & 0.277 & 0.28 \\
\hline 466.69 & 0.055 & 0.057 & 0.274 & 0.278 & 0.28 & 0.275 & 0.278 & 0.278 & 0.276 & 0.278 \\
\hline 467.04 & 0.055 & 0.056 & 0.274 & 0.277 & 0.28 & 0.274 & 0.276 & 0.276 & 0.277 & 0.277 \\
\hline 467.4 & 0.052 & 0.053 & 0.274 & 0.275 & 0.278 & 0.275 & 0.276 & 0.275 & 0.276 & 0.277 \\
\hline 467.75 & 0.051 & 0.052 & 0.273 & 0.275 & 0.278 & 0.275 & 0.275 & 0.275 & 0.275 & 0.276 \\
\hline 468.11 & 0.049 & 0.049 & 0.271 & 0.273 & 0.275 & 0.273 & 0.274 & 0.274 & 0.273 & 0.273 \\
\hline 468.46 & 0.048 & 0.048 & 0.269 & 0.272 & 0.273 & 0.272 & 0.272 & 0.272 & 0.272 & 0.272 \\
\hline 468.82 & 0.046 & 0.046 & 0.269 & 0.271 & 0.272 & 0.271 & 0.271 & 0.271 & 0.271 & 0.27 \\
\hline 469.17 & 0.044 & 0.044 & 0.267 & 0.269 & 0.27 & 0.27 & 0.27 & 0.27 & 0.271 & 0.268 \\
\hline 469.53 & 0.042 & 0.041 & 0.267 & 0.268 & 0.268 & 0.268 & 0.269 & 0.27 & 0.269 & 0.266 \\
\hline 469.88 & 0.041 & 0.039 & 0.267 & 0.267 & 0.266 & 0.267 & 0.269 & 0.268 & 0.268 & 0.266 \\
\hline 470.24 & 0.038 & 0.037 & 0.266 & 0.265 & 0.266 & 0.266 & 0.268 & 0.267 & 0.266 & 0.267 \\
\hline 470.59 & 0.036 & 0.036 & 0.265 & 0.262 & 0.263 & 0.265 & 0.267 & 0.265 & 0.264 & 0.265 \\
\hline 470.94 & 0.034 & 0.035 & 0.263 & 0.262 & 0.261 & 0.264 & 0.266 & 0.263 & 0.262 & 0.264 \\
\hline 471.3 & 0.032 & 0.033 & 0.262 & 0.26 & 0.26 & 0.261 & 0.264 & 0.262 & 0.261 & 0.261 \\
\hline 471.65 & 0.029 & 0.031 & 0.259 & 0.258 & 0.258 & 0.259 & 0.262 & 0.26 & 0.259 & 0.26 \\
\hline 472.01 & 0.027 & 0.029 & 0.258 & 0.257 & 0.257 & 0.257 & 0.259 & 0.259 & 0.257 & 0.259 \\
\hline 472.36 & 0.025 & 0.028 & 0.257 & 0.255 & 0.255 & 0.257 & 0.258 & 0.258 & 0.256 & 0.258 \\
\hline 472.72 & 0.023 & 0.027 & 0.256 & 0.254 & 0.253 & 0.256 & 0.257 & 0.257 & 0.255 & 0.258 \\
\hline
\end{tabular}




\begin{tabular}{|c|c|c|c|c|c|c|c|c|c|c|}
\hline 473.07 & 0.02 & 0.025 & 0.255 & 0.253 & 0.252 & 0.254 & 0.255 & 0.255 & 0.253 & 0.257 \\
\hline 473.43 & 0.018 & 0.023 & 0.253 & 0.252 & 0.25 & 0.253 & 0.253 & 0.252 & 0.252 & 0.256 \\
\hline 473.78 & 0.016 & 0.021 & 0.251 & 0.251 & 0.249 & 0.253 & 0.251 & 0.25 & 0.251 & 0.255 \\
\hline 474.13 & 0.015 & 0.019 & 0.25 & 0.249 & 0.248 & 0.252 & 0.25 & 0.249 & 0.249 & 0.252 \\
\hline 474.49 & 0.014 & 0.017 & 0.249 & 0.249 & 0.247 & 0.251 & 0.248 & 0.249 & 0.248 & 0.251 \\
\hline 474.84 & 0.012 & 0.015 & 0.247 & 0.247 & 0.245 & 0.249 & 0.246 & 0.248 & 0.247 & 0.25 \\
\hline 475.2 & 0.011 & 0.014 & 0.246 & 0.247 & 0.243 & 0.247 & 0.245 & 0.246 & 0.245 & 0.248 \\
\hline 475.55 & 0.009 & 0.012 & 0.245 & 0.245 & 0.241 & 0.246 & 0.244 & 0.243 & 0.243 & 0.246 \\
\hline 475.9 & 0.007 & 0.01 & 0.243 & 0.243 & 0.24 & 0.244 & 0.243 & 0.241 & 0.241 & 0.245 \\
\hline 476.26 & 0.006 & 0.007 & 0.242 & 0.241 & 0.239 & 0.243 & 0.241 & 0.24 & 0.24 & 0.243 \\
\hline 476.61 & 0.004 & 0.005 & 0.24 & 0.24 & 0.238 & 0.241 & 0.238 & 0.239 & 0.238 & 0.24 \\
\hline 476.96 & 0.003 & 0.004 & 0.238 & 0.238 & 0.237 & 0.241 & 0.237 & 0.238 & 0.237 & 0.239 \\
\hline 477.32 & 0.003 & 0.003 & 0.236 & 0.237 & 0.236 & 0.239 & 0.236 & 0.238 & 0.235 & 0.236 \\
\hline 477.67 & 0 & 0.001 & 0.235 & 0.234 & 0.234 & 0.237 & 0.235 & 0.236 & 0.234 & 0.235 \\
\hline 478.03 & -0.001 & -0.001 & 0.234 & 0.233 & 0.233 & 0.236 & 0.233 & 0.235 & 0.233 & 0.233 \\
\hline 478.38 & -0.003 & -0.002 & 0.232 & 0.231 & 0.232 & 0.233 & 0.232 & 0.233 & 0.231 & 0.231 \\
\hline 478.73 & -0.004 & -0.004 & 0.23 & 0.23 & 0.232 & 0.232 & 0.23 & 0.232 & 0.23 & 0.23 \\
\hline 479.09 & -0.007 & -0.006 & 0.228 & 0.228 & 0.231 & 0.232 & 0.229 & 0.231 & 0.228 & 0.229 \\
\hline 479.44 & -0.008 & -0 . & 0.227 & 0.228 & 0.23 & 0.231 & 0.227 & 0.231 & 0.228 & 0.227 \\
\hline 479.79 & -0.008 & -0.009 & 0.227 & 0.227 & 0.229 & 0.23 & 0.226 & 0.231 & 0.228 & 0.227 \\
\hline 480.15 & -0.011 & -0.01 & 0.225 & 0.225 & 0.228 & 0.228 & 0.224 & 0.229 & 0.227 & 0.226 \\
\hline 480.5 & -0.013 & -0.012 & 0.224 & 0.224 & 0.226 & 0.227 & 0.223 & 0.227 & 0.226 & 0.225 \\
\hline 480.85 & -0.015 & -0.013 & 0.222 & 0.222 & 0.225 & 0.224 & 0.222 & 0.226 & 0.225 & 0.223 \\
\hline 481.21 & -0.016 & -0.015 & 0.221 & 0.221 & 0.224 & 0.223 & 0.221 & 0.225 & 0.223 & 0.223 \\
\hline 481.56 & -0.018 & -0.016 & 0.22 & 0.221 & 0.224 & 0.222 & 0.219 & 0.223 & 0.222 & 0.222 \\
\hline 481.91 & -0.02 & -0.018 & 0.218 & 0.218 & 0.222 & 0.22 & 0.218 & 0.221 & 0.22 & 0.219 \\
\hline 482.27 & -0.022 & -0.02 & 0.217 & 0.217 & 0.22 & 0.22 & 0.217 & 0.219 & 0.219 & 0.218 \\
\hline 482.62 & -0.024 & -0.022 & 0.216 & 0.216 & 0.219 & 0.219 & 0.216 & 0.218 & 0.218 & 0.217 \\
\hline 482.97 & -0.025 & -0.024 & 0.216 & 0.214 & 0.218 & 0.218 & 0.215 & 0.217 & 0.217 & 0.216 \\
\hline 483.33 & -0.027 & -0.025 & 0.215 & 0.212 & 0.217 & 0.216 & 0.216 & 0.215 & 0.217 & 0.215 \\
\hline 483.68 & -0.028 & -0.027 & 0.214 & 0.211 & 0.215 & 0.215 & 0.216 & 0.213 & 0.215 & 0.213 \\
\hline 484.03 & -0.029 & -0.028 & 0.214 & 0.212 & 0.215 & 0.215 & 0.216 & 0.213 & 0.214 & 0.213 \\
\hline 484.38 & -0.031 & -0.03 & 0.213 & 0.21 & 0.215 & 0.213 & 0.214 & 0.212 & 0.212 & 0.211 \\
\hline 484.74 & -0.032 & -0.031 & 0.212 & 0.209 & 0.213 & 0.213 & 0.214 & 0.21 & 0.211 & 0.21 \\
\hline 485.09 & -0.032 & -0.033 & 0.211 & 0.208 & 0.212 & 0.213 & 0.213 & 0.209 & 0.21 & 0.209 \\
\hline 485.44 & -0.034 & -0.034 & 0.21 & 0.208 & 0.212 & 0.212 & 0.213 & 0.209 & 0.21 & 0.209 \\
\hline 485.8 & -0.034 & -0.034 & 0.21 & 0.209 & 0.211 & 0.211 & 0.212 & 0.209 & 0.21 & 0.209 \\
\hline 486.15 & -0.035 & -0.034 & 0.21 & 0.208 & 0.211 & 0.21 & 0.211 & 0.208 & 0.209 & 0.208 \\
\hline 486.5 & -0.036 & -0.035 & 0.208 & 0.207 & 0.209 & 0.208 & 0.21 & 0.207 & 0.208 & 0.206 \\
\hline
\end{tabular}




\begin{tabular}{|c|c|c|c|c|c|c|c|c|c|c|}
\hline 486.85 & -0.037 & -0.036 & 0.207 & 0.207 & 0.208 & 0.207 & 0.209 & 0.207 & 0.207 & 0.205 \\
\hline 487.21 & -0.038 & -0.036 & 0.205 & 0.207 & 0.207 & 0.207 & 0.207 & 0.205 & 0.207 & 0.205 \\
\hline 487.56 & -0.039 & -0.036 & 0.204 & 0.206 & 0.206 & 0.206 & 0.207 & 0.206 & 0.207 & 0.205 \\
\hline 487.91 & -0.04 & -0.037 & 0.202 & 0.204 & 0.205 & 0.206 & 0.205 & 0.204 & 0.206 & 0.204 \\
\hline 488.26 & -0.041 & -0.038 & 0.201 & 0.203 & 0.204 & 0.204 & 0.205 & 0.203 & 0.206 & 0.203 \\
\hline 488.62 & -0.043 & -0.04 & 0.201 & 0.203 & 0.203 & 0.203 & 0.203 & 0.203 & 0.206 & 0.202 \\
\hline 488.97 & -0.043 & -0.04 & 0.201 & 0.202 & 0.203 & 0.202 & 0.203 & 0.203 & 0.205 & 0.201 \\
\hline 489.32 & -0.044 & -0.041 & 0.2 & 0.2 & 0.202 & 0.201 & 0.202 & 0.202 & 0.204 & 0.2 \\
\hline 489.67 & -0.046 & -0.043 & 0.198 & 0.199 & 0.201 & 0.2 & 0.2 & 0.201 & 0.202 & 0.199 \\
\hline 490.02 & -0.046 & -0.044 & 0.198 & 0.198 & 0.2 & 0.199 & 0.199 & 0.2 & 0.203 & 0.199 \\
\hline 490.38 & -0.047 & -0.045 & 0.197 & 0.197 & 0.199 & 0.198 & 0.2 & 0.198 & 0.201 & 0.199 \\
\hline 490.73 & -0.047 & -0.045 & 0.196 & 0.197 & 0.198 & 0.197 & 0.198 & 0.197 & 0.202 & 0.199 \\
\hline 491.08 & -0.048 & -0.046 & 0.196 & 0.196 & 0.197 & 0.196 & 0.197 & 0.197 & 0.2 & 0.197 \\
\hline 491.43 & -0.049 & -0.048 & 0.196 & 0.196 & 0.197 & 0.196 & 0.196 & 0.196 & 0.199 & 0.196 \\
\hline 491.79 & -0.05 & -0.048 & 0.195 & 0.195 & 0.196 & 0.195 & 0.195 & 0.196 & 0.199 & 0.195 \\
\hline 492.14 & -0.051 & -0.05 & 0.194 & 0.193 & 0.194 & 0.194 & 0.194 & 0.195 & 0.197 & 0.194 \\
\hline 492.49 & -0.052 & -0.051 & 0.193 & 0.192 & 0.193 & 0.193 & 0.194 & 0.194 & 0.196 & 0.193 \\
\hline 492.84 & -0.053 & -0.052 & 0.192 & 0.192 & 0.192 & 0.191 & 0.193 & 0.193 & 0.195 & 0.193 \\
\hline 493.19 & -0.054 & -0.053 & 0.192 & 0.191 & 0.19 & 0.19 & 0.192 & 0.192 & 0.193 & 0.192 \\
\hline 493.54 & -0.055 & -0.054 & 0.191 & 0.191 & 0.19 & 0.19 & 0.191 & 0.192 & 0.193 & 0.191 \\
\hline 493.9 & -0.057 & -0.055 & 0.189 & 0.189 & 0.188 & 0.189 & 0.19 & 0.191 & 0.191 & 0.19 \\
\hline 494.25 & -0.058 & -0.056 & 0.188 & 0.188 & 0.189 & 0.188 & 0.189 & 0.191 & 0.189 & 0.189 \\
\hline 494.6 & -0.059 & -0.057 & 0.187 & 0.188 & 0.188 & 0.188 & 0.189 & 0.19 & 0.188 & 0.189 \\
\hline 494.95 & -0.059 & -0.057 & 0.186 & 0.187 & 0.187 & 0.187 & 0.189 & 0.189 & 0.186 & 0.188 \\
\hline 495.3 & -0.061 & -0.058 & 0.185 & 0.186 & 0.185 & 0.185 & 0.187 & 0.188 & 0.186 & 0.187 \\
\hline 495.65 & -0.061 & -0.059 & 0.184 & 0.185 & 0.185 & 0.185 & 0.188 & 0.188 & 0.185 & 0.186 \\
\hline 496.01 & -0.062 & -0.059 & 0.184 & 0.185 & 0.185 & 0.185 & 0.187 & 0.187 & 0.185 & 0.186 \\
\hline 496.36 & -0.062 & -0.059 & 0.184 & 0.184 & 0.185 & 0.185 & 0.186 & 0.186 & 0.185 & 0.184 \\
\hline 496.71 & -0.063 & -0.06 & 0.182 & 0.183 & 0.184 & 0.185 & 0.184 & 0.185 & 0.184 & 0.184 \\
\hline 497.06 & -0.063 & -0.06 & 0.182 & 0.183 & 0.183 & 0.185 & 0.184 & 0.184 & 0.184 & 0.183 \\
\hline 497.41 & -0.064 & -0.061 & 0.181 & 0.182 & 0.182 & 0.185 & 0.183 & 0.183 & 0.183 & 0.183 \\
\hline 497.76 & -0.064 & -0.062 & 0.182 & 0.181 & 0.182 & 0.184 & 0.182 & 0.182 & 0.183 & 0.182 \\
\hline 498.11 & -0.064 & -0.062 & 0.182 & 0.182 & 0.181 & 0.184 & 0.182 & 0.182 & 0.183 & 0.182 \\
\hline 498.47 & -0.065 & -0.063 & 0.182 & 0.181 & 0.18 & 0.182 & 0.181 & 0.182 & 0.182 & 0.181 \\
\hline 498.82 & -0.065 & -0.063 & 0.182 & 0.181 & 0.181 & 0.182 & 0.18 & 0.181 & 0.183 & 0.181 \\
\hline 499.17 & -0.066 & -0.064 & 0.182 & 0.181 & 0.181 & 0.182 & 0.18 & 0.181 & 0.182 & 0.181 \\
\hline 499.52 & -0.066 & -0.064 & 0.18 & 0.181 & 0.181 & 0.18 & 0.179 & 0.18 & 0.181 & 0.18 \\
\hline 499.87 & -0.067 & -0.064 & 0.18 & 0.18 & 0.179 & 0.179 & 0.179 & 0.179 & 0.179 & 0.18 \\
\hline 500.22 & -0.067 & -0.065 & 0.179 & 0.18 & 0.179 & 0.178 & 0.178 & 0.179 & 0.179 & 0.18 \\
\hline
\end{tabular}




\begin{tabular}{|c|c|c|c|c|c|c|c|c|c|c|}
\hline 500.57 & -0.067 & -0.065 & 0.179 & 0.179 & 0.179 & 0.178 & 0.178 & 0.179 & 0.179 & 0.18 \\
\hline 500.92 & -0.067 & -0.065 & 0.178 & 0.179 & 0.179 & 0.176 & 0.178 & 0.178 & 0.178 & 0.178 \\
\hline 501.27 & -0.068 & -0.065 & 0.178 & 0.179 & 0.18 & 0.176 & 0.177 & 0.178 & 0.178 & 0.179 \\
\hline 501.62 & -0.068 & -0.065 & 0.177 & 0.178 & 0.179 & 0.176 & 0.177 & 0.178 & 0.177 & 0.177 \\
\hline 501.97 & -0.069 & -0.066 & 0.177 & 0.178 & 0.179 & 0.175 & 0.176 & 0.178 & 0.177 & 0.176 \\
\hline 502.33 & -0.069 & -0.066 & 0.176 & 0.177 & 0.178 & 0.175 & 0.176 & 0.177 & 0.177 & 0.176 \\
\hline 502.68 & -0.069 & -0.067 & 0.175 & 0.176 & 0.177 & 0.175 & 0.176 & 0.178 & 0.177 & 0.176 \\
\hline 503.03 & -0.069 & -0.067 & 0.175 & 0.175 & 0.176 & 0.175 & 0.176 & 0.178 & 0.177 & 0.177 \\
\hline 503.38 & -0.07 & -0.068 & 0.175 & 0.174 & 0.175 & 0.175 & 0.176 & 0.178 & 0.177 & 0.177 \\
\hline 503.73 & -0.07 & -0.068 & 0.174 & 0.174 & 0.175 & 0.176 & 0.176 & 0.177 & 0.177 & 0.176 \\
\hline 504.08 & -0.07 & -0.069 & 0.173 & 0.173 & 0.174 & 0.175 & 0.176 & 0.176 & 0.176 & 0.176 \\
\hline 504.43 & -0.07 & -0.069 & 0.174 & 0.173 & 0.174 & 0.175 & 0.177 & 0.176 & 0.176 & 0.176 \\
\hline 504.78 & -0.071 & -0.069 & 0.173 & 0.172 & 0.173 & 0.175 & 0.176 & 0.176 & 0.177 & 0.176 \\
\hline 505.13 & -0.071 & -0.069 & 0.171 & 0.172 & 0.172 & 0.174 & 0.176 & 0.175 & 0.176 & 0.175 \\
\hline 505.48 & -0.071 & -0.069 & 0.171 & 0.172 & 0.172 & 0.174 & 0.177 & 0.175 & 0.177 & 0.176 \\
\hline 505.83 & -0.07 & -0.069 & 0.171 & 0.173 & 0.171 & 0.174 & 0.177 & 0.175 & 0.176 & 0.176 \\
\hline 506.18 & -0.07 & -0.069 & 0.171 & 0.172 & 0.171 & 0.174 & 0.177 & 0.174 & 0.175 & 0.176 \\
\hline 506.53 & -0.071 & -0.069 & 0.171 & 0.171 & 0.171 & 0.173 & 0.175 & 0.172 & 0.174 & 0.175 \\
\hline 506.88 & -0.072 & -0.071 & 0.171 & 0.17 & 71 & 0.173 & 0.174 & 0.171 & 0.173 & 0.172 \\
\hline 507.23 & -0.072 & -0.071 & 0.171 & 0.171 & 0.171 & 0.172 & 0.173 & 0.172 & 0.173 & 0.171 \\
\hline 507.58 & -0.072 & -0.071 & 0.171 & 0.172 & 0.171 & 0.172 & 0.172 & 0.172 & 0.174 & 0.172 \\
\hline 507.93 & -0.071 & -0.07 & 0.172 & 0.172 & 0.172 & 0.173 & 0.172 & 0.173 & 0.174 & 0.172 \\
\hline 508.28 & -0.071 & -0.07 & 0.171 & 0.172 & 0.171 & 0.173 & 0.172 & 0.173 & 0.174 & 0.171 \\
\hline 508.63 & -0.071 & -0.07 & 0.171 & 0.172 & 0.171 & 0.173 & 0.172 & 0.172 & 0.172 & 0.171 \\
\hline 508.98 & -0.071 & -0.069 & 0.172 & 0.172 & 0.172 & 0.174 & 0.172 & 0.173 & 0.173 & 0.171 \\
\hline 509.33 & -0.071 & -0.07 & 0.172 & 0.171 & 0.172 & 0.173 & 0.172 & 0.173 & 0.173 & 0.171 \\
\hline 509.68 & -0.071 & -0.07 & 0.171 & 0.17 & 0.172 & 0.172 & 0.17 & 0.172 & 0.172 & 0.17 \\
\hline 510.03 & -0.072 & -0.071 & 0.171 & 0.17 & 0.171 & 0.172 & 0.169 & 0.172 & 0.172 & 0.17 \\
\hline 510.38 & -0.071 & -0.071 & 0.17 & 0.17 & 0.171 & 0.172 & 0.17 & 0.172 & 0.173 & 0.171 \\
\hline 510.73 & -0.07 & -0.07 & 0.171 & 0.171 & 0.172 & 0.173 & 0.171 & 0.173 & 0.173 & 0.173 \\
\hline 511.08 & -0.071 & -0.07 & 0.17 & 0.171 & 0.172 & 0.174 & 0.171 & 0.172 & 0.172 & 0.173 \\
\hline 511.43 & -0.071 & -0.071 & 0.17 & 0.17 & 0.172 & 0.173 & 0.171 & 0.172 & 0.172 & 0.173 \\
\hline 511.78 & -0.07 & -0.071 & 0.17 & 0.17 & 0.172 & 0.173 & 0.172 & 0.172 & 0.173 & 0.174 \\
\hline 512.13 & -0.07 & -0.071 & 0.171 & 0.171 & 0.173 & 0.173 & 0.172 & 0.173 & 0.173 & 0.174 \\
\hline 512.48 & -0.069 & -0.07 & 0.172 & 0.171 & 0.174 & 0.173 & 0.173 & 0.174 & 0.174 & 0.175 \\
\hline 512.83 & -0.07 & -0.071 & 0.171 & 0.172 & 0.174 & 0.173 & 0.173 & 0.174 & 0.174 & 0.175 \\
\hline 513.18 & -0.07 & -0.071 & 0.171 & 0.172 & 0.173 & 0.173 & 0.173 & 0.174 & 0.174 & 0.175 \\
\hline 513.53 & -0.069 & -0.07 & 0.171 & 0.172 & 0.174 & 0.173 & 0.173 & 0.175 & 0.175 & 0.175 \\
\hline 513.88 & -0.069 & -0.07 & 0.171 & 0.173 & 0.175 & 0.173 & 0.173 & 0.175 & 0.176 & 0.175 \\
\hline
\end{tabular}




\begin{tabular}{|c|c|c|c|c|c|c|c|c|c|c|}
\hline 514.22 & -0.07 & -0.07 & 0.171 & 0.173 & 0.175 & 0.173 & 0.173 & 0.175 & 0.175 & 0.175 \\
\hline 514.57 & -0.07 & -0.07 & 0.171 & 0.173 & 0.174 & 0.172 & 0.173 & 0.175 & 0.175 & 0.175 \\
\hline 514.92 & -0.07 & -0.07 & 0.172 & 0.173 & 0.174 & 0.171 & 0.174 & 0.174 & 0.176 & 0.174 \\
\hline 515.27 & -0.07 & -0.07 & 0.172 & 0.173 & 0.175 & 0.171 & 0.174 & 0.175 & 0.175 & 0.175 \\
\hline 515.62 & -0.071 & -0.07 & 0.172 & 0.172 & 0.174 & 0.171 & 0.173 & 0.173 & 0.174 & 0.174 \\
\hline 515.97 & -0.071 & -0.07 & 0.171 & 0.171 & 0.174 & 0.17 & 0.172 & 0.173 & 0.174 & 0.174 \\
\hline 516.32 & -0.072 & -0.072 & 0.17 & 0.17 & 0.173 & 0.17 & 0.172 & 0.172 & 0.173 & 0.173 \\
\hline 516.67 & -0.072 & -0.072 & 0.17 & 0.17 & 0.173 & 0.172 & 0.172 & 0.171 & 0.172 & 0.173 \\
\hline 517.02 & -0.072 & -0.072 & 0.17 & 0.171 & 0.174 & 0.172 & 0.172 & 0.171 & 0.172 & 0.173 \\
\hline 517.37 & -0.072 & -0.072 & 0.17 & 0.171 & 0.175 & 0.173 & 0.172 & 0.172 & 0.172 & 0.173 \\
\hline 517.72 & -0.072 & -0.072 & 0.17 & 0.171 & 0.174 & 0.173 & 0.173 & 0.172 & 0.171 & 0.173 \\
\hline 518.06 & -0.072 & -0.072 & 0.171 & 0.171 & 0.173 & 0.173 & 0.173 & 0.172 & 0.171 & 0.173 \\
\hline 518.41 & -0.072 & -0.072 & 0.17 & 0.172 & 0.173 & 0.174 & 0.173 & 0.172 & 0.171 & 0.172 \\
\hline 518.76 & -0.071 & -0.071 & 0.171 & 0.172 & 0.173 & 0.175 & 0.173 & 0.173 & 0.172 & 0.173 \\
\hline 519.11 & -0.071 & -0.072 & 0.171 & 0.172 & 0.172 & 0.174 & 0.174 & 0.172 & 0.172 & 0.172 \\
\hline 519.46 & -0.071 & -0.071 & 0.172 & 0.173 & 0.172 & 0.174 & 0.174 & 0.173 & 0.172 & 0.172 \\
\hline 519.81 & -0.071 & -0.071 & 0.172 & 0.173 & 0.172 & 0.174 & 0.174 & 0.173 & 0.171 & 0.173 \\
\hline 520.16 & -0.07 & -0.07 & 0.173 & 0.174 & 0.172 & 0.175 & 0.175 & 0.174 & 0.172 & 0.174 \\
\hline 520.5 & -0.07 & -0.069 & 0.173 & 0.174 & 172 & 0. & 0.175 & 0.174 & 0.172 & 0.173 \\
\hline 520.85 & -0.069 & -0.069 & 0.174 & 0.174 & 0.172 & 0.174 & 0.176 & 0.175 & 0.173 & 0.174 \\
\hline 521.2 & -0.069 & -0.068 & 0.174 & 0.174 & 0.172 & 0.175 & 0.176 & 0.174 & 0.173 & 0.174 \\
\hline 521.55 & -0.068 & -0.067 & 0.174 & 0.176 & 0.172 & 0.175 & 0.177 & 0.175 & 0.174 & 0.175 \\
\hline 521.9 & -0.068 & -0.067 & 0.175 & 0.176 & 0.173 & 0.175 & 0.176 & 0.175 & 0.174 & 0.174 \\
\hline 522.25 & -0.068 & -0.066 & 0.175 & 0.175 & 0.172 & 0.176 & 0.176 & 0.175 & 0.174 & 0.175 \\
\hline 522.59 & -0.069 & -0.066 & 0.174 & 0.175 & 0.173 & 0.176 & 0.176 & 0.176 & 0.174 & 0.175 \\
\hline 522.94 & -0.068 & -0.066 & 0.174 & 0.175 & 0.174 & 0.177 & 0.176 & 0.176 & 0.174 & 0.176 \\
\hline 523.29 & -0.068 & -0.066 & 0.175 & 0.177 & 0.175 & 0.178 & 0.177 & 0.177 & 0.175 & 0.177 \\
\hline 523.64 & -0.068 & -0.065 & 0.175 & 0.177 & 0.175 & 0.179 & 0.177 & 0.177 & 0.176 & 0.177 \\
\hline 523.99 & -0.068 & -0.066 & 0.175 & 0.177 & 0.174 & 0.178 & 0.177 & 0.176 & 0.176 & 0.177 \\
\hline 524.33 & -0.069 & -0.066 & 0.175 & 0.177 & 0.174 & 0.178 & 0.177 & 0.177 & 0.177 & 0.178 \\
\hline 524.68 & -0.069 & -0.066 & 0.175 & 0.176 & 0.174 & 0.177 & 0.176 & 0.177 & 0.177 & 0.177 \\
\hline 525.03 & -0.068 & -0.066 & 0.176 & 0.177 & 0.175 & 0.178 & 0.177 & 0.178 & 0.178 & 0.178 \\
\hline 525.38 & -0.068 & -0.066 & 0.177 & 0.177 & 0.175 & 0.178 & 0.176 & 0.179 & 0.178 & 0.178 \\
\hline 525.73 & -0.067 & -0.065 & 0.177 & 0.178 & 0.177 & 0.178 & 0.177 & 0.18 & 0.18 & 0.179 \\
\hline 526.07 & -0.066 & -0.065 & 0.178 & 0.179 & 0.178 & 0.179 & 0.178 & 0.181 & 0.18 & 0.18 \\
\hline 526.42 & -0.065 & -0.064 & 0.179 & 0.18 & 0.179 & 0.179 & 0.178 & 0.18 & 0.18 & 0.18 \\
\hline 526.77 & -0.064 & -0.063 & 0.179 & 0.18 & 0.179 & 0.18 & 0.178 & 0.181 & 0.181 & 0.18 \\
\hline 527.12 & -0.064 & -0.063 & 0.179 & 0.18 & 0.178 & 0.18 & 0.179 & 0.18 & 0.182 & 0.179 \\
\hline 527.46 & -0.063 & -0.063 & 0.179 & 0.179 & 0.179 & 0.18 & 0.178 & 0.181 & 0.182 & 0.179 \\
\hline
\end{tabular}




\begin{tabular}{|c|c|c|c|c|c|c|c|c|c|c|}
\hline 527.81 & -0.062 & -0.062 & 0.18 & 0.18 & 0.18 & 0.181 & 0.179 & 0.182 & 0.182 & 0.179 \\
\hline 528.16 & -0.062 & -0.061 & 0.181 & 0.18 & 0.18 & 0.181 & 0.179 & 0.182 & 0.182 & 0.179 \\
\hline 528.51 & -0.062 & -0.062 & 0.18 & 0.18 & 0.181 & 0.182 & 0.179 & 0.181 & 0.182 & 0.179 \\
\hline 528.85 & -0.062 & -0.062 & 0.18 & 0.18 & 0.181 & 0.182 & 0.179 & 0.181 & 0.182 & 0.179 \\
\hline 529.2 & -0.061 & -0.061 & 0.181 & 0.18 & 0.182 & 0.183 & 0.18 & 0.181 & 0.183 & 0.18 \\
\hline 529.55 & -0.062 & -0.062 & 0.181 & 0.181 & 0.181 & 0.183 & 0.181 & 0.18 & 0.183 & 0.18 \\
\hline 529.9 & -0.062 & -0.062 & 0.181 & 0.18 & 0.181 & 0.182 & 0.181 & 0.179 & 0.183 & 0.18 \\
\hline 530.24 & -0.062 & -0.061 & 0.182 & 0.181 & 0.181 & 0.183 & 0.182 & 0.179 & 0.184 & 0.18 \\
\hline 530.59 & -0.062 & -0.061 & 0.181 & 0.18 & 0.182 & 0.183 & 0.183 & 0.18 & 0.183 & 0.18 \\
\hline 530.94 & -0.061 & -0.061 & 0.182 & 0.18 & 0.182 & 0.182 & 0.183 & 0.18 & 0.183 & 0.181 \\
\hline 531.29 & -0.061 & -0.06 & 0.182 & 0.181 & 0.183 & 0.183 & 0.183 & 0.18 & 0.184 & 0.182 \\
\hline 531.63 & -0.06 & -0.06 & 0.182 & 0.18 & 0.183 & 0.183 & 0.182 & 0.18 & 0.184 & 0.182 \\
\hline 531.98 & -0.059 & -0.059 & 0.183 & 0.181 & 0.183 & 0.183 & 0.184 & 0.181 & 0.184 & 0.183 \\
\hline 532.33 & -0.058 & -0.058 & 0.184 & 0.182 & 0.185 & 0.184 & 0.185 & 0.183 & 0.186 & 0.184 \\
\hline 532.67 & -0.057 & -0.057 & 0.184 & 0.182 & 0.184 & 0.183 & 0.186 & 0.183 & 0.186 & 0.184 \\
\hline 533.02 & -0.057 & -0.057 & 0.185 & 0.182 & 0.185 & 84 & 86 & 0.184 & 0.186 & 0.185 \\
\hline 533.37 & -0.056 & -0.056 & 0.185 & 0.183 & 0.186 & 0.184 & 0.186 & 0.185 & 0.186 & 0.185 \\
\hline 533.71 & -0.055 & -0.055 & 0.186 & 0.184 & 0.187 & 0.185 & 0.187 & 0.186 & 0.186 & 0.186 \\
\hline 534.06 & -0.055 & -0.054 & 0.186 & 0.184 & 0.187 & 0.186 & 0.187 & 0.187 & 0.186 & 0.185 \\
\hline 534.41 & -0.054 & -0.053 & 0.187 & 0.186 & 0.189 & 0.188 & 0.187 & 0.188 & 0.188 & 0.186 \\
\hline 534.75 & -0.053 & -0.052 & 0.189 & 0.188 & 0.189 & 0.189 & 0.187 & 0.189 & 0.189 & 0.188 \\
\hline 535.1 & -0.051 & -0.051 & 0.19 & 0.189 & 0.19 & 0.19 & 0.189 & 0.191 & 0.189 & 0.188 \\
\hline 535.45 & -0.051 & -0.05 & 0.19 & 0.19 & 0.191 & 0.19 & 0.191 & 0.192 & 0.19 & 0.189 \\
\hline 535.79 & -0.05 & -0.049 & 0.191 & 0.191 & 0.192 & 0.192 & 0.192 & 0.193 & 0.191 & 0.191 \\
\hline 536.14 & -0.049 & -0.049 & 0.193 & 0.191 & 0.192 & 0.192 & 0.192 & 0.193 & 0.192 & 0.192 \\
\hline 536.49 & -0.048 & -0.048 & 0.194 & 0.192 & 0.193 & 0.194 & 0.194 & 0.195 & 0.194 & 0.193 \\
\hline 536.83 & -0.047 & -0.047 & 0.194 & 0.194 & 0.194 & 0.194 & 0.195 & 0.196 & 0.194 & 0.193 \\
\hline 537.18 & -0.046 & -0.046 & 0.195 & 0.195 & 0.194 & 0.196 & 0.195 & 0.197 & 0.195 & 0.193 \\
\hline 537.53 & -0.045 & -0.046 & 0.195 & 0.195 & 0.196 & 0.196 & 0.196 & 0.199 & 0.196 & 0.195 \\
\hline 537.87 & -0.044 & -0.045 & 0.196 & 0.195 & 0.195 & 0.197 & 0.197 & 0.199 & 0.197 & 0.196 \\
\hline 538.22 & -0.043 & -0.044 & 0.197 & 0.194 & 0.195 & 0.197 & 0.197 & 0.2 & 0.197 & 0.197 \\
\hline 538.57 & -0.043 & -0.044 & 0.197 & 0.194 & 0.196 & 0.197 & 0.198 & 0.199 & 0.198 & 0.197 \\
\hline 538.91 & -0.042 & -0.043 & 0.197 & 0.195 & 0.197 & 0.198 & 0.199 & 0.2 & 0.198 & 0.198 \\
\hline 539.26 & -0.042 & -0.042 & 0.198 & 0.196 & 0.198 & 0.199 & 0.2 & 0.2 & 0.2 & 0.199 \\
\hline 539.6 & -0.041 & -0.042 & 0.199 & 0.196 & 0.199 & 0.199 & 0.201 & 0.201 & 0.2 & 0.199 \\
\hline 539.95 & -0.041 & -0.041 & 0.199 & 0.198 & 0.2 & 0.199 & 0.202 & 0.202 & 0.2 & 0.199 \\
\hline 540.3 & -0.041 & -0.041 & 0.199 & 0.199 & 0.2 & 0.2 & 0.202 & 0.201 & 0.2 & 0.199 \\
\hline 540.64 & -0.04 & -0.04 & 0.201 & 0.2 & 0.201 & 0.202 & 0.202 & 0.201 & 0.201 & 0.201 \\
\hline 540.99 & -0.039 & -0.038 & 0.201 & 0.2 & 0.202 & 0.202 & 0.203 & 0.202 & 0.203 & 0.201 \\
\hline
\end{tabular}




\begin{tabular}{|c|c|c|c|c|c|c|c|c|c|c|}
\hline 541.33 & -0.039 & -0.037 & 0.202 & 0.202 & 0.203 & 0.203 & 0.204 & 0.202 & 0.204 & 0.202 \\
\hline 541.68 & -0.038 & -0.037 & 0.203 & 0.203 & 0.205 & 0.205 & 0.205 & 0.203 & 0.205 & 0.203 \\
\hline 542.02 & -0.039 & -0.036 & 0.204 & 0.205 & 0.205 & 0.206 & 0.206 & 0.204 & 0.206 & 0.204 \\
\hline 542.37 & -0.038 & -0.036 & 0.204 & 0.205 & 0.206 & 0.207 & 0.207 & 0.205 & 0.206 & 0.204 \\
\hline 542.72 & -0.037 & -0.035 & 0.205 & 0.205 & 0.207 & 0.207 & 0.206 & 0.206 & 0.206 & 0.205 \\
\hline 543.06 & -0.035 & -0.034 & 0.206 & 0.207 & 0.207 & 0.208 & 0.206 & 0.207 & 0.207 & 0.206 \\
\hline 543.41 & -0.035 & -0.033 & 0.207 & 0.207 & 0.208 & 0.209 & 0.207 & 0.207 & 0.207 & 0.206 \\
\hline 543.75 & -0.033 & -0.031 & 0.207 & 0.208 & 0.208 & 0.21 & 0.208 & 0.208 & 0.208 & 0.208 \\
\hline 544.1 & -0.032 & -0.03 & 0.208 & 0.209 & 0.209 & 0.211 & 0.208 & 0.21 & 0.209 & 0.209 \\
\hline 544.44 & -0.031 & -0.029 & 0.208 & 0.209 & 0.209 & 0.21 & 0.208 & 0.211 & 0.209 & 0.209 \\
\hline 544.79 & -0.03 & -0.028 & 0.208 & 0.209 & 0.209 & 0.211 & 0.209 & 0.21 & 0.209 & 0.209 \\
\hline 545.13 & -0.028 & -0.027 & 0.21 & 0.21 & 0.209 & 0.212 & 0.21 & 0.211 & 0.21 & 0.21 \\
\hline 545.48 & -0.027 & -0.026 & 0.211 & 0.211 & 0.21 & 0.212 & 0.21 & 0.212 & 0.21 & 0.211 \\
\hline 545.82 & -0.026 & -0.025 & 0.211 & 0.211 & 0.21 & 0.212 & 0.211 & 0.212 & 0.21 & 0.211 \\
\hline 546.17 & -0.024 & -0.024 & 0.212 & 0.212 & 0.21 & 0.213 & 0.212 & 0.212 & 0.211 & 0.212 \\
\hline 546.52 & -0.023 & -0.023 & 0.213 & 0.213 & 0.212 & 0.214 & 0.214 & 0.214 & 0.212 & 0.212 \\
\hline 546.86 & -0.023 & -0.022 & 0.214 & 0.214 & 0.213 & 0.215 & 0.215 & 0.215 & 0.214 & 0.213 \\
\hline 547.21 & -0.021 & -0.021 & 0.215 & 0.216 & 0.214 & 0.216 & 0.216 & 0.216 & 0.215 & 0.215 \\
\hline 547.55 & -0.021 & -0.02 & 0.216 & 0.216 & 0.214 & 0.216 & 0.217 & 0.216 & 0.216 & 0.216 \\
\hline 547.9 & -0.019 & -0.019 & 0.217 & 0.217 & 0.216 & 0.218 & 0.219 & 0.218 & 0.217 & 0.217 \\
\hline 548.24 & -0.018 & -0.018 & 0.218 & 0.217 & 0.218 & 0.22 & 0.22 & 0.219 & 0.219 & 0.218 \\
\hline 548.59 & -0.017 & -0.017 & 0.22 & 0.218 & 0.219 & 0.221 & 0.222 & 0.221 & 0.22 & 0.219 \\
\hline 548.93 & -0.016 & -0.016 & 0.221 & 0.22 & 0.22 & 0.222 & 0.223 & 0.222 & 0.222 & 0.221 \\
\hline 549.28 & -0.015 & -0.015 & 0.222 & 0.221 & 0.221 & 0.223 & 0.224 & 0.224 & 0.223 & 0.222 \\
\hline 549.62 & -0.013 & -0.013 & 0.223 & 0.222 & 0.223 & 0.224 & 0.225 & 0.226 & 0.225 & 0.225 \\
\hline 549.96 & -0.011 & -0.012 & 0.224 & 0.223 & 0.225 & 0.226 & 0.227 & 0.228 & 0.226 & 0.227 \\
\hline 550.31 & -0.011 & -0.01 & 0.224 & 0.225 & 0.224 & 0.226 & 0.227 & 0.227 & 0.226 & 0.228 \\
\hline 550.65 & -0.008 & -0.009 & 0.225 & 0.226 & 0.225 & 0.227 & 0.228 & 0.228 & 0.227 & 0.229 \\
\hline 551 & -0.007 & -0.007 & 0.226 & 0.226 & 0.225 & 0.227 & 0.229 & 0.229 & 0.228 & 0.23 \\
\hline 551.34 & -0.006 & -0.006 & 0.228 & 0.227 & 0.227 & 0.229 & 0.23 & 0.23 & 0.229 & 0.231 \\
\hline 551.69 & -0.005 & -0.005 & 0.229 & 0.229 & 0.229 & 0.231 & 0.231 & 0.231 & 0.23 & 0.233 \\
\hline 552.03 & -0.003 & -0.003 & 0.231 & 0.231 & 0.23 & 0.232 & 0.233 & 0.233 & 0.232 & 0.234 \\
\hline 552.38 & -0.002 & -0.002 & 0.233 & 0.232 & 0.232 & 0.234 & 0.234 & 0.234 & 0.233 & 0.236 \\
\hline 552.72 & -0.001 & 0 & 0.235 & 0.232 & 0.234 & 0.234 & 0.235 & 0.236 & 0.234 & 0.236 \\
\hline 553.07 & 0 & 0.001 & 0.236 & 0.233 & 0.235 & 0.236 & 0.236 & 0.236 & 0.235 & 0.237 \\
\hline 553.41 & 0.002 & 0.003 & 0.237 & 0.235 & 0.237 & 0.238 & 0.238 & 0.238 & 0.237 & 0.238 \\
\hline 553.75 & 0.003 & 0.005 & 0.239 & 0.236 & 0.238 & 0.239 & 0.24 & 0.24 & 0.239 & 0.239 \\
\hline 554.1 & 0.005 & 0.006 & 0.24 & 0.237 & 0.241 & 0.241 & 0.242 & 0.243 & 0.241 & 0.24 \\
\hline 554.44 & 0.006 & 0.008 & 0.242 & 0.238 & 0.243 & 0.243 & 0.243 & 0.245 & 0.242 & 0.242 \\
\hline
\end{tabular}




\begin{tabular}{|c|c|c|c|c|c|c|c|c|c|c|}
\hline 554.79 & 0.008 & 0.009 & 0.243 & 0.239 & 0.244 & 0.245 & 0.245 & 0.247 & 0.244 & 0.243 \\
\hline 555.13 & 0.009 & 0.01 & 0.244 & 0.24 & 0.246 & 0.246 & 0.246 & 0.248 & 0.246 & 0.244 \\
\hline 555.47 & 0.011 & 0.012 & 0.246 & 0.243 & 0.246 & 0.247 & 0.248 & 0.249 & 0.247 & 0.245 \\
\hline 555.82 & 0.013 & 0.013 & 0.248 & 0.244 & 0.247 & 0.248 & 0.25 & 0.251 & 0.248 & 0.247 \\
\hline 556.16 & 0.015 & 0.015 & 0.249 & 0.246 & 0.249 & 0.25 & 0.252 & 0.253 & 0.251 & 0.249 \\
\hline 556.51 & 0.017 & 0.017 & 0.25 & 0.248 & 0.25 & 0.252 & 0.253 & 0.254 & 0.252 & 0.251 \\
\hline 556.85 & 0.02 & 0.019 & 0.252 & 0.25 & 0.252 & 0.253 & 0.255 & 0.255 & 0.253 & 0.253 \\
\hline 557.19 & 0.022 & 0.022 & 0.254 & 0.252 & 0.252 & 0.255 & 0.256 & 0.256 & 0.254 & 0.254 \\
\hline 557.54 & 0.024 & 0.023 & 0.256 & 0.253 & 0.254 & 0.257 & 0.258 & 0.257 & 0.256 & 0.256 \\
\hline 557.88 & 0.025 & 0.025 & 0.257 & 0.255 & 0.254 & 0.258 & 0.26 & 0.258 & 0.257 & 0.258 \\
\hline 558.22 & 0.028 & 0.028 & 0.259 & 0.258 & 0.256 & 0.26 & 0.262 & 0.259 & 0.258 & 0.26 \\
\hline 558.57 & 0.03 & 0.03 & 0.261 & 0.26 & 0.258 & 0.261 & 0.264 & 0.26 & 0.26 & 0.262 \\
\hline 558.91 & 0.033 & 0.033 & 0.263 & 0.262 & 0.26 & 0.263 & 0.266 & 0.262 & 0.261 & 0.265 \\
\hline 559.26 & 0.035 & 0.035 & 0.264 & 0.264 & 0.262 & 0.264 & 0.267 & 0.263 & 0.263 & 0.266 \\
\hline 559.6 & 0.036 & 0.036 & 0.265 & 0.266 & 0.265 & 0.266 & 0.268 & 0.264 & 0.264 & 0.267 \\
\hline 559.94 & 0.038 & 0.038 & 0.268 & 0.267 & 0.266 & 0.268 & 0.269 & 0.265 & 0.265 & 0.268 \\
\hline 560.29 & 0.04 & 0.04 & 0.269 & 0.269 & 0.267 & 0.269 & 0.272 & 0.266 & 0.267 & 0.269 \\
\hline 560.63 & 0.041 & 0.041 & 0.27 & 0.27 & 0.268 & 0.271 & 0.272 & 0.268 & 0.269 & 0.27 \\
\hline 560.97 & 0.043 & 0.043 & 0.272 & 0.273 & 0.271 & 0.273 & 0.275 & 0.27 & 0.271 & 0.272 \\
\hline 561.32 & 0.045 & 0.045 & 0.273 & 0.275 & 0.273 & 0.274 & 0.276 & 0.272 & 0.273 & 0.274 \\
\hline 561.66 & 0.047 & 0.047 & 0.276 & 0.277 & 0.277 & 0.277 & 0.277 & 0.274 & 0.275 & 0.275 \\
\hline 562 & 0.048 & 0.049 & 0.276 & 0.279 & 0.278 & 0.278 & 0.279 & 0.277 & 0.277 & 0.276 \\
\hline 562.34 & 0.051 & 0.051 & 0.278 & 0.281 & 0.28 & 0.28 & 0.282 & 0.28 & 0.28 & 0.278 \\
\hline 562.69 & 0.052 & 0.052 & 0.28 & 0.282 & 0.281 & 0.282 & 0.283 & 0.282 & 0.281 & 0.279 \\
\hline 563.03 & 0.054 & 0.055 & 0.282 & 0.283 & 0.282 & 0.284 & 0.285 & 0.284 & 0.284 & 0.28 \\
\hline 563.37 & 0.056 & 0.057 & 0.284 & 0.284 & 0.283 & 0.285 & 0.287 & 0.286 & 0.285 & 0.282 \\
\hline 563.72 & 0.058 & 0.059 & 0.286 & 0.286 & 0.285 & 0.286 & 0.289 & 0.289 & 0.288 & 0.284 \\
\hline 564.06 & 0.06 & 0.062 & 0.288 & 0.288 & 0.287 & 0.287 & 0.291 & 0.292 & 0.29 & 0.286 \\
\hline 564.4 & 0.063 & 0.064 & 0.29 & 0.291 & 0.29 & 0.29 & 0.293 & 0.295 & 0.294 & 0.29 \\
\hline 564.75 & 0.065 & 0.066 & 0.292 & 0.293 & 0.292 & 0.291 & 0.295 & 0.296 & 0.295 & 0.292 \\
\hline 565.09 & 0.067 & 0.068 & 0.294 & 0.294 & 0.293 & 0.293 & 0.297 & 0.298 & 0.297 & 0.293 \\
\hline 565.43 & 0.069 & 0.07 & 0.294 & 0.296 & 0.294 & 0.295 & 0.3 & 0.3 & 0.299 & 0.295 \\
\hline 565.77 & 0.071 & 0.072 & 0.297 & 0.297 & 0.296 & 0.298 & 0.303 & 0.302 & 0.3 & 0.298 \\
\hline 566.12 & 0.072 & 0.073 & 0.298 & 0.299 & 0.298 & 0.299 & 0.303 & 0.301 & 0.301 & 0.298 \\
\hline 566.46 & 0.075 & 0.076 & 0.299 & 0.302 & 0.3 & 0.301 & 0.306 & 0.303 & 0.303 & 0.302 \\
\hline 566.8 & 0.078 & 0.078 & 0.3 & 0.304 & 0.302 & 0.304 & 0.307 & 0.304 & 0.305 & 0.304 \\
\hline 567.14 & 0.081 & 0.081 & 0.302 & 0.306 & 0.304 & 0.306 & 0.309 & 0.306 & 0.307 & 0.306 \\
\hline 567.49 & 0.084 & 0.083 & 0.305 & 0.308 & 0.307 & 0.31 & 0.311 & 0.307 & 0.308 & 0.308 \\
\hline 567.83 & 0.086 & 0.085 & 0.306 & 0.31 & 0.309 & 0.313 & 0.312 & 0.31 & 0.31 & 0.31 \\
\hline
\end{tabular}




\begin{tabular}{|c|c|c|c|c|c|c|c|c|c|c|}
\hline 568.17 & 0.088 & 0.087 & 0.308 & 0.311 & 0.311 & 0.314 & 0.313 & 0.311 & 0.311 & 0.311 \\
\hline 568.51 & 0.089 & 0.089 & 0.31 & 0.312 & 0.312 & 0.316 & 0.314 & 0.313 & 0.312 & 0.312 \\
\hline 568.85 & 0.092 & 0.092 & 0.313 & 0.315 & 0.314 & 0.318 & 0.316 & 0.315 & 0.314 & 0.313 \\
\hline 569.2 & 0.094 & 0.094 & 0.316 & 0.316 & 0.317 & 0.318 & 0.317 & 0.317 & 0.316 & 0.316 \\
\hline 569.54 & 0.097 & 0.097 & 0.317 & 0.319 & 0.319 & 0.319 & 0.318 & 0.318 & 0.319 & 0.317 \\
\hline 569.88 & 0.1 & 0.099 & 0.319 & 0.32 & 0.321 & 0.321 & 0.319 & 0.321 & 0.321 & 0.319 \\
\hline 570.22 & 0.101 & 0.101 & 0.321 & 0.321 & 0.323 & 0.322 & 0.32 & 0.322 & 0.322 & 0.32 \\
\hline 570.57 & 0.103 & 0.102 & 0.323 & 0.323 & 0.324 & 0.323 & 0.322 & 0.323 & 0.324 & 0.322 \\
\hline 570.91 & 0.104 & 0.104 & 0.324 & 0.325 & 0.326 & 0.325 & 0.323 & 0.325 & 0.325 & 0.323 \\
\hline 571.25 & 0.105 & 0.106 & 0.325 & 0.327 & 0.326 & 0.325 & 0.325 & 0.326 & 0.326 & 0.324 \\
\hline 571.59 & 0.108 & 0.108 & 0.326 & 0.329 & 0.328 & 0.326 & 0.327 & 0.327 & 0.327 & 0.326 \\
\hline 571.93 & 0.109 & 0.111 & 0.328 & 0.331 & 0.329 & 0.327 & 0.329 & 0.329 & 0.33 & 0.329 \\
\hline 572.27 & 0.111 & 0.112 & 0.329 & 0.332 & 0.331 & 0.328 & 0.33 & 0.33 & 0.332 & 0.332 \\
\hline 572.62 & 0.113 & 0.114 & 0.33 & 0.334 & 0.335 & 0.331 & 0.332 & 0.333 & 0.334 & 0.335 \\
\hline 572.96 & 0.116 & 0.117 & 0.332 & 0.336 & 0.336 & 0.334 & 0.333 & 0.335 & 0.336 & 0.336 \\
\hline 573.3 & 0.118 & 0.119 & 0.335 & 0.337 & 0.338 & 0.336 & 0.335 & 0.337 & 0.336 & 0.338 \\
\hline 573.64 & 0.119 & 0.121 & 0.337 & 0.339 & 0.339 & 0.336 & 0.337 & 0.338 & 0.337 & 0.34 \\
\hline 573.98 & 0.122 & 0.123 & 0.339 & 0.342 & 0.341 & 0.338 & 0.34 & 0.341 & 0.34 & 0.342 \\
\hline 574.32 & 0.125 & 0.126 & 0.341 & 0.342 & 0.344 & 0.341 & 0.342 & 0.343 & 0.342 & 0.344 \\
\hline 574.67 & 0.128 & 0.128 & 0.343 & 0.343 & 0.346 & 0.343 & 0.344 & 0.345 & 0.344 & 0.346 \\
\hline 575.01 & 0.13 & 0.131 & 0.344 & 0.345 & 0.349 & 0.344 & 0.346 & 0.348 & 0.345 & 0.349 \\
\hline 575.35 & 0.133 & 0.133 & 0.346 & 0.347 & 0.351 & 0.346 & 0.348 & 0.351 & 0.348 & 0.352 \\
\hline 575.69 & 0.135 & 0.134 & 0.349 & 0.349 & 0.353 & 0.348 & 0.35 & 0.352 & 0.349 & 0.353 \\
\hline 576.03 & 0.137 & 0.137 & 0.351 & 0.352 & 0.357 & 0.351 & 0.353 & 0.355 & 0.352 & 0.356 \\
\hline 576.37 & 0.139 & 0.138 & 0.352 & 0.354 & 0.356 & 0.353 & 0.355 & 0.355 & 0.353 & 0.357 \\
\hline 576.71 & 0.14 & 0.139 & 0.352 & 0.355 & 0.356 & 0.353 & 0.355 & 0.355 & 0.353 & 0.358 \\
\hline 577.05 & 0.141 & 0.14 & 0.353 & 0.358 & 0.358 & 0.355 & 0.357 & 0.357 & 0.355 & 0.361 \\
\hline 577.4 & 0.144 & 0.142 & 0.354 & 0.361 & 0.36 & 0.358 & 0.358 & 0.358 & 0.357 & 0.361 \\
\hline 577.74 & 0.146 & 0.144 & 0.356 & 0.363 & 0.361 & 0.361 & 0.36 & 0.36 & 0.36 & 0.365 \\
\hline 578.08 & 0.148 & 0.147 & 0.36 & 0.365 & 0.363 & 0.362 & 0.363 & 0.363 & 0.363 & 0.366 \\
\hline 578.42 & 0.15 & 0.15 & 0.362 & 0.367 & 0.366 & 0.364 & 0.365 & 0.365 & 0.366 & 0.367 \\
\hline 578.76 & 0.151 & 0.151 & 0.364 & 0.368 & 0.365 & 0.366 & 0.366 & 0.366 & 0.367 & 0.368 \\
\hline 579.1 & 0.153 & 0.154 & 0.366 & 0.369 & 0.367 & 0.368 & 0.367 & 0.367 & 0.368 & 0.369 \\
\hline 579.44 & 0.156 & 0.157 & 0.367 & 0.372 & 0.369 & 0.37 & 0.369 & 0.369 & 0.371 & 0.37 \\
\hline 579.78 & 0.158 & 0.159 & 0.368 & 0.373 & 0.369 & 0.371 & 0.37 & 0.37 & 0.372 & 0.369 \\
\hline 580.12 & 0.16 & 0.162 & 0.371 & 0.375 & 0.371 & 0.372 & 0.37 & 0.371 & 0.374 & 0.372 \\
\hline 580.46 & 0.164 & 0.165 & 0.373 & 0.378 & 0.375 & 0.375 & 0.374 & 0.373 & 0.376 & 0.373 \\
\hline 580.8 & 0.166 & 0.168 & 0.377 & 0.378 & 0.377 & 0.378 & 0.378 & 0.375 & 0.379 & 0.375 \\
\hline 581.14 & 0.168 & 0.172 & 0.381 & 0.379 & 0.378 & 0.379 & 0.381 & 0.378 & 0.381 & 0.377 \\
\hline
\end{tabular}




\begin{tabular}{|c|c|c|c|c|c|c|c|c|c|c|}
\hline 581.49 & 0.17 & 0.174 & 0.382 & 0.379 & 0.38 & 0.38 & 0.383 & 0.38 & 0.382 & 0.377 \\
\hline 581.83 & 0.173 & 0.176 & 0.383 & 0.382 & 0.382 & 0.382 & 0.385 & 0.382 & 0.383 & 0.379 \\
\hline 582.17 & 0.175 & 0.178 & 0.385 & 0.384 & 0.383 & 0.382 & 0.385 & 0.383 & 0.384 & 0.38 \\
\hline 582.51 & 0.177 & 0.18 & 0.387 & 0.388 & 0.385 & 0.385 & 0.388 & 0.386 & 0.386 & 0.383 \\
\hline 582.85 & 0.18 & 0.183 & 0.389 & 0.389 & 0.387 & 0.386 & 0.391 & 0.388 & 0.387 & 0.385 \\
\hline 583.19 & 0.181 & 0.185 & 0.389 & 0.391 & 0.389 & 0.388 & 0.392 & 0.39 & 0.388 & 0.387 \\
\hline 583.53 & 0.183 & 0.187 & 0.392 & 0.393 & 0.391 & 0.391 & 0.395 & 0.392 & 0.391 & 0.389 \\
\hline 583.87 & 0.186 & 0.19 & 0.394 & 0.394 & 0.393 & 0.394 & 0.397 & 0.394 & 0.394 & 0.39 \\
\hline 584.21 & 0.188 & 0.193 & 0.395 & 0.395 & 0.394 & 0.395 & 0.398 & 0.396 & 0.397 & 0.393 \\
\hline 584.55 & 0.19 & 0.195 & 0.396 & 0.398 & 0.395 & 0.396 & 0.399 & 0.397 & 0.398 & 0.394 \\
\hline 584.89 & 0.193 & 0.196 & 0.396 & 0.4 & 0.398 & 0.398 & 0.4 & 0.398 & 0.4 & 0.396 \\
\hline 585.23 & 0.196 & 0.199 & 0.399 & 0.402 & 0.4 & 0.4 & 0.401 & 0.399 & 0.402 & 0.397 \\
\hline 585.57 & 0.199 & 0.202 & 0.4 & 0.404 & 0.402 & 0.402 & 0.403 & 0.402 & 0.403 & 0.399 \\
\hline 585.91 & 0.201 & 0.203 & 0.401 & 0.406 & 0.403 & 0.404 & 0.405 & 0.403 & 0.404 & 0.402 \\
\hline 586.25 & 0.204 & 0.206 & 0.403 & 0.406 & 0.405 & 0.405 & 0.405 & 0.405 & 0.406 & 0.403 \\
\hline 586.59 & 0.206 & 0.207 & 0.405 & 0.408 & 0.407 & 0.408 & 0.407 & 0.406 & 0.409 & 0.405 \\
\hline 586.93 & 0.209 & 0.209 & 0.409 & 0.41 & 0.409 & 0.41 & 0.41 & 0.409 & 0.412 & 0.408 \\
\hline 587.27 & 0.212 & 0.212 & 0.411 & 0.413 & 0.411 & 0.413 & 0.412 & 0.411 & 0.412 & 0.409 \\
\hline 587.61 & 0.214 & 0.214 & 0.413 & 0.414 & 0.412 & 0.413 & 0.414 & 0.413 & 0.413 & 0.41 \\
\hline 587.95 & 0.216 & 0.216 & 0.417 & 0.417 & 0.414 & 0.416 & 0.416 & 0.415 & 0.415 & 0.413 \\
\hline 588.29 & 0.219 & 0.218 & 0.418 & 0.418 & 0.416 & 0.418 & 0.417 & 0.416 & 0.416 & 0.414 \\
\hline 588.63 & 0.222 & 0.222 & 0.421 & 0.42 & 0.418 & 0.419 & 0.419 & 0.418 & 0.419 & 0.417 \\
\hline 588.97 & 0.224 & 0.224 & 0.424 & 0.423 & 0.42 & 0.422 & 0.423 & 0.421 & 0.423 & 0.422 \\
\hline 589.3 & 0.227 & 0.226 & 0.425 & 0.424 & 0.422 & 0.424 & 0.425 & 0.423 & 0.426 & 0.424 \\
\hline 589.64 & 0.23 & 0.23 & 0.427 & 0.426 & 0.425 & 0.425 & 0.426 & 0.425 & 0.428 & 0.426 \\
\hline 589.98 & 0.233 & 0.233 & 0.428 & 0.43 & 0.427 & 0.426 & 0.43 & 0.426 & 0.43 & 0.428 \\
\hline 590.32 & 0.236 & 0.235 & 0.43 & 0.432 & 0.429 & 0.427 & 0.433 & 0.429 & 0.432 & 0.431 \\
\hline 590.66 & 0.239 & 0.237 & 0.432 & 0.434 & 0.43 & 0.429 & 0.434 & 0.43 & 0.432 & 0.433 \\
\hline 591 & 0.242 & 0.239 & 0.433 & 0.435 & 0.431 & 0.431 & 0.436 & 0.431 & 0.433 & 0.435 \\
\hline 591.34 & 0.246 & 0.242 & 0.437 & 0.439 & 0.435 & 0.434 & 0.439 & 0.434 & 0.437 & 0.438 \\
\hline 591.68 & 0.249 & 0.245 & 0.438 & 0.441 & 0.437 & 0.437 & 0.441 & 0.436 & 0.438 & 0.44 \\
\hline 592.02 & 0.252 & 0.249 & 0.44 & 0.443 & 0.44 & 0.44 & 0.445 & 0.44 & 0.44 & 0.442 \\
\hline 592.36 & 0.255 & 0.252 & 0.442 & 0.445 & 0.442 & 0.442 & 0.446 & 0.442 & 0.44 & 0.444 \\
\hline 592.7 & 0.259 & 0.255 & 0.443 & 0.447 & 0.444 & 0.445 & 0.447 & 0.444 & 0.442 & 0.446 \\
\hline 593.04 & 0.261 & 0.258 & 0.446 & 0.448 & 0.446 & 0.448 & 0.449 & 0.447 & 0.443 & 0.448 \\
\hline 593.37 & 0.264 & 0.26 & 0.449 & 0.451 & 0.449 & 0.452 & 0.452 & 0.449 & 0.446 & 0.45 \\
\hline 593.71 & 0.267 & 0.264 & 0.452 & 0.451 & 0.451 & 0.456 & 0.453 & 0.451 & 0.448 & 0.454 \\
\hline 594.05 & 0.27 & 0.269 & 0.455 & 0.452 & 0.454 & 0.459 & 0.456 & 0.453 & 0.451 & 0.455 \\
\hline 594.39 & 0.273 & 0.274 & 0.457 & 0.455 & 0.458 & 0.461 & 0.459 & 0.456 & 0.454 & 0.457 \\
\hline
\end{tabular}




\begin{tabular}{|c|c|c|c|c|c|c|c|c|c|c|}
\hline 594.73 & 0.276 & 0.278 & 0.462 & 0.459 & 0.461 & 0.464 & 0.461 & 0.46 & 0.459 & 0.46 \\
\hline 595.07 & 0.279 & 0.282 & 0.464 & 0.46 & 0.463 & 0.466 & 0.462 & 0.462 & 0.46 & 0.461 \\
\hline 595.41 & 0.283 & 0.285 & 0.467 & 0.463 & 0.465 & 0.468 & 0.465 & 0.466 & 0.462 & 0.464 \\
\hline 595.75 & 0.286 & 0.288 & 0.47 & 0.466 & 0.468 & 0.47 & 0.468 & 0.468 & 0.467 & 0.466 \\
\hline 596.08 & 0.29 & 0.29 & 0.472 & 0.468 & 0.47 & 0.472 & 0.47 & 0.47 & 0.469 & 0.468 \\
\hline 596.42 & 0.292 & 0.293 & 0.475 & 0.47 & 0.471 & 0.472 & 0.472 & 0.471 & 0.47 & 0.47 \\
\hline 596.76 & 0.297 & 0.297 & 0.477 & 0.474 & 0.473 & 0.474 & 0.477 & 0.473 & 0.474 & 0.471 \\
\hline 597.1 & 0.301 & 0.301 & 0.481 & 0.477 & 0.475 & 0.478 & 0.48 & 0.476 & 0.476 & 0.474 \\
\hline 597.44 & 0.305 & 0.304 & 0.483 & 0.482 & 0.478 & 0.481 & 0.481 & 0.479 & 0.479 & 0.477 \\
\hline 597.78 & 0.309 & 0.307 & 0.486 & 0.486 & 0.481 & 0.485 & 0.483 & 0.483 & 0.482 & 0.478 \\
\hline 598.11 & 0.313 & 0.311 & 0.49 & 0.488 & 0.484 & 0.487 & 0.486 & 0.487 & 0.485 & 0.482 \\
\hline 598.45 & 0.316 & 0.314 & 0.492 & 0.491 & 0.487 & 0.49 & 0.49 & 0.49 & 0.488 & 0.486 \\
\hline 598.79 & 0.319 & 0.316 & 0.493 & 0.493 & 0.49 & 0.493 & 0.493 & 0.491 & 0.491 & 0.49 \\
\hline 599.13 & 0.321 & 0.319 & 0.494 & 0.495 & 0.49 & 0.495 & 0.495 & 0.492 & 0.494 & 0.491 \\
\hline 599.47 & 0.325 & 0.322 & 0.496 & 0.496 & 0.492 & 0.498 & 0.494 & 0.493 & 0.495 & 0.495 \\
\hline 599.81 & 0.328 & 0.328 & 0.499 & 0.501 & 0.495 & 0.5 & 0.497 & 0.498 & 0.5 & 0.499 \\
\hline 600.14 & 0.333 & 0.332 & 0.5 & 0.504 & 0.499 & 0.504 & 0.499 & 0.503 & 0.503 & 0.502 \\
\hline 600.48 & 0.336 & 0.335 & 0.501 & 0.505 & 0.501 & 0.505 & 0.5 & 0.503 & 0.505 & 0.505 \\
\hline 600.82 & 0.339 & 0.338 & 0.503 & 0.507 & 0.503 & 0.505 & 0.5 & 0.504 & 0.507 & 0.505 \\
\hline 601.16 & 0.342 & 0.341 & 0.505 & 0.509 & 0.507 & 0.508 & 0.505 & 0.507 & 0.51 & 0.506 \\
\hline 601.49 & 0.343 & 0.344 & 0.507 & 0.511 & 0.509 & 0.508 & 0.506 & 0.507 & 0.511 & 0.509 \\
\hline 601.83 & 0.345 & 0.347 & 0.505 & 0.514 & 0.509 & 0.509 & 0.507 & 0.509 & 0.513 & 0.509 \\
\hline 602.17 & 0.348 & 0.351 & 0.506 & 0.516 & 0.513 & 0.512 & 0.51 & 0.512 & 0.515 & 0.511 \\
\hline 602.51 & 0.35 & 0.354 & 0.51 & 0.519 & 0.514 & 0.513 & 0.511 & 0.515 & 0.517 & 0.513 \\
\hline 602.85 & 0.355 & 0.359 & 0.513 & 0.522 & 0.517 & 0.516 & 0.514 & 0.518 & 0.519 & 0.516 \\
\hline 603.18 & 0.357 & 0.362 & 0.515 & 0.524 & 0.518 & 0.518 & 0.517 & 0.52 & 0.521 & 0.515 \\
\hline 603.52 & 0.359 & 0.365 & 0.517 & 0.524 & 0.521 & 0.52 & 0.52 & 0.521 & 0.523 & 0.516 \\
\hline 603.86 & 0.362 & 0.369 & 0.521 & 0.528 & 0.523 & 0.522 & 0.523 & 0.523 & 0.526 & 0.519 \\
\hline 604.2 & 0.363 & 0.373 & 0.525 & 0.53 & 0.526 & 0.527 & 0.526 & 0.527 & 0.529 & 0.522 \\
\hline 604.53 & 0.366 & 0.376 & 0.525 & 0.532 & 0.526 & 0.529 & 0.53 & 0.531 & 0.531 & 0.524 \\
\hline 604.87 & 0.369 & 0.38 & 0.525 & 0.533 & 0.525 & 0.529 & 0.531 & 0.531 & 0.53 & 0.525 \\
\hline 605.21 & 0.374 & 0.384 & 0.528 & 0.535 & 0.527 & 0.532 & 0.534 & 0.534 & 0.532 & 0.529 \\
\hline 605.54 & 0.376 & 0.388 & 0.532 & 0.536 & 0.531 & 0.534 & 0.534 & 0.536 & 0.534 & 0.531 \\
\hline 605.88 & 0.378 & 0.39 & 0.535 & 0.537 & 0.532 & 0.535 & 0.533 & 0.536 & 0.535 & 0.532 \\
\hline 606.22 & 0.381 & 0.393 & 0.534 & 0.539 & 0.533 & 0.537 & 0.535 & 0.537 & 0.537 & 0.534 \\
\hline 606.56 & 0.381 & 0.394 & 0.534 & 0.54 & 0.536 & 0.537 & 0.538 & 0.537 & 0.538 & 0.535 \\
\hline 606.89 & 0.385 & 0.396 & 0.537 & 0.543 & 0.539 & 0.537 & 0.54 & 0.54 & 0.538 & 0.538 \\
\hline 607.23 & 0.387 & 0.398 & 0.54 & 0.545 & 0.538 & 0.539 & 0.543 & 0.542 & 0.537 & 0.54 \\
\hline 607.57 & 0.387 & 0.399 & 0.54 & 0.543 & 0.538 & 0.538 & 0.543 & 0.541 & 0.538 & 0.539 \\
\hline
\end{tabular}




\begin{tabular}{|c|c|c|c|c|c|c|c|c|c|c|}
\hline 607.9 & 0.389 & 0.398 & 0.541 & 0.544 & 0.538 & 0.538 & 0.543 & 0.539 & 0.537 & 0.54 \\
\hline 608.24 & 0.39 & 0.399 & 0.543 & 0.546 & 0.541 & 0.539 & 0.544 & 0.54 & 0.539 & 0.543 \\
\hline 608.58 & 0.392 & 0.4 & 0.545 & 0.547 & 0.543 & 0.54 & 0.544 & 0.541 & 0.542 & 0.544 \\
\hline 608.91 & 0.391 & 0.398 & 0.543 & 0.545 & 0.542 & 0.54 & 0.543 & 0.542 & 0.543 & 0.544 \\
\hline 609.25 & 0.393 & 0.397 & 0.543 & 0.547 & 0.542 & 0.541 & 0.548 & 0.544 & 0.545 & 0.545 \\
\hline 609.59 & 0.394 & 0.398 & 0.542 & 0.546 & 0.543 & 0.541 & 0.55 & 0.545 & 0.544 & 0.545 \\
\hline 609.92 & 0.397 & 0.398 & 0.545 & 0.546 & 0.545 & 0.542 & 0.551 & 0.546 & 0.546 & 0.546 \\
\hline 610.26 & 0.399 & 0.4 & 0.548 & 0.547 & 0.546 & 0.545 & 0.55 & 0.548 & 0.547 & 0.546 \\
\hline 610.6 & 0.4 & 0.403 & 0.548 & 0.547 & 0.548 & 0.546 & 0.55 & 0.549 & 0.55 & 0.547 \\
\hline 610.93 & 0.4 & 0.402 & 0.547 & 0.547 & 0.547 & 0.546 & 0.549 & 0.548 & 0.553 & 0.547 \\
\hline 611.27 & 0.401 & 0.404 & 0.549 & 0.547 & 0.547 & 0.548 & 0.549 & 0.55 & 0.554 & 0.547 \\
\hline 611.61 & 0.403 & 0.407 & 0.552 & 0.55 & 0.551 & 0.55 & 0.552 & 0.555 & 0.557 & 0.549 \\
\hline 611.94 & 0.405 & 0.408 & 0.553 & 0.549 & 0.552 & 0.551 & 0.552 & 0.555 & 0.558 & 0.547 \\
\hline 612.28 & 0.406 & 0.407 & 0.554 & 0.549 & 0.55 & 0.551 & 0.552 & 0.556 & 0.559 & 0.547 \\
\hline 612.61 & 0.41 & 0.411 & 0.557 & 0.552 & 0.554 & 0.555 & 0.555 & 0.558 & 0.56 & 0.549 \\
\hline 612.95 & 0.412 & 0.412 & 0.557 & 0.553 & 0.555 & 0.556 & 0.552 & 0.556 & 0.56 & 0.548 \\
\hline 613.29 & 0.413 & 0.415 & 0.557 & 0.555 & 0.556 & 0.556 & 0.555 & 0.556 & 0.562 & 0.549 \\
\hline 613.62 & 0.415 & 0.416 & 0.556 & 0.556 & 0.557 & 0.557 & 0.555 & 0.557 & 0.56 & 0.549 \\
\hline 613.96 & 0.416 & 0.416 & 0.556 & 0.557 & 0.559 & 0.559 & 0.557 & 0.558 & 0.562 & 0.553 \\
\hline 614.3 & 0.417 & 0.416 & 0.554 & 0.557 & 0.558 & 0.56 & 0.557 & 0.558 & 0.561 & 0.554 \\
\hline 614.63 & 0.418 & 0.417 & 0.556 & 0.559 & 0.561 & 0.563 & 0.56 & 0.561 & 0.561 & 0.555 \\
\hline 614.97 & 0.419 & 0.419 & 0.556 & 0.56 & 0.563 & 0.564 & 0.561 & 0.561 & 0.56 & 0.557 \\
\hline 615.3 & 0.42 & 0.419 & 0.554 & 0.559 & 0.563 & 0.564 & 0.561 & 0.56 & 0.558 & 0.556 \\
\hline 615.64 & 0.42 & 0.419 & 0.554 & 0.56 & 0.563 & 0.563 & 0.56 & 0.56 & 0.559 & 0.558 \\
\hline 615.97 & 0.421 & 0.423 & 0.556 & 0.562 & 0.566 & 0.563 & 0.56 & 0.562 & 0.561 & 0.559 \\
\hline 616.31 & 0.42 & 0.421 & 0.557 & 0.561 & 0.565 & 0.562 & 0.56 & 0.562 & 0.56 & 0.559 \\
\hline 616.65 & 0.421 & 0.422 & 0.557 & 0.561 & 0.564 & 0.561 & 0.562 & 0.563 & 0.561 & 0.564 \\
\hline 616.98 & 0.421 & 0.422 & 0.561 & 0.562 & 0.565 & 0.565 & 0.561 & 0.565 & 0.562 & 0.565 \\
\hline 617.32 & 0.421 & 0.424 & 0.562 & 0.564 & 0.564 & 0.566 & 0.563 & 0.567 & 0.563 & 0.567 \\
\hline 617.65 & 0.424 & 0.428 & 0.564 & 0.566 & 0.563 & 0.566 & 0.563 & 0.566 & 0.565 & 0.566 \\
\hline 617.99 & 0.425 & 0.429 & 0.567 & 0.568 & 0.563 & 0.567 & 0.565 & 0.568 & 0.564 & 0.567 \\
\hline 618.32 & 0.426 & 0.429 & 0.568 & 0.569 & 0.562 & 0.566 & 0.564 & 0.567 & 0.566 & 0.568 \\
\hline 618.66 & 0.428 & 0.428 & 0.568 & 0.57 & 0.563 & 0.566 & 0.564 & 0.568 & 0.568 & 0.566 \\
\hline 618.99 & 0.428 & 0.428 & 0.57 & 0.57 & 0.564 & 0.567 & 0.564 & 0.568 & 0.568 & 0.567 \\
\hline 619.33 & 0.428 & 0.428 & 0.571 & 0.569 & 0.565 & 0.569 & 0.565 & 0.568 & 0.567 & 0.567 \\
\hline 619.66 & 0.429 & 0.429 & 0.57 & 0.569 & 0.565 & 0.567 & 0.565 & 0.568 & 0.567 & 0.567 \\
\hline 620 & 0.431 & 0.432 & 0.569 & 0.569 & 0.565 & 0.567 & 0.567 & 0.567 & 0.567 & 0.569 \\
\hline 620.33 & 0.432 & 0.434 & 0.571 & 0.572 & 0.568 & 0.57 & 0.569 & 0.57 & 0.568 & 0.567 \\
\hline 620.67 & 0.434 & 0.436 & 0.571 & 0.573 & 0.569 & 0.569 & 0.569 & 0.567 & 0.572 & 0.568 \\
\hline
\end{tabular}




\begin{tabular}{|c|c|c|c|c|c|c|c|c|c|c|}
\hline 621 & 0.432 & 0.435 & 0.572 & 0.574 & 0.569 & 0.567 & 0.567 & 0.568 & 0.57 & 0.568 \\
\hline 621.34 & 0.431 & 0.434 & 0.572 & 0.574 & 0.571 & 0.567 & 0.567 & 0.569 & 0.568 & 0.568 \\
\hline 621.67 & 0.431 & 0.434 & 0.572 & 0.574 & 0.572 & 0.566 & 0.567 & 0.57 & 0.568 & 0.569 \\
\hline 622.01 & 0.433 & 0.436 & 0.574 & 0.576 & 0.577 & 0.568 & 0.569 & 0.572 & 0.571 & 0.571 \\
\hline 622.34 & 0.434 & 0.439 & 0.574 & 0.575 & 0.578 & 0.57 & 0.569 & 0.571 & 0.571 & 0.574 \\
\hline 622.68 & 0.435 & 0.439 & 0.572 & 0.574 & 0.576 & 0.569 & 0.569 & 0.569 & 0.573 & 0.572 \\
\hline 623.01 & 0.436 & 0.44 & 0.574 & 0.576 & 0.577 & 0.569 & 0.568 & 0.57 & 0.574 & 0.574 \\
\hline 623.35 & 0.436 & 0.439 & 0.575 & 0.577 & 0.576 & 0.572 & 0.568 & 0.569 & 0.574 & 0.577 \\
\hline 623.68 & 0.436 & 0.438 & 0.575 & 0.577 & 0.576 & 0.574 & 0.567 & 0.568 & 0.573 & 0.575 \\
\hline 624.02 & 0.435 & 0.438 & 0.574 & 0.576 & 0.575 & 0.574 & 0.565 & 0.568 & 0.574 & 0.575 \\
\hline 624.35 & 0.436 & 0.439 & 0.574 & 0.576 & 0.575 & 0.574 & 0.565 & 0.569 & 0.57 & 0.574 \\
\hline 624.69 & 0.44 & 0.44 & 0.572 & 0.576 & 0.579 & 0.575 & 0.567 & 0.571 & 0.575 & 0.575 \\
\hline 625.02 & 0.44 & 0.439 & 0.571 & 0.572 & 0.576 & 0.572 & 0.567 & 0.569 & 0.574 & 0.574 \\
\hline 625.36 & 0.44 & 0.439 & 0.571 & 0.573 & 0.575 & 0.575 & 0.566 & 0.567 & 0.575 & 0.573 \\
\hline 625.69 & 0.437 & 0.437 & 0.568 & 0.571 & 0.572 & 0.574 & 0.566 & 0.566 & 0.572 & 0.572 \\
\hline 626.02 & 0.436 & 0.436 & 0.569 & 0.57 & 0.572 & 0.573 & 0.568 & 0.566 & 0.572 & 0.571 \\
\hline 626.36 & 0.436 & 0.436 & 0.57 & 0.572 & 0.572 & 0.573 & 0.568 & 0.568 & 0.572 & 0.571 \\
\hline 626.69 & 0.437 & 0.437 & 0.572 & 0.574 & 0.571 & 0.573 & 0.569 & 0.569 & 0.574 & 0.57 \\
\hline 627.03 & 0.439 & 0.438 & 0.573 & 0.573 & 0.572 & 0.572 & 0.572 & 0.57 & 0.575 & 0.57 \\
\hline 627.36 & 0.439 & 0.439 & 0.575 & 0.575 & 0.574 & 0.571 & 0.573 & 0.573 & 0.576 & 0.572 \\
\hline 627.7 & 0.441 & 0.44 & 0.576 & 0.574 & 0.574 & 0.571 & 0.576 & 0.574 & 0.574 & 0.572 \\
\hline 628.03 & 0.441 & 0.439 & 0.576 & 0.573 & 0.572 & 0.573 & 0.577 & 0.573 & 0.577 & 0.572 \\
\hline 628.36 & 0.439 & 0.439 & 0.578 & 0.574 & 0.571 & 0.573 & 0.578 & 0.572 & 0.576 & 0.572 \\
\hline 628.7 & 0.44 & 0.439 & 0.578 & 0.575 & 0.572 & 0.574 & 0.579 & 0.574 & 0.578 & 0.574 \\
\hline 629.03 & 0.44 & 0.44 & 0.578 & 0.575 & 0.571 & 0.572 & 0.578 & 0.576 & 0.578 & 0.575 \\
\hline 629.36 & 0.442 & 0.441 & 0.58 & 0.575 & 0.573 & 0.572 & 0.579 & 0.578 & 0.579 & 0.575 \\
\hline 629.7 & 0.443 & 0.441 & 0.579 & 0.576 & 0.573 & 0.572 & 0.578 & 0.579 & 0.578 & 0.574 \\
\hline 630.03 & 0.443 & 0.443 & 0.581 & 0.577 & 0.575 & 0.572 & 0.58 & 0.579 & 0.578 & 0.576 \\
\hline 630.37 & 0.442 & 0.442 & 0.579 & 0.574 & 0.575 & 0.572 & 0.579 & 0.578 & 0.575 & 0.575 \\
\hline 630.7 & 0.442 & 0.441 & 0.576 & 0.574 & 0.575 & 0.572 & 0.577 & 0.578 & 0.574 & 0.574 \\
\hline 631.03 & 0.44 & 0.441 & 0.574 & 0.572 & 0.573 & 0.571 & 0.576 & 0.577 & 0.573 & 0.572 \\
\hline 631.37 & 0.439 & 0.441 & 0.573 & 0.57 & 0.572 & 0.569 & 0.573 & 0.576 & 0.573 & 0.57 \\
\hline 631.7 & 0.439 & 0.442 & 0.572 & 0.57 & 0.572 & 0.568 & 0.573 & 0.576 & 0.57 & 0.57 \\
\hline 632.03 & 0.439 & 0.44 & 0.57 & 0.57 & 0.57 & 0.567 & 0.57 & 0.575 & 0.569 & 0.568 \\
\hline 632.37 & 0.439 & 0.441 & 0.569 & 0.568 & 0.571 & 0.567 & 0.569 & 0.573 & 0.569 & 0.568 \\
\hline 632.7 & 0.438 & 0.44 & 0.57 & 0.569 & 0.573 & 0.57 & 0.57 & 0.571 & 0.571 & 0.568 \\
\hline 633.03 & 0.438 & 0.442 & 0.569 & 0.568 & 0.572 & 0.571 & 0.571 & 0.571 & 0.572 & 0.567 \\
\hline 633.37 & 0.439 & 0.442 & 0.571 & 0.568 & 0.572 & 0.571 & 0.571 & 0.571 & 0.574 & 0.569 \\
\hline 633.7 & 0.438 & 0.442 & 0.568 & 0.566 & 0.571 & 0.57 & 0.568 & 0.572 & 0.573 & 0.568 \\
\hline
\end{tabular}




\begin{tabular}{|c|c|c|c|c|c|c|c|c|c|c|}
\hline 634.03 & 0.438 & 0.442 & 0.568 & 0.567 & 0.571 & 0.569 & 0.569 & 0.573 & 0.573 & 0.571 \\
\hline 634.37 & 0.438 & 0.443 & 0.568 & 0.567 & 0.571 & 0.569 & 0.571 & 0.573 & 0.573 & 0.572 \\
\hline 634.7 & 0.438 & 0.443 & 0.569 & 0.567 & 0.571 & 0.569 & 0.571 & 0.572 & 0.574 & 0.57 \\
\hline 635.03 & 0.439 & 0.443 & 0.569 & 0.568 & 0.573 & 0.571 & 0.57 & 0.573 & 0.575 & 0.572 \\
\hline 635.37 & 0.437 & 0.442 & 0.569 & 0.567 & 0.573 & 0.567 & 0.569 & 0.572 & 0.574 & 0.57 \\
\hline 635.7 & 0.439 & 0.442 & 0.568 & 0.566 & 0.571 & 0.567 & 0.571 & 0.573 & 0.575 & 0.571 \\
\hline 636.03 & 0.438 & 0.441 & 0.57 & 0.567 & 0.569 & 0.568 & 0.572 & 0.57 & 0.573 & 0.569 \\
\hline 636.36 & 0.436 & 0.439 & 0.567 & 0.564 & 0.568 & 0.564 & 0.571 & 0.57 & 0.568 & 0.568 \\
\hline 636.7 & 0.435 & 0.436 & 0.563 & 0.563 & 0.565 & 0.561 & 0.567 & 0.566 & 0.564 & 0.565 \\
\hline 637.03 & 0.434 & 0.434 & 0.562 & 0.562 & 0.564 & 0.56 & 0.567 & 0.565 & 0.562 & 0.563 \\
\hline 637.36 & 0.434 & 0.433 & 0.564 & 0.563 & 0.565 & 0.563 & 0.567 & 0.565 & 0.563 & 0.564 \\
\hline 637.69 & 0.433 & 0.432 & 0.564 & 0.564 & 0.566 & 0.564 & 0.568 & 0.563 & 0.562 & 0.563 \\
\hline 638.03 & 0.433 & 0.43 & 0.562 & 0.562 & 0.565 & 0.563 & 0.565 & 0.561 & 0.561 & 0.564 \\
\hline 638.36 & 0.433 & 0.43 & 0.562 & 0.563 & 0.565 & 0.563 & 0.563 & 0.564 & 0.561 & 0.565 \\
\hline 638.69 & 0.432 & 0.429 & 0.563 & 0.564 & 0.564 & 0.562 & 0.566 & 0.564 & 0.561 & 0.564 \\
\hline 639.02 & 0.432 & 0.428 & 0.563 & 0.565 & 0.564 & 0.566 & 0.569 & 0.565 & 0.561 & 0.566 \\
\hline 639.36 & 0.43 & 0.427 & 0.564 & 0.564 & 0.565 & 0.564 & 0.565 & 0.565 & 0.562 & 0.563 \\
\hline 639.69 & 0.43 & 0.427 & 0.562 & 0.562 & 0.565 & 0.563 & 0.563 & 0.568 & 0.563 & 0.563 \\
\hline 640.02 & 0.43 & 0.428 & 0.561 & 0.561 & 0.566 & 0.563 & 0.562 & 0.566 & 0.564 & 0.563 \\
\hline 640.35 & 0.43 & 0.429 & 0.565 & 0.565 & 0.567 & 0.565 & 0.565 & 0.57 & 0.566 & 0.567 \\
\hline 640.69 & 0.429 & 0.431 & 0.564 & 0.565 & 0.566 & 0.564 & 0.561 & 0.57 & 0.566 & 0.569 \\
\hline 641.02 & 0.429 & 0.433 & 0.562 & 0.564 & 0.564 & 0.563 & 0.56 & 0.568 & 0.566 & 0.569 \\
\hline 641.35 & 0.429 & 0.434 & 0.562 & 0.563 & 0.564 & 0.565 & 0.561 & 0.567 & 0.567 & 0.567 \\
\hline 641.68 & 0.429 & 0.433 & 0.56 & 0.564 & 0.564 & 0.564 & 0.562 & 0.57 & 0.57 & 0.564 \\
\hline 642.01 & 0.428 & 0.433 & 0.559 & 0.563 & 0.564 & 0.563 & 0.561 & 0.569 & 0.568 & 0.562 \\
\hline 642.35 & 0.425 & 0.432 & 0.559 & 0.564 & 0.564 & 0.561 & 0.558 & 0.568 & 0.568 & 0.564 \\
\hline 642.68 & 0.424 & 0.432 & 0.559 & 0.566 & 0.565 & 0.561 & 0.557 & 0.568 & 0.569 & 0.565 \\
\hline 643.01 & 0.425 & 0.433 & 0.559 & 0.566 & 0.567 & 0.563 & 0.559 & 0.567 & 0.569 & 0.566 \\
\hline 643.34 & 0.426 & 0.434 & 0.56 & 0.568 & 0.568 & 0.562 & 0.561 & 0.566 & 0.569 & 0.569 \\
\hline 643.67 & 0.428 & 0.435 & 0.563 & 0.569 & 0.567 & 0.566 & 0.566 & 0.567 & 0.571 & 0.568 \\
\hline 644 & 0.427 & 0.436 & 0.564 & 0.569 & 0.572 & 0.566 & 0.567 & 0.569 & 0.571 & 0.568 \\
\hline 644.34 & 0.429 & 0.435 & 0.565 & 0.569 & 0.575 & 0.567 & 0.569 & 0.569 & 0.572 & 0.568 \\
\hline 644.67 & 0.428 & 0.434 & 0.564 & 0.567 & 0.573 & 0.568 & 0.57 & 0.568 & 0.572 & 0.566 \\
\hline 645 & 0.429 & 0.433 & 0.564 & 0.567 & 0.571 & 0.569 & 0.567 & 0.57 & 0.57 & 0.567 \\
\hline 645.33 & 0.429 & 0.433 & 0.565 & 0.567 & 0.572 & 0.571 & 0.569 & 0.568 & 0.568 & 0.569 \\
\hline 645.66 & 0.429 & 0.433 & 0.569 & 0.567 & 0.574 & 0.572 & 0.569 & 0.568 & 0.57 & 0.572 \\
\hline 645.99 & 0.43 & 0.434 & 0.568 & 0.567 & 0.574 & 0.573 & 0.569 & 0.568 & 0.571 & 0.572 \\
\hline 646.32 & 0.433 & 0.435 & 0.569 & 0.564 & 0.575 & 0.572 & 0.568 & 0.567 & 0.572 & 0.574 \\
\hline 646.66 & 0.432 & 0.437 & 0.569 & 0.564 & 0.572 & 0.572 & 0.568 & 0.569 & 0.574 & 0.575 \\
\hline
\end{tabular}




\begin{tabular}{|c|c|c|c|c|c|c|c|c|c|c|}
\hline 646.99 & 0.434 & 0.439 & 0.572 & 0.566 & 0.572 & 0.575 & 0.568 & 0.571 & 0.576 & 0.574 \\
\hline 647.32 & 0.434 & 0.439 & 0.571 & 0.566 & 0.573 & 0.575 & 0.565 & 0.573 & 0.573 & 0.576 \\
\hline 647.65 & 0.435 & 0.438 & 0.571 & 0.564 & 0.572 & 0.573 & 0.565 & 0.573 & 0.573 & 0.576 \\
\hline 647.98 & 0.437 & 0.44 & 0.573 & 0.568 & 0.575 & 0.576 & 0.567 & 0.576 & 0.575 & 0.579 \\
\hline 648.31 & 0.439 & 0.442 & 0.574 & 0.572 & 0.577 & 0.574 & 0.569 & 0.581 & 0.577 & 0.58 \\
\hline 648.64 & 0.441 & 0.445 & 0.578 & 0.575 & 0.581 & 0.575 & 0.574 & 0.585 & 0.58 & 0.582 \\
\hline 648.97 & 0.444 & 0.448 & 0.582 & 0.578 & 0.584 & 0.577 & 0.576 & 0.586 & 0.583 & 0.58 \\
\hline 649.3 & 0.446 & 0.449 & 0.582 & 0.579 & 0.582 & 0.577 & 0.578 & 0.587 & 0.583 & 0.58 \\
\hline 649.64 & 0.449 & 0.451 & 0.584 & 0.581 & 0.586 & 0.582 & 0.581 & 0.588 & 0.585 & 0.58 \\
\hline 649.97 & 0.451 & 0.452 & 0.586 & 0.585 & 0.587 & 0.586 & 0.583 & 0.591 & 0.584 & 0.581 \\
\hline 650.3 & 0.453 & 0.455 & 0.587 & 0.588 & 0.59 & 0.588 & 0.584 & 0.591 & 0.583 & 0.581 \\
\hline 650.63 & 0.454 & 0.458 & 0.588 & 0.589 & 0.592 & 0.591 & 0.587 & 0.592 & 0.583 & 0.585 \\
\hline 650.96 & 0.458 & 0.46 & 0.594 & 0.592 & 0.593 & 0.591 & 0.588 & 0.589 & 0.586 & 0.585 \\
\hline 651.29 & 0.461 & 0.463 & 0.596 & 0.594 & 0.59 & 0.595 & 0.591 & 0.59 & 0.587 & 0.587 \\
\hline 651.62 & 0.464 & 0.464 & 0.596 & 0.591 & 0.588 & 0.593 & 0.591 & 0.589 & 0.586 & 0.586 \\
\hline 651.95 & 0.467 & 0.467 & 0.602 & 0.595 & 0.59 & 0.597 & 0.593 & 0.59 & 0.589 & 0.59 \\
\hline 652.28 & 0.468 & 0.469 & 0.603 & 0.596 & 0.59 & 0.598 & 0.594 & 0.589 & 0.589 & 0.591 \\
\hline 652.61 & 0.469 & 0.47 & 0.605 & 0.599 & 0.59 & 0.6 & 0.596 & 0.591 & 0.59 & 0.597 \\
\hline 652.94 & 0.472 & 0.472 & 0.605 & 0.597 & 0.595 & 0.602 & 0.595 & 0.593 & 0.592 & 0.599 \\
\hline 653.27 & 0.476 & 0.476 & 0.608 & 0.6 & 0.594 & 0.6 & 0.595 & 0.594 & 0.593 & 0.6 \\
\hline 653.6 & 0.479 & 0.48 & 0.61 & 0.601 & 0.594 & 0.6 & 0.595 & 0.595 & 0.594 & 0.6 \\
\hline 653.93 & 0.482 & 0.483 & 0.612 & 0.604 & 0.598 & 0.602 & 0.599 & 0.598 & 0.597 & 0.604 \\
\hline 654.26 & 0.485 & 0.485 & 0.611 & 0.604 & 0.601 & 0.601 & 0.598 & 0.6 & 0.599 & 0.604 \\
\hline 654.59 & 0.488 & 0.488 & 0.611 & 0.606 & 0.605 & 0.603 & 0.6 & 0.604 & 0.603 & 0.608 \\
\hline 654.92 & 0.491 & 0.49 & 0.613 & 0.611 & 0.613 & 0.604 & 0.601 & 0.608 & 0.607 & 0.609 \\
\hline 655.25 & 0.491 & 0.494 & 0.614 & 0.615 & 0.615 & 0.607 & 0.605 & 0.61 & 0.609 & 0.612 \\
\hline 655.58 & 0.494 & 0.496 & 0.614 & 0.615 & 0.617 & 0.607 & 0.606 & 0.612 & 0.608 & 0.613 \\
\hline 655.91 & 0.501 & 0.5 & 0.618 & 0.618 & 0.62 & 0.61 & 0.608 & 0.617 & 0.614 & 0.615 \\
\hline 656.24 & 0.504 & 0.505 & 0.615 & 0.619 & 0.623 & 0.61 & 0.608 & 0.617 & 0.616 & 0.614 \\
\hline 656.57 & 0.508 & 0.511 & 0.619 & 0.626 & 0.627 & 0.615 & 0.616 & 0.621 & 0.621 & 0.617 \\
\hline 656.9 & 0.509 & 0.515 & 0.618 & 0.624 & 0.629 & 0.62 & 0.619 & 0.625 & 0.623 & 0.621 \\
\hline 657.23 & 0.514 & 0.519 & 0.619 & 0.627 & 0.633 & 0.623 & 0.624 & 0.627 & 0.627 & 0.622 \\
\hline 657.56 & 0.519 & 0.522 & 0.621 & 0.631 & 0.635 & 0.626 & 0.627 & 0.631 & 0.631 & 0.626 \\
\hline 657.89 & 0.523 & 0.526 & 0.625 & 0.636 & 0.64 & 0.631 & 0.629 & 0.632 & 0.634 & 0.625 \\
\hline 658.22 & 0.528 & 0.531 & 0.627 & 0.64 & 0.641 & 0.633 & 0.635 & 0.635 & 0.637 & 0.627 \\
\hline 658.55 & 0.533 & 0.538 & 0.629 & 0.641 & 0.642 & 0.636 & 0.635 & 0.637 & 0.641 & 0.629 \\
\hline 658.88 & 0.539 & 0.545 & 0.632 & 0.646 & 0.646 & 0.638 & 0.636 & 0.639 & 0.644 & 0.632 \\
\hline 659.21 & 0.542 & 0.549 & 0.635 & 0.649 & 0.648 & 0.639 & 0.64 & 0.64 & 0.647 & 0.636 \\
\hline 659.54 & 0.543 & 0.552 & 0.635 & 0.65 & 0.649 & 0.638 & 0.638 & 0.64 & 0.646 & 0.638 \\
\hline
\end{tabular}




\begin{tabular}{|c|c|c|c|c|c|c|c|c|c|c|}
\hline 659.87 & 0.551 & 0.559 & 0.642 & 0.656 & 0.651 & 0.643 & 0.644 & 0.645 & 0.651 & 0.644 \\
\hline 660.2 & 0.556 & 0.563 & 0.647 & 0.66 & 0.654 & 0.642 & 0.645 & 0.648 & 0.651 & 0.647 \\
\hline 660.53 & 0.561 & 0.566 & 0.651 & 0.663 & 0.654 & 0.643 & 0.646 & 0.653 & 0.653 & 0.652 \\
\hline 660.85 & 0.564 & 0.568 & 0.654 & 0.662 & 0.656 & 0.642 & 0.648 & 0.656 & 0.656 & 0.655 \\
\hline 661.18 & 0.569 & 0.569 & 0.659 & 0.662 & 0.659 & 0.646 & 0.652 & 0.653 & 0.657 & 0.654 \\
\hline 661.51 & 0.574 & 0.573 & 0.663 & 0.662 & 0.659 & 0.646 & 0.658 & 0.658 & 0.659 & 0.663 \\
\hline 661.84 & 0.576 & 0.575 & 0.661 & 0.66 & 0.659 & 0.651 & 0.653 & 0.662 & 0.659 & 0.665 \\
\hline 662.17 & 0.579 & 0.58 & 0.663 & 0.664 & 0.662 & 0.658 & 0.658 & 0.665 & 0.658 & 0.67 \\
\hline 662.5 & 0.582 & 0.583 & 0.668 & 0.664 & 0.663 & 0.66 & 0.662 & 0.67 & 0.659 & 0.671 \\
\hline 662.83 & 0.586 & 0.587 & 0.67 & 0.668 & 0.662 & 0.665 & 0.662 & 0.674 & 0.66 & 0.673 \\
\hline 663.16 & 0.592 & 0.591 & 0.673 & 0.671 & 0.664 & 0.67 & 0.667 & 0.677 & 0.664 & 0.675 \\
\hline 663.49 & 0.592 & 0.591 & 0.672 & 0.672 & 0.665 & 0.673 & 0.668 & 0.679 & 0.664 & 0.672 \\
\hline 663.81 & 0.595 & 0.595 & 0.675 & 0.674 & 0.663 & 0.676 & 0.668 & 0.678 & 0.667 & 0.676 \\
\hline 664.14 & 0.596 & 0.597 & 0.673 & 0.677 & 0.667 & 0.676 & 0.672 & 0.674 & 0.667 & 0.672 \\
\hline 664.47 & 0.598 & 0.6 & 0.675 & 0.682 & 0.669 & 0.682 & 0.676 & 0.675 & 0.667 & 0.676 \\
\hline 664.8 & 0.6 & 0.607 & 0.678 & 0.684 & 0.673 & 0.683 & 0.678 & 0.68 & 0.67 & 0.678 \\
\hline 665.13 & 0.602 & 0.609 & 0.677 & 0.682 & 0.673 & 0.681 & 0.677 & 0.681 & 0.669 & 0.674 \\
\hline 665.46 & 0.606 & 0.613 & 0.678 & 0.682 & 0.673 & 0.68 & 0.685 & 0.679 & 0.674 & 0.674 \\
\hline 665.79 & 0.61 & 0.616 & 0.678 & 0.685 & 0.673 & 0.676 & 0.687 & 0.677 & 0.677 & 0.675 \\
\hline 666.11 & 0.613 & 0.617 & 0.677 & 0.688 & 0.674 & 0.678 & 0.688 & 0.677 & 0.679 & 0.677 \\
\hline 666.44 & 0.616 & 0.621 & 0.68 & 0.688 & 0.682 & 0.682 & 0.69 & 0.678 & 0.688 & 0.677 \\
\hline 666.77 & 0.617 & 0.62 & 0.678 & 0.688 & 0.681 & 0.677 & 0.689 & 0.678 & 0.686 & 0.676 \\
\hline 667.1 & 0.62 & 0.626 & 0.682 & 0.686 & 0.684 & 0.676 & 0.694 & 0.678 & 0.689 & 0.681 \\
\hline 667.43 & 0.624 & 0.63 & 0.681 & 0.685 & 0.686 & 0.673 & 0.695 & 0.682 & 0.69 & 0.681 \\
\hline 667.76 & 0.628 & 0.634 & 0.685 & 0.685 & 0.688 & 0.676 & 0.7 & 0.688 & 0.692 & 0.684 \\
\hline 668.08 & 0.63 & 0.637 & 0.683 & 0.685 & 0.688 & 0.674 & 0.698 & 0.687 & 0.694 & 0.683 \\
\hline 668.41 & 0.632 & 0.635 & 0.682 & 0.681 & 0.684 & 0.671 & 0.698 & 0.688 & 0.694 & 0.684 \\
\hline 668.74 & 0.635 & 0.637 & 0.684 & 0.686 & 0.685 & 0.676 & 0.698 & 0.686 & 0.698 & 0.69 \\
\hline 669.07 & 0.64 & 0.641 & 0.689 & 0.692 & 0.689 & 0.681 & 0.699 & 0.691 & 0.698 & 0.693 \\
\hline 669.4 & 0.64 & 0.641 & 0.693 & 0.691 & 0.69 & 0.683 & 0.7 & 0.693 & 0.701 & 0.692 \\
\hline 669.72 & 0.643 & 0.642 & 0.695 & 0.691 & 0.691 & 0.685 & 0.701 & 0.694 & 0.705 & 0.692 \\
\hline 670.05 & 0.645 & 0.641 & 0.691 & 0.686 & 0.685 & 0.684 & 0.7 & 0.694 & 0.704 & 0.689 \\
\hline 670.38 & 0.647 & 0.641 & 0.693 & 0.684 & 0.688 & 0.687 & 0.703 & 0.694 & 0.706 & 0.692 \\
\hline 670.71 & 0.645 & 0.64 & 0.696 & 0.684 & 0.689 & 0.686 & 0.702 & 0.695 & 0.707 & 0.691 \\
\hline 671.03 & 0.643 & 0.639 & 0.693 & 0.684 & 0.691 & 0.692 & 0.702 & 0.693 & 0.709 & 0.692 \\
\hline 671.36 & 0.651 & 0.64 & 0.696 & 0.684 & 0.694 & 0.695 & 0.701 & 0.697 & 0.713 & 0.696 \\
\hline 671.69 & 0.655 & 0.644 & 0.703 & 0.683 & 0.696 & 0.701 & 0.705 & 0.706 & 0.714 & 0.7 \\
\hline 672.02 & 0.657 & 0.649 & 0.706 & 0.691 & 0.702 & 0.705 & 0.706 & 0.707 & 0.718 & 0.704 \\
\hline 672.34 & 0.659 & 0.65 & 0.708 & 0.694 & 0.706 & 0.705 & 0.708 & 0.708 & 0.717 & 0.705 \\
\hline
\end{tabular}




\begin{tabular}{|c|c|c|c|c|c|c|c|c|c|c|}
\hline 672.67 & 0.657 & 0.645 & 0.706 & 0.693 & 0.707 & 0.704 & 0.706 & 0.708 & 0.715 & 0.704 \\
\hline 673 & 0.659 & 0.648 & 0.705 & 0.696 & 0.712 & 0.706 & 0.703 & 0.711 & 0.713 & 0.705 \\
\hline 673.33 & 0.657 & 0.648 & 0.706 & 0.695 & 0.711 & 0.705 & 0.702 & 0.712 & 0.709 & 0.707 \\
\hline 673.65 & 0.659 & 0.65 & 0.703 & 0.701 & 0.715 & 0.707 & 0.701 & 0.714 & 0.708 & 0.713 \\
\hline 673.98 & 0.66 & 0.657 & 0.707 & 0.704 & 0.714 & 0.711 & 0.702 & 0.716 & 0.713 & 0.715 \\
\hline 674.31 & 0.663 & 0.66 & 0.702 & 0.709 & 0.715 & 0.716 & 0.699 & 0.715 & 0.711 & 0.715 \\
\hline 674.63 & 0.666 & 0.662 & 0.705 & 0.71 & 0.714 & 0.717 & 0.699 & 0.715 & 0.711 & 0.715 \\
\hline 674.96 & 0.66 & 0.663 & 0.703 & 0.711 & 0.71 & 0.715 & 0.696 & 0.709 & 0.709 & 0.711 \\
\hline 675.29 & 0.661 & 0.664 & 0.705 & 0.716 & 0.714 & 0.712 & 0.694 & 0.709 & 0.713 & 0.71 \\
\hline 675.61 & 0.66 & 0.661 & 0.705 & 0.715 & 0.711 & 0.716 & 0.693 & 0.712 & 0.707 & 0.708 \\
\hline 675.94 & 0.66 & 0.664 & 0.707 & 0.711 & 0.713 & 0.714 & 0.695 & 0.713 & 0.709 & 0.705 \\
\hline 676.27 & 0.662 & 0.669 & 0.712 & 0.712 & 0.715 & 0.714 & 0.698 & 0.714 & 0.708 & 0.709 \\
\hline 676.6 & 0.66 & 0.664 & 0.709 & 0.708 & 0.713 & 0.711 & 0.702 & 0.714 & 0.707 & 0.705 \\
\hline 676.92 & 0.662 & 0.666 & 0.709 & 0.712 & 0.712 & 0.71 & 0.702 & 0.713 & 0.706 & 0.706 \\
\hline 677.25 & 0.657 & 0.661 & 0.711 & 0.706 & 0.71 & 0.707 & 0.703 & 0.708 & 0.704 & 0.705 \\
\hline 677.58 & 0.656 & 0.658 & 0.711 & 0.706 & 0.709 & 0.705 & 0.702 & 0.71 & 0.702 & 0.701 \\
\hline 677.9 & 0.658 & 0.657 & 0.714 & 0.705 & 0.71 & 0.704 & 0.702 & 0.71 & 0.699 & 0.702 \\
\hline 678.23 & 0.659 & 0.656 & 0.713 & 0.702 & 0.71 & 0.701 & 0.7 & 0.709 & 0.697 & 0.704 \\
\hline 678.55 & 0.658 & 0.653 & 0.71 & 0.703 & 0.71 & 0.697 & 0.699 & 0.711 & 0.695 & 0.701 \\
\hline 678.88 & 0.654 & 0.648 & 0.706 & 0.701 & 0.704 & 0.698 & 0.697 & 0.703 & 0.69 & 0.7 \\
\hline 679.21 & 0.652 & 0.649 & 0.703 & 0.702 & 0.704 & 0.695 & 0.696 & 0.701 & 0.691 & 0.701 \\
\hline 679.53 & 0.651 & 0.645 & 0.702 & 0.703 & 0.702 & 0.691 & 0.693 & 0.7 & 0.69 & 0.7 \\
\hline 679.86 & 0.651 & 0.644 & 0.699 & 0.703 & 0.704 & 0.687 & 0.694 & 0.698 & 0.693 & 0.697 \\
\hline 680.19 & 0.651 & 0.644 & 0.701 & 0.708 & 0.705 & 0.69 & 0.693 & 0.697 & 0.694 & 0.699 \\
\hline 680.51 & 0.649 & 0.645 & 0.698 & 0.707 & 0.703 & 0.691 & 0.693 & 0.695 & 0.697 & 0.697 \\
\hline 680.84 & 0.65 & 0.653 & 0.704 & 0.712 & 0.708 & 0.697 & 0.695 & 0.7 & 0.702 & 0.698 \\
\hline 681.16 & 0.652 & 0.654 & 0.703 & 0.713 & 0.712 & 0.7 & 0.696 & 0.7 & 0.703 & 0.7 \\
\hline 681.49 & 0.648 & 0.652 & 0.701 & 0.71 & 0.711 & 0.693 & 0.694 & 0.701 & 0.703 & 0.698 \\
\hline 681.82 & 0.645 & 0.649 & 0.701 & 0.711 & 0.711 & 0.691 & 0.696 & 0.703 & 0.704 & 0.696 \\
\hline 682.14 & 0.646 & 0.651 & 0.702 & 0.712 & 0.71 & 0.693 & 0.696 & 0.703 & 0.707 & 0.7 \\
\hline 682.47 & 0.647 & 0.652 & 0.701 & 0.71 & 0.711 & 0.692 & 0.696 & 0.705 & 0.712 & 0.701 \\
\hline 682.79 & 0.646 & 0.653 & 0.703 & 0.703 & 0.708 & 0.691 & 0.692 & 0.704 & 0.714 & 0.701 \\
\hline 683.12 & 0.644 & 0.653 & 0.7 & 0.704 & 0.706 & 0.695 & 0.695 & 0.705 & 0.713 & 0.701 \\
\hline 683.45 & 0.638 & 0.65 & 0.698 & 0.697 & 0.697 & 0.694 & 0.691 & 0.702 & 0.712 & 0.702 \\
\hline 683.77 & 0.64 & 0.652 & 0.702 & 0.695 & 0.695 & 0.694 & 0.692 & 0.702 & 0.714 & 0.706 \\
\hline 684.1 & 0.639 & 0.648 & 0.706 & 0.693 & 0.697 & 0.694 & 0.692 & 0.703 & 0.713 & 0.707 \\
\hline 684.42 & 0.637 & 0.642 & 0.702 & 0.69 & 0.694 & 0.692 & 0.691 & 0.701 & 0.711 & 0.708 \\
\hline 684.75 & 0.632 & 0.636 & 0.699 & 0.688 & 0.689 & 0.69 & 0.69 & 0.694 & 0.705 & 0.708 \\
\hline 685.07 & 0.628 & 0.631 & 0.696 & 0.685 & 0.687 & 0.69 & 0.686 & 0.694 & 0.705 & 0.71 \\
\hline
\end{tabular}




\begin{tabular}{|c|c|c|c|c|c|c|c|c|c|c|}
\hline 685.4 & 0.629 & 0.632 & 0.693 & 0.686 & 0.684 & 0.691 & 0.684 & 0.694 & 0.703 & 0.706 \\
\hline 685.72 & 0.623 & 0.627 & 0.69 & 0.681 & 0.68 & 0.692 & 0.682 & 0.688 & 0.699 & 0.703 \\
\hline 686.05 & 0.616 & 0.622 & 0.69 & 0.681 & 0.678 & 0.689 & 0.675 & 0.685 & 0.694 & 0.699 \\
\hline 686.37 & 0.609 & 0.613 & 0.685 & 0.682 & 0.677 & 0.685 & 0.676 & 0.684 & 0.687 & 0.695 \\
\hline 686.7 & 0.604 & 0.61 & 0.682 & 0.68 & 0.675 & 0.683 & 0.669 & 0.68 & 0.685 & 0.69 \\
\hline 687.02 & 0.6 & 0.606 & 0.681 & 0.682 & 0.677 & 0.684 & 0.668 & 0.678 & 0.681 & 0.689 \\
\hline 687.35 & 0.594 & 0.599 & 0.676 & 0.678 & 0.676 & 0.681 & 0.663 & 0.675 & 0.677 & 0.684 \\
\hline 687.67 & 0.591 & 0.596 & 0.671 & 0.675 & 0.674 & 0.678 & 0.66 & 0.673 & 0.676 & 0.683 \\
\hline 688 & 0.587 & 0.593 & 0.67 & 0.67 & 0.676 & 0.675 & 0.657 & 0.672 & 0.673 & 0.678 \\
\hline 688.32 & 0.583 & 0.591 & 0.669 & 0.666 & 0.674 & 0.671 & 0.656 & 0.677 & 0.671 & 0.675 \\
\hline 688.65 & 0.579 & 0.59 & 0.664 & 0.664 & 0.672 & 0.668 & 0.654 & 0.669 & 0.666 & 0.67 \\
\hline 688.97 & 0.571 & 0.584 & 0.664 & 0.659 & 0.674 & 0.668 & 0.652 & 0.663 & 0.665 & 0.667 \\
\hline 689.3 & 0.567 & 0.578 & 0.662 & 0.659 & 0.676 & 0.666 & 0.652 & 0.662 & 0.66 & 0.666 \\
\hline 689.62 & 0.563 & 0.572 & 0.656 & 0.658 & 0.674 & 0.664 & 0.653 & 0.657 & 0.658 & 0.665 \\
\hline 689.95 & 0.559 & 0.57 & 0.653 & 0.655 & 0.674 & 0.662 & 0.651 & 0.653 & 0.656 & 0.665 \\
\hline 690.27 & 0.559 & 0.566 & 0.651 & 0.651 & 0.671 & 0.658 & 0.648 & 0.656 & 0.654 & 0.664 \\
\hline 690.6 & 0.556 & 0.565 & 0.649 & 0.65 & 0.666 & 0.655 & 0.648 & 0.652 & 0.655 & 0.658 \\
\hline 690.92 & 0.551 & 0.562 & 0.645 & 0.649 & 0.661 & 0.652 & 0.648 & 0.65 & 0.655 & 0.656 \\
\hline 691.25 & 0.543 & 0.553 & 0.646 & 0.644 & 0.656 & 0.645 & 0.645 & 0.648 & 0.651 & 0.65 \\
\hline 691.57 & 0.542 & 0.549 & 0.644 & 0.648 & 0.651 & 0.645 & 0.645 & 0.647 & 0.65 & 0.651 \\
\hline 691.89 & 0.537 & 0.542 & 0.639 & 0.646 & 0.646 & 0.642 & 0.642 & 0.64 & 0.647 & 0.648 \\
\hline 692.22 & 0.531 & 0.534 & 0.64 & 0.644 & 0.645 & 0.643 & 0.64 & 0.642 & 0.643 & 0.645 \\
\hline 692.54 & 0.525 & 0.527 & 0.635 & 0.638 & 0.639 & 0.639 & 0.639 & 0.64 & 0.643 & 0.643 \\
\hline 692.87 & 0.521 & 0.522 & 0.632 & 0.635 & 0.631 & 0.633 & 0.633 & 0.639 & 0.644 & 0.64 \\
\hline 693.19 & 0.516 & 0.519 & 0.627 & 0.627 & 0.624 & 0.628 & 0.63 & 0.638 & 0.64 & 0.637 \\
\hline 693.52 & 0.51 & 0.512 & 0.623 & 0.62 & 0.619 & 0.625 & 0.624 & 0.632 & 0.634 & 0.628 \\
\hline 693.84 & 0.5 & 0.503 & 0.618 & 0.615 & 0.611 & 0.617 & 0.618 & 0.618 & 0.624 & 0.621 \\
\hline 694.16 & 0.492 & 0.493 & 0.613 & 0.613 & 0.608 & 0.613 & 0.615 & 0.614 & 0.62 & 0.616 \\
\hline 694.49 & 0.483 & 0.485 & 0.607 & 0.612 & 0.605 & 0.61 & 0.612 & 0.609 & 0.612 & 0.617 \\
\hline 694.81 & 0.476 & 0.482 & 0.601 & 0.61 & 0.602 & 0.609 & 0.608 & 0.606 & 0.605 & 0.611 \\
\hline 695.14 & 0.465 & 0.472 & 0.595 & 0.602 & 0.596 & 0.605 & 0.599 & 0.599 & 0.599 & 0.604 \\
\hline 695.46 & 0.455 & 0.463 & 0.588 & 0.596 & 0.588 & 0.598 & 0.592 & 0.592 & 0.596 & 0.594 \\
\hline 695.78 & 0.448 & 0.454 & 0.582 & 0.589 & 0.58 & 0.589 & 0.587 & 0.587 & 0.592 & 0.59 \\
\hline 696.11 & 0.442 & 0.448 & 0.576 & 0.59 & 0.576 & 0.581 & 0.584 & 0.582 & 0.587 & 0.584 \\
\hline 696.43 & 0.433 & 0.439 & 0.572 & 0.587 & 0.571 & 0.576 & 0.582 & 0.577 & 0.579 & 0.578 \\
\hline 696.75 & 0.425 & 0.431 & 0.569 & 0.581 & 0.565 & 0.572 & 0.576 & 0.572 & 0.574 & 0.572 \\
\hline 697.08 & 0.414 & 0.419 & 0.562 & 0.577 & 0.558 & 0.564 & 0.567 & 0.564 & 0.569 & 0.566 \\
\hline 697.4 & 0.407 & 0.411 & 0.56 & 0.573 & 0.559 & 0.566 & 0.567 & 0.563 & 0.572 & 0.566 \\
\hline 697.72 & 0.398 & 0.401 & 0.554 & 0.563 & 0.551 & 0.559 & 0.558 & 0.558 & 0.564 & 0.56 \\
\hline
\end{tabular}




\begin{tabular}{|r|r|r|r|r|r|r|r|r|r|r|}
\hline 698.05 & 0.391 & 0.39 & 0.553 & 0.553 & 0.543 & 0.551 & 0.548 & 0.552 & 0.56 & 0.55 \\
\hline 698.37 & 0.382 & 0.38 & 0.546 & 0.546 & 0.536 & 0.541 & 0.54 & 0.544 & 0.553 & 0.544 \\
\hline 698.69 & 0.372 & 0.372 & 0.538 & 0.539 & 0.53 & 0.532 & 0.538 & 0.534 & 0.546 & 0.537 \\
\hline 699.02 & 0.366 & 0.365 & 0.533 & 0.532 & 0.526 & 0.53 & 0.534 & 0.529 & 0.538 & 0.535 \\
\hline 699.34 & 0.355 & 0.356 & 0.525 & 0.524 & 0.518 & 0.524 & 0.526 & 0.52 & 0.532 & 0.527 \\
\hline 699.66 & 0.344 & 0.347 & 0.522 & 0.516 & 0.512 & 0.52 & 0.517 & 0.514 & 0.522 & 0.521 \\
\hline 699.99 & 0.334 & 0.338 & 0.515 & 0.507 & 0.507 & 0.514 & 0.507 & 0.505 & 0.514 & 0.513 \\
\hline 700.31 & 0.325 & 0.327 & 0.509 & 0.503 & 0.504 & 0.511 & 0.5 & 0.5 & 0.508 & 0.507 \\
\hline
\end{tabular}

Absorbance values of Kelly Green A_F from 1-10 minutes for Part I of Phase III

\begin{tabular}{|c|c|c|c|c|c|c|c|c|c|c|}
\hline$\lambda$ & $30 \mathrm{~m}$ & $31 \mathrm{~m}$ & $32 \mathrm{~m}$ & $33 \mathrm{~m}$ & $34 \mathrm{~m}$ & $35 \mathrm{~m}$ & $36 \mathrm{~m}$ & $37 \mathrm{~m}$ & $38 \mathrm{~m}$ & $39 \mathrm{~m}$ \\
\hline 380.34 & 0.334 & 0.309 & 0.307 & 0.322 & 0.305 & 0.303 & 0.294 & 0.362 & 0.287 & 0.29 \\
\hline 380.71 & 0.332 & 0.306 & 0.307 & 0.327 & 0.305 & 0.3 & 0.296 & 0.36 & 0.285 & 0.289 \\
\hline 381.07 & 0.336 & 0.3 & 0.304 & 0.328 & 0.312 & 0.295 & 0.292 & 0.356 & 0.282 & 0.294 \\
\hline 381.44 & 0.329 & 0.293 & 0.306 & 0.321 & 0.309 & 0.296 & 0.288 & 0.359 & 0.279 & 0.289 \\
\hline 381.8 & 0.328 & 0.287 & 0.303 & 0.318 & 0.306 & 0.289 & 0.286 & 0.358 & 0.281 & 0.279 \\
\hline 382.17 & 0.32 & 0.285 & 0.299 & 0.312 & 0.306 & 0.283 & 0.28 & 0.347 & 0.281 & 0.276 \\
\hline 382.53 & 0.317 & 0.292 & 0.3 & 0.315 & 0.304 & 0.285 & 0.28 & 0.347 & 0.272 & 0.272 \\
\hline 382.89 & 0.315 & 0.293 & 0.295 & 0.313 & 0.3 & 0.285 & 0.281 & 0.351 & 0.271 & 0.268 \\
\hline 383.26 & 0.31 & 0.295 & 0.29 & 0.308 & 0.3 & 0.285 & 0.28 & 0.351 & 0.27 & 0.267 \\
\hline 383.62 & 0.306 & 0.288 & 0.285 & 0.306 & 0.298 & 0.286 & 0.281 & 0.346 & 0.267 & 0.265 \\
\hline 383.99 & 0.305 & 0.289 & 0.283 & 0.302 & 0.297 & 0.285 & 0.278 & 0.344 & 0.263 & 0.268 \\
\hline 384.35 & 0.302 & 0.29 & 0.28 & 0.297 & 0.299 & 0.284 & 0.272 & 0.336 & 0.259 & 0.262 \\
\hline 384.72 & 0.3 & 0.29 & 0.28 & 0.292 & 0.299 & 0.284 & 0.275 & 0.335 & 0.261 & 0.264 \\
\hline 385.08 & 0.295 & 0.284 & 0.28 & 0.286 & 0.293 & 0.282 & 0.271 & 0.329 & 0.258 & 0.256 \\
\hline 385.44 & 0.295 & 0.285 & 0.279 & 0.291 & 0.291 & 0.277 & 0.268 & 0.324 & 0.262 & 0.257 \\
\hline 385.81 & 0.294 & 0.287 & 0.278 & 0.285 & 0.291 & 0.282 & 0.265 & 0.32 & 0.256 & 0.261 \\
\hline 386.17 & 0.292 & 0.286 & 0.278 & 0.281 & 0.287 & 0.282 & 0.265 & 0.32 & 0.253 & 0.26 \\
\hline 386.53 & 0.295 & 0.279 & 0.276 & 0.28 & 0.286 & 0.28 & 0.263 & 0.318 & 0.253 & 0.26 \\
\hline 386.9 & 0.292 & 0.275 & 0.272 & 0.278 & 0.289 & 0.277 & 0.258 & 0.314 & 0.252 & 0.257 \\
\hline 387.26 & 0.291 & 0.276 & 0.269 & 0.275 & 0.289 & 0.27 & 0.252 & 0.302 & 0.248 & 0.254 \\
\hline 387.63 & 0.288 & 0.276 & 0.27 & 0.277 & 0.284 & 0.267 & 0.247 & 0.298 & 0.248 & 0.252 \\
\hline 387.99 & 0.29 & 0.275 & 0.27 & 0.274 & 0.283 & 0.269 & 0.247 & 0.295 & 0.246 & 0.25 \\
\hline 388.35 & 0.289 & 0.268 & 0.268 & 0.273 & 0.277 & 0.264 & 0.249 & 0.293 & 0.242 & 0.248 \\
\hline 388.72 & 0.29 & 0.266 & 0.263 & 0.266 & 0.272 & 0.262 & 0.243 & 0.291 & 0.238 & 0.244 \\
\hline 389.08 & 0.288 & 0.26 & 0.257 & 0.265 & 0.27 & 0.263 & 0.244 & 0.291 & 0.234 & 0.243 \\
\hline 389.44 & 0.284 & 0.258 & 0.254 & 0.26 & 0.269 & 0.263 & 0.246 & 0.29 & 0.233 & 0.242 \\
\hline
\end{tabular}




\begin{tabular}{|c|c|c|c|c|c|c|c|c|c|c|}
\hline 389.81 & 0.282 & 0.258 & 0.255 & 0.262 & 0.268 & 0.261 & 0.242 & 0.286 & 0.234 & 0.24 \\
\hline 390.17 & 0.284 & 0.253 & 0.257 & 0.263 & 0.269 & 0.26 & 0.24 & 0.285 & 0.235 & 0.239 \\
\hline 390.53 & 0.282 & 0.254 & 0.256 & 0.258 & 0.267 & 0.261 & 0.238 & 0.287 & 0.235 & 0.241 \\
\hline 390.9 & 0.281 & 0.254 & 0.257 & 0.257 & 0.263 & 0.261 & 0.238 & 0.286 & 0.234 & 0.239 \\
\hline 391.26 & 0.284 & 0.251 & 0.258 & 0.258 & 0.254 & 0.256 & 0.243 & 0.286 & 0.23 & 0.241 \\
\hline 391.62 & 0.283 & 0.251 & 0.255 & 0.256 & 0.256 & 0.254 & 0.244 & 0.286 & 0.226 & 0.243 \\
\hline 391.99 & 0.278 & 0.245 & 0.253 & 0.256 & 0.252 & 0.251 & 0.243 & 0.288 & 0.228 & 0.241 \\
\hline 392.35 & 0.277 & 0.246 & 0.254 & 0.256 & 0.256 & 0.254 & 0.242 & 0.288 & 0.229 & 0.242 \\
\hline 392.71 & 0.273 & 0.249 & 0.254 & 0.257 & 0.256 & 0.255 & 0.239 & 0.289 & 0.228 & 0.242 \\
\hline 393.08 & 0.272 & 0.252 & 0.254 & 0.254 & 0.252 & 0.251 & 0.238 & 0.286 & 0.23 & 0.239 \\
\hline 393.44 & 0.275 & 0.254 & 0.253 & 0.254 & 0.254 & 0.249 & 0.232 & 0.284 & 0.23 & 0.237 \\
\hline 393.8 & 0.276 & 0.249 & 0.252 & 0.254 & 0.252 & 0.245 & 0.232 & 0.283 & 0.228 & 0.235 \\
\hline 394.17 & 0.274 & 0.249 & 0.251 & 0.254 & 0.247 & 0.24 & 0.231 & 0.281 & 0.225 & 0.235 \\
\hline 394.53 & 0.27 & 0.25 & 0.253 & 0.252 & 0.246 & 0.239 & 0.231 & 0.275 & 0.227 & 0.232 \\
\hline 394.89 & 0.27 & 0.244 & 0.251 & 0.253 & 0.245 & 0.236 & 0.226 & 0.274 & 0.23 & 0.232 \\
\hline 395.25 & 0.27 & 0.245 & 0.251 & 0.248 & 0.248 & 0.238 & 0.22 & 0.273 & 0.232 & 0.229 \\
\hline 395.62 & 0.274 & 0.242 & 0.25 & 0.243 & 0.248 & 0.237 & 0.217 & 0.273 & 0.231 & 0.226 \\
\hline 395.98 & 0.273 & 0.245 & 0.252 & 0.244 & 0.251 & 0.236 & 0.217 & 0.273 & 0.229 & 0.23 \\
\hline 396.34 & 0.274 & 0.247 & 0.252 & 0.244 & 0.25 & 0.236 & 0.218 & 0.273 & 0.229 & 0.227 \\
\hline 396.71 & 0.277 & 0.244 & 0.252 & 0.245 & 0.25 & 0.236 & 0.222 & 0.275 & 0.229 & 0.228 \\
\hline 397.07 & 0.278 & 0.243 & 0.253 & 0.246 & 0.251 & 0.237 & 0.221 & 0.276 & 0.228 & 0.231 \\
\hline 397.43 & 0.278 & 0.245 & 0.251 & 0.243 & 0.249 & 0.237 & 0.225 & 0.277 & 0.226 & 0.234 \\
\hline 397.79 & 0.274 & 0.246 & 0.251 & 0.241 & 0.251 & 0.238 & 0.228 & 0.281 & 0.226 & 0.235 \\
\hline 398.16 & 0.273 & 0.248 & 0.251 & 0.241 & 0.257 & 0.238 & 0.228 & 0.283 & 0.229 & 0.235 \\
\hline 398.52 & 0.275 & 0.248 & 0.25 & 0.242 & 0.258 & 0.236 & 0.226 & 0.284 & 0.227 & 0.234 \\
\hline 398.88 & 0.277 & 0.253 & 0.25 & 0.241 & 0.258 & 0.238 & 0.226 & 0.284 & 0.225 & 0.232 \\
\hline 399.24 & 0.277 & 0.251 & 0.247 & 0.241 & 0.259 & 0.238 & 0.229 & 0.29 & 0.227 & 0.235 \\
\hline 399.61 & 0.276 & 0.253 & 0.249 & 0.243 & 0.259 & 0.24 & 0.233 & 0.294 & 0.227 & 0.238 \\
\hline 399.97 & 0.276 & 0.25 & 0.247 & 0.241 & 0.255 & 0.241 & 0.233 & 0.293 & 0.226 & 0.237 \\
\hline 400.33 & 0.277 & 0.251 & 0.247 & 0.241 & 0.252 & 0.241 & 0.229 & 0.291 & 0.225 & 0.239 \\
\hline 400.69 & 0.276 & 0.252 & 0.246 & 0.24 & 0.25 & 0.239 & 0.225 & 0.288 & 0.223 & 0.236 \\
\hline 401.06 & 0.279 & 0.254 & 0.248 & 0.239 & 0.251 & 0.24 & 0.224 & 0.286 & 0.221 & 0.236 \\
\hline 401.42 & 0.278 & 0.252 & 0.252 & 0.241 & 0.249 & 0.241 & 0.223 & 0.286 & 0.222 & 0.235 \\
\hline 401.78 & 0.28 & 0.251 & 0.251 & 0.24 & 0.246 & 0.241 & 0.218 & 0.285 & 0.223 & 0.237 \\
\hline 402.14 & 0.281 & 0.25 & 0.249 & 0.242 & 0.243 & 0.243 & 0.219 & 0.284 & 0.221 & 0.237 \\
\hline 402.5 & 0.28 & 0.246 & 0.246 & 0.242 & 0.243 & 0.242 & 0.218 & 0.285 & 0.219 & 0.239 \\
\hline 402.87 & 0.279 & 0.245 & 0.245 & 0.241 & 0.244 & 0.241 & 0.219 & 0.286 & 0.216 & 0.24 \\
\hline 403.23 & 0.276 & 0.249 & 0.248 & 0.245 & 0.246 & 0.245 & 0.221 & 0.285 & 0.214 & 0.242 \\
\hline 403.59 & 0.276 & 0.25 & 0.251 & 0.244 & 0.247 & 0.243 & 0.22 & 0.285 & 0.215 & 0.236 \\
\hline
\end{tabular}




\begin{tabular}{|c|c|c|c|c|c|c|c|c|c|c|}
\hline 403.95 & 0.279 & 0.252 & 0.254 & 0.244 & 0.251 & 0.241 & 0.22 & 0.285 & 0.212 & 0.233 \\
\hline 404.31 & 0.277 & 0.249 & 0.255 & 0.248 & 0.256 & 0.243 & 0.222 & 0.288 & 0.212 & 0.23 \\
\hline 404.68 & 0.275 & 0.25 & 0.257 & 0.245 & 0.257 & 0.245 & 0.222 & 0.29 & 0.212 & 0.231 \\
\hline 405.04 & 0.275 & 0.252 & 0.257 & 0.247 & 0.259 & 0.247 & 0.223 & 0.29 & 0.214 & 0.23 \\
\hline 405.4 & 0.274 & 0.253 & 0.255 & 0.248 & 0.263 & 0.245 & 0.223 & 0.292 & 0.214 & 0.229 \\
\hline 405.76 & 0.276 & 0.254 & 0.259 & 0.252 & 0.264 & 0.245 & 0.222 & 0.293 & 0.214 & 0.229 \\
\hline 406.12 & 0.277 & 0.254 & 0.262 & 0.252 & 0.265 & 0.247 & 0.221 & 0.294 & 0.213 & 0.226 \\
\hline 406.49 & 0.279 & 0.255 & 0.264 & 0.251 & 0.266 & 0.25 & 0.225 & 0.295 & 0.214 & 0.226 \\
\hline 406.85 & 0.279 & 0.256 & 0.264 & 0.254 & 0.266 & 0.25 & 0.224 & 0.292 & 0.212 & 0.228 \\
\hline 407.21 & 0.282 & 0.255 & 0.26 & 0.25 & 0.262 & 0.25 & 0.223 & 0.293 & 0.211 & 0.225 \\
\hline 407.57 & 0.283 & 0.255 & 0.259 & 0.25 & 0.265 & 0.252 & 0.222 & 0.293 & 0.213 & 0.229 \\
\hline 407.93 & 0.281 & 0.256 & 0.257 & 0.251 & 0.261 & 0.254 & 0.222 & 0.294 & 0.214 & 0.227 \\
\hline 408.29 & 0.281 & 0.257 & 0.255 & 0.249 & 0.26 & 0.252 & 0.219 & 0.294 & 0.214 & 0.229 \\
\hline 408.65 & 0.284 & 0.254 & 0.253 & 0.253 & 0.261 & 0.253 & 0.22 & 0.293 & 0.217 & 0.229 \\
\hline 409.02 & 0.283 & 0.251 & 0.249 & 0.252 & 0.259 & 0.25 & 0.221 & 0.295 & 0.217 & 0.229 \\
\hline 409.38 & 0.282 & 0.249 & 0.25 & 0.252 & 0.258 & 0.252 & 0.224 & 0.293 & 0.215 & 0.231 \\
\hline 409.74 & 0.282 & 0.248 & 0.248 & 0.252 & 0.259 & 0.256 & 0.229 & 0.295 & 0.215 & 0.231 \\
\hline 410.1 & 0.283 & 0.248 & 0.247 & 0.252 & 0.259 & 0.255 & 0.232 & 0.295 & 0.216 & 0.23 \\
\hline 410.46 & 0.28 & 0.248 & 0.247 & 0.255 & 0.26 & 0.254 & 0.23 & 0.295 & 0.217 & 0.231 \\
\hline 410.82 & 0.28 & 0.248 & 0.246 & 0.254 & 0.26 & 0.255 & 0.232 & 0.297 & 0.22 & 0.23 \\
\hline 411.18 & 0.279 & 0.247 & 0.25 & 0.255 & 0.262 & 0.255 & 0.232 & 0.298 & 0.222 & 0.233 \\
\hline 411.54 & 0.279 & 0.245 & 0.248 & 0.256 & 0.26 & 0.251 & 0.231 & 0.298 & 0.221 & 0.234 \\
\hline 411.91 & 0.28 & 0.244 & 0.247 & 0.256 & 0.262 & 0.249 & 0.23 & 0.295 & 0.222 & 0.237 \\
\hline 412.27 & 0.279 & 0.244 & 0.247 & 0.256 & 0.26 & 0.249 & 0.234 & 0.294 & 0.222 & 0.238 \\
\hline 412.63 & 0.28 & 0.247 & 0.246 & 0.254 & 0.259 & 0.248 & 0.233 & 0.293 & 0.219 & 0.236 \\
\hline 412.99 & 0.282 & 0.25 & 0.248 & 0.255 & 0.259 & 0.251 & 0.233 & 0.296 & 0.219 & 0.239 \\
\hline 413.35 & 0.283 & 0.25 & 0.246 & 0.253 & 0.259 & 0.251 & 0.23 & 0.296 & 0.219 & 0.236 \\
\hline 413.71 & 0.282 & 0.251 & 0.245 & 0.253 & 0.259 & 0.249 & 0.229 & 0.296 & 0.218 & 0.235 \\
\hline 414.07 & 0.281 & 0.256 & 0.245 & 0.255 & 0.258 & 0.249 & 0.227 & 0.296 & 0.219 & 0.238 \\
\hline 414.43 & 0.282 & 0.254 & 0.243 & 0.254 & 0.256 & 0.25 & 0.228 & 0.294 & 0.218 & 0.237 \\
\hline 414.79 & 0.284 & 0.254 & 0.244 & 0.254 & 0.258 & 0.25 & 0.226 & 0.296 & 0.219 & 0.235 \\
\hline 415.15 & 0.284 & 0.254 & 0.244 & 0.257 & 0.259 & 0.248 & 0.227 & 0.299 & 0.22 & 0.235 \\
\hline 415.51 & 0.286 & 0.255 & 0.245 & 0.257 & 0.261 & 0.251 & 0.229 & 0.299 & 0.222 & 0.235 \\
\hline 415.88 & 0.284 & 0.255 & 0.248 & 0.257 & 0.261 & 0.253 & 0.232 & 0.301 & 0.22 & 0.232 \\
\hline 416.24 & 0.285 & 0.255 & 0.249 & 0.255 & 0.262 & 0.254 & 0.23 & 0.304 & 0.22 & 0.232 \\
\hline 416.6 & 0.285 & 0.255 & 0.25 & 0.255 & 0.262 & 0.257 & 0.232 & 0.306 & 0.223 & 0.236 \\
\hline 416.96 & 0.282 & 0.253 & 0.249 & 0.256 & 0.264 & 0.255 & 0.232 & 0.305 & 0.224 & 0.236 \\
\hline 417.32 & 0.283 & 0.252 & 0.251 & 0.259 & 0.264 & 0.254 & 0.234 & 0.307 & 0.225 & 0.239 \\
\hline 417.68 & 0.285 & 0.253 & 0.25 & 0.257 & 0.265 & 0.255 & 0.233 & 0.309 & 0.228 & 0.241 \\
\hline
\end{tabular}




\begin{tabular}{|c|c|c|c|c|c|c|c|c|c|c|}
\hline 418.04 & 0.284 & 0.25 & 0.249 & 0.256 & 0.264 & 0.256 & 0.233 & 0.308 & 0.228 & 0.24 \\
\hline 418.4 & 0.284 & 0.252 & 0.251 & 0.253 & 0.264 & 0.256 & 0.233 & 0.311 & 0.227 & 0.24 \\
\hline 418.76 & 0.285 & 0.25 & 0.251 & 0.254 & 0.264 & 0.255 & 0.233 & 0.309 & 0.224 & 0.241 \\
\hline 419.12 & 0.283 & 0.25 & 0.249 & 0.252 & 0.262 & 0.255 & 0.232 & 0.304 & 0.221 & 0.239 \\
\hline 419.48 & 0.282 & 0.25 & 0.248 & 0.252 & 0.259 & 0.254 & 0.23 & 0.302 & 0.22 & 0.24 \\
\hline 419.84 & 0.285 & 0.25 & 0.247 & 0.254 & 0.258 & 0.254 & 0.23 & 0.302 & 0.221 & 0.243 \\
\hline 420.2 & 0.286 & 0.248 & 0.244 & 0.258 & 0.258 & 0.254 & 0.231 & 0.3 & 0.219 & 0.243 \\
\hline 420.56 & 0.286 & 0.248 & 0.244 & 0.26 & 0.259 & 0.252 & 0.232 & 0.301 & 0.221 & 0.241 \\
\hline 420.92 & 0.288 & 0.25 & 0.246 & 0.261 & 0.258 & 0.253 & 0.234 & 0.299 & 0.221 & 0.241 \\
\hline 421.28 & 0.284 & 0.252 & 0.245 & 0.261 & 0.259 & 0.252 & 0.233 & 0.299 & 0.221 & 0.241 \\
\hline 421.64 & 0.283 & 0.252 & 0.247 & 0.263 & 0.259 & 0.252 & 0.234 & 0.298 & 0.22 & 0.242 \\
\hline 422 & 0.285 & 0.255 & 0.247 & 0.264 & 0.263 & 0.254 & 0.237 & 0.299 & 0.221 & 0.243 \\
\hline 422.36 & 0.285 & 0.255 & 0.247 & 0.265 & 0.265 & 0.253 & 0.238 & 0.299 & 0.223 & 0.244 \\
\hline 422.72 & 0.284 & 0.255 & 0.246 & 0.267 & 0.265 & 0.255 & 0.238 & 0.3 & 0.225 & 0.245 \\
\hline 423.08 & 0.286 & 0.257 & 0.248 & 0.268 & 0.267 & 0.256 & 0.238 & 0.303 & 0.226 & 0.249 \\
\hline 423.44 & 0.284 & 0.258 & 0.249 & 0.268 & 0.266 & 0.254 & 0.24 & 0.305 & 0.226 & 0.249 \\
\hline 423.8 & 0.286 & 0.258 & 0.248 & 0.268 & 0.267 & 0.255 & 0.24 & 0.306 & 0.225 & 0.248 \\
\hline 424.16 & 0.285 & 0.261 & 0.251 & 0.267 & 0.266 & 0.256 & 0.239 & 0.306 & 0.228 & 0.248 \\
\hline 424.52 & 0.284 & 0.265 & 0.251 & 0.268 & 0.267 & 0.255 & 0.24 & 0.307 & 0.229 & 0.249 \\
\hline 424.88 & 0.286 & 0.265 & 0.249 & 0.268 & 0.268 & 0.254 & 0.238 & 0.308 & 0.228 & 0.25 \\
\hline 425.24 & 0.288 & 0.263 & 0.248 & 0.268 & 0.266 & 0.254 & 0.239 & 0.305 & 0.227 & 0.247 \\
\hline 425.6 & 0.289 & 0.259 & 0.248 & 0.269 & 0.266 & 0.253 & 0.238 & 0.302 & 0.227 & 0.244 \\
\hline 425.96 & 0.287 & 0.256 & 0.248 & 0.269 & 0.265 & 0.253 & 0.236 & 0.303 & 0.229 & 0.245 \\
\hline 426.32 & 0.289 & 0.257 & 0.249 & 0.271 & 0.264 & 0.253 & 0.237 & 0.302 & 0.228 & 0.246 \\
\hline 426.68 & 0.292 & 0.256 & 0.252 & 0.27 & 0.263 & 0.25 & 0.239 & 0.303 & 0.23 & 0.247 \\
\hline 427.04 & 0.294 & 0.255 & 0.253 & 0.269 & 0.26 & 0.249 & 0.24 & 0.301 & 0.231 & 0.247 \\
\hline 427.4 & 0.296 & 0.255 & 0.253 & 0.271 & 0.264 & 0.252 & 0.239 & 0.3 & 0.232 & 0.246 \\
\hline 427.76 & 0.294 & 0.255 & 0.254 & 0.27 & 0.263 & 0.25 & 0.236 & 0.299 & 0.232 & 0.247 \\
\hline 428.12 & 0.294 & 0.253 & 0.253 & 0.271 & 0.264 & 0.248 & 0.236 & 0.298 & 0.23 & 0.247 \\
\hline 428.47 & 0.294 & 0.25 & 0.253 & 0.271 & 0.261 & 0.25 & 0.233 & 0.297 & 0.228 & 0.245 \\
\hline 428.83 & 0.294 & 0.247 & 0.253 & 0.27 & 0.26 & 0.249 & 0.234 & 0.298 & 0.229 & 0.243 \\
\hline 429.19 & 0.295 & 0.247 & 0.253 & 0.268 & 0.261 & 0.25 & 0.234 & 0.299 & 0.232 & 0.244 \\
\hline 429.55 & 0.296 & 0.25 & 0.254 & 0.268 & 0.262 & 0.251 & 0.236 & 0.302 & 0.234 & 0.245 \\
\hline 429.91 & 0.298 & 0.252 & 0.254 & 0.267 & 0.263 & 0.249 & 0.234 & 0.3 & 0.232 & 0.245 \\
\hline 430.27 & 0.297 & 0.25 & 0.254 & 0.268 & 0.261 & 0.249 & 0.234 & 0.301 & 0.233 & 0.243 \\
\hline 430.63 & 0.297 & 0.249 & 0.253 & 0.267 & 0.26 & 0.251 & 0.231 & 0.297 & 0.229 & 0.242 \\
\hline 430.99 & 0.296 & 0.248 & 0.25 & 0.268 & 0.26 & 0.252 & 0.232 & 0.298 & 0.229 & 0.24 \\
\hline 431.35 & 0.293 & 0.248 & 0.25 & 0.266 & 0.258 & 0.252 & 0.232 & 0.298 & 0.228 & 0.239 \\
\hline 431.71 & 0.292 & 0.249 & 0.249 & 0.266 & 0.258 & 0.253 & 0.233 & 0.299 & 0.231 & 0.238 \\
\hline
\end{tabular}




\begin{tabular}{|c|c|c|c|c|c|c|c|c|c|c|}
\hline 432.07 & 0.291 & 0.248 & 0.249 & 0.264 & 0.257 & 0.255 & 0.233 & 0.299 & 0.233 & 0.238 \\
\hline 432.42 & 0.29 & 0.25 & 0.248 & 0.264 & 0.258 & 0.255 & 0.234 & 0.299 & 0.232 & 0.239 \\
\hline 432.78 & 0.29 & 0.252 & 0.247 & 0.264 & 0.257 & 0.258 & 0.235 & 0.298 & 0.233 & 0.241 \\
\hline 433.14 & 0.287 & 0.254 & 0.248 & 0.265 & 0.255 & 0.259 & 0.235 & 0.298 & 0.23 & 0.24 \\
\hline 433.5 & 0.286 & 0.253 & 0.247 & 0.265 & 0.254 & 0.257 & 0.234 & 0.295 & 0.229 & 0.24 \\
\hline 433.86 & 0.284 & 0.252 & 0.247 & 0.265 & 0.252 & 0.259 & 0.235 & 0.296 & 0.228 & 0.242 \\
\hline 434.22 & 0.283 & 0.253 & 0.245 & 0.263 & 0.251 & 0.259 & 0.233 & 0.295 & 0.228 & 0.242 \\
\hline 434.58 & 0.28 & 0.253 & 0.244 & 0.26 & 0.252 & 0.258 & 0.235 & 0.296 & 0.23 & 0.242 \\
\hline 434.94 & 0.279 & 0.252 & 0.244 & 0.259 & 0.252 & 0.259 & 0.233 & 0.295 & 0.23 & 0.241 \\
\hline 435.29 & 0.278 & 0.251 & 0.243 & 0.258 & 0.252 & 0.258 & 0.233 & 0.295 & 0.229 & 0.241 \\
\hline 435.65 & 0.279 & 0.25 & 0.244 & 0.257 & 0.251 & 0.256 & 0.233 & 0.294 & 0.227 & 0.24 \\
\hline 436.01 & 0.278 & 0.251 & 0.245 & 0.257 & 0.251 & 0.254 & 0.233 & 0.293 & 0.227 & 0.239 \\
\hline 436.37 & 0.278 & 0.25 & 0.246 & 0.255 & 0.252 & 0.253 & 0.231 & 0.291 & 0.227 & 0.236 \\
\hline 436.73 & 0.276 & 0.248 & 0.247 & 0.255 & 0.252 & 0.252 & 0.229 & 0.289 & 0.226 & 0.236 \\
\hline 437.09 & 0.275 & 0.245 & 0.247 & 0.254 & 0.252 & 0.25 & 0.225 & 0.287 & 0.226 & 0.235 \\
\hline 437.44 & 0.275 & 0.246 & 0.247 & 0.254 & 0.252 & 0.251 & 0.225 & 0.288 & 0.227 & 0.234 \\
\hline 437.8 & 0.274 & 0.244 & 0.248 & 0.252 & 0.25 & 0.249 & 0.223 & 0.287 & 0.228 & 0.232 \\
\hline 438.16 & 0.273 & 0.244 & 0.249 & 0.253 & 0.251 & 0.25 & 0.224 & 0.287 & 0.229 & 0.23 \\
\hline 438.52 & 0.272 & 0.245 & 0.25 & 0.255 & 0.252 & 0.252 & 0.222 & 0.284 & 0.228 & 0.229 \\
\hline 438.88 & 0.272 & 0.246 & 0.252 & 0.254 & 0.253 & 0.251 & 0.222 & 0.285 & 0.229 & 0.23 \\
\hline 439.24 & 0.272 & 0.245 & 0.252 & 0.253 & 0.253 & 0.249 & 0.22 & 0.285 & 0.23 & 0.232 \\
\hline 439.59 & 0.271 & 0.245 & 0.253 & 0.256 & 0.253 & 0.25 & 0.22 & 0.286 & 0.231 & 0.233 \\
\hline 439.95 & 0.27 & 0.245 & 0.252 & 0.256 & 0.253 & 0.249 & 0.219 & 0.286 & 0.228 & 0.233 \\
\hline 440.31 & 0.269 & 0.244 & 0.251 & 0.255 & 0.252 & 0.247 & 0.221 & 0.286 & 0.227 & 0.235 \\
\hline 440.67 & 0.27 & 0.242 & 0.25 & 0.253 & 0.253 & 0.247 & 0.224 & 0.286 & 0.227 & 0.234 \\
\hline 441.03 & 0.269 & 0.243 & 0.251 & 0.251 & 0.253 & 0.247 & 0.225 & 0.287 & 0.227 & 0.234 \\
\hline 441.38 & 0.27 & 0.242 & 0.252 & 0.252 & 0.252 & 0.248 & 0.224 & 0.286 & 0.227 & 0.235 \\
\hline 441.74 & 0.269 & 0.242 & 0.25 & 0.252 & 0.253 & 0.248 & 0.224 & 0.286 & 0.226 & 0.233 \\
\hline 442.1 & 0.268 & 0.241 & 0.25 & 0.251 & 0.251 & 0.246 & 0.224 & 0.285 & 0.224 & 0.235 \\
\hline 442.46 & 0.266 & 0.24 & 0.248 & 0.251 & 0.251 & 0.243 & 0.223 & 0.286 & 0.223 & 0.234 \\
\hline 442.81 & 0.264 & 0.24 & 0.246 & 0.251 & 0.249 & 0.242 & 0.222 & 0.285 & 0.222 & 0.233 \\
\hline 443.17 & 0.262 & 0.24 & 0.247 & 0.253 & 0.248 & 0.243 & 0.222 & 0.282 & 0.22 & 0.232 \\
\hline 443.53 & 0.26 & 0.239 & 0.245 & 0.251 & 0.248 & 0.243 & 0.22 & 0.28 & 0.22 & 0.232 \\
\hline 443.89 & 0.26 & 0.238 & 0.244 & 0.25 & 0.247 & 0.242 & 0.219 & 0.28 & 0.221 & 0.231 \\
\hline 444.24 & 0.258 & 0.238 & 0.243 & 0.249 & 0.248 & 0.244 & 0.218 & 0.279 & 0.221 & 0.229 \\
\hline 444.6 & 0.258 & 0.239 & 0.243 & 0.25 & 0.246 & 0.243 & 0.217 & 0.279 & 0.222 & 0.229 \\
\hline 444.96 & 0.257 & 0.238 & 0.242 & 0.25 & 0.244 & 0.241 & 0.217 & 0.277 & 0.221 & 0.228 \\
\hline 445.32 & 0.255 & 0.238 & 0.241 & 0.248 & 0.245 & 0.239 & 0.216 & 0.277 & 0.22 & 0.227 \\
\hline 445.67 & 0.257 & 0.238 & 0.241 & 0.245 & 0.243 & 0.239 & 0.216 & 0.277 & 0.219 & 0.228 \\
\hline
\end{tabular}




\begin{tabular}{|c|c|c|c|c|c|c|c|c|c|c|}
\hline 446.03 & 0.256 & 0.237 & 0.239 & 0.245 & 0.242 & 0.238 & 0.214 & 0.276 & 0.22 & 0.227 \\
\hline 446.39 & 0.258 & 0.238 & 0.239 & 0.242 & 0.241 & 0.239 & 0.213 & 0.277 & 0.22 & 0.226 \\
\hline 446.75 & 0.257 & 0.236 & 0.239 & 0.241 & 0.241 & 0.239 & 0.212 & 0.276 & 0.219 & 0.225 \\
\hline 447.1 & 0.257 & 0.236 & 0.239 & 0.239 & 0.242 & 0.235 & 0.213 & 0.276 & 0.22 & 0.225 \\
\hline 447.46 & 0.258 & 0.236 & 0.238 & 0.238 & 0.24 & 0.235 & 0.213 & 0.275 & 0.219 & 0.225 \\
\hline 447.82 & 0.256 & 0.235 & 0.239 & 0.237 & 0.24 & 0.235 & 0.212 & 0.274 & 0.219 & 0.224 \\
\hline 448.18 & 0.256 & 0.233 & 0.238 & 0.237 & 0.238 & 0.234 & 0.21 & 0.274 & 0.219 & 0.224 \\
\hline 448.53 & 0.256 & 0.232 & 0.238 & 0.237 & 0.238 & 0.233 & 0.209 & 0.274 & 0.218 & 0.222 \\
\hline 448.89 & 0.255 & 0.232 & 0.238 & 0.236 & 0.239 & 0.234 & 0.208 & 0.276 & 0.217 & 0.22 \\
\hline 449.25 & 0.255 & 0.23 & 0.238 & 0.234 & 0.238 & 0.235 & 0.206 & 0.274 & 0.217 & 0.219 \\
\hline 449.6 & 0.252 & 0.228 & 0.237 & 0.234 & 0.237 & 0.234 & 0.204 & 0.274 & 0.215 & 0.218 \\
\hline 449.96 & 0.251 & 0.226 & 0.238 & 0.233 & 0.238 & 0.232 & 0.203 & 0.273 & 0.213 & 0.217 \\
\hline 450.32 & 0.248 & 0.223 & 0.237 & 0.233 & 0.237 & 0.231 & 0.202 & 0.272 & 0.213 & 0.217 \\
\hline 450.67 & 0.25 & 0.223 & 0.238 & 0.232 & 0.236 & 0.231 & 0.202 & 0.272 & 0.213 & 0.217 \\
\hline 451.03 & 0.247 & 0.223 & 0.236 & 0.23 & 0.235 & 0.231 & 0.202 & 0.271 & 0.213 & 0.215 \\
\hline 451.39 & 0.246 & 0.221 & 0.234 & 0.229 & 0.233 & 0.228 & 0.2 & 0.271 & 0.211 & 0.214 \\
\hline 451.74 & 0.247 & 0.221 & 0.233 & 0.228 & 0.232 & 0.227 & 0.199 & 0.27 & 0.211 & 0.213 \\
\hline 452.1 & 0.246 & 0.22 & 0.233 & 0.226 & 0.23 & 0.226 & 0.198 & 0.269 & 0.21 & 0.211 \\
\hline 452.46 & 0.246 & 0.218 & 0.231 & 0.226 & 0.229 & 0.225 & 0.196 & 0.266 & 0.208 & 0.211 \\
\hline 452.81 & 0.245 & 0.217 & 0.229 & 0.225 & 0.228 & 0.222 & 0.194 & 0.264 & 0.207 & 0.209 \\
\hline 453.17 & 0.245 & 0.215 & 0.226 & 0.225 & 0.227 & 0.22 & 0.194 & 0.262 & 0.207 & 0.209 \\
\hline 453.53 & 0.244 & 0.214 & 0.225 & 0.225 & 0.226 & 0.219 & 0.194 & 0.261 & 0.209 & 0.208 \\
\hline 453.88 & 0.242 & 0.214 & 0.223 & 0.223 & 0.225 & 0.219 & 0.195 & 0.261 & 0.21 & 0.209 \\
\hline 454.24 & 0.241 & 0.214 & 0.223 & 0.223 & 0.223 & 0.217 & 0.196 & 0.26 & 0.209 & 0.207 \\
\hline 454.6 & 0.239 & 0.21 & 0.221 & 0.222 & 0.222 & 0.216 & 0.193 & 0.259 & 0.208 & 0.207 \\
\hline 454.95 & 0.238 & 0.208 & 0.22 & 0.222 & 0.221 & 0.216 & 0.192 & 0.257 & 0.209 & 0.205 \\
\hline 455.31 & 0.237 & 0.208 & 0.221 & 0.22 & 0.221 & 0.215 & 0.19 & 0.255 & 0.208 & 0.204 \\
\hline 455.66 & 0.236 & 0.207 & 0.22 & 0.219 & 0.221 & 0.213 & 0.19 & 0.254 & 0.207 & 0.203 \\
\hline 456.02 & 0.234 & 0.206 & 0.219 & 0.219 & 0.221 & 0.212 & 0.19 & 0.254 & 0.208 & 0.203 \\
\hline 456.38 & 0.232 & 0.206 & 0.218 & 0.218 & 0.219 & 0.212 & 0.19 & 0.255 & 0.209 & 0.2 \\
\hline 456.73 & 0.23 & 0.205 & 0.218 & 0.216 & 0.219 & 0.212 & 0.19 & 0.254 & 0.208 & 0.199 \\
\hline 457.09 & 0.229 & 0.204 & 0.218 & 0.215 & 0.219 & 0.211 & 0.189 & 0.254 & 0.207 & 0.197 \\
\hline 457.45 & 0.227 & 0.204 & 0.216 & 0.214 & 0.218 & 0.21 & 0.188 & 0.254 & 0.206 & 0.196 \\
\hline 457.8 & 0.226 & 0.203 & 0.216 & 0.213 & 0.217 & 0.209 & 0.186 & 0.253 & 0.203 & 0.194 \\
\hline 458.16 & 0.227 & 0.201 & 0.214 & 0.211 & 0.216 & 0.208 & 0.186 & 0.253 & 0.202 & 0.193 \\
\hline 458.51 & 0.226 & 0.2 & 0.213 & 0.21 & 0.215 & 0.208 & 0.184 & 0.251 & 0.202 & 0.191 \\
\hline 458.87 & 0.224 & 0.197 & 0.211 & 0.208 & 0.214 & 0.207 & 0.184 & 0.252 & 0.2 & 0.19 \\
\hline 459.23 & 0.222 & 0.194 & 0.209 & 0.209 & 0.212 & 0.207 & 0.183 & 0.253 & 0.2 & 0.19 \\
\hline 459.58 & 0.221 & 0.193 & 0.207 & 0.206 & 0.212 & 0.208 & 0.183 & 0.252 & 0.2 & 0.188 \\
\hline
\end{tabular}




\begin{tabular}{|c|c|c|c|c|c|c|c|c|c|c|}
\hline 459.94 & 0.22 & 0.191 & 0.205 & 0.205 & 0.21 & 0.208 & 0.182 & 0.25 & 0.197 & 0.185 \\
\hline 460.29 & 0.219 & 0.189 & 0.204 & 0.203 & 0.209 & 0.207 & 0.18 & 0.248 & 0.196 & 0.185 \\
\hline 460.65 & 0.218 & 0.188 & 0.201 & 0.202 & 0.208 & 0.205 & 0.178 & 0.247 & 0.196 & 0.185 \\
\hline 461 & 0.217 & 0.187 & 0.2 & 0.203 & 0.206 & 0.204 & 0.175 & 0.247 & 0.195 & 0.185 \\
\hline 461.36 & 0.217 & 0.185 & 0.198 & 0.202 & 0.206 & 0.204 & 0.173 & 0.245 & 0.194 & 0.183 \\
\hline 461.71 & 0.215 & 0.183 & 0.199 & 0.2 & 0.205 & 0.203 & 0.172 & 0.243 & 0.193 & 0.181 \\
\hline 462.07 & 0.214 & 0.182 & 0.198 & 0.2 & 0.204 & 0.202 & 0.17 & 0.241 & 0.193 & 0.181 \\
\hline 462.43 & 0.213 & 0.181 & 0.198 & 0.199 & 0.204 & 0.2 & 0.17 & 0.24 & 0.19 & 0.181 \\
\hline 462.78 & 0.212 & 0.181 & 0.196 & 0.198 & 0.203 & 0.199 & 0.168 & 0.238 & 0.187 & 0.181 \\
\hline 463.14 & 0.212 & 0.179 & 0.195 & 0.198 & 0.201 & 0.198 & 0.166 & 0.236 & 0.187 & 0.179 \\
\hline 463.49 & 0.21 & 0.176 & 0.196 & 0.197 & 0.199 & 0.198 & 0.165 & 0.235 & 0.185 & 0.179 \\
\hline 463.85 & 0.208 & 0.175 & 0.195 & 0.197 & 0.199 & 0.197 & 0.165 & 0.235 & 0.184 & 0.18 \\
\hline 464.2 & 0.206 & 0.175 & 0.193 & 0.196 & 0.198 & 0.195 & 0.164 & 0.234 & 0.183 & 0.18 \\
\hline 464.56 & 0.206 & 0.173 & 0.192 & 0.195 & 0.197 & 0.195 & 0.163 & 0.233 & 0.182 & 0.178 \\
\hline 464.91 & 0.204 & 0.171 & 0.191 & 0.193 & 0.196 & 0.194 & 0.164 & 0.231 & 0.18 & 0.177 \\
\hline 465.27 & 0.201 & 0.17 & 0.189 & 0.192 & 0.196 & 0.194 & 0.164 & 0.23 & 0.178 & 0.176 \\
\hline 465.62 & 0.2 & 0.168 & 0.187 & 0.191 & 0.195 & 0.193 & 0.163 & 0.23 & 0.177 & 0.175 \\
\hline 465.98 & 0.198 & 0.167 & 0.186 & 0.189 & 0.194 & 0.193 & 0.161 & 0.228 & 0.175 & 0.174 \\
\hline 466.33 & 0.196 & 0.167 & 0.185 & 0.188 & 0.193 & 0.192 & 0.16 & 0.228 & 0.175 & 0.172 \\
\hline 466.69 & 0.195 & 0.165 & 0.185 & 0.187 & 0.192 & 0.191 & 0.159 & 0.228 & 0.174 & 0.172 \\
\hline 467.04 & 0.194 & 0.164 & 0.184 & 0.186 & 0.193 & 0.19 & 0.158 & 0.228 & 0.173 & 0.17 \\
\hline 467.4 & 0.193 & 0.163 & 0.183 & 0.184 & 0.192 & 0.188 & 0.156 & 0.227 & 0.172 & 0.169 \\
\hline 467.75 & 0.192 & 0.161 & 0.182 & 0.183 & 0.191 & 0.187 & 0.154 & 0.226 & 0.17 & 0.168 \\
\hline 468.11 & 0.192 & 0.16 & 0.181 & 0.182 & 0.189 & 0.186 & 0.154 & 0.226 & 0.169 & 0.167 \\
\hline 468.46 & 0.19 & 0.158 & 0.181 & 0.18 & 0.187 & 0.185 & 0.153 & 0.226 & 0.167 & 0.166 \\
\hline 468.82 & 0.19 & 0.155 & 0.18 & 0.179 & 0.186 & 0.184 & 0.151 & 0.226 & 0.165 & 0.165 \\
\hline 469.17 & 0.189 & 0.153 & 0.179 & 0.178 & 0.184 & 0.182 & 0.15 & 0.225 & 0.164 & 0.164 \\
\hline 469.53 & 0.189 & 0.152 & 0.178 & 0.177 & 0.183 & 0.18 & 0.149 & 0.226 & 0.163 & 0.164 \\
\hline 469.88 & 0.189 & 0.15 & 0.176 & 0.177 & 0.182 & 0.179 & 0.148 & 0.226 & 0.162 & 0.162 \\
\hline 470.24 & 0.187 & 0.149 & 0.175 & 0.175 & 0.181 & 0.179 & 0.147 & 0.226 & 0.16 & 0.161 \\
\hline 470.59 & 0.186 & 0.146 & 0.174 & 0.174 & 0.179 & 0.179 & 0.146 & 0.225 & 0.158 & 0.159 \\
\hline 470.94 & 0.185 & 0.145 & 0.173 & 0.173 & 0.179 & 0.178 & 0.145 & 0.224 & 0.156 & 0.159 \\
\hline 471.3 & 0.184 & 0.144 & 0.172 & 0.173 & 0.178 & 0.178 & 0.145 & 0.223 & 0.155 & 0.157 \\
\hline 471.65 & 0.182 & 0.143 & 0.171 & 0.171 & 0.178 & 0.177 & 0.144 & 0.223 & 0.154 & 0.156 \\
\hline 472.01 & 0.18 & 0.141 & 0.17 & 0.171 & 0.177 & 0.175 & 0.142 & 0.222 & 0.153 & 0.155 \\
\hline 472.36 & 0.18 & 0.14 & 0.169 & 0.171 & 0.177 & 0.174 & 0.14 & 0.221 & 0.152 & 0.155 \\
\hline 472.72 & 0.178 & 0.14 & 0.169 & 0.171 & 0.177 & 0.173 & 0.139 & 0.22 & 0.152 & 0.154 \\
\hline 473.07 & 0.178 & 0.138 & 0.168 & 0.17 & 0.176 & 0.173 & 0.138 & 0.22 & 0.15 & 0.153 \\
\hline 473.43 & 0.176 & 0.136 & 0.168 & 0.169 & 0.175 & 0.172 & 0.138 & 0.219 & 0.149 & 0.152 \\
\hline
\end{tabular}




\begin{tabular}{|c|c|c|c|c|c|c|c|c|c|c|}
\hline 473.78 & 0.175 & 0.135 & 0.168 & 0.168 & 0.174 & 0.171 & 0.137 & 0.217 & 0.148 & 0.151 \\
\hline 474.13 & 0.174 & 0.133 & 0.168 & 0.168 & 0.173 & 0.169 & 0.135 & 0.216 & 0.146 & 0.151 \\
\hline 474.49 & 0.172 & 0.132 & 0.167 & 0.167 & 0.173 & 0.169 & 0.134 & 0.215 & 0.145 & 0.149 \\
\hline 474.84 & 0.171 & 0.131 & 0.165 & 0.165 & 0.173 & 0.167 & 0.134 & 0.214 & 0.144 & 0.147 \\
\hline 475.2 & 0.17 & 0.129 & 0.164 & 0.164 & 0.171 & 0.166 & 0.132 & 0.212 & 0.142 & 0.146 \\
\hline 475.55 & 0.168 & 0.127 & 0.164 & 0.163 & 0.17 & 0.166 & 0.131 & 0.211 & 0.14 & 0.145 \\
\hline 475.9 & 0.168 & 0.125 & 0.163 & 0.162 & 0.17 & 0.166 & 0.131 & 0.21 & 0.137 & 0.144 \\
\hline 476.26 & 0.166 & 0.125 & 0.162 & 0.161 & 0.169 & 0.165 & 0.132 & 0.21 & 0.135 & 0.142 \\
\hline 476.61 & 0.165 & 0.123 & 0.162 & 0.16 & 0.167 & 0.165 & 0.131 & 0.21 & 0.133 & 0.141 \\
\hline 476.96 & 0.164 & 0.123 & 0.161 & 0.16 & 0.166 & 0.164 & 0.129 & 0.209 & 0.132 & 0.14 \\
\hline 477.32 & 0.161 & 0.121 & 0.159 & 0.159 & 0.165 & 0.164 & 0.127 & 0.207 & 0.13 & 0.139 \\
\hline 477.67 & 0.16 & 0.12 & 0.158 & 0.158 & 0.164 & 0.164 & 0.126 & 0.206 & 0.129 & 0.138 \\
\hline 478.03 & 0.158 & 0.118 & 0.157 & 0.158 & 0.163 & 0.163 & 0.126 & 0.206 & 0.128 & 0.137 \\
\hline 478.38 & 0.157 & 0.117 & 0.156 & 0.156 & 0.161 & 0.162 & 0.124 & 0.204 & 0.126 & 0.137 \\
\hline 478.73 & 0.157 & 0.115 & 0.156 & 0.156 & 0.16 & 0.162 & 0.124 & 0.203 & 0.127 & 0.136 \\
\hline 479.09 & 0.157 & 0.114 & 0.155 & 0.156 & 0.16 & 0.161 & 0.123 & 0.203 & 0.126 & 0.136 \\
\hline 479.44 & 0.156 & 0.114 & 0.154 & 0.156 & 0.16 & 0.16 & 0.122 & 0.202 & 0.125 & 0.136 \\
\hline 479.79 & 0.155 & 0.114 & 0.154 & 0.154 & 0.159 & 0.16 & 0.121 & 0.202 & 0.125 & 0.134 \\
\hline 480.15 & 0.154 & 0.112 & 0.153 & 0.154 & 0.159 & 0.159 & 0.12 & 0.2 & 0.124 & 0.133 \\
\hline 480.5 & 0.153 & 0.111 & 0.151 & 0.152 & 0.158 & 0.158 & 0.119 & 0.199 & 0.123 & 0.132 \\
\hline 480.85 & 0.152 & 0.11 & 0.15 & 0.151 & 0.157 & 0.156 & 0.118 & 0.199 & 0.122 & 0.13 \\
\hline 481.21 & 0.152 & 0.109 & 0.149 & 0.15 & 0.157 & 0.156 & 0.118 & 0.198 & 0.121 & 0.13 \\
\hline 481.56 & 0.151 & 0.109 & 0.148 & 0.149 & 0.156 & 0.155 & 0.117 & 0.196 & 0.12 & 0.13 \\
\hline 481.91 & 0.151 & 0.108 & 0.148 & 0.148 & 0.157 & 0.154 & 0.116 & 0.197 & 0.12 & 0.13 \\
\hline 482.27 & 0.15 & 0.107 & 0.148 & 0.147 & 0.156 & 0.154 & 0.116 & 0.195 & 0.119 & 0.13 \\
\hline 482.62 & 0.149 & 0.107 & 0.147 & 0.146 & 0.156 & 0.152 & 0.115 & 0.195 & 0.117 & 0.129 \\
\hline 482.97 & 0.147 & 0.106 & 0.146 & 0.145 & 0.155 & 0.151 & 0.115 & 0.194 & 0.117 & 0.128 \\
\hline 483.33 & 0.146 & 0.105 & 0.145 & 0.144 & 0.153 & 0.149 & 0.115 & 0.193 & 0.116 & 0.127 \\
\hline 483.68 & 0.145 & 0.104 & 0.144 & 0.143 & 0.152 & 0.148 & 0.114 & 0.193 & 0.114 & 0.127 \\
\hline 484.03 & 0.143 & 0.103 & 0.144 & 0.142 & 0.15 & 0.148 & 0.113 & 0.192 & 0.113 & 0.127 \\
\hline 484.38 & 0.142 & 0.102 & 0.144 & 0.141 & 0.15 & 0.148 & 0.113 & 0.191 & 0.113 & 0.126 \\
\hline 484.74 & 0.141 & 0.102 & 0.143 & 0.142 & 0.15 & 0.146 & 0.112 & 0.19 & 0.113 & 0.126 \\
\hline 485.09 & 0.139 & 0.101 & 0.143 & 0.14 & 0.15 & 0.145 & 0.11 & 0.188 & 0.112 & 0.124 \\
\hline 485.44 & 0.138 & 0.099 & 0.142 & 0.14 & 0.148 & 0.145 & 0.11 & 0.188 & 0.112 & 0.123 \\
\hline 485.8 & 0.136 & 0.097 & 0.14 & 0.138 & 0.146 & 0.144 & 0.109 & 0.186 & 0.111 & 0.122 \\
\hline 486.15 & 0.135 & 0.096 & 0.139 & 0.138 & 0.145 & 0.142 & 0.109 & 0.185 & 0.111 & 0.121 \\
\hline 486.5 & 0.135 & 0.095 & 0.139 & 0.137 & 0.144 & 0.142 & 0.108 & 0.185 & 0.112 & 0.119 \\
\hline 486.85 & 0.134 & 0.093 & 0.139 & 0.136 & 0.143 & 0.142 & 0.107 & 0.184 & 0.111 & 0.119 \\
\hline 487.21 & 0.133 & 0.092 & 0.137 & 0.135 & 0.143 & 0.142 & 0.106 & 0.183 & 0.11 & 0.118 \\
\hline
\end{tabular}




\begin{tabular}{|c|c|c|c|c|c|c|c|c|c|c|}
\hline 487.56 & 0.132 & 0.091 & 0.136 & 0.136 & 0.142 & 0.142 & 0.104 & 0.182 & 0.111 & 0.118 \\
\hline 487.91 & 0.131 & 0.09 & 0.135 & 0.135 & 0.141 & 0.141 & 0.104 & 0.181 & 0.111 & 0.117 \\
\hline 488.26 & 0.13 & 0.089 & 0.134 & 0.134 & 0.141 & 0.14 & 0.103 & 0.179 & 0.109 & 0.117 \\
\hline 488.62 & 0.13 & 0.089 & 0.133 & 0.133 & 0.139 & 0.14 & 0.102 & 0.179 & 0.108 & 0.116 \\
\hline 488.97 & 0.13 & 0.087 & 0.131 & 0.134 & 0.138 & 0.139 & 0.103 & 0.178 & 0.108 & 0.116 \\
\hline 489.32 & 0.131 & 0.087 & 0.13 & 0.133 & 0.137 & 0.138 & 0.103 & 0.178 & 0.107 & 0.115 \\
\hline 489.67 & 0.13 & 0.086 & 0.13 & 0.133 & 0.137 & 0.137 & 0.102 & 0.177 & 0.106 & 0.115 \\
\hline 490.02 & 0.13 & 0.085 & 0.129 & 0.132 & 0.136 & 0.137 & 0.102 & 0.177 & 0.106 & 0.115 \\
\hline 490.38 & 0.128 & 0.084 & 0.128 & 0.131 & 0.135 & 0.136 & 0.101 & 0.176 & 0.104 & 0.115 \\
\hline 490.73 & 0.127 & 0.083 & 0.128 & 0.132 & 0.134 & 0.134 & 0.1 & 0.175 & 0.104 & 0.114 \\
\hline 491.08 & 0.127 & 0.083 & 0.128 & 0.131 & 0.134 & 0.134 & 0.1 & 0.174 & 0.104 & 0.113 \\
\hline 491.43 & 0.126 & 0.083 & 0.127 & 0.13 & 0.133 & 0.133 & 0.1 & 0.173 & 0.103 & 0.112 \\
\hline 491.79 & 0.126 & 0.083 & 0.127 & 0.13 & 0.133 & 0.132 & 0.099 & 0.173 & 0.104 & 0.111 \\
\hline 492.14 & 0.126 & 0.082 & 0.126 & 0.129 & 0.132 & 0.132 & 0.099 & 0.173 & 0.103 & 0.111 \\
\hline 492.49 & 0.125 & 0.081 & 0.127 & 0.128 & 0.132 & 0.131 & 0.098 & 0.172 & 0.103 & 0.111 \\
\hline 492.84 & 0.125 & 0.081 & 0.126 & 0.128 & 0.131 & 0.131 & 0.098 & 0.172 & 0.103 & 0.111 \\
\hline 493.19 & 0.123 & 0.08 & 0.126 & 0.128 & 0.131 & 0.131 & 0.097 & 0.172 & 0.103 & 0.11 \\
\hline 493.54 & 0.123 & 0.079 & 0.125 & 0.127 & 0.13 & 0.13 & 0.097 & 0.17 & 0.103 & 0.11 \\
\hline 493.9 & 0.122 & 0.079 & 0.125 & 0.126 & 0.13 & 0.128 & 0.096 & 0.17 & 0.102 & 0.109 \\
\hline 494.25 & 0.121 & 0.079 & 0.124 & 0.127 & 0.129 & 0.128 & 0.095 & 0.17 & 0.103 & 0.11 \\
\hline 494.6 & 0.121 & 0.078 & 0.123 & 0.126 & 0.129 & 0.128 & 0.095 & 0.17 & 0.103 & 0.11 \\
\hline 494.95 & 0.12 & 0.078 & 0.123 & 0.125 & 0.128 & 0.127 & 0.094 & 0.168 & 0.104 & 0.109 \\
\hline 495.3 & 0.12 & 0.077 & 0.123 & 0.124 & 0.128 & 0.127 & 0.094 & 0.168 & 0.103 & 0.109 \\
\hline 495.65 & 0.119 & 0.076 & 0.122 & 0.124 & 0.128 & 0.125 & 0.094 & 0.167 & 0.102 & 0.109 \\
\hline 496.01 & 0.118 & 0.075 & 0.122 & 0.123 & 0.127 & 0.124 & 0.092 & 0.166 & 0.102 & 0.109 \\
\hline 496.36 & 0.117 & 0.074 & 0.121 & 0.122 & 0.127 & 0.123 & 0.091 & 0.164 & 0.102 & 0.109 \\
\hline 496.71 & 0.116 & 0.074 & 0.121 & 0.121 & 0.126 & 0.123 & 0.089 & 0.164 & 0.101 & 0.108 \\
\hline 497.06 & 0.116 & 0.074 & 0.12 & 0.121 & 0.127 & 0.122 & 0.089 & 0.164 & 0.101 & 0.108 \\
\hline 497.41 & 0.116 & 0.075 & 0.12 & 0.12 & 0.127 & 0.122 & 0.088 & 0.163 & 0.101 & 0.107 \\
\hline 497.76 & 0.116 & 0.074 & 0.121 & 0.119 & 0.127 & 0.122 & 0.088 & 0.163 & 0.101 & 0.106 \\
\hline 498.11 & 0.115 & 0.075 & 0.12 & 0.12 & 0.127 & 0.121 & 0.088 & 0.161 & 0.101 & 0.105 \\
\hline 498.47 & 0.115 & 0.074 & 0.12 & 0.119 & 0.127 & 0.121 & 0.087 & 0.16 & 0.1 & 0.104 \\
\hline 498.82 & 0.114 & 0.074 & 0.119 & 0.119 & 0.126 & 0.121 & 0.087 & 0.161 & 0.1 & 0.106 \\
\hline 499.17 & 0.113 & 0.073 & 0.119 & 0.119 & 0.126 & 0.12 & 0.086 & 0.16 & 0.1 & 0.106 \\
\hline 499.52 & 0.112 & 0.073 & 0.117 & 0.118 & 0.126 & 0.122 & 0.086 & 0.16 & 0.101 & 0.105 \\
\hline 499.87 & 0.112 & 0.073 & 0.118 & 0.117 & 0.126 & 0.122 & 0.086 & 0.161 & 0.1 & 0.104 \\
\hline 500.22 & 0.112 & 0.073 & 0.118 & 0.118 & 0.125 & 0.122 & 0.086 & 0.161 & 0.101 & 0.104 \\
\hline 500.57 & 0.111 & 0.072 & 0.118 & 0.118 & 0.124 & 0.121 & 0.086 & 0.16 & 0.101 & 0.105 \\
\hline 500.92 & 0.109 & 0.072 & 0.118 & 0.117 & 0.124 & 0.121 & 0.086 & 0.159 & 0.102 & 0.105 \\
\hline
\end{tabular}




\begin{tabular}{|c|c|c|c|c|c|c|c|c|c|c|}
\hline 501.27 & 0.108 & 0.071 & 0.117 & 0.118 & 0.125 & 0.12 & 0.086 & 0.158 & 0.102 & 0.105 \\
\hline 501.62 & 0.108 & 0.071 & 0.117 & 0.117 & 0.124 & 0.12 & 0.086 & 0.158 & 0.102 & 0.105 \\
\hline 501.97 & 0.107 & 0.07 & 0.117 & 0.117 & 0.123 & 0.12 & 0.086 & 0.158 & 0.101 & 0.105 \\
\hline 502.33 & 0.106 & 0.069 & 0.117 & 0.116 & 0.122 & 0.12 & 0.086 & 0.158 & 0.102 & 0.105 \\
\hline 502.68 & 0.106 & 0.069 & 0.116 & 0.116 & 0.121 & 0.12 & 0.085 & 0.158 & 0.103 & 0.104 \\
\hline 503.03 & 0.106 & 0.07 & 0.117 & 0.116 & 0.121 & 0.12 & 0.085 & 0.158 & 0.103 & 0.103 \\
\hline 503.38 & 0.105 & 0.069 & 0.118 & 0.115 & 0.121 & 0.119 & 0.085 & 0.157 & 0.103 & 0.103 \\
\hline 503.73 & 0.104 & 0.069 & 0.117 & 0.114 & 0.12 & 0.119 & 0.085 & 0.157 & 0.103 & 0.104 \\
\hline 504.08 & 0.103 & 0.07 & 0.117 & 0.114 & 0.121 & 0.119 & 0.084 & 0.157 & 0.103 & 0.104 \\
\hline 504.43 & 0.102 & 0.07 & 0.116 & 0.113 & 0.122 & 0.12 & 0.084 & 0.156 & 0.104 & 0.103 \\
\hline 504.78 & 0.101 & 0.07 & 0.117 & 0.113 & 0.122 & 0.121 & 0.083 & 0.156 & 0.104 & 0.104 \\
\hline 505.13 & 0.102 & 0.07 & 0.117 & 0.112 & 0.121 & 0.121 & 0.083 & 0.156 & 0.104 & 0.105 \\
\hline 505.48 & 0.101 & 0.069 & 0.117 & 0.112 & 0.122 & 0.122 & 0.082 & 0.156 & 0.104 & 0.106 \\
\hline 505.83 & 0.1 & 0.07 & 0.117 & 0.111 & 0.122 & 0.122 & 0.081 & 0.156 & 0.104 & 0.106 \\
\hline 506.18 & 0.1 & 0.071 & 0.117 & 0.11 & 0.123 & 0.121 & 0.081 & 0.156 & 0.104 & 0.106 \\
\hline 506.53 & 0.098 & 0.07 & 0.117 & 0.11 & 0.123 & 0.121 & 0.081 & 0.155 & 0.104 & 0.107 \\
\hline 506.88 & 0.098 & 0.07 & 0.117 & 0.11 & 0.123 & 0.12 & 0.08 & 0.155 & 0.103 & 0.107 \\
\hline 507.23 & 0.097 & 0.07 & 0.118 & 0.109 & 0.122 & 0.119 & 0.079 & 0.155 & 0.103 & 0.107 \\
\hline 507.58 & 0.098 & 0.07 & 0.118 & 0.109 & 0.123 & 0.119 & 0.079 & 0.154 & 0.103 & 0.107 \\
\hline 507.93 & 0.098 & 0.069 & 0.119 & 0.109 & 0.123 & 0.119 & 0.08 & 0.153 & 0.104 & 0.107 \\
\hline 508.28 & 0.098 & 0.069 & 0.119 & 0.11 & 0.124 & 0.119 & 0.08 & 0.154 & 0.103 & 0.107 \\
\hline 508.63 & 0.098 & 0.069 & 0.119 & 0.11 & 0.123 & 0.118 & 0.08 & 0.154 & 0.103 & 0.105 \\
\hline 508.98 & 0.097 & 0.069 & 0.119 & 0.11 & 0.123 & 0.118 & 0.08 & 0.155 & 0.103 & 0.105 \\
\hline 509.33 & 0.097 & 0.069 & 0.119 & 0.111 & 0.122 & 0.118 & 0.08 & 0.154 & 0.103 & 0.105 \\
\hline 509.68 & 0.098 & 0.068 & 0.118 & 0.111 & 0.122 & 0.118 & 0.08 & 0.153 & 0.104 & 0.105 \\
\hline 510.03 & 0.098 & 0.068 & 0.119 & 0.111 & 0.122 & 0.118 & 0.08 & 0.153 & 0.103 & 0.106 \\
\hline 510.38 & 0.098 & 0.068 & 0.119 & 0.112 & 0.122 & 0.119 & 0.08 & 0.152 & 0.103 & 0.106 \\
\hline 510.73 & 0.098 & 0.068 & 0.12 & 0.112 & 0.122 & 0.12 & 0.08 & 0.152 & 0.103 & 0.106 \\
\hline 511.08 & 0.098 & 0.068 & 0.12 & 0.114 & 0.123 & 0.121 & 0.081 & 0.151 & 0.103 & 0.107 \\
\hline 511.43 & 0.097 & 0.069 & 0.119 & 0.113 & 0.123 & 0.121 & 0.081 & 0.151 & 0.102 & 0.108 \\
\hline 511.78 & 0.098 & 0.069 & 0.119 & 0.114 & 0.122 & 0.121 & 0.081 & 0.152 & 0.101 & 0.109 \\
\hline 512.13 & 0.097 & 0.069 & 0.12 & 0.113 & 0.121 & 0.12 & 0.081 & 0.152 & 0.103 & 0.109 \\
\hline 512.48 & 0.097 & 0.069 & 0.12 & 0.114 & 0.121 & 0.121 & 0.081 & 0.152 & 0.103 & 0.11 \\
\hline 512.83 & 0.097 & 0.069 & 0.12 & 0.114 & 0.121 & 0.121 & 0.081 & 0.151 & 0.103 & 0.109 \\
\hline 513.18 & 0.097 & 0.069 & 0.12 & 0.114 & 0.122 & 0.121 & 0.081 & 0.151 & 0.103 & 0.109 \\
\hline 513.53 & 0.096 & 0.069 & 0.121 & 0.114 & 0.122 & 0.121 & 0.081 & 0.152 & 0.104 & 0.11 \\
\hline 513.88 & 0.095 & 0.07 & 0.121 & 0.115 & 0.122 & 0.122 & 0.082 & 0.152 & 0.105 & 0.11 \\
\hline 514.22 & 0.095 & 0.07 & 0.121 & 0.115 & 0.122 & 0.122 & 0.082 & 0.152 & 0.106 & 0.111 \\
\hline 514.57 & 0.095 & 0.071 & 0.12 & 0.116 & 0.123 & 0.122 & 0.082 & 0.153 & 0.107 & 0.111 \\
\hline
\end{tabular}




\begin{tabular}{|c|c|c|c|c|c|c|c|c|c|c|}
\hline 514.92 & 0.096 & 0.072 & 0.12 & 0.116 & 0.123 & 0.122 & 0.082 & 0.153 & 0.107 & 0.111 \\
\hline 515.27 & 0.096 & 0.073 & 0.121 & 0.116 & 0.124 & 0.122 & 0.082 & 0.153 & 0.108 & 0.111 \\
\hline 515.62 & 0.095 & 0.073 & 0.121 & 0.117 & 0.125 & 0.122 & 0.082 & 0.153 & 0.109 & 0.11 \\
\hline 515.97 & 0.096 & 0.073 & 0.121 & 0.117 & 0.127 & 0.124 & 0.083 & 0.153 & 0.108 & 0.11 \\
\hline 516.32 & 0.096 & 0.073 & 0.12 & 0.117 & 0.127 & 0.125 & 0.083 & 0.152 & 0.108 & 0.11 \\
\hline 516.67 & 0.095 & 0.074 & 0.121 & 0.117 & 0.127 & 0.126 & 0.083 & 0.153 & 0.109 & 0.112 \\
\hline 517.02 & 0.095 & 0.074 & 0.121 & 0.119 & 0.127 & 0.127 & 0.083 & 0.153 & 0.109 & 0.112 \\
\hline 517.37 & 0.094 & 0.074 & 0.122 & 0.119 & 0.128 & 0.127 & 0.083 & 0.153 & 0.109 & 0.112 \\
\hline 517.72 & 0.095 & 0.074 & 0.121 & 0.119 & 0.128 & 0.126 & 0.083 & 0.153 & 0.109 & 0.113 \\
\hline 518.06 & 0.095 & 0.074 & 0.121 & 0.119 & 0.129 & 0.127 & 0.083 & 0.153 & 0.109 & 0.113 \\
\hline 518.41 & 0.095 & 0.074 & 0.123 & 0.119 & 0.129 & 0.128 & 0.083 & 0.153 & 0.11 & 0.113 \\
\hline 518.76 & 0.095 & 0.073 & 0.123 & 0.119 & 0.13 & 0.127 & 0.083 & 0.154 & 0.11 & 0.113 \\
\hline 519.11 & 0.095 & 0.072 & 0.123 & 0.119 & 0.13 & 0.128 & 0.084 & 0.154 & 0.11 & 0.113 \\
\hline 519.46 & 0.096 & 0.073 & 0.124 & 0.118 & 0.13 & 0.129 & 0.085 & 0.154 & 0.11 & 0.114 \\
\hline 519.81 & 0.095 & 0.073 & 0.124 & 0.119 & 0.13 & 0.128 & 0.085 & 0.155 & 0.111 & 0.114 \\
\hline 520.16 & 0.095 & 0.073 & 0.125 & 0.12 & 0.131 & 0.128 & 0.086 & 0.155 & 0.112 & 0.115 \\
\hline 520.5 & 0.095 & 0.074 & 0.126 & 0.12 & 0.132 & 0.128 & 0.086 & 0.155 & 0.112 & 0.115 \\
\hline 520.85 & 0.095 & 0.075 & 0.126 & 0.12 & 0.132 & 0.128 & 0.087 & 0.155 & 0.114 & 0.115 \\
\hline 521.2 & 0.095 & 0.074 & 0.126 & 0.12 & 0.131 & 0.129 & 0.087 & 0.154 & 0.114 & 0.115 \\
\hline 521.55 & 0.094 & 0.075 & 0.126 & 0.12 & 0.132 & 0.129 & 0.087 & 0.154 & 0.115 & 0.115 \\
\hline 521.9 & 0.094 & 0.074 & 0.127 & 0.119 & 0.132 & 0.129 & 0.087 & 0.154 & 0.115 & 0.115 \\
\hline 522.25 & 0.093 & 0.074 & 0.127 & 0.121 & 0.132 & 0.129 & 0.087 & 0.153 & 0.116 & 0.117 \\
\hline 522.59 & 0.093 & 0.075 & 0.127 & 0.122 & 0.132 & 0.129 & 0.088 & 0.154 & 0.117 & 0.118 \\
\hline 522.94 & 0.092 & 0.076 & 0.127 & 0.123 & 0.133 & 0.129 & 0.088 & 0.154 & 0.118 & 0.118 \\
\hline 523.29 & 0.092 & 0.075 & 0.128 & 0.124 & 0.133 & 0.129 & 0.089 & 0.154 & 0.118 & 0.118 \\
\hline 523.64 & 0.091 & 0.075 & 0.128 & 0.124 & 0.133 & 0.129 & 0.088 & 0.153 & 0.118 & 0.119 \\
\hline 523.99 & 0.091 & 0.075 & 0.129 & 0.122 & 0.132 & 0.129 & 0.088 & 0.153 & 0.119 & 0.12 \\
\hline 524.33 & 0.091 & 0.075 & 0.128 & 0.122 & 0.133 & 0.13 & 0.088 & 0.153 & 0.118 & 0.12 \\
\hline 524.68 & 0.090 & 0.076 & 0.129 & 0.122 & 0.133 & 0.131 & 0.087 & 0.154 & 0.119 & 0.12 \\
\hline 525.03 & 0.091 & 0.077 & 0.128 & 0.123 & 0.133 & 0.13 & 0.088 & 0.155 & 0.12 & 0.122 \\
\hline 525.38 & 0.091 & 0.078 & 0.129 & 0.124 & 0.133 & 0.13 & 0.088 & 0.155 & 0.121 & 0.123 \\
\hline 525.73 & 0.091 & 0.078 & 0.129 & 0.125 & 0.133 & 0.13 & 0.089 & 0.155 & 0.121 & 0.123 \\
\hline 526.07 & 0.092 & 0.08 & 0.129 & 0.125 & 0.133 & 0.13 & 0.09 & 0.156 & 0.122 & 0.123 \\
\hline 526.42 & 0.092 & 0.08 & 0.13 & 0.126 & 0.133 & 0.13 & 0.09 & 0.156 & 0.123 & 0.123 \\
\hline 526.77 & 0.092 & 0.08 & 0.129 & 0.127 & 0.133 & 0.131 & 0.09 & 0.156 & 0.125 & 0.124 \\
\hline 527.12 & 0.093 & 0.082 & 0.129 & 0.127 & 0.134 & 0.131 & 0.09 & 0.156 & 0.126 & 0.124 \\
\hline 527.46 & 0.094 & 0.083 & 0.128 & 0.127 & 0.134 & 0.131 & 0.091 & 0.156 & 0.126 & 0.124 \\
\hline 527.81 & 0.094 & 0.084 & 0.129 & 0.129 & 0.135 & 0.131 & 0.091 & 0.157 & 0.126 & 0.125 \\
\hline 528.16 & 0.095 & 0.085 & 0.129 & 0.13 & 0.136 & 0.132 & 0.092 & 0.157 & 0.128 & 0.126 \\
\hline
\end{tabular}




\begin{tabular}{|c|c|c|c|c|c|c|c|c|c|c|}
\hline 528.51 & 0.096 & 0.085 & 0.129 & 0.13 & 0.136 & 0.133 & 0.093 & 0.156 & 0.128 & 0.126 \\
\hline 528.85 & 0.096 & 0.085 & 0.13 & 0.131 & 0.138 & 0.134 & 0.093 & 0.156 & 0.128 & 0.126 \\
\hline 529.2 & 0.096 & 0.085 & 0.13 & 0.132 & 0.138 & 0.136 & 0.094 & 0.156 & 0.129 & 0.126 \\
\hline 529.55 & 0.098 & 0.086 & 0.131 & 0.133 & 0.139 & 0.137 & 0.094 & 0.157 & 0.129 & 0.126 \\
\hline 529.9 & 0.098 & 0.086 & 0.132 & 0.132 & 0.139 & 0.138 & 0.094 & 0.157 & 0.131 & 0.127 \\
\hline 530.24 & 0.098 & 0.086 & 0.131 & 0.132 & 0.139 & 0.138 & 0.095 & 0.156 & 0.13 & 0.128 \\
\hline 530.59 & 0.099 & 0.087 & 0.133 & 0.132 & 0.14 & 0.139 & 0.095 & 0.156 & 0.13 & 0.129 \\
\hline 530.94 & 0.098 & 0.087 & 0.132 & 0.134 & 0.14 & 0.139 & 0.096 & 0.157 & 0.13 & 0.128 \\
\hline 531.29 & 0.098 & 0.088 & 0.134 & 0.134 & 0.14 & 0.14 & 0.096 & 0.158 & 0.129 & 0.128 \\
\hline 531.63 & 0.099 & 0.088 & 0.134 & 0.135 & 0.14 & 0.141 & 0.096 & 0.159 & 0.13 & 0.129 \\
\hline 531.98 & 0.099 & 0.088 & 0.134 & 0.135 & 0.14 & 0.141 & 0.097 & 0.159 & 0.13 & 0.129 \\
\hline 532.33 & 0.1 & 0.088 & 0.135 & 0.136 & 0.14 & 0.141 & 0.099 & 0.161 & 0.131 & 0.129 \\
\hline 532.67 & 0.1 & 0.089 & 0.135 & 0.136 & 0.14 & 0.141 & 0.099 & 0.161 & 0.132 & 0.129 \\
\hline 533.02 & 0.1 & 0.09 & 0.135 & 0.135 & 0.142 & 0.141 & 0.1 & 0.162 & 0.132 & 0.129 \\
\hline 533.37 & 0.1 & 0.091 & 0.134 & 0.136 & 0.142 & 0.141 & 0.101 & 0.162 & 0.132 & 0.129 \\
\hline 533.71 & 0.101 & 0.092 & 0.134 & 0.137 & 0.143 & 0.14 & 0.101 & 0.163 & 0.131 & 0.129 \\
\hline 534.06 & 0.102 & 0.092 & 0.135 & 0.138 & 0.144 & 0.141 & 0.102 & 0.164 & 0.132 & 0.129 \\
\hline 534.41 & 0.103 & 0.092 & 0.135 & 0.138 & 0.144 & 0.14 & 0.104 & 0.165 & 0.132 & 0.129 \\
\hline 534.75 & 0.105 & 0.092 & 0.136 & 0.139 & 0.145 & 0.14 & 0.105 & 0.165 & 0.133 & 0.131 \\
\hline 535.1 & 0.106 & 0.092 & 0.135 & 0.14 & 0.146 & 0.141 & 0.105 & 0.165 & 0.135 & 0.131 \\
\hline 535.45 & 0.106 & 0.094 & 0.136 & 0.141 & 0.147 & 0.142 & 0.106 & 0.165 & 0.136 & 0.132 \\
\hline 535.79 & 0.108 & 0.095 & 0.136 & 0.141 & 0.147 & 0.143 & 0.107 & 0.165 & 0.136 & 0.133 \\
\hline 536.14 & 0.107 & 0.096 & 0.137 & 0.143 & 0.148 & 0.144 & 0.107 & 0.165 & 0.137 & 0.134 \\
\hline 536.49 & 0.108 & 0.098 & 0.137 & 0.144 & 0.149 & 0.143 & 0.107 & 0.166 & 0.137 & 0.134 \\
\hline 536.83 & 0.109 & 0.098 & 0.139 & 0.145 & 0.149 & 0.144 & 0.107 & 0.166 & 0.138 & 0.135 \\
\hline 537.18 & 0.11 & 0.099 & 0.14 & 0.145 & 0.149 & 0.145 & 0.108 & 0.167 & 0.138 & 0.136 \\
\hline 537.53 & 0.11 & 0.1 & 0.14 & 0.145 & 0.149 & 0.146 & 0.108 & 0.168 & 0.139 & 0.136 \\
\hline 537.87 & 0.112 & 0.101 & 0.14 & 0.145 & 0.149 & 0.147 & 0.108 & 0.168 & 0.14 & 0.137 \\
\hline 538.22 & 0.111 & 0.103 & 0.14 & 0.146 & 0.15 & 0.148 & 0.108 & 0.169 & 0.141 & 0.137 \\
\hline 538.57 & 0.112 & 0.105 & 0.14 & 0.146 & 0.152 & 0.15 & 0.109 & 0.17 & 0.141 & 0.137 \\
\hline 538.91 & 0.112 & 0.106 & 0.141 & 0.147 & 0.153 & 0.15 & 0.109 & 0.17 & 0.141 & 0.137 \\
\hline 539.26 & 0.112 & 0.106 & 0.141 & 0.147 & 0.153 & 0.149 & 0.109 & 0.17 & 0.141 & 0.137 \\
\hline 539.6 & 0.112 & 0.107 & 0.141 & 0.149 & 0.154 & 0.149 & 0.109 & 0.17 & 0.142 & 0.137 \\
\hline 539.95 & 0.113 & 0.108 & 0.141 & 0.149 & 0.153 & 0.15 & 0.111 & 0.172 & 0.142 & 0.137 \\
\hline 540.3 & 0.115 & 0.108 & 0.141 & 0.15 & 0.154 & 0.151 & 0.111 & 0.173 & 0.142 & 0.139 \\
\hline 540.64 & 0.115 & 0.11 & 0.141 & 0.151 & 0.154 & 0.152 & 0.113 & 0.173 & 0.142 & 0.139 \\
\hline 540.99 & 0.115 & 0.112 & 0.142 & 0.151 & 0.154 & 0.153 & 0.112 & 0.173 & 0.143 & 0.14 \\
\hline 541.33 & 0.117 & 0.112 & 0.142 & 0.152 & 0.155 & 0.153 & 0.114 & 0.174 & 0.143 & 0.14 \\
\hline 541.68 & 0.117 & 0.113 & 0.143 & 0.153 & 0.156 & 0.154 & 0.116 & 0.174 & 0.144 & 0.14 \\
\hline
\end{tabular}




\begin{tabular}{|c|c|c|c|c|c|c|c|c|c|c|}
\hline 542.02 & 0.118 & 0.114 & 0.145 & 0.154 & 0.157 & 0.155 & 0.116 & 0.175 & 0.145 & 0.141 \\
\hline 542.37 & 0.12 & 0.116 & 0.145 & 0.154 & 0.156 & 0.156 & 0.117 & 0.175 & 0.145 & 0.141 \\
\hline 542.72 & 0.12 & 0.117 & 0.146 & 0.154 & 0.156 & 0.156 & 0.119 & 0.177 & 0.145 & 0.142 \\
\hline 543.06 & 0.121 & 0.117 & 0.147 & 0.155 & 0.157 & 0.156 & 0.121 & 0.178 & 0.146 & 0.143 \\
\hline 543.41 & 0.123 & 0.118 & 0.148 & 0.156 & 0.158 & 0.158 & 0.122 & 0.179 & 0.146 & 0.144 \\
\hline 543.75 & 0.124 & 0.12 & 0.149 & 0.156 & 0.159 & 0.158 & 0.122 & 0.178 & 0.146 & 0.143 \\
\hline 544.1 & 0.125 & 0.122 & 0.15 & 0.157 & 0.16 & 0.158 & 0.123 & 0.179 & 0.147 & 0.144 \\
\hline 544.44 & 0.126 & 0.122 & 0.149 & 0.157 & 0.161 & 0.16 & 0.123 & 0.179 & 0.147 & 0.144 \\
\hline 544.79 & 0.127 & 0.123 & 0.15 & 0.158 & 0.161 & 0.16 & 0.125 & 0.179 & 0.147 & 0.145 \\
\hline 545.13 & 0.128 & 0.124 & 0.151 & 0.158 & 0.162 & 0.161 & 0.126 & 0.179 & 0.147 & 0.147 \\
\hline 545.48 & 0.128 & 0.125 & 0.152 & 0.16 & 0.163 & 0.162 & 0.127 & 0.18 & 0.147 & 0.149 \\
\hline 545.82 & 0.129 & 0.127 & 0.153 & 0.16 & 0.164 & 0.163 & 0.128 & 0.181 & 0.147 & 0.15 \\
\hline 546.17 & 0.129 & 0.127 & 0.154 & 0.162 & 0.165 & 0.163 & 0.129 & 0.182 & 0.147 & 0.151 \\
\hline 546.52 & 0.13 & 0.128 & 0.155 & 0.164 & 0.166 & 0.164 & 0.13 & 0.182 & 0.148 & 0.151 \\
\hline 546.86 & 0.132 & 0.129 & 0.155 & 0.164 & 0.167 & 0.165 & 0.132 & 0.183 & 0.148 & 0.152 \\
\hline 547.21 & 0.132 & 0.131 & 0.156 & 0.166 & 0.167 & 0.165 & 0.133 & 0.184 & 0.15 & 0.153 \\
\hline 547.55 & 0.133 & 0.133 & 0.156 & 0.166 & 0.167 & 0.167 & 0.134 & 0.185 & 0.15 & 0.154 \\
\hline 547.9 & 0.135 & 0.134 & 0.158 & 0.167 & 0.168 & 0.169 & 0.136 & 0.187 & 0.151 & 0.155 \\
\hline 548.24 & 0.135 & 0.135 & 0.159 & 0.168 & 0.169 & 0.17 & 0.137 & 0.189 & 0.152 & 0.156 \\
\hline 548.59 & 0.136 & 0.136 & 0.161 & 0.17 & 0.17 & 0.17 & 0.138 & 0.191 & 0.153 & 0.157 \\
\hline 548.93 & 0.136 & 0.138 & 0.161 & 0.171 & 0.171 & 0.171 & 0.139 & 0.193 & 0.153 & 0.158 \\
\hline 549.28 & 0.137 & 0.139 & 0.162 & 0.172 & 0.172 & 0.172 & 0.141 & 0.195 & 0.155 & 0.158 \\
\hline 549.62 & 0.138 & 0.14 & 0.163 & 0.174 & 0.173 & 0.173 & 0.142 & 0.196 & 0.156 & 0.159 \\
\hline 549.96 & 0.139 & 0.142 & 0.164 & 0.175 & 0.173 & 0.174 & 0.144 & 0.197 & 0.158 & 0.16 \\
\hline 550.31 & 0.141 & 0.143 & 0.165 & 0.176 & 0.174 & 0.175 & 0.144 & 0.198 & 0.159 & 0.161 \\
\hline 550.65 & 0.142 & 0.145 & 0.166 & 0.177 & 0.175 & 0.176 & 0.146 & 0.2 & 0.16 & 0.162 \\
\hline 551 & 0.144 & 0.146 & 0.168 & 0.178 & 0.176 & 0.178 & 0.146 & 0.2 & 0.16 & 0.164 \\
\hline 551.34 & 0.145 & 0.147 & 0.169 & 0.179 & 0.178 & 0.179 & 0.147 & 0.201 & 0.161 & 0.165 \\
\hline 551.69 & 0.145 & 0.147 & 0.171 & 0.181 & 0.179 & 0.179 & 0.148 & 0.201 & 0.163 & 0.165 \\
\hline 552.03 & 0.147 & 0.149 & 0.173 & 0.182 & 0.18 & 0.181 & 0.15 & 0.201 & 0.164 & 0.167 \\
\hline 552.38 & 0.148 & 0.151 & 0.174 & 0.183 & 0.181 & 0.182 & 0.151 & 0.201 & 0.165 & 0.168 \\
\hline 552.72 & 0.15 & 0.152 & 0.175 & 0.185 & 0.182 & 0.183 & 0.152 & 0.202 & 0.167 & 0.169 \\
\hline 553.07 & 0.153 & 0.154 & 0.177 & 0.185 & 0.182 & 0.184 & 0.153 & 0.202 & 0.167 & 0.171 \\
\hline 553.41 & 0.154 & 0.155 & 0.177 & 0.186 & 0.184 & 0.185 & 0.154 & 0.203 & 0.168 & 0.171 \\
\hline 553.75 & 0.156 & 0.157 & 0.18 & 0.187 & 0.185 & 0.186 & 0.155 & 0.204 & 0.168 & 0.173 \\
\hline 554.1 & 0.157 & 0.159 & 0.181 & 0.188 & 0.186 & 0.189 & 0.157 & 0.206 & 0.169 & 0.174 \\
\hline 554.44 & 0.158 & 0.161 & 0.181 & 0.189 & 0.188 & 0.19 & 0.158 & 0.206 & 0.169 & 0.175 \\
\hline 554.79 & 0.159 & 0.163 & 0.183 & 0.191 & 0.19 & 0.191 & 0.161 & 0.209 & 0.171 & 0.176 \\
\hline 555.13 & 0.161 & 0.164 & 0.184 & 0.192 & 0.192 & 0.191 & 0.163 & 0.21 & 0.172 & 0.178 \\
\hline
\end{tabular}




\begin{tabular}{|c|c|c|c|c|c|c|c|c|c|c|}
\hline 555.47 & 0.163 & 0.167 & 0.185 & 0.193 & 0.192 & 0.193 & 0.164 & 0.212 & 0.172 & 0.179 \\
\hline 555.82 & 0.165 & 0.169 & 0.185 & 0.196 & 0.194 & 0.193 & 0.165 & 0.214 & 0.172 & 0.18 \\
\hline 556.16 & 0.166 & 0.17 & 0.187 & 0.197 & 0.194 & 0.195 & 0.166 & 0.216 & 0.173 & 0.182 \\
\hline 556.51 & 0.167 & 0.172 & 0.188 & 0.198 & 0.195 & 0.196 & 0.168 & 0.217 & 0.174 & 0.182 \\
\hline 556.85 & 0.168 & 0.174 & 0.189 & 0.199 & 0.197 & 0.198 & 0.17 & 0.219 & 0.176 & 0.184 \\
\hline 557.19 & 0.17 & 0.176 & 0.191 & 0.199 & 0.198 & 0.2 & 0.172 & 0.221 & 0.177 & 0.186 \\
\hline 557.54 & 0.17 & 0.178 & 0.193 & 0.201 & 0.2 & 0.201 & 0.173 & 0.222 & 0.178 & 0.188 \\
\hline 557.88 & 0.173 & 0.18 & 0.193 & 0.204 & 0.201 & 0.202 & 0.175 & 0.222 & 0.179 & 0.189 \\
\hline 558.22 & 0.175 & 0.183 & 0.195 & 0.205 & 0.202 & 0.203 & 0.177 & 0.224 & 0.181 & 0.191 \\
\hline 558.57 & 0.176 & 0.184 & 0.196 & 0.208 & 0.203 & 0.204 & 0.178 & 0.225 & 0.183 & 0.192 \\
\hline 558.91 & 0.178 & 0.186 & 0.198 & 0.209 & 0.204 & 0.206 & 0.18 & 0.227 & 0.185 & 0.193 \\
\hline 559.26 & 0.179 & 0.187 & 0.198 & 0.21 & 0.206 & 0.206 & 0.182 & 0.228 & 0.187 & 0.194 \\
\hline 559.6 & 0.182 & 0.188 & 0.2 & 0.211 & 0.207 & 0.208 & 0.184 & 0.229 & 0.189 & 0.195 \\
\hline 559.94 & 0.184 & 0.189 & 0.201 & 0.212 & 0.209 & 0.21 & 0.187 & 0.23 & 0.19 & 0.197 \\
\hline 560.29 & 0.186 & 0.192 & 0.202 & 0.213 & 0.212 & 0.21 & 0.189 & 0.232 & 0.191 & 0.199 \\
\hline 560.63 & 0.188 & 0.193 & 0.204 & 0.216 & 0.213 & 0.21 & 0.19 & 0.233 & 0.193 & 0.2 \\
\hline 560.97 & 0.191 & 0.195 & 0.205 & 0.22 & 0.214 & 0.212 & 0.192 & 0.235 & 0.194 & 0.202 \\
\hline 561.32 & 0.194 & 0.198 & 0.206 & 0.221 & 0.215 & 0.213 & 0.194 & 0.237 & 0.197 & 0.203 \\
\hline 561.66 & 0.195 & 0.198 & 0.207 & 0.221 & 0.216 & 0.214 & 0.195 & 0.238 & 0.199 & 0.205 \\
\hline 562 & 0.198 & 0.2 & 0.208 & 0.223 & 0.217 & 0.216 & 0.196 & 0.24 & 0.199 & 0.206 \\
\hline 562.34 & 0.201 & 0.202 & 0.209 & 0.223 & 0.218 & 0.216 & 0.198 & 0.24 & 0.2 & 0.208 \\
\hline 562.69 & 0.203 & 0.204 & 0.211 & 0.224 & 0.219 & 0.218 & 0.2 & 0.242 & 0.202 & 0.209 \\
\hline 563.03 & 0.205 & 0.206 & 0.212 & 0.226 & 0.221 & 0.219 & 0.201 & 0.243 & 0.203 & 0.21 \\
\hline 563.37 & 0.207 & 0.208 & 0.214 & 0.227 & 0.223 & 0.22 & 0.203 & 0.245 & 0.205 & 0.212 \\
\hline 563.72 & 0.209 & 0.212 & 0.215 & 0.228 & 0.226 & 0.221 & 0.204 & 0.246 & 0.207 & 0.214 \\
\hline 564.06 & 0.211 & 0.213 & 0.217 & 0.23 & 0.227 & 0.224 & 0.206 & 0.247 & 0.208 & 0.215 \\
\hline 564.4 & 0.213 & 0.216 & 0.219 & 0.231 & 0.228 & 0.225 & 0.207 & 0.249 & 0.209 & 0.217 \\
\hline 564.75 & 0.214 & 0.218 & 0.22 & 0.231 & 0.23 & 0.226 & 0.209 & 0.25 & 0.21 & 0.218 \\
\hline 565.09 & 0.215 & 0.219 & 0.222 & 0.233 & 0.231 & 0.227 & 0.21 & 0.252 & 0.211 & 0.219 \\
\hline 565.43 & 0.216 & 0.221 & 0.225 & 0.235 & 0.233 & 0.229 & 0.212 & 0.253 & 0.212 & 0.22 \\
\hline 565.77 & 0.218 & 0.222 & 0.226 & 0.236 & 0.235 & 0.231 & 0.214 & 0.254 & 0.213 & 0.222 \\
\hline 566.12 & 0.22 & 0.223 & 0.228 & 0.238 & 0.237 & 0.233 & 0.216 & 0.256 & 0.215 & 0.223 \\
\hline 566.46 & 0.221 & 0.225 & 0.23 & 0.24 & 0.239 & 0.235 & 0.217 & 0.257 & 0.216 & 0.225 \\
\hline 566.8 & 0.223 & 0.226 & 0.232 & 0.241 & 0.24 & 0.237 & 0.22 & 0.259 & 0.217 & 0.228 \\
\hline 567.14 & 0.224 & 0.229 & 0.234 & 0.244 & 0.241 & 0.239 & 0.221 & 0.26 & 0.219 & 0.228 \\
\hline 567.49 & 0.226 & 0.23 & 0.235 & 0.245 & 0.242 & 0.241 & 0.224 & 0.262 & 0.22 & 0.23 \\
\hline 567.83 & 0.229 & 0.231 & 0.237 & 0.247 & 0.243 & 0.242 & 0.225 & 0.263 & 0.221 & 0.231 \\
\hline 568.17 & 0.23 & 0.233 & 0.237 & 0.248 & 0.244 & 0.243 & 0.228 & 0.264 & 0.223 & 0.232 \\
\hline 568.51 & 0.232 & 0.234 & 0.239 & 0.25 & 0.246 & 0.244 & 0.229 & 0.266 & 0.225 & 0.234 \\
\hline
\end{tabular}




\begin{tabular}{|c|c|c|c|c|c|c|c|c|c|c|}
\hline 568.85 & 0.235 & 0.236 & 0.241 & 0.252 & 0.247 & 0.245 & 0.231 & 0.267 & 0.226 & 0.235 \\
\hline 569.2 & 0.237 & 0.238 & 0.242 & 0.253 & 0.249 & 0.246 & 0.233 & 0.268 & 0.227 & 0.237 \\
\hline 569.54 & 0.239 & 0.24 & 0.243 & 0.255 & 0.25 & 0.248 & 0.235 & 0.268 & 0.229 & 0.238 \\
\hline 569.88 & 0.24 & 0.243 & 0.244 & 0.257 & 0.251 & 0.25 & 0.238 & 0.271 & 0.23 & 0.239 \\
\hline 570.22 & 0.24 & 0.245 & 0.246 & 0.258 & 0.253 & 0.251 & 0.239 & 0.273 & 0.231 & 0.239 \\
\hline 570.57 & 0.242 & 0.248 & 0.247 & 0.259 & 0.254 & 0.251 & 0.241 & 0.275 & 0.234 & 0.24 \\
\hline 570.91 & 0.244 & 0.25 & 0.248 & 0.26 & 0.256 & 0.252 & 0.242 & 0.277 & 0.235 & 0.241 \\
\hline 571.25 & 0.245 & 0.251 & 0.25 & 0.263 & 0.256 & 0.253 & 0.244 & 0.278 & 0.236 & 0.242 \\
\hline 571.59 & 0.247 & 0.253 & 0.251 & 0.264 & 0.258 & 0.254 & 0.246 & 0.279 & 0.237 & 0.242 \\
\hline 571.93 & 0.248 & 0.254 & 0.252 & 0.265 & 0.26 & 0.256 & 0.247 & 0.28 & 0.239 & 0.244 \\
\hline 572.27 & 0.249 & 0.256 & 0.252 & 0.266 & 0.262 & 0.258 & 0.25 & 0.281 & 0.239 & 0.246 \\
\hline 572.62 & 0.25 & 0.258 & 0.254 & 0.268 & 0.263 & 0.26 & 0.251 & 0.282 & 0.24 & 0.248 \\
\hline 572.96 & 0.253 & 0.26 & 0.255 & 0.27 & 0.264 & 0.262 & 0.253 & 0.283 & 0.242 & 0.249 \\
\hline 573.3 & 0.255 & 0.262 & 0.257 & 0.271 & 0.265 & 0.263 & 0.255 & 0.285 & 0.244 & 0.251 \\
\hline 573.64 & 0.257 & 0.263 & 0.258 & 0.272 & 0.266 & 0.263 & 0.256 & 0.285 & 0.245 & 0.252 \\
\hline 573.98 & 0.259 & 0.264 & 0.259 & 0.275 & 0.266 & 0.265 & 0.257 & 0.286 & 0.246 & 0.254 \\
\hline 574.32 & 0.26 & 0.265 & 0.259 & 0.277 & 0.267 & 0.266 & 0.259 & 0.286 & 0.246 & 0.256 \\
\hline 574.67 & 0.262 & 0.267 & 0.26 & 0.279 & 0.269 & 0.268 & 0.261 & 0.287 & 0.247 & 0.258 \\
\hline 575.01 & 0.264 & 0.269 & 0.262 & 0.28 & 0.271 & 0.27 & 0.263 & 0.289 & 0.249 & 0.26 \\
\hline 575.35 & 0.266 & 0.272 & 0.263 & 0.283 & 0.273 & 0.272 & 0.263 & 0.29 & 0.251 & 0.262 \\
\hline 575.69 & 0.268 & 0.275 & 0.266 & 0.284 & 0.275 & 0.273 & 0.265 & 0.292 & 0.252 & 0.264 \\
\hline 576.03 & 0.27 & 0.276 & 0.268 & 0.286 & 0.276 & 0.275 & 0.266 & 0.294 & 0.254 & 0.266 \\
\hline 576.37 & 0.271 & 0.277 & 0.27 & 0.287 & 0.278 & 0.276 & 0.269 & 0.296 & 0.258 & 0.267 \\
\hline 576.71 & 0.272 & 0.279 & 0.272 & 0.288 & 0.279 & 0.277 & 0.27 & 0.299 & 0.257 & 0.269 \\
\hline 577.05 & 0.276 & 0.28 & 0.273 & 0.29 & 0.282 & 0.278 & 0.271 & 0.299 & 0.259 & 0.271 \\
\hline 577.4 & 0.277 & 0.283 & 0.275 & 0.291 & 0.284 & 0.28 & 0.273 & 0.301 & 0.26 & 0.272 \\
\hline 577.74 & 0.28 & 0.285 & 0.278 & 0.292 & 0.287 & 0.282 & 0.276 & 0.302 & 0.262 & 0.275 \\
\hline 578.08 & 0.283 & 0.287 & 0.28 & 0.293 & 0.289 & 0.283 & 0.276 & 0.304 & 0.263 & 0.276 \\
\hline 578.42 & 0.285 & 0.29 & 0.282 & 0.295 & 0.29 & 0.285 & 0.278 & 0.306 & 0.266 & 0.277 \\
\hline 578.76 & 0.287 & 0.291 & 0.284 & 0.296 & 0.291 & 0.287 & 0.281 & 0.307 & 0.269 & 0.278 \\
\hline 579.1 & 0.289 & 0.293 & 0.287 & 0.298 & 0.292 & 0.29 & 0.284 & 0.308 & 0.27 & 0.279 \\
\hline 579.44 & 0.29 & 0.294 & 0.289 & 0.299 & 0.293 & 0.291 & 0.285 & 0.309 & 0.272 & 0.28 \\
\hline 579.78 & 0.293 & 0.296 & 0.291 & 0.301 & 0.294 & 0.293 & 0.286 & 0.31 & 0.273 & 0.281 \\
\hline 580.12 & 0.296 & 0.298 & 0.292 & 0.303 & 0.296 & 0.295 & 0.289 & 0.311 & 0.273 & 0.282 \\
\hline 580.46 & 0.298 & 0.299 & 0.294 & 0.305 & 0.298 & 0.297 & 0.292 & 0.312 & 0.275 & 0.283 \\
\hline 580.8 & 0.3 & 0.301 & 0.297 & 0.306 & 0.298 & 0.299 & 0.294 & 0.315 & 0.277 & 0.285 \\
\hline 581.14 & 0.302 & 0.301 & 0.298 & 0.308 & 0.299 & 0.3 & 0.295 & 0.315 & 0.279 & 0.287 \\
\hline 581.49 & 0.302 & 0.303 & 0.299 & 0.31 & 0.3 & 0.302 & 0.296 & 0.317 & 0.281 & 0.289 \\
\hline 581.83 & 0.303 & 0.304 & 0.302 & 0.313 & 0.302 & 0.304 & 0.299 & 0.319 & 0.283 & 0.292 \\
\hline
\end{tabular}




\begin{tabular}{|c|c|c|c|c|c|c|c|c|c|c|}
\hline 582.17 & 0.306 & 0.305 & 0.305 & 0.315 & 0.303 & 0.306 & 0.301 & 0.321 & 0.284 & 0.294 \\
\hline 582.51 & 0.308 & 0.307 & 0.306 & 0.317 & 0.305 & 0.308 & 0.302 & 0.323 & 0.285 & 0.296 \\
\hline 582.85 & 0.31 & 0.309 & 0.307 & 0.318 & 0.308 & 0.309 & 0.304 & 0.326 & 0.287 & 0.298 \\
\hline 583.19 & 0.312 & 0.311 & 0.308 & 0.321 & 0.31 & 0.311 & 0.307 & 0.328 & 0.288 & 0.301 \\
\hline 583.53 & 0.314 & 0.313 & 0.309 & 0.322 & 0.313 & 0.313 & 0.309 & 0.331 & 0.289 & 0.304 \\
\hline 583.87 & 0.315 & 0.314 & 0.31 & 0.323 & 0.313 & 0.313 & 0.31 & 0.333 & 0.292 & 0.305 \\
\hline 584.21 & 0.317 & 0.316 & 0.31 & 0.325 & 0.315 & 0.315 & 0.311 & 0.335 & 0.294 & 0.306 \\
\hline 584.55 & 0.318 & 0.317 & 0.311 & 0.327 & 0.318 & 0.316 & 0.312 & 0.337 & 0.294 & 0.308 \\
\hline 584.89 & 0.32 & 0.321 & 0.312 & 0.328 & 0.32 & 0.318 & 0.314 & 0.338 & 0.295 & 0.309 \\
\hline 585.23 & 0.322 & 0.323 & 0.313 & 0.329 & 0.322 & 0.319 & 0.317 & 0.341 & 0.297 & 0.31 \\
\hline 585.57 & 0.324 & 0.325 & 0.314 & 0.331 & 0.323 & 0.321 & 0.319 & 0.341 & 0.298 & 0.311 \\
\hline 585.91 & 0.325 & 0.327 & 0.314 & 0.332 & 0.324 & 0.321 & 0.32 & 0.342 & 0.299 & 0.313 \\
\hline 586.25 & 0.327 & 0.328 & 0.316 & 0.334 & 0.325 & 0.323 & 0.321 & 0.344 & 0.3 & 0.316 \\
\hline 586.59 & 0.328 & 0.331 & 0.318 & 0.335 & 0.325 & 0.325 & 0.322 & 0.345 & 0.302 & 0.318 \\
\hline 586.93 & 0.331 & 0.333 & 0.32 & 0.336 & 0.327 & 0.326 & 0.323 & 0.346 & 0.303 & 0.319 \\
\hline 587.27 & 0.332 & 0.335 & 0.323 & 0.338 & 0.328 & 0.326 & 0.325 & 0.347 & 0.306 & 0.319 \\
\hline 587.61 & 0.334 & 0.338 & 0.324 & 0.34 & 0.331 & 0.329 & 0.328 & 0.35 & 0.306 & 0.322 \\
\hline 587.95 & 0.337 & 0.34 & 0.327 & 0.343 & 0.332 & 0.33 & 0.33 & 0.353 & 0.308 & 0.323 \\
\hline 588.29 & 0.339 & 0.344 & 0.329 & 0.345 & 0.333 & 0.332 & 0.333 & 0.355 & 0.309 & 0.325 \\
\hline 588.63 & 0.342 & 0.346 & 0.332 & 0.346 & 0.335 & 0.333 & 0.337 & 0.358 & 0.311 & 0.326 \\
\hline 588.97 & 0.346 & 0.347 & 0.335 & 0.348 & 0.337 & 0.335 & 0.338 & 0.358 & 0.313 & 0.328 \\
\hline 589.3 & 0.35 & 0.349 & 0.337 & 0.35 & 0.339 & 0.337 & 0.341 & 0.361 & 0.315 & 0.33 \\
\hline 589.64 & 0.353 & 0.352 & 0.339 & 0.353 & 0.342 & 0.34 & 0.344 & 0.364 & 0.318 & 0.331 \\
\hline 589.98 & 0.357 & 0.355 & 0.342 & 0.355 & 0.345 & 0.341 & 0.347 & 0.366 & 0.32 & 0.332 \\
\hline 590.32 & 0.361 & 0.355 & 0.344 & 0.358 & 0.348 & 0.343 & 0.35 & 0.368 & 0.322 & 0.334 \\
\hline 590.66 & 0.363 & 0.358 & 0.346 & 0.361 & 0.35 & 0.345 & 0.353 & 0.372 & 0.323 & 0.337 \\
\hline 591 & 0.366 & 0.361 & 0.347 & 0.363 & 0.352 & 0.348 & 0.356 & 0.375 & 0.324 & 0.338 \\
\hline 591.34 & 0.369 & 0.363 & 0.35 & 0.366 & 0.353 & 0.351 & 0.357 & 0.377 & 0.327 & 0.341 \\
\hline 591.68 & 0.371 & 0.365 & 0.352 & 0.367 & 0.356 & 0.353 & 0.359 & 0.378 & 0.33 & 0.343 \\
\hline 592.02 & 0.374 & 0.367 & 0.354 & 0.369 & 0.359 & 0.355 & 0.361 & 0.381 & 0.332 & 0.344 \\
\hline 592.36 & 0.376 & 0.369 & 0.355 & 0.371 & 0.36 & 0.358 & 0.364 & 0.383 & 0.334 & 0.346 \\
\hline 592.7 & 0.378 & 0.372 & 0.357 & 0.373 & 0.361 & 0.36 & 0.367 & 0.386 & 0.337 & 0.349 \\
\hline 593.04 & 0.38 & 0.375 & 0.359 & 0.374 & 0.362 & 0.361 & 0.368 & 0.388 & 0.339 & 0.35 \\
\hline 593.37 & 0.382 & 0.377 & 0.361 & 0.375 & 0.362 & 0.363 & 0.371 & 0.388 & 0.341 & 0.353 \\
\hline 593.71 & 0.384 & 0.381 & 0.362 & 0.377 & 0.364 & 0.365 & 0.375 & 0.392 & 0.342 & 0.355 \\
\hline 594.05 & 0.386 & 0.384 & 0.364 & 0.379 & 0.366 & 0.366 & 0.377 & 0.396 & 0.343 & 0.357 \\
\hline 594.39 & 0.389 & 0.385 & 0.366 & 0.381 & 0.367 & 0.368 & 0.38 & 0.397 & 0.346 & 0.358 \\
\hline 594.73 & 0.391 & 0.388 & 0.368 & 0.384 & 0.37 & 0.37 & 0.383 & 0.398 & 0.349 & 0.36 \\
\hline 595.07 & 0.393 & 0.391 & 0.369 & 0.385 & 0.372 & 0.371 & 0.385 & 0.4 & 0.351 & 0.362 \\
\hline
\end{tabular}




\begin{tabular}{|c|c|c|c|c|c|c|c|c|c|c|}
\hline 595.41 & 0.395 & 0.394 & 0.371 & 0.389 & 0.374 & 0.372 & 0.388 & 0.402 & 0.353 & 0.365 \\
\hline 595.75 & 0.398 & 0.396 & 0.373 & 0.391 & 0.377 & 0.373 & 0.391 & 0.406 & 0.356 & 0.368 \\
\hline 596.08 & 0.4 & 0.399 & 0.376 & 0.395 & 0.379 & 0.376 & 0.393 & 0.409 & 0.358 & 0.371 \\
\hline 596.42 & 0.402 & 0.402 & 0.377 & 0.397 & 0.383 & 0.378 & 0.397 & 0.413 & 0.36 & 0.373 \\
\hline 596.76 & 0.405 & 0.406 & 0.379 & 0.4 & 0.387 & 0.381 & 0.4 & 0.416 & 0.362 & 0.376 \\
\hline 597.1 & 0.407 & 0.409 & 0.382 & 0.403 & 0.39 & 0.384 & 0.404 & 0.418 & 0.365 & 0.378 \\
\hline 597.44 & 0.409 & 0.412 & 0.383 & 0.405 & 0.391 & 0.386 & 0.405 & 0.419 & 0.366 & 0.379 \\
\hline 597.78 & 0.412 & 0.416 & 0.386 & 0.407 & 0.393 & 0.389 & 0.408 & 0.42 & 0.369 & 0.38 \\
\hline 598.11 & 0.414 & 0.421 & 0.39 & 0.41 & 0.395 & 0.391 & 0.41 & 0.423 & 0.372 & 0.385 \\
\hline 598.45 & 0.417 & 0.423 & 0.393 & 0.411 & 0.397 & 0.393 & 0.413 & 0.425 & 0.374 & 0.388 \\
\hline 598.79 & 0.42 & 0.426 & 0.396 & 0.415 & 0.399 & 0.397 & 0.417 & 0.428 & 0.376 & 0.391 \\
\hline 599.13 & 0.423 & 0.429 & 0.399 & 0.418 & 0.402 & 0.399 & 0.42 & 0.431 & 0.378 & 0.393 \\
\hline 599.47 & 0.425 & 0.434 & 0.402 & 0.421 & 0.404 & 0.403 & 0.424 & 0.433 & 0.382 & 0.396 \\
\hline 599.81 & 0.43 & 0.437 & 0.404 & 0.423 & 0.407 & 0.405 & 0.427 & 0.435 & 0.384 & 0.4 \\
\hline 600.14 & 0.432 & 0.439 & 0.408 & 0.426 & 0.408 & 0.407 & 0.43 & 0.437 & 0.387 & 0.401 \\
\hline 600.48 & 0.434 & 0.44 & 0.412 & 0.429 & 0.409 & 0.41 & 0.434 & 0.439 & 0.39 & 0.403 \\
\hline 600.82 & 0.438 & 0.441 & 0.412 & 0.433 & 0.411 & 0.412 & 0.436 & 0.442 & 0.392 & 0.405 \\
\hline 601.16 & 0.44 & 0.444 & 0.415 & 0.437 & 0.414 & 0.415 & 0.438 & 0.446 & 0.396 & 0.409 \\
\hline 601.49 & 0.442 & 0.446 & 0.416 & 0.44 & 0.415 & 0.416 & 0.44 & 0.448 & 0.397 & 0.412 \\
\hline 601.83 & 0.447 & 0.447 & 0.417 & 0.443 & 0.417 & 0.418 & 0.444 & 0.449 & 0.399 & 0.413 \\
\hline 602.17 & 0.45 & 0.45 & 0.419 & 0.447 & 0.42 & 0.421 & 0.446 & 0.451 & 0.402 & 0.417 \\
\hline 602.51 & 0.452 & 0.451 & 0.421 & 0.448 & 0.422 & 0.422 & 0.448 & 0.453 & 0.406 & 0.419 \\
\hline 602.85 & 0.455 & 0.455 & 0.423 & 0.45 & 0.424 & 0.425 & 0.452 & 0.454 & 0.408 & 0.42 \\
\hline 603.18 & 0.458 & 0.457 & 0.425 & 0.451 & 0.426 & 0.426 & 0.454 & 0.456 & 0.409 & 0.422 \\
\hline 603.52 & 0.46 & 0.458 & 0.427 & 0.453 & 0.427 & 0.428 & 0.455 & 0.456 & 0.412 & 0.425 \\
\hline 603.86 & 0.464 & 0.462 & 0.429 & 0.454 & 0.43 & 0.43 & 0.458 & 0.457 & 0.414 & 0.428 \\
\hline 604.2 & 0.468 & 0.463 & 0.429 & 0.456 & 0.432 & 0.43 & 0.459 & 0.458 & 0.418 & 0.428 \\
\hline 604.53 & 0.47 & 0.468 & 0.433 & 0.456 & 0.434 & 0.432 & 0.461 & 0.46 & 0.42 & 0.432 \\
\hline 604.87 & 0.472 & 0.471 & 0.435 & 0.457 & 0.436 & 0.433 & 0.463 & 0.463 & 0.422 & 0.435 \\
\hline 605.21 & 0.474 & 0.474 & 0.436 & 0.459 & 0.44 & 0.435 & 0.466 & 0.465 & 0.424 & 0.436 \\
\hline 605.54 & 0.476 & 0.476 & 0.439 & 0.461 & 0.443 & 0.438 & 0.468 & 0.467 & 0.427 & 0.439 \\
\hline 605.88 & 0.477 & 0.477 & 0.439 & 0.463 & 0.443 & 0.439 & 0.47 & 0.469 & 0.428 & 0.44 \\
\hline 606.22 & 0.479 & 0.479 & 0.439 & 0.466 & 0.444 & 0.441 & 0.473 & 0.47 & 0.429 & 0.442 \\
\hline 606.56 & 0.481 & 0.481 & 0.441 & 0.469 & 0.446 & 0.443 & 0.475 & 0.472 & 0.432 & 0.444 \\
\hline 606.89 & 0.483 & 0.483 & 0.44 & 0.471 & 0.445 & 0.444 & 0.476 & 0.473 & 0.434 & 0.447 \\
\hline 607.23 & 0.485 & 0.486 & 0.441 & 0.473 & 0.448 & 0.447 & 0.479 & 0.477 & 0.436 & 0.448 \\
\hline 607.57 & 0.484 & 0.487 & 0.44 & 0.475 & 0.449 & 0.447 & 0.48 & 0.479 & 0.436 & 0.449 \\
\hline 607.9 & 0.483 & 0.491 & 0.442 & 0.477 & 0.449 & 0.45 & 0.481 & 0.481 & 0.436 & 0.452 \\
\hline 608.24 & 0.485 & 0.493 & 0.444 & 0.479 & 0.451 & 0.45 & 0.483 & 0.482 & 0.438 & 0.452 \\
\hline
\end{tabular}




\begin{tabular}{|c|c|c|c|c|c|c|c|c|c|c|}
\hline 608.58 & 0.487 & 0.494 & 0.444 & 0.481 & 0.452 & 0.452 & 0.487 & 0.483 & 0.438 & 0.454 \\
\hline 608.91 & 0.488 & 0.496 & 0.447 & 0.481 & 0.453 & 0.454 & 0.49 & 0.485 & 0.441 & 0.456 \\
\hline 609.25 & 0.489 & 0.497 & 0.446 & 0.482 & 0.455 & 0.454 & 0.49 & 0.485 & 0.443 & 0.457 \\
\hline 609.59 & 0.49 & 0.5 & 0.449 & 0.484 & 0.457 & 0.457 & 0.492 & 0.488 & 0.444 & 0.459 \\
\hline 609.92 & 0.492 & 0.501 & 0.452 & 0.483 & 0.458 & 0.458 & 0.492 & 0.49 & 0.445 & 0.46 \\
\hline 610.26 & 0.494 & 0.501 & 0.453 & 0.483 & 0.459 & 0.458 & 0.493 & 0.491 & 0.446 & 0.461 \\
\hline 610.6 & 0.494 & 0.502 & 0.454 & 0.486 & 0.462 & 0.46 & 0.493 & 0.493 & 0.446 & 0.462 \\
\hline 610.93 & 0.496 & 0.503 & 0.455 & 0.488 & 0.461 & 0.46 & 0.496 & 0.494 & 0.448 & 0.462 \\
\hline 611.27 & 0.499 & 0.505 & 0.458 & 0.489 & 0.463 & 0.463 & 0.497 & 0.496 & 0.45 & 0.463 \\
\hline 611.61 & 0.502 & 0.503 & 0.459 & 0.49 & 0.465 & 0.464 & 0.499 & 0.497 & 0.451 & 0.465 \\
\hline 611.94 & 0.503 & 0.503 & 0.458 & 0.492 & 0.466 & 0.466 & 0.501 & 0.497 & 0.451 & 0.464 \\
\hline 612.28 & 0.504 & 0.504 & 0.46 & 0.492 & 0.467 & 0.467 & 0.502 & 0.499 & 0.454 & 0.464 \\
\hline 612.61 & 0.506 & 0.505 & 0.459 & 0.494 & 0.468 & 0.467 & 0.503 & 0.5 & 0.454 & 0.467 \\
\hline 612.95 & 0.507 & 0.507 & 0.461 & 0.496 & 0.469 & 0.467 & 0.505 & 0.502 & 0.454 & 0.469 \\
\hline 613.29 & 0.507 & 0.508 & 0.461 & 0.496 & 0.469 & 0.466 & 0.505 & 0.502 & 0.455 & 0.468 \\
\hline 613.62 & 0.506 & 0.508 & 0.46 & 0.496 & 0.468 & 0.467 & 0.507 & 0.501 & 0.454 & 0.469 \\
\hline 613.96 & 0.507 & 0.51 & 0.461 & 0.496 & 0.468 & 0.466 & 0.507 & 0.5 & 0.454 & 0.471 \\
\hline 614.3 & 0.51 & 0.512 & 0.463 & 0.495 & 0.467 & 0.467 & 0.507 & 0.499 & 0.455 & 0.47 \\
\hline 614.63 & 0.51 & 0.512 & 0.463 & 0.493 & 0.468 & 0.467 & 0.506 & 0.499 & 0.454 & 0.471 \\
\hline 614.97 & 0.511 & 0.515 & 0.465 & 0.494 & 0.468 & 0.469 & 0.508 & 0.5 & 0.455 & 0.473 \\
\hline 615.3 & 0.511 & 0.515 & 0.464 & 0.494 & 0.468 & 0.469 & 0.508 & 0.5 & 0.455 & 0.473 \\
\hline 615.64 & 0.513 & 0.518 & 0.465 & 0.495 & 0.469 & 0.469 & 0.508 & 0.5 & 0.455 & 0.475 \\
\hline 615.97 & 0.513 & 0.518 & 0.465 & 0.495 & 0.468 & 0.469 & 0.507 & 0.5 & 0.456 & 0.476 \\
\hline 616.31 & 0.512 & 0.519 & 0.468 & 0.495 & 0.469 & 0.471 & 0.507 & 0.498 & 0.456 & 0.475 \\
\hline 616.65 & 0.513 & 0.518 & 0.467 & 0.494 & 0.47 & 0.471 & 0.506 & 0.497 & 0.456 & 0.474 \\
\hline 616.98 & 0.516 & 0.519 & 0.469 & 0.494 & 0.471 & 0.472 & 0.506 & 0.496 & 0.456 & 0.475 \\
\hline 617.32 & 0.518 & 0.52 & 0.47 & 0.496 & 0.471 & 0.472 & 0.506 & 0.497 & 0.456 & 0.476 \\
\hline 617.65 & 0.518 & 0.519 & 0.47 & 0.498 & 0.472 & 0.473 & 0.506 & 0.499 & 0.457 & 0.475 \\
\hline 617.99 & 0.518 & 0.52 & 0.471 & 0.501 & 0.475 & 0.473 & 0.508 & 0.501 & 0.456 & 0.475 \\
\hline 618.32 & 0.517 & 0.521 & 0.472 & 0.502 & 0.476 & 0.476 & 0.508 & 0.501 & 0.459 & 0.475 \\
\hline 618.66 & 0.518 & 0.52 & 0.472 & 0.503 & 0.476 & 0.475 & 0.508 & 0.501 & 0.458 & 0.474 \\
\hline 618.99 & 0.518 & 0.521 & 0.474 & 0.506 & 0.477 & 0.476 & 0.509 & 0.502 & 0.46 & 0.476 \\
\hline 619.33 & 0.518 & 0.521 & 0.473 & 0.506 & 0.478 & 0.476 & 0.508 & 0.502 & 0.46 & 0.475 \\
\hline 619.66 & 0.515 & 0.521 & 0.473 & 0.505 & 0.479 & 0.477 & 0.51 & 0.503 & 0.46 & 0.474 \\
\hline 620 & 0.516 & 0.521 & 0.471 & 0.506 & 0.478 & 0.475 & 0.51 & 0.505 & 0.46 & 0.474 \\
\hline 620.33 & 0.517 & 0.523 & 0.473 & 0.507 & 0.479 & 0.476 & 0.512 & 0.505 & 0.461 & 0.475 \\
\hline 620.67 & 0.515 & 0.522 & 0.472 & 0.509 & 0.479 & 0.476 & 0.513 & 0.507 & 0.461 & 0.474 \\
\hline 621 & 0.517 & 0.524 & 0.473 & 0.509 & 0.479 & 0.476 & 0.515 & 0.507 & 0.462 & 0.477 \\
\hline 621.34 & 0.517 & 0.525 & 0.474 & 0.51 & 0.48 & 0.477 & 0.517 & 0.509 & 0.464 & 0.478 \\
\hline
\end{tabular}




\begin{tabular}{|c|c|c|c|c|c|c|c|c|c|c|}
\hline 621.67 & 0.518 & 0.524 & 0.474 & 0.51 & 0.479 & 0.478 & 0.517 & 0.509 & 0.467 & 0.478 \\
\hline 622.01 & 0.518 & 0.525 & 0.474 & 0.51 & 0.479 & 0.477 & 0.518 & 0.509 & 0.464 & 0.479 \\
\hline 622.34 & 0.519 & 0.526 & 0.477 & 0.512 & 0.48 & 0.479 & 0.52 & 0.51 & 0.467 & 0.481 \\
\hline 622.68 & 0.518 & 0.527 & 0.476 & 0.513 & 0.48 & 0.48 & 0.52 & 0.51 & 0.467 & 0.481 \\
\hline 623.01 & 0.52 & 0.528 & 0.479 & 0.515 & 0.48 & 0.48 & 0.522 & 0.512 & 0.467 & 0.483 \\
\hline 623.35 & 0.522 & 0.53 & 0.479 & 0.517 & 0.482 & 0.48 & 0.522 & 0.511 & 0.468 & 0.485 \\
\hline 623.68 & 0.522 & 0.53 & 0.48 & 0.519 & 0.482 & 0.482 & 0.522 & 0.513 & 0.469 & 0.486 \\
\hline 624.02 & 0.523 & 0.53 & 0.479 & 0.52 & 0.483 & 0.482 & 0.523 & 0.513 & 0.469 & 0.486 \\
\hline 624.35 & 0.524 & 0.532 & 0.482 & 0.521 & 0.482 & 0.483 & 0.523 & 0.511 & 0.471 & 0.488 \\
\hline 624.69 & 0.523 & 0.532 & 0.482 & 0.522 & 0.484 & 0.486 & 0.523 & 0.511 & 0.473 & 0.488 \\
\hline 625.02 & 0.525 & 0.532 & 0.482 & 0.522 & 0.484 & 0.485 & 0.522 & 0.509 & 0.472 & 0.488 \\
\hline 625.36 & 0.524 & 0.531 & 0.482 & 0.523 & 0.485 & 0.485 & 0.524 & 0.509 & 0.469 & 0.489 \\
\hline 625.69 & 0.524 & 0.53 & 0.483 & 0.524 & 0.485 & 0.485 & 0.523 & 0.509 & 0.47 & 0.488 \\
\hline 626.02 & 0.523 & 0.53 & 0.48 & 0.523 & 0.483 & 0.484 & 0.522 & 0.508 & 0.469 & 0.486 \\
\hline 626.36 & 0.524 & 0.531 & 0.48 & 0.521 & 0.484 & 0.485 & 0.522 & 0.508 & 0.469 & 0.487 \\
\hline 626.69 & 0.522 & 0.531 & 0.48 & 0.521 & 0.485 & 0.486 & 0.522 & 0.508 & 0.469 & 0.487 \\
\hline 627.03 & 0.523 & 0.531 & 0.48 & 0.522 & 0.483 & 0.486 & 0.521 & 0.508 & 0.468 & 0.487 \\
\hline 627.36 & 0.524 & 0.53 & 0.481 & 0.52 & 0.484 & 0.485 & 0.52 & 0.506 & 0.467 & 0.487 \\
\hline 627.7 & 0.522 & 0.531 & 0.481 & 0.518 & 0.483 & 0.484 & 0.52 & 0.508 & 0.466 & 0.488 \\
\hline 628.03 & 0.521 & 0.532 & 0.479 & 0.517 & 0.484 & 0.484 & 0.522 & 0.51 & 0.465 & 0.487 \\
\hline 628.36 & 0.521 & 0.532 & 0.477 & 0.516 & 0.483 & 0.484 & 0.522 & 0.511 & 0.466 & 0.488 \\
\hline 628.7 & 0.518 & 0.534 & 0.478 & 0.517 & 0.486 & 0.485 & 0.522 & 0.514 & 0.465 & 0.487 \\
\hline 629.03 & 0.519 & 0.536 & 0.478 & 0.517 & 0.487 & 0.485 & 0.522 & 0.513 & 0.467 & 0.487 \\
\hline 629.36 & 0.522 & 0.537 & 0.476 & 0.517 & 0.487 & 0.484 & 0.523 & 0.513 & 0.467 & 0.488 \\
\hline 629.7 & 0.522 & 0.536 & 0.476 & 0.516 & 0.488 & 0.483 & 0.523 & 0.512 & 0.467 & 0.488 \\
\hline 630.03 & 0.524 & 0.536 & 0.476 & 0.517 & 0.487 & 0.483 & 0.523 & 0.51 & 0.466 & 0.488 \\
\hline 630.37 & 0.524 & 0.535 & 0.475 & 0.516 & 0.486 & 0.483 & 0.523 & 0.509 & 0.466 & 0.488 \\
\hline 630.7 & 0.523 & 0.535 & 0.474 & 0.516 & 0.487 & 0.483 & 0.524 & 0.51 & 0.465 & 0.488 \\
\hline 631.03 & 0.522 & 0.535 & 0.474 & 0.516 & 0.487 & 0.482 & 0.525 & 0.511 & 0.465 & 0.488 \\
\hline 631.37 & 0.523 & 0.534 & 0.474 & 0.519 & 0.486 & 0.483 & 0.525 & 0.509 & 0.466 & 0.486 \\
\hline 631.7 & 0.522 & 0.534 & 0.474 & 0.52 & 0.485 & 0.483 & 0.523 & 0.508 & 0.465 & 0.486 \\
\hline 632.03 & 0.522 & 0.533 & 0.474 & 0.52 & 0.484 & 0.481 & 0.522 & 0.507 & 0.464 & 0.486 \\
\hline 632.37 & 0.525 & 0.532 & 0.474 & 0.519 & 0.482 & 0.481 & 0.522 & 0.506 & 0.464 & 0.485 \\
\hline 632.7 & 0.522 & 0.531 & 0.471 & 0.517 & 0.481 & 0.48 & 0.52 & 0.505 & 0.463 & 0.484 \\
\hline 633.03 & 0.519 & 0.532 & 0.471 & 0.517 & 0.482 & 0.482 & 0.521 & 0.507 & 0.465 & 0.486 \\
\hline 633.37 & 0.519 & 0.533 & 0.472 & 0.519 & 0.481 & 0.483 & 0.52 & 0.508 & 0.463 & 0.486 \\
\hline 633.7 & 0.518 & 0.532 & 0.472 & 0.519 & 0.482 & 0.483 & 0.522 & 0.508 & 0.463 & 0.486 \\
\hline 634.03 & 0.519 & 0.532 & 0.475 & 0.519 & 0.482 & 0.481 & 0.521 & 0.509 & 0.463 & 0.486 \\
\hline 634.37 & 0.521 & 0.531 & 0.476 & 0.519 & 0.481 & 0.482 & 0.519 & 0.509 & 0.464 & 0.485 \\
\hline
\end{tabular}




\begin{tabular}{|c|c|c|c|c|c|c|c|c|c|c|}
\hline 634.7 & 0.52 & 0.528 & 0.476 & 0.518 & 0.48 & 0.482 & 0.518 & 0.509 & 0.464 & 0.483 \\
\hline 635.03 & 0.521 & 0.529 & 0.474 & 0.515 & 0.48 & 0.481 & 0.516 & 0.509 & 0.463 & 0.483 \\
\hline 635.37 & 0.52 & 0.528 & 0.474 & 0.514 & 0.479 & 0.481 & 0.515 & 0.508 & 0.462 & 0.482 \\
\hline 635.7 & 0.52 & 0.528 & 0.476 & 0.515 & 0.481 & 0.481 & 0.515 & 0.508 & 0.461 & 0.482 \\
\hline 636.03 & 0.518 & 0.527 & 0.474 & 0.514 & 0.48 & 0.48 & 0.514 & 0.506 & 0.461 & 0.481 \\
\hline 636.36 & 0.518 & 0.528 & 0.476 & 0.514 & 0.48 & 0.478 & 0.513 & 0.506 & 0.458 & 0.48 \\
\hline 636.7 & 0.52 & 0.528 & 0.476 & 0.513 & 0.479 & 0.477 & 0.511 & 0.504 & 0.457 & 0.479 \\
\hline 637.03 & 0.518 & 0.528 & 0.475 & 0.512 & 0.479 & 0.477 & 0.51 & 0.505 & 0.458 & 0.479 \\
\hline 637.36 & 0.518 & 0.529 & 0.475 & 0.513 & 0.481 & 0.477 & 0.51 & 0.506 & 0.459 & 0.481 \\
\hline 637.69 & 0.516 & 0.531 & 0.473 & 0.514 & 0.481 & 0.478 & 0.511 & 0.506 & 0.459 & 0.48 \\
\hline 638.03 & 0.515 & 0.531 & 0.472 & 0.512 & 0.48 & 0.476 & 0.511 & 0.505 & 0.458 & 0.479 \\
\hline 638.36 & 0.517 & 0.534 & 0.471 & 0.513 & 0.479 & 0.478 & 0.512 & 0.505 & 0.458 & 0.48 \\
\hline 638.69 & 0.515 & 0.533 & 0.472 & 0.515 & 0.48 & 0.478 & 0.514 & 0.505 & 0.457 & 0.482 \\
\hline 639.02 & 0.516 & 0.534 & 0.472 & 0.516 & 0.48 & 0.478 & 0.516 & 0.506 & 0.459 & 0.483 \\
\hline 639.36 & 0.517 & 0.532 & 0.471 & 0.515 & 0.478 & 0.477 & 0.515 & 0.504 & 0.457 & 0.482 \\
\hline 639.69 & 0.518 & 0.531 & 0.471 & 0.515 & 0.479 & 0.479 & 0.517 & 0.505 & 0.456 & 0.482 \\
\hline 640.02 & 0.519 & 0.531 & 0.471 & 0.517 & 0.476 & 0.481 & 0.518 & 0.507 & 0.458 & 0.484 \\
\hline 640.35 & 0.519 & 0.53 & 0.473 & 0.518 & 0.476 & 0.482 & 0.521 & 0.507 & 0.458 & 0.484 \\
\hline 640.69 & 0.519 & 0.529 & 0.474 & 0.519 & 0.478 & 0.482 & 0.522 & 0.505 & 0.457 & 0.483 \\
\hline 641.02 & 0.518 & 0.526 & 0.472 & 0.517 & 0.475 & 0.482 & 0.52 & 0.504 & 0.455 & 0.48 \\
\hline 641.35 & 0.517 & 0.525 & 0.473 & 0.516 & 0.473 & 0.48 & 0.52 & 0.503 & 0.453 & 0.48 \\
\hline 641.68 & 0.518 & 0.524 & 0.472 & 0.518 & 0.474 & 0.481 & 0.523 & 0.503 & 0.454 & 0.482 \\
\hline 642.01 & 0.517 & 0.523 & 0.473 & 0.519 & 0.474 & 0.479 & 0.523 & 0.505 & 0.452 & 0.482 \\
\hline 642.35 & 0.517 & 0.523 & 0.472 & 0.519 & 0.474 & 0.48 & 0.523 & 0.504 & 0.452 & 0.481 \\
\hline 642.68 & 0.515 & 0.523 & 0.473 & 0.518 & 0.474 & 0.481 & 0.522 & 0.505 & 0.451 & 0.479 \\
\hline 643.01 & 0.513 & 0.524 & 0.472 & 0.517 & 0.475 & 0.481 & 0.522 & 0.507 & 0.451 & 0.48 \\
\hline 643.34 & 0.513 & 0.526 & 0.473 & 0.516 & 0.476 & 0.48 & 0.523 & 0.507 & 0.451 & 0.48 \\
\hline 643.67 & 0.512 & 0.526 & 0.472 & 0.517 & 0.477 & 0.48 & 0.524 & 0.507 & 0.45 & 0.478 \\
\hline 644 & 0.513 & 0.526 & 0.472 & 0.516 & 0.478 & 0.479 & 0.521 & 0.506 & 0.451 & 0.479 \\
\hline 644.34 & 0.513 & 0.527 & 0.471 & 0.516 & 0.476 & 0.479 & 0.522 & 0.507 & 0.45 & 0.478 \\
\hline 644.67 & 0.513 & 0.529 & 0.473 & 0.516 & 0.477 & 0.48 & 0.523 & 0.509 & 0.45 & 0.48 \\
\hline 645 & 0.515 & 0.528 & 0.472 & 0.514 & 0.477 & 0.481 & 0.522 & 0.51 & 0.449 & 0.478 \\
\hline 645.33 & 0.514 & 0.528 & 0.473 & 0.514 & 0.478 & 0.482 & 0.519 & 0.512 & 0.449 & 0.477 \\
\hline 645.66 & 0.513 & 0.53 & 0.472 & 0.513 & 0.479 & 0.482 & 0.519 & 0.511 & 0.449 & 0.478 \\
\hline 645.99 & 0.513 & 0.529 & 0.472 & 0.514 & 0.478 & 0.483 & 0.519 & 0.511 & 0.45 & 0.479 \\
\hline 646.32 & 0.514 & 0.527 & 0.472 & 0.514 & 0.478 & 0.484 & 0.519 & 0.511 & 0.452 & 0.479 \\
\hline 646.66 & 0.515 & 0.529 & 0.475 & 0.516 & 0.479 & 0.484 & 0.518 & 0.511 & 0.454 & 0.48 \\
\hline 646.99 & 0.516 & 0.53 & 0.478 & 0.517 & 0.48 & 0.484 & 0.517 & 0.51 & 0.452 & 0.479 \\
\hline 647.32 & 0.516 & 0.531 & 0.481 & 0.518 & 0.48 & 0.483 & 0.518 & 0.512 & 0.454 & 0.48 \\
\hline
\end{tabular}




\begin{tabular}{|c|c|c|c|c|c|c|c|c|c|c|}
\hline 647.65 & 0.516 & 0.53 & 0.479 & 0.52 & 0.481 & 0.483 & 0.521 & 0.515 & 0.452 & 0.48 \\
\hline 647.98 & 0.518 & 0.53 & 0.481 & 0.524 & 0.483 & 0.484 & 0.522 & 0.516 & 0.454 & 0.484 \\
\hline 648.31 & 0.519 & 0.53 & 0.479 & 0.524 & 0.485 & 0.485 & 0.523 & 0.515 & 0.455 & 0.484 \\
\hline 648.64 & 0.52 & 0.532 & 0.481 & 0.527 & 0.486 & 0.485 & 0.525 & 0.517 & 0.456 & 0.486 \\
\hline 648.97 & 0.52 & 0.533 & 0.482 & 0.528 & 0.485 & 0.485 & 0.527 & 0.516 & 0.458 & 0.486 \\
\hline 649.3 & 0.523 & 0.532 & 0.484 & 0.529 & 0.486 & 0.486 & 0.526 & 0.516 & 0.459 & 0.485 \\
\hline 649.64 & 0.523 & 0.535 & 0.487 & 0.531 & 0.49 & 0.487 & 0.527 & 0.517 & 0.459 & 0.486 \\
\hline 649.97 & 0.525 & 0.537 & 0.487 & 0.533 & 0.489 & 0.485 & 0.53 & 0.518 & 0.46 & 0.488 \\
\hline 650.3 & 0.526 & 0.537 & 0.486 & 0.533 & 0.49 & 0.486 & 0.532 & 0.52 & 0.461 & 0.489 \\
\hline 650.63 & 0.526 & 0.535 & 0.486 & 0.535 & 0.49 & 0.488 & 0.533 & 0.522 & 0.462 & 0.492 \\
\hline 650.96 & 0.529 & 0.534 & 0.486 & 0.535 & 0.49 & 0.49 & 0.534 & 0.519 & 0.461 & 0.492 \\
\hline 651.29 & 0.532 & 0.537 & 0.488 & 0.536 & 0.492 & 0.492 & 0.536 & 0.519 & 0.464 & 0.493 \\
\hline 651.62 & 0.532 & 0.537 & 0.487 & 0.534 & 0.492 & 0.494 & 0.536 & 0.52 & 0.463 & 0.493 \\
\hline 651.95 & 0.533 & 0.54 & 0.489 & 0.536 & 0.492 & 0.495 & 0.538 & 0.519 & 0.465 & 0.494 \\
\hline 652.28 & 0.534 & 0.542 & 0.49 & 0.538 & 0.494 & 0.496 & 0.537 & 0.519 & 0.466 & 0.495 \\
\hline 652.61 & 0.536 & 0.544 & 0.492 & 0.541 & 0.498 & 0.499 & 0.539 & 0.522 & 0.467 & 0.496 \\
\hline 652.94 & 0.536 & 0.546 & 0.494 & 0.544 & 0.5 & 0.503 & 0.543 & 0.526 & 0.47 & 0.499 \\
\hline 653.27 & 0.54 & 0.548 & 0.494 & 0.546 & 0.499 & 0.505 & 0.543 & 0.527 & 0.472 & 0.501 \\
\hline 653.6 & 0.543 & 0.551 & 0.496 & 0.547 & 0.502 & 0.508 & 0.543 & 0.528 & 0.473 & 0.502 \\
\hline 653.93 & 0.545 & 0.557 & 0.498 & 0.55 & 0.504 & 0.511 & 0.546 & 0.53 & 0.476 & 0.506 \\
\hline 654.26 & 0.549 & 0.561 & 0.498 & 0.555 & 0.505 & 0.514 & 0.549 & 0.534 & 0.481 & 0.508 \\
\hline 654.59 & 0.551 & 0.564 & 0.499 & 0.557 & 0.509 & 0.517 & 0.552 & 0.538 & 0.485 & 0.512 \\
\hline 654.92 & 0.549 & 0.568 & 0.501 & 0.558 & 0.511 & 0.519 & 0.553 & 0.538 & 0.488 & 0.514 \\
\hline 655.25 & 0.552 & 0.572 & 0.504 & 0.56 & 0.513 & 0.52 & 0.554 & 0.54 & 0.489 & 0.516 \\
\hline 655.58 & 0.556 & 0.575 & 0.507 & 0.562 & 0.516 & 0.522 & 0.556 & 0.545 & 0.491 & 0.52 \\
\hline 655.91 & 0.56 & 0.576 & 0.508 & 0.566 & 0.518 & 0.524 & 0.563 & 0.549 & 0.494 & 0.523 \\
\hline 656.24 & 0.564 & 0.58 & 0.511 & 0.567 & 0.52 & 0.524 & 0.565 & 0.549 & 0.497 & 0.528 \\
\hline 656.57 & 0.566 & 0.583 & 0.514 & 0.568 & 0.524 & 0.524 & 0.567 & 0.551 & 0.497 & 0.53 \\
\hline 656.9 & 0.569 & 0.586 & 0.519 & 0.571 & 0.529 & 0.527 & 0.573 & 0.555 & 0.5 & 0.533 \\
\hline 657.23 & 0.571 & 0.589 & 0.523 & 0.574 & 0.532 & 0.53 & 0.578 & 0.556 & 0.504 & 0.538 \\
\hline 657.56 & 0.575 & 0.589 & 0.526 & 0.575 & 0.533 & 0.533 & 0.579 & 0.557 & 0.506 & 0.537 \\
\hline 657.89 & 0.578 & 0.593 & 0.532 & 0.577 & 0.538 & 0.536 & 0.582 & 0.558 & 0.509 & 0.538 \\
\hline 658.22 & 0.58 & 0.595 & 0.539 & 0.579 & 0.539 & 0.536 & 0.586 & 0.561 & 0.512 & 0.54 \\
\hline 658.55 & 0.584 & 0.6 & 0.543 & 0.585 & 0.543 & 0.539 & 0.591 & 0.567 & 0.516 & 0.546 \\
\hline 658.88 & 0.589 & 0.6 & 0.545 & 0.589 & 0.545 & 0.542 & 0.595 & 0.569 & 0.52 & 0.552 \\
\hline 659.21 & 0.592 & 0.602 & 0.548 & 0.591 & 0.548 & 0.544 & 0.599 & 0.572 & 0.525 & 0.553 \\
\hline 659.54 & 0.596 & 0.607 & 0.553 & 0.593 & 0.552 & 0.549 & 0.601 & 0.577 & 0.53 & 0.557 \\
\hline 659.87 & 0.598 & 0.608 & 0.557 & 0.595 & 0.554 & 0.553 & 0.601 & 0.58 & 0.53 & 0.558 \\
\hline 660.2 & 0.603 & 0.614 & 0.563 & 0.597 & 0.556 & 0.558 & 0.609 & 0.586 & 0.537 & 0.565 \\
\hline
\end{tabular}




\begin{tabular}{|c|c|c|c|c|c|c|c|c|c|c|}
\hline 660.53 & 0.604 & 0.616 & 0.565 & 0.598 & 0.558 & 0.56 & 0.611 & 0.587 & 0.541 & 0.567 \\
\hline 660.85 & 0.608 & 0.62 & 0.567 & 0.601 & 0.562 & 0.563 & 0.614 & 0.591 & 0.544 & 0.57 \\
\hline 661.18 & 0.609 & 0.624 & 0.57 & 0.605 & 0.568 & 0.567 & 0.619 & 0.597 & 0.549 & 0.574 \\
\hline 661.51 & 0.613 & 0.626 & 0.573 & 0.609 & 0.572 & 0.572 & 0.623 & 0.604 & 0.554 & 0.581 \\
\hline 661.84 & 0.616 & 0.63 & 0.574 & 0.615 & 0.581 & 0.579 & 0.627 & 0.608 & 0.558 & 0.585 \\
\hline 662.17 & 0.622 & 0.629 & 0.578 & 0.619 & 0.583 & 0.58 & 0.626 & 0.61 & 0.562 & 0.586 \\
\hline 662.5 & 0.624 & 0.634 & 0.582 & 0.619 & 0.587 & 0.584 & 0.631 & 0.615 & 0.566 & 0.587 \\
\hline 662.83 & 0.628 & 0.638 & 0.587 & 0.624 & 0.592 & 0.588 & 0.632 & 0.617 & 0.57 & 0.593 \\
\hline 663.16 & 0.63 & 0.64 & 0.592 & 0.628 & 0.592 & 0.59 & 0.633 & 0.617 & 0.573 & 0.597 \\
\hline 663.49 & 0.633 & 0.646 & 0.593 & 0.636 & 0.598 & 0.594 & 0.641 & 0.623 & 0.581 & 0.601 \\
\hline 663.81 & 0.638 & 0.65 & 0.595 & 0.641 & 0.601 & 0.596 & 0.642 & 0.623 & 0.585 & 0.601 \\
\hline 664.14 & 0.642 & 0.653 & 0.596 & 0.646 & 0.606 & 0.601 & 0.644 & 0.627 & 0.587 & 0.604 \\
\hline 664.47 & 0.644 & 0.657 & 0.6 & 0.646 & 0.608 & 0.606 & 0.646 & 0.628 & 0.589 & 0.607 \\
\hline 664.8 & 0.646 & 0.659 & 0.603 & 0.648 & 0.608 & 0.605 & 0.647 & 0.628 & 0.589 & 0.61 \\
\hline 665.13 & 0.65 & 0.664 & 0.603 & 0.651 & 0.611 & 0.607 & 0.647 & 0.628 & 0.588 & 0.613 \\
\hline 665.46 & 0.654 & 0.668 & 0.606 & 0.651 & 0.611 & 0.606 & 0.65 & 0.629 & 0.587 & 0.617 \\
\hline 665.79 & 0.657 & 0.672 & 0.609 & 0.653 & 0.615 & 0.611 & 0.655 & 0.631 & 0.589 & 0.622 \\
\hline 666.11 & 0.66 & 0.676 & 0.611 & 0.66 & 0.618 & 0.613 & 0.656 & 0.634 & 0.592 & 0.624 \\
\hline 666.44 & 0.663 & 0.68 & 0.613 & 0.664 & 0.621 & 0.616 & 0.661 & 0.637 & 0.592 & 0.624 \\
\hline 666.77 & 0.668 & 0.681 & 0.614 & 0.667 & 0.628 & 0.62 & 0.666 & 0.639 & 0.593 & 0.626 \\
\hline 667.1 & 0.673 & 0.681 & 0.618 & 0.667 & 0.63 & 0.622 & 0.665 & 0.637 & 0.592 & 0.63 \\
\hline 667.43 & 0.676 & 0.682 & 0.62 & 0.671 & 0.633 & 0.624 & 0.667 & 0.638 & 0.592 & 0.633 \\
\hline 667.76 & 0.681 & 0.688 & 0.624 & 0.677 & 0.639 & 0.624 & 0.672 & 0.643 & 0.598 & 0.637 \\
\hline 668.08 & 0.683 & 0.69 & 0.628 & 0.681 & 0.647 & 0.626 & 0.678 & 0.645 & 0.603 & 0.639 \\
\hline 668.41 & 0.685 & 0.694 & 0.632 & 0.684 & 0.652 & 0.63 & 0.68 & 0.648 & 0.608 & 0.642 \\
\hline 668.74 & 0.688 & 0.693 & 0.633 & 0.685 & 0.653 & 0.63 & 0.683 & 0.65 & 0.613 & 0.641 \\
\hline 669.07 & 0.69 & 0.695 & 0.635 & 0.689 & 0.656 & 0.637 & 0.685 & 0.652 & 0.617 & 0.642 \\
\hline 669.4 & 0.691 & 0.698 & 0.639 & 0.691 & 0.659 & 0.639 & 0.686 & 0.656 & 0.622 & 0.644 \\
\hline 669.72 & 0.696 & 0.696 & 0.641 & 0.692 & 0.658 & 0.639 & 0.688 & 0.654 & 0.624 & 0.648 \\
\hline 670.05 & 0.699 & 0.699 & 0.643 & 0.692 & 0.66 & 0.642 & 0.689 & 0.655 & 0.63 & 0.649 \\
\hline 670.38 & 0.702 & 0.706 & 0.645 & 0.695 & 0.662 & 0.644 & 0.693 & 0.658 & 0.636 & 0.653 \\
\hline 670.71 & 0.699 & 0.709 & 0.647 & 0.697 & 0.664 & 0.65 & 0.696 & 0.663 & 0.639 & 0.655 \\
\hline 671.03 & 0.7 & 0.706 & 0.648 & 0.695 & 0.667 & 0.65 & 0.696 & 0.666 & 0.643 & 0.657 \\
\hline 671.36 & 0.701 & 0.704 & 0.647 & 0.695 & 0.664 & 0.653 & 0.695 & 0.666 & 0.641 & 0.658 \\
\hline 671.69 & 0.702 & 0.705 & 0.648 & 0.696 & 0.66 & 0.656 & 0.696 & 0.669 & 0.641 & 0.661 \\
\hline 672.02 & 0.701 & 0.699 & 0.647 & 0.698 & 0.655 & 0.654 & 0.697 & 0.666 & 0.641 & 0.66 \\
\hline 672.34 & 0.702 & 0.701 & 0.652 & 0.698 & 0.657 & 0.655 & 0.697 & 0.665 & 0.639 & 0.664 \\
\hline 672.67 & 0.701 & 0.699 & 0.653 & 0.698 & 0.657 & 0.653 & 0.694 & 0.666 & 0.639 & 0.665 \\
\hline 673 & 0.705 & 0.694 & 0.649 & 0.696 & 0.655 & 0.651 & 0.694 & 0.662 & 0.635 & 0.664 \\
\hline
\end{tabular}




\begin{tabular}{|c|c|c|c|c|c|c|c|c|c|c|}
\hline 673.33 & 0.702 & 0.701 & 0.652 & 0.698 & 0.657 & 0.654 & 0.698 & 0.666 & 0.635 & 0.663 \\
\hline 673.65 & 0.7 & 0.703 & 0.657 & 0.698 & 0.661 & 0.656 & 0.7 & 0.666 & 0.634 & 0.669 \\
\hline 673.98 & 0.7 & 0.703 & 0.654 & 0.697 & 0.659 & 0.657 & 0.695 & 0.665 & 0.638 & 0.667 \\
\hline 674.31 & 0.7 & 0.702 & 0.653 & 0.694 & 0.656 & 0.655 & 0.694 & 0.661 & 0.636 & 0.666 \\
\hline 674.63 & 0.7 & 0.702 & 0.652 & 0.695 & 0.655 & 0.656 & 0.697 & 0.662 & 0.635 & 0.665 \\
\hline 674.96 & 0.701 & 0.703 & 0.652 & 0.691 & 0.655 & 0.655 & 0.693 & 0.657 & 0.635 & 0.662 \\
\hline 675.29 & 0.704 & 0.704 & 0.653 & 0.687 & 0.658 & 0.653 & 0.693 & 0.654 & 0.639 & 0.66 \\
\hline 675.61 & 0.708 & 0.709 & 0.653 & 0.684 & 0.662 & 0.655 & 0.694 & 0.656 & 0.64 & 0.661 \\
\hline 675.94 & 0.707 & 0.71 & 0.651 & 0.687 & 0.663 & 0.658 & 0.696 & 0.659 & 0.642 & 0.659 \\
\hline 676.27 & 0.708 & 0.71 & 0.648 & 0.689 & 0.663 & 0.658 & 0.698 & 0.657 & 0.642 & 0.659 \\
\hline 676.6 & 0.704 & 0.712 & 0.654 & 0.692 & 0.665 & 0.665 & 0.701 & 0.661 & 0.649 & 0.662 \\
\hline 676.92 & 0.707 & 0.71 & 0.654 & 0.693 & 0.663 & 0.663 & 0.702 & 0.662 & 0.65 & 0.666 \\
\hline 677.25 & 0.709 & 0.71 & 0.653 & 0.691 & 0.661 & 0.662 & 0.7 & 0.664 & 0.65 & 0.662 \\
\hline 677.58 & 0.709 & 0.705 & 0.655 & 0.69 & 0.662 & 0.658 & 0.7 & 0.661 & 0.644 & 0.663 \\
\hline 677.9 & 0.714 & 0.707 & 0.655 & 0.692 & 0.665 & 0.658 & 0.703 & 0.665 & 0.646 & 0.664 \\
\hline 678.23 & 0.713 & 0.709 & 0.653 & 0.695 & 0.663 & 0.66 & 0.701 & 0.663 & 0.644 & 0.666 \\
\hline 678.55 & 0.71 & 0.708 & 0.656 & 0.698 & 0.66 & 0.661 & 0.702 & 0.666 & 0.646 & 0.665 \\
\hline 678.88 & 0.705 & 0.71 & 0.654 & 0.701 & 0.655 & 0.66 & 0.699 & 0.67 & 0.642 & 0.664 \\
\hline 679.21 & 0.705 & 0.708 & 0.655 & 0.701 & 0.653 & 0.659 & 0.695 & 0.669 & 0.643 & 0.663 \\
\hline 679.53 & 0.706 & 0.705 & 0.654 & 0.696 & 0.648 & 0.656 & 0.694 & 0.666 & 0.646 & 0.662 \\
\hline 679.86 & 0.709 & 0.705 & 0.654 & 0.692 & 0.646 & 0.653 & 0.694 & 0.666 & 0.644 & 0.661 \\
\hline 680.19 & 0.708 & 0.705 & 0.647 & 0.689 & 0.646 & 0.647 & 0.691 & 0.663 & 0.64 & 0.655 \\
\hline 680.51 & 0.704 & 0.702 & 0.645 & 0.686 & 0.645 & 0.645 & 0.686 & 0.658 & 0.639 & 0.649 \\
\hline 680.84 & 0.704 & 0.699 & 0.642 & 0.686 & 0.641 & 0.643 & 0.686 & 0.658 & 0.638 & 0.649 \\
\hline 681.16 & 0.704 & 0.703 & 0.641 & 0.688 & 0.639 & 0.644 & 0.688 & 0.66 & 0.638 & 0.65 \\
\hline 681.49 & 0.698 & 0.7 & 0.639 & 0.683 & 0.638 & 0.645 & 0.683 & 0.658 & 0.635 & 0.646 \\
\hline 681.82 & 0.694 & 0.699 & 0.643 & 0.682 & 0.637 & 0.643 & 0.684 & 0.658 & 0.638 & 0.644 \\
\hline 682.14 & 0.697 & 0.7 & 0.64 & 0.678 & 0.638 & 0.643 & 0.687 & 0.658 & 0.64 & 0.647 \\
\hline 682.47 & 0.696 & 0.7 & 0.64 & 0.677 & 0.643 & 0.643 & 0.684 & 0.656 & 0.643 & 0.65 \\
\hline 682.79 & 0.695 & 0.699 & 0.637 & 0.678 & 0.643 & 0.644 & 0.685 & 0.657 & 0.642 & 0.65 \\
\hline 683.12 & 0.691 & 0.702 & 0.637 & 0.679 & 0.646 & 0.643 & 0.686 & 0.656 & 0.638 & 0.652 \\
\hline 683.45 & 0.685 & 0.704 & 0.637 & 0.68 & 0.647 & 0.643 & 0.683 & 0.655 & 0.64 & 0.651 \\
\hline 683.77 & 0.683 & 0.704 & 0.638 & 0.682 & 0.643 & 0.645 & 0.682 & 0.658 & 0.641 & 0.653 \\
\hline 684.1 & 0.685 & 0.706 & 0.639 & 0.682 & 0.645 & 0.648 & 0.682 & 0.66 & 0.641 & 0.655 \\
\hline 684.42 & 0.685 & 0.703 & 0.633 & 0.678 & 0.641 & 0.646 & 0.678 & 0.656 & 0.642 & 0.653 \\
\hline 684.75 & 0.682 & 0.699 & 0.631 & 0.678 & 0.64 & 0.646 & 0.675 & 0.652 & 0.644 & 0.652 \\
\hline 685.07 & 0.679 & 0.698 & 0.631 & 0.678 & 0.641 & 0.641 & 0.675 & 0.653 & 0.644 & 0.653 \\
\hline 685.4 & 0.677 & 0.697 & 0.628 & 0.676 & 0.638 & 0.641 & 0.673 & 0.654 & 0.643 & 0.651 \\
\hline 685.72 & 0.674 & 0.693 & 0.626 & 0.675 & 0.636 & 0.637 & 0.671 & 0.648 & 0.64 & 0.647 \\
\hline
\end{tabular}




\begin{tabular}{|c|c|c|c|c|c|c|c|c|c|c|}
\hline 686.05 & 0.669 & 0.69 & 0.624 & 0.672 & 0.632 & 0.635 & 0.671 & 0.646 & 0.633 & 0.645 \\
\hline 686.37 & 0.662 & 0.687 & 0.624 & 0.671 & 0.629 & 0.634 & 0.668 & 0.645 & 0.63 & 0.642 \\
\hline 686.7 & 0.663 & 0.685 & 0.622 & 0.67 & 0.628 & 0.635 & 0.666 & 0.647 & 0.63 & 0.639 \\
\hline 687.02 & 0.66 & 0.68 & 0.62 & 0.666 & 0.626 & 0.632 & 0.666 & 0.645 & 0.629 & 0.635 \\
\hline 687.35 & 0.656 & 0.678 & 0.616 & 0.662 & 0.624 & 0.629 & 0.665 & 0.641 & 0.627 & 0.634 \\
\hline 687.67 & 0.65 & 0.678 & 0.611 & 0.661 & 0.621 & 0.627 & 0.661 & 0.64 & 0.626 & 0.631 \\
\hline 688 & 0.644 & 0.676 & 0.609 & 0.661 & 0.621 & 0.624 & 0.663 & 0.638 & 0.619 & 0.629 \\
\hline 688.32 & 0.638 & 0.675 & 0.608 & 0.656 & 0.619 & 0.622 & 0.662 & 0.636 & 0.616 & 0.625 \\
\hline 688.65 & 0.635 & 0.673 & 0.606 & 0.654 & 0.617 & 0.622 & 0.659 & 0.634 & 0.616 & 0.623 \\
\hline 688.97 & 0.63 & 0.669 & 0.603 & 0.651 & 0.615 & 0.616 & 0.655 & 0.629 & 0.613 & 0.62 \\
\hline 689.3 & 0.623 & 0.665 & 0.599 & 0.648 & 0.61 & 0.612 & 0.649 & 0.629 & 0.609 & 0.618 \\
\hline 689.62 & 0.621 & 0.663 & 0.595 & 0.644 & 0.606 & 0.605 & 0.644 & 0.627 & 0.607 & 0.614 \\
\hline 689.95 & 0.619 & 0.658 & 0.59 & 0.637 & 0.605 & 0.601 & 0.639 & 0.621 & 0.606 & 0.61 \\
\hline 690.27 & 0.611 & 0.654 & 0.586 & 0.632 & 0.6 & 0.597 & 0.633 & 0.613 & 0.601 & 0.608 \\
\hline 690.6 & 0.608 & 0.65 & 0.58 & 0.628 & 0.597 & 0.591 & 0.628 & 0.61 & 0.594 & 0.601 \\
\hline 690.92 & 0.603 & 0.645 & 0.578 & 0.623 & 0.592 & 0.588 & 0.623 & 0.607 & 0.59 & 0.595 \\
\hline 691.25 & 0.6 & 0.637 & 0.576 & 0.616 & 0.586 & 0.582 & 0.619 & 0.604 & 0.584 & 0.591 \\
\hline 691.57 & 0.596 & 0.633 & 0.573 & 0.612 & 0.581 & 0.578 & 0.611 & 0.6 & 0.58 & 0.586 \\
\hline 691.89 & 0.592 & 0.625 & 0.567 & 0.607 & 0.577 & 0.576 & 0.606 & 0.597 & 0.574 & 0.582 \\
\hline 692.22 & 0.587 & 0.619 & 0.559 & 0.6 & 0.57 & 0.568 & 0.598 & 0.591 & 0.568 & 0.575 \\
\hline 692.54 & 0.581 & 0.613 & 0.555 & 0.591 & 0.566 & 0.565 & 0.592 & 0.587 & 0.561 & 0.569 \\
\hline 692.87 & 0.579 & 0.608 & 0.552 & 0.585 & 0.563 & 0.564 & 0.589 & 0.583 & 0.557 & 0.562 \\
\hline 693.19 & 0.575 & 0.599 & 0.55 & 0.581 & 0.557 & 0.56 & 0.585 & 0.578 & 0.553 & 0.556 \\
\hline 693.52 & 0.566 & 0.597 & 0.543 & 0.579 & 0.548 & 0.555 & 0.581 & 0.576 & 0.549 & 0.553 \\
\hline 693.84 & 0.56 & 0.588 & 0.54 & 0.574 & 0.544 & 0.551 & 0.576 & 0.572 & 0.546 & 0.548 \\
\hline 694.16 & 0.553 & 0.582 & 0.539 & 0.569 & 0.539 & 0.545 & 0.569 & 0.567 & 0.544 & 0.548 \\
\hline 694.49 & 0.546 & 0.574 & 0.534 & 0.564 & 0.534 & 0.541 & 0.564 & 0.563 & 0.54 & 0.543 \\
\hline 694.81 & 0.541 & 0.565 & 0.528 & 0.559 & 0.528 & 0.535 & 0.555 & 0.553 & 0.533 & 0.536 \\
\hline 695.14 & 0.533 & 0.558 & 0.522 & 0.554 & 0.524 & 0.53 & 0.549 & 0.549 & 0.529 & 0.53 \\
\hline 695.46 & 0.529 & 0.549 & 0.517 & 0.549 & 0.519 & 0.522 & 0.542 & 0.542 & 0.521 & 0.525 \\
\hline 695.78 & 0.522 & 0.541 & 0.512 & 0.543 & 0.511 & 0.517 & 0.535 & 0.536 & 0.517 & 0.519 \\
\hline 696.11 & 0.517 & 0.534 & 0.504 & 0.541 & 0.504 & 0.512 & 0.529 & 0.53 & 0.514 & 0.514 \\
\hline 696.43 & 0.508 & 0.528 & 0.5 & 0.537 & 0.498 & 0.504 & 0.519 & 0.524 & 0.511 & 0.513 \\
\hline 696.75 & 0.498 & 0.519 & 0.492 & 0.53 & 0.492 & 0.498 & 0.51 & 0.52 & 0.505 & 0.507 \\
\hline 697.08 & 0.495 & 0.511 & 0.488 & 0.524 & 0.489 & 0.493 & 0.506 & 0.516 & 0.5 & 0.502 \\
\hline 697.4 & 0.49 & 0.505 & 0.479 & 0.519 & 0.481 & 0.486 & 0.501 & 0.513 & 0.496 & 0.494 \\
\hline 697.72 & 0.482 & 0.5 & 0.472 & 0.512 & 0.474 & 0.482 & 0.495 & 0.508 & 0.491 & 0.488 \\
\hline 698.05 & 0.476 & 0.492 & 0.465 & 0.506 & 0.469 & 0.474 & 0.488 & 0.498 & 0.483 & 0.483 \\
\hline 698.37 & 0.465 & 0.487 & 0.46 & 0.502 & 0.463 & 0.469 & 0.484 & 0.494 & 0.48 & 0.479 \\
\hline
\end{tabular}




\begin{tabular}{|c|c|c|c|c|c|c|c|c|c|c|}
\hline 698.69 & 0.459 & 0.482 & 0.453 & 0.494 & 0.456 & 0.463 & 0.477 & 0.486 & 0.475 & 0.474 \\
\hline 699.02 & 0.449 & 0.476 & 0.445 & 0.484 & 0.448 & 0.457 & 0.47 & 0.479 & 0.473 & 0.466 \\
\hline 699.34 & 0.444 & 0.471 & 0.441 & 0.479 & 0.447 & 0.453 & 0.465 & 0.475 & 0.465 & 0.464 \\
\hline 699.66 & 0.438 & 0.468 & 0.438 & 0.473 & 0.445 & 0.448 & 0.46 & 0.471 & 0.46 & 0.461 \\
\hline 699.99 & 0.432 & 0.461 & 0.434 & 0.467 & 0.441 & 0.446 & 0.456 & 0.465 & 0.453 & 0.454 \\
\hline 700.31 & 0.429 & 0.455 & 0.429 & 0.462 & 0.436 & 0.443 & 0.455 & 0.459 & 0.447 & 0.451 \\
\hline 700.63 & 0.424 & 0.45 & 0.422 & 0.454 & 0.429 & 0.437 & 0.445 & 0.45 & 0.442 & 0.446 \\
\hline 700.96 & 0.417 & 0.447 & 0.419 & 0.45 & 0.428 & 0.431 & 0.437 & 0.446 & 0.436 & 0.44 \\
\hline 701.28 & 0.41 & 0.437 & 0.413 & 0.445 & 0.422 & 0.424 & 0.428 & 0.44 & 0.431 & 0.434 \\
\hline 701.6 & 0.405 & 0.429 & 0.407 & 0.439 & 0.416 & 0.419 & 0.421 & 0.437 & 0.425 & 0.429 \\
\hline 701.92 & 0.402 & 0.424 & 0.403 & 0.43 & 0.412 & 0.413 & 0.415 & 0.431 & 0.419 & 0.423 \\
\hline 702.25 & 0.394 & 0.416 & 0.404 & 0.428 & 0.408 & 0.409 & 0.41 & 0.428 & 0.415 & 0.416 \\
\hline 702.57 & 0.39 & 0.409 & 0.401 & 0.425 & 0.403 & 0.405 & 0.406 & 0.426 & 0.411 & 0.413 \\
\hline 702.89 & 0.384 & 0.4 & 0.396 & 0.419 & 0.396 & 0.398 & 0.401 & 0.42 & 0.41 & 0.406 \\
\hline 703.21 & 0.378 & 0.392 & 0.391 & 0.416 & 0.39 & 0.393 & 0.395 & 0.414 & 0.405 & 0.399 \\
\hline 703.54 & 0.371 & 0.386 & 0.384 & 0.411 & 0.384 & 0.386 & 0.388 & 0.407 & 0.4 & 0.394 \\
\hline 703.86 & 0.364 & 0.381 & 0.382 & 0.407 & 0.38 & 0.38 & 0.381 & 0.401 & 0.396 & 0.389 \\
\hline 704.18 & 0.355 & 0.376 & 0.381 & 0.403 & 0.378 & 0.378 & 0.377 & 0.396 & 0.391 & 0.386 \\
\hline 704.5 & 0.35 & 0.367 & 0.375 & 0.399 & 0.375 & 0.373 & 0.374 & 0.391 & 0.388 & 0.384 \\
\hline 704.83 & 0.344 & 0.363 & 0.372 & 0.394 & 0.373 & 0.371 & 0.371 & 0.388 & 0.384 & 0.38 \\
\hline 705.15 & 0.339 & 0.361 & 0.369 & 0.389 & 0.369 & 0.368 & 0.367 & 0.384 & 0.383 & 0.376 \\
\hline 705.47 & 0.33 & 0.355 & 0.362 & 0.386 & 0.364 & 0.364 & 0.362 & 0.379 & 0.38 & 0.376 \\
\hline 705.79 & 0.324 & 0.348 & 0.357 & 0.379 & 0.359 & 0.358 & 0.357 & 0.375 & 0.376 & 0.375 \\
\hline 706.12 & 0.316 & 0.344 & 0.351 & 0.376 & 0.355 & 0.355 & 0.352 & 0.37 & 0.373 & 0.371 \\
\hline 706.44 & 0.309 & 0.339 & 0.348 & 0.371 & 0.354 & 0.349 & 0.349 & 0.365 & 0.37 & 0.365 \\
\hline 706.76 & 0.306 & 0.333 & 0.34 & 0.364 & 0.348 & 0.342 & 0.342 & 0.359 & 0.364 & 0.358 \\
\hline 707.08 & 0.302 & 0.327 & 0.336 & 0.362 & 0.344 & 0.34 & 0.336 & 0.356 & 0.361 & 0.355 \\
\hline 707.4 & 0.296 & 0.322 & 0.332 & 0.357 & 0.34 & 0.339 & 0.332 & 0.353 & 0.356 & 0.349 \\
\hline 707.73 & 0.291 & 0.314 & 0.325 & 0.35 & 0.334 & 0.331 & 0.326 & 0.349 & 0.353 & 0.344 \\
\hline 708.05 & 0.285 & 0.311 & 0.32 & 0.345 & 0.327 & 0.328 & 0.319 & 0.343 & 0.348 & 0.338 \\
\hline 708.37 & 0.28 & 0.307 & 0.314 & 0.341 & 0.324 & 0.324 & 0.315 & 0.338 & 0.346 & 0.334 \\
\hline 708.69 & 0.276 & 0.302 & 0.309 & 0.337 & 0.319 & 0.322 & 0.311 & 0.335 & 0.342 & 0.329 \\
\hline 709.01 & 0.275 & 0.298 & 0.307 & 0.332 & 0.316 & 0.318 & 0.309 & 0.334 & 0.338 & 0.323 \\
\hline 709.33 & 0.272 & 0.296 & 0.303 & 0.33 & 0.312 & 0.316 & 0.306 & 0.332 & 0.333 & 0.319 \\
\hline 709.66 & 0.268 & 0.29 & 0.3 & 0.327 & 0.31 & 0.312 & 0.301 & 0.329 & 0.33 & 0.315 \\
\hline 709.98 & 0.265 & 0.288 & 0.298 & 0.328 & 0.306 & 0.309 & 0.297 & 0.326 & 0.328 & 0.313 \\
\hline 710.3 & 0.259 & 0.284 & 0.295 & 0.323 & 0.304 & 0.308 & 0.294 & 0.324 & 0.326 & 0.311 \\
\hline 710.62 & 0.253 & 0.281 & 0.29 & 0.319 & 0.3 & 0.305 & 0.293 & 0.321 & 0.323 & 0.308 \\
\hline 710.94 & 0.25 & 0.277 & 0.287 & 0.317 & 0.297 & 0.3 & 0.289 & 0.317 & 0.323 & 0.307 \\
\hline
\end{tabular}




\begin{tabular}{|c|c|c|c|c|c|c|c|c|c|c|}
\hline 711.26 & 0.246 & 0.275 & 0.287 & 0.315 & 0.294 & 0.3 & 0.288 & 0.314 & 0.319 & 0.305 \\
\hline 711.58 & 0.245 & 0.269 & 0.283 & 0.313 & 0.291 & 0.299 & 0.285 & 0.313 & 0.316 & 0.303 \\
\hline 711.9 & 0.243 & 0.264 & 0.281 & 0.308 & 0.288 & 0.295 & 0.282 & 0.308 & 0.314 & 0.3 \\
\hline 712.23 & 0.239 & 0.258 & 0.282 & 0.307 & 0.285 & 0.29 & 0.277 & 0.303 & 0.312 & 0.298 \\
\hline 712.55 & 0.234 & 0.253 & 0.279 & 0.304 & 0.282 & 0.288 & 0.273 & 0.298 & 0.308 & 0.295 \\
\hline 712.87 & 0.231 & 0.25 & 0.278 & 0.302 & 0.281 & 0.286 & 0.269 & 0.294 & 0.308 & 0.292 \\
\hline 713.19 & 0.228 & 0.247 & 0.277 & 0.3 & 0.279 & 0.284 & 0.269 & 0.291 & 0.305 & 0.29 \\
\hline 713.51 & 0.225 & 0.243 & 0.275 & 0.296 & 0.276 & 0.282 & 0.266 & 0.288 & 0.303 & 0.289 \\
\hline 713.83 & 0.222 & 0.241 & 0.275 & 0.296 & 0.274 & 0.283 & 0.262 & 0.286 & 0.303 & 0.288 \\
\hline 714.15 & 0.22 & 0.236 & 0.272 & 0.293 & 0.274 & 0.28 & 0.258 & 0.283 & 0.3 & 0.285 \\
\hline 714.47 & 0.217 & 0.234 & 0.272 & 0.291 & 0.27 & 0.278 & 0.255 & 0.282 & 0.296 & 0.282 \\
\hline 714.79 & 0.213 & 0.23 & 0.268 & 0.288 & 0.268 & 0.276 & 0.25 & 0.279 & 0.293 & 0.278 \\
\hline 715.11 & 0.21 & 0.228 & 0.267 & 0.286 & 0.266 & 0.27 & 0.248 & 0.274 & 0.291 & 0.275 \\
\hline 715.43 & 0.206 & 0.226 & 0.265 & 0.285 & 0.265 & 0.268 & 0.245 & 0.272 & 0.287 & 0.274 \\
\hline 715.75 & 0.203 & 0.225 & 0.263 & 0.28 & 0.263 & 0.267 & 0.242 & 0.271 & 0.284 & 0.272 \\
\hline 716.07 & 0.199 & 0.224 & 0.261 & 0.278 & 0.261 & 0.264 & 0.239 & 0.269 & 0.282 & 0.27 \\
\hline 716.39 & 0.198 & 0.221 & 0.257 & 0.277 & 0.258 & 0.263 & 0.236 & 0.265 & 0.279 & 0.268 \\
\hline 716.72 & 0.194 & 0.218 & 0.254 & 0.273 & 0.255 & 0.259 & 0.232 & 0.263 & 0.275 & 0.264 \\
\hline 717.04 & 0.192 & 0.215 & 0.251 & 0.271 & 0.253 & 0.255 & 0.229 & 0.262 & 0.272 & 0.262 \\
\hline 717.36 & 0.19 & 0.213 & 0.246 & 0.268 & 0.251 & 0.251 & 0.228 & 0.26 & 0.267 & 0.26 \\
\hline 717.68 & 0.188 & 0.211 & 0.245 & 0.266 & 0.248 & 0.247 & 0.227 & 0.257 & 0.266 & 0.259 \\
\hline 718 & 0.184 & 0.209 & 0.242 & 0.263 & 0.247 & 0.245 & 0.226 & 0.254 & 0.266 & 0.258 \\
\hline 718.32 & 0.184 & 0.208 & 0.241 & 0.261 & 0.245 & 0.242 & 0.225 & 0.252 & 0.264 & 0.257 \\
\hline 718.64 & 0.183 & 0.206 & 0.239 & 0.259 & 0.242 & 0.242 & 0.223 & 0.252 & 0.262 & 0.254 \\
\hline 718.96 & 0.182 & 0.203 & 0.237 & 0.258 & 0.236 & 0.24 & 0.219 & 0.249 & 0.26 & 0.251 \\
\hline 719.28 & 0.182 & 0.199 & 0.234 & 0.256 & 0.234 & 0.237 & 0.22 & 0.247 & 0.259 & 0.251 \\
\hline 719.6 & 0.179 & 0.196 & 0.231 & 0.254 & 0.235 & 0.235 & 0.219 & 0.246 & 0.259 & 0.251 \\
\hline 719.92 & 0.176 & 0.194 & 0.228 & 0.253 & 0.23 & 0.232 & 0.218 & 0.246 & 0.258 & 0.248 \\
\hline 720.24 & 0.172 & 0.192 & 0.228 & 0.251 & 0.229 & 0.229 & 0.216 & 0.244 & 0.258 & 0.248 \\
\hline 720.56 & 0.169 & 0.191 & 0.227 & 0.248 & 0.225 & 0.23 & 0.214 & 0.242 & 0.255 & 0.244 \\
\hline 720.88 & 0.167 & 0.185 & 0.226 & 0.247 & 0.224 & 0.231 & 0.214 & 0.239 & 0.257 & 0.245 \\
\hline 721.19 & 0.162 & 0.184 & 0.226 & 0.247 & 0.223 & 0.232 & 0.212 & 0.239 & 0.257 & 0.243 \\
\hline 721.51 & 0.16 & 0.18 & 0.224 & 0.245 & 0.221 & 0.229 & 0.21 & 0.237 & 0.255 & 0.238 \\
\hline 721.83 & 0.157 & 0.177 & 0.22 & 0.242 & 0.22 & 0.229 & 0.205 & 0.235 & 0.253 & 0.235 \\
\hline 722.15 & 0.153 & 0.176 & 0.22 & 0.239 & 0.221 & 0.228 & 0.201 & 0.232 & 0.251 & 0.234 \\
\hline 722.47 & 0.148 & 0.175 & 0.219 & 0.238 & 0.223 & 0.23 & 0.2 & 0.232 & 0.251 & 0.234 \\
\hline 722.79 & 0.144 & 0.173 & 0.217 & 0.237 & 0.222 & 0.23 & 0.194 & 0.231 & 0.251 & 0.229 \\
\hline 723.11 & 0.143 & 0.17 & 0.218 & 0.236 & 0.218 & 0.23 & 0.192 & 0.232 & 0.247 & 0.226 \\
\hline 723.43 & 0.14 & 0.164 & 0.219 & 0.234 & 0.219 & 0.227 & 0.187 & 0.229 & 0.245 & 0.225 \\
\hline
\end{tabular}




\begin{tabular}{|c|c|c|c|c|c|c|c|c|c|c|}
\hline 723.75 & 0.139 & 0.161 & 0.216 & 0.232 & 0.218 & 0.229 & 0.183 & 0.226 & 0.242 & 0.223 \\
\hline 724.07 & 0.139 & 0.159 & 0.215 & 0.228 & 0.219 & 0.227 & 0.179 & 0.224 & 0.241 & 0.223 \\
\hline 724.39 & 0.136 & 0.158 & 0.213 & 0.226 & 0.215 & 0.222 & 0.177 & 0.224 & 0.235 & 0.22 \\
\hline 724.71 & 0.136 & 0.154 & 0.212 & 0.223 & 0.214 & 0.218 & 0.173 & 0.222 & 0.232 & 0.218 \\
\hline 725.03 & 0.136 & 0.152 & 0.212 & 0.225 & 0.213 & 0.217 & 0.171 & 0.222 & 0.23 & 0.217 \\
\hline 725.35 & 0.134 & 0.15 & 0.213 & 0.225 & 0.213 & 0.216 & 0.171 & 0.221 & 0.229 & 0.217 \\
\hline 725.66 & 0.133 & 0.146 & 0.213 & 0.224 & 0.213 & 0.214 & 0.171 & 0.219 & 0.23 & 0.216 \\
\hline 725.98 & 0.132 & 0.143 & 0.214 & 0.223 & 0.214 & 0.212 & 0.17 & 0.219 & 0.229 & 0.215 \\
\hline 726.3 & 0.13 & 0.143 & 0.214 & 0.222 & 0.212 & 0.209 & 0.169 & 0.216 & 0.228 & 0.217 \\
\hline 726.62 & 0.13 & 0.143 & 0.211 & 0.219 & 0.213 & 0.206 & 0.167 & 0.214 & 0.226 & 0.217 \\
\hline 726.94 & 0.128 & 0.143 & 0.209 & 0.217 & 0.213 & 0.208 & 0.169 & 0.214 & 0.225 & 0.216 \\
\hline 727.26 & 0.127 & 0.142 & 0.21 & 0.216 & 0.212 & 0.205 & 0.17 & 0.213 & 0.224 & 0.215 \\
\hline 727.58 & 0.124 & 0.141 & 0.208 & 0.213 & 0.21 & 0.203 & 0.17 & 0.209 & 0.225 & 0.212 \\
\hline 727.89 & 0.124 & 0.141 & 0.208 & 0.213 & 0.21 & 0.203 & 0.17 & 0.206 & 0.223 & 0.212 \\
\hline 728.21 & 0.123 & 0.141 & 0.208 & 0.209 & 0.208 & 0.204 & 0.17 & 0.206 & 0.223 & 0.212 \\
\hline 728.53 & 0.12 & 0.143 & 0.205 & 0.207 & 0.207 & 0.202 & 0.168 & 0.204 & 0.222 & 0.212 \\
\hline 728.85 & 0.117 & 0.143 & 0.202 & 0.204 & 0.204 & 0.2 & 0.17 & 0.206 & 0.221 & 0.214 \\
\hline 729.17 & 0.118 & 0.142 & 0.198 & 0.203 & 0.2 & 0.197 & 0.169 & 0.206 & 0.217 & 0.213 \\
\hline 729.49 & 0.118 & 0.142 & 0.197 & 0.202 & 0.199 & 0.196 & 0.169 & 0.204 & 0.215 & 0.212 \\
\hline 729.8 & 0.115 & 0.141 & 0.195 & 0.201 & 0.197 & 0.195 & 0.168 & 0.202 & 0.215 & 0.21 \\
\hline 730.12 & 0.114 & 0.14 & 0.194 & 0.201 & 0.197 & 0.196 & 0.167 & 0.203 & 0.215 & 0.21 \\
\hline 730.44 & 0.115 & 0.137 & 0.191 & 0.201 & 0.197 & 0.195 & 0.165 & 0.203 & 0.215 & 0.208 \\
\hline 730.76 & 0.115 & 0.136 & 0.188 & 0.201 & 0.194 & 0.194 & 0.164 & 0.205 & 0.215 & 0.208 \\
\hline 731.08 & 0.115 & 0.136 & 0.188 & 0.203 & 0.193 & 0.194 & 0.162 & 0.206 & 0.214 & 0.204 \\
\hline 731.39 & 0.113 & 0.135 & 0.186 & 0.204 & 0.191 & 0.193 & 0.161 & 0.205 & 0.216 & 0.202 \\
\hline 731.71 & 0.111 & 0.135 & 0.184 & 0.205 & 0.189 & 0.192 & 0.162 & 0.204 & 0.215 & 0.201 \\
\hline 732.03 & 0.111 & 0.133 & 0.183 & 0.205 & 0.187 & 0.191 & 0.162 & 0.204 & 0.216 & 0.201 \\
\hline 732.35 & 0.111 & 0.132 & 0.183 & 0.203 & 0.187 & 0.194 & 0.159 & 0.2 & 0.216 & 0.198 \\
\hline 732.67 & 0.11 & 0.133 & 0.185 & 0.202 & 0.187 & 0.195 & 0.157 & 0.199 & 0.216 & 0.197 \\
\hline 732.98 & 0.11 & 0.134 & 0.182 & 0.202 & 0.186 & 0.192 & 0.155 & 0.197 & 0.217 & 0.195 \\
\hline 733.3 & 0.109 & 0.132 & 0.182 & 0.201 & 0.186 & 0.192 & 0.155 & 0.196 & 0.215 & 0.193 \\
\hline 733.62 & 0.108 & 0.132 & 0.181 & 0.2 & 0.186 & 0.191 & 0.154 & 0.194 & 0.214 & 0.192 \\
\hline 733.94 & 0.107 & 0.133 & 0.182 & 0.198 & 0.184 & 0.191 & 0.152 & 0.19 & 0.213 & 0.192 \\
\hline 734.25 & 0.106 & 0.131 & 0.182 & 0.195 & 0.186 & 0.193 & 0.151 & 0.187 & 0.212 & 0.191 \\
\hline 734.57 & 0.104 & 0.13 & 0.181 & 0.193 & 0.187 & 0.192 & 0.15 & 0.187 & 0.21 & 0.193 \\
\hline 734.89 & 0.102 & 0.129 & 0.183 & 0.191 & 0.189 & 0.19 & 0.148 & 0.187 & 0.208 & 0.192 \\
\hline 735.21 & 0.103 & 0.128 & 0.184 & 0.19 & 0.188 & 0.188 & 0.147 & 0.186 & 0.207 & 0.188 \\
\hline 735.52 & 0.102 & 0.125 & 0.182 & 0.188 & 0.187 & 0.188 & 0.142 & 0.183 & 0.204 & 0.186 \\
\hline 735.84 & 0.101 & 0.125 & 0.182 & 0.188 & 0.185 & 0.184 & 0.142 & 0.185 & 0.202 & 0.185 \\
\hline
\end{tabular}




\begin{tabular}{|c|c|c|c|c|c|c|c|c|c|c|}
\hline 736.16 & 0.101 & 0.122 & 0.179 & 0.189 & 0.184 & 0.184 & 0.143 & 0.183 & 0.201 & 0.183 \\
\hline 736.47 & 0.098 & 0.119 & 0.179 & 0.187 & 0.183 & 0.187 & 0.141 & 0.183 & 0.199 & 0.183 \\
\hline 736.79 & 0.097 & 0.118 & 0.178 & 0.184 & 0.184 & 0.186 & 0.141 & 0.184 & 0.199 & 0.184 \\
\hline 737.11 & 0.094 & 0.117 & 0.175 & 0.182 & 0.183 & 0.184 & 0.14 & 0.182 & 0.199 & 0.181 \\
\hline 737.43 & 0.092 & 0.117 & 0.177 & 0.181 & 0.182 & 0.182 & 0.142 & 0.185 & 0.198 & 0.179 \\
\hline 737.74 & 0.09 & 0.116 & 0.179 & 0.183 & 0.181 & 0.178 & 0.141 & 0.185 & 0.195 & 0.179 \\
\hline 738.06 & 0.089 & 0.116 & 0.179 & 0.182 & 0.179 & 0.18 & 0.142 & 0.184 & 0.194 & 0.178 \\
\hline 738.38 & 0.089 & 0.115 & 0.178 & 0.184 & 0.177 & 0.18 & 0.142 & 0.183 & 0.196 & 0.178 \\
\hline 738.69 & 0.089 & 0.114 & 0.175 & 0.183 & 0.177 & 0.18 & 0.139 & 0.18 & 0.193 & 0.178 \\
\hline 739.01 & 0.085 & 0.113 & 0.176 & 0.185 & 0.176 & 0.177 & 0.141 & 0.179 & 0.193 & 0.176 \\
\hline 739.33 & 0.083 & 0.11 & 0.176 & 0.184 & 0.179 & 0.181 & 0.141 & 0.175 & 0.19 & 0.174 \\
\hline 739.64 & 0.081 & 0.109 & 0.176 & 0.178 & 0.177 & 0.18 & 0.139 & 0.173 & 0.188 & 0.173 \\
\hline 739.96 & 0.082 & 0.109 & 0.178 & 0.179 & 0.178 & 0.177 & 0.138 & 0.172 & 0.189 & 0.174 \\
\hline 740.28 & 0.081 & 0.108 & 0.176 & 0.182 & 0.175 & 0.177 & 0.138 & 0.169 & 0.186 & 0.171 \\
\hline 740.59 & 0.081 & 0.107 & 0.178 & 0.183 & 0.174 & 0.178 & 0.137 & 0.169 & 0.186 & 0.169 \\
\hline 740.91 & 0.078 & 0.107 & 0.176 & 0.184 & 0.172 & 0.178 & 0.135 & 0.169 & 0.183 & 0.169 \\
\hline 741.22 & 0.076 & 0.109 & 0.173 & 0.183 & 0.168 & 0.179 & 0.135 & 0.168 & 0.186 & 0.169 \\
\hline 741.54 & 0.076 & 0.109 & 0.173 & 0.182 & 0.164 & 0.173 & 0.133 & 0.166 & 0.184 & 0.167 \\
\hline 741.86 & 0.076 & 0.108 & 0.172 & 0.18 & 0.165 & 0.175 & 0.133 & 0.168 & 0.183 & 0.169 \\
\hline 742.17 & 0.076 & 0.106 & 0.173 & 0.176 & 0.166 & 0.178 & 0.133 & 0.169 & 0.184 & 0.169 \\
\hline 742.49 & 0.077 & 0.107 & 0.173 & 0.177 & 0.168 & 0.178 & 0.134 & 0.173 & 0.186 & 0.171 \\
\hline 742.81 & 0.076 & 0.107 & 0.171 & 0.176 & 0.167 & 0.174 & 0.133 & 0.171 & 0.186 & 0.171 \\
\hline 743.12 & 0.076 & 0.107 & 0.171 & 0.178 & 0.169 & 0.175 & 0.134 & 0.173 & 0.187 & 0.171 \\
\hline 743.44 & 0.075 & 0.107 & 0.171 & 0.177 & 0.167 & 0.176 & 0.135 & 0.173 & 0.185 & 0.168 \\
\hline 743.75 & 0.075 & 0.104 & 0.172 & 0.174 & 0.167 & 0.178 & 0.133 & 0.171 & 0.185 & 0.168 \\
\hline 744.07 & 0.075 & 0.102 & 0.173 & 0.172 & 0.168 & 0.18 & 0.132 & 0.17 & 0.183 & 0.17 \\
\hline 744.38 & 0.078 & 0.102 & 0.169 & 0.171 & 0.168 & 0.18 & 0.136 & 0.169 & 0.185 & 0.169 \\
\hline 744.7 & 0.08 & 0.098 & 0.169 & 0.173 & 0.169 & 0.182 & 0.135 & 0.169 & 0.183 & 0.168 \\
\hline 745.02 & 0.08 & 0.097 & 0.171 & 0.174 & 0.173 & 0.183 & 0.132 & 0.168 & 0.185 & 0.17 \\
\hline 745.33 & 0.079 & 0.094 & 0.169 & 0.172 & 0.172 & 0.182 & 0.13 & 0.166 & 0.181 & 0.168 \\
\hline 745.65 & 0.075 & 0.095 & 0.168 & 0.173 & 0.174 & 0.176 & 0.131 & 0.167 & 0.18 & 0.167 \\
\hline 745.96 & 0.075 & 0.094 & 0.167 & 0.171 & 0.171 & 0.176 & 0.13 & 0.163 & 0.178 & 0.167 \\
\hline 746.28 & 0.076 & 0.094 & 0.168 & 0.174 & 0.17 & 0.174 & 0.127 & 0.164 & 0.179 & 0.167 \\
\hline 746.59 & 0.076 & 0.093 & 0.167 & 0.173 & 0.167 & 0.17 & 0.125 & 0.164 & 0.179 & 0.17 \\
\hline 746.91 & 0.075 & 0.092 & 0.163 & 0.169 & 0.166 & 0.169 & 0.121 & 0.166 & 0.178 & 0.169 \\
\hline 747.22 & 0.075 & 0.096 & 0.163 & 0.17 & 0.167 & 0.166 & 0.121 & 0.169 & 0.178 & 0.168 \\
\hline 747.54 & 0.075 & 0.096 & 0.159 & 0.171 & 0.164 & 0.159 & 0.12 & 0.171 & 0.176 & 0.167 \\
\hline 747.85 & 0.075 & 0.095 & 0.162 & 0.173 & 0.165 & 0.157 & 0.117 & 0.169 & 0.176 & 0.168 \\
\hline 748.17 & 0.074 & 0.097 & 0.159 & 0.173 & 0.167 & 0.154 & 0.114 & 0.166 & 0.174 & 0.166 \\
\hline
\end{tabular}




\begin{tabular}{|c|c|c|c|c|c|c|c|c|c|c|}
\hline 748.48 & 0.072 & 0.098 & 0.16 & 0.17 & 0.164 & 0.157 & 0.116 & 0.166 & 0.171 & 0.165 \\
\hline 748.8 & 0.072 & 0.101 & 0.157 & 0.171 & 0.163 & 0.153 & 0.117 & 0.167 & 0.171 & 0.164 \\
\hline 749.11 & 0.073 & 0.097 & 0.159 & 0.173 & 0.161 & 0.157 & 0.113 & 0.163 & 0.17 & 0.165 \\
\hline 749.43 & 0.074 & 0.095 & 0.157 & 0.172 & 0.164 & 0.154 & 0.112 & 0.164 & 0.17 & 0.163 \\
\hline 749.74 & 0.073 & 0.095 & 0.154 & 0.171 & 0.161 & 0.156 & 0.116 & 0.162 & 0.169 & 0.161 \\
\hline 750.06 & 0.073 & 0.093 & 0.155 & 0.173 & 0.162 & 0.16 & 0.117 & 0.159 & 0.165 & 0.158 \\
\hline 750.37 & 0.074 & 0.094 & 0.155 & 0.174 & 0.162 & 0.157 & 0.119 & 0.155 & 0.163 & 0.158 \\
\hline 750.69 & 0.073 & 0.089 & 0.155 & 0.17 & 0.158 & 0.156 & 0.118 & 0.152 & 0.162 & 0.157 \\
\hline 751 & 0.072 & 0.089 & 0.156 & 0.168 & 0.157 & 0.158 & 0.118 & 0.149 & 0.164 & 0.157 \\
\hline 751.32 & 0.07 & 0.086 & 0.155 & 0.168 & 0.154 & 0.159 & 0.116 & 0.147 & 0.164 & 0.156 \\
\hline 751.63 & 0.069 & 0.084 & 0.156 & 0.166 & 0.152 & 0.159 & 0.119 & 0.15 & 0.166 & 0.155 \\
\hline 751.95 & 0.07 & 0.081 & 0.154 & 0.169 & 0.153 & 0.159 & 0.117 & 0.149 & 0.168 & 0.154 \\
\hline 752.26 & 0.069 & 0.078 & 0.155 & 0.167 & 0.151 & 0.162 & 0.116 & 0.145 & 0.169 & 0.153 \\
\hline 752.58 & 0.071 & 0.08 & 0.154 & 0.166 & 0.149 & 0.162 & 0.118 & 0.147 & 0.17 & 0.154 \\
\hline 752.89 & 0.069 & 0.081 & 0.152 & 0.166 & 0.15 & 0.166 & 0.117 & 0.145 & 0.17 & 0.153 \\
\hline 753.2 & 0.068 & 0.079 & 0.156 & 0.166 & 0.15 & 0.163 & 0.115 & 0.146 & 0.169 & 0.155 \\
\hline 753.52 & 0.067 & 0.079 & 0.153 & 0.167 & 0.151 & 0.158 & 0.114 & 0.146 & 0.172 & 0.156 \\
\hline 753.83 & 0.066 & 0.077 & 0.152 & 0.167 & 0.151 & 0.162 & 0.116 & 0.148 & 0.174 & 0.158 \\
\hline 754.15 & 0.063 & 0.079 & 0.147 & 0.168 & 0.154 & 0.163 & 0.119 & 0.149 & 0.175 & 0.16 \\
\hline 754.46 & 0.064 & 0.079 & 0.145 & 0.168 & 0.155 & 0.162 & 0.119 & 0.149 & 0.173 & 0.159 \\
\hline 754.77 & 0.063 & 0.082 & 0.146 & 0.163 & 0.158 & 0.163 & 0.12 & 0.15 & 0.173 & 0.158 \\
\hline 755.09 & 0.061 & 0.082 & 0.144 & 0.164 & 0.16 & 0.161 & 0.12 & 0.148 & 0.173 & 0.155 \\
\hline 755.4 & 0.059 & 0.083 & 0.141 & 0.159 & 0.161 & 0.16 & 0.122 & 0.151 & 0.168 & 0.154 \\
\hline 755.72 & 0.056 & 0.082 & 0.141 & 0.159 & 0.163 & 0.156 & 0.119 & 0.151 & 0.165 & 0.152 \\
\hline 756.03 & 0.051 & 0.079 & 0.141 & 0.158 & 0.164 & 0.156 & 0.117 & 0.15 & 0.166 & 0.149 \\
\hline 756.34 & 0.049 & 0.08 & 0.144 & 0.156 & 0.163 & 0.152 & 0.12 & 0.153 & 0.165 & 0.148 \\
\hline 756.66 & 0.049 & 0.082 & 0.142 & 0.156 & 0.164 & 0.155 & 0.122 & 0.153 & 0.165 & 0.147 \\
\hline 756.97 & 0.047 & 0.082 & 0.145 & 0.153 & 0.163 & 0.155 & 0.124 & 0.153 & 0.166 & 0.145 \\
\hline 757.28 & 0.048 & 0.084 & 0.144 & 0.154 & 0.166 & 0.154 & 0.122 & 0.152 & 0.163 & 0.143 \\
\hline 757.6 & 0.052 & 0.083 & 0.148 & 0.152 & 0.162 & 0.152 & 0.118 & 0.153 & 0.161 & 0.14 \\
\hline 757.91 & 0.05 & 0.08 & 0.15 & 0.154 & 0.162 & 0.154 & 0.115 & 0.152 & 0.161 & 0.142 \\
\hline 758.22 & 0.053 & 0.077 & 0.148 & 0.158 & 0.161 & 0.153 & 0.117 & 0.152 & 0.16 & 0.141 \\
\hline 758.54 & 0.055 & 0.076 & 0.149 & 0.158 & 0.161 & 0.154 & 0.115 & 0.155 & 0.159 & 0.145 \\
\hline 758.85 & 0.055 & 0.074 & 0.149 & 0.158 & 0.16 & 0.153 & 0.114 & 0.153 & 0.16 & 0.145 \\
\hline 759.16 & 0.058 & 0.074 & 0.149 & 0.156 & 0.16 & 0.153 & 0.115 & 0.153 & 0.16 & 0.149 \\
\hline 759.48 & 0.062 & 0.076 & 0.151 & 0.157 & 0.157 & 0.151 & 0.116 & 0.152 & 0.157 & 0.147 \\
\hline 759.79 & 0.064 & 0.075 & 0.15 & 0.156 & 0.157 & 0.15 & 0.115 & 0.149 & 0.156 & 0.148 \\
\hline 760.1 & 0.063 & 0.074 & 0.152 & 0.156 & 0.157 & 0.149 & 0.11 & 0.148 & 0.155 & 0.149 \\
\hline 760.42 & 0.065 & 0.074 & 0.148 & 0.156 & 0.157 & 0.149 & 0.112 & 0.149 & 0.153 & 0.151 \\
\hline
\end{tabular}




\begin{tabular}{|c|c|c|c|c|c|c|c|c|c|c|}
\hline 760.73 & 0.063 & 0.074 & 0.151 & 0.154 & 0.152 & 0.149 & 0.111 & 0.147 & 0.152 & 0.15 \\
\hline 761.04 & 0.06 & 0.073 & 0.153 & 0.159 & 0.154 & 0.151 & 0.111 & 0.144 & 0.154 & 0.15 \\
\hline 761.35 & 0.061 & 0.073 & 0.153 & 0.156 & 0.156 & 0.149 & 0.113 & 0.142 & 0.154 & 0.148 \\
\hline 761.67 & 0.06 & 0.074 & 0.153 & 0.154 & 0.156 & 0.149 & 0.11 & 0.142 & 0.153 & 0.152 \\
\hline 761.98 & 0.059 & 0.074 & 0.154 & 0.155 & 0.154 & 0.151 & 0.111 & 0.14 & 0.153 & 0.155 \\
\hline 762.29 & 0.058 & 0.07 & 0.155 & 0.155 & 0.154 & 0.15 & 0.111 & 0.14 & 0.154 & 0.155 \\
\hline 762.61 & 0.058 & 0.072 & 0.155 & 0.157 & 0.153 & 0.15 & 0.112 & 0.142 & 0.154 & 0.153 \\
\hline 762.92 & 0.059 & 0.071 & 0.154 & 0.157 & 0.155 & 0.15 & 0.113 & 0.144 & 0.152 & 0.154 \\
\hline 763.23 & 0.057 & 0.071 & 0.153 & 0.16 & 0.153 & 0.153 & 0.112 & 0.147 & 0.153 & 0.156 \\
\hline 763.54 & 0.057 & 0.07 & 0.152 & 0.162 & 0.154 & 0.153 & 0.113 & 0.147 & 0.151 & 0.157 \\
\hline 763.86 & 0.056 & 0.07 & 0.151 & 0.161 & 0.155 & 0.153 & 0.11 & 0.149 & 0.152 & 0.156 \\
\hline 764.17 & 0.056 & 0.07 & 0.15 & 0.162 & 0.157 & 0.153 & 0.113 & 0.152 & 0.154 & 0.157 \\
\hline 764.48 & 0.058 & 0.07 & 0.15 & 0.162 & 0.156 & 0.152 & 0.115 & 0.154 & 0.155 & 0.159 \\
\hline 764.79 & 0.057 & 0.071 & 0.15 & 0.163 & 0.156 & 0.153 & 0.116 & 0.157 & 0.156 & 0.16 \\
\hline 765.1 & 0.057 & 0.069 & 0.15 & 0.165 & 0.156 & 0.154 & 0.117 & 0.159 & 0.155 & 0.158 \\
\hline 765.42 & 0.058 & 0.07 & 0.152 & 0.162 & 0.155 & 0.155 & 0.117 & 0.162 & 0.155 & 0.157 \\
\hline 765.73 & 0.059 & 0.071 & 0.152 & 0.162 & 0.154 & 0.157 & 0.115 & 0.162 & 0.155 & 0.156 \\
\hline 766.04 & 0.058 & 0.068 & 0.148 & 0.163 & 0.154 & 0.159 & 0.116 & 0.163 & 0.155 & 0.156 \\
\hline 766.35 & 0.056 & 0.066 & 0.149 & 0.161 & 0.152 & 0.159 & 0.116 & 0.161 & 0.158 & 0.156 \\
\hline 766.66 & 0.056 & 0.066 & 0.148 & 0.16 & 0.153 & 0.156 & 0.115 & 0.159 & 0.157 & 0.155 \\
\hline 766.98 & 0.056 & 0.068 & 0.147 & 0.158 & 0.153 & 0.154 & 0.114 & 0.16 & 0.158 & 0.153 \\
\hline 767.29 & 0.057 & 0.064 & 0.148 & 0.158 & 0.152 & 0.155 & 0.118 & 0.159 & 0.157 & 0.152 \\
\hline 767.6 & 0.054 & 0.064 & 0.147 & 0.161 & 0.151 & 0.153 & 0.118 & 0.157 & 0.157 & 0.152 \\
\hline 767.91 & 0.051 & 0.062 & 0.146 & 0.155 & 0.152 & 0.152 & 0.117 & 0.157 & 0.154 & 0.149 \\
\hline 768.22 & 0.052 & 0.061 & 0.146 & 0.153 & 0.151 & 0.15 & 0.115 & 0.153 & 0.15 & 0.148 \\
\hline 768.53 & 0.052 & 0.064 & 0.144 & 0.151 & 0.149 & 0.147 & 0.115 & 0.153 & 0.15 & 0.149 \\
\hline 768.85 & 0.05 & 0.061 & 0.141 & 0.152 & 0.151 & 0.144 & 0.116 & 0.152 & 0.149 & 0.147 \\
\hline 769.16 & 0.049 & 0.063 & 0.141 & 0.154 & 0.152 & 0.143 & 0.118 & 0.15 & 0.149 & 0.149 \\
\hline 769.47 & 0.05 & 0.064 & 0.144 & 0.153 & 0.153 & 0.142 & 0.118 & 0.149 & 0.146 & 0.149 \\
\hline 769.78 & 0.049 & 0.066 & 0.143 & 0.156 & 0.155 & 0.143 & 0.118 & 0.152 & 0.145 & 0.15 \\
\hline 770.09 & 0.048 & 0.065 & 0.145 & 0.155 & 0.153 & 0.145 & 0.12 & 0.152 & 0.144 & 0.151 \\
\hline 770.4 & 0.047 & 0.063 & 0.143 & 0.154 & 0.151 & 0.146 & 0.122 & 0.153 & 0.146 & 0.152 \\
\hline 770.71 & 0.047 & 0.064 & 0.144 & 0.152 & 0.151 & 0.145 & 0.121 & 0.152 & 0.144 & 0.151 \\
\hline 771.03 & 0.048 & 0.06 & 0.145 & 0.147 & 0.149 & 0.15 & 0.114 & 0.152 & 0.141 & 0.151 \\
\hline 771.34 & 0.051 & 0.061 & 0.145 & 0.152 & 0.145 & 0.153 & 0.113 & 0.152 & 0.142 & 0.149 \\
\hline 771.65 & 0.05 & 0.061 & 0.143 & 0.152 & 0.144 & 0.156 & 0.113 & 0.156 & 0.14 & 0.149 \\
\hline 771.96 & 0.05 & 0.055 & 0.145 & 0.154 & 0.143 & 0.155 & 0.112 & 0.153 & 0.14 & 0.146 \\
\hline 772.27 & 0.051 & 0.057 & 0.141 & 0.153 & 0.144 & 0.154 & 0.111 & 0.15 & 0.139 & 0.144 \\
\hline 772.58 & 0.048 & 0.054 & 0.141 & 0.152 & 0.143 & 0.152 & 0.111 & 0.151 & 0.139 & 0.142 \\
\hline
\end{tabular}




\begin{tabular}{|r|c|c|c|c|c|c|c|c|c|c|}
\hline 772.89 & 0.047 & 0.056 & 0.143 & 0.153 & 0.146 & 0.154 & 0.112 & 0.15 & 0.142 & 0.141 \\
\hline 773.2 & 0.048 & 0.054 & 0.141 & 0.147 & 0.143 & 0.152 & 0.112 & 0.146 & 0.142 & 0.144 \\
\hline 773.51 & 0.046 & 0.056 & 0.14 & 0.146 & 0.141 & 0.151 & 0.11 & 0.145 & 0.14 & 0.146 \\
\hline 773.82 & 0.048 & 0.055 & 0.142 & 0.15 & 0.142 & 0.155 & 0.108 & 0.145 & 0.139 & 0.144 \\
\hline 774.13 & 0.047 & 0.057 & 0.148 & 0.155 & 0.144 & 0.156 & 0.107 & 0.146 & 0.144 & 0.146 \\
\hline 774.44 & 0.045 & 0.055 & 0.149 & 0.158 & 0.146 & 0.155 & 0.11 & 0.148 & 0.148 & 0.147 \\
\hline 774.75 & 0.046 & 0.056 & 0.146 & 0.158 & 0.148 & 0.151 & 0.113 & 0.149 & 0.149 & 0.147 \\
\hline 775.07 & 0.048 & 0.056 & 0.145 & 0.159 & 0.149 & 0.153 & 0.111 & 0.149 & 0.152 & 0.144 \\
\hline 775.38 & 0.047 & 0.062 & 0.144 & 0.154 & 0.15 & 0.156 & 0.113 & 0.151 & 0.157 & 0.143 \\
\hline 775.69 & 0.045 & 0.064 & 0.149 & 0.157 & 0.147 & 0.16 & 0.112 & 0.153 & 0.161 & 0.146 \\
\hline 776 & 0.047 & 0.064 & 0.146 & 0.156 & 0.147 & 0.161 & 0.111 & 0.15 & 0.163 & 0.142 \\
\hline 776.31 & 0.046 & 0.065 & 0.139 & 0.154 & 0.14 & 0.159 & 0.11 & 0.147 & 0.161 & 0.141 \\
\hline 776.62 & 0.047 & 0.067 & 0.144 & 0.157 & 0.141 & 0.163 & 0.111 & 0.148 & 0.158 & 0.137 \\
\hline 776.93 & 0.047 & 0.063 & 0.142 & 0.157 & 0.14 & 0.163 & 0.114 & 0.147 & 0.159 & 0.133 \\
\hline 777.24 & 0.046 & 0.064 & 0.136 & 0.155 & 0.138 & 0.157 & 0.117 & 0.144 & 0.155 & 0.131 \\
\hline 777.55 & 0.046 & 0.064 & 0.132 & 0.153 & 0.138 & 0.154 & 0.111 & 0.142 & 0.149 & 0.131 \\
\hline 777.86 & 0.044 & 0.069 & 0.127 & 0.155 & 0.141 & 0.15 & 0.112 & 0.143 & 0.147 & 0.132 \\
\hline 778.17 & 0.042 & 0.067 & 0.125 & 0.152 & 0.14 & 0.156 & 0.111 & 0.136 & 0.148 & 0.133 \\
\hline 778.48 & 0.039 & 0.067 & 0.125 & 0.151 & 0.14 & 0.151 & 0.117 & 0.134 & 0.146 & 0.136 \\
\hline 778.79 & 0.034 & 0.062 & 0.123 & 0.153 & 0.139 & 0.149 & 0.115 & 0.127 & 0.141 & 0.137 \\
\hline 779.1 & 0.038 & 0.058 & 0.124 & 0.147 & 0.143 & 0.151 & 0.115 & 0.129 & 0.138 & 0.139 \\
\hline 779.41 & 0.04 & 0.061 & 0.125 & 0.146 & 0.146 & 0.148 & 0.114 & 0.132 & 0.133 & 0.145 \\
\hline 779.71 & 0.04 & 0.059 & 0.129 & 0.147 & 0.152 & 0.153 & 0.114 & 0.135 & 0.133 & 0.148 \\
\hline 780.02 & 0.042 & 0.057 & 0.129 & 0.145 & 0.15 & 0.152 & 0.113 & 0.129 & 0.134 & 0.149 \\
\hline
\end{tabular}

Absorbance values of Kelly Green A_F from 30-39 minutes for Part II of Phase III

\begin{tabular}{|c|c|c|c|c|c|c|c|}
\hline $\boldsymbol{\lambda}$ & $\mathbf{1}$ & $\mathbf{2}$ & $\mathbf{3}$ & $\mathbf{4}$ & $\mathbf{5}$ & $\mathbf{6}$ & $\mathbf{7}$ \\
\hline 380 & 0.215 & 0.254 & 0.278 & 0.227 & 0.279 & 0.257 & 0.237 \\
\hline 390 & 0.21 & 0.254 & 0.296 & 0.261 & 0.269 & 0.286 & 0.256 \\
\hline 400 & 0.23 & 0.275 & 0.315 & 0.258 & 0.289 & 0.302 & 0.277 \\
\hline 410 & 0.259 & 0.288 & 0.332 & 0.282 & 0.297 & 0.322 & 0.283 \\
\hline 420 & 0.269 & 0.301 & 0.345 & 0.307 & 0.324 & 0.341 & 0.3 \\
\hline 430 & 0.278 & 0.314 & 0.342 & 0.317 & 0.323 & 0.338 & 0.307 \\
\hline 440 & 0.283 & 0.311 & 0.339 & 0.323 & 0.327 & 0.346 & 0.303 \\
\hline 450 & 0.295 & 0.313 & 0.355 & 0.328 & 0.334 & 0.352 & 0.314 \\
\hline 460 & 0.322 & 0.346 & 0.376 & 0.35 & 0.346 & 0.38 & 0.333 \\
\hline 470 & 0.355 & 0.377 & 0.405 & 0.376 & 0.371 & 0.406 & 0.362 \\
\hline 480 & 0.387 & 0.409 & 0.432 & 0.404 & 0.391 & 0.431 & 0.389 \\
\hline
\end{tabular}




\begin{tabular}{|c|c|c|c|c|c|c|c|}
\hline 490 & 0.405 & 0.432 & 0.452 & 0.423 & 0.403 & 0.457 & 0.405 \\
\hline 500 & 0.419 & 0.435 & 0.458 & 0.434 & 0.405 & 0.466 & 0.407 \\
\hline 510 & 0.424 & 0.426 & 0.45 & 0.429 & 0.399 & 0.458 & 0.404 \\
\hline 520 & 0.413 & 0.412 & 0.432 & 0.412 & 0.382 & 0.444 & 0.381 \\
\hline 530 & 0.401 & 0.394 & 0.409 & 0.391 & 0.365 & 0.431 & 0.361 \\
\hline 540 & 0.354 & 0.343 & 0.359 & 0.341 & 0.315 & 0.38 & 0.317 \\
\hline 550 & 0.27 & 0.254 & 0.278 & 0.249 & 0.235 & 0.306 & 0.237 \\
\hline 560 & 0.183 & 0.154 & 0.18 & 0.149 & 0.159 & 0.22 & 0.152 \\
\hline 570 & 0.13 & 0.092 & 0.117 & 0.082 & 0.112 & 0.169 & 0.1 \\
\hline 580 & 0.103 & 0.059 & 0.083 & 0.051 & 0.088 & 0.147 & 0.07 \\
\hline 590 & 0.088 & 0.044 & 0.066 & 0.033 & 0.07 & 0.128 & 0.048 \\
\hline 600 & 0.078 & 0.035 & 0.057 & 0.018 & 0.057 & 0.12 & 0.033 \\
\hline 610 & 0.074 & 0.03 & 0.05 & 0.013 & 0.046 & 0.11 & 0.023 \\
\hline 620 & 0.076 & 0.03 & 0.05 & 0.015 & 0.047 & 0.112 & 0.021 \\
\hline 630 & 0.083 & 0.029 & 0.05 & 0.02 & 0.052 & 0.116 & 0.027 \\
\hline 640 & 0.091 & 0.03 & 0.051 & 0.027 & 0.06 & 0.114 & 0.03 \\
\hline 650 & 0.098 & 0.033 & 0.055 & 0.031 & 0.069 & 0.119 & 0.034 \\
\hline 660 & 0.102 & 0.035 & 0.054 & 0.026 & 0.075 & 0.127 & 0.035 \\
\hline 670 & 0.106 & 0.04 & 0.062 & 0.034 & 0.085 & 0.133 & 0.037 \\
\hline 680 & 0.107 & 0.046 & 0.06 & 0.038 & 0.091 & 0.137 & 0.039 \\
\hline 690 & 0.11 & 0.045 & 0.064 & 0.041 & 0.087 & 0.139 & 0.043 \\
\hline 700 & 0.115 & 0.052 & 0.068 & 0.042 & 0.091 & 0.142 & 0.05 \\
\hline 710 & 0.123 & 0.053 & 0.068 & 0.038 & 0.083 & 0.142 & 0.043 \\
\hline 720 & 0.123 & 0.047 & 0.072 & 0.033 & 0.072 & 0.141 & 0.039 \\
\hline 730 & 0.126 & 0.041 & 0.065 & 0.028 & 0.064 & 0.137 & 0.04 \\
\hline 740 & 0.128 & 0.03 & 0.062 & 0.01 & 0.056 & 0.138 & 0.036 \\
\hline 750 & 0.125 & 0.023 & 0.061 & 0.012 & 0.055 & 0.128 & 0.025 \\
\hline 760 & 0.124 & 0.015 & 0.056 & 0.022 & 0.057 & 0.129 & 0.035 \\
\hline 770 & 0.14 & 0.028 & 0.061 & 0.019 & 0.059 & 0.138 & 0.037 \\
\hline 780 & 0.132 & 0.033 & 0.059 & 0.034 & 0.07 & 0.138 & 0.04 \\
\hline
\end{tabular}

Absorbance values for Orange A_F for Run 1-7 for Phase IV

\begin{tabular}{|c|c|c|}
\hline Red Average of the Average Abs & Red UCL & Red LCL \\
\hline 0.2993375 & 0.400940446 & 0.197734554 \\
\hline 0.3058825 & 0.409800042 & 0.201964958 \\
\hline 0.319055 & 0.428327849 & 0.209782151 \\
\hline 0.33185 & 0.441741942 & 0.221958058 \\
\hline 0.3428425 & 0.455345483 & 0.230339517 \\
\hline 0.348465 & 0.460742009 & 0.236187991 \\
\hline 0.356185 & 0.470634624 & 0.241735376 \\
\hline
\end{tabular}




\begin{tabular}{|c|c|c|}
\hline 0.3759625 & 0.494593436 & 0.257331564 \\
\hline 0.40406 & 0.52869949 & 0.27942051 \\
\hline 0.4390125 & 0.568631823 & 0.309393177 \\
\hline 0.4782825 & 0.612138376 & 0.344426624 \\
\hline 0.5037425 & 0.639145559 & 0.368339441 \\
\hline 0.512925 & 0.648872187 & 0.376977813 \\
\hline 0.5245625 & 0.661223264 & 0.387901736 \\
\hline 0.5286625 & 0.664000229 & 0.393324771 \\
\hline 0.448715 & 0.636156259 & 0.369793741 \\
\hline 0.4075525 & 0.579954802 & 0.317475198 \\
\hline 0.38003 & 0.53510969 & 0.27999531 \\
\hline 0.3284225 & 0.500191459 & 0.259868541 \\
\hline 0.24693 & 0.438961113 & 0.217883887 \\
\hline 0.1746225 & 0.346474117 & 0.147385883 \\
\hline 0.135635 & 0.265217605 & 0.084027395 \\
\hline 0.1164025 & 0.215836395 & 0.055433605 \\
\hline 0.10829 & 0.188515797 & 0.044289203 \\
\hline 0.1044925 & 0.178828062 & 0.037751938 \\
\hline 0.1043775 & 0.175440285 & 0.033544715 \\
\hline 0.1034025 & 0.176486972 & 0.032268028 \\
\hline 0.10398 & 0.175636991 & 0.031168009 \\
\hline 0.1045975 & 0.175883781 & 0.032076219 \\
\hline 0.1036725 & 0.175798395 & 0.033396605 \\
\hline 0.102472778 & 0.173283249 & 0.034061751 \\
\hline 0.1031725 & 0.171995768 & 0.032949787 \\
\hline 0.1023625 & 0.170590159 & 0.035754841 \\
\hline 0.104205 & 0.16863006 & 0.03609494 \\
\hline 0.102675 & 0.1706924 & 0.0377176 \\
\hline 0.1024625 & 0.169988751 & 0.035361249 \\
\hline 0.1021125 & 0.171316424 & 0.033608576 \\
\hline 0.100933611 & 0.173039467 & 0.031185533 \\
\hline 0.0991175 & 0.172972469 & 0.028894753 \\
\hline 0.171751785 & 0.029533215 \\
\hline & 0.169912155 & 0.028322845 \\
\hline
\end{tabular}

The absorbance values, upper confidence bound and lower confidence bounds for all Red shirt for Phase IV 


\begin{tabular}{|c|c|c|c|c|c|c|}
\hline \multirow[b]{2}{*}{$\lambda$} & \multicolumn{2}{|c|}{ Average Absorbance } & UCL & $\mathbf{L C L}$ & UCL & LCL \\
\hline & I & $\mathrm{J}$ & \multicolumn{2}{|c|}{1} & \multicolumn{2}{|c|}{$\mathbf{J}$} \\
\hline 380 & 0.182325 & 0.1899 & 0.26127 & 0.10338 & 0.22767 & 0.15213 \\
\hline 390 & 0.1726 & 0.180175 & 0.25005 & 0.09515 & 0.2204 & 0.13995 \\
\hline 400 & 0.15915 & 0.1684 & 0.23452 & 0.08378 & 0.21387 & 0.12293 \\
\hline 410 & 0.14395 & 0.1527 & 0.2222 & 0.0657 & 0.20281 & 0.10259 \\
\hline 420 & 0.131825 & 0.138225 & 0.21095 & 0.0527 & 0.18768 & 0.08877 \\
\hline 430 & 0.12235 & 0.1245 & 0.20374 & 0.04096 & 0.17552 & 0.07348 \\
\hline 440 & 0.114575 & 0.1164 & 0.19518 & 0.03397 & 0.17072 & 0.06208 \\
\hline 450 & 0.11115 & 0.11265 & 0.18885 & 0.03345 & 0.16667 & 0.05863 \\
\hline 460 & 0.111125 & 0.113375 & 0.18907 & 0.03318 & 0.16907 & 0.05768 \\
\hline 470 & 0.11195 & 0.117975 & 0.19054 & 0.03336 & 0.17511 & 0.06084 \\
\hline 480 & 0.11525 & 0.12345 & 0.19062 & 0.03988 & 0.18238 & 0.06452 \\
\hline 490 & 0.1229 & 0.13115 & 0.19718 & 0.04862 & 0.18727 & 0.07503 \\
\hline 500 & 0.13425 & 0.141025 & 0.21026 & 0.05824 & 0.19328 & 0.08877 \\
\hline 510 & 0.144475 & 0.153825 & 0.22018 & 0.06877 & 0.20337 & 0.10428 \\
\hline 520 & 0.157 & 0.1685 & 0.23133 & 0.08267 & 0.21986 & 0.11714 \\
\hline 530 & 0.17405 & 0.18715 & 0.24805 & 0.10005 & 0.23884 & 0.13546 \\
\hline 540 & 0.193825 & 0.208525 & 0.26896 & 0.11869 & 0.26254 & 0.15451 \\
\hline 550 & 0.2135 & 0.231975 & 0.29161 & 0.13539 & 0.28619 & 0.17776 \\
\hline 560 & 0.232275 & 0.2527 & 0.31594 & 0.14861 & 0.30797 & 0.19743 \\
\hline 570 & 0.2533 & 0.27445 & 0.34472 & 0.16188 & 0.33332 & 0.21558 \\
\hline 580 & 0.276425 & 0.2961 & 0.37361 & 0.17924 & 0.35369 & 0.23851 \\
\hline 590 & 0.277975 & 0.2984 & 0.37508 & 0.18087 & 0.35614 & 0.24066 \\
\hline 600 & 0.296925 & 0.320525 & 0.40562 & 0.18823 & 0.37774 & 0.26331 \\
\hline 610 & 0.29055 & 0.31685 & 0.39946 & 0.18164 & 0.37593 & 0.25777 \\
\hline 620 & 0.286975 & 0.31425 & 0.39213 & 0.18182 & 0.37636 & 0.25214 \\
\hline 630 & 0.285725 & 0.314375 & 0.38795 & 0.1835 & 0.37918 & 0.24957 \\
\hline 640 & 0.282775 & 0.312675 & 0.38548 & 0.18007 & 0.3786 & 0.24675 \\
\hline 650 & 0.264675 & 0.292525 & 0.36275 & 0.1666 & 0.3588 & 0.22625 \\
\hline 660 & 0.230275 & 0.254825 & 0.32 & 0.14055 & 0.31931 & 0.19034 \\
\hline 670 & 0.189 & 0.2092 & 0.27465 & 0.10335 & 0.27418 & 0.14422 \\
\hline 680 & 0.147725 & 0.163775 & 0.23142 & 0.06403 & 0.22753 & 0.10002 \\
\hline 690 & 0.11775 & 0.129725 & 0.2035 & 0.032 & 0.19583 & 0.06362 \\
\hline 700 & 0.100975 & 0.10985 & 0.18674 & 0.01521 & 0.1767 & 0.043 \\
\hline 710 & 0.09185 & 0.098275 & 0.17313 & 0.01057 & 0.16357 & 0.03298 \\
\hline 720 & 0.0873 & 0.0948 & 0.16674 & 0.00786 & 0.15753 & 0.03207 \\
\hline 730 & 0.086175 & 0.091625 & 0.16263 & 0.00972 & 0.14899 & 0.03426 \\
\hline 740 & 0.08545 & 0.09375 & 0.15526 & 0.01564 & 0.14618 & 0.04132 \\
\hline
\end{tabular}




\begin{tabular}{|l|l|l|l|l|l|l|}
\hline 750 & 0.0836 & 0.093925 & 0.15247 & 0.01473 & 0.14146 & 0.04639 \\
\hline 760 & 0.0822 & 0.093275 & 0.15395 & 0.01045 & 0.13693 & 0.04962 \\
\hline 770 & 0.0821 & 0.092975 & 0.15469 & 0.00951 & 0.13831 & 0.04764 \\
\hline 780 & 0.0801 & 0.094725 & 0.15131 & 0.00889 & 0.14169 & 0.04776 \\
\hline
\end{tabular}

The absorbance values, upper confidence bound and lower confidence bounds for Royal shirt I and J for Phase IV

\begin{tabular}{|c|c|c|c|c|c|c|}
\hline Wavelength & $\begin{array}{c}\text { Gold FRONT } \\
\text { Average of the } \\
\text { Averages }\end{array}$ & UCL & LCL & $\begin{array}{c}\text { Gold BACK } \\
\text { Averages }\end{array}$ & UCL & LCL \\
\hline 380 & 0.22181 & 0.28228 & 0.16134 & 0.21754 & 0.26425 & 0.17083 \\
\hline 390 & 0.23148 & 0.28815 & 0.17481 & 0.22598 & 0.26992 & 0.18204 \\
\hline 400 & 0.24397 & 0.30889 & 0.17905 & 0.23706 & 0.28443 & 0.18969 \\
\hline 410 & 0.2565 & 0.32869 & 0.18431 & 0.24946 & 0.29784 & 0.20108 \\
\hline 420 & 0.26609 & 0.33806 & 0.19412 & 0.2605 & 0.30817 & 0.21283 \\
\hline 430 & 0.26983 & 0.34257 & 0.19709 & 0.2636 & 0.31121 & 0.21599 \\
\hline 440 & 0.26939 & 0.34013 & 0.19865 & 0.26202 & 0.30736 & 0.21668 \\
\hline 450 & 0.26067 & 0.32796 & 0.19338 & 0.25055 & 0.29311 & 0.20799 \\
\hline 460 & 0.24195 & 0.30411 & 0.17979 & 0.23267 & 0.26931 & 0.19603 \\
\hline 470 & 0.22322 & 0.28193 & 0.16451 & 0.21526 & 0.25342 & 0.1771 \\
\hline 480 & 0.20405 & 0.26218 & 0.14592 & 0.19543 & 0.23515 & 0.15571 \\
\hline 490 & 0.18303 & 0.2333 & 0.13276 & 0.17543 & 0.2113 & 0.13956 \\
\hline 500 & 0.16097 & 0.20294 & 0.119 & 0.15424 & 0.18623 & 0.12225 \\
\hline 510 & 0.14036 & 0.17596 & 0.10476 & 0.13477 & 0.1642 & 0.10534 \\
\hline 520 & 0.12271 & 0.15472 & 0.0907 & 0.11809 & 0.14798 & 0.0882 \\
\hline 530 & 0.1121 & 0.14488 & 0.07932 & 0.104 & 0.13122 & 0.07678 \\
\hline 540 & 0.10492 & 0.13559 & 0.07425 & 0.0963 & 0.12532 & 0.06728 \\
\hline 550 & 0.10205 & 0.12924 & 0.07486 & 0.09314 & 0.12294 & 0.06334 \\
\hline 560 & 0.0982 & 0.12273 & 0.07367 & 0.09129 & 0.1199 & 0.06268 \\
\hline 570 & 0.09587 & 0.11738 & 0.07436 & 0.09153 & 0.12014 & 0.06292 \\
\hline 580 & 0.09315 & 0.11178 & 0.07452 & 0.08964 & 0.11722 & 0.06206 \\
\hline 590 & 0.09213 & 0.11062 & 0.07364 & 0.08904 & 0.11744 & 0.06064 \\
\hline 600 & 0.09064 & 0.11157 & 0.06971 & 0.08801 & 0.11724 & 0.05878 \\
\hline 610 & 0.08798 & 0.10979 & 0.06617 & 0.0858 & 0.11482 & 0.05678 \\
\hline 620 & 0.08665 & 0.10827 & 0.06503 & 0.08465 & 0.11282 & 0.05648 \\
\hline 630 & 0.08519 & 0.10749 & 0.06289 & 0.08302 & 0.10982 & 0.05622 \\
\hline 640 & 0.08593 & 0.10997 & 0.06189 & 0.08159 & 0.10781 & 0.05537 \\
\hline 650 & 0.08594 & 0.11076 & 0.06112 & 0.07907 & 0.10611 & 0.05203 \\
\hline
\end{tabular}




\begin{tabular}{|c|c|c|c|c|c|c|}
\hline 660 & 0.08849 & 0.11405 & 0.06293 & 0.08026 & 0.1102 & 0.05032 \\
\hline 670 & 0.09108 & 0.11682 & 0.06534 & 0.08077 & 0.11095 & 0.05059 \\
\hline 680 & 0.09223 & 0.11751 & 0.06695 & 0.08039 & 0.10978 & 0.051 \\
\hline 690 & 0.0929 & 0.1161 & 0.0697 & 0.08093 & 0.1114 & 0.05046 \\
\hline 700 & 0.09339 & 0.11647 & 0.07031 & 0.08115 & 0.11307 & 0.04923 \\
\hline 710 & 0.09294 & 0.11729 & 0.06859 & 0.08107 & 0.11187 & 0.05027 \\
\hline 720 & 0.09495 & 0.12261 & 0.06729 & 0.08311 & 0.11589 & 0.05033 \\
\hline 730 & 0.09486 & 0.12519 & 0.06453 & 0.08431 & 0.11699 & 0.05163 \\
\hline 740 & 0.09547 & 0.12779 & 0.06315 & 0.08472 & 0.11777 & 0.05167 \\
\hline 750 & 0.0969 & 0.13306 & 0.06074 & 0.08664 & 0.11936 & 0.05392 \\
\hline 760 & 0.09281 & 0.12819 & 0.05743 & 0.08442 & 0.11532 & 0.05352 \\
\hline 770 & 0.08998 & 0.12519 & 0.05477 & 0.0836 & 0.11641 & 0.05079 \\
\hline 780 & 0.08462 & 0.11686 & 0.05238 & 0.08258 & 0.11276 & 0.0524 \\
\hline
\end{tabular}

The absorbance values, upper confidence bound and lower confidence bounds of Gold Front and Gold Back for Phase IV

\begin{tabular}{|c|c|c|c|c|c|}
\hline \multicolumn{7}{|c|}{ Kelly Green } \\
\hline $\begin{array}{c}\text { Front- } \\
\text { Back }\end{array}$ & $\begin{array}{c}\text { Front- } \\
\text { Sleeve }\end{array}$ & $\begin{array}{c}\text { Front- } \\
\text { Armpit }\end{array}$ & $\begin{array}{c}\text { Back- } \\
\text { Sleeve }\end{array}$ & $\begin{array}{c}\text { Back- } \\
\text { Armpit }\end{array}$ & $\begin{array}{c}\text { Sleeve- } \\
\text { Armpit }\end{array}$ \\
\hline FALSE & FALSE & FALSE & FALSE & FALSE & FALSE \\
\hline FALSE & FALSE & FALSE & FALSE & FALSE & FALSE \\
\hline FALSE & FALSE & FALSE & FALSE & FALSE & FALSE \\
\hline FALSE & FALSE & FALSE & FALSE & FALSE & FALSE \\
\hline FALSE & FALSE & FALSE & FALSE & FALSE & FALSE \\
\hline FALSE & FALSE & FALSE & FALSE & FALSE & FALSE \\
\hline FALSE & FALSE & FALSE & FALSE & FALSE & FALSE \\
\hline FALSE & FALSE & FALSE & FALSE & FALSE & FALSE \\
\hline FALSE & FALSE & FALSE & FALSE & FALSE & FALSE \\
\hline FALSE & FALSE & FALSE & FALSE & FALSE & FALSE \\
\hline FALSE & FALSE & FALSE & FALSE & FALSE & FALSE \\
\hline FALSE & FALSE & FALSE & FALSE & FALSE & FALSE \\
\hline FALSE & FALSE & FALSE & FALSE & FALSE & FALSE \\
\hline FALSE & FALSE & FALSE & FALSE & FALSE & FALSE \\
\hline FALSE & FALSE & FALSE & FALSE & FALSE & FALSE \\
\hline FALSE & FALSE & FALSE & FALSE & FALSE & FALSE \\
\hline FALSE & FALSE & FALSE & FALSE & FALSE & FALSE \\
\hline FALSE & FALSE & FALSE & FALSE & FALSE & FALSE \\
\hline FALSE & FALSE & FALSE & FALSE & FALSE & FALSE \\
\hline FALSE & FALSE & FALSE & FALSE & FALSE & FALSE \\
\hline FALSE & FALSE & FALSE & FALSE & FALSE & FALSE \\
\hline
\end{tabular}




\begin{tabular}{|c|c|c|c|c|c|}
\hline FALSE & FALSE & FALSE & FALSE & FALSE & FALSE \\
\hline FALSE & FALSE & FALSE & FALSE & FALSE & FALSE \\
\hline FALSE & FALSE & FALSE & FALSE & FALSE & FALSE \\
\hline FALSE & FALSE & FALSE & FALSE & FALSE & FALSE \\
\hline FALSE & FALSE & FALSE & FALSE & FALSE & FALSE \\
\hline FALSE & FALSE & FALSE & FALSE & FALSE & FALSE \\
\hline FALSE & FALSE & FALSE & FALSE & FALSE & FALSE \\
\hline FALSE & FALSE & FALSE & FALSE & FALSE & FALSE \\
\hline FALSE & FALSE & FALSE & FALSE & FALSE & FALSE \\
\hline FALSE & FALSE & FALSE & FALSE & FALSE & FALSE \\
\hline FALSE & FALSE & FALSE & FALSE & FALSE & FALSE \\
\hline FALSE & FALSE & FALSE & FALSE & FALSE & FALSE \\
\hline FALSE & FALSE & FALSE & FALSE & FALSE & FALSE \\
\hline FALSE & FALSE & FALSE & FALSE & FALSE & FALSE \\
\hline FALSE & FALSE & FALSE & FALSE & FALSE & FALSE \\
\hline FALSE & FALSE & FALSE & FALSE & FALSE & FALSE \\
\hline FALSE & FALSE & FALSE & FALSE & FALSE & FALSE \\
\hline FALSE & FALSE & FALSE & FALSE & FALSE & FALSE \\
\hline FALSE & FALSE & FALSE & FALSE & FALSE & FALSE \\
\hline FALSE & FALSE & FALSE & FALSE & FALSE & FALSE \\
\hline
\end{tabular}

The OR function results for the six comparisons of the Kelly Green shirts

\begin{tabular}{|c|c|c|c|c|c|c|c|}
\hline Wavelength & $\mathbf{1}$ & $\mathbf{2}$ & $\mathbf{3}$ & $\mathbf{4}$ & $\mathbf{5}$ & $\mathbf{6}$ & $\mathbf{7}$ \\
\hline 380 & 0.204 & 0.183 & 0.248 & 0.209 & 0.212 & 0.187 & 0.173 \\
\hline 390 & 0.220 & 0.197 & 0.249 & 0.227 & 0.221 & 0.199 & 0.182 \\
\hline 400 & 0.233 & 0.211 & 0.261 & 0.234 & 0.242 & 0.222 & 0.187 \\
\hline 410 & 0.249 & 0.224 & 0.279 & 0.247 & 0.255 & 0.231 & 0.207 \\
\hline 420 & 0.248 & 0.235 & 0.294 & 0.258 & 0.261 & 0.244 & 0.211 \\
\hline 430 & 0.261 & 0.239 & 0.306 & 0.255 & 0.261 & 0.248 & 0.221 \\
\hline 440 & 0.267 & 0.250 & 0.313 & 0.266 & 0.273 & 0.260 & 0.233 \\
\hline 450 & 0.282 & 0.265 & 0.331 & 0.280 & 0.289 & 0.279 & 0.253 \\
\hline 460 & 0.312 & 0.290 & 0.359 & 0.313 & 0.309 & 0.297 & 0.271 \\
\hline 470 & 0.349 & 0.326 & 0.405 & 0.349 & 0.345 & 0.332 & 0.313 \\
\hline 480 & 0.392 & 0.367 & 0.445 & 0.394 & 0.388 & 0.370 & 0.359 \\
\hline 490 & 0.424 & 0.395 & 0.473 & 0.425 & 0.416 & 0.392 & 0.387 \\
\hline 500 & 0.438 & 0.404 & 0.484 & 0.435 & 0.423 & 0.401 & 0.405 \\
\hline 510 & 0.454 & 0.424 & 0.498 & 0.458 & 0.437 & 0.414 & 0.416 \\
\hline 520 & 0.460 & 0.435 & 0.511 & 0.473 & 0.448 & 0.415 & 0.416 \\
\hline 530 & 0.426 & 0.401 & 0.485 & 0.444 & 0.418 & 0.390 & 0.381 \\
\hline
\end{tabular}




\begin{tabular}{|c|c|c|c|c|c|c|c|}
\hline 540 & 0.369 & 0.336 & 0.415 & 0.374 & 0.347 & 0.335 & 0.322 \\
\hline 550 & 0.325 & 0.284 & 0.363 & 0.323 & 0.294 & 0.291 & 0.280 \\
\hline 560 & 0.299 & 0.255 & 0.332 & 0.287 & 0.259 & 0.263 & 0.254 \\
\hline 570 & 0.265 & 0.211 & 0.280 & 0.244 & 0.213 & 0.217 & 0.209 \\
\hline 580 & 0.210 & 0.149 & 0.195 & 0.169 & 0.144 & 0.137 & 0.134 \\
\hline 590 & 0.169 & 0.095 & 0.124 & 0.104 & 0.080 & 0.071 & 0.073 \\
\hline 600 & 0.153 & 0.068 & 0.094 & 0.076 & 0.056 & 0.041 & 0.044 \\
\hline 610 & 0.145 & 0.058 & 0.074 & 0.055 & 0.046 & 0.033 & 0.034 \\
\hline 620 & 0.143 & 0.051 & 0.067 & 0.047 & 0.043 & 0.034 & 0.038 \\
\hline 630 & 0.147 & 0.050 & 0.068 & 0.042 & 0.040 & 0.034 & 0.040 \\
\hline 640 & 0.151 & 0.049 & 0.071 & 0.040 & 0.041 & 0.036 & 0.040 \\
\hline 650 & 0.155 & 0.050 & 0.075 & 0.037 & 0.040 & 0.043 & 0.044 \\
\hline 660 & 0.156 & 0.041 & 0.076 & 0.030 & 0.034 & 0.044 & 0.048 \\
\hline 670 & 0.157 & 0.036 & 0.075 & 0.028 & 0.030 & 0.045 & 0.052 \\
\hline 680 & 0.159 & 0.031 & 0.068 & 0.022 & 0.028 & 0.043 & 0.054 \\
\hline 690 & 0.155 & 0.026 & 0.064 & 0.018 & 0.027 & 0.034 & 0.042 \\
\hline 700 & 0.154 & 0.025 & 0.065 & 0.017 & 0.030 & 0.029 & 0.041 \\
\hline 710 & 0.160 & 0.033 & 0.062 & 0.019 & 0.036 & 0.027 & 0.041 \\
\hline 720 & 0.154 & 0.031 & 0.061 & 0.022 & 0.041 & 0.027 & 0.031 \\
\hline 730 & 0.151 & 0.031 & 0.058 & 0.026 & 0.039 & 0.024 & 0.030 \\
\hline 740 & 0.148 & 0.023 & 0.060 & 0.025 & 0.033 & 0.033 & 0.029 \\
\hline 750 & 0.141 & 0.020 & 0.059 & 0.023 & 0.033 & 0.040 & 0.032 \\
\hline 760 & 0.140 & 0.018 & 0.058 & 0.023 & 0.029 & 0.033 & 0.018 \\
\hline 770 & 0.143 & 0.012 & 0.048 & 0.014 & 0.032 & 0.022 & 0.005 \\
\hline 780 & 0.154 & 0.012 & 0.048 & 0.007 & 0.023 & 0.031 & 0.013 \\
\hline
\end{tabular}

Absorbance values for Red Section 1 for Run 1-7 for Phase $V$

\begin{tabular}{|c|c|c|}
\hline Gold Average of Average Abs & Gold UCL & Gold LCL \\
\hline 0.244373333 & 0.299183094 & 0.189563573 \\
\hline 0.256193333 & 0.314714871 & 0.197671796 \\
\hline 0.269246667 & 0.330829199 & 0.207664135 \\
\hline 0.281916667 & 0.347727193 & 0.21610614 \\
\hline 0.292343333 & 0.362017684 & 0.222668983 \\
\hline 0.296976667 & 0.368809322 & 0.225144012 \\
\hline 0.295653333 & 0.367528336 & 0.22377833 \\
\hline 0.287303333 & 0.357157972 & 0.217448695 \\
\hline 0.271543333 & 0.337902711 & 0.205183956 \\
\hline 0.253856667 & 0.315822917 & 0.191890417 \\
\hline
\end{tabular}




\begin{tabular}{|c|c|c|}
\hline 0.23168 & 0.287412458 & 0.175947542 \\
\hline 0.210113333 & 0.259974007 & 0.16025266 \\
\hline 0.186366667 & 0.229313986 & 0.143419347 \\
\hline 0.16445 & 0.20103675 & 0.12786325 \\
\hline 0.144966667 & 0.177267108 & 0.112666225 \\
\hline 0.131533333 & 0.161355569 & 0.101711098 \\
\hline 0.121983333 & 0.150407182 & 0.093559484 \\
\hline 0.111476667 & 0.143221797 & 0.088018203 \\
\hline 0.108553333 & 0.138875973 & 0.08407736 \\
\hline 0.106576667 & 0.135518965 & 0.081587702 \\
\hline 0.104446667 & 0.133359966 & 0.079793368 \\
\hline 0.102526667 & 0.131467704 & 0.07742563 \\
\hline 0.09995 & 0.129698825 & 0.075354508 \\
\hline 0.09944 & 0.126509253 & 0.073390747 \\
\hline 0.098863333 & 0.126339815 & 0.072540185 \\
\hline 0.098386667 & 0.126228282 & 0.071498385 \\
\hline 0.09767 & 0.125550283 & 0.071223051 \\
\hline 0.09522 & 0.124710954 & 0.070629046 \\
\hline 0.09543 & 0.12286582 & 0.06757418 \\
\hline 0.094916667 & 0.123395527 & 0.067464473 \\
\hline 0.093076667 & 0.122794943 & 0.06703839 \\
\hline 0.090996667 & 0.12127957 & 0.064873763 \\
\hline 0.090136667 & 0.119326938 & 0.062666396 \\
\hline 0.090086667 & 0.118962042 & 0.061311291 \\
\hline 0.091482963 & 0.118480563 & 0.06169277 \\
\hline 0.090226667 & 0.119660906 & 0.063305019 \\
\hline 0.089183333 & 0.118913634 & 0.0615397 \\
\hline 0.087776667 & 0.117621999 & 0.060744668 \\
\hline 0.086176667 & 0.117322188 & 0.058231145 \\
\hline 0.085233333 & 0.114028677 & 0.056437989 \\
\hline
\end{tabular}

The absorbance values, upper confidence bound and lower confidence bounds for all front squares of the Gold Shirts for Phase V 


\begin{tabular}{|c|c|c|c|c|c|c|}
\hline Wavelength & \multicolumn{2}{|c|}{ Average of Average Abs } & UCL & LCL & UCL & LCL \\
\hline & $\mathrm{G}$ & $\mathrm{H}$ & \multicolumn{2}{|c|}{ G } & \multicolumn{2}{|c|}{$\mathrm{H}$} \\
\hline 380 & 0.271233333 & 0.249733333 & 0.322993 & 0.219474 & 0.30551 & 0.19395 \\
\hline 390 & 0.2527 & 0.226533333 & 0.286615 & 0.218785 & 0.27577 & 0.1773 \\
\hline 400 & 0.2478 & 0.2146 & 0.28195 & 0.21365 & 0.27353 & 0.15567 \\
\hline 410 & 0.256833333 & 0.213666667 & 0.294135 & 0.219531 & 0.2763 & 0.15103 \\
\hline 420 & 0.265433333 & 0.218566667 & 0.297949 & 0.232918 & 0.27882 & 0.15832 \\
\hline 430 & 0.265233333 & 0.2144 & 0.299624 & 0.230843 & 0.27484 & 0.15396 \\
\hline 440 & 0.254933333 & 0.205833333 & 0.298504 & 0.211363 & 0.26586 & 0.1458 \\
\hline 450 & 0.242366667 & 0.194433333 & 0.295782 & 0.188951 & 0.24696 & 0.1419 \\
\hline 460 & 0.219133333 & 0.174533333 & 0.274419 & 0.163847 & 0.2161 & 0.13297 \\
\hline 470 & 0.194233333 & 0.148366667 & 0.256403 & 0.132064 & 0.18005 & 0.11668 \\
\hline 480 & 0.171366667 & 0.124666667 & 0.233157 & 0.109576 & 0.14442 & 0.10491 \\
\hline 490 & 0.158766667 & 0.1136 & 0.222017 & 0.095516 & 0.12875 & 0.09845 \\
\hline 500 & 0.1523 & 0.111766667 & 0.214632 & 0.089968 & 0.12696 & 0.09657 \\
\hline 510 & 0.149433333 & 0.111633333 & 0.205872 & 0.092994 & 0.12851 & 0.09476 \\
\hline 520 & 0.150033333 & 0.112066667 & 0.199313 & 0.100754 & 0.1274 & 0.09673 \\
\hline 530 & 0.154166667 & 0.116833333 & 0.197248 & 0.111086 & 0.13058 & 0.10309 \\
\hline 540 & 0.160633333 & 0.121266667 & 0.203875 & 0.117391 & 0.12857 & 0.11396 \\
\hline 550 & 0.175166667 & 0.127633333 & 0.222056 & 0.128277 & 0.13313 & 0.12214 \\
\hline 560 & 0.192566667 & 0.140233333 & 0.237469 & 0.147664 & 0.15665 & 0.12382 \\
\hline 570 & 0.2167 & 0.160933333 & 0.265812 & 0.167588 & 0.1956 & 0.12627 \\
\hline 580 & 0.240266667 & 0.1828 & 0.301201 & 0.179332 & 0.23479 & 0.13081 \\
\hline 590 & 0.2699 & 0.211633333 & 0.345587 & 0.194213 & 0.28116 & 0.14211 \\
\hline 600 & 0.3155 & 0.2535 & 0.405134 & 0.225866 & 0.35142 & 0.15558 \\
\hline 610 & 0.350466667 & 0.286433333 & 0.450856 & 0.250077 & 0.40304 & 0.16982 \\
\hline 620 & 0.359833333 & 0.299166667 & 0.464598 & 0.255068 & 0.42042 & 0.17791 \\
\hline 630 & 0.367066667 & 0.307133333 & 0.474798 & 0.259335 & 0.42772 & 0.18655 \\
\hline 640 & 0.3643 & 0.3076 & 0.465334 & 0.263266 & 0.42507 & 0.19013 \\
\hline 650 & 0.374166667 & 0.3195 & 0.469227 & 0.279107 & 0.43957 & 0.19943 \\
\hline 660 & 0.4373 & 0.384133333 & 0.533375 & 0.341225 & 0.51927 & 0.24899 \\
\hline 670 & 0.5072 & 0.4593 & 0.59585 & 0.41855 & 0.60294 & 0.31566 \\
\hline 680 & 0.517866667 & 0.471633333 & 0.607542 & 0.428192 & 0.6192 & 0.32407 \\
\hline 690 & 0.456666667 & 0.402933333 & 0.550966 & 0.362368 & 0.53326 & 0.27261 \\
\hline 700 & 0.326766667 & 0.2737 & 0.406404 & 0.24713 & 0.37274 & 0.17466 \\
\hline 710 & 0.2273 & 0.178966667 & 0.274626 & 0.179974 & 0.24907 & 0.10886 \\
\hline
\end{tabular}




\begin{tabular}{|c|c|c|c|c|c|c|}
\hline 720 & 0.179366667 & 0.136333333 & 0.210625 & 0.148108 & 0.18581 & 0.08686 \\
\hline 730 & 0.1536 & 0.109466667 & 0.186544 & 0.120656 & 0.14782 & 0.07111 \\
\hline 740 & 0.140266667 & 0.093566667 & 0.17733 & 0.103203 & 0.12559 & 0.06154 \\
\hline 750 & 0.137733333 & 0.087833333 & 0.179611 & 0.095856 & 0.11597 & 0.0597 \\
\hline 760 & 0.129066667 & 0.078666667 & 0.169441 & 0.088692 & 0.10928 & 0.04806 \\
\hline 770 & 0.129933333 & 0.071366667 & 0.163768 & 0.096098 & 0.09745 & 0.04529 \\
\hline 780 & 0.126033333 & 0.066133333 & 0.151751 & 0.100315 & 0.09521 & 0.03705 \\
\hline
\end{tabular}

The absorbance values, upper confidence bound and lower confidence bounds for the front of Kelly Green $G$ and $H$ for Phase V

\begin{tabular}{|c|c|c|c|c|c|c|}
\hline & Gold Section 2 & & & $\begin{array}{c}\text { Kelly Green Section } \\
\mathbf{2}\end{array}$ & & \\
\hline Wavelength & $\begin{array}{c}\text { Average of Average } \\
\text { Abs }\end{array}$ & UCL & LCL & $\begin{array}{c}\text { Average of Average } \\
\text { Abs }\end{array}$ & UCL & LCL \\
\hline 380 & 0.24514 & 0.29879 & 0.19149 & 0.2697 & 0.36624 & 0.17316 \\
\hline 390 & 0.25755 & 0.31376 & 0.20134 & 0.24064 & 0.31848 & 0.1628 \\
\hline 400 & 0.27054 & 0.33011 & 0.21097 & 0.22986 & 0.30357 & 0.15615 \\
\hline 410 & 0.28376 & 0.34674 & 0.22078 & 0.23299 & 0.30913 & 0.15685 \\
\hline 420 & 0.29469 & 0.36184 & 0.22754 & 0.23819 & 0.31415 & 0.16223 \\
\hline 430 & 0.29936 & 0.36699 & 0.23173 & 0.23591 & 0.31338 & 0.15844 \\
\hline 440 & 0.29813 & 0.36723 & 0.22903 & 0.22552 & 0.30068 & 0.15036 \\
\hline 450 & 0.29011 & 0.35774 & 0.22248 & 0.20971 & 0.27755 & 0.14187 \\
\hline 460 & 0.2743 & 0.34042 & 0.20818 & 0.18551 & 0.24322 & 0.1278 \\
\hline 470 & 0.25664 & 0.31995 & 0.19333 & 0.15743 & 0.20602 & 0.10884 \\
\hline 480 & 0.23426 & 0.29268 & 0.17584 & 0.13037 & 0.17136 & 0.08938 \\
\hline 490 & 0.2125 & 0.26583 & 0.15917 & 0.11374 & 0.15237 & 0.07511 \\
\hline 500 & 0.1883 & 0.23548 & 0.14112 & 0.1056 & 0.14681 & 0.06439 \\
\hline 510 & 0.16588 & 0.20729 & 0.12447 & 0.10271 & 0.14673 & 0.05869 \\
\hline 520 & 0.14613 & 0.18393 & 0.10833 & 0.10247 & 0.14977 & 0.05517 \\
\hline 530 & 0.13246 & 0.16728 & 0.09764 & 0.10608 & 0.15603 & 0.05613 \\
\hline 540 & 0.12276 & 0.15632 & 0.0892 & 0.11318 & 0.1628 & 0.06356 \\
\hline 550 & 0.11629 & 0.14819 & 0.08439 & 0.12721 & 0.17703 & 0.07739 \\
\hline 560 & 0.11223 & 0.14383 & 0.08063 & 0.14922 & 0.20585 & 0.09259 \\
\hline 570 & 0.10933 & 0.14052 & 0.07814 & 0.17805 & 0.25203 & 0.10407 \\
\hline 580 & 0.10778 & 0.13799 & 0.07757 & 0.20761 & 0.3016 & 0.11362 \\
\hline 590 & 0.10587 & 0.13597 & 0.07577 & 0.24327 & 0.35979 & 0.12675 \\
\hline 600 & 0.10395 & 0.13388 & 0.07402 & 0.29506 & 0.43999 & 0.15013 \\
\hline 610 & 0.10144 & 0.13004 & 0.07284 & 0.3341 & 0.50161 & 0.16659 \\
\hline & & & & & & \\
\hline
\end{tabular}




\begin{tabular}{|c|c|c|c|c|c|c|}
\hline 620 & 0.10102 & 0.12993 & 0.07211 & 0.34655 & 0.52083 & 0.17227 \\
\hline 630 & 0.10044 & 0.13049 & 0.07039 & 0.35316 & 0.52756 & 0.17876 \\
\hline 640 & 0.10001 & 0.12992 & 0.0701 & 0.35313 & 0.52279 & 0.18347 \\
\hline 650 & 0.099 & 0.12824 & 0.06976 & 0.36453 & 0.53095 & 0.19811 \\
\hline 660 & 0.09652 & 0.12656 & 0.06648 & 0.42731 & 0.59705 & 0.25757 \\
\hline 670 & 0.09695 & 0.1274 & 0.0665 & 0.4981 & 0.66641 & 0.32979 \\
\hline 680 & 0.09657 & 0.12549 & 0.06765 & 0.50916 & 0.67619 & 0.34213 \\
\hline 690 & 0.09518 & 0.12446 & 0.0659 & 0.44464 & 0.61785 & 0.27143 \\
\hline 700 & 0.09302 & 0.12255 & 0.06349 & 0.31315 & 0.45887 & 0.16743 \\
\hline 710 & 0.09213 & 0.12134 & 0.06292 & 0.20781 & 0.30129 & 0.11433 \\
\hline 720 & 0.09204 & 0.12192 & 0.06216 & 0.15556 & 0.2163 & 0.09482 \\
\hline 730 & 0.09361 & 0.12138 & 0.06584 & 0.1241 & 0.1695 & 0.0787 \\
\hline 740 & 0.09261 & 0.1205 & 0.06472 & 0.10703 & 0.14963 & 0.06443 \\
\hline 750 & 0.0916 & 0.11923 & 0.06397 & 0.09808 & 0.14039 & 0.05577 \\
\hline 760 & 0.09027 & 0.11948 & 0.06106 & 0.08976 & 0.13231 & 0.04721 \\
\hline 770 & 0.08905 & 0.11767 & 0.06043 & 0.08344 & 0.1283 & 0.03858 \\
\hline 780 & 0.08763 & 0.11608 & 0.05918 & 0.07978 & 0.12609 & 0.03347 \\
\hline
\end{tabular}

The absorbance values, upper confidence bound and lower confidence bounds of Section 2 for Gold and Kelly Green

\begin{tabular}{|c|c|c|}
\hline \multicolumn{3}{|c|}{ Red } \\
\hline $\begin{array}{l}\text { Section 1- } \\
\text { Section 2 }\end{array}$ & $\begin{array}{l}\text { Section 1- } \\
\text { Section 3 }\end{array}$ & $\begin{array}{l}\text { Section 2 - } \\
\text { Section 3 }\end{array}$ \\
\hline FALSE & FALSE & FALSE \\
\hline FALSE & FALSE & FALSE \\
\hline FALSE & FALSE & FALSE \\
\hline FALSE & FALSE & FALSE \\
\hline FALSE & FALSE & FALSE \\
\hline FALSE & FALSE & FALSE \\
\hline FALSE & FALSE & FALSE \\
\hline FALSE & FALSE & FALSE \\
\hline FALSE & FALSE & FALSE \\
\hline FALSE & FALSE & FALSE \\
\hline FALSE & FALSE & FALSE \\
\hline FALSE & FALSE & FALSE \\
\hline FALSE & FALSE & FALSE \\
\hline
\end{tabular}




\begin{tabular}{|l|l|l|}
\hline FALSE & FALSE & FALSE \\
\hline FALSE & FALSE & FALSE \\
\hline FALSE & FALSE & FALSE \\
\hline FALSE & FALSE & FALSE \\
\hline FALSE & FALSE & FALSE \\
\hline FALSE & FALSE & FALSE \\
\hline FALSE & FALSE & FALSE \\
\hline FALSE & FALSE & FALSE \\
\hline FALSE & FALSE & FALSE \\
\hline FALSE & FALSE & FALSE \\
\hline FALSE & FALSE & FALSE \\
\hline FALSE & FALSE & FALSE \\
\hline FALSE & FALSE & FALSE \\
\hline FALSE & FALSE & FALSE \\
\hline FALSE & FALSE & FALSE \\
\hline FALSE & FALSE & FALSE \\
\hline FALSE & FALSE & FALSE \\
\hline FALSE & FALSE & FALSE \\
\hline FALSE & FALSE & FALSE \\
\hline FALSE & FALSE & FALSE \\
\hline FALSE & FALSE & FALSE \\
\hline FALSE & FALSE & FALSE \\
\hline FALSE & FALSE & FALSE \\
\hline FALSE & FALSE & FALSE \\
\hline FALSE & FALSE & FALSE \\
\hline FALSE & FALSE & FALSE \\
\hline FALSE & FALSE & FALSE \\
\hline FALSE & FALSE & FALSE \\
\hline
\end{tabular}

The OR functions results for the three comparisons of the Red shirts 
